\title{
Theoretical Studies on the Excited-State Decay Mechanism of Homomenthyl Salicylate in a Gas Phase and an Acetonitrile Solution
}

\author{
Xue-Ping Chang ${ }^{1 *}$, Teng-Shuo Zhang ${ }^{3}$, and Ganglong Cui²
}

1 College of Chemistry and Chemical Engineering, Xinyang Normal University, Xinyang 464000, P. R. China

2 Key Laboratory of Theoretical and Computational Photochemistry, Ministry of Education, Chemistry College,

Beijing Normal University, Beijing 100875, P. R. China

3 China College of Chemical Engineering, Zhejiang University of Technology, Hangzhou 310014, P. R. China

\section{E-mail: xuepingchang@xynu.edu.cn}

\section{Contents}

1. Active Orbitals

2. QM(CASSCF)/MM Optimized So Minimum Structures of Different Snapshots

3. Vertical Excitation Energies of Different Snapshots

4. Bond Lengths of Minima and Intersection Structures

5. Spin Orbit Couplings

6. MEPTs and Isomerization Path

7. CASPT2 and QM(CASPT2)/MM Computed LIICs

8. CASPT2 Computed Plausible Relaxation Pathways of HMS

9. Inclusion the Menthone into the MM Region by means of a Link-Atom Scheme

10. Cartesian Coordinates of All Optimized Structures and LIIC Structures 


\section{Active Orbitals}

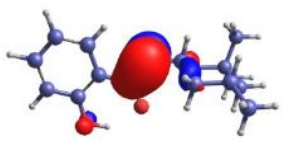

65 [2.00]

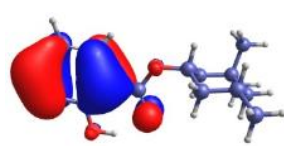

70 [1.93]

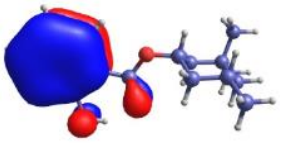

66 [1.99]

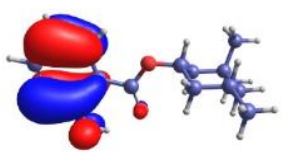

71 [1.94]

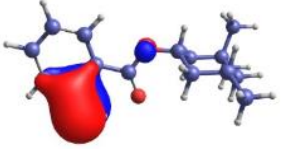

67 [2.00]

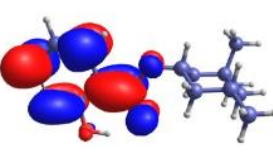

$72[0.08]$

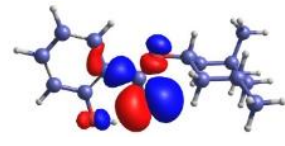

68 [2.00]

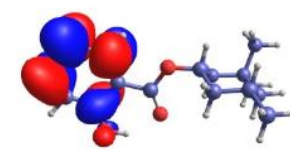

$73[0.06]$

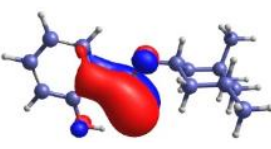

69 [1.96]

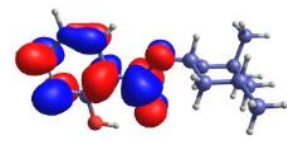

74 [0.04]

Figure S1. Active space used in CASSCF and CASPT2 computations of HMS molecule in gas phase in this work. The occupation numbers of active-space orbitals are calculated at the Franck-Condon point and state-averaged.

\section{QM(CASSCF)/MM Optimized S 0 Minimum Structures of Different Snapshots}

Snapshot1

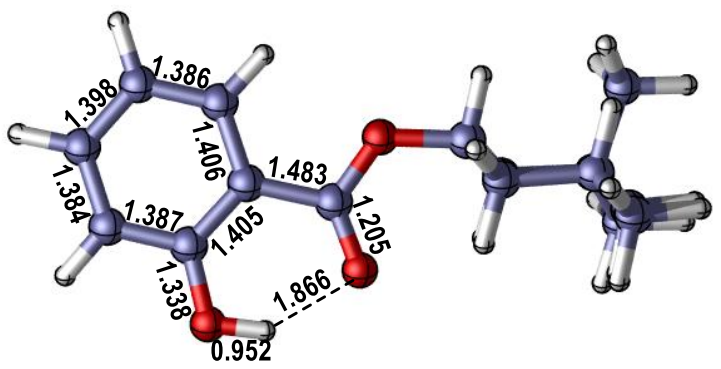

Snapshot3

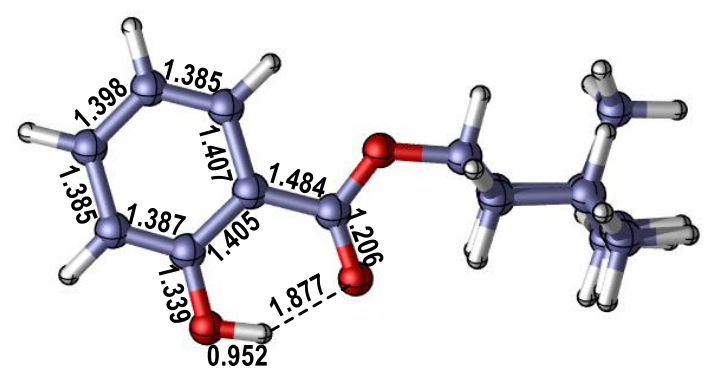

Snapshot2

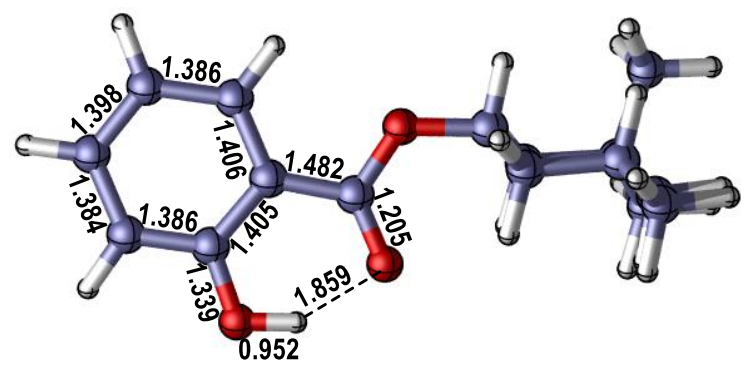

Snapshot4

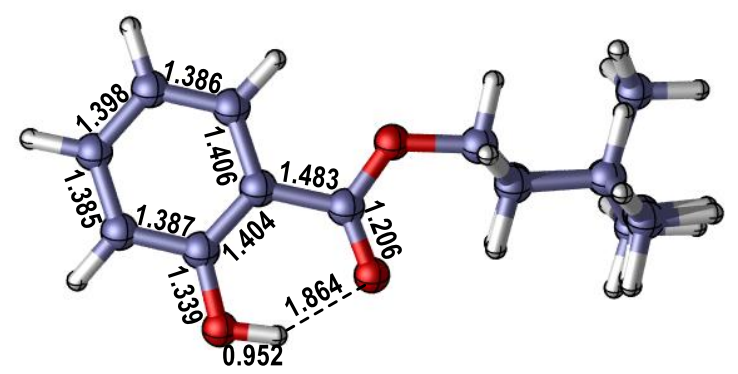

Figure S2. QM(CASSCF)/MM optimized $S_{0}$ minimum structures of different snapshots in acetonitrile solution. 


\section{Vertical Excitation Energies of Different Snapshots}

Table S1: QM(CASPT2)/MM computed vertical excitation energies (in $\mathrm{kcal} / \mathrm{mol}[\mathrm{eV}]$ ) to the lowest three singlet and triplet excited states of different snapshots

\begin{tabular}{|c|c|c|c|c|}
\hline & snapshot1 & snapshot2 & snapshot3 & snapshot4 \\
\hline $\mathrm{S}_{0} \rightarrow \mathrm{S}_{1}$ & $93.8[4.07]$ & $93.8[4.07]$ & $93.9[4.07]$ & $93.8[4.07]$ \\
\hline $\mathrm{S}_{0} \rightarrow \mathrm{T}_{1}$ & $84.5[3.66]$ & $85.1[3.69]$ & $85.0[3.69]$ & $84.8[3.68]$ \\
\hline $\mathrm{S}_{0} \rightarrow \mathrm{T}_{2}$ & $88.6[3.84]$ & $90.2[3.91]$ & $90.1[3.91]$ & $88.5[3.84]$ \\
\hline
\end{tabular}

\section{Bond Lengths of Minima and Intersection Structures}

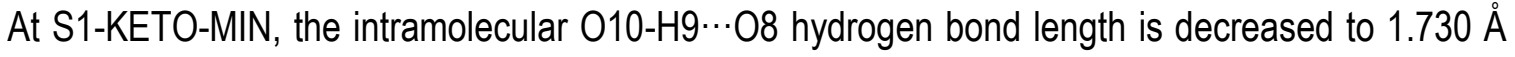
from $1.867 \AA$ in S0-KETO-MIN in gas phase. The $\mathrm{C} 2-\mathrm{C} 3, \mathrm{C} 4-\mathrm{C} 5$, and $\mathrm{C} 6-\mathrm{C} 1$ bond lengths are elongated, that is, $1.429,1.456$, and $1.454 \AA$ in S1-KETO-MIN versus $1.385,1.386$, and $1.405 \AA$ in SO-KETO-MIN in gas phase, while the C6-C7 and C1-010 bond lengths are shortened (1.433 and $1.303 \AA$ vs. 1.484 and $1.339 \AA$ ). For comparison, the intramolecular 010-H $\cdots 08$ hydrogen bond lengths of T1-KETO-MIN in gas phase is little decreased to $1.860 \AA$ from $1.867 \AA$ in SO-KETO-MIN, which would not effectively facilitate the following $T_{1}$ ESIPT. Other obvious bond length changes are elongation of $\mathrm{C} 2-\mathrm{C} 3, \mathrm{C} 5-\mathrm{C} 6$, and $\mathrm{C} 6-\mathrm{C} 1$ bonds and shortening of the $\mathrm{C} 1-\mathrm{C} 2, \mathrm{C} 4-\mathrm{C} 5$, and $\mathrm{C} 6-\mathrm{C} 7$ bonds in both environments (see Figure $5 \mathrm{~b}$ and Tables S2-S3). In T2-KETO-MIN, the whole phenyl has a little expansion with the elongation of the contained six $\mathrm{C}-\mathrm{C}$ bonds in different environments. For example, the $\mathrm{C} 1-\mathrm{C} 2 / \mathrm{C} 5-\mathrm{C} 6 / \mathrm{C} 6-\mathrm{C} 1$ bond lengths are increased to 1.431/1.441/1.446 $\AA$ in T2KETO-MIN from 1.387/1.406/1.405 $\AA$ in SO-KETO-MIN $\AA$ in gas phase. However, beyond those bonds of T2-KETO-MIN, the variations of remain $\mathrm{C} 1-010, \mathrm{C} 6-\mathrm{C} 7, \mathrm{C} 7-08, \mathrm{O} 8 \cdots \mathrm{H} 9$, and $\mathrm{H} 9-010$ bonds are very similar to counterparts of S1-KETO-MIN (see panels a and $\mathbf{c}$ in Figure 5). At the CASPT2 level, the adiabatic excitation energies of S1-KETO-MIN, T1-KETO-MIN, and T2-KETOMIN relative to SO-KETO-MIN are computed to be $86.8,82.0$, and $83.5 \mathrm{kcal} / \mathrm{mol}$, respectively (see Table 2). 

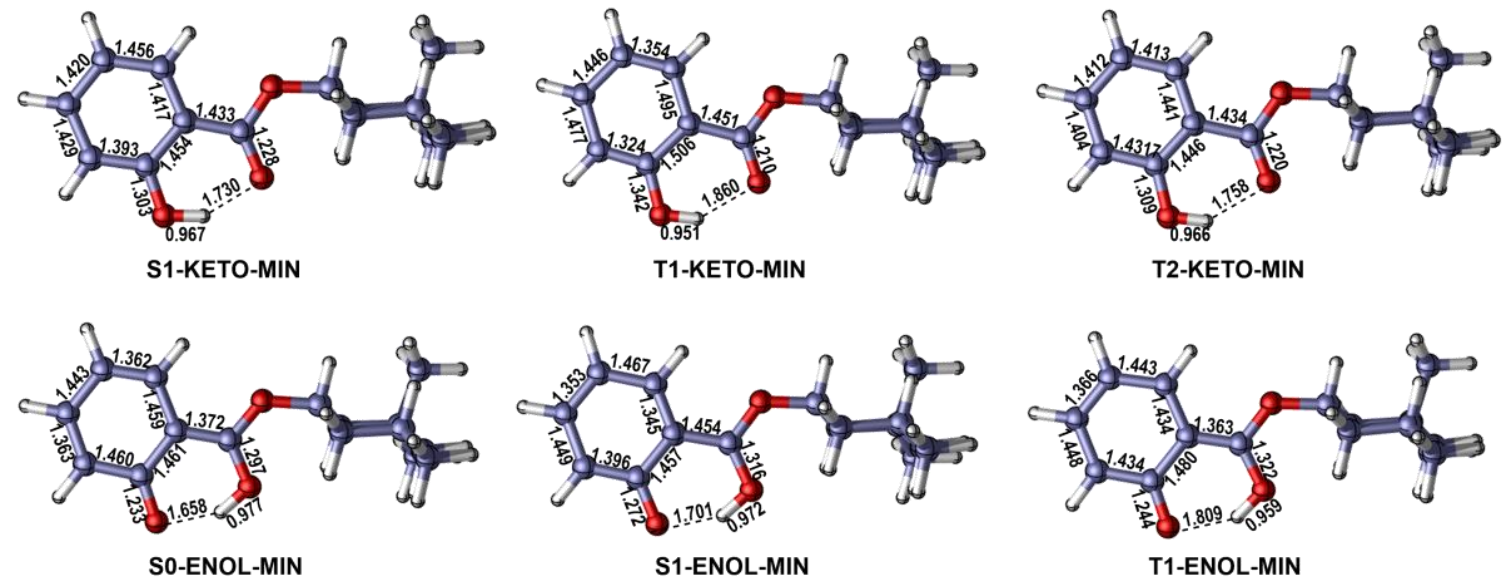

Figure S3. CASSCF optimized $S_{0}, S_{1}, T_{1}$, and $T_{2}$ minima in gas phase with selected bond lengths (in $\AA$ ) and given atomic numbering.
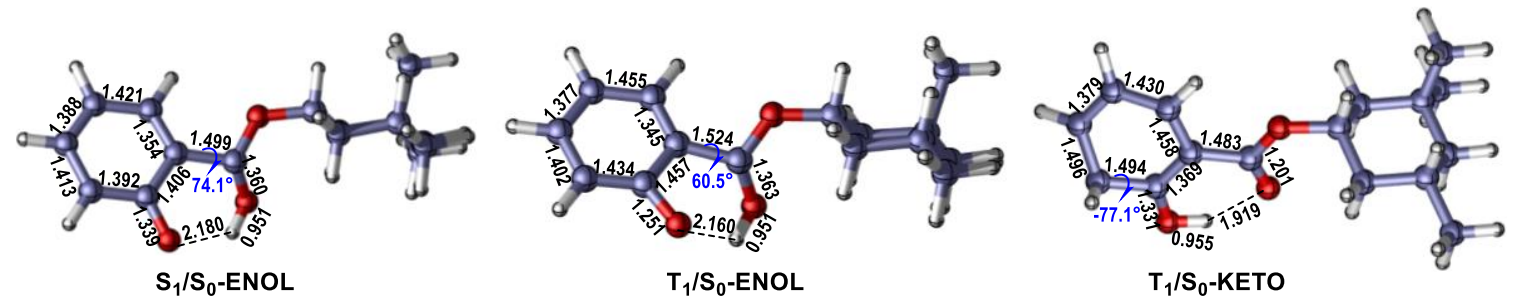

Figure S4. CASSCF optimized minimum-energy conical intersection and crossing points in gas phase. Also shown are selected bond lengths (in $\AA$ ).

Table S2: Selected bond lengths (in $\AA$ ) of $\mathrm{S}_{0}, \mathrm{~S}_{1}, \mathrm{~T}_{1}$, and $\mathrm{T}_{2}$ minima in acetonitrile solution optimized at the QM(CASSCF)/MM level

\begin{tabular}{rrrrrrrr}
\hline & $\begin{array}{r}\text { S0- } \\
\text { KETO- } \\
\text { MIN }\end{array}$ & $\begin{array}{r}\text { SETO- } \\
\text { MIN }\end{array}$ & $\begin{array}{r}\text { T1- } \\
\text { KETO- } \\
\text { MIN }\end{array}$ & $\begin{array}{r}\text { T2- } \\
\text { KETO- } \\
\text { MIN }\end{array}$ & $\begin{array}{r}\text { S0- } \\
\text { ENOL- } \\
\text { MIN }\end{array}$ & $\begin{array}{r}\text { S1- } \\
\text { ENOL- } \\
\text { MIN }\end{array}$ & $\begin{array}{r}\text { T1- } \\
\text { ENOL- } \\
\text { MIN }\end{array}$ \\
\hline C1-C2 & 1.387 & 1.392 & 1.324 & 1.430 & 1.458 & 1.395 & 1.433 \\
C2-C3 & 1.384 & 1.420 & 1.472 & 1.403 & 1.363 & 1.447 & 1.447 \\
C3-C4 & 1.398 & 1.414 & 1.447 & 1.412 & 1.442 & 1.353 & 1.366 \\
C4-C5 & 1.386 & 1.454 & 1.355 & 1.412 & 1.362 & 1.466 & 1.443 \\
C5-C6 & 1.406 & 1.424 & 1.493 & 1.441 & 1.458 & 1.345 & 1.435 \\
C6-C1 & 1.405 & 1.447 & 1.504 & 1.445 & 1.459 & 1.457 & 1.478 \\
C1-010 & 1.338 & 1.306 & 1.341 & 1.308 & 1.234 & 1.272 & 1.243 \\
C6-C7 & 1.483 & 1.438 & 1.452 & 1.434 & 1.374 & 1.454 & 1.363 \\
C7-08 & 1.205 & 1.224 & 1.210 & 1.220 & 1.295 & 1.315 & 1.321 \\
O8-H9 & 1.866 & 1.751 & 1.882 & 1.754 & 0.979 & 0.972 & 0.959 \\
H9-010 & 0.952 & 0.964 & 0.951 & 0.967 & 1.630 & 1.703 & 1.789 \\
\hline
\end{tabular}


Table S3: Selected bond lengths (in $\AA$ ) of $S_{0}, S_{1}, T_{1}$, and $T_{2}$ minima in gas phase optimized at the CASSCF level

\begin{tabular}{rrrrrrrr}
\hline & $\begin{array}{r}\text { S0- } \\
\text { KETO- } \\
\text { MIN }\end{array}$ & $\begin{array}{r}\text { SETO- } \\
\text { MIN }\end{array}$ & $\begin{array}{r}\text { T1- } \\
\text { KETO- } \\
\text { MIN }\end{array}$ & $\begin{array}{r}\text { T2- } \\
\text { KETO- } \\
\text { MIN }\end{array}$ & $\begin{array}{r}\text { S0- } \\
\text { ENOL- } \\
\text { MIN }\end{array}$ & $\begin{array}{r}\text { S1- } \\
\text { ENOL- } \\
\text { MIN }\end{array}$ & $\begin{array}{r}\text { T1- } \\
\text { ENOL- } \\
\text { MIN }\end{array}$ \\
\hline C1-C2 & 1.387 & 1.393 & 1.324 & 1.431 & 1.460 & 1.396 & 1.434 \\
C2-C3 & 1.385 & 1.429 & 1.477 & 1.404 & 1.363 & 1.449 & 1.448 \\
C3-C4 & 1.398 & 1.420 & 1.446 & 1.412 & 1.443 & 1.353 & 1.366 \\
C4-C5 & 1.386 & 1.456 & 1.354 & 1.413 & 1.362 & 1.467 & 1.443 \\
C5-C6 & 1.406 & 1.417 & 1.495 & 1.441 & 1.459 & 1.345 & 1.434 \\
C6-C1 & 1.405 & 1.454 & 1.506 & 1.446 & 1.461 & 1.457 & 1.480 \\
C1-010 & 1.339 & 1.303 & 1.342 & 1.309 & 1.233 & 1.272 & 1.244 \\
C6-C7 & 1.484 & 1.433 & 1.451 & 1.434 & 1.372 & 1.454 & 1.363 \\
C7-08 & 1.206 & 1.228 & 1.210 & 1.220 & 1.297 & 1.316 & 1.322 \\
O8-H9 & 1.867 & 1.730 & 1.860 & 1.758 & 0.977 & 0.972 & 0.959 \\
H9-010 & 0.952 & 0.967 & 0.951 & 0.966 & 1.658 & 1.701 & 1.809 \\
\hline
\end{tabular}

Table S4: Selected bond lengths (in $\AA$ ) of minimum-energy conical intersection and crossing points (left) in acetonitrile solution optimized at the QM(CASSCF)/MM level and (right) in gas phase optimized at the CASSCF level

\begin{tabular}{|c|c|c|c|c|c|c|c|}
\hline & $\begin{array}{l}\mathrm{S}_{1} / \mathrm{S}_{0-}^{-} \\
\mathrm{ENOL}\end{array}$ & $\begin{array}{l}\mathrm{T}_{1} / \mathrm{S}_{0-} \\
\text { ENOL }\end{array}$ & $\begin{array}{l}\mathrm{T}_{1} / \mathrm{S}_{0}^{-} \\
\text {KETO }\end{array}$ & & $\begin{array}{l}\mathrm{S}_{1} / \mathrm{S}_{0^{-}} \\
\mathrm{ENOL}\end{array}$ & $\begin{array}{l}\mathrm{T}_{1} / \mathrm{S}_{0^{-}} \\
\mathrm{ENOL}\end{array}$ & $\begin{array}{l}\mathrm{T}_{1} / \mathrm{S}_{0}- \\
\text { KETO }\end{array}$ \\
\hline C1-C2 & 1.387 & 1.44 & 1.494 & C1-C2 & 1.392 & 1.434 & 1.494 \\
\hline C2-C3 & 1.415 & 1.407 & 1.494 & C2-C3 & 1.413 & 1.402 & 1.496 \\
\hline C3-C4 & 1.360 & 1.396 & 1.378 & C3-C4 & 1.388 & 1.377 & 1.379 \\
\hline C4-C5 & 1.435 & 1.446 & 1.430 & C4-C5 & 1.421 & 1.455 & 1.430 \\
\hline C5-C6 & 1.361 & 1.324 & 1.457 & C5-C6 & 1.354 & 1.345 & 1.458 \\
\hline C6-C1 & 1.406 & 1.461 & 1.371 & C6-C1 & 1.406 & 1.457 & 1.369 \\
\hline C1-010 & 1.334 & 1.242 & 1.331 & C1-010 & 1.339 & 1.251 & 1.331 \\
\hline $\mathrm{C} 6-\mathrm{C} 7$ & 1.510 & 1.519 & 1.481 & C6-C7 & 1.499 & 1.524 & 1.483 \\
\hline C7-08 & 1.353 & 1.364 & 1.201 & C7-08 & 1.360 & 1.363 & 1.201 \\
\hline O8-H9 & 0.954 & 0.951 & 1.914 & O8-H9 & 0.951 & 0.951 & 1.919 \\
\hline $\mathrm{H} 9-010$ & 1.999 & 2.173 & 0.955 & $\mathrm{H} 9-010$ & 2.180 & 2.160 & 0.955 \\
\hline
\end{tabular}

\section{Spin-Orbit Couplings}

Table S5: The spin-orbit couplings ( $\mathrm{cm}^{-1}$ ) of Conformer 1 (SOC1) and Conformer 2 (SOC2) of HMS based on the QM(CASPT2)/MM [CASPT2] calculations

\begin{tabular}{|c|c|c|c|}
\hline Structures & States & SOC1 & SOC2 \\
\hline \multirow{2}{*}{ S1-KETO-MIN } & $\mathrm{S}_{1} / \mathrm{T}_{1}$ & $0.025[0.003]$ & $0.122[0.003]$ \\
\cline { 2 - 4 } & $\mathrm{S}_{1} / \mathrm{T}_{2}$ & $0.185[0.005]$ & $0.327[0.002]$ \\
\hline
\end{tabular}




\begin{tabular}{|c|c|c|c|}
\hline $\mathrm{T}_{1} / \mathrm{S}_{0}-\mathrm{ENOL}$ & $\mathrm{T}_{1} / \mathrm{S}_{0}$ & $2.205[2.709]$ & - \\
\hline $\mathrm{T}_{1} / \mathrm{S}_{0}-\mathrm{KETO}$ & $\mathrm{T}_{1} / \mathrm{S}_{0}$ & $1.332[1.364]$ & $1.423[1.431]$ \\
\hline
\end{tabular}

Table S6: CASPT2 and QM(CASPT2)/MM computed adiabatic excitation energies (in kcal/mol) of the minimum and intersection structures relative to their $\mathrm{S}_{0}$ keto minima for $\mathrm{HMS}$ in gas phase and acetonitrile solution and for MS in methanol solution.

\begin{tabular}{|l|l|l|l|}
\hline Structures & Gas phase $^{\mathrm{a}}$ & Acetonitrile solution $^{\mathrm{a}}$ & Methanol solution $^{\mathrm{b}}$ \\
\hline S0-KETO-MIN & 0.0 & 0.0 & 0.0 \\
\hline S1-KETO-MIN & 86.8 & 86.9 & 85.0 \\
\hline T1-KETO-MIN & 82.0 & 81.5 & 81.4 \\
\hline T2-KETO-MIN & 83.5 & 84.5 & - \\
\hline S0-ENOL-MIN & 21.9 & 21.4 & 21.4 \\
\hline S1- ENOL -MIN & 84.2 & 83.9 & 82.6 \\
\hline T1- ENOL -MIN & 70.2 & 69.6 & 67.5 \\
\hline S $_{1} / \mathrm{S}_{0}$-ENOL & $89.4 / 85.4$ & $87.4 / 84.6$ & $84.4 / 83.5$ \\
\hline $\mathrm{T}_{1} / \mathrm{S}_{0}-$ ENOL & $79.6 / 73.5$ & $79.8 / 72.8$ & $81.6 / 76.3$ \\
\hline $\mathrm{T}_{1} / \mathrm{S}_{0}-$ KETO & $87.6 / 82.8$ & $87.8 / 83.0$ & $91.1 / 85.0$ \\
\hline
\end{tabular}

a This work. bJ. Phys. Chem. A 2021, 125, 1880-1891.

\section{MEPTs and Isomerization Path}

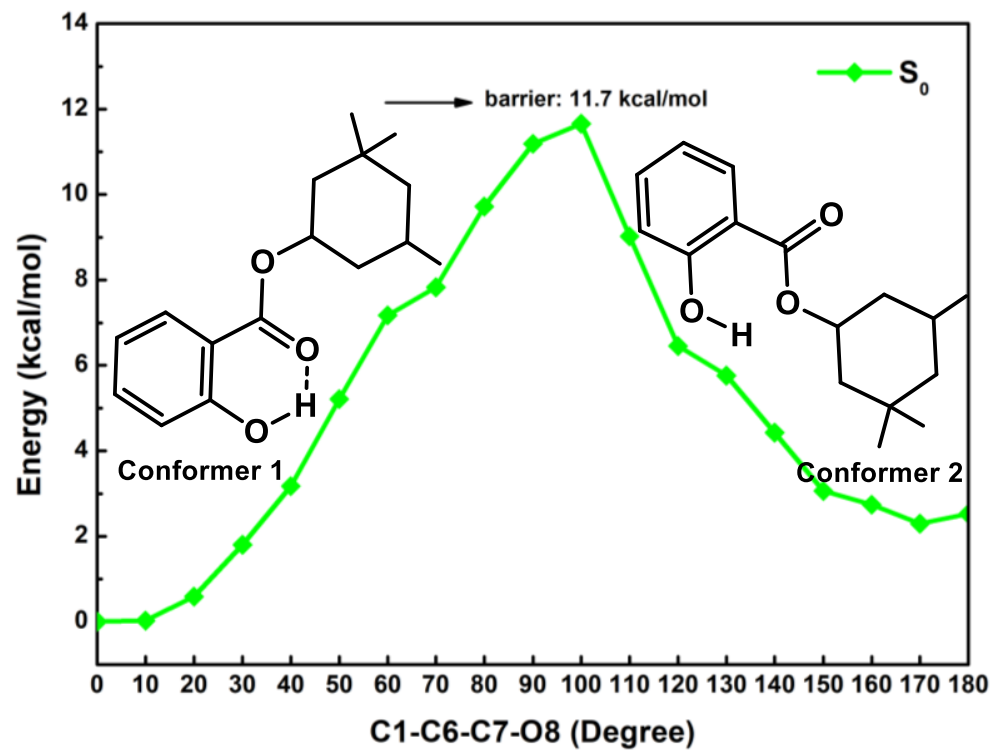

Figure S5. QM(CASPT2//CASSCF)/MM computed $\mathrm{S}_{0}$ state isomerization path of the solvated HMS molecule along the rotation of the central $\mathrm{C} 1-\mathrm{C} 6-\mathrm{C} 7-08$ dihedral angle with geometric structure optimizations by fixing the dihedral angle shown at 10 degree increments between 0 and 180 degrees and relaxing all other internal coordinates in the ground state at the QM(CASSCF)/MM level. 

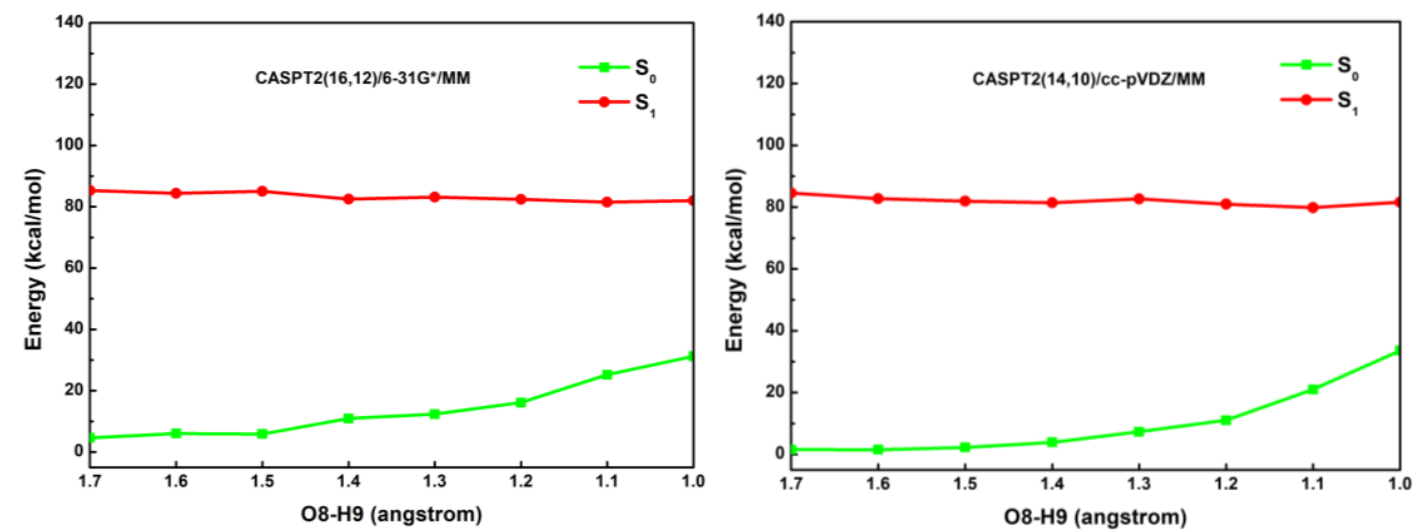

Figure S6. QM(CASPT2)/MM computed relaxed $\mathrm{S}_{1}$ state MEPTs (left plane: CASPT2(16,12)/6$31 \mathrm{G}^{*} / \mathrm{MM}$ and left plane: CASPT2(14,10)/cc-pVDZ/MM ) of the solvated HMS molecule along the O8-H9 bond with geometric structure optimizations (left plane: $\operatorname{CASSCF}(16,12) / 6-31 G^{*} / \mathrm{MM}$ and left plane: CASSCF $(14,10) / c c-p V D Z / M M)$ by fixing the dihedral angle shown at 0.1 angstrom increments between 1.7 and 1.0 angstroms and relaxing all other internal coordinates in the $S_{1}$ state at the QM(CASSCF)/MM level.

\section{CASPT2 and QM(CASPT2)/MM Computed LIICs}
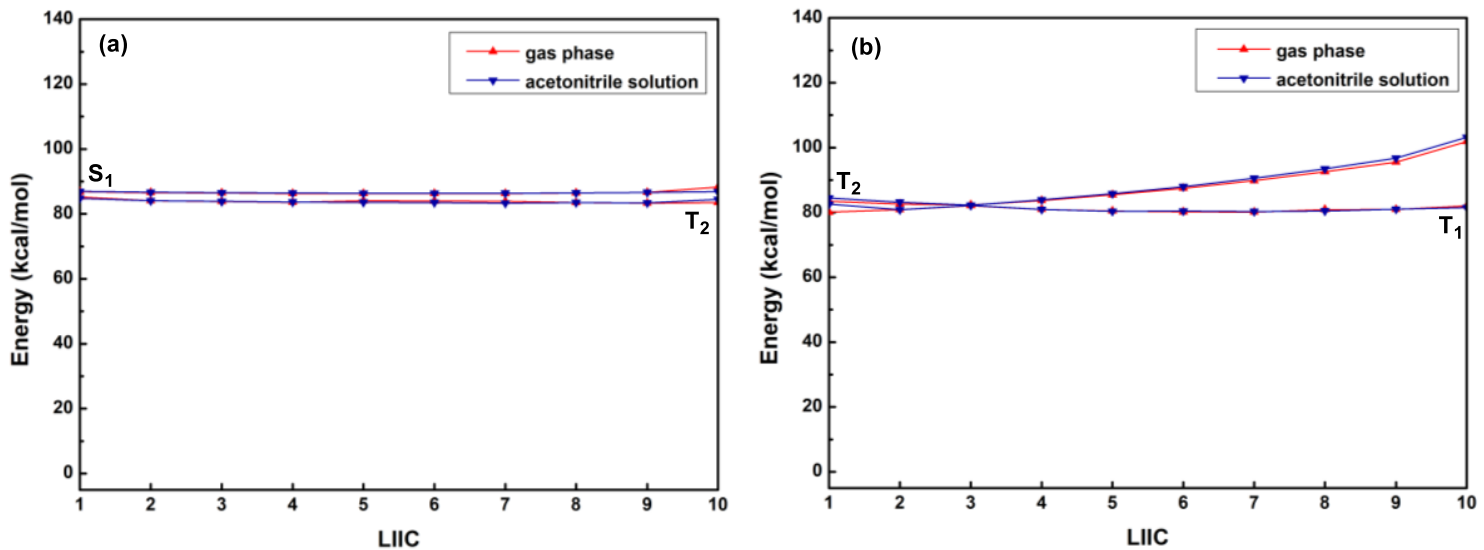

Figure S7. CASPT2 and QM(CASPT2)/MM computed LIICs (in kcal/mol) connecting (a) S1-KETOMIN and T2-KETO-MIN and (b) T2-KETO-MIN and T1-KETO-MIN, in gas phase and acetonitrile solution, respectively. 


\section{CASPT2 Computed Plausible Relaxation Pathways of HMS}

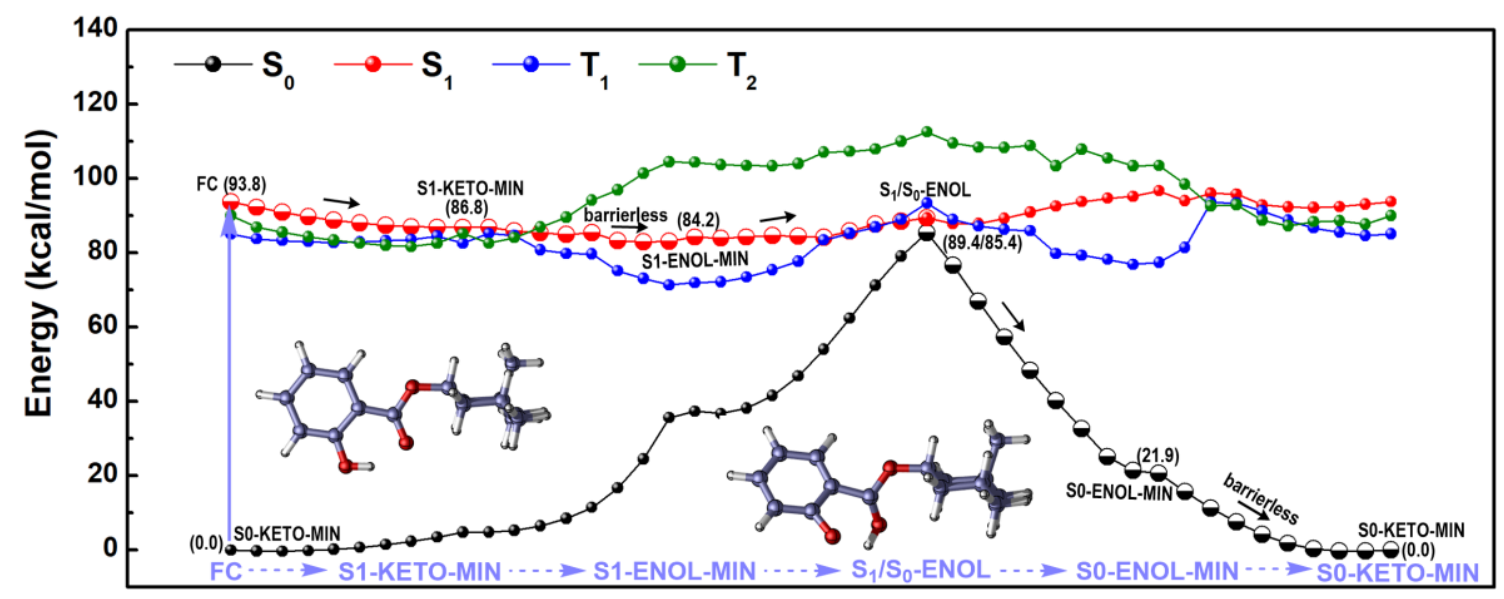

Figure S8. Plausible relaxation pathway I of HMS in gas phase calculated at the CASPT2 level. Relative energies of minima and intersection structures are in $\mathrm{kcal} / \mathrm{mol}$.

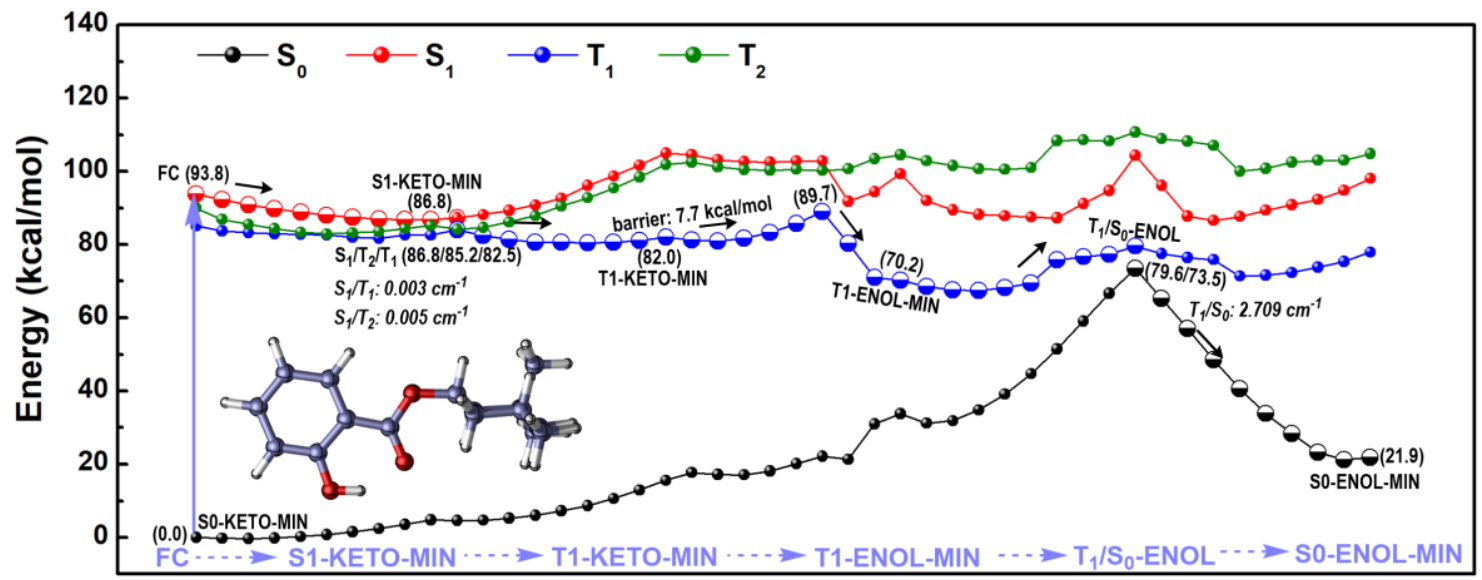

Figure S9. Plausible relaxation pathway II of HMS in gas phase calculated at the CASPT2 level. Relative energies of minima and intersection structures are in $\mathrm{kcal} / \mathrm{mol}$. Spin-orbit couplings at the S1-KETO-MIN and $\mathrm{T}_{1} / \mathrm{S}_{0}$-ENOL are given in $\mathrm{cm}^{-1}$ 


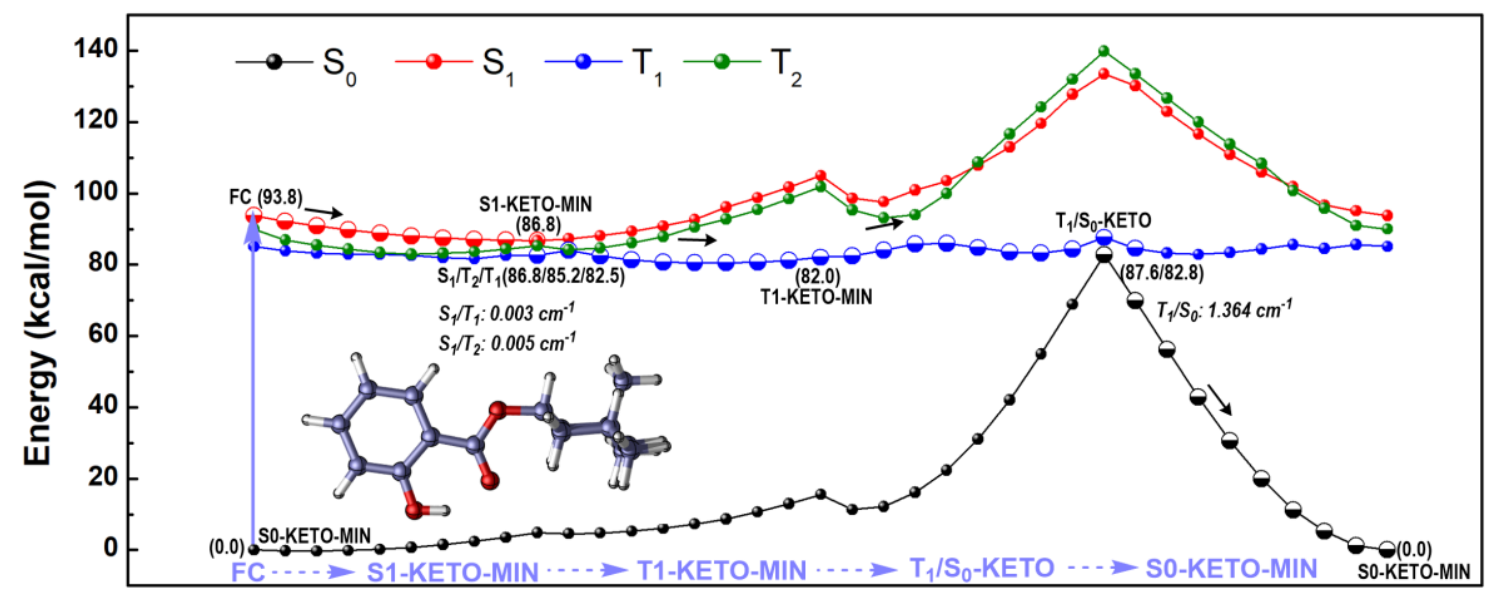

Figure S10. Plausible relaxation pathway III of HMS in gas phase calculated at the CASPT2 level. Relative energies of minima and intersection structures are in $\mathrm{kcal} / \mathrm{mol}$. Spin-orbit couplings at the S1-KETO-MIN and $\mathrm{T}_{1} / \mathrm{S}_{0}-\mathrm{KETO}$ are given in $\mathrm{cm}^{-1}$

\section{Inclusion the Menthone into the MM Region by means of a Link-Atom Scheme}

We put the menthone into MM region by means of a link-atom scheme and give the computed results as follows, including the energies of the minimum and intersection structures in table S7, the minimum-energy proton transfer paths in $S_{1}, T_{1}$, and $S_{0}$ states in Figure $S 11$ of panels a-c and $S_{0}$ isomerization path in Figure $\mathrm{S} 11$ of panel $\mathbf{d}$. Based on these results, we proposed that the substituent effect may have a slight influence on the key geometric structures and potential energy surfaces but give virtually the same excited-state relaxation pathways.

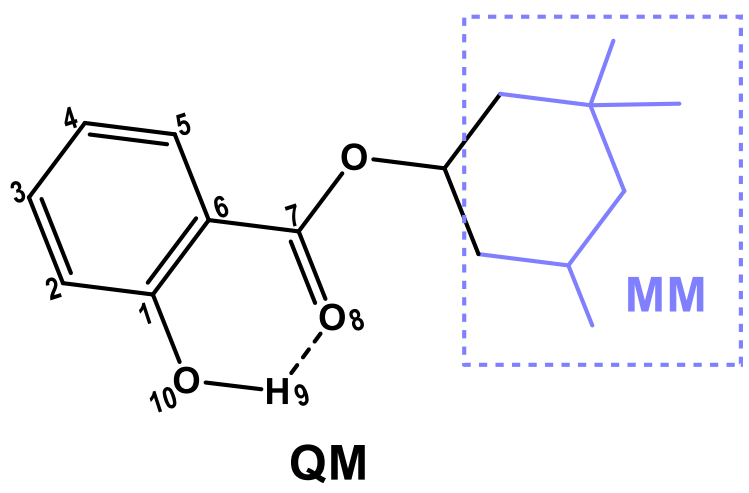

Illustration of link-atom scheme 
Table S7: QM(CASPT2)/MM computed adiabatic excitation energies (in kcal $/ \mathrm{mol}$ ) of the minimum and intersection structures relative to their $\mathrm{S}_{0}$ keto minima for HMS in acetonitrile solution

\begin{tabular}{|c|c|c|c|}
\hline Structures & Energy $(\mathrm{kcal} / \mathrm{mol})$ & Structures & Energy $(\mathrm{kcal} / \mathrm{mol})$ \\
\hline SO-KETO-MIN & 0.0 & S0-ENOL-MIN & 22.8 \\
\hline S1-KETO-MIN & 88.5 & S1-ENOL-MIN & 82.5 \\
\hline T2-KETO-MIN & 83.6 & T1-ENOL-MIN & 70.4 \\
\hline T1-KETO-MIN & 80.9 & $\mathrm{~S}_{1} / \mathrm{S}_{0}$-ENOL & $92.7 / 89.8$ \\
\hline $\mathrm{T}_{1} / \mathrm{S}_{0}-$ KETO & $89.1 / 84.4$ & $\mathrm{~T}_{1} / \mathrm{S}_{0}$-ENOL & $81.7 / 75.1$ \\
\hline
\end{tabular}
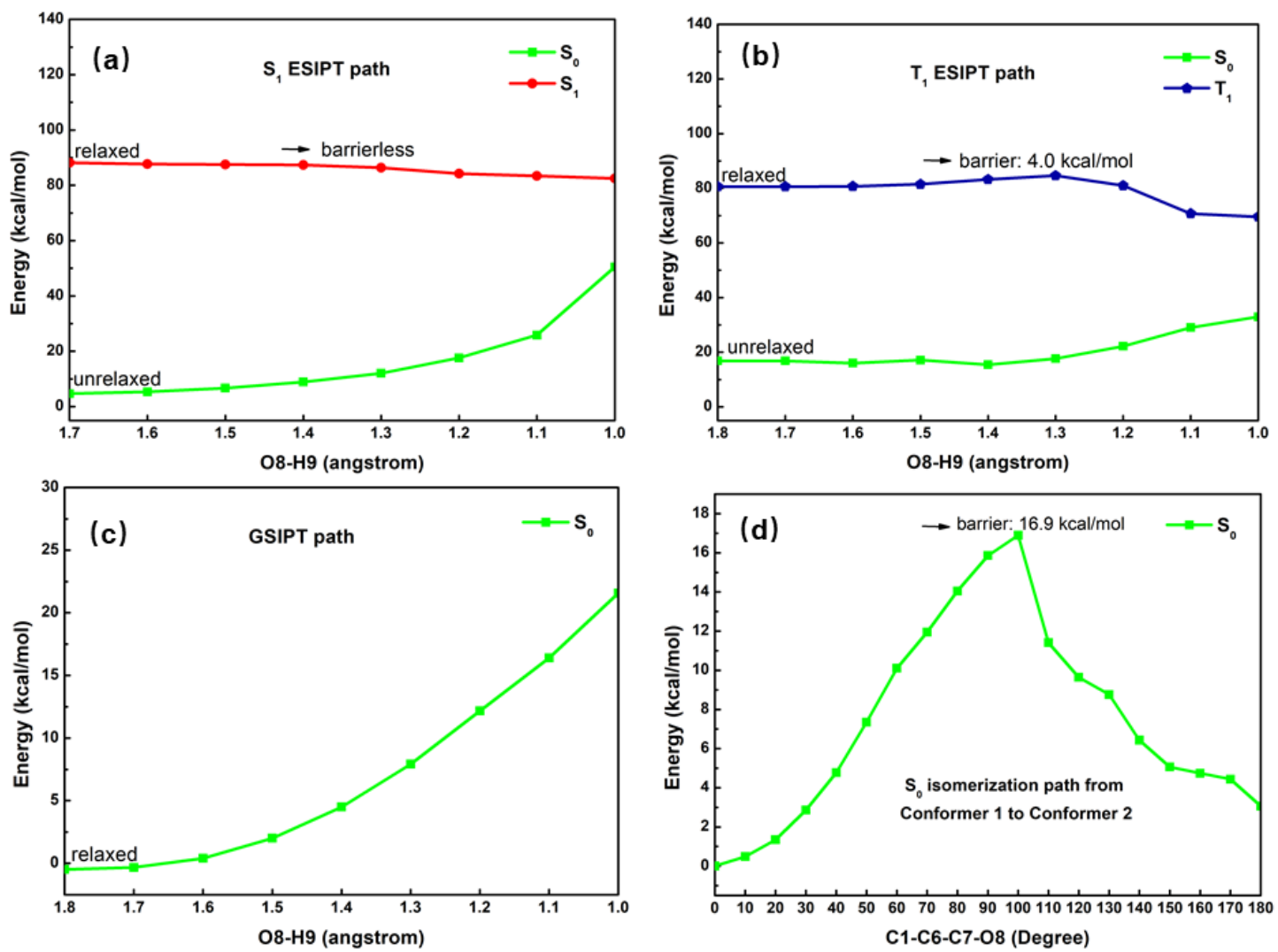

Figure S11. QM(CASPT2//CASSCF)/MM computed minimum-energy proton transfer (MEPT) paths with (a) relaxed $\mathrm{S}_{1}$ state, (b) relaxed $\mathrm{T}_{1}$ state, and (c) relaxed $\mathrm{S}_{0}$ state, and (d) $\mathrm{S}_{0}$ state isomerization path from conformer 1 to conformer 2 of the solvated HMS molecule in acetonitrile solution. 


\section{Cartesian coordinates of All Optimized Structures and LIIC Structures}

In xyz format (unit: Ångstrom)

\section{CASSCF/6-31G*}

In gas phase

\begin{tabular}{|c|c|c|c|}
\hline \multicolumn{4}{|c|}{ SO-KETO-MIN } \\
\hline C & -4.353296986 & -0.892718616 & -1.519764319 \\
\hline C & -3.009458654 & -0.690621967 & -1.248352811 \\
\hline C & -2.595716437 & -0.036108013 & -0.074326527 \\
\hline C & -3.570223387 & 0.416338599 & 0.831222817 \\
\hline C & -4.913443725 & 0.210719693 & 0.553122207 \\
\hline C & -5.307989413 & -0.435488772 & -0.606656831 \\
\hline $\mathrm{H}$ & -4.654575316 & -1.394575169 & -2.420762464 \\
\hline$H$ & -2.263179612 & -1.034073576 & -1.936354700 \\
\hline$H$ & -5.633609706 & 0.568373291 & 1.265270560 \\
\hline$H$ & -6.355427688 & -0.582847162 & -0.799257130 \\
\hline C & -1.153916151 & 0.174371640 & 0.207157637 \\
\hline 0 & -0.749944653 & 0.727831685 & 1.199209247 \\
\hline 0 & -3.286516668 & 1.049884333 & 1.976034305 \\
\hline $\mathrm{H}$ & -2.344481029 & 1.142190541 & 2.076648289 \\
\hline 0 & -0.365321229 & -0.303890931 & -0.735529843 \\
\hline C & 1.070074938 & -0.222262557 & -0.691951631 \\
\hline C & 1.659974451 & -1.031863393 & 0.459133073 \\
\hline C & 1.583692243 & 1.212218435 & -0.721552452 \\
\hline C & 3.204743712 & -1.047668515 & 0.435249409 \\
\hline$H$ & 1.328436406 & -0.604575164 & 1.397099429 \\
\hline C & 3.116136136 & 1.223198191 & -0.815065411 \\
\hline $\mathrm{H}$ & 1.149215152 & 1.730866596 & -1.571920965 \\
\hline C & 3.723014068 & 0.402811749 & 0.332541873 \\
\hline$H$ & 3.501277693 & 0.915125754 & 1.267605491 \\
\hline C & 3.654532192 & 2.654976161 & -0.813425819 \\
\hline$H$ & 3.399136927 & 3.167289409 & 0.110065687 \\
\hline $\mathrm{H}$ & 4.735983192 & 2.666339364 & -0.910920324 \\
\hline$H$ & 3.241745135 & 3.230486815 & -1.636676856 \\
\hline $\mathrm{H}$ & 1.273230164 & 1.737329137 & 0.173708703 \\
\hline$H$ & 1.276276812 & -2.047752845 & 0.413894329 \\
\hline$H$ & 4.806640346 & 0.390990299 & 0.239587518 \\
\hline$H$ & 3.403350374 & 0.767953253 & -1.760870867 \\
\hline C & 3.705967824 & -1.665456309 & 1.748547244 \\
\hline$H$ & 3.350263873 & -2.685749892 & 1.861514596 \\
\hline
\end{tabular}




$\begin{array}{llll}\mathrm{H} & 4.791663344 & -1.688976693 & 1.777748817 \\ \mathrm{H} & 3.362692442 & -1.097403789 & 2.607705128 \\ \mathrm{C} & 3.735888310 & -1.907570728 & -0.725359385 \\ \mathrm{H} & 4.821505306 & -1.936543511 & -0.710302112 \\ \mathrm{H} & 3.378826215 & -2.929698773 & -0.638193414 \\ \mathrm{H} & 3.437888916 & -1.541639332 & -1.700849551 \\ \mathrm{H} & 1.331277474 & -0.695253432 & -1.627701158\end{array}$

$\begin{array}{lrrr}\text { S1-KETO-MIN } & & \\ \text { C } & -4.448234819 & -0.875158774 & -1.513384860 \\ \text { C } & -3.021068136 & -0.701922643 & -1.284932024 \\ \text { C } & -2.542862605 & -0.058493666 & -0.116164730 \\ \text { C } & -3.551943187 & 0.406985952 & 0.822139548 \\ \text { C } & -4.917795244 & 0.241240726 & 0.604832694 \\ \text { C } & -5.402564690 & -0.406176628 & -0.572854401 \\ \text { H } & -4.770065012 & -1.366851711 & -2.411507625 \\ \text { H } & -2.324941207 & -1.065868699 & -2.010885897 \\ \text { H } & -5.584571702 & 0.618637753 & 1.355374967 \\ \text { H } & -6.454016796 & -0.531060908 & -0.736451903 \\ \text { C } & -1.152636320 & 0.144486969 & 0.166750974 \\ \text { O } & -0.735099754 & 0.702612642 & 1.177478874 \\ \text { O } & -3.195841760 & 1.008255404 & 1.921773909 \\ \text { H } & -2.231766211 & 1.067276215 & 1.964787844 \\ \text { O } & -0.344045504 & -0.334706383 & -0.781596945 \\ \mathrm{C} & 1.081638646 & -0.240226018 & -0.716299801 \\ \text { C } & 1.667179241 & -1.043994495 & 0.442554521 \\ \text { C } & 1.586300336 & 1.198608435 & -0.737637032 \\ \mathrm{C} & 3.211869269 & -1.042377584 & 0.445991093 \\ \text { H } & 1.312409542 & -0.621850256 & 1.373831908 \\ \text { C } & 3.119319793 & 1.226469707 & -0.806341956 \\ \text { H } & 1.160366891 & 1.712894224 & -1.595325014 \\ \mathrm{C} & 3.716207949 & 0.413614230 & 0.351778165 \\ \mathrm{H} & 3.472016620 & 0.923552039 & 1.282537354 \\ \mathrm{C} & 3.643132370 & 2.663699362 & -0.797348679 \\ \mathrm{H} & 3.364258273 & 3.174930316 & 0.120002096 \\ \mathrm{H} & 4.726260650 & 2.687219714 & -0.874225734 \\ \mathrm{H} & 3.239592855 & 3.233355003 & -1.629385519 \\ \mathrm{H} & 1.253296179 & 1.718969835 & 0.151872881 \\ \mathrm{H} & 1.296504205 & -2.064660180 & 0.389635928 \\ \mathrm{H} & 4.801667133 & 0.413967890 & 0.277898988 \\ \mathrm{H} & 3.427774127 & 0.773418008 & -1.746716138 \\ \mathrm{C} & 3.697814356 & -1.654045268 & 1.767973198 \\ \mathrm{H} & 3.352999064 & -2.678893655 & 1.874417816 \\ \mathrm{H} & 4.783197323 & -1.663780098 & 1.817576252 \\ \mathrm{H} & 3.331051919 & -1.090781347 & 2.620568548 \\ \mathrm{C} & 3.774607500 & -1.895781278 & -0.704520390 \\ \mathrm{H} & 4.860265862 & -1.912340208 & -0.670061658\end{array}$




\begin{tabular}{|c|c|c|c|}
\hline & 427724776 & 512 & -0 . \\
\hline & 3.489362892 & -1.532868171 & \\
\hline & 1.366968167 & -0.709741184 & 55 \\
\hline \multicolumn{4}{|c|}{ T1-KETO-MIN } \\
\hline C & -4.331635094 & -0.907906115 & -1.556418861 \\
\hline & -3.013746127 & 9014343 & \\
\hline & -2.563806853 & -0.026841500 & -0.0605207 \\
\hline 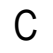 & -3.631000622 & 0.446616073 & 0.890686364 \\
\hline U & & & \\
\hline C & -5.338082262 & -0.446048763 & -0.6 \\
\hline H & -4.638850824 & -1.407779501 & -2.457101 \\
\hline & -2.259137042 & -1.055778505 & -1.986747895 \\
\hline & -5.649118097 & 0.589011294 & 91496 \\
\hline H & -6.381051037 & -0.593949830 & -0.8 \\
\hline C & -1.152793131 & 0.174192090 & 0.212127664 \\
\hline 0 & -0.745740355 & 0.727217913 & 1.208071638 \\
\hline 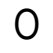 & -3.277485825 & 1.075975058 & 2.021780773 \\
\hline-1 & -2.331111080 & 1.14 & 2.0 \\
\hline 0 & -0.363072755 & -0.308407914 & -0.7 \\
\hline C & 1.070373299 & -0.224894864 & -0.693154562 \\
\hline U & 1.66 & -1.0 & \\
\hline & & & \\
\hline C & 3.206509111 & -1.0 & 0.4 \\
\hline $\mathrm{H}$ & 1.329098507 & -0.607323092 & 1.3 \\
\hline C & 3.11 & 1.22255 & \\
\hline H & & & 31 \\
\hline C & 3.723366278 & $0.4 C$ & 0.3 \\
\hline $\mathrm{H}$ & 3.500866083 & 0.91565 & 41709 \\
\hline C & 3.653578774 & 2.654582511 & -0.815334630 \\
\hline $\mathrm{H}$ & 3.39 & & \\
\hline $\mathrm{H}$ & 4.735 & 2.66 & -0.6 \\
\hline $\mathrm{H}$ & 3.241576904 & $3.22 \varepsilon$ & -1.640141750 \\
\hline H & 1.272885923 & 1.734550787 & 0.174017742 \\
\hline $\mathrm{H}$ & 1.2794 & -2.0 & \\
\hline & & & 076647 \\
\hline H & 3.403135856 & 0.766 & -1.760873349 \\
\hline C & 3.707661879 & -1.664837960 & 1.749138976 \\
\hline H & 3.353828750 & -2.685853484 & 1.861480305 \\
\hline$\because$ & & & 77977 \\
\hline $\mathrm{H}$ & 3.362406041 & -1.09 & 2.608077430 \\
\hline & 3.739611977 & -1.907159589 & -0.724630131 \\
\hline $\mathrm{H}$ & 4.825231396 & -1.935301960 & -0.708341023 \\
\hline H & & -2 & 236 \\
\hline & 3.4 & -1.541327339 & -1.700371350 \\
\hline & & -0.697114 & \\
\hline
\end{tabular}




\begin{tabular}{|c|c|c|c|}
\hline \multicolumn{4}{|c|}{ 2-KETO-MIN } \\
\hline & -4.424029505 & -0.874561132 & -1.490465987 \\
\hline & -3.035109357 & -0.711145636 & -1.291947925 \\
\hline & -2.539081766 & -0.052813382 & -0.110065576 \\
\hline & -3.544528181 & 0.409266727 & 0.820052118 \\
\hline & -4.944881076 & 0.227743156 & 0.588425606 \\
\hline & -5.396201974 & -0.418363765 & -0.573495 \\
\hline & -4.746983750 & -1.372433853 & -2.3876597 \\
\hline & -2.344103927 & -1.077741288 & -2.022038043 \\
\hline & -5.614872809 & 0.604996945 & 1.336422119 \\
\hline & -6.441489721 & -0.561874741 & -0.760933807 \\
\hline & -1.147323889 & 0.153694193 & 0.1692834 \\
\hline & -0.738617434 & 0.712250458 & 1.174434756 \\
\hline & -3.215725955 & 1.021983463 & 1.929521339 \\
\hline & -2.253259343 & 1.089329211 & 1.983020095 \\
\hline & -0.346811974 & -0.321180782 & -0.7711188 \\
\hline & 1.084837036 & -0.230503746 & -0.709351450 \\
\hline & 1.666904307 & -1.036604853 & 0.4484107 \\
\hline & 1.590975828 & 1.206924969 & -0.730341059 \\
\hline & 3.211643602 & -1.042939141 & 0.444112266 \\
\hline & 1.316342642 & -0.610303355 & 1.379215080 \\
\hline & 3.124043457 & 1.227747967 & -0.805906 \\
\hline & 1.163961417 & 25474 & 874 \\
\hline & 3.722756634 & 0.410639958 & 0.348390779 \\
\hline & 3.486304845 & 0.920923219 & 1.280852634 \\
\hline & 3.654395913 & 2.662539972 & -0.797629751 \\
\hline & 3.382992788 & 3.173708536 & 0.121916120 \\
\hline & 4.737156749 & 2.680632949 & -0.880380748 \\
\hline & 3.249309274 & 3.235270392 & -1.626760815 \\
\hline & 1.261723395 & 1.725000682 & 0.161596883 \\
\hline & 1.290952818 & -2.055429096 & 0.397476742 \\
\hline & 4.807698754 & 0.40 & 328124 \\
\hline & 3.425918720 & 0.774555083 & -1.748346162 \\
\hline & 3.700725503 & -1.658141661 & 1.763201972 \\
\hline & 3.350955333 & -2.681169471 & 1.870703759 \\
\hline & 4.786253015 & -1.673850325 & 812088 \\
\hline & 3.341595691 & -1.093422439 & 2.618005176 \\
\hline & 3.763619706 & -1.898666464 & -0.709934836 \\
\hline & 4.849249465 & -1.921401384 & -0.681050347 \\
\hline & 3.411329055 & -2.923010332 & -0.628326 \\
\hline & 3.475771609 & -1.533533543 & -1.688744 \\
\hline & 1.36193 & -0.700877359 & $-1.64255 \varsigma$ \\
\hline
\end{tabular}

\begin{tabular}{|c|c|c|}
\hline MIN & & \\
\hline-4.421829091 & -0.887806963 & -1.517440839 \\
\hline-3.082865233 & -0.730446932 & 39353 \\
\hline & & \\
\hline
\end{tabular}




\begin{tabular}{|c|c|c|c|}
\hline & 071 & 50 & 05 \\
\hline C & -4.929703685 & 0.234317135 & \\
\hline & -5.351169387 & -0.393671408 & -0.5297747 \\
\hline$H_{2}$ & -4.797580297 & -1.376860162 & -2.3971839 \\
\hline & -2.376579561 & -1.090662256 & -2.048353197 \\
\hline $\mathrm{H}$ & -5.618072887 & 0.603404762 & 1.341636 \\
\hline H & -6.405591198 & 937908 & \\
\hline C & -1.232324847 & 0.102358427 & 0.0808459 \\
\hline O & -0.754140356 & 0.689411120 & 1.13389998 \\
\hline U & -3.148328846 & 1.009325951 & 10001461 \\
\hline $\mathrm{H}$ & -1.504651631 & 0.96 & 1.696690 \\
\hline 0 & -0.370583156 & 78605 & -0.78903 \\
\hline C & 1.070126797 & 69877 & -0.701143 \\
\hline & 1.641259319 & 51964 & 950332 \\
\hline C & 675814 & 1.2 & -0.7 \\
\hline C & 3.186916218 & -1.03 & $0.4 t$ \\
\hline$\Pi$ & 1.290993252 & -0.612617113 & 1.3922 \\
\hline U & 3.0 & 1.2 & \\
\hline $\mathrm{H}$ & & & \\
\hline C & 3. & 0.4 & 0.3 \\
\hline $\mathrm{H}$ & $3.4^{5}-5$ & 0.92 & 1.29867237 \\
\hline U & & & -0.77 \\
\hline$\Pi$ & & & \\
\hline $\mathrm{H}$ & & & -0.8 \\
\hline $\mathrm{H}$ & 3.22 & 3.24 & 49076 \\
\hline$H$ & 1.23 & 1.73 & 66090 \\
\hline$\Pi$ & & & 977 \\
\hline H & & & \\
\hline $\mathrm{H}$ & & & -1.7 \\
\hline C & 3. & -1.6 & 1.7 \\
\hline$H$ & & & 106 \\
\hline $\mathrm{H}$ & & & \\
\hline $\mathrm{H}$ & & & \\
\hline C & 62 & -1.8 & -0.6 \\
\hline $\mathrm{H}$ & 4. & -1.9 & -0.6 \\
\hline H & & & \\
\hline H & & & \\
\hline $\mathrm{H}$ & 1.35126 & -0.6 & -1.6 \\
\hline \multicolumn{4}{|c|}{ S1-ENOL-MIN } \\
\hline C & & & \\
\hline C & & & -1.2 \\
\hline C & -2.625965219 & -0.053649974 & -0.10354755 \\
\hline C & -J. & 0.429260652 & 0.86650 \\
\hline$C$ & & 0.226310 & 0.5919 \\
\hline C & & & $-0.6171114 \varepsilon$ \\
\hline & .750 & -1.387981800 & -2.420 \\
\hline
\end{tabular}




\begin{tabular}{|c|c|c|c|}
\hline $\mathrm{H}$ & -2.334498878 & -1.024966313 & -1.929797771 \\
\hline $\mathrm{H}$ & -5.671428427 & 0.581390983 & 1.305589093 \\
\hline ト & -6.442056872 & -0.578019769 & -0.789151966 \\
\hline C & -1.204989434 & 0.135950160 & 18246 \\
\hline & -0.711573254 & 0.729335834 & 1.205688149 \\
\hline C & -3.161706716 & 1.009262783 & 1.91022948 \\
\hline r & -1.468207516 & 0.994835961 & 1.755692524 \\
\hline C & -0.365302178 & -0.320817602 & -0.7728 \\
\hline C & 1.066071876 & -0.227930146 & -0.70 \\
\hline C & 1.646456676 & -1.036193454 & 0.45 \\
\hline 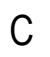 & 1.571240851 & 1.210166778 & -0.72 \\
\hline C & 3.191531120 & -1.039337233 & 0.45 \\
\hline ⺊ & 1.296392104 & -0.615250215 & \\
\hline C & 3.104774182 & 1.231233689 & -0.79 \\
\hline ト & 1.145716031 & 1.723922166 & $-1.5 \varepsilon$ \\
\hline C & 3.699677065 & 96478 & 0.3 \\
\hline r & 3.458444499 & 0.925194065 & 1.29 \\
\hline 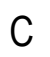 & 3.633845209 & 2.666 & -0.75 \\
\hline $\mathrm{H}$ & 3.360818732 & 3.178479677 & 0.12 \\
\hline דו & 4.716588862 & 94665 & -0.8 \\
\hline $\mathrm{H}$ & 3.230076544 & 3.238077635 & -1.62 \\
\hline $\mathrm{H}$ & 1.242260351 & 1.734 & 0.1 \\
\hline $\mathrm{H}$ & 1.271488821 & -2.054837656 & 0.39 \\
\hline $\mathrm{H}$ & 4.784752713 & 0.412399488 & 0.28 \\
\hline $\mathrm{H}$ & 3.409268465 & 0.777203986 & -1.73 \\
\hline C & 3.678832615 & -1.655296318 & 1.77 \\
\hline $\mathrm{H}$ & 3.329380761 & -2.678446103 & 1.87 \\
\hline 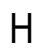 & 4.764169512 & -1.6705 & 1.81 \\
\hline $\mathrm{H}$ & 3.318990398 & -1.09 & 2.6 \\
\hline C & 3.746720060 & -1.893072044 & -0.70 \\
\hline $\mathrm{H}$ & 4.832246307 & -1.912411668 & -0.6 \\
\hline $\mathrm{H}$ & 3.397622136 & -2.91 & -0.6 \\
\hline $\mathrm{H}$ & 3.459150771 & -1.528695942 & -1.6 \\
\hline $\mathrm{H}$ & 1.343265646 & $-0.6 s$ & -1.6 \\
\hline \multicolumn{4}{|c|}{ T1-ENOL-MIN } \\
\hline$C$ & -4.477498252 & -0.883893342 & -1.50379274 \\
\hline C & & -0.7 & \\
\hline C & -2.564498705 & -0.055690032 & -0.111813539 \\
\hline C & -3.560581534 & 0.431477000 & 0.86 \\
\hline C & -4.954082045 & 0.228546256 & 0.596970893 \\
\hline 0 & -5.406188649 & -0.438177691 & $-0.6060907 \varepsilon$ \\
\hline $\mathrm{H}$ & -4.794176238 & -1.381331021 & -2.4029741 \\
\hline 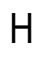 & -2.369108822 & -1.074401375 & -2.01733703 \\
\hline 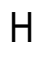 & -5.646171775 & 0.595375100 & 1.33 \\
\hline 11 & -6.458312403 & -0.570957121 & -0.77269796 \\
\hline & -1.230836970 & 0.119048508 & 0.1 \\
\hline
\end{tabular}

S16 


$\begin{array}{lrrr}\text { O } & -0.713264642 & 0.712266783 & 1.172539666 \\ \text { O } & -3.226298562 & 1.010420374 & 1.916751160 \\ \text { H } & -1.425230471 & 0.994263899 & 1.749666057 \\ \text { O } & -0.364588075 & -0.322517605 & -0.776133973 \\ \text { C } & 1.066247637 & -0.221545908 & -0.693446112 \\ \text { C } & 1.647132467 & -1.030816924 & 0.462771934 \\ \text { C } & 1.571519124 & 1.216220475 & -0.716904672 \\ \text { C } & 3.192350259 & -1.036849893 & 0.456264101 \\ \text { H } & 1.302039616 & -0.609779326 & 1.398282352 \\ \text { C } & 3.104924664 & 1.234734858 & -0.793137957 \\ \text { H } & 1.143505261 & 1.731001494 & -1.572693104 \\ \text { C } & 3.703064837 & 0.416968050 & 0.361053097 \\ \text { H } & 3.466918331 & 0.927100585 & 1.293887915 \\ \text { C } & 3.636789033 & 2.668958474 & -0.785988206 \\ \text { H } & 3.367991925 & 3.180884438 & 0.133970201 \\ \text { H } & 4.719301287 & 2.685450224 & -0.870555012 \\ \text { H } & 3.231091215 & 3.241886695 & -1.614547547 \\ \text { H } & 1.247813759 & 1.740838037 & 0.173588273 \\ \text { H } & 1.269830951 & -2.048526735 & 0.408974092 \\ \text { H } & 4.787851257 & 0.411881947 & 0.281376402 \\ \text { H } & 3.405027204 & 0.780927928 & -1.735572527 \\ \text { C } & 3.682818473 & -1.653258027 & 1.774329977 \\ \text { H } & 3.332367808 & -2.675951964 & 1.881804305 \\ \text { H } & 4.768271752 & -1.669867398 & 1.815921409 \\ \text { H } & 3.326198829 & -1.088755387 & 2.630446779 \\ \text { C } & 3.742804292 & -1.892000206 & -0.698911328 \\ \text { H } & 4.828323358 & -1.915327535 & -0.670062749 \\ \text { H } & 3.389900895 & -2.916050990 & -0.617946833 \\ \text { H } & 3.455261106 & -1.526126982 & -1.677467442 \\ \text { H } & 1.349467158 & -0.690401808 & -1.624654256\end{array}$

\begin{tabular}{lrrr}
\multicolumn{1}{l}{$\mathrm{T}_{1} / \mathrm{S}_{0}-\mathrm{KETO}$} \\
$\mathrm{C}$ & -4.394672466 & -1.243125111 & -1.195853205 \\
$\mathrm{C}$ & -3.082864233 & -0.674589336 & -1.220075325 \\
$\mathrm{C}$ & -2.601763996 & 0.115796158 & -0.092671695 \\
$\mathrm{C}$ & -3.529845025 & 0.628822875 & 0.772969335 \\
$\mathrm{C}$ & -4.933608495 & 0.573871073 & 0.264508639 \\
$\mathrm{C}$ & -5.306330511 & -0.769842373 & -0.275912901 \\
$\mathrm{H}$ & -4.606068262 & -2.090668221 & -1.824387663 \\
$\mathrm{H}$ & -2.370078798 & -1.012423691 & -1.944929811 \\
$\mathrm{H}$ & -5.106573947 & 1.358698618 & -0.466017515 \\
$\mathrm{H}$ & -6.224336238 & -1.267773029 & -0.029294390 \\
$\mathrm{C}$ & -1.156201562 & 0.244609426 & 0.211809036 \\
$\mathrm{O}$ & -0.751722503 & 0.710382049 & 1.242624330 \\
$\mathrm{O}$ & -3.315842507 & 1.190786703 & 1.960819660 \\
$\mathrm{H}$ & -2.378328111 & 1.181196683 & 2.145012417 \\
$\mathrm{O}$ & -0.385024350 & -0.184423976 & -0.757470506
\end{tabular}




$\begin{array}{lrrr}\text { C } & 1.055847976 & -0.137517392 & -0.709814390 \\ \mathrm{C} & 1.624543097 & -1.034355045 & 0.385352667 \\ \mathrm{C} & 1.594111733 & 1.286052790 & -0.639451866 \\ \mathrm{C} & 3.168930340 & -1.076029119 & 0.365428874 \\ \mathrm{H} & 1.293422155 & -0.663955218 & 1.346968278 \\ \mathrm{C} & 3.126852446 & 1.275297929 & -0.727807962 \\ \mathrm{H} & 1.172706955 & 1.869711086 & -1.453591265 \\ \mathrm{C} & 3.713551029 & 0.368471199 & 0.363949278 \\ \mathrm{H} & 3.495923764 & 0.820017468 & 1.330732125 \\ \mathrm{C} & 3.690421665 & 2.693814432 & -0.627867097 \\ \mathrm{H} & 3.437562048 & 3.147605476 & 0.326391966 \\ \mathrm{H} & 4.772533703 & 2.691894926 & -0.718426131 \\ \mathrm{H} & 3.293112746 & 3.329983108 & -1.413233530 \\ \mathrm{H} & 1.286774752 & 1.750667324 & 0.289436179 \\ \mathrm{H} & 1.223926349 & -2.038144077 & 0.269265271 \\ \mathrm{H} & 4.797275724 & 0.343214177 & 0.274136400 \\ \mathrm{H} & 3.409998004 & 0.879439421 & -1.701297214 \\ \mathrm{C} & 3.652624064 & -1.791178610 & 1.635104348 \\ \mathrm{H} & 3.279314524 & -2.810664732 & 1.675668033 \\ \mathrm{H} & 4.737654752 & -1.834903182 & 1.666959818 \\ \mathrm{H} & 3.313802820 & -1.277618414 & 2.529557824 \\ \mathrm{C} & 3.690259854 & -1.863689562 & -0.849645763 \\ \mathrm{H} & 4.775114093 & -1.913502890 & -0.832412148 \\ \mathrm{H} & 3.314179547 & -2.882642326 & -0.834286362 \\ \mathrm{H} & 3.403655857 & -1.425685325 & -1.798645211 \\ \mathrm{H} & 1.305493884 & -0.551045557 & -1.676330642 \\ & & & \\ \mathrm{~S} / \mathrm{S} \mathrm{-}-\mathrm{ENOL} & & & \\ \mathrm{C} & -4.553591829 & -1.194345986 & -1.149229080 \\ \mathrm{C} & -3.155119126 & -1.173478882 & -0.897882566 \\ \mathrm{C} & -2.643903688 & -0.162056511 & -0.156236111 \\ \mathrm{C} & -3.458468847 & 0.897796263 & 0.281098420 \\ \mathrm{C} & -4.828087847 & 0.925267965 & 0.035463205 \\ \mathrm{C} & -5.366146289 & -0.186953316 & -0.649135148 \\ \mathrm{H} & -4.981174957 & -1.980411442 & -1.745283226 \\ \mathrm{H} & -2.518129479 & -1.932408038 & -1.307876996 \\ \mathrm{H} & -5.435744691 & 1.756032726 & 0.349328725 \\ \mathrm{H} & -6.423253860 & -0.225394318 & -0.831594961 \\ \mathrm{C} & -1.216906188 & 0.090414209 & 0.226675318 \\ \mathrm{O} & -0.90811065 & -0.072999705 & 1.540595508 \\ \mathrm{O} & -2.733253197 & 1.850131332 & 0.880772165 \\ \mathrm{H} & -1.371793898 & 0.595622141 & 2.032508344 \\ \mathrm{O} & -0.370301226 & -0.503921535 & -0.619761842 \\ \mathrm{C} & 1.041073273 & -0.316442423 & -0.612827742 \\ \mathrm{C} & 1.741375125 & -1.178744084 & 0.436961430 \\ \mathrm{C} & 1.442473404 & 1.146798982 & -0.515611536 \\ \mathrm{C} & 3.281751036 & -1.056323574 & 0.364294127\end{array}$




\begin{tabular}{|c|c|c|c|}
\hline $\mathrm{H}$ & 1.404283402 & -0.878009764 & 1.421242104 \\
\hline C & 2.958750011 & 1.300195994 & -0.668628848 \\
\hline $\mathrm{H}$ & 0.923805568 & 1.711714825 & -1.285036891 \\
\hline C & 3.680287365 & 0.436055431 & 0.374873529 \\
\hline & 3.460477651 & 0.843734562 & 1.360893196 \\
\hline & 3.373948545 & 2.768072002 & -0.568266339 \\
\hline & 3.117177022 & 3.178882730 & 0.404447827 \\
\hline & 4.444439221 & 2.885587605 & -0.707407052 \\
\hline $\mathrm{H}$ & 2.872868105 & 3.366839144 & -1.323372095 \\
\hline $\mathrm{H}$ & 1.133177880 & 1.547927010 & 0.446210332 \\
\hline F & 1.441234103 & -2.214885457 & 0.30 \\
\hline ト & 4.756419541 & 0.522687340 & 0.240475017 \\
\hline F & 3.232123931 & 0.950754369 & -1.66 \\
\hline & 3.888997381 & -1.738092226 & 1.59 \\
\hline $\mathrm{H}$ & 3.630554764 & -2.792854801 & 1.6317 \\
\hline $\mathrm{H}$ & 4.973198658 & -1.663334620 & 1.58 \\
\hline $\mathrm{H}$ & 3.533552155 & -1.281072793 & 2.517918 \\
\hline$u$ & 45676 & 18027 & $-0.8 \varepsilon$ \\
\hline $\mathrm{H}$ & 4.923554153 & -1.699268222 & -0.904636863 \\
\hline ト & 52558 & -2.817732905 & -0.87 \\
\hline $\mathrm{H}$ & 3.472119752 & -1.345485295 & -1.813263230 \\
\hline & 1.310278995 & -0.6793 & \\
\hline \multicolumn{4}{|c|}{$\mathrm{T}_{1} / \mathrm{S}_{0}-\mathrm{ENOL}$} \\
\hline$C$ & -4.495464523 & -0.996443469 & 37896 \\
\hline C & -3.087359324 & -0.950532570 & -0.981666771 \\
\hline C & -2.622083090 & -0.034742804 & -0.113 \\
\hline C & -3.560264954 & 0.856322964 & 0.556964442 \\
\hline 乙 & -4.945807856 & 0.797578955 & 0.19 \\
\hline C & -5.390407287 & -0.122545708 & -0.767865055 \\
\hline $\mathrm{H}$ & -4.807617043 & -1.667810920 & -2.123584554 \\
\hline $\mathrm{H}$ & -2.401913371 & -1.608287613 & -1.478954307 \\
\hline $\mathrm{H}$ & -5.623177902 & 1.465354108 & 0.690071348 \\
\hline $\mathrm{H}$ & -6.427470164 & -0.136104611 & -1.051407374 \\
\hline C & -1.153240382 & 0.112105507 & 0.266691220 \\
\hline 0 & -0.910929765 & -0.119889810 & 1.587416815 \\
\hline 0 & -3.168147629 & 1.618934718 & 1.467997306 \\
\hline $\mathrm{H}$ & -1.416081303 & 0.515564439 & 2.082896152 \\
\hline 0 & -0.362216323 & -0.592675841 & -0.557721842 \\
\hline C & 1.049606378 & -0.387277830 & -0.599764641 \\
\hline C & 1.776584230 & -1.180965983 & 0.484437937 \\
\hline C & 1.438263302 & 1.085965078 & -0.577389842 \\
\hline C & 3.313277141 & -1.049885678 & 0.393835527 \\
\hline $\mathrm{H}$ & 1.443169304 & -0.829637661 & 1.453200505 \\
\hline 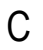 & 2.954188112 & 1.247745088 & -0.750110555 \\
\hline $\mathrm{H}$ & 0.907063664 & 1.612646415 & -1.365387900 \\
\hline & 3.695169767 & 0.444709431 & 0.327964024 \\
\hline
\end{tabular}




$\begin{array}{lrrr}\text { H } & 3.479238149 & 0.899358463 & 1.294042594 \\ \mathrm{C} & 3.358345542 & 2.722691994 & -0.718807350 \\ \mathrm{H} & 3.107372326 & 3.176899728 & 0.235978916 \\ \mathrm{H} & 4.427388119 & 2.839257904 & -0.870712865 \\ \mathrm{H} & 2.849693306 & 3.284744337 & -1.496634209 \\ \mathrm{H} & 1.136682584 & 1.531534009 & 0.367304527 \\ \mathrm{H} & 1.486742209 & -2.225729161 & 0.411713129 \\ \mathrm{H} & 4.769329942 & 0.535620040 & 0.180597410 \\ \mathrm{H} & 3.227261231 & 0.855862860 & -1.728154923 \\ \mathrm{C} & 3.935421085 & -1.665281919 & 1.655545221 \\ \mathrm{H} & 3.683897265 & -2.718670697 & 1.741616425 \\ \mathrm{H} & 5.018928960 & -1.584462016 & 1.634661019 \\ \mathrm{H} & 3.581703359 & -1.165857782 & 2.552361284 \\ \mathrm{C} & 3.865784878 & -1.811768331 & -0.824211160 \\ \mathrm{H} & 4.949089258 & -1.737796823 & -0.861034160 \\ \mathrm{H} & 3.610255562 & -2.865724805 & -0.762190957 \\ \mathrm{H} & 3.484934551 & -1.440752605 & -1.768571528 \\ \mathrm{H} & 1.309123595 & -0.793495660 & -1.568577015\end{array}$

$S_{0}$ state minimum-energy proton transfer path 1.9 angstrom

$\begin{array}{lrrr}\text { C } & -4.353296986 & -0.892718616 & -1.519764319 \\ \text { C } & -3.009458654 & -0.690621967 & -1.248352811 \\ \text { C } & -2.595716437 & -0.036108013 & -0.074326527 \\ \text { C } & -3.570223387 & 0.416338599 & 0.831222817 \\ \text { C } & -4.913443725 & 0.210719693 & 0.553122207 \\ \text { C } & -5.307989413 & -0.435488772 & -0.606656831 \\ \text { H } & -4.654575316 & -1.394575169 & -2.420762464 \\ \text { H } & -2.263179612 & -1.034073576 & -1.936354700 \\ \text { H } & -5.633609706 & 0.568373291 & 1.265270560 \\ \text { H } & -6.355427688 & -0.582847162 & -0.799257130 \\ \text { C } & -1.153916151 & 0.174371640 & 0.207157637 \\ \text { O } & -0.749944653 & 0.727831685 & 1.199209247 \\ \text { O } & -3.286516668 & 1.049884333 & 1.976034305 \\ \text { H } & -2.344481029 & 1.142190541 & 2.076648289 \\ \text { O } & -0.365321229 & -0.303890931 & -0.735529843 \\ \text { C } & 1.070074938 & -0.222262557 & -0.691951631 \\ \text { C } & 1.659974451 & -1.031863393 & 0.459133073 \\ \text { C } & 1.583692243 & 1.212218435 & -0.721552452 \\ \text { C } & 3.204743712 & -1.047668515 & 0.435249409 \\ \text { H } & 1.328436406 & -0.604575164 & 1.397099429 \\ \text { C } & 3.116136136 & 1.223198191 & -0.815065411 \\ \text { H } & 1.149215152 & 1.730866596 & -1.571920965 \\ \text { C } & 3.723014068 & 0.402811749 & 0.332541873 \\ \text { H } & 3.501277693 & 0.915125754 & 1.267605491 \\ \text { C } & 3.654532192 & 2.654976161 & -0.813425819 \\ \text { H } & 3.399136927 & 3.167289409 & 0.110065687\end{array}$




$\begin{array}{llll}\mathrm{H} & 4.735983192 & 2.666339364 & -0.910920324 \\ \mathrm{H} & 3.241745135 & 3.230486815 & -1.636676856 \\ \mathrm{H} & 1.273230164 & 1.737329137 & 0.173708703 \\ \mathrm{H} & 1.276276812 & -2.047752845 & 0.413894329 \\ \mathrm{H} & 4.806640346 & 0.390990299 & 0.239587518 \\ \mathrm{H} & 3.403350374 & 0.767953253 & -1.760870867 \\ \mathrm{C} & 3.705967824 & -1.665456309 & 1.748547244 \\ \mathrm{H} & 3.350263873 & -2.685749892 & 1.861514596 \\ \mathrm{H} & 4.791663344 & -1.688976693 & 1.777748817 \\ \mathrm{H} & 3.362692442 & -1.097403789 & 2.607705128 \\ \mathrm{C} & 3.735888310 & -1.907570728 & -0.725359385 \\ \mathrm{H} & 4.821505306 & -1.936543511 & -0.710302112 \\ \mathrm{H} & 3.378826215 & -2.929698773 & -0.638193414 \\ \mathrm{H} & 3.437888916 & -1.541639332 & -1.700849551 \\ \mathrm{H} & 1.331277474 & -0.695253432 & -1.627701158\end{array}$

\section{8 angstrom}

$\begin{array}{lrrr}\text { C } & -4.367238483 & -0.887065395 & -1.518982772 \\ \text { C } & -3.019390200 & -0.694306062 & -1.259687089 \\ \text { C } & -2.595037979 & -0.044476924 & -0.087772339 \\ \text { C } & -3.557473465 & 0.412698849 & 0.827542342 \\ \text { C } & -4.904534174 & 0.216684383 & 0.562223119 \\ \text { C } & -5.311158734 & -0.425356351 & -0.596405054 \\ \text { H } & -4.680409686 & -1.385080962 & -2.418040244 \\ \text { H } & -2.280346614 & -1.040669463 & -1.954334730 \\ \text { H } & -5.617047426 & 0.577285753 & 1.280520290 \\ \text { H } & -6.360900936 & -0.566126479 & -0.781271787 \\ \text { C } & -1.154890753 & 0.164409111 & 0.193948256 \\ \text { O } & -0.757876056 & 0.717868690 & 1.189832126 \\ \text { O } & -3.244728144 & 1.040696837 & 1.966246441 \\ \text { H } & -2.295531445 & 1.115830968 & 2.036733877 \\ \text { O } & -0.362556225 & -0.311654004 & -0.745136495 \\ \text { C } & 1.073111918 & -0.226797828 & -0.696877552 \\ \text { C } & 1.660831628 & -1.034809502 & 0.456372995 \\ \text { C } & 1.582733543 & 1.209088748 & -0.724178192 \\ \text { C } & 3.205813481 & -1.046371327 & 0.438051403 \\ \text { H } & 1.324867698 & -0.608081286 & 1.392995972 \\ \text { C } & 3.115412264 & 1.223837516 & -0.813154097 \\ \text { H } & 1.149345653 & 1.727169664 & -1.575413075 \\ \text { C } & 3.720702929 & 0.405495799 & 0.336809735 \\ \text { H } & 3.494776539 & 0.917563086 & 1.270983912 \\ \text { C } & 3.650333721 & 2.656886591 & -0.811268718 \\ \text { H } & 3.389294495 & 3.170191756 & 0.110069429 \\ \text { H } & 4.732187743 & 2.670651021 & -0.903663653 \\ \text { H } & 3.240108003 & 3.229910451 & -1.637515999 \\ \text { H } & 1.268288850 & 1.732642674 & 0.170618202 \\ \text { H } & 1.280045295 & -2.051713986 & 0.410091137\end{array}$




$\begin{array}{lrrr}\mathrm{H} & 4.804614656 & 0.396301360 & 0.247217217 \\ \mathrm{H} & 3.406333436 & 0.768550837 & -1.757769058 \\ \mathrm{C} & 3.703765108 & -1.662676768 & 1.753273877 \\ \mathrm{H} & 3.351249810 & -2.684253183 & 1.864472697 \\ \mathrm{H} & 4.789386496 & -1.682333200 & 1.786940610 \\ \mathrm{H} & 3.354918993 & -1.096094361 & 2.611135995 \\ \mathrm{C} & 3.743573139 & -1.904933616 & -0.720512110 \\ \mathrm{H} & 4.829156029 & -1.931589089 & -0.700827500 \\ \mathrm{H} & 3.388324624 & -2.927824962 & -0.635142178 \\ \mathrm{H} & 3.449003628 & -1.539342178 & -1.697161662 \\ \mathrm{H} & 1.338146427 & -0.699068668 & -1.631853109\end{array}$

\section{7 angstrom}

$\begin{array}{lrrr}\text { C } & -4.386758610 & -0.886830419 & -1.514498762 \\ \text { C } & -3.033557317 & -0.703587815 & -1.275293066 \\ \text { C } & -2.593917483 & -0.055202421 & -0.109480232 \\ \text { C } & -3.538338357 & 0.410057365 & 0.819660792 \\ \text { C } & -4.890635868 & 0.223966190 & 0.574966247 \\ \text { C } & -5.314665804 & -0.417286001 & -0.578790770 \\ \text { H } & -4.717219784 & -1.383597683 & -2.408036963 \\ \text { H } & -2.305221141 & -1.055486167 & -1.978936905 \\ \text { H } & -5.592009039 & 0.589419554 & 1.301720696 \\ \text { H } & -6.367512927 & -0.551950873 & -0.750298238 \\ \text { C } & -1.156359500 & 0.153727187 & 0.171665290 \\ \text { O } & -0.770874835 & 0.709962586 & 1.172480626 \\ \text { O } & -3.183447498 & 1.033483342 & 1.945706543 \\ \text { H } & -2.224525341 & 1.084623259 & 1.970308930 \\ \text { O } & -0.357575947 & -0.318308196 & -0.760965938 \\ \text { C } & 1.078412618 & -0.229590032 & -0.704644334 \\ \text { C } & 1.661127634 & -1.036264327 & 0.451870168 \\ \text { C } & 1.583831486 & 1.207697250 & -0.727708286 \\ \text { C } & 3.206211343 & -1.045041637 & 0.440751688 \\ \text { H } & 1.319992795 & -0.609225870 & 1.386562251 \\ \text { C } & 3.116932743 & 1.226018515 & -0.809506277 \\ \text { H } & 1.153107479 & 1.725965362 & -1.580139691 \\ \text { C } & 3.718666422 & 0.407924733 & 0.342546398 \\ \text { H } & 3.487492181 & 0.918914457 & 1.276014774 \\ \text { C } & 3.648546647 & 2.660285766 & -0.803632452 \\ \text { H } & 3.383084817 & 3.171627583 & 0.117523488 \\ \text { H } & 4.730677947 & 2.676541345 & -0.892129245 \\ \text { H } & 3.240014895 & 3.233600161 & -1.630505411 \\ \text { H } & 1.263945220 & 1.728966300 & 0.166553475 \\ \text { H } & 1.282171471 & -2.053764130 & 0.404429258 \\ \text { H } & 4.802970486 & 0.400981986 & 0.257881880 \\ \text { H } & 3.413175772 & 0.772347583 & -1.753238851 \\ \text { C } & 3.699105348 & -1.660895822 & 1.758086347 \\ \text { H } & 3.347429605 & -2.682934759 & 1.867582396\end{array}$




$\begin{array}{llll}\mathrm{H} & 4.784579508 & -1.679151691 & 1.796457444 \\ \mathrm{H} & 3.345807920 & -1.094882095 & 2.614491413 \\ \mathrm{C} & 3.750865937 & -1.902246581 & -0.715584946 \\ \mathrm{H} & 4.836384283 & -1.926622474 & -0.691007933 \\ \mathrm{H} & 3.397395299 & -2.925893787 & -0.631960949 \\ \mathrm{H} & 3.460001196 & -1.537134604 & -1.693530432 \\ \mathrm{H} & 1.349326905 & -0.700637081 & -1.638406250\end{array}$

$\begin{array}{lrrr}\text { 1.6 angstrom } & & \\ \mathrm{C} & -4.408040340 & -0.881840235 & -1.508926959 \\ \mathrm{C} & -3.049458649 & -0.711004287 & -1.290943774 \\ \mathrm{C} & -2.592169738 & -0.066572181 & -0.130779405 \\ \mathrm{C} & -3.516453195 & 0.407471541 & 0.813837942 \\ \mathrm{C} & -4.874433593 & 0.233742422 & 0.590429225 \\ \mathrm{C} & -5.317947237 & -0.404173909 & -0.558640690 \\ \mathrm{H} & -4.757422036 & -1.375200223 & -2.397131271 \\ \mathrm{H} & -2.333494129 & -1.068596660 & -2.004816467 \\ \mathrm{H} & -5.563199318 & 0.604368558 & 1.326512333 \\ \mathrm{H} & -6.374085647 & -0.530443718 & -0.716001044 \\ \mathrm{C} & -1.156775524 & 0.139889017 & 0.147862617 \\ \mathrm{O} & -0.781012802 & 0.697750048 & 1.153698734 \\ \mathrm{O} & -3.118329052 & 1.023682196 & 1.925984228 \\ \mathrm{H} & -2.149920001 & 1.049496679 & 1.903610688 \\ \mathrm{O} & -0.352666764 & -0.328772771 & -0.778646978 \\ \mathrm{C} & 1.083851036 & -0.236233736 & -0.715529975 \\ \mathrm{C} & 1.661759863 & -1.041622453 & 0.444117520 \\ \mathrm{C} & 1.583803624 & 1.202875705 & -0.735838851 \\ \mathrm{C} & 3.206927934 & -1.043448633 & 0.444289175 \\ \mathrm{H} & 1.311840000 & -0.617446343 & 1.376845805 \\ \mathrm{C} & 3.117406886 & 1.227487130 & -0.805869724 \\ \mathrm{H} & 1.157567670 & 1.718756285 & -1.591938269 \\ \mathrm{C} & 3.713899323 & 0.411703144 & 0.350503318 \\ \mathrm{H} & 3.474027393 & 0.921452766 & 1.282437837 \\ \mathrm{C} & 3.642617978 & 2.664082211 & -0.795797893 \\ \mathrm{H} & 3.366452220 & 3.174669876 & 0.122607728 \\ \mathrm{H} & 4.725416518 & 2.684999133 & -0.874354351 \\ \mathrm{H} & 3.239229225 & 3.235187200 & -1.626701775 \\ \mathrm{H} & 1.254975205 & 1.723534057 & 0.155522140 \\ \mathrm{H} & 1.287832106 & -2.060755984 & 0.392544329 \\ \mathrm{H} & 4.798767004 & 0.409157468 & 0.273505198 \\ \mathrm{H} & 3.422730332 & 0.775286057 & -1.747388071 \\ \mathrm{C} & 3.692610268 & -1.657946866 & 1.764904357 \\ \mathrm{H} & 3.345553820 & -2.681944700 & 1.870672093 \\ \mathrm{H} & 4.777811214 & -1.670441323 & 1.811784497 \\ \mathrm{H} & 3.329571270 & -1.094635282 & 2.618998860 \\ \mathrm{C} & 3.763614346 & -1.897557837 & -0.708593541 \\ \mathrm{H} & 4.848962874 & -1.918210879 & -0.675434538\end{array}$




$\begin{array}{llll}\mathrm{H} & 3.413044430 & -2.922500417 & -0.628905173 \\ \mathrm{H} & 3.479267449 & -1.532306852 & -1.688425163 \\ \mathrm{H} & 1.359931861 & -0.706558923 & -1.648048309\end{array}$

1.5 angstrom

\begin{tabular}{|c|c|c|c|}
\hline & -4.428280984 & -0.879033807 & -1.50350143 \\
\hline & -3.065119030 & -0.719275349 & -1.3( \\
\hline & -2.591012369 & -0.077672324 & -0.152178999 \\
\hline & -3.495213994 & 0.404988948 & 0.807602705 \\
\hline & -4.858668106 & 0.241925799 & 0.6044547 \\
\hline & -5.320594520 & -0.393540807 & -0.5393108 \\
\hline & -4.795801558 & -1.369698536 & -2.385847725 \\
\hline & -2.361297276 & -1.082307098 & -2.030022878 \\
\hline & -5.535340104 & 0.617412586 & 190494 \\
\hline & -6.379636959 & -0.512420253 & -0.682700803 \\
\hline & -1.158146807 & 0.127028517 & 0.123448527 \\
\hline & -0.792508326 & 0.687104209 & 1.134551101 \\
\hline & -3.056612046 & 1.014029178 & 1.904095087 \\
\hline & -2.077500523 & 1.015284942 & 1.835331329 \\
\hline & -0.347667421 & -0.337262267 & -0.795769291 \\
\hline & 1.089462458 & -0.240579043 & -0.724603026 \\
\hline & 1.662108406 & -1.044733354 & 0.438264830 \\
\hline & 1.584305163 & 1.200150294 & -0.741141974 \\
\hline & 3.207296792 & -1.041905685 & 0.447545911 \\
\hline & 1.305410972 & -0.621660573 & 1.368960582 \\
\hline & 3.118267762 & 1.229462041 & -0.8021212 \\
\hline & 1.16 & 1.71 & -1. \\
\hline & 3.710473218 & 0.414801164 & 0.357264209 \\
\hline & 3.463971385 & 0.923429466 & 1.288065770 \\
\hline & 3.638783576 & 2.667726454 & -0.788089876 \\
\hline & 3.355652035 & 3.17 & 0.1 \\
\hline & 4.721932081 & 2.692036539 & -0.860296953 \\
\hline & 3.238490086 & 3.238058929 & -1.621002610 \\
\hline & 1.248659273 & 1.719018032 & 0.148754529 \\
\hline & 1.291454647 & -2.06489 & 0.384206353 \\
\hline & 4.795725055 & 0.415395033 & 0.286302614 \\
\hline & 3.430407016 & 0.778817119 & -1.742132367 \\
\hline & 3.686768797 & -1.655447657 & 1.770862702 \\
\hline & 3.342190242 & -2.680521489 & 1.874149766 \\
\hline & 738 & -1.664 & 1.824220835 \\
\hline & 3.316920928 & -1.093551204 & 2.622952275 \\
\hline & 3.773184765 & -1.893986505 & -0.702341894 \\
\hline & 4.858340117 & -1.911716239 & -0.662569620 \\
\hline & 3. & -2.91 & -0.625250141 \\
\hline & 3.493890051 & -1.529037568 & -1.683758709 \\
\hline & & -0.70977 & \\
\hline
\end{tabular}




$\begin{array}{lrrr}1.4 \text { angstrom } & & \\ 6 & -4.447852329 & -0.876009093 & -1.497641159 \\ 6 & -3.080781931 & -0.727429674 & -1.321385045 \\ 6 & -2.589640069 & -0.088419570 & -0.172868893 \\ 6 & -3.472979241 & 0.403390637 & 0.802442121 \\ 6 & -4.842317513 & 0.250539127 & 0.618446535 \\ 6 & -5.322401354 & -0.382386571 & -0.519582218 \\ 1 & -4.833435596 & -1.363909485 & -2.373776811 \\ 1 & -2.389379263 & -1.096035384 & -2.054457733 \\ 1 & -5.506782308 & 0.630974701 & 1.371544883 \\ 1 & -6.384137358 & -0.493742831 & -0.648993752 \\ 6 & -1.159789056 & 0.113483961 & 0.098037732 \\ 8 & -0.802622560 & 0.675928610 & 1.114389844 \\ 8 & -2.996904067 & 1.004414323 & 1.881642482 \\ 1 & -2.002958362 & 0.981399368 & 1.766986188 \\ 8 & -0.342886966 & -0.346165055 & -0.813078949 \\ 6 & 1.095166428 & -0.245362827 & -0.733953493 \\ 6 & 1.662375608 & -1.048453814 & 0.432031371 \\ 6 & 1.584638142 & 1.197012384 & -0.747200948 \\ 6 & 3.207525296 & -1.040395873 & 0.450845490 \\ 1 & 1.298521434 & -0.627042443 & 1.360734866 \\ 6 & 3.118927493 & 1.231330343 & -0.798280195 \\ 1 & 1.165524094 & 1.710828702 & -1.607937303 \\ 6 & 3.706285151 & 0.418073702 & 0.364549371 \\ 1 & 3.452148602 & 0.925400455 & 1.293992100 \\ 6 & 3.634269233 & 2.671402235 & -0.780212104 \\ 1 & 3.343393507 & 3.179113044 & 0.135203861 \\ 1 & 4.717750517 & 2.699379085 & -0.845299689 \\ 1 & 3.237531244 & 3.240690236 & -1.615513190 \\ 1 & 1.241698460 & 1.714517676 & 0.140725815 \\ 1 & 1.295399669 & -2.069728640 & 0.374968379 \\ 1 & 4.791920561 & 0.422320450 & 0.300512997 \\ 1 & 3.438537089 & 0.782238165 & -1.736493375 \\ 6 & 3.680628055 & -1.653145111 & 1.776810204 \\ 1 & 3.339024133 & -2.679479163 & 1.877254365 \\ 1 & 4.765183193 & -1.658582307 & 1.836940578 \\ 1 & 3.303492595 & -1.093095588 & 2.626903646 \\ 6 & 3.783168773 & -1.889948687 & -0.696068392 \\ 1 & 4.868066395 & -1.904485069 & -0.649352587 \\ 1 & 3.437483349 & -2.916942130 & -0.621956912 \\ 1 & 3.509156798 & -1.525092710 & -1.679030579 \\ 1 & 1.381966717 & -0.713605432 & -1.663938555\end{array}$

1.3 angstrom

$\begin{array}{llll}\text { C } & -4.466925714 & -0.873243502 & -1.492540330 \\ \text { C } & -3.097126348 & -0.734792001 & -1.334855892 \\ \text { C } & -2.590402037 & -0.097893584 & -0.190637517\end{array}$




$\begin{array}{lrrr}\text { C } & -3.453200136 & 0.402626045 & 0.799656040 \\ \text { C } & -4.829738303 & 0.258187199 & 0.631355190 \\ \text { C } & -5.325541259 & -0.372036721 & -0.501256487 \\ \text { H } & -4.869083505 & -1.358291131 & -2.362748905 \\ \text { H } & -2.417008856 & -1.108231237 & -2.076321098 \\ \text { H } & -5.482838693 & 0.643007017 & 1.391995382 \\ \text { H } & -6.389522842 & -0.476594689 & -0.618232906 \\ \text { C } & -1.165106890 & 0.100236855 & 0.073987187 \\ \text { O } & -0.813851870 & 0.665281278 & 1.096084883 \\ \text { O } & -2.946941950 & 0.994730078 & 1.860800249 \\ \text { H } & -1.928340485 & 0.948945641 & 1.702261465 \\ \text { O } & -0.340876060 & -0.353573525 & -0.827138574 \\ \text { C } & 1.098514394 & -0.248466800 & -0.739998623 \\ \text { C } & 1.660496892 & -1.050484082 & 0.428883068 \\ \text { C } & 1.582618389 & 1.195458317 & -0.750072883 \\ \text { C } & 3.205603287 & -1.038278767 & 0.455257596 \\ \text { H } & 1.291130463 & -0.629838382 & 1.355792007 \\ \text { C } & 3.117160189 & 1.233869351 & -0.793305151 \\ \text { H } & 1.166328835 & 1.708503026 & -1.612551046 \\ \text { C } & 3.700667183 & 0.421654877 & 0.372213778 \\ \text { H } & 3.440381235 & 0.927839333 & 1.300557417 \\ \text { C } & 3.628231554 & 2.675396582 & -0.772027119 \\ \text { H } & 3.331750322 & 3.181776536 & 0.142300890 \\ \text { H } & 4.711886376 & 2.706282181 & -0.832085159 \\ \text { H } & 3.233848519 & 3.244002245 & -1.608875582 \\ \text { H } & 1.233932276 & 1.711382724 & 0.136567487 \\ \text { H } & 1.296283982 & -2.072564374 & 0.369880456 \\ \text { H } & 4.786545978 & 0.428932096 & 0.313747016 \\ \text { H } & 3.442605822 & 0.786089734 & -1.730099834 \\ \text { C } & 3.673482199 & -1.650496844 & 1.783298029 \\ \text { H } & 3.333870438 & -2.677677727 & 1.881572266 \\ \text { H } & 4.757707352 & -1.653351962 & 1.848647397 \\ \text { H } & 3.290910369 & -1.091736396 & 2.631791064 \\ \text { C } & 3.789039000 & -1.885741553 & -0.689253864 \\ \text { H } & 4.873692062 & -1.897201230 & -0.637218992 \\ \text { H } & 3.445930219 & -2.913724547 & -0.617236010 \\ \text { H } & 3.518931877 & -1.521276072 & -1.673458755 \\ \text { H } & 1.389870302 & -0.715726263 & -1.668793195 \\ & & & \end{array}$

1.2 angstrom

$\begin{array}{llll}\text { C } & -4.482465607 & -0.872283686 & -1.490199477 \\ \text { C } & -3.111482842 & -0.742429388 & -1.348770925 \\ \text { C } & -2.590060966 & -0.104781604 & -0.203754276 \\ \text { C } & -3.430228091 & 0.403100034 & 0.799261035 \\ \text { C } & -4.821991598 & 0.263910055 & 0.642043819 \\ \text { C } & -5.329176034 & -0.363290643 & -0.484615238 \\ \text { H } & -4.899863809 & -1.354611322 & -2.354622531\end{array}$




\begin{tabular}{|c|c|c|c|}
\hline & 2.440654284 & -1.119523361 & \\
\hline & .463724848 & 0.652782132 & \\
\hline & -6.394859050 & -0.462723249 & -0 \\
\hline & -1.172972856 & 0.087051002 & $9635^{\prime}$ \\
\hline & -0.821458656 & 0.655555328 & 785102 \\
\hline & -2.909171024 & 73 & 1. \\
\hline & $-1 . \varepsilon$ & & \\
\hline & 929489 & 9466549 & \\
\hline & 342307 & -0.250014310 & \\
\hline & & & \\
\hline & 1.58037 & 1.1 & -0.1 \\
\hline & 3.203373572 & -1.036317721 & 0.45927 \\
\hline & 1.284974623 & 19007 & \\
\hline & 3.1 & 3594 & \\
\hline & 1.16 & 1.7 & \\
\hline & 3.695992103 & 0.424614201 & 0.37 \\
\hline & 3.431876885 & 0.929994291 & 1.306065 \\
\hline & & & \\
\hline & 3.32 & 3.18 & \\
\hline & 4.706 & 2.711861641 & -0.8 \\
\hline & 3.230355939 & 3.247298755 & -1.60 \\
\hline & 1.227 & 1.70 & \\
\hline & & & \\
\hline & 4.78 & 0.4 & 0.3 \\
\hline & 3.444425668 & 0.789854793 & -1.72474 \\
\hline 0 & 3.667061332 & -1.647983104 & 1.789013 \\
\hline $\mathrm{H}$ & & & \\
\hline & & & \\
\hline & 3.280467 & -1.089985650 & 2.63616 \\
\hline 0 & 3.792348670 & -1.882635849 & -0.683225 \\
\hline H & 4.87 & -1.892394713 & \\
\hline & 3.450 & -2.91 & \\
\hline$F_{-}$ & & & \\
\hline & 1.396 & -0.7161 & \\
\hline
\end{tabular}

1.1 angstrom

$\begin{array}{lrrr}\text { C } & -4.465064777 & -0.876759927 & -1.498027972 \\ \text { C } & -3.112220785 & -0.747192174 & -1.356809440 \\ \text { C } & -2.570289729 & -0.096664776 & -0.190009754 \\ \text { C } & -3.428179392 & 0.421682511 & 0.834020307 \\ \text { C } & -4.850793150 & 0.260193231 & 0.638790892 \\ \text { C } & -5.332722586 & -0.362523945 & -0.480782249 \\ \text { H } & -4.885201223 & -1.358811111 & -2.361270458 \\ \text { H } & -2.437709836 & -1.122593367 & -2.102384353 \\ \text { H } & -5.500195988 & 0.646063317 & 1.401951651 \\ \text { H } & -6.396454085 & -0.470059355 & -0.602312297 \\ \text { C } & -1.190899037 & 0.079024044 & 0.035185032\end{array}$




$\begin{array}{lrrr}\text { O } & -0.790719040 & 0.658059717 & 1.079678838 \\ \text { O } & -2.964992477 & 0.990587927 & 1.855014643 \\ \text { H } & -1.706093243 & 0.919366739 & 1.630867458 \\ \text { O } & -0.344912868 & -0.358877612 & -0.839075559 \\ \text { C } & 1.097891373 & -0.247458517 & -0.739828212 \\ \text { C } & 1.654817566 & -1.050325662 & 0.430168117 \\ \text { C } & 1.577436775 & 1.197397651 & -0.748922282 \\ \text { C } & 3.200020197 & -1.036180527 & 0.459555266 \\ \text { H } & 1.281397089 & -0.630690691 & 1.355410158 \\ \text { C } & 3.112253060 & 1.236548539 & -0.788518203 \\ \text { H } & 1.163073537 & 1.708845150 & -1.613228298 \\ \text { C } & 3.693444940 & 0.424465459 & 0.378318610 \\ \text { H } & 3.429914458 & 0.929935012 & 1.306078820 \\ \text { C } & 3.621769681 & 2.678595664 & -0.765446842 \\ \text { H } & 3.322796653 & 3.184175556 & 0.148474478 \\ \text { H } & 4.705506065 & 2.710430666 & -0.823186610 \\ \text { H } & 3.228655587 & 3.247183632 & -1.602886037 \\ \text { H } & 1.224688296 & 1.712009810 & 0.136313286 \\ \text { H } & 1.292112933 & -2.072720888 & 0.368112379 \\ \text { H } & 4.779442408 & 0.433038586 & 0.322777235 \\ \text { H } & 3.440505120 & 0.789461869 & -1.724659104 \\ \text { C } & 3.665218731 & -1.648318772 & 1.788561744 \\ \text { H } & 3.326280978 & -2.675809022 & 1.885764424 \\ \text { H } & 4.749284798 & -1.650226535 & 1.856309820 \\ \text { H } & 3.280154270 & -1.090074943 & 2.636224945 \\ \text { C } & 3.787227568 & -1.882635489 & -0.683780124 \\ \text { H } & 4.871727470 & -1.892966039 & -0.628905517 \\ \text { H } & 3.445016579 & -2.910988828 & -0.613007741 \\ \text { H } & 3.519520915 & -1.518197843 & -1.668685777 \\ \text { H } & 1.391205723 & -0.714009347 & -1.667910369\end{array}$

1.0 angstrom

$\begin{array}{lrrr}\text { C } & -4.418373034 & -0.888838287 & -1.517300057 \\ \text { C } & -3.077727726 & -0.734653023 & -1.332301448 \\ \text { C } & -2.567408777 & -0.070042213 & -0.139586539 \\ \text { C } & -3.491153109 & 0.437118521 & 0.868955499 \\ \text { C } & -4.911649941 & 0.237446727 & 0.605814773 \\ \text { C } & -5.340428481 & -0.391007106 & -0.526047520 \\ \text { H } & -4.799780600 & -1.377925602 & -2.394564602 \\ \text { H } & -2.375377053 & -1.097555790 & -2.057114393 \\ \text { H } & -5.595335215 & 0.609326313 & 1.345889761 \\ \text { H } & -6.396134224 & -0.521258313 & -0.688104089 \\ \text { C } & -1.218580569 & 0.097787384 & 0.070322058 \\ \text { O } & -0.746261230 & 0.684733135 & 1.121983199 \\ \text { O } & -3.119471265 & 1.008367574 & 1.898980636 \\ \text { H } & -1.522894979 & 0.959580998 & 1.688815495 \\ \text { O } & -0.359264631 & -0.337086675 & -0.800277273\end{array}$




$\begin{array}{lrrr}\text { C } & 1.081767818 & -0.231811846 & -0.711686836 \\ \text { C } & 1.651334489 & -1.039449572 & 0.449426311 \\ \text { C } & 1.574921268 & 1.208781831 & -0.730570034 \\ \text { C } & 3.196970591 & -1.038933873 & 0.453405428 \\ \text { H } & 1.298149872 & -0.618225503 & 1.381856484 \\ \text { C } & 3.109190039 & 1.233356425 & -0.795609374 \\ \text { H } & 1.150915198 & 1.722374995 & -1.588834555 \\ \text { C } & 3.701882084 & 0.417241079 & 0.362624324 \\ \text { H } & 3.457577791 & 0.925956583 & 1.293982818 \\ \text { C } & 3.633284286 & 2.670316835 & -0.783387168 \\ \text { H } & 3.355450953 & 3.180037596 & 0.134965213 \\ \text { H } & 4.716116418 & 2.691505785 & -0.860095707 \\ \text { H } & 3.231142571 & 3.241776700 & -1.614558152 \\ \text { H } & 1.242960262 & 1.729961264 & 0.158865905 \\ \text { H } & 1.278437009 & -2.058439675 & 0.393006544 \\ \text { H } & 4.786924059 & 0.416360623 & 0.290070737 \\ \text { H } & 3.417540532 & 0.781784078 & -1.736313280 \\ \text { C } & 3.679014774 & -1.654103229 & 1.775038731 \\ \text { H } & 3.331875202 & -2.678157405 & 1.879282526 \\ \text { H } & 4.764014881 & -1.666420618 & 1.824389303 \\ \text { H } & 3.313974078 & -1.091315446 & 2.628615561 \\ \text { C } & 3.758197915 & -1.891513728 & -0.698424876 \\ \text { H } & 4.843363596 & -1.910623091 & -0.661531813 \\ \text { H } & 3.408798317 & -2.916917804 & -0.620540405 \\ \text { H } & 3.477077148 & -1.526213323 & -1.679192533 \\ \text { H } & 1.363874221 & -0.700342594 & -1.642309699\end{array}$

$\mathrm{S}_{1}$ state minimum-energy proton transfer path 1.7 angstrom

$\begin{array}{lrrr}\text { C } & -4.448234819 & -0.875158774 & -1.513384860 \\ \text { C } & -3.021068136 & -0.701922643 & -1.284932024 \\ \text { C } & -2.542862605 & -0.058493666 & -0.116164730 \\ \text { C } & -3.551943187 & 0.406985952 & 0.822139548 \\ \text { C } & -4.917795244 & 0.241240726 & 0.604832694 \\ \text { C } & -5.402564690 & -0.406176628 & -0.572854401 \\ \text { H } & -4.770065012 & -1.366851711 & -2.411507625 \\ \text { H } & -2.324941207 & -1.065868699 & -2.010885897 \\ \text { H } & -5.584571702 & 0.618637753 & 1.355374967 \\ \text { H } & -6.454016796 & -0.531060908 & -0.736451903 \\ \text { C } & -1.152636320 & 0.144486969 & 0.166750974 \\ \text { O } & -0.735099754 & 0.702612642 & 1.177478874 \\ \text { O } & -3.195841760 & 1.008255404 & 1.921773909 \\ \text { H } & -2.231766211 & 1.067276215 & 1.964787844 \\ \text { O } & -0.344045504 & -0.334706383 & -0.781596945 \\ \text { C } & 1.081638646 & -0.240226018 & -0.716299801 \\ \text { C } & 1.667179241 & -1.043994495 & 0.442554521 \\ \text { C } & 1.586300336 & 1.198608435 & -0.737637032\end{array}$




$\begin{array}{lrrr}\mathrm{C} & 3.211869269 & -1.042377584 & 0.445991093 \\ \mathrm{H} & 1.312409542 & -0.621850256 & 1.373831908 \\ \mathrm{C} & 3.119319793 & 1.226469707 & -0.806341956 \\ \mathrm{H} & 1.160366891 & 1.712894224 & -1.595325014 \\ \mathrm{C} & 3.716207949 & 0.413614230 & 0.351778165 \\ \mathrm{H} & 3.472016620 & 0.923552039 & 1.282537354 \\ \mathrm{C} & 3.643132370 & 2.663699362 & -0.797348679 \\ \mathrm{H} & 3.364258273 & 3.174930316 & 0.120002096 \\ \mathrm{H} & 4.726260650 & 2.687219714 & -0.874225734 \\ \mathrm{H} & 3.239592855 & 3.233355003 & -1.629385519 \\ \mathrm{H} & 1.253296179 & 1.718969835 & 0.151872881 \\ \mathrm{H} & 1.296504205 & -2.064660180 & 0.389635928 \\ \mathrm{H} & 4.801667133 & 0.413967890 & 0.277898988 \\ \mathrm{H} & 3.427774127 & 0.773418008 & -1.746716138 \\ \mathrm{C} & 3.697814356 & -1.654045268 & 1.767973198 \\ \mathrm{H} & 3.352999064 & -2.678893655 & 1.874417816 \\ \mathrm{H} & 4.783197323 & -1.663780098 & 1.817576252 \\ \mathrm{H} & 3.331051919 & -1.090781347 & 2.620568548 \\ \mathrm{C} & 3.774607500 & -1.895781278 & -0.704520390 \\ \mathrm{H} & 4.860265862 & -1.912340208 & -0.670061658 \\ \mathrm{H} & 3.427724776 & -2.922059512 & -0.623943316 \\ \mathrm{H} & 3.489362892 & -1.532868171 & -1.684866853 \\ \mathrm{H} & 1.366968167 & -0.709741184 & -1.648055299\end{array}$

1.6 angstrom

$\begin{array}{lrrr}\text { C } & -4.473707861 & -0.873493062 & -1.501144440 \\ \text { C } & -3.043137399 & -0.712988099 & -1.302923813 \\ \text { C } & -2.543857564 & -0.073046453 & -0.145894512 \\ \text { C } & -3.533025232 & 0.405498038 & 0.815218878 \\ \text { C } & -4.902377343 & 0.250839255 & 0.623609173 \\ \text { C } & -5.406126048 & -0.395466567 & -0.544845362 \\ \text { H } & -4.818295068 & -1.364330107 & -2.391373833 \\ \text { H } & -2.362691992 & -1.083471208 & -2.040975778 \\ \text { H } & -5.555513330 & 0.633958874 & 1.383301291 \\ \text { H } & -6.460816554 & -0.514512456 & -0.692310530 \\ \text { C } & -1.157440345 & 0.130599379 & 0.135813039 \\ \text { O } & -0.754054873 & 0.693445588 & 1.155655303 \\ \text { O } & -3.123283675 & 0.999710414 & 1.893089708 \\ \text { H } & -2.142186383 & 1.028472123 & 1.877353723 \\ \text { O } & -0.339129945 & -0.342975232 & -0.804129508 \\ \text { C } & 1.086050550 & -0.243076045 & -0.726778733 \\ \text { C } & 1.664126291 & -1.045640046 & 0.436468004 \\ \text { C } & 1.585205653 & 1.197654916 & -0.742106992 \\ \text { C } & 3.208748161 & -1.039624423 & 0.451456812 \\ \text { H } & 1.300687705 & -0.624546356 & 1.364932496 \\ \text { C } & 3.118724067 & 1.230306380 & -0.799140277 \\ \text { H } & 1.164194271 & 1.711747205 & -1.602308895\end{array}$




$\begin{array}{lrrr}\mathrm{C} & 3.709449339 & 0.417949679 & 0.362510424 \\ \mathrm{H} & 3.456945509 & 0.926136006 & 1.291991535 \\ \mathrm{C} & 3.638433303 & 2.668971264 & -0.784935514 \\ \mathrm{H} & 3.351794921 & 3.178517810 & 0.130942137 \\ \mathrm{H} & 4.722008367 & 2.695367985 & -0.854186152 \\ \mathrm{H} & 3.239305072 & 3.238388574 & -1.619250589 \\ \mathrm{H} & 1.243317377 & 1.715328455 & 0.145637781 \\ \mathrm{H} & 1.296707943 & -2.067283767 & 0.380342269 \\ \mathrm{H} & 4.795398923 & 0.421577131 & 0.296554629 \\ \mathrm{H} & 3.435437349 & 0.779104505 & -1.737651866 \\ \mathrm{C} & 3.686833828 & -1.651432779 & 1.776203190 \\ \mathrm{H} & 3.343959340 & -2.677281432 & 1.879118693 \\ \mathrm{H} & 4.771860875 & -1.658431132 & 1.833324424 \\ \mathrm{H} & 3.312696617 & -1.090029667 & 2.626802050 \\ \mathrm{C} & 3.782060840 & -1.890232437 & -0.695917349 \\ \mathrm{H} & 4.867477299 & -1.903703189 & -0.653909068 \\ \mathrm{H} & 3.437558016 & -2.917575058 & -0.618741075 \\ \mathrm{H} & 3.502666630 & -1.527245430 & -1.677920650 \\ \mathrm{H} & 1.380328342 & -0.710632879 & -1.656608841\end{array}$

\begin{tabular}{lrrr}
\multicolumn{4}{l}{1.5 angstrom } \\
C & -4.492547433 & -0.869197704 & -1.491715416 \\
C & -3.060658590 & -0.719898561 & -1.315443644 \\
C & -2.544687573 & -0.084454234 & -0.167809514 \\
C & -3.519468431 & 0.404400757 & 0.812478830 \\
C & -4.890656003 & 0.258363669 & 0.637826646 \\
C & -5.407788389 & -0.385083379 & -0.524368150 \\
H & -4.853961858 & -1.357831366 & -2.376600262 \\
H & -2.392627374 & -1.095451178 & -2.062830867 \\
H & -5.534089277 & 0.645021774 & 1.404019940 \\
H & -6.464792004 & -0.498480465 & -0.660440909 \\
C & -1.161057584 & 0.118474817 & 0.112013467 \\
O & -0.767352393 & 0.685109168 & 1.139887954 \\
O & -3.070296458 & 0.990273622 & 1.872268425 \\
H & -2.071782408 & 0.996122523 & 1.811999158 \\
O & -0.335175885 & -0.350692293 & -0.821296267 \\
C & 1.089643436 & -0.246655585 & -0.735390733 \\
C & 1.662355907 & -1.047823844 & 0.431343473 \\
C & 1.584030102 & 1.195654726 & -0.746416406 \\
C & 3.206833063 & -1.037557134 & 0.455614272 \\
H & 1.291913931 & -0.627419048 & 1.357394826 \\
C & 3.117776993 & 1.232864317 & -0.794341428 \\
H & 1.166491054 & 1.709352170 & -1.608526235 \\
C & 3.703954638 & 0.421468141 & 0.370288467 \\
H & 3.444536176 & 0.928424867 & 1.298519952 \\
C & 3.633183319 & 2.673025730 & -0.776155405 \\
H & 3.339724271 & 3.181130109 & 0.138350047
\end{tabular}




$\begin{array}{llrr}\mathrm{H} & 4.717058729 & 2.702539824 & -0.839029362 \\ \mathrm{H} & 3.237329632 & 3.241836751 & -1.612435188 \\ \mathrm{H} & 1.235112579 & 1.710991726 & 0.139993709 \\ \mathrm{H} & 1.297997252 & -2.070422788 & 0.372968507 \\ \mathrm{H} & 4.790247573 & 0.428188301 & 0.310738903 \\ \mathrm{H} & 3.441273496 & 0.783191086 & -1.731275084 \\ \mathrm{C} & 3.678643672 & -1.648606501 & 1.782951058 \\ \mathrm{H} & 3.338004779 & -2.675439471 & 1.883407315 \\ \mathrm{H} & 4.763320150 & -1.652621178 & 1.846501975 \\ \mathrm{H} & 3.297903647 & -1.088591326 & 2.631520347 \\ \mathrm{C} & 3.789251824 & -1.886135414 & -0.688676701 \\ \mathrm{H} & 4.874419400 & -1.896678048 & -0.640190688 \\ \mathrm{H} & 3.447094795 & -2.914439677 & -0.613949825 \\ \mathrm{H} & 3.514779624 & -1.523508819 & -1.672208500 \\ \mathrm{H} & 1.390394558 & -0.712890302 & -1.663714887\end{array}$

1.4 angstrom

$\begin{array}{lrrr}\text { C } & -4.510618916 & -0.863583361 & -1.480863737 \\ \text { C } & -3.078524731 & -0.724953183 & -1.324681163 \\ \text { C } & -2.545553604 & -0.094958799 & -0.187878662 \\ \text { C } & -3.506844855 & 0.405020921 & 0.813121087 \\ \text { C } & -4.879325381 & 0.266003200 & 0.651859988 \\ \text { C } & -5.408266247 & -0.374470439 & -0.504631108 \\ \text { H } & -4.887215980 & -1.349881229 & -2.360826340 \\ \text { H } & -2.422463054 & -1.105369050 & -2.080842240 \\ \text { H } & -5.514341276 & 0.655598916 & 1.423647024 \\ \text { H } & -6.467387406 & -0.482335595 & -0.630099627 \\ \text { C } & -1.163702195 & 0.105351147 & 0.086798658 \\ \text { O } & -0.775541215 & 0.676374759 & 1.123798970 \\ \text { O } & -3.021064765 & 0.981113670 & 1.852732926 \\ \text { H } & -1.994636012 & 0.964824928 & 1.748782686 \\ \text { O } & -0.331134764 & -0.359745505 & -0.840432523 \\ \text { C } & 1.093300490 & -0.251452006 & -0.745719056 \\ \text { C } & 1.660406000 & -1.051309365 & 0.424586001 \\ \text { C } & 1.582503272 & 1.192596336 & -0.752684113 \\ \text { C } & 3.204634839 & -1.035647534 & 0.459579714 \\ \text { H } & 1.281783383 & -0.632376666 & 1.347956506 \\ \text { C } & 3.116418722 & 1.235130360 & -0.789806225 \\ \text { H } & 1.169301176 & 1.705196653 & -1.617525085 \\ \text { C } & 3.697357565 & 0.425112331 & 0.378417847 \\ \text { H } & 3.430056267 & 0.930716459 & 1.305123655 \\ \text { C } & 3.626430991 & 2.677143142 & -0.767117878 \\ \text { H } & 3.324747999 & 3.183569050 & 0.145623599 \\ \text { H } & 4.710598861 & 2.710570295 & -0.822437819 \\ \text { H } & 3.234388201 & 3.245043851 & -1.605804984 \\ \text { H } & 1.225228390 & 1.705891991 & 0.131529349 \\ \text { H } & 1.300053555 & -2.075143218 & 0.363167477\end{array}$




$\begin{array}{lrrr}\mathrm{H} & 4.783986136 & 0.435437743 & 0.326064865 \\ \mathrm{H} & 3.448122230 & 0.787258089 & -1.724731612 \\ \mathrm{C} & 3.669102093 & -1.645739599 & 1.789930551 \\ \mathrm{H} & 3.331310739 & -2.673794524 & 1.887452505 \\ \mathrm{H} & 4.753310001 & -1.646064598 & 1.861099562 \\ \mathrm{H} & 3.280449016 & -1.087474778 & 2.636046998 \\ \mathrm{C} & 3.797962352 & -1.881638106 & -0.681019390 \\ \mathrm{H} & 4.882778803 & -1.888583838 & -0.624849807 \\ \mathrm{H} & 3.458720677 & -2.911121662 & -0.609331293 \\ \mathrm{H} & 3.529312022 & -1.519348878 & -1.666298637 \\ \mathrm{H} & 1.400689579 & -0.716406139 & -1.672466881\end{array}$

\section{3 angstrom}

$\begin{array}{lrrr}\text { C } & -4.523681545 & -0.859300274 & -1.472701665 \\ \text { C } & -3.091494780 & -0.724877172 & -1.324481854 \\ \text { C } & -2.549078972 & -0.101009316 & -0.199135352 \\ \text { C } & -3.503207560 & 0.408355547 & 0.819683859 \\ \text { C } & -4.876027268 & 0.269502527 & 0.658789807 \\ \text { C } & -5.407907629 & -0.370166159 & -0.496667298 \\ \text { H } & -4.905274223 & -1.344707796 & -2.351126580 \\ \text { H } & -2.441543037 & -1.107824448 & -2.085492338 \\ \text { H } & -5.509838137 & 0.659356016 & 1.431564362 \\ \text { H } & -6.468361484 & -0.475000934 & -0.616320772 \\ \mathrm{C} & -1.167009775 & 0.095871815 & 0.069024187 \\ \text { O } & -0.778215857 & 0.673452356 & 1.118956580 \\ \text { O } & -2.994618598 & 0.974591100 & 1.839986051 \\ \text { H } & -1.910362419 & 0.940932358 & 1.699214320 \\ \text { O } & -0.328296125 & -0.365888205 & -0.853361873 \\ \text { C } & 1.095594849 & -0.254407969 & -0.752244325 \\ \mathrm{C} & 1.658686952 & -1.052957668 & 0.420850433 \\ \mathrm{C} & 1.581004504 & 1.190873917 & -0.755982687 \\ \mathrm{C} & 3.202704972 & -1.034399845 & 0.462115722 \\ \mathrm{H} & 1.274660472 & -0.633959763 & 1.341890838 \\ \mathrm{C} & 3.114975846 & 1.236671440 & -0.786958199 \\ \mathrm{H} & 1.170154056 & 1.703210672 & -1.622111856 \\ \mathrm{C} & 3.692841418 & 0.427374770 & 0.383301597 \\ \mathrm{H} & 3.420569384 & 0.932070179 & 1.309020665 \\ \mathrm{C} & 3.621871812 & 2.679741714 & -0.761566195 \\ \mathrm{H} & 3.315507078 & 3.185052577 & 0.150215852 \\ \mathrm{H} & 4.706185507 & 2.715474747 & -0.812618506 \\ \mathrm{H} & 3.231918742 & 3.247228263 & -1.601517796 \\ \mathrm{H} & 1.218295369 & 1.701724821 & 0.127356379 \\ \mathrm{H} & 1.300513453 & -2.077484801 & 0.358045597 \\ \mathrm{H} & 4.779663276 & 0.439969543 & 0.335467996 \\ \mathrm{H} & 3.451449861 & 0.789901885 & -1.720736736 \\ \mathrm{C} & 3.662957234 & -1.643812960 & 1.794242531 \\ \mathrm{H} & 3.326734748 & -2.672530552 & 1.890245555\end{array}$




$\begin{array}{llll}\mathrm{H} & 4.746878290 & -1.642081857 & 1.869779276 \\ \mathrm{H} & 3.269767356 & -1.086416656 & 2.638808790 \\ \mathrm{C} & 3.802259874 & -1.879078195 & -0.676211968 \\ \mathrm{H} & 4.886855251 & -1.884062214 & -0.615639213 \\ \mathrm{H} & 3.464621342 & -2.909208617 & -0.606041255 \\ \mathrm{H} & 3.536966296 & -1.517146061 & -1.662544638 \\ \mathrm{H} & 1.407612451 & -0.718479011 & -1.677827510\end{array}$

$\begin{array}{lrrr}\text { 1.2 angstrom } & & \\ \mathrm{C} & -4.534143914 & -0.858890331 & -1.467836838 \\ \mathrm{C} & -3.095032664 & -0.712875940 & -1.301156816 \\ \mathrm{C} & -2.563527416 & -0.094315986 & -0.186587443 \\ \mathrm{C} & -3.509473971 & 0.417128480 & 0.837035528 \\ \mathrm{C} & -4.883846651 & 0.266015318 & 0.655864389 \\ \mathrm{C} & -5.408547251 & -0.378572446 & -0.507899535 \\ \mathrm{H} & -4.900178992 & -1.346946677 & -2.351320110 \\ \mathrm{H} & -2.438119938 & -1.093146659 & -2.058596359 \\ \mathrm{H} & -5.527126056 & 0.651818146 & 1.422879553 \\ \mathrm{H} & -6.469632007 & -0.484292694 & -0.627281917 \\ \mathrm{C} & -1.167747792 & 0.095247957 & 0.063567025 \\ \mathrm{O} & -0.759550384 & 0.682711799 & 1.129092214 \\ \mathrm{O} & -3.003617548 & 0.977655909 & 1.846006824 \\ \mathrm{H} & -1.795353348 & 0.936442077 & 1.679304401 \\ \mathrm{O} & -0.329786453 & -0.365579144 & -0.857954772 \\ \mathrm{C} & 1.095026148 & -0.257021538 & -0.759487496 \\ \mathrm{C} & 1.655981808 & -1.056869314 & 0.413871929 \\ \mathrm{C} & 1.580401285 & 1.188352774 & -0.764723573 \\ \mathrm{C} & 3.199739468 & -1.034717796 & 0.461807233 \\ \mathrm{H} & 1.266670303 & -0.641635648 & 1.334318576 \\ \mathrm{C} & 3.114456056 & 1.236003391 & -0.787174135 \\ \mathrm{H} & 1.173954565 & 1.697548232 & -1.634757768 \\ \mathrm{C} & 3.687036783 & 0.428088911 & 0.386504776 \\ \mathrm{H} & 3.408364635 & 0.931991088 & 1.310717752 \\ \mathrm{C} & 3.618608122 & 2.680012494 & -0.759501234 \\ \mathrm{H} & 3.305822356 & 3.185184977 & 0.150143381 \\ \mathrm{H} & 4.703131228 & 2.717547984 & -0.804023308 \\ \mathrm{H} & 3.232780391 & 3.246393772 & -1.602090953 \\ \mathrm{H} & 1.211927387 & 1.701618261 & 0.114742969 \\ \mathrm{H} & 1.300688843 & -2.082107472 & 0.346579316 \\ \mathrm{H} & 4.774063353 & 0.442920500 & 0.344823326 \\ \mathrm{H} & 3.456914441 & 0.789494969 & -1.718875144 \\ \mathrm{C} & 3.655320005 & -1.644467578 & 1.795378298 \\ \mathrm{H} & 3.321169798 & -2.674088683 & 1.888814707 \\ \mathrm{H} & 4.738872452 & -1.640186296 & 1.875753766 \\ \mathrm{H} & 3.256976782 & -1.088861799 & 2.638679121 \\ \mathrm{C} & 3.805803372 & -1.876894588 & -0.674903318 \\ \mathrm{H} & 4.890143431 & -1.879434035 & -0.610094414\end{array}$




$\begin{array}{llll}H & 3.470305801 & -2.907877509 & -0.607108103 \\ H & 3.543531835 & -1.514548600 & -1.661939011 \\ H & 1.404326699 & -0.722290553 & -1.685301022\end{array}$

1.1 angstrom

$\begin{array}{lrrr}\text { C } & -4.509920783 & -0.870377594 & -1.484091719 \\ \text { C } & -3.075056752 & -0.699804838 & -1.273023663 \\ \text { C } & -2.576490893 & -0.074843517 & -0.148157030 \\ \text { C } & -3.539186967 & 0.420729558 & 0.843396302 \\ \text { C } & -4.905360045 & 0.254994038 & 0.636044885 \\ \text { C } & -5.401688558 & -0.407759577 & -0.558732873 \\ \text { H } & -4.835791800 & -1.365362939 & -2.379816692 \\ \text { H } & -2.396704262 & -1.072529728 & -2.015865407 \\ \text { H } & -5.580082612 & 0.629344697 & 1.381287041 \\ \text { H } & -6.459188683 & -0.526562627 & -0.701489903 \\ \text { C } & -1.191129986 & 0.105806959 & 0.085456405 \\ \text { O } & -0.748374404 & 0.691285788 & 1.140717381 \\ \text { O } & -3.033647611 & 0.995337589 & 1.873690493 \\ \text { H } & -1.667648458 & 0.949031606 & 1.687071173 \\ \text { O } & -0.352351666 & -0.344479255 & -0.813535909 \\ \text { C } & 1.083578038 & -0.241557270 & -0.728110884 \\ \text { C } & 1.650431750 & -1.046461525 & 0.437308062 \\ \text { C } & 1.573970125 & 1.200825249 & -0.743292424 \\ \text { C } & 3.195442152 & -1.036071945 & 0.459311496 \\ \text { H } & 1.281868342 & -0.628535146 & 1.365174668 \\ \text { C } & 3.108345626 & 1.235165439 & -0.789941176 \\ \text { H } & 1.157509313 & 1.711429895 & -1.607311334 \\ \text { C } & 3.692158595 & 0.423182400 & 0.375483347 \\ \text { H } & 3.432390955 & 0.929870267 & 1.303687156 \\ \text { C } & 3.623536873 & 2.675324031 & -0.771642454 \\ \text { H } & 3.330452758 & 3.183510459 & 0.142796920 \\ \text { H } & 4.707204189 & 2.703560157 & -0.834418482 \\ \text { H } & 3.228430702 & 3.244086162 & -1.608121547 \\ \text { H } & 1.226253718 & 1.719839786 & 0.141325151 \\ \text { H } & 1.285378375 & -2.068240657 & 0.375581468 \\ \text { H } & 4.778154253 & 0.429480573 & 0.316655665 \\ \text { H } & 3.431454417 & 0.785304515 & -1.726655843 \\ \text { C } & 3.666837146 & -1.649234481 & 1.785718586 \\ \text { H } & 3.325409619 & -2.675757533 & 1.885176085 \\ \text { H } & 4.751356463 & -1.654315948 & 1.847670584 \\ \text { H } & 3.287923158 & -1.089733820 & 2.635357179 \\ \text { C } & 3.775309544 & -1.883657166 & -0.686954928 \\ \text { H } & 4.860272582 & -1.894480238 & -0.639540165 \\ \text { H } & 3.433085415 & -2.911857499 & -0.612987432 \\ \text { H } & 3.500649920 & -1.519921649 & -1.670165240 \\ \text { H } & 1.371552429 & -0.710008471 & -1.657783228 \\ & & & \end{array}$




\begin{tabular}{lrrr}
\multicolumn{1}{l}{1.0 angstrom } & & \\
$\mathrm{C}$ & -4.480423131 & -0.884524005 & -1.508067999 \\
$\mathrm{C}$ & -3.058021192 & -0.671706849 & -1.219960716 \\
$\mathrm{C}$ & -2.621342120 & -0.055282322 & -0.107108835 \\
$\mathrm{C}$ & -3.593447897 & 0.428406941 & 0.864771771 \\
$\mathrm{C}$ & -4.948612665 & 0.228841077 & 0.595999175 \\
$\mathrm{C}$ & -5.390858829 & -0.438918221 & -0.611483806 \\
$\mathrm{H}$ & -4.756480413 & -1.384477388 & -2.416389322 \\
$\mathrm{H}$ & -2.339440441 & -1.026861011 & -1.934537310 \\
$\mathrm{H}$ & -5.662715888 & 0.585656040 & 1.312908054 \\
$\mathrm{H}$ & -6.443689934 & -0.572784889 & -0.781046977 \\
$\mathrm{C}$ & -1.201191609 & 0.133367902 & 0.135564479 \\
$\mathrm{O}$ & -0.711908360 & 0.726625203 & 1.201614178 \\
$\mathrm{O}$ & -3.151182296 & 1.007783171 & 1.907991657 \\
$\mathrm{H}$ & -1.499892507 & 0.993336410 & 1.756543266 \\
$\mathrm{O}$ & -0.362814955 & -0.324548582 & -0.779293818 \\
$\mathrm{C}$ & 1.067831914 & -0.230681899 & -0.708866163 \\
$\mathrm{C}$ & 1.647019467 & -1.038160316 & 0.449946551 \\
$\mathrm{C}$ & 1.571745982 & 1.207916869 & -0.731425433 \\
$\mathrm{C}$ & 3.192066430 & -1.039281876 & 0.452169963 \\
$\mathrm{H}$ & 1.294154875 & -0.617581023 & 1.382795652 \\
$\mathrm{C}$ & 3.105399405 & 1.231042148 & -0.798241618 \\
$\mathrm{H}$ & 1.147600162 & 1.721223251 & -1.590172987 \\
$\mathrm{C}$ & 3.698353514 & 0.416186979 & 0.360525734 \\
$\mathrm{H}$ & 3.453391079 & 0.925582206 & 1.291337302 \\
$\mathrm{C}$ & 3.632455510 & 2.667029501 & -0.788889415 \\
$\mathrm{H}$ & 3.356328922 & 3.178565356 & 0.129008301 \\
$\mathrm{H}$ & 4.715392657 & 2.686889671 & -0.866985362 \\
$\mathrm{H}$ & 3.229937074 & 3.238133331 & -1.620283919 \\
$\mathrm{H}$ & 1.239914217 & 1.731466604 & 0.156752980 \\
$\mathrm{H}$ & 1.273536071 & -2.057334806 & 0.394583921 \\
$\mathrm{H}$ & 4.783682425 & 0.414947429 & 0.288622993 \\
$\mathrm{H}$ & 3.413057437 & 0.777342556 & -1.738378474 \\
$\mathrm{C}$ & 3.676976097 & -1.654799870 & 1.772655417 \\
$\mathrm{H}$ & 3.328570099 & -2.678423571 & 1.878046823 \\
$\mathrm{H}$ & 4.762234713 & -1.668674940 & 1.819938902 \\
$\mathrm{H}$ & 3.314227379 & -1.091424118 & 2.626814147 \\
$\mathrm{C}$ & 3.751306180 & -1.891924148 & -0.700615332 \\
$\mathrm{H}$ & 4.836805370 & -1.909509369 & -0.668008170 \\
$\mathrm{H}$ & 3.403694297 & -2.917900999 & -0.620456874 \\
$\mathrm{H}$ & 3.465293037 & -1.527833282 & -1.680387749 \\
$\mathrm{H}$ & 1.347380925 & -0.701153383 & -1.640719231 \\
& & & \\
& & &
\end{tabular}

$\mathrm{T}_{1}$ state minimum-energy proton transfer path 1.9 angstrom

$\begin{array}{llll}\text { C } & -4.331635094 & -0.907906115 & -1.556418861 \\ \text { C } & -3.013746127 & -0.719014343 & -1.307136122\end{array}$




$\begin{array}{lrrr}\text { C } & -2.563806853 & -0.026841500 & -0.060520764 \\ \text { C } & -3.631000622 & 0.446616073 & 0.890686364 \\ \text { C } & -4.908148565 & 0.241180538 & 0.607486343 \\ \text { C } & -5.338082262 & -0.446048763 & -0.626812796 \\ \text { H } & -4.638850824 & -1.407779501 & -2.457101367 \\ \text { H } & -2.259137042 & -1.055778505 & -1.986747895 \\ \text { H } & -5.649118097 & 0.589011294 & 1.302891496 \\ \text { H } & -6.381051037 & -0.593949830 & -0.819541558 \\ \text { C } & -1.152793131 & 0.174192090 & 0.212127664 \\ \text { O } & -0.745740355 & 0.727217913 & 1.208071638 \\ \text { O } & -3.277485825 & 1.075975058 & 2.021780773 \\ \text { H } & -2.331111080 & 1.145358382 & 2.085647890 \\ \text { O } & -0.363072755 & -0.308407914 & -0.738911513 \\ \text { C } & 1.070373299 & -0.224894864 & -0.693154562 \\ \text { C } & 1.661608481 & -1.033840325 & 0.458058092 \\ \text { C } & 1.583487642 & 1.210079225 & -0.721703463 \\ \text { C } & 3.206509111 & -1.047773504 & 0.435480321 \\ \text { H } & 1.329098507 & -0.607323092 & 1.396115059 \\ \text { C } & 3.115899774 & 1.222550621 & -0.815412271 \\ \text { H } & 1.148473733 & 1.729065372 & -1.571658731 \\ \text { C } & 3.723366278 & 0.403267630 & 0.332709072 \\ \text { H } & 3.500866083 & 0.915653434 & 1.267541709 \\ \text { C } & 3.653578774 & 2.654582511 & -0.815334630 \\ \text { H } & 3.396693192 & 3.168360596 & 0.106920808 \\ \text { H } & 4.735153633 & 2.666407003 & -0.911457243 \\ \text { H } & 3.241576904 & 3.228445064 & -1.640141750 \\ \text { H } & 1.272885923 & 1.734550787 & 0.174017742 \\ \text { H } & 1.279434074 & -2.050335685 & 0.412527438 \\ \text { H } & 4.807040738 & 0.392453787 & 0.240076647 \\ \text { H } & 3.403135856 & 0.766632807 & -1.760873349 \\ \text { C } & 3.707661879 & -1.664837960 & 1.749138976 \\ \text { H } & 3.353828750 & -2.685853484 & 1.861480305 \\ \text { H } & 4.793365496 & -1.686299331 & 1.779477977 \\ \text { H } & 3.362406041 & -1.097675098 & 2.608077430 \\ \text { C } & 3.739611977 & -1.907159589 & -0.724630131 \\ \text { H } & 4.825231396 & -1.935301960 & -0.708341023 \\ \text { H } & 3.383272622 & -2.929581401 & -0.637989236 \\ \text { H } & 3.442403819 & -1.541327339 & -1.700371350 \\ \text { H } & 1.334148694 & -0.697114300 & -1.628783336\end{array}$

1.8 angstrom
C -4.344453910
$-0.906239227$
$-1.553690301$
C $\quad-3.024203390$
$-0.722557634$
$-1.316649776$
C $\quad-2.564246615$
$-0.032470940$
$-0.072674753$
C $\quad-3.619439729$
0.444944910
0.887750023
C $\quad-4.900106373$
0.244793628
0.616951334
C $\quad-5.341161465$
$-0.441685961$
$-0.615582575$ 


$\begin{array}{lrrr}\text { H } & -4.661810635 & -1.404433604 & -2.451852696 \\ \mathrm{H} & -2.275779492 & -1.061143749 & -2.002500666 \\ \mathrm{H} & -5.634631457 & 0.594790873 & 1.318032686 \\ \mathrm{H} & -6.386035178 & -0.586591314 & -0.800153218 \\ \mathrm{C} & -1.154577512 & 0.168286112 & 0.199630366 \\ \mathrm{O} & -0.753738356 & 0.722445422 & 1.198845798 \\ \mathrm{O} & -3.240244981 & 1.070953965 & 2.010812453 \\ \mathrm{H} & -2.288800485 & 1.126212130 & 2.047702256 \\ \mathrm{O} & -0.361552365 & -0.311981204 & -0.748283105 \\ \mathrm{C} & 1.072026200 & -0.226732507 & -0.698020220 \\ \mathrm{C} & 1.660107878 & -1.034873184 & 0.455285303 \\ \mathrm{C} & 1.582670174 & 1.209069867 & -0.724351133 \\ \mathrm{C} & 3.205090382 & -1.046499208 & 0.438198551 \\ \mathrm{H} & 1.323642187 & -0.608649723 & 1.392075711 \\ \mathrm{C} & 3.115371956 & 1.223991708 & -0.812587571 \\ \mathrm{H} & 1.149798454 & 1.727615543 & -1.575650421 \\ \mathrm{C} & 3.720076436 & 0.405362277 & 0.337464926 \\ \mathrm{H} & 3.493594029 & 0.917221476 & 1.271622111 \\ \mathrm{C} & 3.650651894 & 2.656910060 & -0.810263218 \\ \mathrm{H} & 3.389574414 & 3.170076729 & 0.111147580 \\ \mathrm{H} & 4.732536529 & 2.670509360 & -0.902472623 \\ \mathrm{H} & 3.240694454 & 3.230242372 & -1.636451607 \\ \mathrm{H} & 1.267930999 & 1.732595434 & 0.170495533 \\ \mathrm{H} & 1.279506545 & -2.051893070 & 0.408491900 \\ \mathrm{H} & 4.804063375 & 0.396214940 & 0.248541346 \\ \mathrm{H} & 3.406611386 & 0.768782564 & -1.757160647 \\ \mathrm{C} & 3.702413737 & -1.662954327 & 1.753594706 \\ \mathrm{H} & 3.349730373 & -2.684513681 & 1.864559646 \\ \mathrm{H} & 4.788025997 & -1.682763210 & 1.787818647 \\ \mathrm{H} & 3.353221000 & -1.096406718 & 2.611340395 \\ \mathrm{C} & 3.743581849 & -1.904920958 & -0.720127652 \\ \mathrm{H} & 4.829164379 & -1.931571938 & -0.699848829 \\ \mathrm{H} & 3.388329604 & -2.927847912 & -0.635016636 \\ \mathrm{H} & 3.449486470 & -1.539263141 & -1.696885723 \\ \mathrm{H} & 1.339214286 & -0.698470423 & -1.632866108\end{array}$

1.7 angstrom

$\begin{array}{llll}\text { C } & -4.366274492 & -0.902789537 & -1.549481543 \\ \text { C } & -3.042022767 & -0.730529954 & -1.331308266 \\ \text { C } & -2.565702297 & -0.043403113 & -0.092103226 \\ \text { C } & -3.600765530 & 0.442462153 & 0.882526784 \\ \text { C } & -4.887234904 & 0.253651806 & 0.630557354 \\ \text { C } & -5.346673846 & -0.430534921 & -0.599005336 \\ \text { H } & -4.700414628 & -1.398325649 & -2.443111984 \\ \text { H } & -2.304006955 & -1.074592159 & -2.026156847 \\ \text { H } & -5.611160694 & 0.608616666 & 1.340043226 \\ \text { H } & -6.394665138 & -0.567799383 & -0.771568756\end{array}$




$\begin{array}{lrrr}\text { C } & -1.158096011 & 0.156590660 & 0.179974881 \\ \text { O } & -0.768296747 & 0.713123701 & 1.184675484 \\ \text { O } & -3.179258488 & 1.060989806 & 1.991720711 \\ \text { H } & -2.220089137 & 1.092267669 & 1.983768183 \\ \text { O } & -0.358999878 & -0.320226564 & -0.761618133 \\ \text { C } & 1.074822605 & -0.230760778 & -0.704293129 \\ \text { C } & 1.658771451 & -1.037366646 & 0.452025191 \\ \text { C } & 1.580475692 & 1.206746514 & -0.727287193 \\ \text { C } & 3.203879450 & -1.044284463 & 0.442758064 \\ \text { H } & 1.316022373 & -0.612115363 & 1.387031779 \\ \text { C } & 3.113579003 & 1.226474941 & -0.807676806 \\ \text { H } & 1.150308332 & 1.724320886 & -1.580514073 \\ \text { C } & 3.714999409 & 0.409168009 & 0.345038844 \\ \text { H } & 3.482572182 & 0.920010896 & 1.278276694 \\ \text { C } & 3.644355151 & 2.661050920 & -0.802112258 \\ \text { H } & 3.377068326 & 3.173084361 & 0.118136487 \\ \text { H } & 4.726634123 & 2.677922382 & -0.888853157 \\ \text { H } & 3.236859321 & 3.233396692 & -1.630190609 \\ \text { H } & 1.259334980 & 1.728588642 & 0.166309731 \\ \text { H } & 1.281466882 & -2.055482897 & 0.403101479 \\ \text { H } & 4.799396603 & 0.403181130 & 0.261300640 \\ \text { H } & 3.410896685 & 0.772585475 & -1.750982275 \\ \text { C } & 3.696414026 & -1.659679609 & 1.760435386 \\ \text { H } & 3.346349999 & -2.682356913 & 1.869282114 \\ \text { H } & 4.781884154 & -1.676142139 & 1.800016781 \\ \text { H } & 3.341230558 & -1.094504549 & 2.616614128 \\ \text { C } & 3.750623192 & -1.900749877 & -0.713170030 \\ \text { H } & 4.836147450 & -1.924382086 & -0.687318578 \\ \text { H } & 3.397799982 & -2.924697048 & -0.630286066 \\ \text { H } & 3.460593197 & -1.535515449 & -1.691316952 \\ \text { H } & 1.347509356 & -0.701438419 & -1.637966948\end{array}$

1.6 angstrom

$\begin{array}{lrrr}\text { C } & -4.389101166 & -0.899703624 & -1.543763571 \\ \text { C } & -3.060780989 & -0.738468735 & -1.346301567 \\ \text { C } & -2.566874293 & -0.054229952 & -0.112470449 \\ \text { C } & -3.580424559 & 0.439839170 & 0.877570415 \\ \text { C } & -4.872592289 & 0.261595138 & 0.645539109 \\ \text { C } & -5.351707835 & -0.420188781 & -0.579846431 \\ \text { H } & -4.740859651 & -1.392605942 & -2.432181177 \\ \text { H } & -2.334246487 & -1.087783220 & -2.051016557 \\ \text { H } & -5.584940464 & 0.621214116 & 1.364223816 \\ \text { H } & -6.402803080 & -0.550166911 & -0.738921033 \\ \text { C } & -1.160904011 & 0.144890382 & 0.157631292 \\ \text { O } & -0.781496857 & 0.703648253 & 1.167839223 \\ \text { O } & -3.116718506 & 1.050445838 & 1.971017821 \\ \text { H } & -2.149900544 & 1.057866988 & 1.917506348\end{array}$




$\begin{array}{lrrr}\text { O } & -0.356087218 & -0.327786633 & -0.777875437 \\ \text { C } & 1.078190109 & -0.234443487 & -0.712693950 \\ \text { C } & 1.656809849 & -1.039489636 & 0.447185552 \\ \text { C } & 1.578929495 & 1.204668345 & -0.731536291 \\ \text { C } & 3.201967500 & -1.041983273 & 0.447312502 \\ \text { H } & 1.307217594 & -0.614699193 & 1.379878731 \\ \text { C } & 3.112460206 & 1.229126346 & -0.802740095 \\ \text { H } & 1.152130443 & 1.721838626 & -1.586660033 \\ \text { C } & 3.709386134 & 0.413012222 & 0.353179643 \\ \text { H } & 3.469919692 & 0.922776318 & 1.285205604 \\ \text { C } & 3.638543149 & 2.665397714 & -0.793056617 \\ \text { H } & 3.364252145 & 3.175865668 & 0.125978767 \\ \text { H } & 4.721232191 & 2.685754475 & -0.873460412 \\ \text { H } & 3.234073372 & 3.237033044 & -1.623092077 \\ \text { H } & 1.250899450 & 1.724319065 & 0.160856813 \\ \text { H } & 1.282548113 & -2.058604646 & 0.396276976 \\ \text { H } & 4.794253545 & 0.410252272 & 0.275876981 \\ \text { H } & 3.416803155 & 0.776756037 & -1.744521904 \\ \text { C } & 3.687968654 & -1.656354138 & 1.767883497 \\ \text { H } & 3.340084001 & -2.680022263 & 1.874295878 \\ \text { H } & 4.773202213 & -1.669812602 & 1.814138161 \\ \text { H } & 3.325946088 & -1.092387707 & 2.621976329 \\ \text { C } & 3.758101218 & -1.896540346 & -0.705500103 \\ \text { H } & 4.843477428 & -1.917173787 & -0.672988591 \\ \text { H } & 3.407616866 & -2.921477062 & -0.625109406 \\ \text { H } & 3.473102571 & -1.531772552 & -1.685308933 \\ \text { H } & 1.356655756 & -0.704049729 & -1.645057050\end{array}$

1.5 angstrom

$\begin{array}{lrrr}\text { C } & -4.411874187 & -0.895633155 & -1.537350583 \\ \text { C } & -3.079605692 & -0.746172444 & -1.360386260 \\ \text { C } & -2.568289847 & -0.065145556 & -0.132243612 \\ \text { C } & -3.559524884 & 0.437520352 & 0.873484764 \\ \text { C } & -4.857370061 & 0.270099572 & 0.660423039 \\ \text { C } & -5.356140917 & -0.408273429 & -0.560112930 \\ \text { H } & -4.781420314 & -1.385565249 & -2.420283257 \\ \text { H } & -2.365051530 & -1.101206367 & -2.074814128 \\ \text { H } & -5.558092530 & 0.634384158 & 1.388054181 \\ \text { H } & -6.410158374 & -0.530343656 & -0.706104921 \\ \text { C } & -1.163697734 & 0.132405572 & 0.134704328 \\ \text { O } & -0.793982889 & 0.693524499 & 1.150519125 \\ \text { O } & -3.054879990 & 1.038765552 & 1.949793172 \\ \text { H } & -2.078909161 & 1.022683485 & 1.850961246 \\ \text { O } & -0.353228036 & -0.335974805 & -0.794098435 \\ \text { C } & 1.081569880 & -0.238756398 & -0.721381342 \\ \text { C } & 1.654956771 & -1.042498326 & 0.441794422 \\ \text { C } & 1.577114163 & 1.202044129 & -0.736516235\end{array}$




$\begin{array}{lrrr}\mathrm{C} & 3.200123593 & -1.039717246 & 0.451874872 \\ \mathrm{H} & 1.298013455 & -0.619011284 & 1.372309989 \\ \mathrm{C} & 3.111009266 & 1.231662158 & -0.797860335 \\ \mathrm{H} & 1.153934662 & 1.718213582 & -1.594004373 \\ \mathrm{C} & 3.703226908 & 0.417037520 & 0.361557334 \\ \mathrm{H} & 3.456318911 & 0.925660959 & 1.292249794 \\ \mathrm{C} & 3.631847681 & 2.669808713 & -0.784073066 \\ \mathrm{H} & 3.349365510 & 3.178971119 & 0.133192972 \\ \mathrm{H} & 4.714971421 & 2.693964353 & -0.856949870 \\ \mathrm{H} & 3.231154531 & 3.240265804 & -1.616732134 \\ \mathrm{H} & 1.241707229 & 1.719937102 & 0.154168701 \\ \mathrm{H} & 1.284378912 & -2.062790406 & 0.388182130 \\ \mathrm{H} & 4.788527302 & 0.417839229 & 0.290999803 \\ \mathrm{H} & 3.422902685 & 0.780840216 & -1.737899674 \\ \mathrm{C} & 3.679242002 & -1.652975209 & 1.775473280 \\ \mathrm{H} & 3.334402129 & -2.677960493 & 1.878968724 \\ \mathrm{H} & 4.764153964 & -1.662453159 & 1.829153931 \\ \mathrm{H} & 3.309282620 & -1.090783289 & 2.627322638 \\ \mathrm{C} & 3.766654289 & -1.891933054 & -0.697598339 \\ \mathrm{H} & 4.851832591 & -1.909079185 & -0.657694310 \\ \mathrm{H} & 3.418953795 & -2.918034727 & -0.620150025 \\ \mathrm{H} & 3.487252803 & -1.527504960 & -1.679177023 \\ \mathrm{H} & 1.365662030 & -0.707259872 & -1.652485767\end{array}$

1.4 angstrom

$\begin{array}{lrrr}\text { C } & -4.433576551 & -0.892132352 & -1.530170362 \\ \text { C } & -3.097666792 & -0.754513902 & -1.372745697 \\ \text { C } & -2.570258714 & -0.075688254 & -0.150508810 \\ \text { C } & -3.539322653 & 0.436134061 & 0.870086351 \\ \text { C } & -4.842617647 & 0.278870388 & 0.673739038 \\ \text { C } & -5.359731724 & -0.396197298 & -0.541153372 \\ \text { H } & -4.819809245 & -1.379692498 & -2.407344402 \\ \text { H } & -2.394777263 & -1.115974640 & -2.095794260 \\ \text { H } & -5.532337610 & 0.648023818 & 1.409266013 \\ \text { H } & -6.416329253 & -0.510204826 & -0.675109761 \\ \text { C } & -1.166840644 & 0.120233167 & 0.112220079 \\ \text { O } & -0.806237560 & 0.684460968 & 1.133700840 \\ \text { O } & -2.996922773 & 1.027738917 & 1.928155358 \\ \text { H } & -2.007542195 & 0.989007042 & 1.784945220 \\ \text { O } & -0.350634486 & -0.343938584 & -0.809191930 \\ \text { C } & 1.085067397 & -0.242976195 & -0.729426032 \\ \text { C } & 1.653229891 & -1.045756206 & 0.436725523 \\ \text { C } & 1.575353373 & 1.199454568 & -0.741894052 \\ \text { C } & 3.198317061 & -1.037501252 & 0.456364465 \\ \text { H } & 1.288791081 & -0.624522326 & 1.365378719 \\ \text { C } & 3.109599072 & 1.234248068 & -0.792668259 \\ \text { H } & 1.156474263 & 1.713569295 & -1.602670656\end{array}$




$\begin{array}{lrrr}\mathrm{C} & 3.696846708 & 0.421044318 & 0.370230898 \\ \mathrm{H} & 3.442271996 & 0.928248979 & 1.299604263 \\ \mathrm{C} & 3.625092512 & 2.674267434 & -0.774656383 \\ \mathrm{H} & 3.334794572 & 3.181869888 & 0.141000781 \\ \mathrm{H} & 4.708552850 & 2.702202156 & -0.840395761 \\ \mathrm{H} & 3.227926513 & 3.243746833 & -1.609650747 \\ \mathrm{H} & 1.231918217 & 1.716705845 & 0.146108320 \\ \mathrm{H} & 1.286655835 & -2.067255091 & 0.379542936 \\ \mathrm{H} & 4.782526443 & 0.425537022 & 0.306573611 \\ \mathrm{H} & 3.429348074 & 0.785075137 & -1.730828205 \\ \mathrm{C} & 3.671276355 & -1.650149291 & 1.782426717 \\ \mathrm{H} & 3.329320382 & -2.676365521 & 1.883069525 \\ \mathrm{H} & 4.755845072 & -1.655987561 & 1.842579893 \\ \mathrm{H} & 3.294323424 & -1.089816599 & 2.632406132 \\ \mathrm{C} & 3.774545280 & -1.886940393 & -0.690346711 \\ \mathrm{H} & 4.859465068 & -1.900775775 & -0.643694889 \\ \mathrm{H} & 3.429559126 & -2.914168050 & -0.615942363 \\ \mathrm{H} & 3.500189271 & -1.522525561 & -1.673369541 \\ \mathrm{H} & 1.373648220 & -0.710799919 & -1.659290722\end{array}$

1.3 angstrom

$\begin{array}{lrrr}\text { C } & -4.452189000 & -0.888913652 & -1.524275068 \\ \text { C } & -3.112980403 & -0.760623513 & -1.384263902 \\ \text { C } & -2.573474503 & -0.084627047 & -0.167127950 \\ \text { C } & -3.521810072 & 0.435351233 & 0.867725490 \\ \text { C } & -4.830567455 & 0.284756331 & 0.683960510 \\ \text { C } & -5.362248697 & -0.386792876 & -0.524183398 \\ \text { H } & -4.852856158 & -1.373966451 & -2.396440705 \\ \text { H } & -2.420434882 & -1.126480179 & -2.115288875 \\ \text { H } & -5.511061446 & 0.657601566 & 1.426080991 \\ \text { H } & -6.420816893 & -0.495326987 & -0.647816597 \\ \text { C } & -1.171336936 & 0.108671575 & 0.091101504 \\ \text { O } & -0.818366271 & 0.675738116 & 1.119124001 \\ \text { O } & -2.948035183 & 1.017646162 & 1.906895980 \\ \text { H } & -1.935263849 & 0.957260429 & 1.721855502 \\ \text { O } & -0.348722896 & -0.350306835 & -0.821637380 \\ \text { C } & 1.088217780 & -0.245625759 & -0.734936244 \\ \text { C } & 1.651487109 & -1.047237135 & 0.434047140 \\ \text { C } & 1.573519721 & 1.198236984 & -0.743973073 \\ \text { C } & 3.196535438 & -1.035520563 & 0.460436944 \\ \text { H } & 1.282146631 & -0.626080293 & 1.360871600 \\ \text { C } & 3.107978122 & 1.236686061 & -0.788133237 \\ \text { H } & 1.156808534 & 1.712257205 & -1.605770857 \\ \text { C } & 3.692009097 & 0.424253249 & 0.376957609 \\ \text { H } & 3.432342419 & 0.930502555 & 1.305418063 \\ \text { C } & 3.619645288 & 2.678011844 & -0.767160297 \\ \text { H } & 3.324371977 & 3.184342268 & 0.147580200\end{array}$




$\begin{array}{llll}\mathrm{H} & 4.703257808 & 2.708557167 & -0.828452172 \\ \mathrm{H} & 3.224529110 & 3.246934692 & -1.603475635 \\ \mathrm{H} & 1.225186939 & 1.713341701 & 0.143444040 \\ \mathrm{H} & 1.287132634 & -2.069389739 & 0.375625215 \\ \mathrm{H} & 4.777880753 & 0.431249443 & 0.317927564 \\ \mathrm{H} & 3.432669048 & 0.788792105 & -1.725176602 \\ \mathrm{C} & 3.664689792 & -1.647511930 & 1.788492300 \\ \mathrm{H} & 3.324498058 & -2.674466971 & 1.887320395 \\ \mathrm{H} & 4.748955750 & -1.651030487 & 1.853406172 \\ \mathrm{H} & 3.282801146 & -1.088198528 & 2.636915410 \\ \mathrm{C} & 3.779518704 & -1.883418936 & -0.683985577 \\ \mathrm{H} & 4.864206671 & -1.894873464 & -0.632474294 \\ \mathrm{H} & 3.436480167 & -2.911407830 & -0.611363297 \\ \mathrm{H} & 3.508880594 & -1.519354482 & -1.668194553 \\ \mathrm{H} & 1.380748328 & -0.712481269 & -1.663785141\end{array}$

1.2 angstrom

$\begin{array}{lrrr}\text { C } & -4.565535661 & -0.871236095 & -1.486024057 \\ \text { C } & -3.121897384 & -0.748382812 & -1.362397716 \\ \text { C } & -2.549060175 & -0.095518049 & -0.188982795 \\ \text { C } & -3.418302706 & 0.407591569 & 0.810402460 \\ \text { C } & -4.860334088 & 0.267673627 & 0.654935698 \\ \text { C } & -5.413251541 & -0.389605019 & -0.528022637 \\ \text { H } & -4.963089605 & -1.355939869 & -2.359149648 \\ \text { H } & -2.478926907 & -1.133536440 & -2.125765304 \\ \text { H } & -5.480688244 & 0.662414968 & 1.434636766 \\ \text { H } & -6.478293908 & -0.481681352 & -0.621906603 \\ \text { C } & -1.185054518 & 0.088710597 & 0.054697253 \\ \text { O } & -0.774232777 & 0.668073450 & 1.102826967 \\ \text { O } & -2.983448396 & 0.979833950 & 1.843466233 \\ \text { H } & -1.789576140 & 0.939686987 & 1.681881489 \\ \text { O } & -0.339223133 & -0.356039497 & -0.834920009 \\ \text { C } & 1.094063797 & -0.244853917 & -0.735075111 \\ \text { C } & 1.655964309 & -1.046890656 & 0.434616640 \\ \text { C } & 1.578543804 & 1.199514916 & -0.743184874 \\ \text { C } & 3.200578378 & -1.031811406 & 0.467062683 \\ \text { H } & 1.278715160 & -0.628461035 & 1.358544649 \\ \text { C } & 3.112832292 & 1.240305322 & -0.780912116 \\ \text { H } & 1.165396694 & 1.710880461 & -1.608499454 \\ \text { C } & 3.693740946 & 0.428907013 & 0.386482466 \\ \text { H } & 3.428099179 & 0.934124709 & 1.313846285 \\ \text { C } & 3.622634600 & 2.682325845 & -0.757259105 \\ \text { H } & 3.321219117 & 3.188137601 & 0.155855040 \\ \text { H } & 4.706681558 & 2.715184503 & -0.812603218 \\ \text { H } & 3.230834431 & 3.250714795 & -1.595613226 \\ \text { H } & 1.222135698 & 1.713225732 & 0.140836260 \\ \text { H } & 1.294824183 & -2.070094291 & 0.371451525\end{array}$




$\begin{array}{rrrr}\mathrm{H} & 4.780123364 & 0.438362924 & 0.332707373 \\ \mathrm{H} & 3.443477096 & 0.793231087 & -1.716426704 \\ \mathrm{C} & 3.665234061 & -1.643326116 & 1.796634031 \\ \mathrm{H} & 3.327335962 & -2.671337252 & 1.893314645 \\ \mathrm{H} & 4.749369842 & -1.643901867 & 1.866765246 \\ \mathrm{H} & 3.277510585 & -1.085700187 & 2.643570269 \\ \mathrm{C} & 3.791560561 & -1.877504777 & -0.674953511 \\ \mathrm{H} & 4.876226594 & -1.886571287 & -0.618659423 \\ \mathrm{H} & 3.450343309 & -2.906336550 & -0.604702135 \\ \mathrm{H} & 3.524140764 & -1.513411170 & -1.659956591 \\ \mathrm{H} & 1.395661893 & -0.710204621 & -1.662247939\end{array}$

\section{1 angstrom}

$\begin{array}{lrrr}\text { C } & -4.496895715 & -0.877471925 & -1.496218847 \\ \text { C } & -3.074532432 & -0.717813571 & -1.304441694 \\ \text { C } & -2.553170353 & -0.065744553 & -0.131615468 \\ \text { C } & -3.524294094 & 0.427792249 & 0.856331151 \\ \text { C } & -4.928710383 & 0.241511527 & 0.613784595 \\ \text { C } & -5.407486240 & -0.422055872 & -0.583378620 \\ \text { H } & -4.834704558 & -1.371757819 & -2.389356254 \\ \text { H } & -2.396505952 & -1.089338376 & -2.044311495 \\ \text { H } & -5.602523892 & 0.616473572 & 1.359570168 \\ \text { H } & -6.463423795 & -0.543816599 & -0.731809494 \\ \mathrm{C} & -1.216082868 & 0.108401705 & 0.093074409 \\ \text { O } & -0.719056323 & 0.699066631 & 1.153193983 \\ \text { O } & -3.169822147 & 1.004648452 & 1.901362728 \\ \text { H } & -1.583654074 & 0.994637599 & 1.765655238 \\ \text { O } & -0.355239836 & -0.336085646 & -0.796849765 \\ \text { C } & 1.075121267 & -0.231921645 & -0.709585669 \\ \mathrm{C} & 1.651376321 & -1.038569444 & 0.450707603 \\ \mathrm{C} & 1.574412072 & 1.207942466 & -0.729829810 \\ \mathrm{C} & 3.196470751 & -1.036376026 & 0.457793424 \\ \mathrm{H} & 1.294809065 & -0.619569105 & 1.382729589 \\ \mathrm{C} & 3.108270562 & 1.235031160 & -0.791465138 \\ \mathrm{H} & 1.152180604 & 1.719718044 & -1.590381456 \\ \mathrm{C} & 3.700470646 & 0.420049502 & 0.367700029 \\ \mathrm{H} & 3.453694027 & 0.928551415 & 1.298624306 \\ \mathrm{C} & 3.631656082 & 2.672359094 & -0.778198458 \\ \mathrm{H} & 3.350153101 & 3.182080786 & 0.139172791 \\ \mathrm{H} & 4.714901337 & 2.695437926 & -0.851488972 \\ \mathrm{H} & 3.231131702 & 3.243362613 & -1.610623396 \\ \mathrm{H} & 1.238306868 & 1.730805027 & 0.156988093 \\ \mathrm{H} & 1.280353763 & -2.058450470 & 0.392952737 \\ \mathrm{H} & 4.785952426 & 0.420491042 & 0.297190563 \\ \mathrm{H} & 3.420128426 & 0.783684206 & -1.731304925 \\ \mathrm{C} & 3.678483622 & -1.650833639 & 1.779879168 \\ \mathrm{H} & 3.333023379 & -2.675649433 & 1.883463127\end{array}$




$\begin{array}{llll}\mathrm{H} & 4.763614045 & -1.661132751 & 1.831588442 \\ \mathrm{H} & 3.310496087 & -1.088997671 & 2.632917280 \\ \mathrm{C} & 3.761706609 & -1.887807154 & -0.692975981 \\ \mathrm{H} & 4.847039679 & -1.906025419 & -0.654184928 \\ \mathrm{H} & 3.412932033 & -2.913622881 & -0.616324656 \\ \mathrm{H} & 3.481332463 & -1.522200101 & -1.673731842 \\ \mathrm{H} & 1.364418707 & -0.700249173 & -1.639330759\end{array}$

$\begin{array}{lrrr}\text { 1.0 angstrom } & & \\ \mathrm{C} & -4.479658321 & -0.882064485 & -1.502106420 \\ \mathrm{C} & -3.062808621 & -0.710179681 & -1.289399773 \\ \mathrm{C} & -2.561726165 & -0.056693542 & -0.113964586 \\ \mathrm{C} & -3.554217167 & 0.431606140 & 0.866683561 \\ \mathrm{C} & -4.949536926 & 0.231463727 & 0.599957373 \\ \mathrm{C} & -5.405720687 & -0.434755782 & -0.602363606 \\ \mathrm{H} & -4.799598193 & -1.378973738 & -2.400435411 \\ \mathrm{H} & -2.372514739 & -1.076285479 & -2.020679063 \\ \mathrm{H} & -5.638825504 & 0.599704262 & 1.335092455 \\ \mathrm{H} & -6.458470505 & -0.565622131 & -0.766309845 \\ \mathrm{C} & -1.227332261 & 0.117460638 & 0.108934000 \\ \mathrm{O} & -0.711800239 & 0.709914950 & 1.169733254 \\ \mathrm{O} & -3.217832382 & 1.010300822 & 1.915344390 \\ \mathrm{H} & -1.464278063 & 0.998633559 & 1.761695119 \\ \mathrm{O} & -0.363005486 & -0.325472965 & -0.779394028 \\ \mathrm{C} & 1.067780627 & -0.224543618 & -0.697267730 \\ \mathrm{C} & 1.648185250 & -1.033552124 & 0.459360921 \\ \mathrm{C} & 1.572061213 & 1.213571948 & -0.721167401 \\ \mathrm{C} & 3.193399322 & -1.037023506 & 0.456205172 \\ \mathrm{H} & 1.300103605 & -0.614100265 & 1.394452580 \\ \mathrm{C} & 3.105611462 & 1.234291100 & -0.793300885 \\ \mathrm{H} & 1.145808791 & 1.726634147 & -1.578899533 \\ \mathrm{C} & 3.702077678 & 0.417555939 & 0.362453628 \\ \mathrm{H} & 3.462937807 & 0.927323088 & 1.294716475 \\ \mathrm{C} & 3.635167713 & 2.669369919 & -0.784830165 \\ \mathrm{H} & 3.362561482 & 3.181047827 & 0.134144739 \\ \mathrm{H} & 4.717921812 & 2.687620872 & -0.865925303 \\ \mathrm{H} & 3.231179265 & 3.241460353 & -1.614808908 \\ \mathrm{H} & 1.245021169 & 1.738926003 & 0.167643845 \\ \mathrm{H} & 1.272903180 & -2.051934007 & 0.403693242 \\ \mathrm{H} & 4.787065773 & 0.414069741 & 0.285331420 \\ \mathrm{H} & 3.408935263 & 0.780921588 & -1.734916654 \\ \mathrm{C} & 3.681909177 & -1.653098412 & 1.775139676 \\ \mathrm{H} & 3.333130881 & -2.676508145 & 1.881292024 \\ \mathrm{H} & 4.767289594 & -1.667654951 & 1.819406533 \\ \mathrm{H} & 3.322066431 & -1.089661476 & 2.630607321 \\ \mathrm{H} & 3.747768318 & -1.890809536 & -0.698111371 \\ & 4.833265307 & -1.912507650 & -0.666804901\end{array}$




$\begin{array}{llll}\mathrm{H} & 3.396225796 & -2.915439341 & -0.618539247 \\ \mathrm{H} & 3.461904382 & -1.524786259 & -1.677108559 \\ \mathrm{H} & 1.351376949 & -0.693653772 & -1.628282539\end{array}$

LIIC path connecting FC point and S1-KETO-MIN Coord_1

\begin{tabular}{|c|c|c|c|}
\hline & -4.3 & -0.892718616 & -1.519764319 \\
\hline & -3.009458654 & -0.690621967 & -1.248352811 \\
\hline & -2.595716437 & -0.036108013 & -0.074326527 \\
\hline C & -3.570223387 & 0.416338599 & 0.831222817 \\
\hline & -4.913443725 & 0.210719693 & 055 \\
\hline & -5.307989413 & -0.435488772 & -0.606656831 \\
\hline & -4.654575316 & -1.394575169 & -2.420762464 \\
\hline & -2.263179612 & -1.034073576 & -1.936354700 \\
\hline & -5.633609706 & 0.568373291 & 1.265270560 \\
\hline & -6.355427688 & -0.582847162 & -0.799257130 \\
\hline & -1.153916151 & 0.174371640 & 0.207157637 \\
\hline & -0.749944653 & 0.727831685 & 1.199209247 \\
\hline & -3.286516668 & 1.049884333 & 1.976034305 \\
\hline & -2.344481029 & 1.142190541 & 2.076648289 \\
\hline & -0.365321229 & -0.303890931 & -0.735529843 \\
\hline & 1.070074938 & -0.222262557 & -0.691951631 \\
\hline & 1.659974451 & -1.031863393 & 0.459133073 \\
\hline & 1.583692243 & 1.212218435 & -0.721552452 \\
\hline & 3.204743712 & -1.047668515 & 0.435249409 \\
\hline & 1.328 & -0.604575164 & 1.397099429 \\
\hline & 3.116136136 & 1.223 & -0.815 \\
\hline & 1.149215152 & 1.730866596 & -1.571920965 \\
\hline & 3.723014068 & 0.402811749 & 0.332541873 \\
\hline & 3.501277693 & 0.915125754 & 1.267605491 \\
\hline & 3.654532192 & 2.654976161 & -0.813425819 \\
\hline & 3.399136927 & 3.167289409 & 0.110065687 \\
\hline & 4.735983192 & 2.666339364 & -0.910920324 \\
\hline & 3.241745135 & 3.230486815 & -1.636676856 \\
\hline & 1.273230164 & 1.737329137 & 0.173708703 \\
\hline & 1.276276812 & -2.047752845 & 0.413894329 \\
\hline & 4.806640346 & 0.390990299 & 0.239587518 \\
\hline & 3.403350374 & 0.767953253 & -1.760870867 \\
\hline & 3.705967824 & -1.665456309 & 1.748547244 \\
\hline & 3.350263873 & -2.685749892 & 1.861514596 \\
\hline & 4.791663344 & -1.688976693 & 1.777748817 \\
\hline & 3.362692442 & -1.097403789 & 2.607705128 \\
\hline & 3.735888310 & -1.907570728 & -0.725359385 \\
\hline & 4.821505306 & -1.936543511 & -0.710302112 \\
\hline & 3.378826215 & -2.929698773 & -0.638193414 \\
\hline & 3.437888916 & -1.541639332 & -1.700849551 \\
\hline & 1.3312 & -0.69 & -1.627 \\
\hline
\end{tabular}


Coord_2

\begin{tabular}{lrrr} 
C & -4.363992113 & -0.890964362 & -1.519324610 \\
C & -3.010796515 & -0.691922849 & -1.252447690 \\
C & -2.589864589 & -0.038545303 & -0.078900608 \\
C & -3.568214858 & 0.415356631 & 0.830280358 \\
C & -4.914148954 & 0.214141316 & 0.558863839 \\
C & -5.318609481 & -0.432387433 & -0.603127944 \\
H & -4.667624038 & -1.391707902 & -2.420000173 \\
H & -2.270005464 & -1.037783473 & -1.944822441 \\
H & -5.628514607 & 0.574108641 & 1.275411591 \\
H & -6.366573161 & -0.577210340 & -0.792449058 \\
C & -1.153790784 & 0.171109813 & 0.202741012 \\
O & -0.748296255 & 0.725111153 & 1.196852387 \\
O & -3.276190436 & 1.045431577 & 1.970243243 \\
H & -2.331478170 & 1.134090483 & 2.064585048 \\
O & -0.363006724 & -0.307276020 & -0.740601954 \\
C & 1.071334576 & -0.224231017 & -0.694621849 \\
$\mathrm{C}$ & 1.660765920 & -1.033194575 & 0.457315534 \\
$\mathrm{C}$ & 1.583983513 & 1.210729986 & -0.723308854 \\
$\mathrm{C}$ & 3.205559931 & -1.047084277 & 0.436440730 \\
$\mathrm{H}$ & 1.326659196 & -0.606459141 & 1.394566999 \\
$\mathrm{C}$ & 3.116519424 & 1.223564387 & -0.814092260 \\
$\mathrm{H}$ & 1.150439584 & 1.728896226 & -1.574489613 \\
$\mathrm{C}$ & 3.722309865 & 0.404009531 & 0.334679823 \\
$\mathrm{H}$ & 3.498096650 & 0.916074765 & 1.269289492 \\
$\mathrm{C}$ & 3.653322864 & 2.655948392 & -0.811631760 \\
$\mathrm{H}$ & 3.395335842 & 3.168157726 & 0.111205107 \\
$\mathrm{H}$ & 4.734985238 & 2.668645894 & -0.906855968 \\
$\mathrm{H}$ & 3.241544032 & 3.230810132 & -1.635860320 \\
$\mathrm{H}$ & 1.271031591 & 1.735329222 & 0.171340412 \\
$\mathrm{H}$ & 1.278489895 & -2.049617550 & 0.411218131 \\
$\mathrm{H}$ & 4.806161748 & 0.393524926 & 0.243824117 \\
$\mathrm{H}$ & 3.406076346 & 0.768548657 & -1.759318325 \\
$\mathrm{C}$ & 3.705112668 & -1.664191221 & 1.750707325 \\
$\mathrm{H}$ & 3.350597943 & -2.684993391 & 1.862945332 \\
$\mathrm{H}$ & 4.790801643 & -1.686194321 & 1.782156630 \\
$\mathrm{H}$ & 3.359241043 & -1.096651680 & 2.609164688 \\
$\mathrm{C}$ & 3.740196067 & -1.906290135 & -0.723085050 \\
$\mathrm{H}$ & 4.825842045 & -1.933898339 & -0.705891350 \\
$\mathrm{H}$ & 3.384244244 & -2.928880511 & -0.636653947 \\
$\mathrm{H}$ & 3.443596050 & -1.540696713 & -1.699120523 \\
$\mathrm{H}$ & 1.335198995 & -0.696853649 & -1.629956416 \\
& & & \\
\hline
\end{tabular}

Coord_3

$\begin{array}{llll}\text { C } & -4.374651817 & -0.889158862 & -1.518815610 \\ \text { C } & -3.012122717 & -0.693211549 & -1.256533690\end{array}$ 


$\begin{array}{lrrr}\text { C } & -2.584007083 & -0.040994303 & -0.083492808 \\ \text { C } & -3.566199555 & 0.414362032 & 0.829321557 \\ \text { C } & -4.914797651 & 0.217558213 & 0.564607841 \\ \text { C } & -5.329202282 & -0.429244533 & -0.599540641 \\ \text { H } & -4.680619938 & -1.388785298 & -2.419168772 \\ \text { H } & -2.276842164 & -1.041448777 & -1.953241942 \\ \text { H } & -5.623328802 & 0.579810341 & 1.285521893 \\ \text { H } & -6.377670759 & -0.571541042 & -0.785596858 \\ \text { C } & -1.153660882 & 0.167833713 & 0.198306613 \\ \text { O } & -0.746646952 & 0.722370851 & 1.194480884 \\ \text { O } & -3.265924434 & 1.040936475 & 1.964391442 \\ \text { H } & -2.318591667 & 1.125933983 & 2.052428147 \\ \text { O } & -0.360680027 & -0.310671020 & -0.745685151 \\ \text { C } & 1.072600077 & -0.226206817 & -0.697300650 \\ \text { C } & 1.661559120 & -1.034529975 & 0.455492032 \\ \text { C } & 1.584274312 & 1.209234589 & -0.725073555 \\ \text { C } & 3.206369228 & -1.046499573 & 0.437632331 \\ \text { H } & 1.324880996 & -0.608351542 & 1.392021301 \\ \text { C } & 3.116895024 & 1.223928487 & -0.813120960 \\ \text { H } & 1.151667582 & 1.726917923 & -1.577066616 \\ \text { C } & 3.721592370 & 0.405207128 & 0.336816826 \\ \text { H } & 3.494898054 & 0.917020066 & 1.270966090 \\ \text { C } & 3.652099263 & 2.656917993 & -0.809841056 \\ \text { H } & 3.391516542 & 3.169019729 & 0.112333910 \\ \text { H } & 4.733966540 & 2.670953594 & -0.902790066 \\ \text { H } & 3.241333435 & 3.231130030 & -1.635046916 \\ \text { H } & 1.268829293 & 1.733318724 & 0.168956813 \\ \text { H } & 1.280710793 & -2.051485450 & 0.408537727 \\ \text { H } & 4.805664146 & 0.396062929 & 0.248064620 \\ \text { H } & 3.408798545 & 0.769145055 & -1.757761226 \\ \text { C } & 3.704244267 & -1.662925318 & 1.752866627 \\ \text { H } & 3.350923641 & -2.684235091 & 1.864377032 \\ \text { H } & 4.789919446 & -1.683407721 & 1.786568328 \\ \text { H } & 3.355773344 & -1.095903180 & 2.610616190 \\ \text { C } & 3.744501167 & -1.905003139 & -0.720800152 \\ \text { H } & 4.830169948 & -1.931243340 & -0.701465550 \\ \text { H } & 3.389664543 & -2.928055312 & -0.635102945 \\ \text { H } & 3.449304947 & -1.539747412 & -1.697379525 \\ \text { H } & 1.339130697 & -0.698457151 & -1.632212817\end{array}$

Coord_4

$\begin{array}{lrrr}C & -4.385276617 & -0.887302662 & -1.518237907 \\ C & -3.013437919 & -0.694488247 & -1.260611091 \\ C & -2.578144588 & -0.043454702 & -0.088103006 \\ C & -3.564178559 & 0.413354928 & 0.828346861 \\ C & -4.915391256 & 0.220970317 & 0.570354139 \\ C & -5.339768586 & -0.426060532 & -0.595895440\end{array}$




$\begin{array}{lrrr}\text { H } & -4.693563640 & -1.385808099 & -2.418269176 \\ \mathrm{H} & -2.283689665 & -1.045069678 & -1.961613844 \\ \mathrm{H} & -5.618054207 & 0.585477840 & 1.295601391 \\ \mathrm{H} & -6.388721859 & -0.565839839 & -0.778700557 \\ \mathrm{C} & -1.153526883 & 0.164543512 & 0.193854317 \\ \mathrm{O} & -0.744996856 & 0.719611154 & 1.192095083 \\ \mathrm{O} & -3.255720135 & 1.036399775 & 1.958480040 \\ \mathrm{H} & -2.305823064 & 1.117722179 & 2.040178948 \\ \mathrm{O} & -0.358341328 & -0.314075725 & -0.750779652 \\ \mathrm{C} & 1.073871779 & -0.228190014 & -0.699988251 \\ \mathrm{C} & 1.662354621 & -1.035869873 & 0.453662634 \\ \mathrm{C} & 1.584565116 & 1.207732784 & -0.726846553 \\ \mathrm{C} & 3.207172731 & -1.045914673 & 0.438824531 \\ \mathrm{H} & 1.323102093 & -0.610252543 & 1.389462602 \\ \mathrm{C} & 3.117263823 & 1.224290788 & -0.812151560 \\ \mathrm{H} & 1.152899486 & 1.724932122 & -1.579652515 \\ \mathrm{C} & 3.720862470 & 0.406404428 & 0.338952924 \\ \mathrm{H} & 3.491682852 & 0.917961864 & 1.272635691 \\ \mathrm{C} & 3.650862364 & 2.657885694 & -0.808054056 \\ \mathrm{H} & 3.387680044 & 3.169876329 & 0.113452110 \\ \mathrm{H} & 4.732928443 & 2.673263193 & -0.898722963 \\ \mathrm{H} & 3.241114234 & 3.231447531 & -1.634237016 \\ \mathrm{H} & 1.266623492 & 1.731298125 & 0.166558110 \\ \mathrm{H} & 1.282939995 & -2.053357049 & 0.405853227 \\ \mathrm{H} & 4.805148744 & 0.398604329 & 0.252309320 \\ \mathrm{H} & 3.411518045 & 0.769742856 & -1.756200227 \\ \mathrm{C} & 3.703363367 & -1.661659219 & 1.755025824 \\ \mathrm{H} & 3.351241840 & -2.683475795 & 1.865810134 \\ \mathrm{H} & 4.789017945 & -1.680617624 & 1.790984429 \\ \mathrm{H} & 3.352290241 & -1.095158580 & 2.612060288 \\ \mathrm{C} & 3.748804669 & -1.903710438 & -0.718505052 \\ \mathrm{H} & 4.834490146 & -1.928579240 & -0.697024848 \\ \mathrm{H} & 3.395088144 & -2.927224012 & -0.633540443 \\ \mathrm{H} & 3.455016346 & -1.538792009 & -1.695626921 \\ \mathrm{H} & 1.343072999 & -0.700064151 & -1.634470817\end{array}$

Coord_5

$\begin{array}{llll}\text { C } & -4.395865914 & -0.885395962 & -1.517591607 \\ \text { C } & -3.014741817 & -0.695753050 & -1.264680094 \\ \text { C } & -2.572277086 & -0.045926606 & -0.092731307 \\ \text { C } & -3.562151656 & 0.412335431 & 0.827356161 \\ \text { C } & -4.915929455 & 0.224377117 & 0.576102444 \\ \text { C } & -5.350307685 & -0.422835530 & -0.592192141 \\ \text { H } & -4.706454541 & -1.382776601 & -2.417301676 \\ \text { H } & -2.290547464 & -1.048646175 & -1.969938241 \\ \text { H } & -5.612691006 & 0.591110842 & 1.305649695 \\ \text { H } & -6.399726062 & -0.560107040 & -0.771760154\end{array}$




\begin{tabular}{|c|c|c|c|}
\hline & 3 & 0.161239413 & 0.1 \\
\hline 0 & -0.743346056 & 0.716831952 & 994984 \\
\hline & -3.245577934 & 1.031821874 & 34t \\
\hline & -2.293173463 & 1.109455981 & 2.02783834 \\
\hline 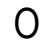 & -0.355990627 & -0.317490225 & .755885456 \\
\hline 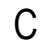 & 1.075149578 & 200180514 & 年 \\
\hline & 2218 & 37214073 & \\
\hline & 1.584856015 & 206224486 & -0.72862795 \\
\hline & 3.207970032 & -1.045329673 & 0.440017132 \\
\hline$\Pi$ & & & \\
\hline C & 3.117625622 & 1.224651391 & 256 \\
\hline$H$ & 1.154135485 & 1.722939124 & -1.582247214 \\
\hline 0 & 3.720120066 & 0.407601427 & 0.341088123 \\
\hline $\mathrm{H}$ & 450950 & 0.9 & 98292 \\
\hline C & $3.649612^{1}$ & 2.65 & -0.80 \\
\hline $\mathrm{H}$ & 3.383826342 & 3.170727527 & 0.114559709 \\
\hline$\Gamma$ & 4.731870940 & 2.675574692 & -0.894654564 \\
\hline & 86735 & 62635 & 430618 \\
\hline $\mathrm{H}$ & 1.26 & & 0.1 \\
\hline $\mathrm{H}$ & 1.285 & -2.05 & 0.403164731 \\
\hline $\mathrm{H}$ & 4.804615444 & 0.40 & 0.256558116 \\
\hline$\pi$ & & & \\
\hline 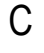 & & & \\
\hline H & & & \\
\hline $\mathrm{H}$ & 4.78 & -1.6 & $1.7 \varsigma$ \\
\hline$H$ & 3. & $-1 .(\mathrm{r}$ & 2.6 \\
\hline U & & & \\
\hline$\Pi$ & & & \\
\hline $\mathrm{H}$ & 3.40 & -2.92 & -0.6 \\
\hline $\mathrm{H}$ & 3.46 & -1.5 & 362824 \\
\hline & & & \\
\hline \multicolumn{4}{|c|}{ Coord_6 } \\
\hline 0 & -4.406 & & \\
\hline C & & & \\
\hline 0 & & -0.0 & \\
\hline C & -3.5 & 0.41 & 0.826349460 \\
\hline C & -4.91 & 0.227778314 & 0.581852642 \\
\hline C & 19088 & -0.419570 & -0.588430845 \\
\hline$\cdots$ & -4.7 & & \\
\hline H & & & \\
\hline $\mathrm{H}$ & -5.6 & 0.596708542 & 1.315666396 \\
\hline $\mathrm{H}$ & -6.410682963 & -0.554343040 & -0.764775755 \\
\hline C & -1.15324 & 0.157921 & 0.184895 \\
\hline 0 & & & \\
\hline 0 & & 1.02 & \\
\hline & & 1.101136378 & \\
\hline
\end{tabular}




$\begin{array}{lrrr}\text { O } & -0.353627724 & -0.320914325 & -0.761002753 \\ \text { C } & 1.076433680 & -0.232178317 & -0.705390050 \\ \text { C } & 1.663951922 & -1.038562676 & 0.449986133 \\ \text { C } & 1.585146814 & 1.204709685 & -0.730417753 \\ \text { C } & 3.208761131 & -1.044744477 & 0.441210332 \\ \text { H } & 1.319542196 & -0.614080246 & 1.384306299 \\ \text { C } & 3.117980626 & 1.225010289 & -0.810218957 \\ \text { H } & 1.155375585 & 1.720938824 & -1.584850914 \\ \text { C } & 3.719365068 & 0.408798230 & 0.343222427 \\ \text { H } & 3.485202352 & 0.919834865 & 1.275953791 \\ \text { C } & 3.648348661 & 2.659815693 & -0.804490958 \\ \text { H } & 3.379955443 & 3.171573226 & 0.115656609 \\ \text { H } & 4.730793943 & 2.677887990 & -0.890584863 \\ \text { H } & 3.240650733 & 3.232075230 & -1.632627719 \\ \text { H } & 1.262201893 & 1.727227122 & 0.161714810 \\ \text { H } & 1.287422491 & -2.057111446 & 0.400472129 \\ \text { H } & 4.804064147 & 0.403697131 & 0.260811019 \\ \text { H } & 3.416948245 & 0.770942655 & -1.753065926 \\ \text { C } & 3.701563667 & -1.659125920 & 1.759343624 \\ \text { H } & 3.351854543 & -2.681954294 & 1.868680534 \\ \text { H } & 4.787156146 & -1.675026818 & 1.799829531 \\ \text { H } & 3.345276939 & -1.093681376 & 2.614925889 \\ \text { C } & 3.757405768 & -1.901107236 & -0.713883753 \\ \text { H } & 4.843106750 & -1.923223437 & -0.688098448 \\ \text { H } & 3.405943946 & -2.925542408 & -0.630380646 \\ \text { H } & 3.466446749 & -1.536862512 & -1.692086921 \\ \text { H } & 1.350989496 & -0.703287952 & -1.638991419\end{array}$

Coord_7

$\begin{array}{lrrr}\text { C } & -4.416935318 & -0.881432361 & -1.516094110 \\ \text { C } & -3.017315116 & -0.698246152 & -1.272792492 \\ \text { C } & -2.560526986 & -0.050904401 & -0.102041905 \\ \text { C } & -3.558080854 & 0.410258929 & 0.825326761 \\ \text { C } & -4.916839852 & 0.231173515 & 0.587604445 \\ \text { C } & -5.371301988 & -0.416264329 & -0.584611741 \\ \text { H } & -4.732076039 & -1.376552397 & -2.415163472 \\ \text { H } & -2.304291165 & -1.055665479 & -1.986444841 \\ \text { H } & -5.601699804 & 0.602270444 & 1.325650997 \\ \text { H } & -6.421592164 & -0.548548237 & -0.757747154 \\ \text { C } & -1.153099181 & 0.154589709 & 0.180389412 \\ \text { O } & -0.740042355 & 0.711215853 & 1.184851584 \\ \text { O } & -3.225482031 & 1.022544576 & 1.940392640 \\ \text { H } & -2.268234361 & 1.092764180 & 2.002885944 \\ \text { O } & -0.351252623 & -0.324347823 & -0.766131354 \\ \text { C } & 1.077723978 & -0.234183317 & -0.708104153 \\ \text { C } & 1.664753720 & -1.039915677 & 0.448139035 \\ \text { C } & 1.585437814 & 1.203188686 & -0.732215955\end{array}$




$\begin{array}{lrrr}\mathrm{C} & 3.209545932 & -1.044159175 & 0.442404034 \\ \mathrm{H} & 1.317761096 & -0.616006943 & 1.381708499 \\ \mathrm{C} & 3.118328724 & 1.225367489 & -0.809255558 \\ \mathrm{H} & 1.156619882 & 1.718931321 & -1.587463414 \\ \mathrm{C} & 3.718597369 & 0.409994827 & 0.345355826 \\ \mathrm{H} & 3.481936953 & 0.920765868 & 1.277602194 \\ \mathrm{C} & 3.647071862 & 2.660777790 & -0.802714859 \\ \mathrm{H} & 3.376067344 & 3.172413427 & 0.116742909 \\ \mathrm{H} & 4.729697339 & 2.680203093 & -0.886513966 \\ \mathrm{H} & 3.240406332 & 3.232385434 & -1.631828317 \\ \mathrm{H} & 1.259986191 & 1.725176724 & 0.159270112 \\ \mathrm{H} & 1.289675791 & -2.058993946 & 0.397775431 \\ \mathrm{H} & 4.803494747 & 0.406248527 & 0.265068017 \\ \mathrm{H} & 3.419659045 & 0.771544758 & -1.751492529 \\ \mathrm{C} & 3.700644566 & -1.657858922 & 1.761502128 \\ \mathrm{H} & 3.352148840 & -2.681192094 & 1.870117833 \\ \mathrm{H} & 4.786195446 & -1.672226222 & 1.804258628 \\ \mathrm{H} & 3.341746538 & -1.092948879 & 2.616347386 \\ \mathrm{C} & 3.761703169 & -1.899796739 & -0.711557452 \\ \mathrm{H} & 4.847402849 & -1.920531739 & -0.683612549 \\ \mathrm{H} & 3.411375844 & -2.924692111 & -0.628783346 \\ \mathrm{H} & 3.472165552 & -1.535888212 & -1.690299323 \\ \mathrm{H} & 1.354963596 & -0.704904652 & -1.641254118\end{array}$

Coord_8

$\begin{array}{lrrr}\text { C } & -4.427414017 & -0.879376164 & -1.515243309 \\ \text { C } & -3.018584019 & -0.699474552 & -1.276835993 \\ \text { C } & -2.554644382 & -0.053410103 & -0.106724108 \\ \text { C } & -3.556036955 & 0.409202030 & 0.824287960 \\ \text { C } & -4.917211652 & 0.234562419 & 0.593357543 \\ \text { C } & -5.381755885 & -0.412918632 & -0.580734840 \\ \text { H } & -4.744805440 & -1.373360300 & -2.413993371 \\ \text { H } & -2.311175867 & -1.059108274 & -1.994627344 \\ \text { H } & -5.596072401 & 0.607796044 & 1.335603297 \\ \text { H } & -6.432453162 & -0.542722837 & -0.750674356 \\ \text { C } & -1.152947985 & 0.151244308 & 0.175864814 \\ \text { O } & -0.738389554 & 0.708379050 & 1.182408288 \\ \text { O } & -3.215529133 & 1.017846175 & 1.934247437 \\ \text { H } & -2.255946564 & 1.084340377 & 1.990275846 \\ \text { O } & -0.348865425 & -0.327790724 & -0.771271454 \\ \text { C } & 1.079020378 & -0.236195318 & -0.710827251 \\ \text { C } & 1.665557519 & -1.041272873 & 0.446286030 \\ \text { C } & 1.585728914 & 1.201661285 & -0.734022651 \\ \text { C } & 3.210324331 & -1.043573673 & 0.443598133 \\ \text { H } & 1.315979192 & -0.617942044 & 1.379097597 \\ \text { C } & 3.118669927 & 1.225722991 & -0.808294160 \\ \text { H } & 1.157868386 & 1.716916823 & -1.590084715\end{array}$




$\begin{array}{lrrr}\mathrm{C} & 3.717816965 & 0.411191027 & 0.347488326 \\ \mathrm{H} & 3.478654551 & 0.921693267 & 1.279243491 \\ \mathrm{C} & 3.645781860 & 2.661737993 & -0.800942358 \\ \mathrm{H} & 3.372162143 & 3.173248030 & 0.117818409 \\ \mathrm{H} & 4.728581241 & 2.682519895 & -0.882441661 \\ \mathrm{H} & 3.240153735 & 3.232693230 & -1.631032419 \\ \mathrm{H} & 1.257767292 & 1.723116425 & 0.156810009 \\ \mathrm{H} & 1.291936892 & -2.060879849 & 0.395074627 \\ \mathrm{H} & 4.802907144 & 0.408803131 & 0.269329121 \\ \mathrm{H} & 3.422366946 & 0.772148258 & -1.749915125 \\ \mathrm{C} & 3.699712367 & -1.656591818 & 1.763660324 \\ \mathrm{H} & 3.352434744 & -2.680428994 & 1.871556433 \\ \mathrm{H} & 4.785214547 & -1.669422223 & 1.808692028 \\ \mathrm{H} & 3.338200040 & -1.092220376 & 2.617761289 \\ \mathrm{C} & 3.765998369 & -1.898480336 & -0.709220653 \\ \mathrm{H} & 4.851690550 & -1.917830840 & -0.679111648 \\ \mathrm{H} & 3.416810347 & -2.923835309 & -0.627174446 \\ \mathrm{H} & 3.477886350 & -1.534907610 & -1.688499920 \\ \mathrm{H} & 1.358948300 & -0.706524453 & -1.643518218\end{array}$

\section{Coord_9}

$\begin{array}{lrrr}\text { C } & -4.437854520 & -0.877270663 & -1.514324610 \\ \text { C } & -3.019840916 & -0.700690649 & -1.280870991 \\ \text { C } & -2.548756581 & -0.055926807 & -0.111424408 \\ \text { C } & -3.553987457 & 0.408132827 & 0.823233162 \\ \text { C } & -4.917527952 & 0.237944619 & 0.599111843 \\ \text { C } & -5.392180190 & -0.409533332 & -0.576800143 \\ \text { H } & -4.757479843 & -1.370115501 & -2.412756076 \\ \text { H } & -2.318068268 & -1.062506375 & -2.002762746 \\ \text { H } & -5.590357403 & 0.613284644 & 1.345522597 \\ \text { H } & -6.443265666 & -0.536867438 & -0.743557351 \\ \text { C } & -1.152792481 & 0.147885308 & 0.171322013 \\ \text { O } & -0.736736156 & 0.705523048 & 1.179950386 \\ \text { O } & -3.205640229 & 1.013108473 & 1.928044940 \\ \text { H } & -2.243781160 & 1.075865878 & 1.977577740 \\ \text { O } & -0.346465924 & -0.331242923 & -0.776423058 \\ \text { C } & 1.080323180 & -0.238214415 & -0.713559250 \\ \text { C } & 1.666363218 & -1.042634277 & 0.444427031 \\ \text { C } & 1.586020216 & 1.200127587 & -0.735837851 \\ \text { C } & 3.211096131 & -1.042987974 & 0.444792830 \\ \text { H } & 1.314196394 & -0.619885643 & 1.376473397 \\ \text { C } & 3.119004123 & 1.226076888 & -0.807334756 \\ \text { H } & 1.159121282 & 1.714895223 & -1.592715017 \\ \text { C } & 3.717023766 & 0.412387031 & 0.349619926 \\ \text { H } & 3.475355252 & 0.922616967 & 1.280877590 \\ \text { C } & 3.644478560 & 2.662696391 & -0.799173557 \\ \text { H } & 3.368239845 & 3.174077028 & 0.118883209\end{array}$




$\begin{array}{llrr}\mathrm{H} & 4.727445642 & 2.684838395 & -0.878368166 \\ \mathrm{H} & 3.239892835 & 3.232998634 & -1.630239918 \\ \mathrm{H} & 1.255545192 & 1.721046226 & 0.154334513 \\ \mathrm{H} & 1.294205993 & -2.062769250 & 0.392369727 \\ \mathrm{H} & 4.802301146 & 0.411360829 & 0.273594517 \\ \mathrm{H} & 3.425071846 & 0.772753356 & -1.748333425 \\ \mathrm{C} & 3.698766965 & -1.655324417 & 1.765818225 \\ \mathrm{H} & 3.352712043 & -2.679664894 & 1.872996335 \\ \mathrm{H} & 4.784213343 & -1.666615022 & 1.813129629 \\ \mathrm{H} & 3.334637438 & -1.091496181 & 2.619167488 \\ \mathrm{C} & 3.770291070 & -1.897157938 & -0.706873350 \\ \mathrm{H} & 4.855969651 & -1.915120638 & -0.674595448 \\ \mathrm{H} & 3.422247246 & -2.922972210 & -0.625553745 \\ \mathrm{H} & 3.483609349 & -1.533920408 & -1.686688620 \\ \mathrm{H} & 1.362943599 & -0.708147148 & -1.645783917\end{array}$

$\begin{array}{lrrr}\text { Coord_10 } & & \\ \text { C } & -4.448234819 & -0.875158774 & -1.513384860 \\ \text { C } & -3.021068136 & -0.701922643 & -1.284932024 \\ \text { C } & -2.542862605 & -0.058493666 & -0.116164730 \\ \text { C } & -3.551943187 & 0.406985952 & 0.822139548 \\ \text { C } & -4.917795244 & 0.241240726 & 0.604832694 \\ \text { C } & -5.402564690 & -0.406176628 & -0.572854401 \\ \text { H } & -4.770065012 & -1.366851711 & -2.411507625 \\ \text { H } & -2.324941207 & -1.065868699 & -2.010885897 \\ \text { H } & -5.584571702 & 0.618637753 & 1.355374967 \\ \text { H } & -6.454016796 & -0.531060908 & -0.736451903 \\ \text { C } & -1.152636320 & 0.144486969 & 0.166750974 \\ \text { O } & -0.735099754 & 0.702612642 & 1.177478874 \\ \text { O } & -3.195841760 & 1.008255404 & 1.921773909 \\ \text { H } & -2.231766211 & 1.067276215 & 1.964787844 \\ \text { O } & -0.344045504 & -0.334706383 & -0.781596945 \\ \text { C } & 1.081638646 & -0.240226018 & -0.716299801 \\ \text { C } & 1.667179241 & -1.043994495 & 0.442554521 \\ \text { C } & 1.586300336 & 1.198608435 & -0.737637032 \\ \text { C } & 3.211869269 & -1.042377584 & 0.445991093 \\ \text { H } & 1.312409542 & -0.621850256 & 1.373831908 \\ \text { C } & 3.119319793 & 1.226469707 & -0.806341956 \\ \text { H } & 1.160366891 & 1.712894224 & -1.595325014 \\ \text { C } & 3.716207949 & 0.413614230 & 0.351778165 \\ \text { H } & 3.472016620 & 0.923552039 & 1.282537354 \\ \text { C } & 3.643132370 & 2.663699362 & -0.797348679 \\ \text { H } & 3.364258273 & 3.174930316 & 0.120002096 \\ \text { H } & 4.726260650 & 2.687219714 & -0.874225734 \\ \text { H } & 3.239592855 & 3.233355003 & -1.629385519 \\ \text { H } & 1.253296179 & 1.718969835 & 0.151872881 \\ \text { H } & 1.296504205 & -2.064660180 & 0.389635928\end{array}$




$\begin{array}{rrrr}\mathrm{H} & 4.801667133 & 0.413967890 & 0.277898988 \\ \mathrm{H} & 3.427774127 & 0.773418008 & -1.746716138 \\ \mathrm{C} & 3.697814356 & -1.654045268 & 1.767973198 \\ \mathrm{H} & 3.352999064 & -2.678893655 & 1.874417816 \\ \mathrm{H} & 4.783197323 & -1.663780098 & 1.817576252 \\ \mathrm{H} & 3.331051919 & -1.090781347 & 2.620568548 \\ \mathrm{C} & 3.774607500 & -1.895781278 & -0.704520390 \\ \mathrm{H} & 4.860265862 & -1.912340208 & -0.670061658 \\ \mathrm{H} & 3.427724776 & -2.922059512 & -0.623943316 \\ \mathrm{H} & 3.489362892 & -1.532868171 & -1.684866853 \\ \mathrm{H} & 1.366968167 & -0.709741184 & -1.648055299\end{array}$

LIIC path connecting S1-KETO-MIN and T1-KETO-MIN Coord_1

$\begin{array}{lrrr}\text { C } & -4.448234819 & -0.875158774 & -1.513384860 \\ \text { C } & -3.021068136 & -0.701922643 & -1.284932024 \\ \text { C } & -2.542862605 & -0.058493666 & -0.116164730 \\ \text { C } & -3.551943187 & 0.406985952 & 0.822139548 \\ \text { C } & -4.917795244 & 0.241240726 & 0.604832694 \\ \text { C } & -5.402564690 & -0.406176628 & -0.572854401 \\ \text { H } & -4.770065012 & -1.366851711 & -2.411507625 \\ \text { H } & -2.324941207 & -1.065868699 & -2.010885897 \\ \text { H } & -5.584571702 & 0.618637753 & 1.355374967 \\ \text { H } & -6.454016796 & -0.531060908 & -0.736451903 \\ \text { C } & -1.152636320 & 0.144486969 & 0.166750974 \\ \text { O } & -0.735099754 & 0.702612642 & 1.177478874 \\ \text { O } & -3.195841760 & 1.008255404 & 1.921773909 \\ \text { H } & -2.231766211 & 1.067276215 & 1.964787844 \\ \text { O } & -0.344045504 & -0.334706383 & -0.781596945 \\ \text { C } & 1.081638646 & -0.240226018 & -0.716299801 \\ \text { C } & 1.667179241 & -1.043994495 & 0.442554521 \\ \text { C } & 1.586300336 & 1.198608435 & -0.737637032 \\ \text { C } & 3.211869269 & -1.042377584 & 0.445991093 \\ \text { H } & 1.312409542 & -0.621850256 & 1.373831908 \\ \text { C } & 3.119319793 & 1.226469707 & -0.806341956 \\ \text { H } & 1.160366891 & 1.712894224 & -1.595325014 \\ \text { C } & 3.716207949 & 0.413614230 & 0.351778165 \\ \text { H } & 3.472016620 & 0.923552039 & 1.282537354 \\ \text { C } & 3.643132370 & 2.663699362 & -0.797348679 \\ \text { H } & 3.364258273 & 3.174930316 & 0.120002096 \\ \text { H } & 4.726260650 & 2.687219714 & -0.874225734 \\ \text { H } & 3.239592855 & 3.233355003 & -1.629385519 \\ \text { H } & 1.253296179 & 1.718969835 & 0.151872881 \\ \text { H } & 1.296504205 & -2.064660180 & 0.389635928 \\ \text { H } & 4.801667133 & 0.413967890 & 0.277898988 \\ \text { H } & 3.427774127 & 0.773418008 & -1.746716138 \\ \text { C } & 3.697814356 & -1.654045268 & 1.767973198\end{array}$

S55 


$\begin{array}{llll}\mathrm{H} & 3.352999064 & -2.678893655 & 1.874417816 \\ \mathrm{H} & 4.783197323 & -1.663780098 & 1.817576252 \\ \mathrm{H} & 3.331051919 & -1.090781347 & 2.620568548 \\ \mathrm{C} & 3.774607500 & -1.895781278 & -0.704520390 \\ \mathrm{H} & 4.860265862 & -1.912340208 & -0.670061658 \\ \mathrm{H} & 3.427724776 & -2.922059512 & -0.623943316 \\ \mathrm{H} & 3.489362892 & -1.532868171 & -1.684866853 \\ \mathrm{H} & 1.366968167 & -0.709741184 & -1.648055299\end{array}$

Coord_2

\begin{tabular}{|c|c|c|c|}
\hline & -4.435629621 & -0.878947361 & -1.518441111 \\
\hline & -3.020596719 & -0.703771048 & -1.287315095 \\
\hline & -2.545182285 & -0.054950104 & -0.109877210 \\
\hline & -3.560590054 & 0.411506231 & 0013557 \\
\hline & -4.916817256 & 0.241150417 & \\
\hline & -5.395786686 & -0.410703829 & -0.579079039 \\
\hline & -4.755803944 & -1.371544899 & -2.416877975 \\
\hline & -2.317744968 & -1.064819478 & -2.0082698 \\
\hline & -5.592053903 & 0.615488545 & 1.349717396 \\
\hline & -6.446352664 & -0.538149439 & -0.7459861 \\
\hline & -1.152635585 & 0.147816012 & 0.171883814 \\
\hline & -0.736244254 & 0.705380250 & 1.18095988 \\
\hline & -3.204639332 & 1.015856374 & 134138 \\
\hline & -2.242496259 & 1.075999776 & 1.978443940 \\
\hline & -0.346166526 & -0.331767422 & -0.776795254 \\
\hline & 1.080 & -0.238508219 & -0.713690650 \\
\hline & 1.666600422 & -1.042857876 & 0.4443020 \\
\hline & 1.586014014 & 1.199897585 & -0.735833855 \\
\hline & 3.211343733 & -1.042976575 & 0.444814934 \\
\hline & 1.314317893 & -0.620214744 & 1.37636199 \\
\hline & 3.11 & 40290 & -0.807350 \\
\hline & 1.159046483 & 1.714705925 & -1.592659813 \\
\hline & 3.717088669 & 0.412469128 & 0.349652523 \\
\hline & 3.475325550 & 0.922689766 & 1.280889692 \\
\hline & 3.644 & 2.662696391 & -0.79934 \\
\hline & 3.36 & 3.17 & 0.118578408 \\
\hline & 4.727342141 & 2.684910294 & -0.878384861 \\
\hline & 3.239861433 & 3.232819735 & -1.630575215 \\
\hline & 1.255513288 & 1.720727523 & 0.154395813 \\
\hline & 1.29 & -2.063064351 & 0.392200829 \\
\hline & 4.802369 & 0.411575130 & 0.27366422 \\
\hline & 3.425072248 & 0.772659755 & -1.748316126 \\
\hline & 3.699014266 & -1.655241717 & 1.765872825 \\
\hline & 186842 & -2.679667693 & 1.872970537 \\
\hline & 4.784 & -1.666285318 & 1.813311729 \\
\hline & 3.334655341 & -1.091532477 & 2.6192011 \\
\hline & & & -0.706 \\
\hline
\end{tabular}




$\begin{array}{llll}H & 4.856447149 & -1.914913936 & -0.674387249 \\ H & 3.422826547 & -2.922915213 & -0.625554645 \\ H & 3.484172552 & -1.533826612 & -1.686640523 \\ H & 1.363314700 & -0.708334953 & -1.645903019\end{array}$

Coord_3

\begin{tabular}{lrrr} 
C & -4.422936421 & -0.882696264 & -1.523424807 \\
C & -3.020040818 & -0.705629852 & -1.289717792 \\
C & -2.547503484 & -0.051412306 & -0.103613006 \\
C & -3.569269355 & 0.415997528 & 0.837828659 \\
C & -4.915811655 & 0.241080719 & 0.605181845 \\
C & -5.388910187 & -0.415206128 & -0.585240943 \\
H & -4.741463744 & -1.376198097 & -2.422168572 \\
H & -2.310521169 & -1.063748476 & -2.005635644 \\
H & -5.599455701 & 0.612302443 & 1.344023396 \\
H & -6.438575563 & -0.545211738 & -0.755438153 \\
C & -1.152638982 & 0.151138113 & 0.176995613 \\
O & -0.737397056 & 0.708137951 & 1.184422484 \\
O & -3.213501331 & 1.023436875 & 1.944437840 \\
H & -2.253299360 & 1.084709980 & 1.992048044 \\
O & -0.348286627 & -0.328831124 & -0.772007656 \\
C & 1.079156376 & -0.236792917 & -0.711091454 \\
C & 1.666011220 & -1.041723875 & 0.446040730 \\
C & 1.585720315 & 1.201183687 & -0.734037754 \\
C & 3.210799828 & -1.043576673 & 0.443636832 \\
H & 1.316213396 & -0.618586545 & 1.378876502 \\
C & 3.118651225 & 1.225609789 & -0.808358857 \\
H & 1.157724481 & 1.716515823 & -1.590001015 \\
C & 3.717947270 & 0.411321628 & 0.347526326 \\
H & 3.478608952 & 0.921820867 & 1.279235691 \\
C & 3.645577762 & 2.661690891 & -0.801339659 \\
H & 3.371621044 & 3.173505628 & 0.117149206 \\
H & 4.728398638 & 2.682599890 & -0.882538162 \\
H & 3.240116333 & 3.232283134 & -1.631763620 \\
H & 1.257720391 & 1.722477825 & 0.156905013 \\
H & 1.292752492 & -2.061469749 & 0.394756729 \\
H & 4.803045047 & 0.409181529 & 0.269434221 \\
H & 3.422358945 & 0.771904455 & -1.749910326 \\
C & 3.700187764 & -1.656441817 & 1.763768726 \\
H & 3.353350644 & -2.680444292 & 1.871518935 \\
H & 4.785684446 & -1.668793020 & 1.809048429 \\
H & 3.338229838 & -1.092291476 & 2.617823891 \\
C & 3.766919370 & -1.898337638 & -0.709079151 \\
H & 4.852607349 & -1.917481535 & -0.678701149 \\
H & 3.417915745 & -2.923764913 & -0.627159947 \\
H & 3.478972449 & -1.534778210 & -1.688405821 \\
H & 1.359660800 & -0.706926950 & -1.643754718 \\
& & \\
\hline
\end{tabular}




$\begin{array}{lrrr}\text { Coord_4 } & & & \\ \text { C } & -4.410156917 & -0.886405161 & -1.528336308 \\ \text { C } & -3.019401217 & -0.707499250 & -1.292139694 \\ \text { C } & -2.549826683 & -0.047880301 & -0.097372106 \\ \text { C } & -3.577982158 & 0.420459731 & 0.845584762 \\ \text { C } & -4.914780855 & 0.241031818 & 0.605418742 \\ \text { C } & -5.381937186 & -0.419682930 & -0.591340341 \\ \text { H } & -4.727046341 & -1.380811200 & -2.427379772 \\ \text { H } & -2.303270763 & -1.062656074 & -2.002983746 \\ \text { H } & -5.606778805 & 0.609079743 & 1.338293396 \\ \text { H } & -6.430687964 & -0.552247239 & -0.764808352 \\ \text { C } & -1.152646782 & 0.154453213 & 0.182086610 \\ \text { O } & -0.738557954 & 0.710885852 & 1.187867084 \\ \text { O } & -3.222429234 & 1.030996675 & 1.955685540 \\ \text { H } & -2.264176460 & 1.093406680 & 2.005600443 \\ \text { O } & -0.350405823 & -0.325897725 & -0.767234355 \\ \text { C } & 1.077909180 & -0.235080219 & -0.708502353 \\ \text { C } & 1.665412218 & -1.040592976 & 0.447770732 \\ \text { C } & 1.585419615 & 1.202467185 & -0.732249151 \\ \text { C } & 3.210238731 & -1.044178173 & 0.442456830 \\ \text { H } & 1.318096695 & -0.616965945 & 1.381376102 \\ \text { C } & 3.118295925 & 1.225178589 & -0.809367860 \\ \text { H } & 1.156401083 & 1.718324323 & -1.587349313 \\ \text { C } & 3.718785069 & 0.410171927 & 0.345399425 \\ \text { H } & 3.481868048 & 0.920945767 & 1.277575693 \\ \text { C } & 3.646772962 & 2.660683591 & -0.803334557 \\ \text { H } & 3.375270044 & 3.172784127 & 0.115714506 \\ \text { H } & 4.729431740 & 2.680289291 & -0.886685964 \\ \text { H } & 3.240358431 & 3.231745934 & -1.632951316 \\ \text { H } & 1.259917990 & 1.724221326 & 0.159400411 \\ \text { H } & 1.290867292 & -2.059877048 & 0.397303728 \\ \text { H } & 4.803694247 & 0.406787129 & 0.265208921 \\ \text { H } & 3.419635343 & 0.771152156 & -1.751499328 \\ \text { C } & 3.701336168 & -1.657645917 & 1.761661225 \\ \text { H } & 3.353491844 & -2.681224194 & 1.870063333 \\ \text { H } & 4.786880545 & -1.671303522 & 1.804786530 \\ \text { H } & 3.341776839 & -1.093058678 & 2.616437388 \\ \text { C } & 3.763053672 & -1.899609034 & -0.711348750 \\ \text { H } & 4.848748049 & -1.920043536 & -0.683003752 \\ \text { H } & 3.412993545 & -2.924609511 & -0.628759348 \\ \text { H } & 3.473763651 & -1.535723310 & -1.690163219 \\ \text { H } & 1.356006995 & -0.705517651 & -1.641610820 \\ & & & \end{array}$

Coord_5

$\begin{array}{llll}\text { C } & -4.397291814 & -0.890073762 & -1.533175311 \\ \text { C } & -3.018677816 & -0.709378851 & -1.294580095\end{array}$




$\begin{array}{lrrr}\text { C } & -2.552151983 & -0.044354203 & -0.091154104 \\ \text { C } & -3.586728357 & 0.424892331 & 0.853281661 \\ \text { C } & -4.913725856 & 0.241003919 & 0.605697041 \\ \text { C } & -5.374868289 & -0.424133628 & -0.597376846 \\ \text { H } & -4.712552240 & -1.385383498 & -2.432511675 \\ \text { H } & -2.295993766 & -1.061542378 & -2.000314042 \\ \text { H } & -5.614023104 & 0.605820742 & 1.332527396 \\ \text { H } & -6.422690662 & -0.559255239 & -0.774096556 \\ \text { C } & -1.152658884 & 0.157761311 & 0.187157012 \\ \text { O } & -0.739727055 & 0.713623852 & 1.191293787 \\ \text { O } & -3.231423332 & 1.038535376 & 1.966877041 \\ \text { H } & -2.275128265 & 1.102089278 & 2.019100945 \\ \text { O } & -0.352524125 & -0.322967422 & -0.762475157 \\ \text { C } & 1.076658279 & -0.233370315 & -0.705923450 \\ \text { C } & 1.664803521 & -1.039465177 & 0.449492130 \\ \text { C } & 1.585112015 & 1.203747789 & -0.730467850 \\ \text { C } & 3.209660632 & -1.044781176 & 0.441274934 \\ \text { H } & 1.319967898 & -0.615352944 & 1.383860700 \\ \text { C } & 3.117927025 & 1.224746389 & -0.810377557 \\ \text { H } & 1.155076283 & 1.720131326 & -1.584704512 \\ \text { C } & 3.719602266 & 0.409019829 & 0.343271826 \\ \text { H } & 3.485102951 & 0.920064364 & 1.275909690 \\ \text { C } & 3.647950265 & 2.659674392 & -0.805329359 \\ \text { H } & 3.378897945 & 3.172056831 & 0.114274208 \\ \text { H } & 4.730441442 & 2.677978490 & -0.890828364 \\ \text { H } & 3.240587835 & 3.231208131 & -1.634138319 \\ \text { H } & 1.262106191 & 1.725957922 & 0.161882210 \\ \text { H } & 1.288976092 & -2.058286447 & 0.399841927 \\ \text { H } & 4.804317745 & 0.404392227 & 0.260988118 \\ \text { H } & 3.416901444 & 0.770402957 & -1.753083426 \\ \text { C } & 3.702459966 & -1.658854018 & 1.759550427 \\ \text { H } & 3.353610840 & -2.682007492 & 1.868603735 \\ \text { H } & 4.788045846 & -1.673816918 & 1.800526129 \\ \text { H } & 3.345296640 & -1.093834176 & 2.615041689 \\ \text { C } & 3.759174173 & -1.900876139 & -0.713612253 \\ \text { H } & 4.844869550 & -1.922600039 & -0.687295247 \\ \text { H } & 3.408060147 & -2.925449109 & -0.630352944 \\ \text { H } & 3.468546149 & -1.536662208 & -1.691913023 \\ \text { H } & 1.352353196 & -0.704107049 & -1.639471521\end{array}$

Coord_6

$\begin{array}{lrrr}C & -4.384341418 & -0.893701362 & -1.537941811 \\ C & -3.017870318 & -0.711268654 & -1.297038293 \\ C & -2.554479284 & -0.040834301 & -0.084958804 \\ C & -3.595508058 & 0.429294932 & 0.860919260 \\ C & -4.912647652 & 0.240997315 & 0.606016844 \\ C & -5.367704086 & -0.428557730 & -0.603350442\end{array}$




$\begin{array}{lrrr}\text { H } & -4.697981937 & -1.389914700 & -2.437563974 \\ \mathrm{H} & -2.288690364 & -1.060407579 & -1.997626345 \\ \mathrm{H} & -5.621188603 & 0.602525544 & 1.326725396 \\ \mathrm{H} & -6.414584460 & -0.566235140 & -0.783302854 \\ \mathrm{C} & -1.152675284 & 0.161062012 & 0.192207014 \\ \mathrm{O} & -0.740903956 & 0.716351851 & 1.194702784 \\ \mathrm{O} & -3.240484032 & 1.046052577 & 1.978012242 \\ \mathrm{H} & -2.286155065 & 1.110757079 & 2.032549546 \\ \mathrm{O} & -0.354641326 & -0.320040325 & -0.757729956 \\ \mathrm{C} & 1.075403679 & -0.231663317 & -0.703354549 \\ \mathrm{C} & 1.664185421 & -1.038340575 & 0.451204934 \\ \mathrm{C} & 1.584797615 & 1.205025588 & -0.728694053 \\ \mathrm{C} & 3.209065630 & -1.045385575 & 0.440091132 \\ \mathrm{H} & 1.321827093 & -0.613747542 & 1.386330498 \\ \mathrm{C} & 3.117544626 & 1.224313490 & -0.811387756 \\ \mathrm{H} & 1.153750085 & 1.721936624 & -1.582066711 \\ \mathrm{C} & 3.720398969 & 0.407865529 & 0.341143327 \\ \mathrm{H} & 3.488313951 & 0.919176765 & 1.274237590 \\ \mathrm{C} & 3.649109761 & 2.658663394 & -0.807324157 \\ \mathrm{H} & 3.382504743 & 3.171323730 & 0.112828210 \\ \mathrm{H} & 4.731427839 & 2.675667594 & -0.894965762 \\ \mathrm{H} & 3.240804332 & 3.230669730 & -1.635324819 \\ \mathrm{H} & 1.264284893 & 1.727687427 & 0.164350409 \\ \mathrm{H} & 1.287079293 & -2.056697751 & 0.402371230 \\ \mathrm{H} & 4.804915646 & 0.401996631 & 0.256771618 \\ \mathrm{H} & 3.414157348 & 0.769656854 & -1.754662528 \\ \mathrm{C} & 3.703559269 & -1.660066221 & 1.757436126 \\ \mathrm{H} & 3.353707743 & -2.682794093 & 1.867140136 \\ \mathrm{H} & 4.789180847 & -1.676333119 & 1.796267031 \\ \mathrm{H} & 3.348789543 & -1.094617777 & 2.613636786 \\ \mathrm{C} & 3.755280968 & -1.902138935 & -0.715869554 \\ \mathrm{H} & 4.840971948 & -1.925150938 & -0.691575852 \\ \mathrm{H} & 3.403115647 & -2.926283611 & -0.631940947 \\ \mathrm{H} & 3.463320148 & -1.537594708 & -1.693655323 \\ \mathrm{H} & 1.348699597 & -0.702695252 & -1.637336618\end{array}$

\section{Coord_7}

$\begin{array}{llll}\text { C } & -4.371306316 & -0.897287565 & -1.542635513 \\ \text { C } & -3.016978718 & -0.713168252 & -1.299513493 \\ \text { C } & -2.556808484 & -0.037320702 & -0.078786004 \\ \text { C } & -3.604321357 & 0.433667132 & 0.868497163 \\ \text { C } & -4.911547254 & 0.241012016 & 0.606377844 \\ \text { C } & -5.360445388 & -0.432954632 & -0.609260844 \\ \text { H } & -4.683336036 & -1.394404202 & -2.442536774 \\ \text { H } & -2.281360767 & -1.059251676 & -1.994920445 \\ \text { H } & -5.628275504 & 0.599194342 & 1.320887496 \\ \text { H } & -6.406369962 & -0.573186143 & -0.792427256\end{array}$




\begin{tabular}{|c|c|c|c|}
\hline & -1.152695784 & 11 & 16 \\
\hline 0 & -0.742088651 & 0.719069753 & 1.198094287 \\
\hline 0 & -3.249611836 & 1.053547574 & 1.989090843 \\
\hline & -2.297257463 & 1.119409482 & 2.045946150 \\
\hline 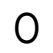 & -0.356757527 & -0.317116425 & .752998656 \\
\hline & 1.074145375 & -0.229959218 & 100 \\
\hline & 1.663557917 & -1.037219175 & 0.4529091 \\
\hline 0 & 1.584476415 & 1.206300487 & -0.72692755 \\
\hline & 3.208453933 & -1.045991473 & 0.438905331 \\
\hline$\Pi$ & 1.323674694 & -0.612149945 & \\
\hline C & 3.117148822 & 1.223879586 & -0.81 \\
\hline $\mathrm{H}$ & 1.152422485 & 1.723740325 & -1.579435811 \\
\hline 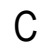 & 3.721175468 & 0.406708827 & 0.339014023 \\
\hline & 3.491501350 & 0.918282968 & 1.272559389 \\
\hline C & 3.650251561 & 2.657650490 & -0.80 \\
\hline$\Pi$ & 3.386090544 & 3.170584728 & 0.111376507 \\
\hline$\Gamma$ & 4.732391142 & 2.673356392 & -0.899098166 \\
\hline & 3.241008134 & 30631 & -1.636510917 \\
\hline & 1.2664 & 1.7 & 4913 \\
\hline П & 1.285176790 & -2.055111346 & 0.404891929 \\
\hline П & 4.805488343 & 0.399600431 & 0.252559420 \\
\hline & 3.411 & 56 & \\
\hline & & & 8327 \\
\hline & 3.353 & -2. & 1.8 \\
\hline H & 4.790285647 & -1.67 & 1.792009127 \\
\hline П & 3.352 & -1.0 & 2.6 \\
\hline 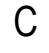 & 3. & & 354 \\
\hline & 4.837 & -1.6 & 5651 \\
\hline H & 3.398160447 & -2.92 & -0.6 \\
\hline $\mathrm{H}$ & 3.45808 & -1.5 & -1.6 \\
\hline & 99 & -0.70 & -1.635206220 \\
\hline \multicolumn{4}{|c|}{ Coord_8 } \\
\hline 0 & -4.358 & & \\
\hline C & & & -1 \\
\hline C & -2.55 & -0.0 & -0.072635404 \\
\hline C & -3.613 & 008629 & 0.876015465 \\
\hline C & -4.910425652 & 0.241048317 & 0.606780145 \\
\hline C & -5.353092587 & -0.437323731 & -0.615107845 \\
\hline$\because$ & -4.66 & -1.3 & 429674 \\
\hline $\mathrm{H}$ & -2.274 & -1.058074775 & -1.9921961 \\
\hline $\mathrm{H}$ & -5.635283805 & 0.595827340 & 1.315013793 \\
\hline $\mathrm{H}$ & -6.398048063 & -0.580107744 & -0.801469758 \\
\hline$\tau$ & -1.152720 & 0.167641511 & 0.202246613 \\
\hline 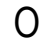 & & 0.721777553 & 1.201468289 \\
\hline 0 & -3.25880 & 1.061020175 & 2.000112743 \\
\hline & -2.3 & 1.128046083 & \\
\hline
\end{tabular}




$\begin{array}{lrrr}\text { O } & -0.358872728 & -0.314196023 & -0.748281052 \\ \text { C } & 1.072883477 & -0.228258214 & -0.698246650 \\ \text { C } & 1.662921317 & -1.036101076 & 0.454604834 \\ \text { C } & 1.584148516 & 1.207572386 & -0.725168452 \\ \text { C } & 3.207825932 & -1.046598778 & 0.437717333 \\ \text { H } & 1.325510696 & -0.610560043 & 1.391225699 \\ \text { C } & 3.116739726 & 1.223444687 & -0.813410658 \\ \text { H } & 1.151093483 & 1.725542227 & -1.576811912 \\ \text { C } & 3.721931869 & 0.405549929 & 0.336883624 \\ \text { H } & 3.494665152 & 0.917382965 & 1.270874891 \\ \text { C } & 3.651375761 & 2.656635692 & -0.811314158 \\ \text { H } & 3.389655643 & 3.169839927 & 0.109919010 \\ \text { H } & 4.733331443 & 2.671045094 & -0.903225865 \\ \text { H } & 3.241199236 & 3.229590733 & -1.637696718 \\ \text { H } & 1.268614690 & 1.731125327 & 0.169246013 \\ \text { H } & 1.283268995 & -2.053527048 & 0.407403827 \\ \text { H } & 4.806035846 & 0.397203528 & 0.248351217 \\ \text { H } & 3.408638744 & 0.768173158 & -1.757806724 \\ \text { C } & 3.705685968 & -1.662502320 & 1.753197024 \\ \text { H } & 3.353836942 & -2.684377095 & 1.864200932 \\ \text { H } & 4.791360845 & -1.681373723 & 1.787752230 \\ \text { H } & 3.355695841 & -1.096209277 & 2.610799089 \\ \text { C } & 3.747454368 & -1.904651935 & -0.720366253 \\ \text { H } & 4.833120550 & -1.930236236 & -0.700104951 \\ \text { H } & 3.393194542 & -2.927937708 & -0.635100548 \\ \text { H } & 3.452843147 & -1.539441209 & -1.697117725 \\ \text { H } & 1.341392596 & -0.699868652 & -1.633080218\end{array}$

Coord_9

$\begin{array}{lrrr}\text { C } & -4.344983913 & -0.904333967 & -1.551804012 \\ \text { C } & -3.014942518 & -0.716996050 & -1.304512392 \\ \text { C } & -2.561472483 & -0.030312601 & -0.066506706 \\ \text { C } & -3.622048562 & 0.442318931 & 0.883473662 \\ \text { C } & -4.909283852 & 0.241106215 & 0.607223643 \\ \text { C } & -5.345646385 & -0.441664530 & -0.620891244 \\ \text { H } & -4.653819735 & -1.403256301 & -2.452242679 \\ \text { H } & -2.266623461 & -1.056876977 & -1.989453345 \\ \text { H } & -5.642213408 & 0.592424641 & 1.309104196 \\ \text { H } & -6.389619359 & -0.586999341 & -0.810430258 \\ \text { C } & -1.152748982 & 0.170920013 & 0.207236516 \\ \text { O } & -0.744480755 & 0.724475151 & 1.204825088 \\ \text { O } & -3.268070036 & 1.068469779 & 2.011077846 \\ \text { H } & -2.319691064 & 1.136666180 & 2.072582149 \\ \text { O } & -0.360986727 & -0.311279124 & -0.743577153 \\ \text { C } & 1.071617976 & -0.226560217 & -0.695707652 \\ \text { C } & 1.662275721 & -1.034986274 & 0.456292031 \\ \text { C } & 1.583814012 & 1.208841189 & -0.723416653\end{array}$

S62 


$\begin{array}{lrrr}\text { C } & 3.207181632 & -1.047207676 & 0.436527229 \\ \text { H } & 1.327335495 & -0.608977945 & 1.393651300 \\ \text { C } & 3.116317522 & 1.223008888 & -0.814423461 \\ \text { H } & 1.149763084 & 1.727342223 & -1.574194914 \\ \text { C } & 3.722668468 & 0.404388628 & 0.334752225 \\ \text { H } & 3.497805851 & 0.916476664 & 1.269184191 \\ \text { C } & 3.652482361 & 2.655618989 & -0.813309659 \\ \text { H } & 3.393200045 & 3.169089326 & 0.108455607 \\ \text { H } & 4.734248740 & 2.668733690 & -0.907348965 \\ \text { H } & 3.241377531 & 3.229050130 & -1.638882419 \\ \text { H } & 1.270765891 & 1.732833527 & 0.171673614 \\ \text { H } & 1.281355892 & -2.051945046 & 0.409907031 \\ \text { H } & 4.806558546 & 0.394806128 & 0.244146920 \\ \text { H } & 3.405864347 & 0.767435654 & -1.759371925 \\ \mathrm{C} & 3.706713869 & -1.663726317 & 1.751072124 \\ \text { H } & 3.353869841 & -2.685173491 & 1.862725136 \\ \text { H } & 4.792406642 & -1.683898020 & 1.783496327 \\ \text { H } & 3.359109944 & -1.097017177 & 2.609366390 \\ \mathrm{C} & 3.743521269 & -1.905902238 & -0.722605954 \\ \text { H } & 4.829167046 & -1.932770540 & -0.704353752 \\ \text { H } & 3.388218244 & -2.928757409 & -0.636672246 \\ \text { H } & 3.447592349 & -1.540355309 & -1.698838122 \\ \text { H } & 1.337739495 & -0.698453949 & -1.630958816 \\ & & & \\ \text { Coord } 10 & & & \\ \text { C } & -4.331635094 & -0.907906115 & -1.556418861 \\ \mathrm{C} & -3.013746127 & -0.719014343 & -1.307136122 \\ \mathrm{C} & -2.563806853 & -0.026841500 & -0.060520764 \\ \mathrm{C} & -3.631000622 & 0.446616073 & 0.890686364 \\ \mathrm{C} & -4.908148565 & 0.241180538 & 0.607486343 \\ \mathrm{C} & -5.338082262 & -0.446048763 & -0.626812796 \\ \mathrm{H} & -4.638850824 & -1.407779501 & -2.457101367 \\ \mathrm{H} & -2.259137042 & -1.055778505 & -1.986747895 \\ \mathrm{H} & -5.649118097 & 0.589011294 & 1.302891496 \\ \mathrm{H} & -6.381051037 & -0.593949830 & -0.819541558 \\ \mathrm{C} & -1.152793131 & 0.174192090 & 0.212127664 \\ \mathrm{O} & -0.745740355 & 0.727217913 & 1.208071638 \\ \mathrm{O} & -3.277485825 & 1.075975058 & 2.021780773 \\ \mathrm{H} & -2.331111080 & 1.145358382 & 2.085647890 \\ \mathrm{O} & -0.363072755 & -0.308407914 & -0.738911513 \\ \mathrm{C} & 1.070373299 & -0.224894864 & -0.693154562 \\ \mathrm{C} & 1.661608481 & -1.033840325 & 0.458058092 \\ \mathrm{C} & 1.583487642 & 1.210079225 & -0.721703463 \\ \mathrm{C} & 3.206509111 & -1.047773504 & 0.435480321 \\ \mathrm{H} & 1.329098507 & -0.607323092 & 1.396115059 \\ \mathrm{C} & 3.115899774 & 1.222550621 & -0.815412271 \\ \mathrm{H} & 1.148473733 & 1.729065372 & -1.571658731\end{array}$

S63 


$\begin{array}{lrrr}\mathrm{C} & 3.723366278 & 0.403267630 & 0.332709072 \\ \mathrm{H} & 3.500866083 & 0.915653434 & 1.267541709 \\ \mathrm{C} & 3.653578774 & 2.654582511 & -0.815334630 \\ \mathrm{H} & 3.396693192 & 3.168360596 & 0.106920808 \\ \mathrm{H} & 4.735153633 & 2.666407003 & -0.911457243 \\ \mathrm{H} & 3.241576904 & 3.228445064 & -1.640141750 \\ \mathrm{H} & 1.272885923 & 1.734550787 & 0.174017742 \\ \mathrm{H} & 1.279434074 & -2.050335685 & 0.412527438 \\ \mathrm{H} & 4.807040738 & 0.392453787 & 0.240076647 \\ \mathrm{H} & 3.403135856 & 0.766632807 & -1.760873349 \\ \mathrm{C} & 3.707661879 & -1.664837960 & 1.749138976 \\ \mathrm{H} & 3.353828750 & -2.685853484 & 1.861480305 \\ \mathrm{H} & 4.793365496 & -1.686299331 & 1.779477977 \\ \mathrm{H} & 3.362406041 & -1.097675098 & 2.608077430 \\ \mathrm{C} & 3.739611977 & -1.907159589 & -0.724630131 \\ \mathrm{H} & 4.825231396 & -1.935301960 & -0.708341023 \\ \mathrm{H} & 3.383272622 & -2.929581401 & -0.637989236 \\ \mathrm{H} & 3.442403819 & -1.541327339 & -1.700371350 \\ \mathrm{H} & 1.334148694 & -0.697114300 & -1.628783336\end{array}$

LIIC path connecting T1-KETO-MIN and T//So-KETO

\begin{tabular}{lrrr}
\multicolumn{4}{l}{ Coord_1 } \\
C & -4.331635094 & -0.907906115 & -1.556418861 \\
C & -3.013746127 & -0.719014343 & -1.307136122 \\
C & -2.563806853 & -0.026841500 & -0.060520764 \\
C & -3.631000622 & 0.446616073 & 0.890686364 \\
C & -4.908148565 & 0.241180538 & 0.607486343 \\
C & -5.338082262 & -0.446048763 & -0.626812796 \\
H & -4.638850824 & -1.407779501 & -2.457101367 \\
H & -2.259137042 & -1.055778505 & -1.986747895 \\
H & -5.649118097 & 0.589011294 & 1.302891496 \\
H & -6.381051037 & -0.593949830 & -0.819541558 \\
C & -1.152793131 & 0.174192090 & 0.212127664 \\
O & -0.745740355 & 0.727217913 & 1.208071638 \\
O & -3.277485825 & 1.075975058 & 2.021780773 \\
H & -2.331111080 & 1.145358382 & 2.085647890 \\
O & -0.363072755 & -0.308407914 & -0.738911513 \\
C & 1.070373299 & -0.224894864 & -0.693154562 \\
C & 1.661608481 & -1.033840325 & 0.458058092 \\
C & 1.583487642 & 1.210079225 & -0.721703463 \\
C & 3.206509111 & -1.047773504 & 0.435480321 \\
H & 1.329098507 & -0.607323092 & 1.396115059 \\
C & 3.115899774 & 1.222550621 & -0.815412271 \\
H & 1.148473733 & 1.729065372 & -1.571658731 \\
C & 3.723366278 & 0.403267630 & 0.332709072 \\
H & 3.500866083 & 0.915653434 & 1.267541709
\end{tabular}




$\begin{array}{lrrr}\mathrm{C} & 3.653578774 & 2.654582511 & -0.815334630 \\ \mathrm{H} & 3.396693192 & 3.168360596 & 0.106920808 \\ \mathrm{H} & 4.735153633 & 2.666407003 & -0.911457243 \\ \mathrm{H} & 3.241576904 & 3.228445064 & -1.640141750 \\ \mathrm{H} & 1.272885923 & 1.734550787 & 0.174017742 \\ \mathrm{H} & 1.279434074 & -2.050335685 & 0.412527438 \\ \mathrm{H} & 4.807040738 & 0.392453787 & 0.240076647 \\ \mathrm{H} & 3.403135856 & 0.766632807 & -1.760873349 \\ \mathrm{C} & 3.707661879 & -1.664837960 & 1.749138976 \\ \mathrm{H} & 3.353828750 & -2.685853484 & 1.861480305 \\ \mathrm{H} & 4.793365496 & -1.686299331 & 1.779477977 \\ \mathrm{H} & 3.362406041 & -1.097675098 & 2.608077430 \\ \mathrm{C} & 3.739611977 & -1.907159589 & -0.724630131 \\ \mathrm{H} & 4.825231396 & -1.935301960 & -0.708341023 \\ \mathrm{H} & 3.383272622 & -2.929581401 & -0.637989236 \\ \mathrm{H} & 3.442403819 & -1.541327339 & -1.700371350 \\ \mathrm{H} & 1.334148694 & -0.697114300 & -1.628783336\end{array}$

Coord_2

$\begin{array}{lrrr}\text { C } & -4.339482914 & -0.954774370 & -1.528684311 \\ \text { C } & -3.016611315 & -0.723154149 & -1.301056092 \\ \text { C } & -2.566397684 & -0.018503503 & -0.067461902 \\ \text { C } & -3.615565559 & 0.460907431 & 0.876735664 \\ \text { C } & -4.913862352 & 0.275898518 & 0.583444842 \\ \text { C } & -5.341492085 & -0.496026633 & -0.604187542 \\ \text { H } & -4.642136036 & -1.497700707 & -2.405840972 \\ \text { H } & -2.264787861 & -1.058952675 & -1.984459341 \\ \text { H } & -5.639346304 & 0.789929159 & 1.188362087 \\ \text { H } & -6.382082960 & -0.695024050 & -0.761967856 \\ \text { C } & -1.151824885 & 0.176646012 & 0.209799115 \\ \text { O } & -0.746224452 & 0.720733952 & 1.210113188 \\ \text { O } & -3.273690835 & 1.086041380 & 2.012369546 \\ \text { H } & -2.327038966 & 1.147679184 & 2.086563848 \\ \text { O } & -0.363078427 & -0.299221524 & -0.742928953 \\ \text { C } & 1.071104675 & -0.217888415 & -0.696049648 \\ \text { C } & 1.660356718 & -1.035808875 & 0.449723534 \\ \text { C } & 1.585169516 & 1.216844587 & -0.713582852 \\ \text { C } & 3.205259830 & -1.050796677 & 0.428379831 \\ \text { H } & 1.326990297 & -0.615703746 & 1.390314398 \\ \text { C } & 3.117677825 & 1.228744190 & -0.805783457 \\ \text { H } & 1.151385181 & 1.742409725 & -1.560121011 \\ \text { C } & 3.723372670 & 0.400550029 & 0.336871125 \\ \text { H } & 3.500301351 & 0.906184368 & 1.275235592 \\ \text { C } & 3.656380164 & 2.660346892 & -0.794564360 \\ \text { H } & 3.398833846 & 3.167419529 & 0.131209308 \\ \text { H } & 4.738066240 & 2.672067993 & -0.889436162 \\ \text { H } & 3.245645732 & 3.240573833 & -1.615542215 \\ & & & \end{array}$




$\begin{array}{lrrr}\mathrm{H} & 1.273825494 & 1.734553824 & 0.185763911 \\ \mathrm{H} & 1.277469990 & -2.051661846 & 0.396309827 \\ \mathrm{H} & 4.807132746 & 0.389544629 & 0.245194516 \\ \mathrm{H} & 3.405505247 & 0.779587056 & -1.754313324 \\ \mathrm{C} & 3.704666466 & -1.678006621 & 1.737886424 \\ \mathrm{H} & 3.349938043 & -2.699560492 & 1.842263133 \\ \mathrm{H} & 4.790329945 & -1.700507221 & 1.769064626 \\ \mathrm{H} & 3.358975242 & -1.116953782 & 2.600650486 \\ \mathrm{C} & 3.738730267 & -1.901948437 & -0.737617855 \\ \mathrm{H} & 4.824315348 & -1.931069638 & -0.720552651 \\ \mathrm{H} & 3.381480045 & -2.924698709 & -0.658894648 \\ \mathrm{H} & 3.442677250 & -1.528580608 & -1.710873121 \\ \mathrm{H} & 1.334373494 & -0.683564547 & -1.635065718\end{array}$

Coord_3

$\begin{array}{lrrr}\text { C } & -4.347434014 & -1.000227372 & -1.497152210 \\ \text { C } & -3.020761017 & -0.725998752 & -1.293256094 \\ \text { C } & -2.569431383 & -0.008898301 & -0.073001007 \\ \text { C } & -3.601016058 & 0.476290232 & 0.863603464 \\ \text { C } & -4.919109155 & 0.311595921 & 0.557121142 \\ \text { C } & -5.343638385 & -0.543492838 & -0.576838439 \\ \text { H } & -4.644151032 & -1.585556212 & -2.348861071 \\ \text { H } & -2.272167063 & -1.061342677 & -1.980651043 \\ \text { H } & -5.615702305 & 0.972355072 & 1.044467273 \\ \text { H } & -6.379136157 & -0.791254456 & -0.696014252 \\ \text { C } & -1.151206785 & 0.180059814 & 0.208410813 \\ \text { O } & -0.746699156 & 0.715340350 & 1.212780389 \\ \text { O } & -3.271369938 & 1.096724976 & 2.004053346 \\ \text { H } & -2.324651366 & 1.150275481 & 2.089175851 \\ \text { O } & -0.363770925 & -0.289463421 & -0.746044452 \\ \text { C } & 1.071175076 & -0.210624515 & -0.698440148 \\ \text { C } & 1.658541518 & -1.037347574 & 0.441867734 \\ \text { C } & 1.586499312 & 1.223668486 & -0.705416350 \\ \text { C } & 3.203436232 & -1.053749074 & 0.421368233 \\ \text { H } & 1.324640194 & -0.623379446 & 1.384933999 \\ \text { C } & 3.119081325 & 1.234621386 & -0.796498656 \\ \text { H } & 1.153855185 & 1.755689925 & -1.548501512 \\ \text { C } & 3.723099070 & 0.397683428 & 0.340662622 \\ \text { H } & 3.499795050 & 0.896806664 & 1.282449294 \\ \text { C } & 3.659121863 & 2.665595293 & -0.774603254 \\ \text { H } & 3.401250546 & 3.166207930 & 0.154587010 \\ \text { H } & 4.740896640 & 2.676938694 & -0.868497560 \\ \text { H } & 3.249583631 & 3.252061832 & -1.591735817 \\ \text { H } & 1.274743992 & 1.734865827 & 0.197462416 \\ \text { H } & 1.274708092 & -2.052427248 & 0.380903525 \\ \text { H } & 4.806918048 & 0.386215628 & 0.249672219 \\ \text { H } & 3.407171346 & 0.791964459 & -1.748017025\end{array}$

S66 


$\begin{array}{llll}\mathrm{C} & 3.701275869 & -1.690884022 & 1.726667025 \\ \mathrm{H} & 3.345454541 & -2.712813996 & 1.823397530 \\ \mathrm{H} & 4.786899945 & -1.714665724 & 1.758411225 \\ \mathrm{H} & 3.355481640 & -1.135692684 & 2.593169787 \\ \mathrm{C} & 3.736804670 & -1.897010938 & -0.750394755 \\ \mathrm{H} & 4.822351248 & -1.927355337 & -0.732824853 \\ \mathrm{H} & 3.378439445 & -2.919933410 & -0.679268348 \\ \mathrm{H} & 3.441753249 & -1.516283809 & -1.721119924 \\ \mathrm{H} & 1.333607595 & -0.670004148 & -1.640756919\end{array}$

\section{Coord_4}

$\begin{array}{lrrr}\text { C } & -4.355403016 & -1.043899175 & -1.461971903 \\ \text { C } & -3.026175517 & -0.727198551 & -1.283981691 \\ \text { C } & -2.572898684 & 0.002269901 & -0.077353003 \\ \text { C } & -3.587404561 & 0.493066437 & 0.851023064 \\ \text { C } & -4.923816853 & 0.348316525 & 0.528006339 \\ \text { C } & -5.344402983 & -0.588008043 & -0.544925537 \\ \text { H } & -4.644770333 & -1.670856923 & -2.286311966 \\ \text { H } & -2.281261265 & -1.062575576 & -1.975578741 \\ \text { H } & -5.578544802 & 1.131720283 & 0.874179865 \\ \text { H } & -6.372060057 & -0.881892666 & -0.621852045 \\ \text { C } & -1.150931285 & 0.184648813 & 0.207806816 \\ \text { O } & -0.747169552 & 0.711209952 & 1.215953685 \\ \text { O } & -3.270688336 & 1.108222881 & 1.996625343 \\ \text { H } & -2.324149769 & 1.153333882 & 2.093349048 \\ \text { O } & -0.365127926 & -0.278935318 & -0.748400254 \\ \text { C } & 1.070608375 & -0.202980915 & -0.700411148 \\ \text { C } & 1.656091020 & -1.038396377 & 0.434413934 \\ \text { C } & 1.587583416 & 1.230653086 & -0.697233852 \\ \text { C } & 3.200966032 & -1.056650177 & 0.414426829 \\ \text { H } & 1.321959694 & -0.630294544 & 1.379899999 \\ \text { C } & 3.120221024 & 1.240207292 & -0.787529657 \\ \text { H } & 1.156056483 & 1.769053926 & -1.536838313 \\ \text { C } & 3.722559970 & 0.394629626 & 0.344120324 \\ \text { H } & 3.499350054 & 0.887472665 & 1.289228991 \\ \text { C } & 3.661995962 & 2.670330292 & -0.755367052 \\ \text { H } & 3.404124343 & 3.164720926 & 0.177146312 \\ \text { H } & 4.743841644 & 2.680968395 & -0.848515959 \\ \text { H } & 3.253651036 & 3.262955633 & -1.568643913 \\ \text { H } & 1.275738490 & 1.735584926 & 0.209089716 \\ \text { H } & 1.271020590 & -2.052554647 & 0.366192027 \\ \text { H } & 4.806414446 & 0.382374728 & 0.253587019 \\ \text { H } & 3.408260144 & 0.803796957 & -1.741961623 \\ \mathrm{C} & 3.697325767 & -1.703550525 & 1.715472925 \\ \text { H } & 3.340151238 & -2.725682697 & 1.804837331 \\ \text { H } & 4.782908546 & -1.728912223 & 1.747550724 \\ \text { H } & 3.351755740 & -1.153973684 & 2.585632785\end{array}$




$\begin{array}{llll}\mathrm{C} & 3.733764468 & -1.892368535 & -0.762986252 \\ \mathrm{H} & 4.819266044 & -1.924237536 & -0.745142554 \\ \mathrm{H} & 3.374018645 & -2.915294510 & -0.699174149 \\ \mathrm{H} & 3.439624448 & -1.504417210 & -1.731144526 \\ \mathrm{H} & 1.331888398 & -0.656302246 & -1.645947819\end{array}$

Coord_5

$\begin{array}{lrrr}\text { C } & -4.363279412 & -1.085436377 & -1.423316500 \\ \text { C } & -3.032828317 & -0.726414352 & -1.273504891 \\ \text { C } & -2.576784883 & 0.015282401 & -0.080752506 \\ \text { C } & -3.574791055 & 0.511506438 & 0.838703463 \\ \text { C } & -4.927889856 & 0.386008127 & 0.495542134 \\ \text { C } & -5.343622086 & -0.629139043 & -0.508629836 \\ \text { H } & -4.643830335 & -1.753116526 & -2.218381160 \\ \text { H } & -2.292052766 & -1.062280575 & -1.969528044 \\ \text { H } & -5.528328897 & 1.263639292 & 0.680855149 \\ \text { H } & -6.360639057 & -0.966181368 & -0.539687037 \\ \text { C } & -1.150990182 & 0.190614715 & 0.207817912 \\ \text { O } & -0.747648156 & 0.708495749 & 1.219502988 \\ \text { O } & -3.271849637 & 1.120739379 & 1.989831342 \\ \text { H } & -2.325781170 & 1.157078785 & 2.098900949 \\ \text { O } & -0.367120527 & -0.267443721 & -0.750150555 \\ \text { C } & 1.069432877 & -0.194837015 & -0.702054350 \\ \text { C } & 1.652934621 & -1.038900974 & 0.427274232 \\ \text { C } & 1.588525912 & 1.237897687 & -0.689061249 \\ \text { C } & 3.197777433 & -1.059520879 & 0.407531930 \\ \text { H } & 1.318855996 & -0.636406345 & 1.375127297 \\ \text { C } & 3.121204527 & 1.245528788 & -0.778838254 \\ \text { H } & 1.158161984 & 1.782649728 & -1.525162012 \\ \text { C } & 3.721763866 & 0.391348526 & 0.347286323 \\ \text { H } & 3.498956949 & 0.878125162 & 1.295626591 \\ \text { C } & 3.665184462 & 2.674555692 & -0.736750555 \\ \text { H } & 3.407619845 & 3.162947526 & 0.199000814 \\ \text { H } & 4.747087543 & 2.684110591 & -0.829341560 \\ \text { H } & 3.258100337 & 3.273305933 & -1.546163712 \\ \text { H } & 1.276897293 & 1.736799425 & 0.220626817 \\ \text { H } & 1.266286490 & -2.051971647 & 0.352039525 \\ \text { H } & 4.805633348 & 0.377931628 & 0.257025618 \\ \text { H } & 3.408900644 & 0.815125759 & -1.736114527 \\ \text { C } & 3.692647665 & -1.716093926 & 1.704286621 \\ \text { H } & 3.333801238 & -2.738242698 & 1.786519131 \\ \text { H } & 4.778183644 & -1.743388925 & 1.736510425 \\ \text { H } & 3.347618040 & -1.171892885 & 2.578028285 \\ \text { C } & 3.729548470 & -1.888033938 & -0.775424754 \\ \text { H } & 4.814994848 & -1.921782736 & -0.757493657 \\ \text { H } & 3.368098745 & -2.910782210 & -0.718690852 \\ \text { H } & 3.436296346 & -1.492947907 & -1.740983926\end{array}$

S68 


$\begin{array}{lrrr}\text { H } & 1.329263997 & -0.642322145 & -1.650736418 \\ \text { Coord } 6 & & \\ \text { C } & -4.370926817 & -1.124490182 & -1.381389601 \\ \text { C } & -3.040687520 & -0.723312850 & -1.262127792 \\ \text { C } & -2.581071187 & 0.030408303 & -0.083455904 \\ \text { C } & -3.563245159 & 0.531855436 & 0.826327261 \\ \text { C } & -4.931205755 & 0.424512530 & 0.459124331 \\ \text { C } & -5.341081385 & -0.666450849 & -0.468158635 \\ \text { H } & -4.641129536 & -1.831840230 & -2.145305354 \\ \text { H } & -2.304522267 & -1.060084675 & -1.962819939 \\ \text { H } & -5.465606391 & 1.363830198 & 0.468292835 \\ \text { H } & -6.344585555 & -1.043343174 & -0.449768033 \\ \text { C } & -1.151375280 & 0.198146912 & 0.208259913 \\ \text { O } & -0.748155256 & 0.707332152 & 1.223286987 \\ \text { O } & -3.275095838 & 1.134498283 & 1.983362642 \\ \text { H } & -2.329836869 & 1.161792182 & 2.105591749 \\ \text { O } & -0.369714024 & -0.254798819 & -0.751463354 \\ \text { C } & 1.067681178 & -0.186072913 & -0.703468952 \\ \text { C } & 1.649002818 & -1.038812374 & 0.420348730 \\ \text { C } & 1.589429916 & 1.245499688 & -0.680920148 \\ \text { C } & 3.193798432 & -1.062384077 & 0.400653531 \\ \text { H } & 1.315230893 & -0.641685349 & 1.370518999 \\ \text { C } & 3.122137424 & 1.250614890 & -0.770376054 \\ \text { H } & 1.160346285 & 1.796627331 & -1.513496908 \\ \text { C } & 3.720713967 & 0.387797027 & 0.350208927 \\ \text { H } & 3.498594151 & 0.868696763 & 1.301701593 \\ \text { C } & 3.668861862 & 2.678275592 & -0.718627054 \\ \text { H } & 3.411887448 & 3.160869526 & 0.220287016 \\ \text { H } & 4.750812443 & 2.686322293 & -0.810797660 \\ \text { H } & 3.263178735 & 3.283167437 & -1.524167412 \\ \text { H } & 1.278301089 & 1.738588224 & 0.232059517 \\ \text { H } & 1.260389890 & -2.050610048 & 0.338290622 \\ \text { H } & 4.804579947 & 0.372795624 & 0.260085019 \\ \text { H } & 3.409226443 & 0.826001457 & -1.730433227 \\ \text { C } & 3.687068364 & -1.728610126 & 1.693080922 \\ \text { H } & 3.326175942 & -2.750577899 & 1.768361429 \\ \text { H } & 4.772547144 & -1.758244026 & 1.725312124 \\ \text { H } & 3.342877141 & -1.189562087 & 2.570336784 \\ \text { C } & 3.724103766 & -1.884011937 & -0.787750758 \\ \text { H } & 4.809480546 & -1.920047537 & -0.769869954 \\ \text { H } & 3.360573442 & -2.906387811 & -0.737913252 \\ \text { H } & 3.431786946 & -1.481829809 & -1.750680527 \\ \text { H } & 1.325793594 & -0.627920147 & -1.655229218\end{array}$

Coord_7

C $\quad-4.378180514 \quad-1.160707184 \quad-1.336434994$ 


\begin{tabular}{|c|c|c|c|}
\hline & 6617 & טכ & -1.2501895 \\
\hline$C$ & -2.585734985 & 047909205 & -0.085745 \\
\hline$C$ & -3.552845355 & 0.554342442 & 0.81354475 \\
\hline 0 & 3611157 & 463555031 & 18107628 \\
\hline C & 36508785 & -0.699496349 & 2754120 \\
\hline & -4.636428135 & 40 & \\
\hline & -2.318650268 & -1.055604777 & -1.9558203 \\
\hline & -5.391039988 & 1.428025405 & 0.240833318 \\
\hline & -6.323528455 & -1.112567982 & -0.352400424 \\
\hline C & -1.152078785 & 0.207425914 & 0.208930714 \\
\hline 0 & -0.748719655 & 0.707837453 & 1.22715028 \\
\hline 0 & -3.280705434 & 1.149750585 & 1.976848941 \\
\hline & -2.336649269 & 1.167829682 & 2.113114052 \\
\hline U & -0.37 & -0.2 & \\
\hline$C$ & 1.0653 & -0.1765 & -0.7 \\
\hline C & 1.644225120 & -1.038086073 & 0.413524228 \\
\hline C & 1.590397914 & 1.253555391 & -0.672828949 \\
\hline & 3.188954831 & -1.065264876 & 55827 \\
\hline H & 8795 & -0.64 & 1.365964698 \\
\hline C & 3.123124223 & 1.255 & -0.762083254 \\
\hline 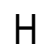 & 1.162787385 & 1.811139531 & -1.501860808 \\
\hline U & 7169 & & \\
\hline & 3.498 & 0.85 & 1.3 \\
\hline & 3.67 & 2.68 & -0.70 \\
\hline $\mathrm{H}$ & 3.417064648 & 3.158460325 & 0.241166319 \\
\hline П & 4.75 & & -0.79267 \\
\hline 11 & & & \\
\hline & 1.280 & 1.74 & 0.2 \\
\hline $\mathrm{H}$ & 1.253 & -2.048403850 & 0.324767323 \\
\hline$H_{2}$ & 4.803 & 0.3668 & 0.26287 \\
\hline $\mathrm{H}$ & & & \\
\hline C & 3.68 & -1.7 & 1.6 \\
\hline$H$ & 3.317 & -2.76 & 1.750264128 \\
\hline $\mathrm{H}$ & 4.765811242 & -1.773633527 & 1.713972724 \\
\hline $\mathrm{H}$ & & & 583 \\
\hline C & 3.71 & -1.8 & -0.8 \\
\hline $\mathrm{H}_{\text {L }}$ & 4.802672348 & -1.919078539 & -0.782265857 \\
\hline $\mathrm{H}$ & 3.351346141 & -2.902093411 & -0.756952954 \\
\hline $\mathrm{H}$ & 3.426126348 & -1.471003006 & -1.760281829 \\
\hline 11 & 1.321546894 & -0.612943743 & -1.659542722 \\
\hline \multicolumn{4}{|c|}{ Coord_8 } \\
\hline$c$ & -4.3 & -1.19 & -1.2 \\
\hline C & 22 & -0.708812049 & -1.238078189 \\
\hline C & -2.5 & 0.068047204 & -0.087937908 \\
\hline C & & 0.579 & 0.79996 \\
\hline & & 0.502 & 0.37181 \\
\hline
\end{tabular}




$\begin{array}{lrrr}\mathrm{C} & -5.329563482 & -0.727802755 & -0.375707824 \\ \mathrm{H} & -4.629448334 & -1.976570943 & -1.985031741 \\ \mathrm{H} & -2.334416866 & -1.048434774 & -1.948953342 \\ \mathrm{H} & -5.305444784 & 1.451848404 & 0.003505402 \\ \mathrm{H} & -6.296998651 & -1.172999082 & -0.247966717 \\ \mathrm{C} & -1.153093186 & 0.218628813 & 0.209604314 \\ \mathrm{O} & -0.749379555 & 0.710118752 & 1.230917987 \\ \mathrm{O} & -3.288989836 & 1.166775984 & 1.969852541 \\ \mathrm{H} & -2.346585768 & 1.175626685 & 2.121085451 \\ \mathrm{O} & -0.376534526 & -0.225285815 & -0.753535755 \\ \mathrm{C} & 1.062600976 & -0.166197212 & -0.706056549 \\ \mathrm{C} & 1.638528119 & -1.036679472 & 0.406671329 \\ \mathrm{C} & 1.591534814 & 1.262161490 & -0.664804050 \\ \mathrm{C} & 3.183168531 & -1.068190474 & 0.386796728 \\ \mathrm{H} & 1.305983796 & -0.649682746 & 1.361337599 \\ \mathrm{C} & 3.124270723 & 1.260202391 & -0.753888452 \\ \mathrm{H} & 1.165671686 & 1.826344232 & -1.490267005 \\ \mathrm{C} & 3.717833767 & 0.379690730 & 0.355550325 \\ \mathrm{H} & 3.497805952 & 0.849268762 & 1.313160297 \\ \mathrm{C} & 3.678354964 & 2.684208293 & -0.683230647 \\ \mathrm{H} & 3.423279544 & 3.155683028 & 0.261826018 \\ \mathrm{H} & 4.760383942 & 2.687783192 & -0.774745857 \\ \mathrm{H} & 3.276206036 & 3.301655038 & -1.480979405 \\ \mathrm{H} & 1.282132391 & 1.744163127 & 0.254574818 \\ \mathrm{H} & 1.244655889 & -2.045286445 & 0.311266021 \\ \mathrm{H} & 4.801645347 & 0.360071124 & 0.265516918 \\ \mathrm{H} & 3.409499244 & 0.846641661 & -1.719343825 \\ \mathrm{C} & 3.672464162 & -1.753991028 & 1.670453922 \\ \mathrm{H} & 3.306149938 & -2.774949401 & 1.732107327 \\ \mathrm{H} & 4.757773240 & -1.789723431 & 1.702505924 \\ \mathrm{H} & 3.330739741 & -1.224680591 & 2.554569186 \\ \mathrm{C} & 3.709351966 & -1.876883337 & -0.812264561 \\ \mathrm{H} & 4.794524045 & -1.918914637 & -0.794676656 \\ \mathrm{H} & 3.340327740 & -2.897870910 & -0.775938654 \\ \mathrm{H} & 3.419357848 & -1.460393807 & -1.769840527 \\ \mathrm{H} & 1.316606992 & -0.597228845 & -1.663805620 \\ & & & \\ \mathrm{Coord} 99 & & & \\ \mathrm{C} & -4.390687317 & -1.223100288 & -1.238732988 \\ \mathrm{C} & -3.071090722 & -0.696688450 & -1.226251586 \\ \mathrm{C} & -2.596077786 & 0.091100107 & -0.090398307 \\ \mathrm{C} & -3.535833354 & 0.606661742 & 0.785127955 \\ \mathrm{C} & -4.934886257 & 0.541523341 & 0.319534024 \\ \mathrm{C} & -5.319820085 & -0.750851953 & -0.324384823 \\ \mathrm{H} & -4.619873332 & -2.041395045 & -1.898772638 \\ \mathrm{H} & -2.351801270 & -1.038120273 & -1.942725841 \\ \mathrm{H} & -5.209879273 & 1.430615304 & -0.237729016\end{array}$




\begin{tabular}{|c|c|c|c|}
\hline & 405253 & -1.223715387 & -0.136958809 \\
\hline C & -1.154409784 & 0.231941415 & \\
\hline 0 & -0.750183052 & 0.714282652 & 005 \\
\hline 0 & -3.300290337 & 1.185878187 & 1.9618610 \\
\hline$\Pi$ & -2.360043968 & 185691885 & 2904475 \\
\hline U & 0656627 & -0.208016116 & S \\
\hline$C$ & 9363877 & 4827113 & -070748 \\
\hline $\mathrm{C}+2+$ & 1.631832418 & -1.034547777 & 0.399641 \\
\hline & 1.592953517 & 1.271418393 & -0.6568630 \\
\hline C & 353528 & -1.071189676 & \\
\hline$\Pi$ & 1.300114691 & -0.652388647 & $1.000+4$ \\
\hline $\mathrm{C}$ & 3.125689923 & 1.264766291 & -0.745707 \\
\hline & 1.169203886 & 1.842408434 & -1.478728708 \\
\hline & 3.715977968 & 0.375025329 & 035810370 \\
\hline$\Pi$ & 3.497266450 & 0.835 & 1.318 \\
\hline U & 3.684511465 & 2.686416491 & -0.665577 \\
\hline$\Pi$ & 3.430658444 & 3.152488126 & 0.2824813 \\
\hline & 4.766576744 & 2.686935593 & -0.75672355 \\
\hline & 3.284 & 3.31 & $-1.4 t$ \\
\hline $\mathrm{H}$ & 1.284695593 & 1.748073223 & 0.265638 \\
\hline$\Pi$ & 1.234585789 & -2.041184348 & 0.297552 \\
\hline & 4.79 & & \\
\hline & & & \\
\hline C & 3.66 & -1.7 & 1.6 \\
\hline $\mathrm{H}$ & 3.293 & -2.787192102 & 1.71375 \\
\hline$H$ & 4.748 & -1.8 & 1.69 \\
\hline$\Pi$ & & & \\
\hline C & 3.699 & -1.8 & -0.8 \\
\hline H & 4.784 & -1.9 & -0.807093 \\
\hline$H$ & 3.327 & $-2.8 s$ & -0.79501 \\
\hline $\mathrm{H}$ & & & \\
\hline & & -0.58 & \\
\hline \multicolumn{4}{|c|}{ Coord_10 } \\
\hline C & -4.39 & -1.2 & -1.1 \\
\hline C & -3.0 & -0.6 & -1.2 \\
\hline C & $-2.60^{\prime}$ & 0.11579 & -0.0926716 \\
\hline C & -3.529845025 & 0.628822875 & 0.77296933 \\
\hline C & -4.933608495 & 0.573871073 & 0.2645086 \\
\hline C & & -0.7 & -02 \\
\hline $\mathrm{H}$ & -4.60 & -2.05 & -1.82438 \\
\hline $\mathrm{H}$ & -2.370078798 & -1.012423691 & $-1.94492 \mathrm{c}$ \\
\hline $\mathrm{H}$ & -5.106573947 & 1.358698618 & -0.4660175 \\
\hline $\mathrm{H}$ & -6.224336238 & -1.267773029 & -0.0292 \\
\hline - & & & \\
\hline 0 & -0.7 & 0.71038 & \\
\hline & & $1.1907 \varepsilon$ & 1.96 \\
\hline
\end{tabular}




$\begin{array}{lrrr}\text { H } & -2.378328111 & 1.181196683 & 2.145012417 \\ \text { O } & -0.385024350 & -0.184423976 & -0.757470506 \\ \text { C } & 1.055847976 & -0.137517392 & -0.709814390 \\ \text { C } & 1.624543097 & -1.034355045 & 0.385352667 \\ \text { C } & 1.594111733 & 1.286052790 & -0.639451866 \\ \text { C } & 3.168930340 & -1.076029119 & 0.365428874 \\ \text { H } & 1.293422155 & -0.663955218 & 1.346968278 \\ \text { C } & 3.126852446 & 1.275297929 & -0.727807962 \\ \text { H } & 1.172706955 & 1.869711086 & -1.453591265 \\ \text { C } & 3.713551029 & 0.368471199 & 0.363949278 \\ \text { H } & 3.495923764 & 0.820017468 & 1.330732125 \\ \text { C } & 3.690421665 & 2.693814432 & -0.627867097 \\ \text { H } & 3.437562048 & 3.147605476 & 0.326391966 \\ \text { H } & 4.772533703 & 2.691894926 & -0.718426131 \\ \text { H } & 3.293112746 & 3.329983108 & -1.413233530 \\ \text { H } & 1.286774752 & 1.750667324 & 0.289436179 \\ \text { H } & 1.223926349 & -2.038144077 & 0.269265271 \\ \text { H } & 4.797275724 & 0.343214177 & 0.274136400 \\ \text { H } & 3.409998004 & 0.879439421 & -1.701297214 \\ \text { C } & 3.652624064 & -1.791178610 & 1.635104348 \\ \text { H } & 3.279314524 & -2.810664732 & 1.675668033 \\ \text { H } & 4.737654752 & -1.834903182 & 1.666959818 \\ \text { H } & 3.313802820 & -1.277618414 & 2.529557824 \\ \text { C } & 3.690259854 & -1.863689562 & -0.849645763 \\ \text { H } & 4.775114093 & -1.913502890 & -0.832412148 \\ \text { H } & 3.314179547 & -2.882642326 & -0.834286362 \\ \text { H } & 3.403655857 & -1.425685325 & -1.798645211 \\ \text { H } & 1.305493884 & -0.551045557 & -1.676330642\end{array}$

LIIC path connecting $\mathrm{T}_{1} / \mathrm{S}_{0}-\mathrm{KETO}$ and SO-KETO-MIN Coord_1

$\begin{array}{lrrr}\text { C } & -4.394672466 & -1.243125111 & -1.195853205 \\ \text { C } & -3.082864233 & -0.674589336 & -1.220075325 \\ \text { C } & -2.601763996 & 0.115796158 & -0.092671695 \\ \text { C } & -3.529845025 & 0.628822875 & 0.772969335 \\ \text { C } & -4.933608495 & 0.573871073 & 0.264508639 \\ \text { C } & -5.306330511 & -0.769842373 & -0.275912901 \\ \text { H } & -4.606068262 & -2.090668221 & -1.824387663 \\ \text { H } & -2.370078798 & -1.012423691 & -1.944929811 \\ \text { H } & -5.106573947 & 1.358698618 & -0.466017515 \\ \text { H } & -6.224336238 & -1.267773029 & -0.029294390 \\ \text { C } & -1.156201562 & 0.244609426 & 0.211809036 \\ \text { O } & -0.751722503 & 0.710382049 & 1.242624330 \\ \text { O } & -3.315842507 & 1.190786703 & 1.960819660 \\ \text { H } & -2.378328111 & 1.181196683 & 2.145012417 \\ \text { O } & -0.385024350 & -0.184423976 & -0.757470506 \\ \text { C } & 1.055847976 & -0.137517392 & -0.709814390\end{array}$




$\begin{array}{lrrr}\text { C } & 1.624543097 & -1.034355045 & 0.385352667 \\ \mathrm{C} & 1.594111733 & 1.286052790 & -0.639451866 \\ \mathrm{C} & 3.168930340 & -1.076029119 & 0.365428874 \\ \mathrm{H} & 1.293422155 & -0.663955218 & 1.346968278 \\ \mathrm{C} & 3.126852446 & 1.275297929 & -0.727807962 \\ \mathrm{H} & 1.172706955 & 1.869711086 & -1.453591265 \\ \mathrm{C} & 3.713551029 & 0.368471199 & 0.363949278 \\ \mathrm{H} & 3.495923764 & 0.820017468 & 1.330732125 \\ \mathrm{C} & 3.690421665 & 2.693814432 & -0.627867097 \\ \mathrm{H} & 3.437562048 & 3.147605476 & 0.326391966 \\ \mathrm{H} & 4.772533703 & 2.691894926 & -0.718426131 \\ \mathrm{H} & 3.293112746 & 3.329983108 & -1.413233530 \\ \mathrm{H} & 1.286774752 & 1.750667324 & 0.289436179 \\ \mathrm{H} & 1.223926349 & -2.038144077 & 0.269265271 \\ \mathrm{H} & 4.797275724 & 0.343214177 & 0.274136400 \\ \mathrm{H} & 3.409998004 & 0.879439421 & -1.701297214 \\ \mathrm{C} & 3.652624064 & -1.791178610 & 1.635104348 \\ \mathrm{H} & 3.279314524 & -2.810664732 & 1.675668033 \\ \mathrm{H} & 4.737654752 & -1.834903182 & 1.666959818 \\ \mathrm{H} & 3.313802820 & -1.277618414 & 2.529557824 \\ \mathrm{C} & 3.690259854 & -1.863689562 & -0.849645763 \\ \mathrm{H} & 4.775114093 & -1.913502890 & -0.832412148 \\ \mathrm{H} & 3.314179547 & -2.882642326 & -0.834286362 \\ \mathrm{H} & 3.403655857 & -1.425685325 & -1.798645211 \\ \mathrm{H} & 1.305493884 & -0.551045557 & -1.676330642\end{array}$

Coord_2

$\begin{array}{rrrr}\text { C } & -4.388801017 & -1.214970585 & -1.240315090 \\ \text { C } & -3.067360123 & -0.682258750 & -1.220314788 \\ \text { C } & -2.597890588 & 0.093616107 & -0.085595706 \\ \text { C } & -3.531955654 & 0.598970341 & 0.783271955 \\ \text { C } & -4.939122654 & 0.532529037 & 0.315705422 \\ \text { C } & -5.316046882 & -0.750195054 & -0.327954425 \\ \text { H } & -4.615118833 & -2.030195644 & -1.904463738 \\ \text { H } & -2.346344971 & -1.018310973 & -1.937866041 \\ \text { H } & -5.227525678 & 1.401353501 & -0.261322421 \\ \text { H } & -6.259696253 & -1.227465587 & -0.144316410 \\ \text { C } & -1.152379082 & 0.232740515 & 0.215182917 \\ \text { O } & -0.747126255 & 0.708417050 & 1.241730888 \\ \text { O } & -3.324717037 & 1.170965586 & 1.968458640 \\ \text { H } & -2.387597772 & 1.178832584 & 2.152667553 \\ \text { O } & -0.380021027 & -0.200942916 & -0.752285553 \\ \text { C } & 1.060289175 & -0.148972111 & -0.706528951 \\ \text { C } & 1.633377918 & -1.036166477 & 0.394254831 \\ \text { C } & 1.594705517 & 1.276604394 & -0.647415645 \\ \text { C } & 3.177840129 & -1.073642677 & 0.372252726 \\ \text { H } & 1.303009095 & -0.659936847 & 1.353906495\end{array}$




$\begin{array}{lrrr}\text { C } & 3.127341125 & 1.269599992 & -0.737972351 \\ \text { H } & 1.170402584 & 1.853056835 & -1.465164705 \\ \text { C } & 3.718322869 & 0.372349126 & 0.359365624 \\ \text { H } & 3.500980650 & 0.830289158 & 1.323204097 \\ \text { C } & 3.687042668 & 2.690387094 & -0.649118246 \\ \text { H } & 3.434624247 & 3.150287627 & 0.302332123 \\ \text { H } & 4.768993745 & 2.690916191 & -0.741602253 \\ \text { H } & 3.286560934 & 3.319846940 & -1.438268906 \\ \text { H } & 1.287713290 & 1.747526827 & 0.278433018 \\ \text { H } & 1.235345989 & -2.041859245 & 0.286149322 \\ \text { H } & 4.801968743 & 0.349538126 & 0.268040919 \\ \text { H } & 3.410144846 & 0.867570960 & -1.709014121 \\ \text { C } & 3.665568565 & -1.778101429 & 1.646361318 \\ \text { H } & 3.295115936 & -2.798268702 & 1.695083223 \\ \text { H } & 4.750759943 & -1.818662833 & 1.676769923 \\ \text { H } & 3.326857438 & -1.258914490 & 2.537607880 \\ \text { C } & 3.699515465 & -1.868726234 & -0.837827262 \\ \text { H } & 4.784532046 & -1.915303638 & -0.821981361 \\ \text { H } & 3.326418538 & -2.888616208 & -0.814382360 \\ \text { H } & 3.410166346 & -1.438593202 & -1.789572727 \\ \text { H } & 1.310446792 & -0.568739339 & -1.670224720 \\ & & & \\ \text { Coord_3 } 3 & & \\ \text { C } & -4.382952015 & -1.183559984 & -1.283619294 \\ \text { C } & -3.053637620 & -0.688112148 & -1.222204290 \\ \text { C } & -2.594614489 & 0.072760104 & -0.080551604 \\ \text { C } & -3.534963953 & 0.570077941 & 0.791571555 \\ \text { C } & -4.942310857 & 0.490313535 & 0.360408924 \\ \text { C } & -5.322880884 & -0.725176353 & -0.377387026 \\ \text { H } & -4.622783733 & -1.964547639 & -1.982578645 \\ \text { H } & -2.325650368 & -1.022902676 & -1.933322541 \\ \text { H } & -5.335657186 & 1.408888302 & -0.046096006 \\ \text { H } & -6.289105453 & -1.176457287 & -0.253962216 \\ \text { C } & -1.149306981 & 0.221823514 & 0.217049616 \\ \text { O } & -0.743651756 & 0.707091953 & 1.239511291 \\ \text { O } & -3.332546940 & 1.150857682 & 1.974246543 \\ \text { H } & -2.395863870 & 1.175119384 & 2.157162755 \\ \text { O } & -0.375434129 & -0.216440718 & -0.748344754 \\ \text { C } & 1.064287475 & -0.159703709 & -0.703915752 \\ \text { C } & 1.641120617 & -1.037537173 & 0.402468831 \\ \text { C } & 1.595087313 & 1.267674094 & -0.655547845 \\ \text { C } & 3.185645928 & -1.071122275 & 0.378995629 \\ \text { H } & 1.311159393 & -0.655619348 & 1.360054000 \\ \text { C } & 3.127641322 & 1.264128791 & -0.747693855 \\ \text { H } & 1.168353583 & 1.837135331 & -1.476910905 \\ \text { C } & 3.722256868 & 0.376167129 & 0.355215727 \\ \text { H } & 3.504865351 & 0.840326158 & 1.316066496\end{array}$




$\begin{array}{lrrr}\mathrm{C} & 3.683692663 & 2.686972493 & -0.669414750 \\ \mathrm{H} & 3.431388047 & 3.152819729 & 0.279171522 \\ \mathrm{H} & 4.765511844 & 2.689745096 & -0.763389655 \\ \mathrm{H} & 3.280501738 & 3.309927136 & -1.462337708 \\ \mathrm{H} & 1.288122794 & 1.744753525 & 0.267180519 \\ \mathrm{H} & 1.245539588 & -2.044971447 & 0.301984522 \\ \mathrm{H} & 4.805848845 & 0.355601523 & 0.262809118 \\ \mathrm{H} & 3.410449647 & 0.856089459 & -1.716208725 \\ \mathrm{C} & 3.676718562 & -1.765201527 & 1.657515819 \\ \mathrm{H} & 3.308917736 & -2.785922002 & 1.714034622 \\ \mathrm{H} & 4.762039944 & -1.802824828 & 1.686900524 \\ \mathrm{H} & 3.337826843 & -1.240526290 & 2.545478884 \\ \mathrm{C} & 3.708044469 & -1.873445934 & -0.825981057 \\ \mathrm{H} & 4.793197043 & -1.917008938 & -0.811103059 \\ \mathrm{H} & 3.337737041 & -2.894139909 & -0.794811057 \\ \mathrm{H} & 3.416448045 & -1.450961503 & -1.780445329 \\ \mathrm{H} & 1.315297892 & -0.585536240 & -1.664729622\end{array}$

Coord_4

$\begin{array}{lrrr}\text { C } & -4.377302715 & -1.148930482 & -1.325454196 \\ \text { C } & -3.041742419 & -0.692108950 & -1.225361589 \\ \text { C } & -2.592012789 & 0.053304506 & -0.077157308 \\ \text { C } & -3.538733854 & 0.542378740 & 0.798380156 \\ \text { C } & -4.943433157 & 0.447782433 & 0.399418527 \\ \text { C } & -5.327182185 & -0.695124149 & -0.423749033 \\ \text { H } & -4.629262731 & -1.893837435 & -2.058241646 \\ \text { H } & -2.307932466 & -1.026107876 & -1.930892638 \\ \text { H } & -5.429430490 & 1.380859202 & 0.173393514 \\ \text { H } & -6.313061456 & -1.115390180 & -0.357489024 \\ \text { C } & -1.147029682 & 0.211918914 & 0.217696916 \\ \text { O } & -0.741256155 & 0.706479848 & 1.236224291 \\ \text { O } & -3.338642442 & 1.130869583 & 1.978568344 \\ \text { H } & -2.402323775 & 1.170277582 & 2.158600757 \\ \text { O } & -0.371384928 & -0.230887617 & -0.745406254 \\ \text { C } & 1.067730175 & -0.169702810 & -0.701829248 \\ \text { C } & 1.647718817 & -1.038453877 & 0.410126132 \\ \text { C } & 1.595183814 & 1.259272590 & -0.663766047 \\ \text { C } & 3.192297728 & -1.068478378 & 0.385687525 \\ \text { H } & 1.317897095 & -0.650967149 & 1.365562100 \\ \text { C } & 3.127677725 & 1.258870892 & -0.756992055 \\ \text { H } & 1.166439782 & 1.821953929 & -1.488735508 \\ \text { C } & 3.725337569 & 0.379915329 & 0.351464923 \\ \text { H } & 3.507642055 & 0.850140359 & 1.309295694 \\ \text { C } & 3.680337664 & 2.683558892 & -0.688840852 \\ \text { H } & 3.427897848 & 3.155211626 & 0.256839221 \\ \text { H } & 4.762051040 & 2.688352492 & -0.783944455 \\ \text { H } & 3.274857835 & 3.300208738 & -1.485514808\end{array}$




$\begin{array}{lrrr}\mathrm{H} & 1.288006893 & 1.742380424 & 0.255777416 \\ \mathrm{H} & 1.254422088 & -2.047469148 & 0.316947723 \\ \mathrm{H} & 4.808897144 & 0.361376524 & 0.258334718 \\ \mathrm{H} & 3.410758945 & 0.844959163 & -1.722916423 \\ \mathrm{C} & 3.686100764 & -1.752479825 & 1.668586922 \\ \mathrm{H} & 3.320724940 & -2.773625701 & 1.732586227 \\ \mathrm{H} & 4.771526345 & -1.787406127 & 1.697299820 \\ \mathrm{H} & 3.346813140 & -1.222432386 & 2.553207084 \\ \mathrm{C} & 3.715692768 & -1.877890833 & -0.814102758 \\ \mathrm{H} & 4.800958544 & -1.918677439 & -0.799843659 \\ \mathrm{H} & 3.347957539 & -2.899256010 & -0.775523155 \\ \mathrm{H} & 3.422287548 & -1.462841003 & -1.771253526 \\ \mathrm{H} & 1.319855797 & -0.601449145 & -1.659712619\end{array}$

\section{Coord_5}

$\begin{array}{rrrr}\text { C } & -4.371985013 & -1.111190783 & -1.365489296 \\ \text { C } & -3.031703519 & -0.694278550 & -1.229426390 \\ \text { C } & -2.590167987 & 0.035257302 & -0.075052707 \\ \text { C } & -3.543146155 & 0.516031137 & 0.804201360 \\ \text { C } & -4.942674857 & 0.405335628 & 0.433492329 \\ \text { C } & -5.329193783 & -0.660426447 & -0.466563335 \\ \text { H } & -4.634732333 & -1.818256531 & -2.130946653 \\ \text { H } & -2.293143766 & -1.027913773 & -1.930205941 \\ \text { H } & -5.507597997 & 1.317830995 & 0.391090430 \\ \text { H } & -6.331915655 & -1.044951677 & -0.454114832 \\ \text { C } & -1.145601581 & 0.203035817 & 0.217396216 \\ \text { O } & -0.739918453 & 0.706607549 & 1.232112488 \\ \text { O } & -3.342237043 & 1.111354880 & 1.981754743 \\ \text { H } & -2.406085675 & 1.164477683 & 2.156944454 \\ \text { O } & -0.367995325 & -0.244296217 & -0.743240554 \\ \text { C } & 1.070502175 & -0.178985812 & -0.700129351 \\ \text { C } & 1.653124521 & -1.038920776 & 0.417353629 \\ \text { C } & 1.594898614 & 1.251390093 & -0.671990647 \\ \text { C } & 3.197749132 & -1.065720375 & 0.392360826 \\ \text { H } & 1.323241197 & -0.645965445 & 1.370574801 \\ \text { C } & 3.127351227 & 1.253809988 & -0.765881556 \\ \text { H } & 1.164514286 & 1.807493230 & -1.500547305 \\ \text { C } & 3.727532570 & 0.383589629 & 0.348084126 \\ \text { H } & 3.509346852 & 0.859746963 & 1.302870895 \\ \text { C } & 3.676898763 & 2.680136592 & -0.707473051 \\ \text { H } & 3.424142246 & 3.157472827 & 0.235269416 \\ \text { H } & 4.758529043 & 2.686723991 & -0.803410256 \\ \text { H } & 3.269502038 & 3.290673239 & -1.507867509 \\ \text { H } & 1.287335595 & 1.740416626 & 0.244317516 \\ \text { H } & 1.261927991 & -2.049362147 & 0.331209426 \\ \text { H } & 4.811077344 & 0.366854424 & 0.254522319 \\ \text { H } & 3.410905147 & 0.834144362 & -1.729165424\end{array}$




$\begin{array}{llll}\mathrm{C} & 3.693749063 & -1.739929625 & 1.679596121 \\ \mathrm{H} & 3.330564340 & -2.761375999 & 1.750802328 \\ \mathrm{H} & 4.779257043 & -1.772402830 & 1.707923323 \\ \mathrm{H} & 3.353914244 & -1.204607384 & 2.560828686 \\ \mathrm{C} & 3.722326169 & -1.882096733 & -0.802181660 \\ \mathrm{H} & 4.807685746 & -1.920347840 & -0.788257959 \\ \mathrm{H} & 3.356936841 & -2.904003709 & -0.756464757 \\ \mathrm{H} & 3.427490048 & -1.474280307 & -1.761980927 \\ \mathrm{H} & 1.323937394 & -0.616514546 & -1.655044117\end{array}$

\section{Coord_6}

$\begin{array}{lrrr}\text { C } & -4.367090414 & -1.070512578 & -1.403392802 \\ \text { C } & -3.023529419 & -0.694712650 & -1.234074688 \\ \text { C } & -2.589168885 & 0.018562300 & -0.073912208 \\ \text { C } & -3.548091157 & 0.491131336 & 0.809522459 \\ \text { C } & -4.940163255 & 0.363255728 & 0.463348536 \\ \text { C } & -5.329067982 & -0.621526347 & -0.505349138 \\ \text { H } & -4.639356432 & -1.738064926 & -2.200187861 \\ \text { H } & -2.281245364 & -1.028375575 & -1.930945038 \\ \text { H } & -5.569161499 & 1.221244288 & 0.601296144 \\ \text { H } & -6.345902455 & -0.965891770 & -0.543030638 \\ \text { C } & -1.145089083 & 0.195135412 & 0.216394113 \\ \text { O } & -0.739643852 & 0.707459049 & 1.227395286 \\ \text { O } & -3.342470241 & 1.092626477 & 1.984059045 \\ \text { H } & -2.406160675 & 1.157840482 & 2.151988254 \\ \text { O } & -0.365385926 & -0.256719018 & -0.741636856 \\ \text { C } & 1.072487579 & -0.187592213 & -0.698684851 \\ \mathrm{C} & 1.657291818 & -1.038957273 & 0.424272029 \\ \mathrm{C} & 1.594114717 & 1.243998190 & -0.680146750 \\ \mathrm{C} & 3.201955631 & -1.062855775 & 0.399052728 \\ \mathrm{H} & 1.327200697 & -0.640618146 & 1.375226597 \\ \mathrm{C} & 3.126542126 & 1.248928588 & -0.774371157 \\ \mathrm{H} & 1.162408383 & 1.793709427 & -1.512262110 \\ \mathrm{C} & 3.728792170 & 0.387192929 & 0.345053227 \\ \mathrm{H} & 3.509985252 & 0.869165662 & 1.296777996 \\ \mathrm{C} & 3.673257267 & 2.676700194 & -0.725377853 \\ \mathrm{H} & 3.420057045 & 3.159614429 & 0.214403317 \\ \mathrm{H} & 4.754823643 & 2.684861393 & -0.821912658 \\ \mathrm{H} & 3.264265633 & 3.281300637 & -1.529455311 \\ \mathrm{H} & 1.286045392 & 1.738849426 & 0.232886616 \\ \mathrm{H} & 1.268007893 & -2.050679248 & 0.344931124 \\ \mathrm{H} & 4.812336045 & 0.372044828 & 0.251292517 \\ \mathrm{H} & 3.410712648 & 0.823610862 & -1.734975123 \\ \mathrm{C} & 3.699698566 & -1.727534822 & 1.690571522 \\ \mathrm{H} & 3.338476942 & -2.749163499 & 1.768751126 \\ \mathrm{H} & 4.785270745 & -1.757789828 & 1.718741024 \\ \mathrm{H} & 3.359215843 & -1.187020184 & 2.568383083\end{array}$




$\begin{array}{llll}\mathrm{C} & 3.727831666 & -1.886091434 & -0.790197356 \\ \mathrm{H} & 4.813268545 & -1.922039440 & -0.776383856 \\ \mathrm{H} & 3.364566640 & -2.908418010 & -0.737575452 \\ \mathrm{H} & 3.431883447 & -1.485324807 & -1.752603028 \\ \mathrm{H} & 1.327370897 & -0.630789245 & -1.650600917\end{array}$

Coord_7

\begin{tabular}{lrrr}
$\mathrm{C}$ & -4.362676716 & -1.027120176 & -1.438839904 \\
$\mathrm{C}$ & -3.017206216 & -0.693558451 & -1.239031089 \\
$\mathrm{C}$ & -2.589111786 & 0.003102799 & -0.073456803 \\
$\mathrm{C}$ & -3.553457756 & 0.467723133 & 0.814809358 \\
$\mathrm{C}$ & -4.935980754 & 0.321744726 & 0.489680533 \\
$\mathrm{C}$ & -5.326879686 & -0.578920842 & -0.539625737 \\
$\mathrm{H}$ & -4.643304036 & -1.653577121 & -2.265465663 \\
$\mathrm{H}$ & -2.272207265 & -1.027609575 & -1.932862940 \\
$\mathrm{H}$ & -5.613307704 & 1.093293479 & 0.798759957 \\
$\mathrm{H}$ & -6.355163655 & -0.879033061 & -0.623403645 \\
$\mathrm{C}$ & -1.145571481 & 0.188134011 & 0.214904014 \\
$\mathrm{O}$ & -0.740467353 & 0.708977750 & 1.222261790 \\
$\mathrm{O}$ & -3.338368842 & 1.074962276 & 1.985628643 \\
$\mathrm{H}$ & -2.401457670 & 1.150427884 & 2.143322452 \\
$\mathrm{O}$ & -0.363675826 & -0.268244419 & -0.740409953 \\
$\mathrm{C}$ & 1.073570275 & -0.195582615 & -0.697376349 \\
$\mathrm{C}$ & 1.660173919 & -1.038596374 & 0.430994231 \\
$\mathrm{C}$ & 1.592697115 & 1.237052691 & -0.688165749 \\
$\mathrm{C}$ & 3.204871429 & -1.059888678 & 0.405807331 \\
$\mathrm{H}$ & 1.329769493 & -0.634946245 & 1.379640501 \\
$\mathrm{C}$ & 3.125112527 & 1.244209491 & -0.782461457 \\
$\mathrm{H}$ & 1.159936982 & 1.780537427 & -1.523804910 \\
$\mathrm{C}$ & 3.729047567 & 0.390735929 & 0.342364324 \\
$\mathrm{H}$ & 3.509528651 & 0.878421962 & 1.291012694 \\
$\mathrm{C}$ & 3.669257465 & 2.673249694 & -0.742607451 \\
$\mathrm{H}$ & 3.415525647 & 3.161649729 & 0.194193816 \\
$\mathrm{H}$ & 4.750775940 & 2.682783494 & -0.839552760 \\
$\mathrm{H}$ & 3.258943237 & 3.272071436 & -1.550326009 \\
$\mathrm{H}$ & 1.284038990 & 1.737646728 & 0.221560519 \\
$\mathrm{H}$ & 1.272625293 & -2.051466347 & 0.358262327 \\
$\mathrm{H}$ & 4.812601745 & 0.376977326 & 0.248589120 \\
$\mathrm{H}$ & 3.410002545 & 0.813328260 & -1.740353527 \\
$\mathrm{C}$ & 3.703978165 & -1.715270124 & 1.701551420 \\
$\mathrm{H}$ & 3.344509838 & -2.736972398 & 1.786506727 \\
$\mathrm{H}$ & 4.789599844 & -1.743520727 & 1.729743026 \\
$\mathrm{H}$ & 3.362782344 & -1.169634383 & 2.575916286 \\
& 3.732117266 & -1.889893737 & -0.778117256 \\
$\mathrm{H}$ & 3.817617647 & -1.923750137 & -0.764236756 \\
\hline
\end{tabular}




$\begin{array}{lrrr}\text { H } & 1.329999897 & -0.644348047 & -1.646268919 \\ \text { Coord_8 } & & \\ \text { C } & -4.358779013 & -0.981279969 & -1.471512308 \\ \text { C } & -3.012699918 & -0.691016548 & -1.244080991 \\ \text { C } & -2.590101385 & -0.011300003 & -0.073469207 \\ \text { C } & -3.559119957 & 0.445803431 & 0.820502258 \\ \text { C } & -4.930173653 & 0.280957421 & 0.513184835 \\ \text { C } & -5.322637081 & -0.533156937 & -0.568906840 \\ \text { H } & -4.646778132 & -1.565144713 & -2.326282666 \\ \text { H } & -2.266019664 & -1.025793074 & -1.935804938 \\ \text { H } & -5.639326508 & 0.936844966 & 0.978725768 \\ \text { H } & -6.359770259 & -0.785270859 & -0.694371950 \\ \text { C } & -1.147143884 & 0.181902012 & 0.213095514 \\ \text { O } & -0.742460451 & 0.711065153 & 1.216862489 \\ \text { O } & -3.328811641 & 1.058596777 & 1.986466743 \\ \text { H } & -2.390767274 & 1.142213481 & 2.130280355 \\ \text { O } & -0.362981826 & -0.278997020 & -0.739408554 \\ \text { C } & 1.073635078 & -0.203036817 & -0.696099952 \\ \text { C } & 1.661719619 & -1.037883774 & 0.437623431 \\ \text { C } & 1.590492414 & 1.230494291 & -0.695986052 \\ \text { C } & 3.206447028 & -1.056818577 & 0.412678428 \\ \text { H } & 1.330919395 & -0.628988243 & 1.383925297 \\ \text { C } & 3.122907123 & 1.239638590 & -0.790140358 \\ \text { H } & 1.156901082 & 1.767892626 & -1.535112211 \\ \text { C } & 3.728207969 & 0.394238929 & 0.340028525 \\ \text { H } & 3.507907152 & 0.887547465 & 1.285586590 \\ \text { C } & 3.664707366 & 2.669792790 & -0.759193057 \\ \text { H } & 3.410376446 & 3.163596429 & 0.174611315 \\ \text { H } & 4.746191244 & 2.680529495 & -0.856397060 \\ \text { H } & 3.253297133 & 3.262968937 & -1.570510512 \\ \text { H } & 1.281182692 & 1.736756927 & 0.210404213 \\ \text { H } & 1.275753493 & -2.051785446 & 0.371336725 \\ \text { H } & 4.811781647 & 0.381703725 & 0.246388319 \\ \text { H } & 3.408596648 & 0.803273157 & -1.745292228 \\ \text { C } & 3.706603466 & -1.703099825 & 1.712589021 \\ \text { H } & 3.348709140 & -2.724779895 & 1.804152528 \\ \text { H } & 4.792262643 & -1.729526323 & 1.740948026 \\ \text { H } & 3.364645842 & -1.152407885 & 2.583484886 \\ \text { C } & 3.735113171 & -1.893509938 & -0.765892457 \\ \text { H } & 4.820665946 & -1.925453040 & -0.751801753 \\ \text { H } & 3.375511941 & -2.916352812 & -0.700018848 \\ \text { H } & 3.437688548 & -1.506378209 & -1.733376325 \\ \text { H } & 1.331686898 & -0.657281446 & -1.641946218\end{array}$

Coord_9

C $\quad-4.355425511 \quad-0.933294870 \quad-1.501092210$ 


\begin{tabular}{|c|c|c|c|}
\hline & 17 & 47 & -12 - \\
\hline$C$ & -2.592255988 & 024894503 & .0738172 \\
\hline$C$ & -3.564912157 & 0.425325531 & 0.827013360 \\
\hline $\mathrm{C}-\mathrm{s}$ & 22751153 & 241031315 & 3460423 \\
\hline 乙 & -5.316288182 & 84824835 & 20688145 \\
\hline & 50057333 & 507 & \\
\hline & -2.262716365 & -1.023172572 & -1.9397414 \\
\hline & -5.646519905 & 0.755430453 & 1.136964784 \\
\hline & -6.359740657 & -0.685564451 & -0.755024257 \\
\hline C & -1.149924583 & 0.176258512 & 0.211079316 \\
\hline 0 & 44451 & 0.713574750 & 1.211296185 \\
\hline 0 & -3.312434839 & 1.043690674 & 1.986377344 \\
\hline & -2.372689473 & 1.133008379 & 2.111832950 \\
\hline & -0.3634 & -0.28 & 7055 \\
\hline $\mathrm{C}$ & 1.07 & -0.21005 & $-0.6 s$ \\
\hline C & 1.661866921 & -1.036878072 & 0.444251132 \\
\hline C & 1.587325516 & 1.224250190 & -0.703553752 \\
\hline & 3.206621530 & -1.0536 & 0.419736028 \\
\hline$H$ & 1.330 & -0.62 & 1.388172601 \\
\hline C & 3.119749924 & 1.235208689 & -0.797375958 \\
\hline H & 1.153 & 1.755671928 & -1.546134211 \\
\hline U & 3.72 & & \\
\hline & 751 & 0.8 & 5890 \\
\hline & 3.659 & 2.66 & 3056 \\
\hline $\mathrm{H}$ & 3.404 & 3.165479729 & 0.155655212 \\
\hline H & 4.74 & 2.6 & -0.8 \\
\hline & & & 715 \\
\hline & 1.277 & $1.73^{2}$ & 0.1 \\
\hline$H$ & 1.277370589 & -2.05 & 267330 \\
\hline$H$ & 4.809 & 0.38 & 0.24 \\
\hline $\mathrm{H}$ & & & 28 \\
\hline$C$ & 3.707 & -1.6 & 60026 \\
\hline H & 3.351106742 & -2.71 & 1.821785831 \\
\hline $\mathrm{H}$ & 4.793250643 & -1.715 & 1.752417128 \\
\hline $\mathrm{H}$ & & -11 & 285 \\
\hline C & 3.736 & & -0.7 \\
\hline $\mathrm{H}$ & 4.822369145 & -1.927 & -0.739020053 \\
\hline $\mathrm{H}$ & 3.378783341 & -2.919899511 & -0.681177249 \\
\hline $\mathrm{H}$ & 3.438904746 & -1.51643 & -1.723409023 \\
\hline 11 & 1.002017091 & -0.669693547 & -1.637546617 \\
\hline \multicolumn{4}{|c|}{ Coord_10 } \\
\hline$c$ & -4.35 & -0.89 & -1.5 \\
\hline C & 00945865 & -0.690621967 & -1.248352811 \\
\hline C & & 36108013 & -0.074326527 \\
\hline C & -3 & 0.4163 & 0.831222817 \\
\hline & & 0.210719693 & 0.55 \\
\hline
\end{tabular}




\begin{tabular}{|c|c|c|c|}
\hline C & -5.307989413 & -0.435488772 & -0.606656831 \\
\hline $\mathrm{H}$ & -4.654575316 & -1.394575169 & -2.420762464 \\
\hline & -2.263179612 & -1.034073576 & -1.936354700 \\
\hline & -5.633609706 & 0.568373291 & 0560 \\
\hline & -6.355427688 & -0.582847162 & $-0.7 \mathrm{~s}$ \\
\hline & -1.153916151 & 0.174371640 & 57637 \\
\hline & -0.749944653 & 0.727831685 & \\
\hline & -3.286516668 & 1.049884333 & 1.976 \\
\hline & 1029 & 1.142 & \\
\hline C & -0.365 & -0.303890931 & -0.7 \\
\hline & 938 & -0.22 & -0.6 \\
\hline C & 451 & -1.03186 & \\
\hline 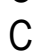 & 1.5 & 1.2122 & -0.7 \\
\hline C & & -1.0 & \\
\hline $\mathrm{H}$ & 106 & -0.60 & \\
\hline C & & & \\
\hline $\mathrm{H}$ & 152 & 1.7 & -1. \\
\hline C & 68 & 0.4 & \\
\hline $\mathrm{H}$ & 693 & 0.91 & 1.2 \\
\hline & 3.6 & 2.6 & \\
\hline $\mathrm{H}$ & 3.3 & 3.1 & \\
\hline ト & 4.7 & 2.66 & -0.5 \\
\hline $\mathrm{H}$ & 35 & 3.2 & -1.6 \\
\hline $\mathrm{H}$ & 164 & 1.7 & \\
\hline $\mathrm{H}$ & 1.2 & -2.0 & \\
\hline $\mathrm{H}$ & & 0.3 & \\
\hline $\mathrm{H}$ & & & \\
\hline C & 3.7 & -1.665 & \\
\hline $\mathrm{H}$ & 3.35 & -2.685 & 1.8 \\
\hline $\mathrm{H}$ & 4.7 & -1.6 & 1.7 \\
\hline $\mathrm{H}$ & 442 & -1.09 & 2.6 \\
\hline C & 3.7 & -1.9 & -0.7 \\
\hline $\mathrm{H}$ & 4.82 & -1.936 & -0.7 \\
\hline $\mathrm{H}$ & 3.3 & -2.9 & \\
\hline $\mathrm{H}$ & 3.43 & -1.54 & -1.7 \\
\hline$\Pi$ & 1.331277474 & -0.695253432 & -1.6277 \\
\hline \multicolumn{4}{|c|}{$\begin{array}{l}\text { LIIC path connecting T1-ENOL-MIN and } \mathrm{T}_{1} / \mathrm{S}_{0}-\mathrm{ENOL} \\
\text { Coord_1 }\end{array}$} \\
\hline C & -4.477498252 & -0.883893342 & -1.503792749 \\
\hline C & -3.06 & -0.70 & -1.2 \\
\hline C & -2.56 & -0.05 & -0.1 \\
\hline C & -3.560581534 & 0.431477000 & 0.86 \\
\hline C & & & \\
\hline C & -5.406188649 & -0.438177691 & -0.606090786 \\
\hline $\mathrm{H}$ & -4.794176238 & -1.381331021 & -2.40 \\
\hline $\mathrm{H}$ & -2.369108822 & -1.074401375 & -2.01 \\
\hline $\mathrm{H}$ & -5.646171775 & 0.595375100 & 1.33 \\
\hline
\end{tabular}

S82 


$\begin{array}{lrrr}\text { H } & -6.458312403 & -0.570957121 & -0.772697967 \\ \mathrm{C} & -1.230836970 & 0.119048508 & 0.110923007 \\ \mathrm{O} & -0.713264642 & 0.712266783 & 1.172539666 \\ \mathrm{O} & -3.226298562 & 1.010420374 & 1.91675160 \\ \mathrm{H} & -1.425230471 & 0.994263899 & 1.749666057 \\ \mathrm{O} & -0.364588075 & -0.322517605 & -0.776133973 \\ \mathrm{C} & 1.066247637 & -0.221545908 & -0.693446112 \\ \mathrm{C} & 1.647132467 & -1.030816924 & 0.462771934 \\ \mathrm{C} & 1.571519124 & 1.216220475 & -0.716904672 \\ \mathrm{C} & 3.192350259 & -1.036849893 & 0.456264101 \\ \mathrm{H} & 1.302039616 & -0.609779326 & 1.398282352 \\ \mathrm{C} & 3.104924664 & 1.234734858 & -0.793137957 \\ \mathrm{H} & 1.143505261 & 1.731001494 & -1.572693104 \\ \mathrm{C} & 3.703064837 & 0.416968050 & 0.361053097 \\ \mathrm{H} & 3.466918331 & 0.927100585 & 1.293887915 \\ \mathrm{C} & 3.636789033 & 2.668958474 & -0.785988206 \\ \mathrm{H} & 3.367991925 & 3.180884438 & 0.133970201 \\ \mathrm{H} & 4.719301287 & 2.685450224 & -0.870555012 \\ \mathrm{H} & 3.231091215 & 3.241886695 & -1.614547547 \\ \mathrm{H} & 1.247813759 & 1.740838037 & 0.173588273 \\ \mathrm{H} & 1.269830951 & -2.048526735 & 0.408974092 \\ \mathrm{H} & 4.787851257 & 0.411881947 & 0.281376402 \\ \mathrm{H} & 3.405027204 & 0.780927928 & -1.735572527 \\ \mathrm{C} & 3.682818473 & -1.653258027 & 1.774329977 \\ \mathrm{H} & 3.332367808 & -2.675951964 & 1.881804305 \\ \mathrm{H} & 4.768271752 & -1.669867398 & 1.815921409 \\ \mathrm{H} & 3.326198829 & -1.088755387 & 2.630446779 \\ \mathrm{C} & 3.742804292 & -1.892000206 & -0.698911328 \\ \mathrm{H} & 4.828323358 & -1.915327535 & -0.670062749 \\ \mathrm{H} & 3.389900895 & -2.916050990 & -0.617946833 \\ \mathrm{H} & 3.455261106 & -1.526126982 & -1.677467442 \\ \mathrm{H} & 1.349467158 & -0.690401808 & -1.624654256\end{array}$

Coord_2

$\begin{array}{lrrr}\text { C } & -4.479019721 & -0.881859862 & -1.501414008 \\ \text { C } & -3.061743520 & -0.730137453 & -1.269137793 \\ \text { C } & -2.571214784 & -0.059294803 & -0.112430210 \\ \text { C } & -3.564552655 & 0.468468035 & 0.844941960 \\ \text { C } & -4.959251355 & 0.289103120 & 0.563848440 \\ \text { C } & -5.408507589 & -0.393508829 & -0.625159043 \\ \text { H } & -4.794836947 & -1.386834899 & -2.396674273 \\ \text { H } & -2.367470870 & -1.121861483 & -1.983569741 \\ \text { H } & -5.652489109 & 0.685020949 & 1.280822995 \\ \text { H } & -6.461157567 & -0.506445138 & -0.803878557 \\ \text { C } & -1.221254189 & 0.108063207 & 0.127298207 \\ \text { O } & -0.733444652 & 0.629818443 & 1.244749088 \\ \text { O } & -3.224139434 & 1.056250076 & 1.887942835\end{array}$




$\begin{array}{lrrr}\text { H } & -1.461710707 & 0.947803767 & 1.779847428 \\ \text { O } & -0.362220224 & -0.362718428 & -0.755879656 \\ \text { C } & 1.067207078 & -0.246917115 & -0.685330348 \\ \text { C } & 1.664693818 & -1.051283378 & 0.466074534 \\ \text { C } & 1.556089712 & 1.196499786 & -0.706465651 \\ \text { C } & 3.209787131 & -1.038604074 & 0.452345930 \\ \text { H } & 1.318050493 & -0.636631748 & 1.403955201 \\ \text { C } & 3.088563022 & 1.234230790 & -0.791434557 \\ \text { H } & 1.116608880 & 1.710357221 & -1.556976613 \\ \text { C } & 3.702983566 & 0.421292428 & 0.357478425 \\ \text { H } & 3.466081252 & 0.926741766 & 1.292694795 \\ \text { C } & 3.602967261 & 2.674804993 & -0.784429557 \\ \text { H } & 3.333162838 & 3.181699430 & 0.138033208 \\ \text { H } & 4.684714336 & 2.704672694 & -0.875076461 \\ \text { H } & 3.185577230 & 3.244293833 & -1.609565815 \\ \text { H } & 1.231581787 & 1.713573724 & 0.188692214 \\ \text { H } & 1.299521695 & -2.073396351 & 0.412197527 \\ \text { H } & 4.787327647 & 0.429138931 & 0.271763617 \\ \text { H } & 3.388578142 & 0.785990058 & -1.736588325 \\ \text { C } & 3.714450567 & -1.651451120 & 1.766702930 \\ \text { H } & 3.377165842 & -2.678590891 & 1.873924533 \\ \text { H } & 4.800258548 & -1.654711720 & 1.802870332 \\ \text { H } & 3.355093743 & -1.093021080 & 2.625656291 \\ \text { C } & 3.764861669 & -1.884830833 & -0.707213950 \\ \text { H } & 4.850756150 & -1.894868437 & -0.684123348 \\ \text { H } & 3.424980446 & -2.913286510 & -0.626235045 \\ \text { H } & 3.467537648 & -1.520786711 & -1.683549922 \\ \text { H } & 1.350755398 & -0.710227154 & -1.619436716\end{array}$

Coord_3

$\begin{array}{lrrr}\text { C } & -4.479762522 & -0.886231861 & -1.495197710 \\ \text { C } & -3.061853023 & -0.754588156 & -1.246899891 \\ \text { C } & -2.577801988 & -0.061182706 & -0.113138710 \\ \text { C } & -3.567828559 & 0.509610836 & 0.818806959 \\ \text { C } & -4.962979457 & 0.351235525 & 0.527538339 \\ \text { C } & -5.409294888 & -0.352529723 & -0.643934146 \\ \text { H } & -4.794302944 & -1.403178099 & -2.384037473 \\ \text { H } & -2.366115970 & -1.175683582 & -1.943312741 \\ \text { H } & -5.656971505 & 0.778440858 & 1.225707486 \\ \text { H } & -6.461753463 & -0.447625230 & -0.834902361 \\ \text { C } & -1.211945088 & 0.100249809 & 0.143683313 \\ \text { O } & -0.755392157 & 0.546877138 & 1.311189196 \\ \text { O } & -3.221764630 & 1.110764779 & 1.853253933 \\ \text { H } & -1.490106305 & 0.905832163 & 1.808670728 \\ \text { O } & -0.360323726 & -0.399579127 & -0.734912150 \\ \text { C } & 1.067516879 & -0.270034821 & -0.676755349 \\ \text { C } & 1.681267919 & -1.070854675 & 0.468785134\end{array}$




$\begin{array}{lrrr}\text { C } & 1.541043912 & 1.178500986 & -0.694213552 \\ \text { C } & 3.225993333 & -1.040574677 & 0.447444431 \\ \text { H } & 1.333746694 & -0.663294947 & 1.409538402 \\ \text { C } & 3.072308523 & 1.234409186 & -0.788270759 \\ \text { H } & 1.090248879 & 1.692422420 & -1.538738609 \\ \text { C } & 3.702662567 & 0.424932231 & 0.354312225 \\ \text { H } & 3.465724052 & 0.924950665 & 1.292460894 \\ \text { C } & 3.570245756 & 2.680746093 & -0.780046858 \\ \text { H } & 3.300153137 & 3.181896331 & 0.145480912 \\ \text { H } & 4.651032934 & 2.723287197 & -0.877021161 \\ \text { H } & 3.141401429 & 3.247757234 & -1.601025317 \\ \text { H } & 1.216528885 & 1.687379821 & 0.206176717 \\ \text { H } & 1.327503895 & -2.096907951 & 0.413856128 \\ \text { H } & 4.786365745 & 0.445004633 & 0.262284719 \\ \text { H } & 3.371544440 & 0.792432257 & -1.736646826 \\ \text { C } & 3.744722771 & -1.651351021 & 1.757275325 \\ \text { H } & 3.419923645 & -2.682637594 & 1.863251234 \\ \text { H } & 4.830709248 & -1.642083619 & 1.787732227 \\ \text { H } & 3.383381341 & -1.099613681 & 2.619716890 \\ \text { C } & 3.784593472 & -1.877081435 & -0.717493953 \\ \text { H } & 4.870676551 & -1.874634334 & -0.700456250 \\ \text { H } & 3.457048651 & -2.909618809 & -0.637493646 \\ \text { H } & 3.477505051 & -1.513810509 & -1.691114521 \\ \text { H } & 1.350688997 & -0.727078454 & -1.614282218\end{array}$

\section{Coord_4}

$\begin{array}{lrrr}\text { C } & -4.480185123 & -0.896279466 & -1.485047608 \\ \text { C } & -3.062317222 & -0.782215855 & -1.220669390 \\ \text { C } & -2.584295887 & -0.061471806 & -0.114026807 \\ \text { C } & -3.570293858 & 0.554441340 & 0.789088656 \\ \text { C } & -4.965273059 & 0.414679332 & 0.487651938 \\ \text { C } & -5.408843288 & -0.314888922 & -0.662636148 \\ \text { H } & -4.793273646 & -1.429280603 & -2.364856972 \\ \text { H } & -2.365550771 & -1.234998891 & -1.896208737 \\ \text { H } & -5.659493109 & 0.875087261 & 1.164290883 \\ \text { H } & -6.460481564 & -0.394066629 & -0.866064361 \\ \text { C } & -1.202903989 & 0.095369806 & 0.159997710 \\ \text { O } & -0.778615656 & 0.462876631 & 1.371628498 \\ \text { O } & -3.218795533 & 1.173111887 & 1.812285231 \\ \text { H } & -1.509636708 & 0.866560263 & 1.837586333 \\ \text { O } & -0.358896625 & -0.433348530 & -0.713311749 \\ \text { C } & 1.067190874 & -0.291038422 & -0.667757346 \\ \text { C } & 1.696896121 & -1.089539776 & 0.470955332 \\ \text { C } & 1.526342310 & 1.162128982 & -0.680304948 \\ \text { C } & 3.241041335 & -1.042678575 & 0.441675134 \\ \text { H } & 1.349128294 & -0.689701448 & 1.415017704 \\ \text { C } & 3.056152519 & 1.235264289 & -0.783739457\end{array}$

S85 


$\begin{array}{lrrr}\text { H } & 1.064377376 & 1.676999321 & -1.518185111 \\ \mathrm{C} & 3.702120668 & 0.428014530 & 0.351550624 \\ \mathrm{H} & 3.465822950 & 0.921934265 & 1.293112194 \\ \mathrm{C} & 3.538562252 & 2.686832695 & -0.773049558 \\ \mathrm{H} & 3.268856833 & 3.181603130 & 0.156032812 \\ \mathrm{H} & 4.618215933 & 2.741404596 & -0.876554363 \\ \mathrm{H} & 3.098482323 & 3.252234635 & -1.589197814 \\ \mathrm{H} & 1.202568787 & 1.662227820 & 0.225804615 \\ \mathrm{H} & 1.353857795 & -2.119115054 & 0.414084531 \\ \mathrm{H} & 4.785006046 & 0.459666134 & 0.252980820 \\ \mathrm{H} & 3.353959541 & 0.800173157 & -1.735773123 \\ \mathrm{C} & 3.773722970 & -1.652730819 & 1.746227226 \\ \mathrm{H} & 3.460765650 & -2.687892494 & 1.850047034 \\ \mathrm{H} & 4.859733747 & -1.631686720 & 1.770733530 \\ \mathrm{H} & 3.411103445 & -1.108237481 & 2.612729390 \\ \mathrm{C} & 3.802139873 & -1.868758133 & -0.729517850 \\ \mathrm{H} & 4.888246754 & -1.854562133 & -0.718780653 \\ \mathrm{H} & 3.486283149 & -2.905085009 & -0.651405949 \\ \mathrm{H} & 3.485297551 & -1.505307609 & -1.699963624 \\ \mathrm{H} & 1.349323598 & -0.741170455 & -1.609157814\end{array}$

\begin{tabular}{lrrr}
\multicolumn{4}{l}{ Coord_5 } \\
C & -4.480676824 & -0.910863665 & -1.471057506 \\
C & -3.063434818 & -0.812095356 & -1.190462884 \\
C & -2.590723385 & -0.060312305 & -0.115151308 \\
C & -3.571829858 & 0.602244144 & 0.755650755 \\
C & -4.966110059 & 0.479016633 & 0.443940833 \\
C & -5.407381489 & -0.280067721 & -0.681523347 \\
H & -4.792351142 & -1.463386304 & -2.339234369 \\
H & -2.366225372 & -1.298406094 & -1.842181534 \\
H & -5.659906105 & 0.974078671 & 1.096248579 \\
H & -6.457640565 & -0.345106224 & -0.897706165 \\
C & -1.194128786 & 0.093119204 & 0.176228915 \\
O & -0.802632656 & 0.377060428 & 1.425798601 \\
O & -3.214881330 & 1.241896690 & 1.764946527 \\
H & -1.519694510 & 0.827694761 & 1.868008633 \\
O & -0.357937227 & -0.464380135 & -0.691058549 \\
C & 1.066235879 & -0.310146325 & -0.658311648 \\
C & 1.711642725 & -1.107341378 & 0.472732335 \\
C & 1.511908307 & 1.147211084 & -0.664936048 \\
C & 3.255018636 & -1.044799977 & 0.435211531 \\
H & 1.364225801 & -0.715727851 & 1.420456403 \\
C & 3.040039818 & 1.236733089 & -0.778011955 \\
H & 1.038887575 & 1.663750818 & -1.495584708 \\
C & 3.701360167 & 0.430694532 & 0.349146524 \\
H & 3.466342448 & 0.917978665 & 1.294505095 \\
C & 3.507777752 & 2.693060391 & -0.763826755
\end{tabular}




$\begin{array}{rrrr}\mathrm{H} & 3.239096635 & 3.180942129 & 0.169202811 \\ \mathrm{H} & 4.586139029 & 2.759093298 & -0.874048661 \\ \mathrm{H} & 3.056640219 & 3.257567434 & -1.574559411 \\ \mathrm{H} & 1.189582788 & 1.638059617 & 0.247275416 \\ \mathrm{H} & 1.378706699 & -2.140068451 & 0.413184829 \\ \mathrm{H} & 4.783265846 & 0.473352034 & 0.243823815 \\ \mathrm{H} & 3.335803438 & 0.809023456 & -1.734049624 \\ \mathrm{C} & 3.801583073 & -1.655237420 & 1.733821522 \\ \mathrm{H} & 3.499883253 & -2.694024695 & 1.834729231 \\ \mathrm{H} & 4.887481252 & -1.623077615 & 1.752157826 \\ \mathrm{H} & 3.438349147 & -1.118427482 & 2.604851285 \\ \mathrm{C} & 3.817652873 & -1.859891435 & -0.742953655 \\ \mathrm{H} & 4.903635853 & -1.834593434 & -0.738743251 \\ \mathrm{H} & 3.512895552 & -2.899741906 & -0.667485550 \\ \mathrm{H} & 3.491040749 & -1.495478809 & -1.709816121 \\ \mathrm{H} & 1.346701498 & -0.752847454 & -1.603947513\end{array}$

\begin{tabular}{lrrr}
\multicolumn{4}{l}{ Coord_6 } \\
C & -4.481538621 & -0.928534968 & -1.453533505 \\
C & -3.065440119 & -0.843053162 & -1.156498383 \\
C & -2.597100389 & -0.057840603 & -0.116540108 \\
C & -3.572326057 & 0.652146749 & 0.718564050 \\
C & -4.965447957 & 0.543733238 & 0.396342028 \\
C & -5.405067386 & -0.247419818 & -0.700872549 \\
H & -4.792009045 & -1.503230811 & -2.307617764 \\
H & -2.368504369 & -1.364096798 & -1.781492629 \\
H & -5.658059409 & 1.074300175 & 1.021578171 \\
H & -6.453439067 & -0.299906920 & -0.930187467 \\
C & -1.185620383 & 0.093178705 & 0.192432714 \\
O & -0.826964157 & 0.288450322 & 1.473360808 \\
O & -3.209724233 & 1.315349397 & 1.711508621 \\
H & -1.519896407 & 0.786320657 & 1.901263539 \\
O & -0.357447024 & -0.493082937 & -0.668026850 \\
C & 1.064648776 & -0.327624922 & -0.648330444 \\
C & 1.725585624 & -1.124239680 & 0.474356232 \\
C & 1.497639609 & 1.133526582 & -0.648332048 \\
C & 3.268018934 & -1.046792076 & 0.428277933 \\
H & 1.379097200 & -0.741179551 & 1.425999000 \\
C & 3.023880216 & 1.238707688 & -0.771332357 \\
H & 1.013629871 & 1.652241420 & -1.471254805 \\
C & 3.700371965 & 0.433152232 & 0.347009823 \\
H & 3.467248252 & 0.913445267 & 1.296433396 \\
C & 3.477702450 & 2.699378995 & -0.752933356 \\
H & 3.210656734 & 3.180039528 & 0.184320014 \\
H & 4.554619628 & 2.776375999 & -0.870072762 \\
H & 3.015627818 & 3.263501035 & -1.557773110 \\
H & 1.177439387 & 1.614818417 & 0.270242719
\end{tabular}




\begin{tabular}{|c|c|c|c|}
\hline $\mathrm{H}$ & 1.402198301 & -2.159799857 & 0.411618131 \\
\hline $\mathrm{H}$ & 4.781144544 & 0.486316337 & 0.234713314 \\
\hline ト & 3.317007941 & 0.818697657 & -1.731616726 \\
\hline C & 3.828466277 & -1.658409118 & 1.720393925 \\
\hline & 3.537511856 & -2.700587096 & 1.817852933 \\
\hline & 4.914127655 & -1.615695317 & 1.732332227 \\
\hline & 3.465249951 & -1.129564981 & 2.596292986 \\
\hline c & 3.831275774 & -1.850541031 & -0.757389256 \\
\hline ト & 4.917000055 & -1.814691130 & -0.759938657 \\
\hline & 3.537101755 & -2.893670206 & -0.685099950 \\
\hline & 3.494834251 & -1.484611905 & -1.720323422 \\
\hline H & 1.342842399 & -0.762552454 & -1.598455717 \\
\hline \multicolumn{4}{|c|}{ Coord_7 } \\
\hline 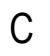 & -4.482971124 & -0.947647368 & -1.43 \\
\hline c & -3.068490318 & -0.8 & 207580 \\
\hline C & -2.603429688 & -0.054140405 & -0.118190606 \\
\hline C & -3.571688356 & 0.703220249 & 0.678134750 \\
\hline C & -4.963232557 & 0.608285342 & 0.345015925 \\
\hline C & -5.401988390 & -0.216208817 & -0.720956852 \\
\hline $\mathrm{H}$ & -4.792573545 & -1.546233310 & -2.270819863 \\
\hline ト & -2.372647070 & -1.4 & 824 \\
\hline $\mathrm{H}$ & -5.653815407 & 1.174524282 & 0.940657270 \\
\hline r & -6.447995664 & -0.257512720 & -0.96 \\
\hline C & -1.177382284 & 255609 & 0.208737215 \\
\hline 0 & -0.851112960 & 0.195825116 & 1.513820711 \\
\hline U & -3.203097130 & 1.391510301 & 3118 \\
\hline $\mathrm{H}$ & -1.510117407 & 0.738833751 & 1.938461540 \\
\hline C & -0.357433826 & -0.51 & -0.6439 \\
\hline C & 1.062414177 & 60124 & -0.637658447 \\
\hline C & 1.738809325 & -1.140181282 & 0.476157134 \\
\hline C & 1.483418505 & 1.120835281 & -0.630731443 \\
\hline$C$ & 3.280134034 & -1.048481675 & 0.421142729 \\
\hline $\mathrm{H}$ & 1.393825701 & -0.765781255 & 1.431875005 \\
\hline C & 3.007558416 & 1.241048191 & -0.764005956 \\
\hline $\mathrm{H}$ & 0.988424873 & 79016 & -1.445542003 \\
\hline C & 3.699140464 & 0.435588331 & 0.345012925 \\
\hline $\mathrm{H}$ & 3.468517552 & 3165 & 1.29 \\
\hline C & 3.448118446 & 2.705704896 & -0.741066355 \\
\hline $\mathrm{H}$ & 3.183311129 & 3.179034429 & 0.200558712 \\
\hline $\mathrm{H}$ & 4.523440124 & 2.793232200 & -0.865371763 \\
\hline 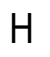 & 2.975156316 & 3.269709237 & -1.539660410 \\
\hline $\mathrm{H}$ & 1.166010386 & 1.592475617 & 0.294336922 \\
\hline s & 1.424487802 & -2.178307958 & 0.409993128 \\
\hline $\mathrm{H}$ & 4.778628042 & 0.498824034 & 0.225479118 \\
\hline $\mathrm{H}$ & 3.297460837 & 0.828830259 & -1.728666325 \\
\hline & 3.854555578 & -1.661695621 & 1.70634122 \\
\hline
\end{tabular}

S88 


$\begin{array}{llll}\mathrm{H} & 3.573911155 & -2.707037894 & 1.800092432 \\ \mathrm{H} & 4.939864058 & -1.608893717 & 1.711612821 \\ \mathrm{H} & 3.491975053 & -1.140899180 & 2.587313684 \\ \mathrm{C} & 3.843126577 & -1.840799132 & -0.772349155 \\ \mathrm{H} & 4.928464453 & -1.794855027 & -0.781930158 \\ \mathrm{H} & 3.559095758 & -2.886977505 & -0.703495649 \\ \mathrm{H} & 3.496734754 & -1.473073306 & -1.731084824 \\ \mathrm{H} & 1.337734897 & -0.770795755 & -1.592409317\end{array}$

Coord_8

$\begin{array}{lrrr}\text { C } & -4.485068524 & -0.966470669 & -1.410187602 \\ \text { C } & -3.072657822 & -0.902811863 & -1.079227577 \\ \text { C } & -2.609698887 & -0.049217802 & -0.120062407 \\ \text { C } & -3.569847656 & 0.754571953 & 0.634925046 \\ \text { C } & -4.959409256 & 0.672166149 & 0.290375220 \\ \text { C } & -5.398164888 & -0.185642311 & -0.742020054 \\ \text { H } & -4.794212846 & -1.589683017 & -2.230011560 \\ \text { H } & -2.378792771 & -1.493983105 & -1.643023819 \\ \text { H } & -5.647068006 & 1.273532890 & 0.854282060 \\ \text { H } & -6.441345562 & -0.216894615 & -0.999019970 \\ \text { C } & -1.169419283 & 0.099112306 & 0.225350714 \\ \text { O } & -0.874526265 & 0.097731307 & 1.546392714 \\ \text { O } & -3.194857131 & 1.468402306 & 1.589446517 \\ \text { H } & -1.490492509 & 0.680932647 & 1.980286244 \\ \text { O } & -0.357915123 & -0.545156740 & -0.618558043 \\ \text { C } & 1.059501077 & -0.358835124 & -0.626065545 \\ \text { C } & 1.751398129 & -1.155072785 & 0.478555333 \\ \text { C } & 1.469117907 & 1.108900281 & -0.612367045 \\ \text { C } & 3.291447838 & -1.049673578 & 0.414114229 \\ \text { H } & 1.408518000 & -0.789174658 & 1.438403904 \\ \text { C } & 2.990939916 & 1.243595391 & -0.756383057 \\ \text { H } & 0.963071668 & 1.632449820 & -1.418799004 \\ \mathrm{C} & 3.697646565 & 0.438220131 & 0.342997722 \\ \text { H } & 3.470147751 & 0.904421763 & 1.300826794 \\ \mathrm{C} & 3.418794544 & 2.711933297 & -0.729038251 \\ \mathrm{H} & 3.156844228 & 3.178087726 & 0.216970716 \\ \mathrm{H} & 4.492361925 & 2.809602600 & -0.860841863 \\ \mathrm{H} & 2.934912211 & 3.275818038 & -1.521169110 \\ \mathrm{H} & 1.155182282 & 1.571050712 & 0.319191022 \\ \mathrm{H} & 1.445725103 & -2.195552861 & 0.409057728 \\ \mathrm{H} & 4.775691044 & 0.511139936 & 0.215885818 \\ \mathrm{H} & 3.277007136 & 0.838992362 & -1.725432724 \\ \mathrm{C} & 3.880047279 & -1.664479120 & 1.692112020 \\ \mathrm{H} & 3.609351759 & -2.712762697 & 1.782223026 \\ \mathrm{H} & 4.964889458 & -1.601970613 & 1.690373123 \\ \mathrm{H} & 3.518729054 & -1.151570981 & 2.578218884 \\ \mathrm{C} & 3.853283478 & -1.830792634 & -0.787308657\end{array}$




$\begin{array}{llll}\mathrm{H} & 4.938109454 & -1.775131829 & -0.804266359 \\ \mathrm{H} & 3.579029156 & -2.879801909 & -0.721820951 \\ \mathrm{H} & 3.496743750 & -1.461299404 & -1.741657828 \\ \mathrm{H} & 1.331328497 & -0.778128358 & -1.585456414\end{array}$

\section{Coord_9}

$\begin{array}{lrrr}\text { C } & -4.487815620 & -0.983292171 & -1.386003799 \\ \text { C } & -3.077925220 & -0.928843864 & -1.037370874 \\ \text { C } & -2.615877587 & -0.042992805 & -0.122066411 \\ \text { C } & -3.566767257 & 0.805418256 & 0.589762043 \\ \text { C } & -4.953933256 & 0.734960951 & 0.233101319 \\ \text { C } & -5.393556686 & -0.154900711 & -0.764248658 \\ \text { H } & -4.796930944 & -1.630904818 & -2.186687755 \\ \text { H } & -2.386950673 & -1.553914709 & -1.567584612 \\ \text { H } & -5.637760006 & 1.370224797 & 0.763681956 \\ \text { H } & -6.433448465 & -0.176986813 & -1.035916277 \\ \text { C } & -1.161738481 & 0.104578606 & 0.242576317 \\ \text { O } & -0.896541964 & -0.007444000 & 1.569823815 \\ \text { O } & -3.184956828 & 1.544172313 & 1.523443710 \\ \text { H } & -1.461370403 & 0.607688145 & 2.026684246 \\ \text { O } & -0.358922428 & -0.569275941 & -0.591268844 \\ \mathrm{C} & 1.055858776 & -0.373116528 & -0.613235245 \\ \mathrm{C} & 1.763432027 & -1.168780984 & 0.482063233 \\ \mathrm{C} & 1.454602205 & 1.097503179 & -0.593443042 \\ \mathrm{C} & 3.302031939 & -1.050157775 & 0.407539730 \\ \mathrm{H} & 1.423304001 & -0.810923361 & 1.446002704 \\ \mathrm{C} & 2.973871415 & 1.246179089 & -0.748840651 \\ \mathrm{H} & 0.937348468 & 1.623143418 & -1.391355602 \\ \mathrm{C} & 3.695867466 & 0.441276934 & 0.340786322 \\ \mathrm{H} & 3.472159752 & 0.900959166 & 1.302677893 \\ \mathrm{C} & 3.389491645 & 2.717947195 & -0.717746053 \\ \mathrm{H} & 3.131059527 & 3.177386026 & 0.232521817 \\ \mathrm{H} & 4.461130619 & 2.825394503 & -0.857502664 \\ \mathrm{H} & 2.894562806 & 3.281424136 & -1.503336006 \\ \mathrm{H} & 1.144860881 & 1.550621909 & 0.344468623 \\ \mathrm{H} & 1.466046907 & -2.211456060 & 0.409693529 \\ \mathrm{H} & 4.772296944 & 0.523518937 & 0.205640713 \\ \mathrm{H} & 3.255445333 & 0.848704960 & -1.722177226 \\ \mathrm{C} & 3.905146579 & -1.666095322 & 1.678198723 \\ \mathrm{H} & 3.644107861 & -2.717096696 & 1.765104730 \\ \mathrm{H} & 4.989407161 & -1.594193612 & 1.668992420 \\ \mathrm{H} & 3.545752855 & -1.160637782 & 2.569357885 \\ \mathrm{C} & 3.861775080 & -1.820684930 & -0.801704659 \\ \mathrm{H} & 4.945960159 & -1.755622127 & -0.826494359 \\ \mathrm{H} & 3.596998057 & -2.872314707 & -0.739144351 \\ \mathrm{H} & 3.494794949 & -1.449788306 & -1.751564528 \\ \mathrm{H} & 1.323525297 & -0.785123658 & -1.577159111\end{array}$




$\begin{array}{lrrr}\text { Coord_10 } & & & \\ \text { C } & -4.495464523 & -0.996443469 & -1.343937896 \\ \text { C } & -3.087359324 & -0.950532570 & -0.981666771 \\ \text { C } & -2.622083090 & -0.034742804 & -0.113286107 \\ \text { C } & -3.560264954 & 0.856322964 & 0.556964442 \\ \text { C } & -4.945807856 & 0.797578955 & 0.191946913 \\ \text { C } & -5.390407287 & -0.122545708 & -0.767865055 \\ \text { H } & -4.807617043 & -1.667810920 & -2.123584554 \\ \text { H } & -2.401913371 & -1.608287613 & -1.478954307 \\ \text { H } & -5.623177902 & 1.465354108 & 0.690071348 \\ \text { H } & -6.427470164 & -0.136104611 & -1.051407374 \\ \text { C } & -1.153240382 & 0.112105507 & 0.266691220 \\ \text { O } & -0.910929765 & -0.119889810 & 1.587416815 \\ \text { O } & -3.168147629 & 1.618934718 & 1.467997306 \\ \text { H } & -1.416081303 & 0.515564439 & 2.082896152 \\ \text { O } & -0.362216323 & -0.592675841 & -0.557721842 \\ \text { C } & 1.049606378 & -0.387277830 & -0.599764641 \\ \text { C } & 1.776584230 & -1.180965983 & 0.484437937 \\ \text { C } & 1.438263302 & 1.085965078 & -0.577389842 \\ \text { C } & 3.313277141 & -1.049885678 & 0.393835527 \\ \text { H } & 1.443169304 & -0.829637661 & 1.453200505 \\ \text { C } & 2.954188112 & 1.247745088 & -0.750110555 \\ \text { H } & 0.907063664 & 1.612646415 & -1.365387900 \\ \text { C } & 3.695169767 & 0.444709431 & 0.327964024 \\ \text { H } & 3.479238149 & 0.899358463 & 1.294042594 \\ \mathrm{C} & 3.358345542 & 2.722691994 & -0.718807350 \\ \text { H } & 3.107372326 & 3.176899728 & 0.235978916 \\ \text { H } & 4.427388119 & 2.839257904 & -0.870712865 \\ \text { H } & 2.849693306 & 3.284744337 & -1.496634209 \\ \text { H } & 1.136682584 & 1.531534009 & 0.367304527 \\ \text { H } & 1.486742209 & -2.225729161 & 0.411713129 \\ \text { H } & 4.769329942 & 0.535620040 & 0.180597410 \\ \text { H } & 3.227261231 & 0.855862860 & -1.728154923 \\ \text { C } & 3.935421085 & -1.665281919 & 1.655545221 \\ \text { H } & 3.683897265 & -2.718670697 & 1.741616425 \\ \text { H } & 5.018928960 & -1.584462016 & 1.634661019 \\ \text { H } & 3.581703359 & -1.165857782 & 2.552361284 \\ \text { C } & 3.865784878 & -1.811768331 & -0.82421160 \\ \text { H } & 4.949089258 & -1.737796823 & -0.861034160 \\ \text { H } & 3.610255562 & -2.865724805 & -0.762190957 \\ \text { H } & 3.484934551 & -1.440752605 & -1.768571528 \\ \text { H } & 1.309123595 & -0.793495660 & -1.568577015 \\ & & & \\ \end{array}$

LIIC path connecting $\mathrm{T}_{1} / \mathrm{S}_{0}-\mathrm{ENOL}$ and S0-ENO-MIN Coord_1

$\begin{array}{llll}\text { C } & -4.495464523 & -0.996443469 & -1.343937896\end{array}$ 


$\begin{array}{lrrr}\text { C } & -3.087359324 & -0.950532570 & -0.981666771 \\ \text { C } & -2.622083090 & -0.034742804 & -0.113286107 \\ \text { C } & -3.560264954 & 0.856322964 & 0.556964442 \\ \text { C } & -4.945807856 & 0.797578955 & 0.191946913 \\ \text { C } & -5.390407287 & -0.122545708 & -0.767865055 \\ \text { H } & -4.807617043 & -1.667810920 & -2.123584554 \\ \text { H } & -2.401913371 & -1.608287613 & -1.478954307 \\ \text { H } & -5.623177902 & 1.465354108 & 0.690071348 \\ \text { H } & -6.427470164 & -0.136104611 & -1.051407374 \\ \text { C } & -1.153240382 & 0.112105507 & 0.266691220 \\ \text { O } & -0.910929765 & -0.119889810 & 1.587416815 \\ \text { O } & -3.168147629 & 1.618934718 & 1.467997306 \\ \text { H } & -1.416081303 & 0.515564439 & 2.082896152 \\ \text { O } & -0.362216323 & -0.592675841 & -0.557721842 \\ \text { C } & 1.049606378 & -0.387277830 & -0.599764641 \\ \text { C } & 1.776584230 & -1.180965983 & 0.484437937 \\ \text { C } & 1.438263302 & 1.085965078 & -0.577389842 \\ \text { C } & 3.313277141 & -1.049885678 & 0.393835527 \\ \text { H } & 1.443169304 & -0.829637661 & 1.453200505 \\ \text { C } & 2.954188112 & 1.247745088 & -0.750110555 \\ \text { H } & 0.907063664 & 1.612646415 & -1.365387900 \\ \text { C } & 3.695169767 & 0.444709431 & 0.327964024 \\ \text { H } & 3.479238149 & 0.899358463 & 1.294042594 \\ \text { C } & 3.358345542 & 2.722691994 & -0.718807350 \\ \text { H } & 3.107372326 & 3.176899728 & 0.235978916 \\ \text { H } & 4.427388119 & 2.839257904 & -0.870712865 \\ \text { H } & 2.849693306 & 3.284744337 & -1.496634209 \\ \text { H } & 1.136682584 & 1.531534009 & 0.367304527 \\ \text { H } & 1.486742209 & -2.225729161 & 0.411713129 \\ \text { H } & 4.769329942 & 0.535620040 & 0.180597410 \\ \text { H } & 3.227261231 & 0.855862860 & -1.728154923 \\ \text { C } & 3.935421085 & -1.665281919 & 1.655545221 \\ \text { H } & 3.683897265 & -2.718670697 & 1.741616425 \\ \text { H } & 5.018928960 & -1.584462016 & 1.634661019 \\ \text { H } & 3.581703359 & -1.165857782 & 2.552361284 \\ \text { C } & 3.865784878 & -1.811768331 & -0.824211160 \\ \text { H } & 4.949089258 & -1.737796823 & -0.861034160 \\ \text { H } & 3.610255562 & -2.865724805 & -0.762190957 \\ \text { H } & 3.484934551 & -1.440752605 & -1.768571528 \\ \text { H } & 1.309123595 & -0.793495660 & -1.568577015\end{array}$

Coord_2

$\begin{array}{llll}\text { C } & -4.523878526 & -0.977430168 & -1.362793397 \\ \text { C } & -3.115180124 & -0.942225270 & -1.045589277 \\ \text { C } & -2.623012987 & -0.060951006 & -0.141068209 \\ \text { C } & -3.540728952 & 0.800768159 & 0.593868141 \\ \text { C } & -4.938614654 & 0.748916853 & 0.265731917\end{array}$

S92 


\begin{tabular}{|c|c|c|c|}
\hline & 991 & 907 & J1 \\
\hline $\mathrm{H}$ & -4.863670551 & -1.619296416 & \\
\hline & -2.441480478 & -1.576141815 & $.58 / 8923$ \\
\hline & -5.602430106 & 1.393376800 & 0.81068265 \\
\hline & -6.451859764 & -0.138370712 & -0.9665638 \\
\hline & 3408681 & 86655807 & \\
\hline & 384713662 & 05 & \\
\hline & -3.126918927 & 1.532558909 & 1.51777380 \\
\hline & -1.446007705 & 0.558629339 & 2.00995544 \\
\hline & -0.351853324 & -0.582653542 & -0.609098344 \\
\hline & 1.063160777 & -0.380148225 & -0.625441248 \\
\hline & 1.768684529 & -1.170344882 & 0.4749187 \\
\hline & 1.454536407 & 1.092313278 & -0.606953943 \\
\hline & 3.307100240 & -1.044994678 & 0.406907030 \\
\hline & 1.422709602 & -0.8 & 178906 \\
\hline & 2.973855614 & 1.24 & -0.155954455 \\
\hline & 0.938478065 & 1.613955118 & -1.408228503 \\
\hline & 3.694523863 & 0.448 & 644123 \\
\hline & & & \\
\hline & 3.3826 & 2.72 & -0.7 \\
\hline H & 3.117973524 & 3.181395132 & 0.221902118 \\
\hline & 4.454 & 2.8329 & -0.861512161 \\
\hline & & & \\
\hline & 1.13 & 1.54 & 13726 \\
\hline & 1.476240707 & -2.21 & 0.403586829 \\
\hline$\Pi$ & 4.771 & 0.53 & 0.208048015 \\
\hline 11 & & & -1 . \\
\hline & & -1.65 & 7119 \\
\hline & 3.6503 & -2.70 & 706026 \\
\hline & 4.991089257 & -1.57 & 1.677026521 \\
\hline П & 3.54 & -1.1 & 2.5 \\
\hline & & & 955 \\
\hline & 4.95 & -1.7 & -0.8 \\
\hline & 3.615 & -2.86 & 387254 \\
\hline $\mathrm{H}$ & 3.511 & -1.44 & 494627 \\
\hline & sous & -0.793225758 & -1.5873123 \\
\hline \multicolumn{4}{|c|}{ Coord_3 } \\
\hline 0 & -4.5448 & -0.96 & -1 . \\
\hline 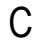 & & -0.92 & 822280 \\
\hline C & -2.6 & -0.08 & 189013 \\
\hline C & -3.521695553 & 0.746830355 & 0.628659847 \\
\hline$C$ & -4.929926352 & 0.696106048 & 0.334009324 \\
\hline C & -5.41632 & -0.142503411 & -0.66653734 \\
\hline & & & \\
\hline & -2. & -1.53731 & -1.686951023 \\
\hline & $55820 c$ & 1.314585 & 0.9215 \\
\hline
\end{tabular}




$\begin{array}{lrrr}\text { H } & -6.468735766 & -0.151539711 & -0.887451366 \\ \mathrm{C} & -1.172760682 & 0.067287702 & 0.171928513 \\ \mathrm{O} & -0.861945562 & 0.026267602 & 1.482493407 \\ \mathrm{O} & -3.090489121 & 1.450207605 & 1.563930510 \\ \mathrm{H} & -1.474530708 & 0.600243743 & 1.941271238 \\ \mathrm{O} & -0.343779322 & -0.567788439 & -0.655712647 \\ \mathrm{C} & 1.074235075 & -0.370120829 & -0.648389347 \\ \mathrm{C} & 1.758636326 & -1.158274783 & 0.466240532 \\ \mathrm{C} & 1.470633005 & 1.100922479 & -0.633625143 \\ \mathrm{C} & 3.298516339 & -1.040806472 & 0.418513829 \\ \mathrm{H} & 1.402287102 & -0.792257560 & 1.421134602 \\ \mathrm{C} & 2.992954716 & 1.247214090 & -0.761126956 \\ \mathrm{H} & 0.969422271 & 1.618556615 & -1.446810102 \\ \mathrm{C} & 3.693617864 & 0.450118030 & 0.348120825 \\ \mathrm{H} & 3.451909149 & 0.916882067 & 1.302158993 \\ \mathrm{C} & 3.408587647 & 2.719016996 & -0.732750451 \\ \mathrm{H} & 3.132562327 & 3.185342529 & 0.209133516 \\ \mathrm{H} & 4.482700821 & 2.824807201 & -0.853000059 \\ \mathrm{H} & 2.929047112 & 3.277047438 & -1.531608211 \\ \mathrm{H} & 1.142894284 & 1.562448412 & 0.293481522 \\ \mathrm{H} & 1.461907307 & -2.201256756 & 0.396159229 \\ \mathrm{H} & 4.772335743 & 0.531206136 & 0.232697218 \\ \mathrm{H} & 3.292787138 & 0.842538860 & -1.725946222 \\ \mathrm{C} & 3.877395577 & -1.647804919 & 1.704631322 \\ \mathrm{H} & 3.613823862 & -2.697902993 & 1.794470130 \\ \mathrm{H} & 4.961635256 & -1.576896512 & 1.714676625 \\ \mathrm{H} & 3.502358850 & -1.135423179 & 2.585302885 \\ \mathrm{C} & 3.879598780 & -1.820528633 & -0.774622855 \\ \mathrm{H} & 4.964020459 & -1.756152829 & -0.779786857 \\ \mathrm{H} & 3.613108658 & -2.871530006 & -0.709324652 \\ \mathrm{H} & 3.530769355 & -1.456401006 & -1.733898422 \\ \mathrm{H} & 1.358296396 & -0.789957459 & -1.603753517\end{array}$

Coord_4

$\begin{array}{lrrr}\text { C } & -4.558273327 & -0.945163966 & -1.395304502 \\ \text { C } & -3.155049328 & -0.913248466 & -1.156174982 \\ \text { C } & -2.619256591 & -0.097313606 & -0.184741514 \\ \text { C } & -3.504141251 & 0.694892650 & 0.662102049 \\ \text { C } & -4.920846353 & 0.639636046 & 0.396591328 \\ \text { C } & -5.421203890 & -0.161659310 & -0.621424046 \\ \text { H } & -4.942407755 & -1.534282408 & -2.208209860 \\ \text { H } & -2.498794078 & -1.492255907 & -1.775834629 \\ \text { H } & -5.563791102 & 1.229704891 & 1.022313172 \\ \text { H } & -6.478895964 & -0.175145414 & -0.815679458 \\ \text { C } & -1.181470083 & 0.053835202 & 0.132681612 \\ \text { O } & -0.842185359 & 0.107400206 & 1.427999803 \\ \text { O } & -3.060249219 & 1.372327700 & 1.607888016\end{array}$




$\begin{array}{lrrr}\text { H } & -1.500107210 & 0.641378648 & 1.878173037 \\ \text { O } & -0.338097922 & -0.548311441 & -0.697172049 \\ \text { C } & 1.082810075 & -0.357401927 & -0.668437449 \\ \text { C } & 1.746688827 & -1.144809080 & 0.458622734 \\ \text { C } & 1.486439408 & 1.111589877 & -0.657369845 \\ \text { C } & 3.287826535 & -1.037335975 & 0.428648928 \\ \text { H } & 1.382258198 & -0.771348253 & 1.407481003 \\ \text { C } & 3.011413715 & 1.247048590 & -0.765830157 \\ \text { H } & 0.999571769 & 1.626141816 & -1.481131607 \\ \text { C } & 3.692648867 & 0.450868631 & 0.356232625 \\ \text { H } & 3.441634946 & 0.923577969 & 1.304883896 \\ \text { C } & 3.436076547 & 2.716290394 & -0.738759253 \\ \text { H } & 3.151079625 & 3.188644828 & 0.197400714 \\ \text { H } & 4.512278225 & 2.814535003 & -0.845698059 \\ \text { H } & 2.970512415 & 3.273736434 & -1.546210513 \\ \text { H } & 1.149245681 & 1.581160014 & 0.261737417 \\ \text { H } & 1.444045606 & -2.186157456 & 0.389755126 \\ \text { H } & 4.773162741 & 0.524874937 & 0.254218820 \\ \text { H } & 3.321138137 & 0.835965558 & -1.724752525 \\ \text { C } & 3.846749774 & -1.642084619 & 1.724608022 \\ \text { H } & 3.575058358 & -2.689957592 & 1.816073029 \\ \text { H } & 4.931184053 & -1.578401016 & 1.747505326 \\ \text { H } & 3.464591951 & -1.123034182 & 2.598270887 \\ \text { C } & 3.878185078 & -1.826381434 & -0.753720555 \\ \text { H } & 4.962930555 & -1.769055329 & -0.745674356 \\ \text { H } & 3.604065262 & -2.875310708 & -0.687017947 \\ \text { H } & 3.543885654 & -1.464414003 & -1.718959325 \\ \text { H } & 1.375630100 & -0.783982254 & -1.617814116\end{array}$

\section{Coord_5}

$\begin{array}{lrrr}\text { C } & -4.563950230 & -0.931860265 & -1.410262200 \\ \text { C } & -3.166354226 & -0.893027465 & -1.202373288 \\ \text { C } & -2.615043287 & -0.107376208 & -0.198675714 \\ \text { C } & -3.489105653 & 0.645257644 & 0.695020552 \\ \text { C } & -4.912486554 & 0.579888040 & 0.453242335 \\ \text { C } & -5.421457493 & -0.186541214 & -0.581293344 \\ \text { H } & -4.963988058 & -1.498067306 & -2.231616560 \\ \text { H } & -2.514782883 & -1.441347003 & -1.854096632 \\ \text { H } & -5.549215100 & 1.139291380 & 1.112635981 \\ \text { H } & -6.482985266 & -0.208806812 & -0.752978553 \\ \text { C } & -1.189708785 & 0.046187903 & 0.099828605 \\ \text { O } & -0.824927058 & 0.193383814 & 1.373156799 \\ \text { O } & -3.037777617 & 1.299245295 & 1.651127618 \\ \text { H } & -1.521223409 & 0.683221551 & 1.821949632 \\ \text { O } & -0.334964526 & -0.524379940 & -0.732965053 \\ \text { C } & 1.088814380 & -0.342148726 & -0.685352150 \\ \text { C } & 1.733057226 & -1.129979380 & 0.452319032\end{array}$




$\begin{array}{lrrr}\text { C } & 1.501819506 & 1.124142179 & -0.678110751 \\ \text { C } & 3.275289437 & -1.034602372 & 0.437277232 \\ \text { H } & 1.362991500 & -0.748919853 & 1.395875199 \\ \text { C } & 3.029128617 & 1.246720289 & -0.770282654 \\ \text { H } & 1.028563073 & 1.636450617 & -1.511127210 \\ \text { C } & 3.691800664 & 0.450257532 & 0.362772626 \\ \text { H } & 3.434052047 & 0.928936068 & 1.306570595 \\ \text { C } & 3.464934747 & 2.712715294 & -0.744634052 \\ \text { H } & 3.173513327 & 3.191174131 & 0.186403316 \\ \text { H } & 4.542952826 & 2.801981103 & -0.840205860 \\ \text { H } & 3.012694716 & 3.270405835 & -1.559420312 \\ \text { H } & 1.157756782 & 1.601898417 & 0.233663517 \\ \text { H } & 1.422900805 & -2.169185757 & 0.384747130 \\ \text { H } & 4.773820043 & 0.515742238 & 0.272198320 \\ \text { H } & 3.346233739 & 0.829121359 & -1.723917224 \\ \text { C } & 3.815767177 & -1.638218720 & 1.741553924 \\ \text { H } & 3.534648854 & -2.683446294 & 1.834637932 \\ \text { H } & 4.900336052 & -1.583249015 & 1.775310029 \\ \text { H } & 3.429002347 & -1.112292180 & 2.609040988 \\ \text { C } & 3.871384780 & -1.833346734 & -0.735627752 \\ \text { H } & 4.956352057 & -1.784550427 & -0.716369452 \\ \text { H } & 3.588284159 & -2.879777610 & -0.667515649 \\ \text { H } & 3.550346553 & -1.472816608 & -1.705883525 \\ \text { H } & 1.387867498 & -0.775525853 & -1.629352916\end{array}$

\section{Coord_6}

$\begin{array}{lrrr}\text { C } & -4.561278430 & -0.920404964 & -1.425203403 \\ \text { C } & -3.171042128 & -0.869197063 & -1.242003991 \\ \text { C } & -2.609539288 & -0.111930408 & -0.205720116 \\ \text { C } & -3.477757151 & 0.598164944 & 0.728323253 \\ \text { C } & -4.906000153 & 0.517125338 & 0.503622835 \\ \text { C } & -5.417474889 & -0.216950914 & -0.547237841 \\ \text { H } & -4.972295860 & -1.466178206 & -2.254811960 \\ \text { H } & -2.520635282 & -1.384822300 & -1.921040337 \\ \text { H } & -5.540218298 & 1.043712174 & 1.191879385 \\ \text { H } & -6.481433369 & -0.252266917 & -0.701319349 \\ \text { C } & -1.197657488 & 0.044320605 & 0.074361305 \\ \text { O } & -0.809598957 & 0.283933620 & 1.318813995 \\ \text { O } & -3.024975920 & 1.231187486 & 1.695217820 \\ \text { H } & -1.536364112 & 0.727191052 & 1.773916827 \\ \text { O } & -0.334613523 & -0.496057733 & -0.762390954 \\ \text { C } & 1.092098877 & -0.324461225 & -0.698801148 \\ \text { C } & 1.717921021 & -1.113788180 & 0.447644730 \\ \text { C } & 1.516598209 & 1.138441580 & -0.695707250 \\ \text { C } & 3.261117737 & -1.032635372 & 0.444328731 \\ \text { H } & 1.344890797 & -0.724920554 & 1.386751897 \\ \text { C } & 3.045944420 & 1.246056991 & -0.774735157\end{array}$




$\begin{array}{lrrr}\text { H } & 1.055961077 & 1.649279318 & -1.536639612 \\ \mathrm{C} & 3.691246965 & 0.448204330 & 0.367462227 \\ \mathrm{H} & 3.429555549 & 0.932933865 & 1.307035795 \\ \mathrm{C} & 3.494996253 & 2.708072896 & -0.750834755 \\ \mathrm{H} & 3.199914631 & 3.192767929 & 0.175782612 \\ \mathrm{H} & 4.574565430 & 2.786900800 & -0.837260460 \\ \mathrm{H} & 3.055195221 & 3.266770033 & -1.571682814 \\ \mathrm{H} & 1.168461086 & 1.624585220 & 0.209520413 \\ \mathrm{H} & 1.398649500 & -2.150314455 & 0.381596927 \\ \mathrm{H} & 4.774491643 & 0.503703037 & 0.286082122 \\ \mathrm{H} & 3.367695744 & 0.821772558 & -1.723805726 \\ \mathrm{C} & 3.785020274 & -1.636137418 & 1.755396528 \\ \mathrm{H} & 3.493207449 & -2.678257891 & 1.850243131 \\ \mathrm{H} & 4.869698753 & -1.591386216 & 1.797750330 \\ \mathrm{H} & 3.396361646 & -1.103078580 & 2.617653390 \\ \mathrm{C} & 3.859146180 & -1.841520131 & -0.720579353 \\ \mathrm{H} & 4.944268056 & -1.802750129 & -0.692375147 \\ \mathrm{H} & 3.565745257 & -2.884986910 & -0.650903648 \\ \mathrm{H} & 3.549836654 & -1.481777505 & -1.694919422 \\ \mathrm{H} & 1.394639099 & -0.764745656 & -1.638141317\end{array}$

\begin{tabular}{lrrr}
\multicolumn{4}{l}{ Coord_7 } \\
C & -4.549178729 & -0.910762566 & -1.440767702 \\
C & -3.168012530 & -0.841791662 & -1.274413891 \\
C & -2.602919889 & -0.110799106 & -0.204243114 \\
C & -3.471499551 & 0.553812138 & 0.763034556 \\
C & -4.902628950 & 0.451468433 & 0.547196639 \\
C & -5.409397088 & -0.252804218 & -0.520580040 \\
H & -4.965403157 & -1.438679505 & -2.279175265 \\
H & -2.514432081 & -1.322715693 & -1.975541942 \\
H & -5.538924200 & 0.943090570 & 1.259044689 \\
H & -6.474333768 & -0.305459624 & -0.663115847 \\
C & -1.205520786 & 0.048344205 & 0.057635007 \\
O & -0.795538157 & 0.378868426 & 1.266029093 \\
O & -3.024298716 & 1.168309185 & 1.741861624 \\
H & -1.543939813 & 0.774976054 & 1.735528627 \\
O & -0.337404827 & -0.463276933 & -0.784442654 \\
C & 1.092393279 & -0.304365121 & -0.708291952 \\
C & 1.701413025 & -1.096195577 & 0.445020529 \\
C & 1.530533208 & 1.154396184 & -0.709922353 \\
C & 3.245464235 & -1.031483174 & 0.449682433 \\
H & 1.328427394 & -0.699255949 & 1.380675900 \\
C & 3.061620820 & 1.244872090 & -0.779496556 \\
H & 1.081203477 & 1.664493418 & -1.557373914 \\
C & 3.691150967 & 0.444582731 & 0.369907629 \\
H & 3.428646147 & 0.935497766 & 1.305994692 \\
C & 3.526068652 & 2.702101093 & -0.757947157
\end{tabular}




$\begin{array}{llll}\mathrm{H} & 3.230423631 & 3.193217729 & 0.165071413 \\ \mathrm{H} & 4.606914533 & 2.768964597 & -0.837833659 \\ \mathrm{H} & 3.097534122 & 3.262522735 & -1.583530214 \\ \mathrm{H} & 1.181436686 & 1.649186516 & 0.189686511 \\ \mathrm{H} & 1.371375599 & -2.129452653 & 0.380919929 \\ \mathrm{H} & 4.775334644 & 0.488564536 & 0.295092222 \\ \mathrm{H} & 3.384945542 & 0.813673257 & -1.724863022 \\ \mathrm{C} & 3.755121569 & -1.635821219 & 1.765962626 \\ \mathrm{H} & 3.451355249 & -2.674312991 & 1.862912232 \\ \mathrm{H} & 4.839914747 & -1.602851513 & 1.814276128 \\ \mathrm{H} & 3.367593343 & -1.095323980 & 2.624073088 \\ \mathrm{C} & 3.841201377 & -1.851018535 & -0.708901750 \\ \mathrm{H} & 4.926438953 & -1.823830831 & -0.674394948 \\ \mathrm{H} & 3.536178653 & -2.890997808 & -0.637314144 \\ \mathrm{H} & 3.541745756 & -1.491466206 & -1.686376019 \\ \mathrm{H} & 1.395339301 & -0.751721656 & -1.643808419\end{array}$

\begin{tabular}{|c|c|c|c|}
\hline \multicolumn{4}{|c|}{ Coord_8 } \\
\hline \multicolumn{4}{|l|}{ C } \\
\hline C & -3.155505828 & -0.810651158 & -1.298512496 \\
\hline$C$ & -2.595355485 & -0.103595210 & -0.191984014 \\
\hline C & -3.472169251 & 0.512389339 & 0.800364756 \\
\hline C & -4.903783954 & 0.382839827 & 0.583056542 \\
\hline C & -5.396948691 & -0.294198023 & -0.503079635 \\
\hline $\mathrm{H}$ & -4.940085354 & -1.415618903 & -2.306196267 \\
\hline $\mathrm{H}$ & -2.493298477 & -1.254717490 & -2.015709343 \\
\hline $\mathrm{H}$ & -5.547942698 & 0.837194260 & 1.312427095 \\
\hline $\mathrm{H}$ & -6.461200165 & -0.368641229 & -0.641605147 \\
\hline C & -1.213554290 & 0.058604502 & 0.051625903 \\
\hline 0 & -0.781915055 & 0.478159736 & 1.216278590 \\
\hline 0 & -3.039187419 & 1.110733679 & 1.792987830 \\
\hline $\mathrm{H}$ & -1.542126212 & 0.828632458 & 1.708595823 \\
\hline 0 & -0.343913225 & -0.425764431 & -0.797589655 \\
\hline C & 1.089218179 & -0.281777519 & -0.713059353 \\
\hline C & 1.683592923 & -1.077092580 & 0.445051232 \\
\hline C & 1.543259609 & 1.171978585 & -0.720363253 \\
\hline C & 3.228386230 & -1.031227676 & 0.453135632 \\
\hline $\mathrm{H}$ & 1.314193092 & -0.671748549 & 1.378436898 \\
\hline C & 3.075764521 & 1.242942487 & -0.784982759 \\
\hline $\mathrm{H}$ & 1.103498281 & 1.682052620 & -1.572810713 \\
\hline C & 3.691657464 & 0.439188732 & 0.369514524 \\
\hline $\mathrm{H}$ & 3.432003946 & 0.936483268 & 1.302984091 \\
\hline C & 3.557899855 & 2.694455593 & -0.766787057 \\
\hline $\mathrm{H}$ & 3.265316738 & 3.192241228 & 0.153599809 \\
\hline $\mathrm{H}$ & 4.639712034 & 2.747688999 & -0.843312660 \\
\hline $\mathrm{H}$ & 3.139074626 & 3.257302634 & -1.595661613 \\
\hline $\mathrm{H}$ & 1.196829786 & 1.675745219 & 0.17473351 \\
\hline
\end{tabular}




$\begin{array}{lrrr}\mathrm{H} & 1.341015594 & -2.106407254 & 0.383603329 \\ \mathrm{H} & 4.776460045 & 0.469984434 & 0.298064822 \\ \mathrm{H} & 3.397055244 & 0.804529656 & -1.727674827 \\ \mathrm{C} & 3.726765368 & -1.637320420 & 1.772916026 \\ \mathrm{H} & 3.409747746 & -2.671572091 & 1.872587434 \\ \mathrm{H} & 4.811699646 & -1.617826319 & 1.823988034 \\ \mathrm{H} & 3.343893040 & -1.089014980 & 2.628143292 \\ \mathrm{C} & 3.816923376 & -1.862016034 & -0.701068852 \\ \mathrm{H} & 4.902255150 & -1.848098333 & -0.663466046 \\ \mathrm{H} & 3.498903254 & -2.897896506 & -0.626944344 \\ \mathrm{H} & 3.524965355 & -1.502087111 & -1.680661021 \\ \mathrm{H} & 1.388958900 & -0.736434255 & -1.645739218\end{array}$

$\begin{array}{lrrr}\text { Coord_9 } & & & \\ \text { C } & -4.487336720 & -0.896712666 & -1.476455506 \\ \text { C } & -3.130499626 & -0.775230858 & -1.312326292 \\ \text { C } & -2.587033485 & -0.089469109 & -0.165453913 \\ \text { C } & -3.482462553 & 0.474150134 & 0.841864058 \\ \text { C } & -4.911209951 & 0.310829821 & 0.609544345 \\ \text { C } & -5.379041089 & -0.341571526 & -0.497382534 \\ \text { H } & -4.890599053 & -1.397056502 & -2.337577171 \\ \text { H } & -2.452528778 & -1.179860283 & -2.038112749 \\ \text { H } & -5.570828799 & 0.725169352 & 1.348904595 \\ \text { H } & -6.440407262 & -0.442701034 & -0.641674845 \\ \text { C } & -1.222121590 & 0.075908804 & 0.059489503 \\ \text { O } & -0.767529557 & 0.582067642 & 1.171897785 \\ \text { O } & -3.075021223 & 1.058637677 & 1.850950636 \\ \text { H } & -1.528482112 & 0.890828162 & 1.695785823 \\ \text { O } & -0.355126227 & -0.382870429 & -0.799313159 \\ \text { C } & 1.081691278 & -0.256425716 & -0.711825052 \\ \text { C } & 1.664378222 & -1.056239874 & 0.448689035 \\ \text { C } & 1.554169312 & 1.191271784 & -0.726351153 \\ \text { C } & 3.209754830 & -1.032019273 & 0.454330731 \\ \text { H } & 1.303006195 & -0.642048248 & 1.381246099 \\ \text { C } & 3.087678820 & 1.239957589 & -0.791821756 \\ \text { H } & 1.121603282 & 1.702072120 & -1.582017112 \\ \text { C } & 3.692866364 & 0.431668530 & 0.365302326 \\ \text { H } & 3.440639145 & 0.935624366 & 1.297192691 \\ \mathrm{C} & 3.590105757 & 2.684620892 & -0.778623054 \\ \text { H } & 3.305110840 & 3.189418231 & 0.140293408 \\ \text { H } & 4.672485934 & 2.722280294 & -0.855888462 \\ \text { H } & 3.178860431 & 3.250619332 & -1.609111114 \\ \text { H } & 1.214905988 & 1.704453825 & 0.165584012 \\ \text { H } & 1.307226495 & -2.080795551 & 0.391053330 \\ \text { H } & 4.777880346 & 0.447331632 & 0.293099022 \\ \text { H } & 3.402420943 & 0.793922859 & -1.733100127 \\ \text { C } & 3.700799165 & -1.640804418 & 1.775613830\end{array}$




$\begin{array}{lrrr}\text { H } & 3.369107542 & -2.670057792 & 1.879065035 \\ \mathrm{H} & 4.785905647 & -1.636749920 & 1.825327133 \\ \mathrm{H} & 3.326970142 & -1.084214379 & 2.629474389 \\ \mathrm{C} & 3.784998472 & -1.874820033 & -0.697827949 \\ \mathrm{H} & 4.870399553 & -1.876130434 & -0.661251148 \\ \mathrm{H} & 3.452456049 & -2.905845209 & -0.620103844 \\ \mathrm{H} & 3.497434252 & -1.513948211 & -1.678363223 \\ \mathrm{H} & 1.373715499 & -0.718706653 & -1.642856816 \\ & & & \\ \mathrm{C} \text { oord } 10 & & & \\ \mathrm{C} & -4.421829091 & -0.887806963 & -1.517440839 \\ \mathrm{C} & -3.082865233 & -0.730446932 & -1.326139353 \\ \mathrm{C} & -2.578014696 & -0.065412854 & -0.129510538 \\ \mathrm{C} & -3.510267071 & 0.438701550 & 0.876455465 \\ \mathrm{C} & -4.929703685 & 0.234317135 & 0.604467493 \\ \mathrm{C} & -5.351169387 & -0.393671408 & -0.529774769 \\ \mathrm{H} & -4.797580297 & -1.376860162 & -2.397183934 \\ \mathrm{H} & -2.376579561 & -1.090662256 & -2.048353197 \\ \mathrm{H} & -5.618072887 & 0.603404762 & 1.341636108 \\ \mathrm{H} & -6.405591198 & -0.526937908 & -0.697592301 \\ \mathrm{C} & -1.232324847 & 0.102358427 & 0.080845964 \\ \mathrm{O} & -0.754140356 & 0.689411120 & 1.133899980 \\ \mathrm{O} & -3.148328846 & 1.009325951 & 1.908146155 \\ \mathrm{H} & -1.504651631 & 0.962437428 & 1.696690864 \\ \mathrm{O} & -0.370583156 & -0.331278605 & -0.789035436 \\ \mathrm{C} & 1.070126797 & -0.226369877 & -0.701143069 \\ \mathrm{C} & 1.641259319 & -1.034451964 & 0.458950332 \\ \mathrm{C} & 1.564675814 & 1.213757706 & -0.720819404 \\ \mathrm{C} & 3.186916218 & -1.035932507 & 0.459160812 \\ \mathrm{H} & 1.290993252 & -0.612617113 & 1.392231268 \\ \mathrm{C} & 3.098797993 & 1.236285077 & -0.790046895 \\ \mathrm{H} & 1.138921083 & 1.728078875 & -1.577769374 \\ \mathrm{C} & 3.693388736 & 0.419619889 & 0.366825674 \\ \mathrm{H} & 3.451854570 & 0.928790867 & 1.298672371 \\ \mathrm{C} & 3.625181133 & 2.672410932 & -0.779836447 \\ \mathrm{H} & 3.351019491 & 3.182887078 & 0.139207187 \\ \mathrm{H} & 4.707805938 & 2.691830985 & -0.859887772 \\ \mathrm{H} & 3.221403159 & 3.244254991 & -1.609949076 \\ \mathrm{H} & 1.235852999 & 1.735223196 & 0.169666090 \\ \mathrm{H} & 1.266838449 & -2.052940169 & 0.403693977 \\ \mathrm{H} & 4.778257046 & 0.417471620 & 0.291768041 \\ \mathrm{H} & 3.403843334 & 0.783910318 & -1.731445463 \\ \mathrm{C} & 3.671600122 & -1.651378840 & 1.779699417 \\ \mathrm{H} & 3.322847951 & -2.674730014 & 1.885436106 \\ \mathrm{H} & 4.756714854 & -1.665704731 & 1.825932702 \\ \mathrm{H} & 3.310092896 & -1.087511048 & 2.634075247 \\ \mathrm{C} & 3.744191662 & -1.889554338 & -0.693824850 \\ & & & \\ & & & 5100\end{array}$




$\begin{array}{llll}H & 4.829466698 & -1.909001729 & -0.660409238 \\ H & 3.394682324 & -2.914770228 & -0.613997964 \\ H & 3.460041889 & -1.524980022 & -1.673976158 \\ H & 1.351261217 & -0.694902498 & -1.632053376\end{array}$

LIIC path connecting S1-ENOL-MIN and $\mathrm{S}_{1} / \mathrm{S}_{0}-\mathrm{ENOL}$ Coord_1

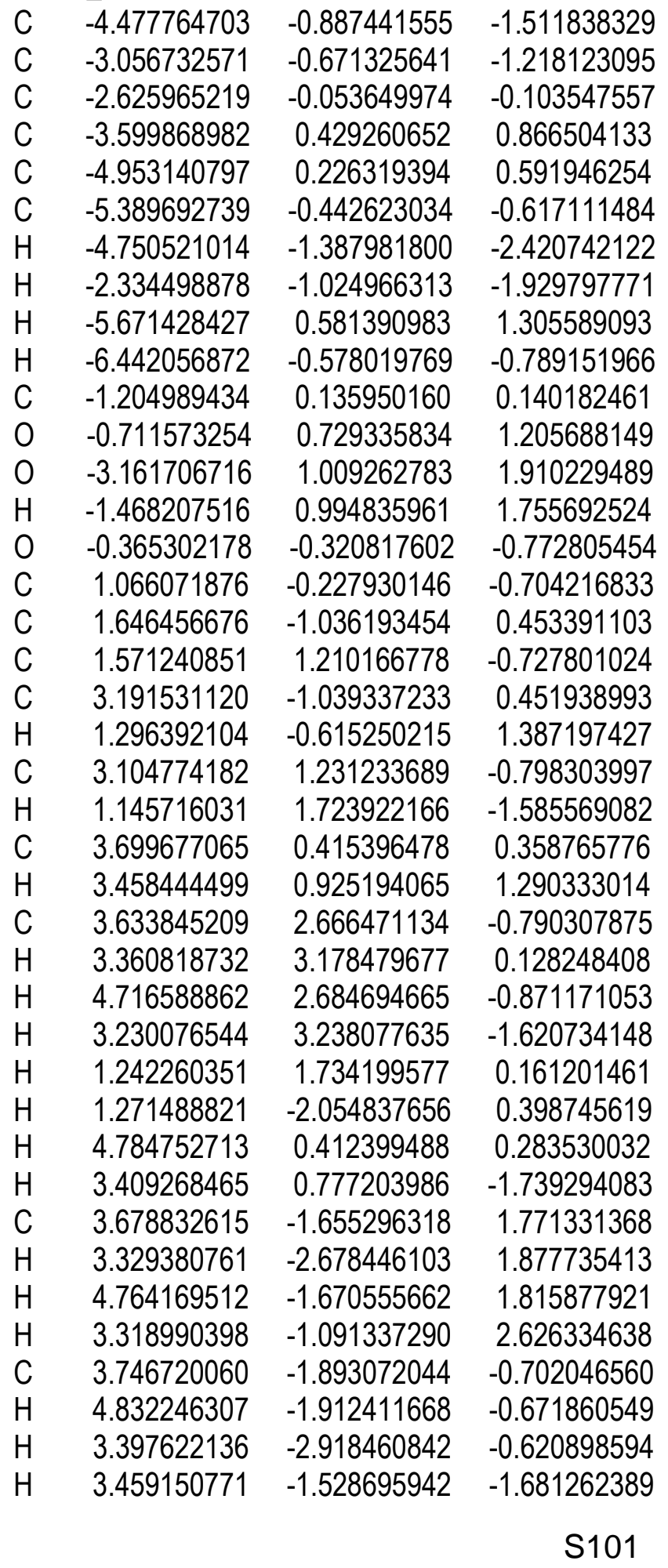


$\begin{array}{llll}H & 1.343265646 & -0.698589048 & -1.636607518\end{array}$

Coord_2

\begin{tabular}{lrrr} 
C & -4.482120223 & -0.921904964 & -1.489528205 \\
C & -3.061472819 & -0.724563052 & -1.206421785 \\
C & -2.626655388 & -0.065942502 & -0.115878710 \\
C & -3.590952959 & 0.487653335 & 0.816880256 \\
C & -4.949086554 & 0.306244320 & 0.553729142 \\
C & -5.390002689 & -0.416829231 & -0.617111146 \\
H & -4.768526143 & -1.455156206 & -2.375648473 \\
H & -2.344096269 & -1.120951880 & -1.899992439 \\
H & -5.662033805 & 0.717803051 & 1.242467889 \\
H & -6.443573266 & -0.542111442 & -0.788411558 \\
C & -1.204186286 & 0.126124211 & 0.146266110 \\
O & -0.738678151 & 0.655683346 & 1.262785290 \\
O & -3.127548923 & 1.118259680 & 1.828965829 \\
H & -1.505370110 & 0.967647268 & 1.768451125 \\
O & -0.362920124 & -0.346987724 & -0.759152056 \\
$\mathrm{C}$ & 1.066611378 & -0.242247116 & -0.696102651 \\
$\mathrm{C}$ & 1.659637619 & -1.055174778 & 0.452078632 \\
$\mathrm{C}$ & 1.558544312 & 1.200105589 & -0.707979352 \\
$\mathrm{C}$ & 3.204856231 & -1.043133773 & 0.444207830 \\
$\mathrm{H}$ & 1.308717494 & -0.646360445 & 1.391007800 \\
$\mathrm{C}$ & 3.090998624 & 1.236962689 & -0.786307057 \\
$\mathrm{H}$ & 1.123271978 & 1.718752523 & -1.557835611 \\
$\mathrm{C}$ & 3.699361168 & 0.416990529 & 0.360695024 \\
$\mathrm{H}$ & 3.458217149 & 0.916693066 & 1.297789095 \\
$\mathrm{C}$ & 3.605896559 & 2.677167690 & -0.769865657 \\
$\mathrm{H}$ & 3.332428838 & 3.179146927 & 0.154105210 \\
$\mathrm{H}$ & 4.687945439 & 2.707128293 & -0.856287663 \\
$\mathrm{H}$ & 3.191731032 & 3.250951634 & -1.593672013 \\
$\mathrm{H}$ & 1.229270986 & 1.711742221 & 0.188633015 \\
$\mathrm{H}$ & 1.294225594 & -2.076855848 & 0.390158830 \\
$\mathrm{H}$ & 4.784045843 & 0.424661530 & 0.279931722 \\
$\mathrm{H}$ & 3.394273344 & 0.793422459 & -1.732704625 \\
$\mathrm{C}$ & 3.704324766 & -1.664840320 & 1.756394028 \\
$\mathrm{H}$ & 3.365928144 & -2.692385891 & 1.856006231 \\
$\mathrm{H}$ & 4.790010845 & -1.669210218 & 1.795822929 \\
$\mathrm{H}$ & 3.342690839 & -1.111603980 & 2.617643886 \\
$\mathrm{C}$ & 3.763467473 & -1.882239034 & -0.718857352 \\
$\mathrm{H}$ & 4.849285349 & -1.891285838 & -0.693263350 \\
$\mathrm{H}$ & 3.424576848 & -2.911567710 & -0.644233046 \\
$\mathrm{H}$ & 3.468067550 & -1.512995708 & -1.693919721 \\
$\mathrm{H}$ & 1.345179098 & -0.702237651 & -1.633576719 \\
& & & \\
\hline
\end{tabular}

Coord_3

C $\quad-4.487024924 \quad-0.958840568 \quad-1.462576208$

S102 


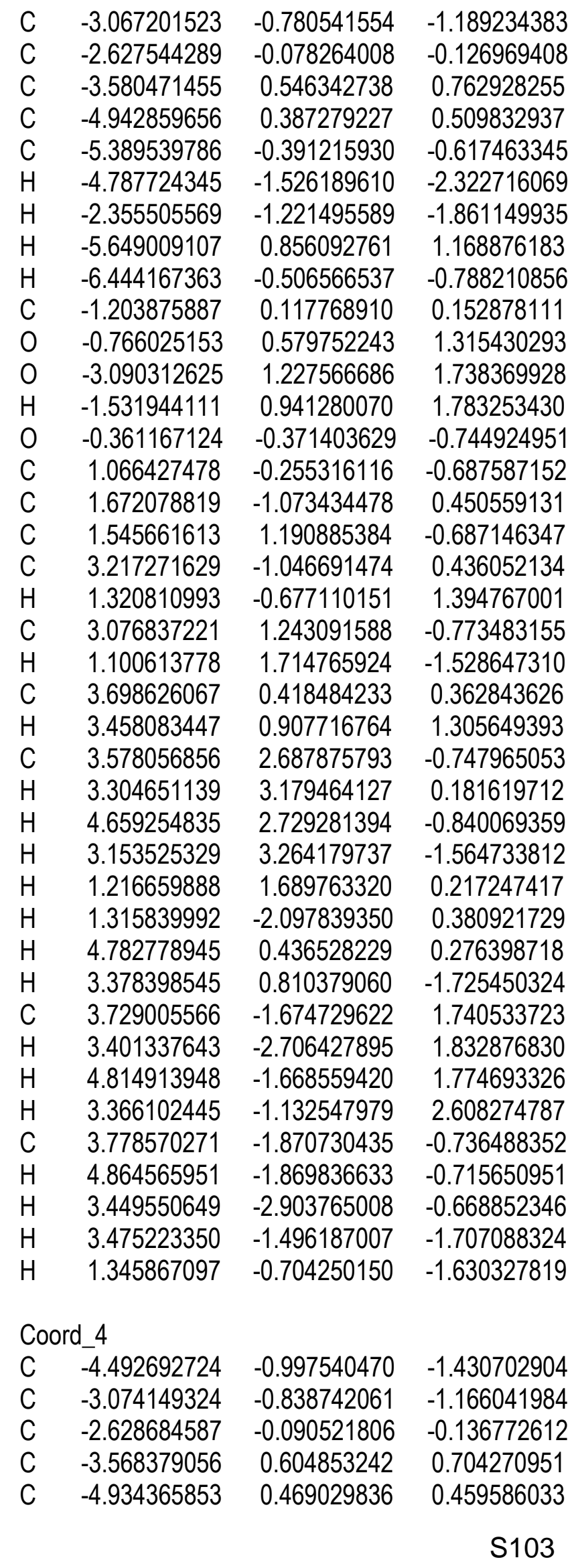




\begin{tabular}{|c|c|c|c|}
\hline & 3889 & 0725 & -0.618466644 \\
\hline & 435448 & -1.599981515 & \\
\hline & -2.369083273 & -1.325846596 & -1 \\
\hline & -5.632148906 & 0.995388771 & 1.08372757 \\
\hline & -6.443890862 & -0.470948236 & -0.7889458 \\
\hline C & -1.204074185 & 0.110854707 & \\
\hline 0 & -0.793018755 & 501043334 & \\
\hline 0 & -3.049879821 & 1.336021595 & 1.6 \\
\hline F & -1.547389414 & 0.914214368 & 1.801471 \\
\hline 0 & -0.3 & -0.394125828 & \\
\hline C & 1.065497576 & -0.267162818 & -0.67866 \\
\hline C & 772319 & -1.090938778 & 0.4 \\
\hline C & 1.532559312 & 1.182486786 & -0.6653490 \\
\hline C & 3.228780134 & -1.049943273 & \\
\hline & 1.332660198 & -0.70 & 1.3 \\
\hline 0 & 3.062265818 & 1.249633489 & $-0.75987 \varepsilon$ \\
\hline $\mathrm{H}$ & 1.077701975 & 1.711897926 & -1.49805610 \\
\hline c & 3.697461967 & $0.41 s$ & \\
\hline & 3.45 & 0.89 & 1.3 \\
\hline C & 3.550289354 & 2.698620192 & -0.72 \\
\hline $\mathrm{H}$ & 3.277444835 & 3.179484331 & 0.210662013 \\
\hline F & 4.63 & 2.75 & \\
\hline F & & & \\
\hline t & 1.20 & 1.66 & 0.2 \\
\hline r & 1.336331698 & -2.1177 & 0.371094224 \\
\hline $\mathrm{H}$ & 4.78 & 0.448 & 0.27 \\
\hline F & & & \\
\hline c & 3.75 & $-1.6 \varepsilon$ & 1.7 \\
\hline $\mathrm{H}$ & 3.435633949 & -2.72 & 40902 \\
\hline $\mathrm{H}$ & 4.838 & -1.66 & 515926 \\
\hline H & & & \\
\hline C & 3.79 & & -0.7 \\
\hline H & 4.878 & -1.848004235 & -0.738955356 \\
\hline ⺊ & 3.472 & -2.89502 & -0.694652049 \\
\hline $\mathrm{H}$ & & & \\
\hline $\mathrm{H}$ & & -0.7 & -1.626 \\
\hline \multicolumn{4}{|c|}{ Coord_5 } \\
\hline C & $-4.4 \mathrm{~s}$ & & \\
\hline C & & -0.89 & 518984 \\
\hline c & 29289 & -0.102675907 & -0.145200713 \\
\hline C & -3.554605557 & 0.662412650 & 0.64078634 \\
\hline 0 & -4.923473554 & 0.550862642 & 0.402507631 \\
\hline c & -5.386471886 & -0.339122526 & -0.620514946 \\
\hline $\mathrm{H}$ & & & 925 \\
\hline $\mathrm{H}$ & -2.38 & -1.43 & -1.752840528 \\
\hline $\mathrm{H}$ & -5.611190302 & 1.134367582 & 0.98629 \\
\hline
\end{tabular}




\begin{tabular}{|c|c|c|c|}
\hline-1 & 6.442765366 & -0.434640929 & 157358 \\
\hline C & -1.204800888 & 0.105272807 & \\
\hline 0 & -0.819050661 & 0.419003832 & 1.40697230 \\
\hline 0 & -3.006097214 & 1.441850404 & 1.527308910 \\
\hline $\mathrm{H}$ & -1.551136910 & 0.884657866 & 1.824295634 \\
\hline 0 & -0.359670626 & -0.415322129 & $-0.11461<8$ \\
\hline C & 1.063791376 & -0.277889622 & -0.669267 \\
\hline$C$ & 1.694733824 & -1.107659577 & 0.4470696 \\
\hline C & 1.519167211 & 1.174823384 & -0.6426652 \\
\hline C & 3.239404134 & -1.052798978 & 0118610 \\
\hline H & 1.344283296 & -0.737239054 & 1.4021710 \\
\hline$C$ & 3.047217721 & 1.256558689 & -0.74559765 \\
\hline $\mathrm{H}$ & 1.054438274 & 1.709964624 & -1.46616640 \\
\hline C & 3.695848267 & 0.421488531 & 0.367644825 \\
\hline $\mathrm{H}$ & 3.458034847 & 0.888817665 & 1.322279 \\
\hline C & 3.522486355 & 2.709393796 & -0.7003268 \\
\hline$H_{-}$ & 3.250702635 & 3.179273030 & 0.240938217 \\
\hline $\mathrm{H}$ & 4.601535631 & 2.772914302 & -0.804180960 \\
\hline $\mathrm{H}$ & 3.0772 & 3.29 & -1.50 \\
\hline $\mathrm{H}$ & 1.192384586 & 1.647238319 & 0.277646819 \\
\hline $\mathrm{H}$ & 1.355743396 & -2.136617951 & 0.3608828 \\
\hline $\mathrm{H}$ & 4.778533546 & 497131 & 0.269357 \\
\hline$\Pi$ & 3.34 & & \\
\hline C & 3.776 & 26920 & 1.70 \\
\hline $\mathrm{H}$ & 3.468914149 & -2.734214897 & 1.7828096 \\
\hline $\mathrm{H}$ & 4.8620 & -1.6 & 1.7293 \\
\hline $\mathrm{H}$ & 3.41 & -1 & 86 \\
\hline C & 3.803905372 & -1.84 & -0.77 \\
\hline $\mathrm{H}$ & 4.889943852 & -1.825735133 & -0.7630353 \\
\hline $\mathrm{H}$ & 3.493676549 & -2.885336108 & -0.721375 \\
\hline $\mathrm{H}$ & 3.48 & 66007 & -1.7 \\
\hline $\mathrm{H}$ & 1.3 & -0.7037 & -1.622 \\
\hline \multicolumn{4}{|c|}{ Coord_6 } \\
\hline C & 26 & -1.0 & -1.352452999 \\
\hline$C$ & 6321 & 109467 & -1.100587182 \\
\hline C & -2.631932291 & -0.114690707 & -0.152143312 \\
\hline C & -3.539068754 & 0.718077154 & 0.572647742 \\
\hline C & -4.910020154 & 0.631968448 & 0.33838222 \\
\hline C & -5.383885490 & -0.311718822 & -0.624060645 \\
\hline 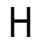 & -4.855708947 & -1.748227727 & -2.1138896 \\
\hline $\mathrm{H}$ & -2.404350174 & -1.540183009 & -1.6825365 \\
\hline $\mathrm{H}$ & -5.585830302 & 1.271380292 & 0.8763540 \\
\hline $\mathrm{H}$ & -6.440765663 & -0.396967129 & -0.795481260 \\
\hline C & -1.206081286 & 0.100889209 & 0.17642881 \\
\hline 0 & -0.8434 & 0.333035125 & 1.44543040 \\
\hline 0 & -2.958807713 & 1.542916612 & 1.40730 \\
\hline
\end{tabular}




\begin{tabular}{|c|c|c|c|}
\hline & 1.542617510 & 46662 & 331 \\
\hline 0 & -0.359999828 & -0.435213833 & \\
\hline C & 1.061268878 & -0.287641120 & -0.6592982 \\
\hline 0 & 1.704987522 & -1.123557581 & 0.44538943 \\
\hline C & 1.505394606 & 1.167775087 & -0.619200242 \\
\hline C & 3.249170233 & -1.055147874 & \\
\hline$H$ & 1.355715895 & -0.766365457 & 1.406012 \\
\hline $\mathrm{C}$ & 3.031600319 & 1.263809189 & -0.7307997 \\
\hline $\mathrm{H}$ & 1.030691175 & 1.708709421 & -1.433129605 \\
\hline $\mathrm{C}$ & 3.693755964 & 0.423225729 & 0.3701603 \\
\hline H & 3.458080049 & 0.879312763 & 1.330793 \\
\hline$C$ & 3.494502552 & 2.720168295 & -0.675196 \\
\hline H & 3.224287530 & 3.178915629 & 0.272017818 \\
\hline H & 4.572253831 & 2.794435099 & -0.785175057 \\
\hline $\mathrm{H}$ & 3.038844719 & 3.305440639 & -1.468566605 \\
\hline $\mathrm{H}$ & 1.180565285 & 1.626660616 & 0.309127 \\
\hline H & 1.374133698 & -2.154392555 & 0.350617123 \\
\hline$\Pi$ & 4.775505346 & 0.470904436 & 0.265658118 \\
\hline H & 3.325061738 & 0.865 & -1.700072423 \\
\hline C & 3.798567773 & -1.704692823 & 1.6880880 \\
\hline $\mathrm{H}$ & 3.501307253 & -2.747426696 & 1.75640842 \\
\hline$\Pi$ & 4.884 & -1.668646719 & 1.70 \\
\hline H & 3.43 & -1.197227288 & \\
\hline$C$ & 3.814 & -1.83 & -0.7 \\
\hline 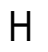 & 4.900103553 & -1.802997731 & -0.7876929 \\
\hline $\mathrm{H}$ & 3.51 & 207 & -0.7 \\
\hline$\Pi$ & 3.486 & -1. & -1.7486 \\
\hline $\mathrm{H}$ & 1.34 & -0.701600252 & -1.61 \\
\hline \multicolumn{4}{|c|}{ Coord_7 } \\
\hline$C$ & -4.5 & 9882 & -1.306925292 \\
\hline C & -3.10 & -1.016866171 & -1.058437478 \\
\hline C & -2.634 & -0.126495112 & -0.15748250 \\
\hline C & -3.521691155 & 0.770866453 & 0.50033423 \\
\hline$c$ & -4.893827151 & 0.711447452 & 0.267310919 \\
\hline C & -5.380545985 & -0.282793618 & -0.62956564 \\
\hline $\mathrm{H}$ & -4.882824254 & -1.818024132 & -2.0290688 \\
\hline $\mathrm{H}$ & -2.426837175 & -1.646320217 & -1.601661917 \\
\hline $\mathrm{H}$ & -5.555753698 & 1.404616699 & 0.7542265 \\
\hline $\mathrm{H}$ & -6.437812965 & -0.357291223 & -0.802580458 \\
\hline C & -1.207945085 & 0.097590207 & 0.18594531 \\
\hline 0 & -0.865716061 & 0.242498517 & 1.47850660 \\
\hline 0 & -2.907884907 & 1.636996315 & 1.278992692 \\
\hline $\mathrm{H}$ & -1.521286312 & 0.808979958 & 1.887247136 \\
\hline 0 & -0.361105427 & -0.454026835 & -0.6808618 \\
\hline C & 1.05788 & -0.296570319 & -0.6486205 \\
\hline C & 1.714552924 & -1.138570884 & 0.44405 \\
\hline
\end{tabular}




$\begin{array}{lrrr}\text { C } & 1.491145006 & 1.161218681 & -0.595079243 \\ \text { C } & 3.258096633 & -1.056863578 & 0.400435028 \\ \text { H } & 1.366997896 & -0.794605355 & 1.410174600 \\ \text { C } & 3.015310719 & 1.271313092 & -0.715682452 \\ \text { H } & 1.006320774 & 1.707850323 & -1.399134603 \\ \text { C } & 3.691149866 & 0.425304232 & 0.372631828 \\ \text { H } & 3.458147149 & 0.870146963 & 1.339220498 \\ \text { C } & 3.466185452 & 2.730906497 & -0.649770447 \\ \text { H } & 3.198063929 & 3.178523228 & 0.303370723 \\ \text { H } & 4.542483028 & 2.815755904 & -0.766103852 \\ \text { H } & 2.999998515 & 3.319220441 & -1.434758603 \\ \text { H } & 1.168850184 & 1.606555817 & 0.341205325 \\ \text { H } & 1.391554202 & -2.171058256 & 0.340719524 \\ \text { H } & 4.771822642 & 0.482490835 & 0.261658718 \\ \text { H } & 3.305048837 & 0.884090164 & -1.690735022 \\ \text { C } & 3.820534175 & -1.713767123 & 1.669584421 \\ \text { H } & 3.532938257 & -2.759720500 & 1.729630925 \\ \text { H } & 4.906190853 & -1.668168522 & 1.680902019 \\ \text { H } & 3.457734047 & -1.218057186 & 2.564802786 \\ \text { C } & 3.822899877 & -1.817668133 & -0.812599959 \\ \text { H } & 4.908590455 & -1.779794431 & -0.812690760 \\ \text { H } & 3.530433253 & -2.863295103 & -0.775992254 \\ \text { H } & 3.486149452 & -1.419259801 & -1.762499124 \\ \text { H } & 1.335767294 & -0.698650248 & -1.613429015\end{array}$

Coord_8

$\begin{array}{lrrr}\text { C } & -4.526919925 & -1.144734683 & -1.258110591 \\ \text { C } & -3.119043923 & -1.073233676 & -1.010521473 \\ \text { C } & -2.636833790 & -0.137958207 & -0.161104711 \\ \text { C } & -3.502418351 & 0.819883258 & 0.424616730 \\ \text { C } & -4.874721053 & 0.788398857 & 0.189738212 \\ \text { C } & -5.376367585 & -0.251966218 & -0.637444147 \\ \text { H } & -4.912498355 & -1.881773835 & -1.938556040 \\ \text { H } & -2.453027777 & -1.748927728 & -1.510948411 \\ \text { H } & -5.520670596 & 1.532276112 & 0.620841644 \\ \text { H } & -6.433778962 & -0.315095122 & -0.813066158 \\ \text { C } & -1.210425789 & 0.095314808 & 0.196572817 \\ \text { O } & -0.885005165 & 0.146729008 & 1.505560709 \\ \text { O } & -2.853269407 & 1.722027426 & 1.144079180 \\ \text { H } & -1.486638707 & 0.757202255 & 1.928077437 \\ \text { O } & -0.363037326 & -0.471954837 & -0.662145648 \\ \text { C } & 1.053574974 & -0.304814419 & -0.637076146 \\ \text { C } & 1.723436022 & -1.152608480 & 0.443336333 \\ \text { C } & 1.476328905 & 1.155052385 & -0.570437940 \\ \text { C } & 3.266184435 & -1.057809477 & 0.391506427 \\ \text { H } & 1.378163102 & -0.821690959 & 1.414885802 \\ \text { C } & 2.998249415 & 1.278998392 & -0.700468649 \\ & & & \\ & & & S 107\end{array}$




$\begin{array}{lrrr}\text { H } & 0.981197772 & 1.707117524 & -1.364394296 \\ \mathrm{C} & 3.687991064 & 0.427879028 & 0.374959727 \\ \mathrm{H} & 3.458222647 & 0.861655261 & 1.347383798 \\ \mathrm{C} & 3.437398649 & 2.741572996 & -0.624563845 \\ \mathrm{H} & 3.171920028 & 3.178237129 & 0.334405022 \\ \mathrm{H} & 4.512078323 & 2.836856503 & -0.747545855 \\ \mathrm{H} & 2.960529312 & 3.332694342 & -1.401009398 \\ \mathrm{H} & 1.157174285 & 1.586994616 & 0.373670429 \\ \mathrm{H} & 1.408033200 & -2.186561356 & 0.331680023 \\ \mathrm{H} & 4.767438441 & 0.494427735 & 0.257201416 \\ \mathrm{H} & 3.283713336 & 0.903043267 & -1.681200221 \\ \mathrm{C} & 3.842013977 & -1.721754122 & 1.651011421 \\ \mathrm{H} & 3.563901857 & -2.770705601 & 1.702974124 \\ \mathrm{H} & 4.927333955 & -1.666731118 & 1.655979417 \\ \mathrm{H} & 3.480579449 & -1.237644587 & 2.553122585 \\ \mathrm{C} & 3.830006775 & -1.802834030 & -0.831770362 \\ \mathrm{H} & 4.915377354 & -1.756168926 & -0.837764459 \\ \mathrm{H} & 3.546122555 & -2.851091705 & -0.802888656 \\ \mathrm{H} & 3.484288050 & -1.398568203 & -1.775965426 \\ \mathrm{H} & 1.329739394 & -0.695178549 & -1.607349917\end{array}$

Coord_9

$\begin{array}{lrrr}\text { C } & -4.538979626 & -1.172581582 & -1.206966086 \\ \text { C } & -3.135589025 & -1.125912483 & -0.957520170 \\ \text { C } & -2.640041191 & -0.148885411 & -0.162904311 \\ \text { C } & -3.481237449 & 0.864402464 & 0.346528023 \\ \text { C } & -4.852558649 & 0.862000062 & 0.106458905 \\ \text { C } & -5.371236984 & -0.218958517 & -0.648004249 \\ \text { H } & -4.944783655 & -1.937373837 & -1.844046434 \\ \text { H } & -2.483183080 & -1.845923431 & -1.411554202 \\ \text { H } & -5.480357095 & 1.652718118 & 0.477707935 \\ \text { H } & -6.428497361 & -0.270019020 & -0.827420361 \\ \text { C } & -1.213558687 & 0.094067209 & 0.208491513 \\ \text { O } & -0.900588964 & 0.045072905 & 1.525626709 \\ \text { O } & -2.795002001 & 1.796299929 & 1.004768174 \\ \text { H } & -1.438216004 & 0.691651852 & 1.974497442 \\ \text { O } & -0.365849025 & -0.489141233 & -0.641842848 \\ \text { C } & 1.048288377 & -0.312481425 & -0.624486744 \\ \text { C } & 1.731625527 & -1.165550584 & 0.443561434 \\ \text { C } & 1.460870003 & 1.149207781 & -0.545412539 \\ \text { C } & 3.273415033 & -1.057844874 & 0.382973730 \\ \text { H } & 1.389232601 & -0.847303963 & 1.420414100 \\ \text { C } & 2.980327716 & 1.286799994 & -0.685390749 \\ \text { H } & 0.955214868 & 1.706275920 & -1.329132993 \\ \text { C } & 3.684238764 & 0.431111232 & 0.377049225 \\ \text { H } & 3.458297049 & 0.854199863 & 1.355111199 \\ \text { C } & 3.408034143 & 2.752142200 & -0.600122842 \\ & & & \\ & & & \text { S108 }\end{array}$




$\begin{array}{lrrr}\text { H } & 3.145778624 & 3.178229027 & 0.364498924 \\ \mathrm{H} & 4.480921923 & 2.857706706 & -0.730117053 \\ \mathrm{H} & 2.920294013 & 3.345673642 & -1.367959300 \\ \mathrm{H} & 1.145492380 & 1.568104714 & 0.406314230 \\ \mathrm{H} & 1.423569505 & -2.200823059 & 0.324037921 \\ \mathrm{H} & 4.762302140 & 0.506873238 & 0.252127516 \\ \mathrm{H} & 3.260934536 & 0.921705766 & -1.671636919 \\ \mathrm{C} & 3.863042576 & -1.728248926 & 1.632697517 \\ \mathrm{H} & 3.594250156 & -2.779982998 & 1.676989521 \\ \mathrm{H} & 4.947912558 & -1.663907121 & 1.630945618 \\ \mathrm{H} & 3.503555150 & -1.255399891 & 2.541552484 \\ \mathrm{C} & 3.835455878 & -1.787548528 & -0.850350961 \\ \mathrm{H} & 4.920404655 & -1.732209225 & -0.862628661 \\ \mathrm{H} & 3.559981156 & -2.838249303 & -0.828764461 \\ \mathrm{H} & 3.480435449 & -1.377848797 & -1.788743829 \\ \mathrm{H} & 1.322144493 & -0.691493051 & -1.600075513 \\ & & & \\ \mathrm{C} 00 \mathrm{r} \mathbf{1} & 10 & & \\ \mathrm{C} & -4.553591829 & -1.194345986 & -1.149229080 \\ \mathrm{C} & -3.155119126 & -1.173478882 & -0.897882566 \\ \mathrm{C} & -2.643903688 & -0.162056511 & -0.156236111 \\ \mathrm{C} & -3.458468847 & 0.897796263 & 0.281098420 \\ \mathrm{C} & -4.828087847 & 0.925267965 & 0.035463205 \\ \mathrm{C} & -5.366146289 & -0.186953316 & -0.649135148 \\ \mathrm{H} & -4.981174957 & -1.980411442 & -1.745283226 \\ \mathrm{H} & -2.518129479 & -1.932408038 & -1.307876996 \\ \mathrm{H} & -5.435744691 & 1.756032726 & 0.349328725 \\ \mathrm{H} & -6.423253860 & -0.225394318 & -0.831594961 \\ \mathrm{C} & -1.216906188 & 0.090414209 & 0.226675318 \\ \mathrm{O} & -0.908111065 & -0.072999705 & 1.540595508 \\ \mathrm{O} & -2.733253197 & 1.850131332 & 0.880772165 \\ \mathrm{H} & -1.371793898 & 0.595622141 & 2.032508344 \\ \mathrm{O} & -0.370301226 & -0.503921535 & -0.619761842 \\ \mathrm{C} & 1.041073273 & -0.316442423 & -0.612827742 \\ \mathrm{C} & 1.741375125 & -1.178744084 & 0.436961430 \\ \mathrm{C} & 1.442473404 & 1.146798982 & -0.515611536 \\ \mathrm{C} & 3.281751036 & -1.056323574 & 0.364294127 \\ \mathrm{H} & 1.404283402 & -0.878009764 & 1.421242104 \\ \mathrm{C} & 2.958750011 & 1.300195994 & -0.668628848 \\ \mathrm{H} & 0.923805568 & 1.711714825 & -1.285036891 \\ \mathrm{C} & 3.680287365 & 0.436055431 & 0.374873529 \\ \mathrm{H} & 3.460477651 & 0.843734562 & 1.360893196 \\ \mathrm{C} & 3.373948545 & 2.768072002 & -0.568266339 \\ \mathrm{H} & 3.117177022 & 3.178882730 & 0.404447827 \\ \mathrm{H} & 4.444439221 & 2.885587605 & -0.707407052 \\ \mathrm{H} & 2.872868105 & 3.366839144 & -1.323372095 \\ \mathrm{H} & 1.133177880 & 1.547927010 & 0.446210332 \\ & & & \\ & & & \end{array}$




$\begin{array}{rrrr}\mathrm{H} & 1.441234103 & -2.214885457 & 0.305552823 \\ \mathrm{H} & 4.756419541 & 0.522687340 & 0.240475017 \\ \mathrm{H} & 3.232123931 & 0.950754369 & -1.662571018 \\ \mathrm{C} & 3.888997381 & -1.738092226 & 1.599409617 \\ \mathrm{H} & 3.630554764 & -2.792854801 & 1.631787417 \\ \mathrm{H} & 4.973198658 & -1.663334620 & 1.588556213 \\ \mathrm{H} & 3.533552155 & -1.281072793 & 2.517918983 \\ \mathrm{C} & 3.839245676 & -1.764418027 & -0.883636564 \\ \mathrm{H} & 4.923554153 & -1.699268222 & -0.904636863 \\ \mathrm{H} & 3.573152558 & -2.817732905 & -0.873547361 \\ \mathrm{H} & 3.472119752 & -1.345485295 & -1.813263230 \\ \mathrm{H} & 1.310278995 & -0.679324949 & -1.595987015\end{array}$

LIIC path connecting $S_{1} / S_{0}-E N O L$ and SO-ENOL-MIN Coord_1

$\begin{array}{lrrr}\text { C } & -4.553591829 & -1.194345986 & -1.149229080 \\ \text { C } & -3.155119126 & -1.173478882 & -0.897882566 \\ \text { C } & -2.643903688 & -0.162056511 & -0.156236111 \\ \text { C } & -3.458468847 & 0.897796263 & 0.281098420 \\ \text { C } & -4.828087847 & 0.925267965 & 0.035463205 \\ \text { C } & -5.366146289 & -0.186953316 & -0.649135148 \\ \text { H } & -4.981174957 & -1.980411442 & -1.745283226 \\ \text { H } & -2.518129479 & -1.932408038 & -1.307876996 \\ \text { H } & -5.435744691 & 1.756032726 & 0.349328725 \\ \text { H } & -6.423253860 & -0.225394318 & -0.831594961 \\ \text { C } & -1.216906188 & 0.090414209 & 0.226675318 \\ \text { O } & -0.908111065 & -0.072999705 & 1.540595508 \\ \text { O } & -2.733253197 & 1.850131332 & 0.880772165 \\ \text { H } & -1.371793898 & 0.595622141 & 2.032508344 \\ \text { O } & -0.370301226 & -0.503921535 & -0.619761842 \\ \text { C } & 1.041073273 & -0.316442423 & -0.612827742 \\ \text { C } & 1.741375125 & -1.178744084 & 0.436961430 \\ \text { C } & 1.442473404 & 1.146798982 & -0.515611536 \\ \text { C } & 3.281751036 & -1.056323574 & 0.364294127 \\ \text { H } & 1.404283402 & -0.878009764 & 1.421242104 \\ \text { C } & 2.958750011 & 1.300195994 & -0.668628848 \\ \text { H } & 0.923805568 & 1.711714825 & -1.285036891 \\ \text { C } & 3.680287365 & 0.436055431 & 0.374873529 \\ \text { H } & 3.460477651 & 0.843734562 & 1.360893196 \\ \text { C } & 3.373948545 & 2.768072002 & -0.568266339 \\ \text { H } & 3.117177022 & 3.178882730 & 0.404447827 \\ \text { H } & 4.444439221 & 2.885587605 & -0.707407052 \\ \text { H } & 2.872868105 & 3.366839144 & -1.323372095 \\ \text { H } & 1.133177880 & 1.547927010 & 0.446210332 \\ \text { H } & 1.441234103 & -2.214885457 & 0.305552823 \\ \text { H } & 4.756419541 & 0.522687340 & 0.240475017 \\ \text { H } & 3.232123931 & 0.950754369 & -1.662571018 \\ & & & \\ & & & \\ & & 5110\end{array}$




$\begin{array}{llll}\mathrm{C} & 3.888997381 & -1.738092226 & 1.599409617 \\ \mathrm{H} & 3.630554764 & -2.792854801 & 1.631787417 \\ \mathrm{H} & 4.973198658 & -1.663334620 & 1.588556213 \\ \mathrm{H} & 3.533552155 & -1.281072793 & 2.517918983 \\ \mathrm{C} & 3.839245676 & -1.764418027 & -0.883636564 \\ \mathrm{H} & 4.923554153 & -1.699268222 & -0.904636863 \\ \mathrm{H} & 3.573152558 & -2.817732905 & -0.873547361 \\ \mathrm{H} & 3.472119752 & -1.345485295 & -1.813263230 \\ \mathrm{H} & 1.310278995 & -0.679324949 & -1.595987015\end{array}$

Coord_2

$\begin{array}{lrrr}\text { C } & -4.571198027 & -1.164411784 & -1.192814886 \\ \text { C } & -3.173422526 & -1.149428183 & -0.976347672 \\ \text { C } & -2.640017892 & -0.176528614 & -0.183726012 \\ \text { C } & -3.450790347 & 0.850418060 & 0.348519428 \\ \text { C } & -4.833265550 & 0.871985261 & 0.133837308 \\ \text { C } & -5.379518285 & -0.190328213 & -0.609564946 \\ \text { H } & -5.011119859 & -1.916351239 & -1.822794929 \\ \text { H } & -2.542476385 & -1.879305334 & -1.444375304 \\ \text { H } & -5.438044289 & 1.672908019 & 0.521395835 \\ \text { H } & -6.440644365 & -0.224233118 & -0.769929857 \\ \text { C } & -1.218544685 & 0.065633907 & 0.181660612 \\ \text { O } & -0.892836464 & -0.010148799 & 1.492460006 \\ \text { O } & -2.746739200 & 1.769960825 & 0.996574673 \\ \text { H } & -1.422451401 & 0.627506547 & 1.963093143 \\ \text { O } & -0.358345424 & -0.504259636 & -0.660957947 \\ \text { C } & 1.055477674 & -0.317266521 & -0.635075644 \\ \text { C } & 1.739064325 & -1.169552683 & 0.433168631 \\ \text { C } & 1.457027403 & 1.146905082 & -0.548974341 \\ \text { C } & 3.280407137 & -1.051432076 & 0.379360328 \\ \text { H } & 1.390657998 & -0.856736961 & 1.409642703 \\ \text { C } & 2.976167113 & 1.295000595 & -0.682201149 \\ \text { H } & 0.950880271 & 1.703351422 & -1.332774394 \\ \text { C } & 3.681345265 & 0.440250134 & 0.380182129 \\ \text { H } & 3.448699547 & 0.858489961 & 1.358759696 \\ \text { C } & 3.393665944 & 2.762858997 & -0.590057440 \\ \text { H } & 3.124652424 & 3.183803327 & 0.374958229 \\ \text { H } & 4.466336723 & 2.875966909 & -0.715262852 \\ \text { H } & 2.905152410 & 3.355801740 & -1.357839499 \\ \text { H } & 1.135150679 & 1.560245712 & 0.402997331 \\ \text { H } & 1.438303403 & -2.206371561 & 0.308871621 \\ \text { H } & 4.759355745 & 0.523536939 & 0.259893817 \\ \text { H } & 3.263155535 & 0.935244565 & -1.668550318 \\ \text { C } & 3.869082176 & -1.722170822 & 1.629347016 \\ \text { H } & 3.607054859 & -2.775785797 & 1.668956319 \\ \text { H } & 4.953483154 & -1.650677018 & 1.632386615 \\ \text { H } & 3.502684850 & -1.254758592 & 2.538256583 \\ & & & \\ & & 5111\end{array}$




$\begin{array}{llll}\text { C } & 3.852376675 & -1.773274126 & -0.854018159 \\ \text { H } & 4.936969553 & -1.710872825 & -0.861373564 \\ H & 3.583623457 & -2.825786501 & -0.837204759 \\ \text { H } & 3.498833752 & -1.362671099 & -1.792575331 \\ \text { H } & 1.336148995 & -0.690961752 & -1.610632117\end{array}$

Coord_3

$\begin{array}{lrrr}\text { C } & -4.583335432 & -1.133571683 & -1.234261588 \\ \text { C } & -3.187646329 & -1.118788979 & -1.049149275 \\ \text { C } & -2.634902092 & -0.185043616 & -0.207694715 \\ \text { C } & -3.443156648 & 0.801074256 & 0.414255429 \\ \text { C } & -4.837160147 & 0.811378157 & 0.226033818 \\ \text { C } & -5.389028590 & -0.199289914 & -0.572689843 \\ \text { H } & -5.033104163 & -1.851292135 & -1.896240138 \\ \text { H } & -2.561217684 & -1.815321931 & -1.571002710 \\ \text { H } & -5.439896293 & 1.576353613 & 0.682613547 \\ \text { H } & -6.453259866 & -0.232557716 & -0.713290450 \\ \text { C } & -1.219784288 & 0.047256401 & 0.140382908 \\ \text { O } & -0.878479065 & 0.058406304 & 1.442268306 \\ \text { O } & -2.762611199 & 1.685549524 & 1.110526279 \\ \text { H } & -1.467514104 & 0.658193149 & 1.896966339 \\ \text { O } & -0.348530128 & -0.499634738 & -0.699300749 \\ \text { C } & 1.067790676 & -0.315086723 & -0.655677446 \\ \text { C } & 1.734163323 & -1.158642186 & 0.429665330 \\ \text { C } & 1.471969908 & 1.149235182 & -0.580444343 \\ \text { C } & 3.276355936 & -1.046973277 & 0.393377529 \\ \text { H } & 1.375982701 & -0.833701060 & 1.398542802 \\ \text { C } & 2.993788414 & 1.289965294 & -0.695137850 \\ \text { H } & 0.978626172 & 1.698132020 & -1.377615700 \\ \text { C } & 3.682090966 & 0.443295433 & 0.384771430 \\ \text { H } & 3.438301050 & 0.871975562 & 1.356044197 \\ \text { C } & 3.415997245 & 2.757046499 & -0.610901346 \\ \text { H } & 3.136421326 & 3.188063728 & 0.346608923 \\ \text { H } & 4.490698425 & 2.864303408 & -0.723048251 \\ \text { H } & 2.940373511 & 3.345087038 & -1.390409602 \\ \text { H } & 1.139231081 & 1.574775813 & 0.361862124 \\ \text { H } & 1.430985601 & -2.195543256 & 0.312286423 \\ \text { H } & 4.761796940 & 0.521783637 & 0.277672019 \\ \text { H } & 3.292781439 & 0.919932768 & -1.674044221 \\ \text { C } & 3.846463077 & -1.707938325 & 1.657033220 \\ \text { H } & 3.579196260 & -2.759936399 & 1.703569321 \\ \text { H } & 4.930984453 & -1.641228119 & 1.673023120 \\ \text { H } & 3.470840249 & -1.230046390 & 2.556663182 \\ \text { C } & 3.860313280 & -1.782781726 & -0.826009162 \\ \text { H } & 4.945099155 & -1.724650124 & -0.820677061 \\ \text { H } & 3.587235356 & -2.834016001 & -0.802744556 \\ \text { H } & 3.520179852 & -1.379675998 & -1.772728025\end{array}$




$\begin{array}{lrrr}\text { H } & 1.358302296 & -0.699532550 & -1.623828216 \\ \text { Coord } 4 & & \\ \text { C } & -4.589680733 & -1.101900182 & -1.273991391 \\ \text { C } & -3.197348831 & -1.081842575 & -1.115673782 \\ \text { C } & -2.628739891 & -0.187553313 & -0.226901616 \\ \text { C } & -3.436544546 & 0.750185952 & 0.478767634 \\ \text { C } & -4.840775449 & 0.744056153 & 0.311385520 \\ \text { C } & -5.395065889 & -0.213607318 & -0.539571539 \\ \text { H } & -5.046362164 & -1.785515731 & -1.966489143 \\ \text { H } & -2.573399387 & -1.741009628 & -1.686749423 \\ \text { H } & -5.442889589 & 1.467345005 & 0.831733860 \\ \text { H } & -6.461545263 & -0.250060719 & -0.663528050 \\ \text { C } & -1.220760890 & 0.035195801 & 0.103825206 \\ \text { O } & -0.864584961 & 0.132482609 & 1.390922698 \\ \text { O } & -2.782363301 & 1.597259014 & 1.223099986 \\ \text { H } & -1.505748906 & 0.688840751 & 1.835900232 \\ \text { O } & -0.341036422 & -0.490179934 & -0.734043252 \\ \text { C } & 1.077883980 & -0.310056724 & -0.674252748 \\ \text { C } & 1.726909626 & -1.146048180 & 0.426777731 \\ \text { C } & 1.487147405 & 1.153643482 & -0.609823042 \\ \text { C } & 3.269849935 & -1.042997674 & 0.406279532 \\ \text { H } & 1.360760499 & -0.808835656 & 1.388384801 \\ \text { C } & 3.011455017 & 1.284915694 & -0.707637551 \\ \text { H } & 1.006613674 & 1.695824521 & -1.419318905 \\ \text { C } & 3.682727365 & 0.445133631 & 0.388381329 \\ \text { H } & 3.429743947 & 0.884242266 & 1.352571397 \\ \text { C } & 3.440764047 & 2.750457596 & -0.631192743 \\ \text { H } & 3.152558226 & 3.191592128 & 0.319096125 \\ \text { H } & 4.517331524 & 2.850398107 & -0.731439853 \\ \text { H } & 2.978098816 & 3.334429641 & -1.421437704 \\ \text { H } & 1.145518384 & 1.591478515 & 0.323112421 \\ \text { H } & 1.419519002 & -2.182442156 & 0.316258523 \\ \text { H } & 4.763938642 & 0.517349137 & 0.293270322 \\ \text { H } & 3.320582639 & 0.904532467 & -1.679354721 \\ \text { C } & 3.821793675 & -1.695307325 & 1.682367422 \\ \text { H } & 3.547681458 & -2.745204898 & 1.735664027 \\ \text { H } & 4.906377452 & -1.634907520 & 1.710090023 \\ \text { H } & 3.438906550 & -1.206751589 & 2.573146488 \\ \text { C } & 3.862996378 & -1.793122929 & -0.799834256 \\ \text { H } & 4.947904657 & -1.740789924 & -0.783045557 \\ \text { H } & 3.583969158 & -2.842593404 & -0.770254253 \\ \text { H } & 3.535805154 & -1.396785098 & -1.753934925 \\ \text { H } & 1.376351702 & -0.705294751 & -1.635289920\end{array}$

Coord_5

C $\quad-4.589665932 \quad-1.069424976 \quad-1.312473192$ 


$\begin{array}{lrrr}\text { C } & -3.201882329 & -1.038839076 & -1.175218785 \\ \text { C } & -2.621691187 & -0.184039116 & -0.239897415 \\ \text { C } & -3.432067548 & 0.698118451 & 0.542649837 \\ \text { C } & -4.845186347 & 0.670593148 & 0.389178227 \\ \text { C } & -5.397925287 & -0.233032018 & -0.511405037 \\ \text { H } & -5.049753466 & -1.719209023 & -2.034520747 \\ \text { H } & -2.577759088 & -1.656855321 & -1.790448430 \\ \text { H } & -5.448793694 & 1.346802799 & 0.967413970 \\ \text { H } & -6.465821465 & -0.276405819 & -0.622751043 \\ \text { C } & -1.221620089 & 0.029379402 & 0.073166507 \\ \text { O } & -0.850645861 & 0.211885216 & 1.339419495 \\ \text { O } & -2.807802100 & 1.505384811 & 1.334919298 \\ \text { H } & -1.535895509 & 0.720690554 & 1.781745830 \\ \text { O } & -0.336114825 & -0.476004334 & -0.764285356 \\ \text { C } & 1.085567380 & -0.302316819 & -0.690345250 \\ \text { C } & 1.717532923 & -1.131794981 & 0.424887531 \\ \text { C } & 1.502349307 & 1.159978385 & -0.636869248 \\ \text { C } & 3.261135332 & -1.039555376 & 0.417974730 \\ \text { H } & 1.345534498 & -0.782072358 & 1.379685497 \\ \text { C } & 3.028950316 & 1.279662694 & -0.719936450 \\ \text { H } & 1.034314674 & 1.696193622 & -1.457583706 \\ \text { C } & 3.683454364 & 0.445689330 & 0.390692425 \\ \text { H } & 3.423534644 & 0.895312262 & 1.348120799 \\ \text { C } & 3.467739651 & 2.742881699 & -0.651399646 \\ \text { H } & 3.173136426 & 3.194273030 & 0.292058321 \\ \text { H } & 4.545992626 & 2.834011705 & -0.741244656 \\ \text { H } & 3.017809018 & 3.323533040 & -1.451338806 \\ \text { H } & 1.154099284 & 1.610298815 & 0.287116520 \\ \text { H } & 1.404126501 & -2.167085358 & 0.321330221 \\ \text { H } & 4.765970942 & 0.510130535 & 0.306033120 \\ \text { H } & 3.346034440 & 0.888751666 & -1.684839923 \\ \text { C } & 3.795783375 & -1.684196121 & 1.705207125 \\ \text { H } & 3.513269051 & -2.731481999 & 1.765261430 \\ \text { H } & 4.880397050 & -1.631647317 & 1.743114023 \\ \text { H } & 3.407872247 & -1.184704186 & 2.587691485 \\ \text { C } & 3.860299776 & -1.804465928 & -0.775772154 \\ \text { H } & 4.945283055 & -1.759475125 & -0.749087655 \\ \text { H } & 3.573736860 & -2.851664005 & -0.739851353 \\ \text { H } & 3.545242856 & -1.414264600 & -1.736455523 \\ \text { H } & 1.389801001 & -0.708482149 & -1.644670819 \\ & & & \\ \text { Coord_6 } 6 & & \\ \text { C } & -4.582354729 & -1.036118475 & -1.350223395 \\ \text { C } & -3.200298930 & -0.989968070 & -1.226916989 \\ \text { C } & -2.613899190 & -0.174484011 & -0.244926615 \\ \text { C } & -3.431052946 & 0.645185946 & 0.606649243 \\ \text { C } & -4.851572247 & 0.591514541 & 0.458581834 \\ & & & \\ & & & \\ & & & \end{array}$




\begin{tabular}{|c|c|c|c|}
\hline C & 751590 & -0.257310120 & -0.4 \\
\hline$H_{-}$ & -5.041568364 & -1.652456721 & -2 \\
\hline $\mathrm{H}$ & -2.572584687 & -1.563238611 & -1.8806432 \\
\hline$H$ & -5.459641695 & 1.215562888 & 1.08809007 \\
\hline & -6.466204563 & -0.311253623 & -0.593462643 \\
\hline C & -1.222526490 & 0.029773004 & 0.049873 \\
\hline 0 & -0.836081458 & 0.296413821 & \\
\hline 0 & -2.841205705 & 1.410157402 & 1.446775 \\
\hline $\mathrm{H}$ & -1.556592711 & 0.755089552 & 1.7365123 \\
\hline 0 & -0.334118324 & -0.457173932 & -0.788897 \\
\hline C & 1.090557881 & -0.291984321 & -0.1033835 \\
\hline C & 1.706249324 & -1.115892279 & 0.4244600 \\
\hline$C$ & 1.517284107 & 1.168089487 & -0.66127804 \\
\hline $\mathrm{U}$ & 3.250444232 & -1.036703073 & 0.428335830 \\
\hline$H$ & 1.330908394 & -0.753333853 & 1.373070 \\
\hline$C$ & 3.045973419 & 1.273997989 & -0.7323266 \\
\hline $\mathrm{H}$ & 1.061059276 & 1.699011020 & -1.492022408 \\
\hline C & 3.684466363 & 0.444859834 & 0.391295926 \\
\hline $\mathrm{H}$ & 3.420249544 & 0.90 & 1.34 \\
\hline C & 3.496629850 & 2.734067998 & -0.672101049 \\
\hline $\mathrm{H}$ & 3.198236928 & 3.195943331 & 0.265042420 \\
\hline H & 4.576366628 & 2.81 & \\
\hline$\Pi$ & 3.05 & 3.3 & 7506 \\
\hline $\mathrm{H}$ & 1.165 & $1.6^{3}$ & 0.254329317 \\
\hline H & 1.385003099 & -2.149458956 & 0.32816622 \\
\hline $\mathrm{H}$ & 4.76807 & 0.499984234 & 0.315131423 \\
\hline $\mathrm{H}$ & 3.36 & 0.8 & 421 \\
\hline C & 3.769221370 & -1.67 & 1.725347923 \\
\hline $\mathrm{H}$ & 3.476794149 & -2.718660595 & 1.79235872 \\
\hline $\mathrm{H}$ & 4.853861351 & -1.6314 & 1.771476325 \\
\hline $\mathrm{H}$ & 3.37 & -1.1 & 2.600246586 \\
\hline C & 3.851987280 & -1.81 & 176054 \\
\hline $\mathrm{H}$ & 4.937023953 & -1.780901327 & -0.7195702 \\
\hline $\mathrm{H}$ & 3.556335257 & -2.861356706 & -0.71169005 \\
\hline $\mathrm{H}$ & 3.54 & -1.43 & -1.720608723 \\
\hline 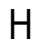 & & -0.709298252 & -1.6515292 \\
\hline \multicolumn{4}{|c|}{ Coord_7 } \\
\hline C & -4.56 & -1.0 & -1.38780 \\
\hline C & -3.19 & -0.93 & -1.269595589 \\
\hline$C$ & -2.605496987 & -0.158818814 & -0.2397459 \\
\hline C & -3.435170850 & 0.591664340 & 0.67170734 \\
\hline C & -4.861262350 & 0.507273835 & 0.51853113 \\
\hline C & -5.394422287 & -0.286208219 & -0.475881432 \\
\hline $\mathrm{H}$ & -5.019168063 & -1.585236515 & -2.1684524 \\
\hline $\mathrm{H}$ & -2.555457886 & -1.460360003 & -1.9553464 \\
\hline $\mathrm{H}$ & -5.477861993 & 1.074323480 & 1.1917 \\
\hline
\end{tabular}




$\begin{array}{lrrr}\text { H } & -6.462442764 & -0.354315628 & -0.578806344 \\ \mathrm{C} & -1.223679890 & 0.036429705 & 0.035862601 \\ \mathrm{O} & -0.820194959 & 0.385887030 & 1.240924692 \\ \mathrm{O} & -2.885602009 & 1.311754993 & 1.559663913 \\ \mathrm{H} & -1.566248111 & 0.793549556 & 1.702503320 \\ \mathrm{O} & -0.335562126 & -0.433674832 & -0.806384158 \\ \mathrm{C} & 1.092421379 & -0.279137019 & -0.712610753 \\ \mathrm{C} & 1.693245724 & -1.098325081 & 0.426091833 \\ \mathrm{C} & 1.531534009 & 1.177838085 & -0.682643350 \\ \mathrm{C} & 3.237975133 & -1.034514576 & 0.437178133 \\ \mathrm{H} & 1.317579795 & -0.722518650 & 1.369330197 \\ \mathrm{C} & 3.062086623 & 1.267683492 & -0.745186751 \\ \mathrm{H} & 1.085958077 & 1.704071124 & -1.522105007 \\ \mathrm{C} & 3.685942767 & 0.442497931 & 0.389640326 \\ \mathrm{H} & 3.420574548 & 0.913761165 & 1.334995394 \\ \mathrm{C} & 3.527036354 & 2.723708697 & -0.694051152 \\ \mathrm{H} & 3.227960031 & 3.196379733 & 0.237446118 \\ \mathrm{H} & 4.608023233 & 2.792536702 & -0.769409157 \\ \mathrm{H} & 3.100418524 & 3.299651636 & -1.509916807 \\ \mathrm{H} & 1.178437484 & 1.654056619 & 0.225339315 \\ \mathrm{H} & 1.362287398 & -2.129503952 & 0.337625922 \\ \mathrm{H} & 4.770401246 & 0.486692635 & 0.319461024 \\ \mathrm{H} & 3.386891046 & 0.854803961 & -1.698202623 \\ \mathrm{C} & 3.743007972 & -1.666303220 & 1.742481725 \\ \mathrm{H} & 3.439193946 & -2.706647996 & 1.816910330 \\ \mathrm{H} & 4.827702146 & -1.634223717 & 1.794322828 \\ \mathrm{H} & 3.353282840 & -1.143797981 & 2.610691090 \\ \mathrm{C} & 3.837628277 & -1.830837630 & -0.735509951 \\ \mathrm{H} & 4.922722652 & -1.805311231 & -0.695502749 \\ \mathrm{H} & 3.531354355 & -2.871799204 & -0.685975352 \\ \mathrm{H} & 3.542663953 & -1.451368805 & -1.706797223 \\ \mathrm{H} & 1.399967199 & -0.707904351 & -1.655263821\end{array}$

Coord_8

$\begin{array}{lrrr}\text { C } & -4.538621828 & -0.966571668 & -1.425829303 \\ \text { C } & -3.172253827 & -0.874765162 & -1.301493494 \\ \text { C } & -2.596619187 & -0.136811210 & -0.221261015 \\ \text { C } & -3.446683249 & 0.537823037 & 0.739047051 \\ \text { C } & -4.875816053 & 0.418203229 & 0.567496339 \\ \text { C } & -5.387302890 & -0.319573723 & -0.472615435 \\ \text { H } & -4.978259656 & -1.517423408 & -2.236981359 \\ \text { H } & -2.522742079 & -1.348100198 & -2.011559243 \\ \text { H } & -5.506527196 & 0.923541466 & 1.275533990 \\ \text { H } & -6.453573763 & -0.405477527 & -0.583052742 \\ \text { C } & -1.225345486 & 0.049592005 & 0.033824502 \\ \text { O } & -0.802064458 & 0.480204837 & 1.197437186 \\ \text { O } & -2.945314312 & 1.210337485 & 1.674868319 \\ & & & \\ & & & S 116\end{array}$




$\begin{array}{lrrr}\text { H } & -1.562789911 & 0.837882963 & 1.682589719 \\ \text { O } & -0.341242224 & -0.405337030 & -0.814621860 \\ \text { C } & 1.090455178 & -0.263778417 & -0.716948954 \\ \text { C } & 1.678642321 & -1.079030676 & 0.430601333 \\ \text { C } & 1.544467213 & 1.189117086 & -0.700383250 \\ \text { C } & 3.223844032 & -1.033102774 & 0.444215831 \\ \text { H } & 1.306398295 & -0.689465948 & 1.369532099 \\ \text { C } & 3.076612221 & 1.260427990 & -0.759041352 \\ \text { H } & 1.107752480 & 1.711220621 & -1.547049312 \\ \text { C } & 3.688023863 & 0.438375132 & 0.384923425 \\ \text { H } & 3.425377546 & 0.920940465 & 1.325275498 \\ \text { C } & 3.558386655 & 2.711398896 & -0.718304854 \\ \text { H } & 3.262450835 & 3.195269228 & 0.208404415 \\ \text { H } & 4.640333135 & 2.766532599 & -0.790914656 \\ \text { H } & 3.141329826 & 3.285832934 & -1.540111110 \\ \text { H } & 1.194327688 & 1.678931818 & 0.200958812 \\ \text { H } & 1.335997497 & -2.107082050 & 0.350900127 \\ \text { H } & 4.773041946 & 0.469897231 & 0.317440721 \\ \text { H } & 3.399943547 & 0.835910058 & -1.707384525 \\ \text { C } & 3.718206069 & -1.659519517 & 1.756110727 \\ \text { H } & 3.401547044 & -2.695384993 & 1.838785735 \\ \text { H } & 4.803013445 & -1.640235420 & 1.810383029 \\ \text { H } & 3.332831939 & -1.124765079 & 2.618768689 \\ \text { C } & 3.816422473 & -1.846290034 & -0.720424050 \\ \text { H } & 4.901600653 & -1.833064731 & -0.678307648 \\ \text { H } & 3.497986354 & -2.883146606 & -0.662994047 \\ \text { H } & 3.528188552 & -1.471563304 & -1.695557324 \\ \text { H } & 1.394317899 & -0.704394652 & -1.654991220\end{array}$

\section{Coord_9}

$\begin{array}{llll}\text { C } & -4.494810423 & -0.929921968 & -1.464939704 \\ \text { C } & -3.139629325 & -0.807806957 & -1.319603194 \\ \text { C } & -2.587422986 & -0.107787108 & -0.184677912 \\ \text { C } & -3.469010248 & 0.484009933 & 0.810382960 \\ \text { C } & -4.897195951 & 0.324383722 & 0.602962343 \\ \text { C } & -5.374661989 & -0.357500523 & -0.483343435 \\ \text { H } & -4.911204352 & -1.448817403 & -2.308658867 \\ \text { H } & -2.468395380 & -1.225675290 & -2.044156546 \\ \text { H } & -5.549916397 & 0.763165056 & 1.334683195 \\ \text { H } & -6.437111065 & -0.465123333 & -0.612719647 \\ \text { C } & -1.227921188 & 0.069945003 & 0.047964102 \\ \text { O } & -0.780263858 & 0.579522442 & 1.161602583 \\ \text { O } & -3.027219118 & 1.106142077 & 1.794170928 \\ \text { H } & -1.543105512 & 0.890533665 & 1.680860823 \\ \text { O } & -0.352511424 & -0.371645225 & -0.810251057 \\ \text { C } & 1.083413978 & -0.245749216 & -0.714689150 \\ \text { C } & 1.662396521 & -1.057838174 & 0.439230732\end{array}$




$\begin{array}{lrrr}\text { C } & 1.555038915 & 1.201900188 & -0.713569453 \\ \text { C } & 3.207962131 & -1.032665874 & 0.448953534 \\ \text { H } & 1.298491794 & -0.653863247 & 1.375264200 \\ \text { C } & 3.088395924 & 1.251827489 & -0.774698056 \\ \text { H } & 1.124478180 & 1.720427522 & -1.565568811 \\ \text { C } & 3.690750867 & 0.432093534 & 0.375844029 \\ \text { H } & 3.435874047 & 0.926432568 & 1.312161395 \\ \text { C } & 3.589779556 & 2.696535694 & -0.746501656 \\ \text { H } & 3.301969036 & 3.192134530 & 0.176532715 \\ \text { H } & 4.672280936 & 2.735968395 & -0.820845561 \\ \text { H } & 3.179840726 & 3.269993934 & -1.572515612 \\ \text { H } & 1.212801890 & 1.705910523 & 0.182433814 \\ \text { H } & 1.305866694 & -2.081885852 & 0.369818928 \\ \text { H } & 4.775927941 & 0.448933430 & 0.306547120 \\ \text { H } & 3.405280844 & 0.815058257 & -1.719598524 \\ \text { C } & 3.696141563 & -1.654348820 & 1.765342230 \\ \text { H } & 3.365136844 & -2.684884492 & 1.857662736 \\ \text { H } & 4.781141745 & -1.649861418 & 1.817547629 \\ \text { H } & 3.319947637 & -1.106684780 & 2.623925887 \\ \text { C } & 3.786779471 & -1.863725033 & -0.709932651 \\ \text { H } & 4.872072150 & -1.864809232 & -0.670215247 \\ \text { H } & 3.454564349 & -2.895647307 & -0.643194747 \\ \text { H } & 3.501763351 & -1.493386606 & -1.687676721 \\ \text { H } & 1.378624698 & -0.698734948 & -1.649264417\end{array}$

\begin{tabular}{lrrr}
\multicolumn{4}{l}{ Coord_10 } \\
C & -4.477764703 & -0.887441555 & -1.511838329 \\
C & -3.056732571 & -0.671325641 & -1.218123095 \\
C & -2.625965219 & -0.053649974 & -0.103547557 \\
C & -3.599868982 & 0.429260652 & 0.866504133 \\
C & -4.953140797 & 0.226319394 & 0.591946254 \\
C & -5.389692739 & -0.442623034 & -0.617111484 \\
H & -4.750521014 & -1.387981800 & -2.420742122 \\
H & -2.334498878 & -1.024966313 & -1.929797771 \\
H & -5.671428427 & 0.581390983 & 1.305589093 \\
H & -6.442056872 & -0.578019769 & -0.789151966 \\
C & -1.204989434 & 0.135950160 & 0.140182461 \\
O & -0.711573254 & 0.729335834 & 1.205688149 \\
O & -3.161706716 & 1.009262783 & 1.910229489 \\
H & -1.468207516 & 0.994835961 & 1.755692524 \\
O & -0.365302178 & -0.320817602 & -0.772805454 \\
C & 1.066071876 & -0.227930146 & -0.704216833 \\
C & 1.646456676 & -1.036193454 & 0.453391103 \\
C & 1.571240851 & 1.210166778 & -0.727801024 \\
C & 3.191531120 & -1.039337233 & 0.451938993 \\
H & 1.296392104 & -0.615250215 & 1.387197427 \\
C & 3.104774182 & 1.231233689 & -0.798303997
\end{tabular}




$\begin{array}{lrrr}\mathrm{H} & 1.145716031 & 1.723922166 & -1.585569082 \\ \mathrm{C} & 3.699677065 & 0.415396478 & 0.358765776 \\ \mathrm{H} & 3.458444499 & 0.925194065 & 1.290333014 \\ \mathrm{C} & 3.633845209 & 2.666471134 & -0.790307875 \\ \mathrm{H} & 3.360818732 & 3.178479677 & 0.128248408 \\ \mathrm{H} & 4.716588862 & 2.684694665 & -0.871171053 \\ \mathrm{H} & 3.230076544 & 3.238077635 & -1.620734148 \\ \mathrm{H} & 1.242260351 & 1.734199577 & 0.161201461 \\ \mathrm{H} & 1.271488821 & -2.054837656 & 0.398745619 \\ \mathrm{H} & 4.784752713 & 0.412399488 & 0.283530032 \\ \mathrm{H} & 3.409268465 & 0.777203986 & -1.739294083 \\ \mathrm{C} & 3.678832615 & -1.655296318 & 1.771331368 \\ \mathrm{H} & 3.329380761 & -2.678446103 & 1.877735413 \\ \mathrm{H} & 4.764169512 & -1.670555662 & 1.815877921 \\ \mathrm{H} & 3.318990398 & -1.091337290 & 2.626334638 \\ \mathrm{C} & 3.746720060 & -1.893072044 & -0.702046560 \\ \mathrm{H} & 4.832246307 & -1.912411668 & -0.671860549 \\ \mathrm{H} & 3.397622136 & -2.918460842 & -0.620898594 \\ \mathrm{H} & 3.459150771 & -1.528695942 & -1.681262389 \\ \mathrm{H} & 1.343265646 & -0.698589048 & -1.636607518\end{array}$

$\underline{\text { In acetonitrile solution }}$

\begin{tabular}{lrrr}
\multicolumn{5}{l}{ SO-KETO-MIN } \\
C & -4.515201326 & -0.400805029 & -1.855736134 \\
C & -3.181451230 & -0.375125026 & -1.481482107 \\
C & -2.778806198 & 0.220923013 & -0.272872021 \\
C & -3.754562271 & 0.783013055 & 0.566889039 \\
C & -5.085772365 & 0.765497056 & 0.179575014 \\
C & -5.467403392 & 0.188203016 & -1.019133071 \\
H & -4.809460348 & -0.854842062 & -2.784067199 \\
H & -2.434378173 & -0.806214059 & -2.117452151 \\
H & -5.806210417 & 1.216708086 & 0.835650062 \\
H & -6.503132467 & 0.201302015 & -1.303526096 \\
C & -1.344494099 & 0.280596019 & 0.100976010 \\
O & -0.954382070 & 0.733908052 & 1.147589081 \\
O & -3.481933248 & 1.359663097 & 1.742872125 \\
H & -2.548349183 & 1.317046093 & 1.923385137 \\
O & -0.544260039 & -0.199703016 & -0.830909057 \\
C & 0.890368064 & -0.207467015 & -0.722219051 \\
C & 1.380843099 & -1.138294082 & 0.382675025 \\
& & & \\
& & & $S 119$
\end{tabular}




$\begin{array}{lrrr}\text { C } & 1.486719105 & 1.192066085 & -0.615617046 \\ \text { C } & 2.921902209 & -1.247819088 & 0.414983032 \\ \text { H } & 1.035212074 & -0.764273053 & 1.338446095 \\ \text { C } & 3.020571217 & 1.118485078 & -0.643317046 \\ \text { H } & 1.122695080 & 1.800113128 & -1.439462105 \\ \text { C } & 3.527056256 & 0.172058014 & 0.455255034 \\ \text { H } & 3.293798237 & 0.619141043 & 1.420531101 \\ \text { C } & 3.638651260 & 2.510523181 & -0.498054038 \\ \text { H } & 3.369749243 & 2.960209212 & 0.453778032 \\ \text { H } & 4.722405340 & 2.464154178 & -0.548779038 \\ \text { H } & 3.298450240 & 3.175256231 & -1.286571093 \\ \text { H } & 1.166035083 & 1.664744121 & 0.304595022 \\ \text { H } & 0.940045065 & -2.121757151 & 0.242279020 \\ \text { H } & 4.611296332 & 0.101698009 & 0.404701028 \\ \text { H } & 3.325843238 & 0.726020052 & -1.611264114 \\ \text { C } & 3.330888239 & -2.002916144 & 1.687826124 \\ \text { H } & 2.912588208 & -3.005464218 & 1.699186120 \\ \text { H } & 4.411244318 & -2.094817150 & 1.755189128 \\ \text { H } & 2.985199216 & -1.488884106 & 2.579495186 \\ \text { C } & 3.451961247 & -2.038805146 & -0.794613060 \\ \text { H } & 4.532188329 & -2.137092152 & -0.738958054 \\ \text { H } & 3.031487218 & -3.040188220 & -0.811097058 \\ \text { H } & 3.220773232 & -1.573731111 & -1.745601124 \\ \text { H } & 1.166358082 & -0.621012045 & -1.681525120 \\ & & & \\ \text { S1-KETO-MIN } & & \\ \text { C } & -4.633063335 & -0.331801025 & -1.868795133 \\ \text { C } & -3.223365232 & -0.360891026 & -1.513388109 \\ \text { C } & -2.763893200 & 0.237763016 & -0.305642021 \\ \text { C } & -3.782943270 & 0.800950058 & 0.553650040 \\ \text { C } & -5.131606370 & 0.820686057 & 0.209667016 \\ \text { C } & -5.588237403 & 0.272216021 & -1.018426074 \\ \text { H } & -4.936955356 & -0.769492058 & -2.800696203 \\ \text { H } & -2.522336179 & -0.824436061 & -2.174906158 \\ \text { H } & -5.808636419 & 1.269934093 & 0.909475067 \\ \text { H } & -6.623034475 & 0.319895024 & -1.287803091 \\ \text { C } & -1.378402099 & 0.302581022 & 0.075209004 \\ \text { O } & -0.979869072 & 0.777618056 & 1.130651080 \\ \text { O } & -3.459293246 & 1.326144095 & 1.704652124 \\ \text { H } & -2.504746179 & 1.274509090 & 1.832740133 \\ \text { O } & -0.562139038 & -0.209438015 & -0.848354063 \\ \text { C } & 0.861324060 & -0.238336019 & -0.706716052 \\ \text { C } & 1.312616096 & -1.164443085 & 0.419964029 \\ \text { C } & 1.485229106 & 1.149940083 & -0.600791044 \\ \text { C } & 2.849608204 & -1.303258094 & 0.490878035 \\ \text { H } & 0.949123069 & -0.772306053 & 1.361601097 \\ \text { C } & 3.017343215 & 1.047402074 & -0.592781042 \\ & & & \\ & & & \\ & & & \end{array}$




$\begin{array}{llll}\mathrm{H} & 1.152350083 & 1.755727127 & -1.439699103 \\ \mathrm{C} & 3.481574249 & 0.104802009 & 0.527530037 \\ \mathrm{H} & 3.234666230 & 0.567817041 & 1.481867105 \\ \mathrm{C} & 3.659307266 & 2.428828173 & -0.449076033 \\ \mathrm{H} & 3.375396242 & 2.895420211 & 0.490266036 \\ \mathrm{H} & 4.743142341 & 2.361879170 & -0.472172032 \\ \mathrm{H} & 3.351188240 & 3.090232222 & -1.253602091 \\ \mathrm{H} & 1.149985084 & 1.637665116 & 0.305871022 \\ \mathrm{H} & 0.856870059 & -2.141453155 & 0.280313021 \\ \mathrm{H} & 4.565442330 & 0.013695001 & 0.502737035 \\ \mathrm{H} & 3.337449238 & 0.637660046 & -1.548986111 \\ \mathrm{C} & 3.214340231 & -2.049568146 & 1.782318130 \\ \mathrm{H} & 2.776546199 & -3.043829219 & 1.796079130 \\ \mathrm{H} & 4.290995308 & -2.161139157 & 1.877156133 \\ \mathrm{H} & 2.857092204 & -1.517691108 & 2.658865190 \\ \mathrm{C} & 3.392817244 & -2.120745153 & -0.695160049 \\ \mathrm{H} & 4.469507321 & -2.239466164 & -0.613111042 \\ \mathrm{H} & 2.952877213 & -3.113903225 & -0.707982050 \\ \mathrm{H} & 3.192196232 & -1.664410120 & -1.657204118 \\ \mathrm{H} & 1.158526084 & -0.667788048 & -1.653815119\end{array}$

\begin{tabular}{lrrr}
\multicolumn{4}{l}{ T1-KETO-MIN } \\
C & -4.476113321 & -0.481194033 & -1.949121138 \\
C & -3.209544228 & -0.603623042 & -1.484494105 \\
C & -2.786358198 & 0.138406008 & -0.260524019 \\
C & -3.866226281 & 0.785224058 & 0.562952040 \\
C & -5.094509367 & 0.878948064 & 0.076434007 \\
C & -5.451021393 & 0.316153021 & -1.236240087 \\
H & -4.771547343 & -0.991176071 & -2.848319207 \\
H & -2.479074177 & -1.206181089 & -1.984044143 \\
H & -5.852999423 & 1.349294096 & 0.674056047 \\
H & -6.414597462 & 0.515493038 & -1.658327117 \\
C & -1.381386098 & 0.319620021 & 0.057922006 \\
O & -1.003388074 & 0.863891061 & 1.070240078 \\
O & -3.574797257 & 1.239668092 & 1.790889129 \\
H & -2.632705191 & 1.276946094 & 1.916856138 \\
O & -0.568331042 & -0.183185013 & -0.861294061 \\
C & 0.862295063 & -0.197715014 & -0.729067054 \\
C & 1.320058096 & -1.103579081 & 0.410293029 \\
C & 1.476384104 & 1.195708083 & -0.647131048 \\
C & 2.858045205 & -1.234964087 & 0.475077035 \\
H & 0.962351072 & -0.697319049 & 1.349033095 \\
C & 3.009424215 & 1.100439081 & -0.644105049 \\
H & 1.136261084 & 1.787896129 & -1.492464107 \\
C & 3.483203253 & 0.176587014 & 0.488094034 \\
H & 3.239897232 & 0.652523047 & 1.436977105 \\
C & 3.643276264 & 2.487666179 & -0.522701040 \\
& & & S121
\end{tabular}




$\begin{array}{llll}\mathrm{H} & 3.361662240 & 2.965313211 & 0.411696031 \\ \mathrm{H} & 4.727130343 & 2.426479176 & -0.550645039 \\ \mathrm{H} & 3.327173240 & 3.136000227 & -1.334546096 \\ \mathrm{H} & 1.145795080 & 1.695632123 & 0.254701016 \\ \mathrm{H} & 0.867174061 & -2.084333149 & 0.290127020 \\ \mathrm{H} & 4.567027327 & 0.089726008 & 0.458472035 \\ \mathrm{H} & 3.327511241 & 0.678922049 & -1.595609114 \\ \mathrm{C} & 3.231870232 & -1.962628139 & 1.774339127 \\ \mathrm{H} & 2.798272200 & -2.958309212 & 1.803291128 \\ \mathrm{H} & 4.309255310 & -2.068659151 & 1.864546136 \\ \mathrm{H} & 2.877638209 & -1.420743100 & 2.645981190 \\ \mathrm{C} & 3.397049244 & -2.066275150 & -0.703109053 \\ \mathrm{H} & 4.474243319 & -2.180604159 & -0.624505043 \\ \mathrm{H} & 2.960265215 & -3.060807220 & -0.701092051 \\ \mathrm{H} & 3.191162230 & -1.622997116 & -1.670141120 \\ \mathrm{H} & 1.151761082 & -0.640378048 & -1.671388121\end{array}$

$\begin{array}{lrrr}\text { T2-KETO-MIN } & & \\ \text { C } & -4.588104562 & -0.338327029 & -1.843482029 \\ \text { C } & -3.211303108 & -0.373526164 & -1.530196609 \\ \text { C } & -2.727743467 & 0.214413863 & -0.306455192 \\ \text { C } & -3.736338889 & 0.778194399 & 0.561751585 \\ \text { C } & -5.123361973 & 0.796500191 & 0.214122070 \\ \text { C } & -5.557779987 & 0.249137554 & -1.002490632 \\ \text { H } & -4.902741173 & -0.777296163 & -2.773832303 \\ \text { H } & -2.519923978 & -0.830500152 & -2.207020274 \\ \text { H } & -5.796477397 & 1.247546551 & 0.916317371 \\ \text { H } & -6.589568565 & 0.277139878 & -1.286560477 \\ \text { C } & -1.343257766 & 0.282469413 & 0.059452365 \\ \text { O } & -0.949241774 & 0.755901765 & 1.112942211 \\ \text { O } & -3.421390963 & 1.313779111 & 1.713108260 \\ \text { H } & -2.464270205 & 1.261917481 & 1.837697889 \\ \text { O } & -0.531265292 & -0.213260183 & -0.860011070 \\ \text { C } & 0.898756582 & -0.219700101 & -0.729151807 \\ \text { C } & 1.370960862 & -1.141539553 & 0.391172146 \\ \text { C } & 1.496759195 & 1.178977901 & -0.621648349 \\ \text { C } & 2.910602930 & -1.254660720 & 0.449270188 \\ \text { H } & 1.006667624 & -0.755873475 & 1.334864967 \\ \text { C } & 3.030450078 & 1.103165788 & -0.625005909 \\ \text { H } & 1.147327852 & 1.782755950 & -1.455321622 \\ \text { C } & 3.518712358 & 0.163933592 & 0.488017774 \\ \text { H } & 3.270823584 & 0.618438042 & 1.446075620 \\ \text { C } & 3.648044003 & 2.495382870 & -0.479187081 \\ \text { H } & 3.362854630 & 2.951857811 & 0.464654191 \\ \text { H } & 4.732642412 & 2.448427215 & -0.511075884 \\ \text { H } & 3.321522500 & 3.154968327 & -1.277892459 \\ \text { H } & 1.157547768 & 1.654007755 & 0.289878513 \\ & & & \\ & & & 5122\end{array}$




$\begin{array}{lrrr}\text { H } & 0.930515789 & -2.125693034 & 0.252290926 \\ \mathrm{H} & 4.603690674 & 0.091283737 & 0.454809504 \\ \mathrm{H} & 3.351384978 & 0.703505301 & -1.585162583 \\ \mathrm{C} & 3.297296533 & -2.000973550 & 1.734189914 \\ \mathrm{H} & 2.876448587 & -3.002547366 & 1.745964510 \\ \mathrm{H} & 4.376303004 & -2.094772943 & 1.820590631 \\ \mathrm{H} & 2.937397286 & -1.479592008 & 2.615883297 \\ \mathrm{C} & 3.458469984 & -2.056822636 & -0.744995898 \\ \mathrm{H} & 4.537539946 & -2.157637305 & -0.671614413 \\ \mathrm{H} & 3.035372099 & -3.057238978 & -0.759764277 \\ \mathrm{H} & 3.243135024 & -1.599152130 & -1.703253387 \\ \mathrm{H} & 1.191034300 & -0.640843523 & -1.680974485 \\ & & & \\ \mathrm{~S} 0-\mathrm{ENOL}-\mathrm{MIN} & & \\ \mathrm{C} & -4.611562334 & -0.333859022 & -1.886868134 \\ \mathrm{C} & -3.282183236 & -0.356643023 & -1.592134113 \\ \mathrm{C} & -2.790555203 & 0.234685019 & -0.353389028 \\ \mathrm{C} & -3.723649266 & 0.836482061 & 0.592918040 \\ \mathrm{C} & -5.128749368 & 0.839068060 & 0.204941014 \\ \mathrm{C} & -5.537092400 & 0.290274020 & -0.973681069 \\ \mathrm{H} & -4.980988359 & -0.766878055 & -2.798165201 \\ \mathrm{H} & -2.575890187 & -0.808617058 & -2.260952162 \\ \mathrm{H} & -5.816977417 & 1.301218095 & 0.887116063 \\ \mathrm{H} & -6.579261472 & 0.325136022 & -1.234926087 \\ \mathrm{C} & -1.450121106 & 0.269661021 & -0.054218003 \\ \mathrm{O} & -0.995568072 & 0.780885054 & 1.044869077 \\ \mathrm{O} & -3.369633242 & 1.337184097 & 1.663357121 \\ \mathrm{H} & -1.758676126 & 1.109712081 & 1.561620112 \\ \mathrm{O} & -0.576987043 & -0.213360018 & -0.884903065 \\ \mathrm{C} & 0.859982061 & -0.228992014 & -0.708958049 \\ \mathrm{C} & 1.288269090 & -1.150774082 & 0.426993032 \\ \mathrm{C} & 1.468879106 & 1.163779084 & -0.603727041 \\ \mathrm{C} & 2.825561204 & -1.284398090 & 0.515862038 \\ \mathrm{H} & 0.914149067 & -0.756897052 & 1.363881100 \\ \mathrm{C} & 3.001741218 & 1.063296077 & -0.575537039 \\ \mathrm{H} & 1.144252084 & 1.763118129 & -1.449730102 \\ \mathrm{C} & 3.452777249 & 0.125919007 & 0.554801038 \\ \mathrm{H} & 3.194472229 & 0.592350043 & 1.504409109 \\ \mathrm{C} & 3.636087264 & 2.447786175 & -0.428619030 \\ \mathrm{H} & 3.339625241 & 2.916377212 & 0.505695035 \\ \mathrm{H} & 4.719917339 & 2.383543174 & -0.438433029 \\ \mathrm{H} & 3.335932240 & 3.105132223 & -1.239145090 \\ \mathrm{H} & 1.124887080 & 1.656453120 & 0.296690019 \\ \mathrm{H} & 0.836079061 & -2.128236154 & 0.283650023 \\ \mathrm{H} & 4.536533329 & 0.036725002 & 0.542798039 \\ \mathrm{H} & 3.334353239 & 0.651227044 & -1.526024110 \\ \mathrm{C} & 3.174392227 & -2.025724144 & 1.814356129 \\ & & & \\ & & & 5123\end{array}$




$\begin{array}{llll}\mathrm{H} & 2.739113195 & -3.020915220 & 1.825318131 \\ \mathrm{H} & 4.249745306 & -2.134081155 & 1.922580137 \\ \mathrm{H} & 2.805284201 & -1.492141108 & 2.684871193 \\ \mathrm{C} & 3.383601241 & -2.104576152 & -0.661274046 \\ \mathrm{H} & 4.458723323 & -2.222561163 & -0.563815038 \\ \mathrm{H} & 2.944370212 & -3.097761223 & -0.677889047 \\ \mathrm{H} & 3.197335231 & -1.650727121 & -1.627500118 \\ \mathrm{H} & 1.161593085 & -0.661679046 & -1.650685120\end{array}$

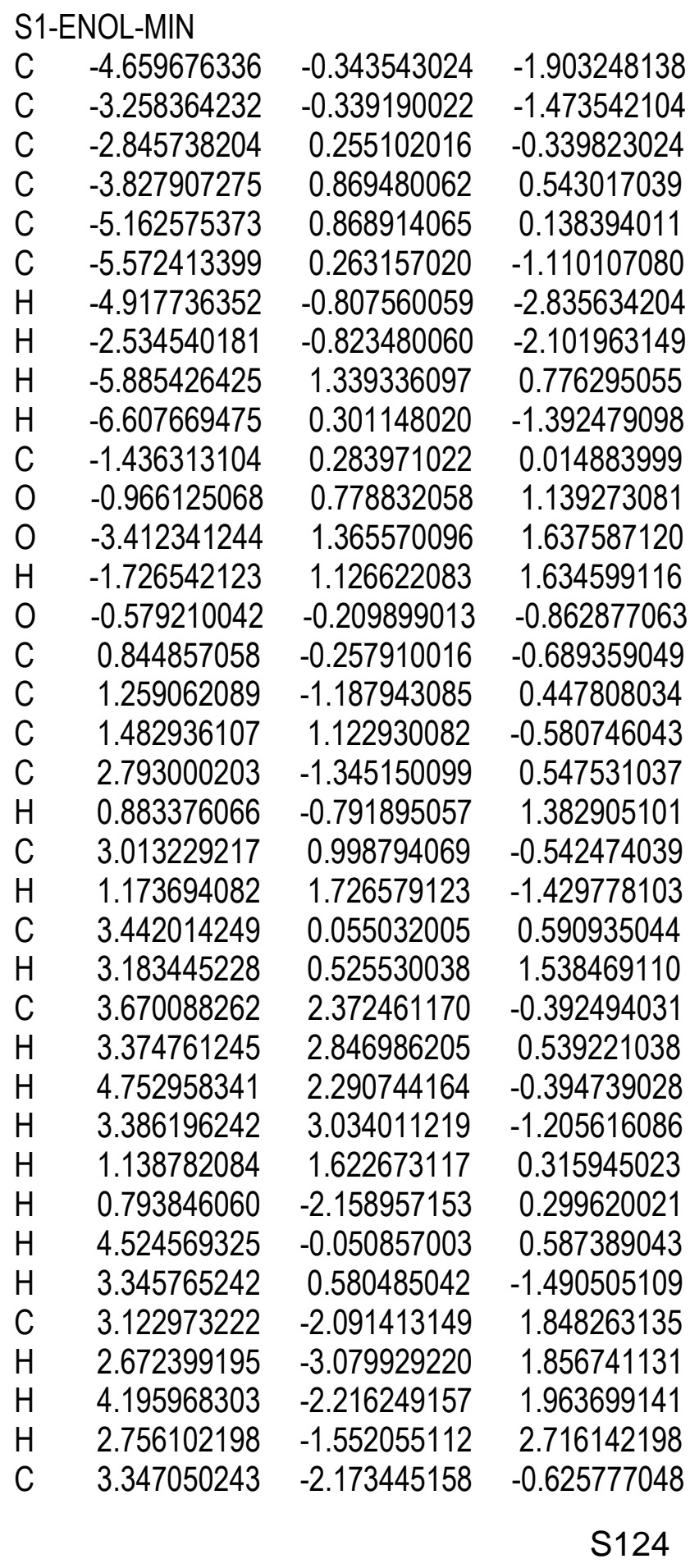




$\begin{array}{llll}\mathrm{H} & 4.420100316 & -2.306197166 & -0.522832038 \\ \mathrm{H} & 2.894325211 & -3.160668227 & -0.644434049 \\ \mathrm{H} & 3.171539226 & -1.717258126 & -1.592892117 \\ \mathrm{H} & 1.147894083 & -0.695794051 & -1.629625120\end{array}$

\section{T1-ENOL-MIN}

$\begin{array}{lrrr}\text { C } & -4.687374336 & -0.251747017 & -1.964008143 \\ \text { C } & -3.293757237 & -0.318752022 & -1.596716116 \\ \text { C } & -2.822382204 & 0.263618018 & -0.373316028 \\ \text { C } & -3.827933274 & 0.869704063 & 0.524756037 \\ \text { C } & -5.199384376 & 0.897453065 & 0.109031008 \\ \text { C } & -5.617393402 & 0.345116024 & -1.161034083 \\ \text { H } & -4.984335357 & -0.676317047 & -2.906313208 \\ \text { H } & -2.596071187 & -0.789496059 & -2.257895164 \\ \text { H } & -5.899634427 & 1.354577095 & 0.780558054 \\ \text { H } & -6.647684477 & 0.413547030 & -1.450334105 \\ \mathrm{C} & -1.501236109 & 0.284686020 & -0.039427002 \\ \text { O } & -1.017183074 & 0.791483056 & 1.079936077 \\ \text { O } & -3.518386253 & 1.363399099 & 1.622983114 \\ \text { H } & -1.745221127 & 1.128793081 & 1.605532113 \\ \text { O } & -0.616322042 & -0.225032017 & -0.869212060 \\ \mathrm{C} & 0.807086059 & -0.260574021 & -0.676633050 \\ \mathrm{C} & 1.215270089 & -1.178419085 & 0.471668033 \\ \mathrm{C} & 1.442306105 & 1.122353083 & -0.578958043 \\ \mathrm{C} & 2.748603200 & -1.333364094 & 0.581283041 \\ \mathrm{H} & 0.835019061 & -0.773070058 & 1.400969101 \\ \mathrm{C} & 2.972519214 & 0.999885074 & -0.531009038 \\ \mathrm{H} & 1.137018083 & 1.716959125 & -1.435685103 \\ \mathrm{C} & 3.395974246 & 0.067892007 & 0.614180044 \\ \mathrm{H} & 3.131542227 & 0.547641037 & 1.555576114 \\ \mathrm{C} & 3.627162261 & 2.375677171 & -0.390737030 \\ \mathrm{H} & 3.326689239 & 2.858663204 & 0.535067039 \\ \mathrm{H} & 4.710131339 & 2.295324167 & -0.386658026 \\ \mathrm{H} & 3.346631241 & 3.029206215 & -1.211456086 \\ \mathrm{H} & 1.094615078 & 1.631647116 & 0.310795022 \\ \mathrm{H} & 0.750983052 & -2.150799155 & 0.330801023 \\ \mathrm{H} & 4.478679322 & -0.036628003 & 0.617961047 \\ \mathrm{H} & 3.310892239 & 0.572576044 & -1.472837108 \\ \mathrm{C} & 3.071753220 & -2.066486149 & 1.891149136 \\ \mathrm{H} & 2.621528190 & -3.055048221 & 1.906860138 \\ \mathrm{H} & 4.144124300 & -2.189634156 & 2.014058145 \\ \mathrm{H} & 2.699771195 & -1.518770111 & 2.751703200 \\ \mathrm{C} & 3.310038237 & -2.172908154 & -0.580447042 \\ \mathrm{H} & 4.382531314 & -2.304112166 & -0.469622033 \\ \mathrm{H} & 2.857691206 & -3.160348228 & -0.592034044 \\ \mathrm{H} & 3.140012227 & -1.726577123 & -1.553032110 \\ \mathrm{H} & 1.120173079 & -0.707047052 & -1.609240116 \\ & & & \\ \end{array}$

S125 


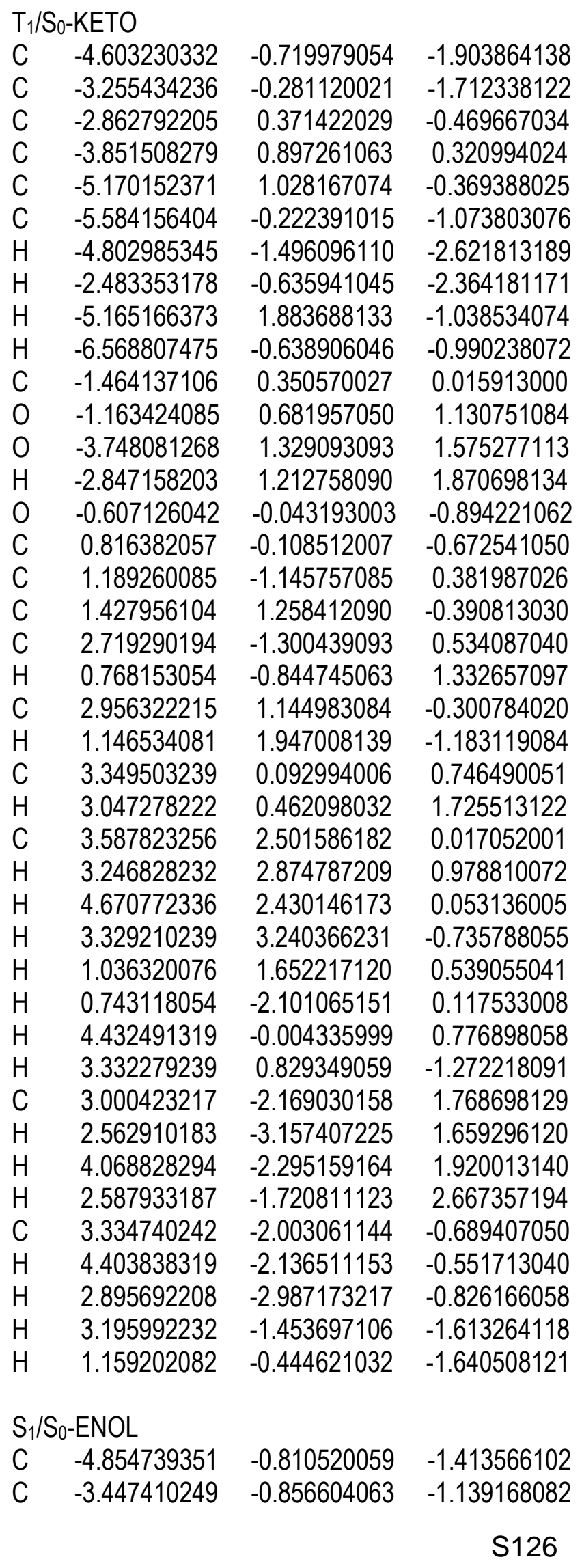




$\begin{array}{lrrr}\text { C } & -2.871991205 & 0.172662011 & -0.460299036 \\ \text { C } & -3.658184262 & 1.255013089 & -0.028111001 \\ \text { C } & -5.012425361 & 1.335946098 & -0.316447021 \\ \text { C } & -5.604595404 & 0.244707017 & -0.995236072 \\ \text { H } & -5.308365382 & -1.604915114 & -1.977851145 \\ \text { H } & -2.855641205 & -1.677337121 & -1.501133110 \\ \text { H } & -5.592391402 & 2.186500157 & -0.012157001 \\ \text { H } & -6.655520481 & 0.281089022 & -1.211321088 \\ \text { C } & -1.425882105 & 0.382213027 & -0.077965007 \\ \text { O } & -1.146903082 & 0.363529027 & 1.245618092 \\ \text { O } & -2.975686213 & 2.182043156 & 0.645294047 \\ \text { H } & -1.711336125 & 1.011798071 & 1.658396121 \\ \text { O } & -0.543849037 & -0.171280012 & -0.909802067 \\ \text { C } & 0.866638063 & -0.175305014 & -0.697884052 \\ \text { C } & 1.283068093 & -1.206431088 & 0.348879025 \\ \text { C } & 1.465544104 & 1.197037086 & -0.410640032 \\ \text { C } & 2.814995202 & -1.358801099 & 0.453853032 \\ \text { H } & 0.890851065 & -0.903863063 & 1.311220095 \\ \text { C } & 2.998481216 & 1.088845077 & -0.395679027 \\ \text { H } & 1.142098084 & 1.901607139 & -1.171803083 \\ \text { C } & 3.449870251 & 0.037984004 & 0.630348044 \\ \text { H } & 3.198064231 & 0.407900031 & 1.623528119 \\ \text { C } & 3.657057262 & 2.442120174 & -0.122374006 \\ \text { H } & 3.375307245 & 2.826961201 & 0.854072061 \\ \text { H } & 4.739793343 & 2.358653168 & -0.146129011 \\ \text { H } & 3.365394242 & 3.177682228 & -0.866305062 \\ \text { H } & 1.114346081 & 1.566526114 & 0.547567037 \\ \text { H } & 0.826721059 & -2.162686154 & 0.107047006 \\ \text { H } & 4.533650324 & -0.053767001 & 0.604584044 \\ \text { H } & 3.318729238 & 0.768631055 & -1.385473098 \\ \text { C } & 3.136076228 & -2.214639157 & 1.687850122 \\ \text { H } & 2.683692192 & -3.199169232 & 1.606689117 \\ \text { H } & 4.207866303 & -2.351115171 & 1.802628127 \\ \text { H } & 2.760275199 & -1.751875124 & 2.595418187 \\ \text { C } & 3.389529243 & -2.074517149 & -0.781424058 \\ \text { H } & 4.461835319 & -2.212822158 & -0.676913049 \\ \text { H } & 2.940155210 & -3.057058222 & -0.895126066 \\ \text { H } & 3.222109230 & -1.532085110 & -1.704514125 \\ \text { H } & 1.221133087 & -0.495696035 & -1.667901118 \\ & & & \end{array}$

\begin{tabular}{lrrr}
\multicolumn{1}{l}{$\mathrm{T}_{1} / \mathrm{S}_{0}$-ENOL } & & \\
$\mathrm{C}$ & -4.712976338 & -0.504409034 & -2.014659143 \\
$\mathrm{C}$ & -3.341945239 & -0.534929041 & -1.557043110 \\
$\mathrm{C}$ & -2.934262212 & 0.185111012 & -0.523456039 \\
$\mathrm{C}$ & -3.898448280 & 0.975761069 & 0.238681017 \\
$\mathrm{C}$ & -5.260825379 & 1.028362076 & -0.224292015 \\
$\mathrm{C}$ & -5.654672405 & 0.285693023 & -1.352816099 \\
& & & $\mathrm{~S} 127$
\end{tabular}




$\begin{array}{lrrr}\text { H } & -4.983413361 & -1.050931078 & -2.898876209 \\ \mathrm{H} & -2.629687187 & -1.125752079 & -2.101667154 \\ \mathrm{H} & -5.954358430 & 1.629772120 & 0.332043023 \\ \mathrm{H} & -6.667247477 & 0.335061027 & -1.704847120 \\ \mathrm{C} & -1.493656105 & 0.228757017 & -0.044331004 \\ \mathrm{O} & -1.327785094 & -0.227073016 & 1.230523089 \\ \mathrm{O} & -3.559260258 & 1.556060110 & 1.282488093 \\ \mathrm{H} & -1.839361130 & 0.334256026 & 1.803562130 \\ \mathrm{O} & -0.651423046 & -0.328637021 & -0.930014066 \\ \mathrm{C} & 0.762687056 & -0.216606017 & -0.774557057 \\ \mathrm{C} & 1.315606096 & -1.292331092 & 0.160055009 \\ \mathrm{C} & 1.216092086 & 1.178796087 & -0.359225027 \\ \mathrm{C} & 2.855673205 & -1.265442093 & 0.268015021 \\ \mathrm{H} & 0.888165062 & -1.145306083 & 1.143347085 \\ \mathrm{C} & 2.748614197 & 1.256461092 & -0.326323024 \\ \mathrm{H} & 0.813765059 & 1.912480138 & -1.052381076 \\ \mathrm{C} & 3.315136241 & 0.170788015 & 0.599828042 \\ \mathrm{H} & 3.007915219 & 0.404095030 & 1.618641114 \\ \mathrm{C} & 3.222724235 & 2.647078190 & 0.099863007 \\ \mathrm{H} & 2.882093210 & 2.885592209 & 1.103957078 \\ \mathrm{H} & 4.307001311 & 2.709988195 & 0.093789005 \\ \mathrm{H} & 2.842503205 & 3.413219244 & -0.569579043 \\ \mathrm{H} & 0.822994058 & 1.416177100 & 0.625302048 \\ \mathrm{H} & 0.981708068 & -2.266831163 & -0.186387012 \\ \mathrm{H} & 4.402349314 & 0.213080014 & 0.591328043 \\ \mathrm{H} & 3.117254224 & 1.081954078 & -1.335600095 \\ \mathrm{C} & 3.282876236 & -2.199538158 & 1.409996103 \\ \mathrm{H} & 2.966359215 & -3.221079234 & 1.218278086 \\ \mathrm{H} & 4.363248312 & -2.204087156 & 1.527585109 \\ \mathrm{H} & 2.846691204 & -1.889724135 & 2.354870170 \\ \mathrm{C} & 3.516085253 & -1.769546125 & -1.027692074 \\ \mathrm{H} & 4.597974332 & -1.773728128 & -0.927943067 \\ \mathrm{H} & 3.201857230 & -2.786681202 & -1.244335091 \\ \mathrm{H} & 3.273801238 & -1.163728082 & -1.893147134 \\ \mathrm{H} & 1.118461080 & -0.403437030 & -1.779500129\end{array}$

$\mathrm{S}_{0}$ state minimum-energy proton transfer path 1.9 angstrom
C -4.515201326
$-0.400805029$
$-1.855736134$
C -3.181451230
$-0.375125026$
$-1.481482107$
C $\quad-2.778806198$
$0.220923013 \quad-0.272872021$
C -3.754562271
0.783013055
0.566889039
C -5.085772365
0.765497056
0.179575014
C $\quad-5.467403392$
0.188203016
$-1.019133071$
H $\quad-4.809460348$
$-0.854842062$
$-2.784067199$
$\mathrm{H} \quad-2.434378173$
$-0.806214059$
$-2.117452151$
H $\quad-5.806210417$
1.216708086
0.835650062 


$\begin{array}{lrrr}\text { H } & -6.503132467 & 0.201302015 & -1.303526096 \\ \mathrm{C} & -1.344494099 & 0.280596019 & 0.100976010 \\ \mathrm{O} & -0.954382070 & 0.733908052 & 1.147589081 \\ \mathrm{O} & -3.481933248 & 1.359663097 & 1.742872125 \\ \mathrm{H} & -2.548349183 & 1.317046093 & 1.923385137 \\ \mathrm{O} & -0.544260039 & -0.199703016 & -0.830909057 \\ \mathrm{C} & 0.890368064 & -0.207467015 & -0.722219051 \\ \mathrm{C} & 1.380843099 & -1.138294082 & 0.382675025 \\ \mathrm{C} & 1.486719105 & 1.192066085 & -0.615617046 \\ \mathrm{C} & 2.921902209 & -1.247819088 & 0.414983032 \\ \mathrm{H} & 1.035212074 & -0.764273053 & 1.338446095 \\ \mathrm{C} & 3.020571217 & 1.118485078 & -0.643317046 \\ \mathrm{H} & 1.122695080 & 1.800113128 & -1.439462105 \\ \mathrm{C} & 3.527056256 & 0.172058014 & 0.455255034 \\ \mathrm{H} & 3.293798237 & 0.619141043 & 1.420531101 \\ \mathrm{C} & 3.638651260 & 2.510523181 & -0.498054038 \\ \mathrm{H} & 3.369749243 & 2.960209212 & 0.453778032 \\ \mathrm{H} & 4.722405340 & 2.464154178 & -0.548779038 \\ \mathrm{H} & 3.298450240 & 3.175256231 & -1.286571093 \\ \mathrm{H} & 1.166035083 & 1.664744121 & 0.304595022 \\ \mathrm{H} & 0.940045065 & -2.121757151 & 0.242279020 \\ \mathrm{H} & 4.611296332 & 0.101698009 & 0.404701028 \\ \mathrm{H} & 3.325843238 & 0.726020052 & -1.611264114 \\ \mathrm{C} & 3.330888239 & -2.002916144 & 1.687826124 \\ \mathrm{H} & 2.912588208 & -3.005464218 & 1.699186120 \\ \mathrm{H} & 4.411244318 & -2.094817150 & 1.755189128 \\ \mathrm{H} & 2.985199216 & -1.488884106 & 2.579495186 \\ \mathrm{C} & 3.451961247 & -2.038805146 & -0.794613060 \\ \mathrm{H} & 4.532188329 & -2.137092152 & -0.738958054 \\ \mathrm{H} & 3.031487218 & -3.040188220 & -0.811097058 \\ \mathrm{H} & 3.220773232 & -1.573731111 & -1.745601124 \\ \mathrm{H} & 1.166358082 & -0.621012045 & -1.681525120\end{array}$

1.8 angstrom

$\begin{array}{lrrr}\text { C } & -4.526413327 & -0.397568031 & -1.854213135 \\ \text { C } & -3.189618228 & -0.377145028 & -1.489494105 \\ \text { C } & -2.780650201 & 0.216727014 & -0.282851018 \\ \text { C } & -3.748136271 & 0.781612059 & 0.563549042 \\ \text { C } & -5.082009364 & 0.769768056 & 0.186824012 \\ \text { C } & -5.471607393 & 0.193960014 & -1.010680073 \\ \text { H } & -4.828647346 & -0.849657061 & -2.780943201 \\ \text { H } & -2.447561174 & -0.809987056 & -2.130315154 \\ \text { H } & -5.796944417 & 1.222882087 & 0.847550063 \\ \text { H } & -6.508907468 & 0.210184017 & -1.289166093 \\ \text { C } & -1.348078095 & 0.278043020 & 0.093672009 \\ \text { O } & -0.966147072 & 0.734085052 & 1.143040083 \\ \text { O } & -3.448989250 & 1.353036099 & 1.735216126 \\ & & & \\ & & & S 129\end{array}$




$\begin{array}{lrrr}\text { H } & -2.507105183 & 1.295002093 & 1.885219133 \\ \text { O } & -0.544263039 & -0.201084016 & -0.834474061 \\ \text { C } & 0.890016066 & -0.207969014 & -0.722269053 \\ \text { C } & 1.377784100 & -1.138704084 & 0.383802030 \\ \text { C } & 1.485088106 & 1.191719087 & -0.613891044 \\ \text { C } & 2.918898212 & -1.247744088 & 0.419567030 \\ \text { H } & 1.029666074 & -0.765031057 & 1.338847095 \\ \text { C } & 3.019049219 & 1.118620082 & -0.638167045 \\ \text { H } & 1.122894082 & 1.799827129 & -1.438490102 \\ \text { C } & 3.523508255 & 0.172241014 & 0.461362031 \\ \text { H } & 3.287981235 & 0.619043045 & 1.426213105 \\ \text { C } & 3.636190263 & 2.510883180 & -0.491650036 \\ \text { H } & 3.364986241 & 2.960468213 & 0.459575031 \\ \text { H } & 4.720058339 & 2.465003179 & -0.540004041 \\ \text { H } & 3.297399235 & 3.175449227 & -1.280906091 \\ \text { H } & 1.161901082 & 1.664054122 & 0.305635020 \\ \text { H } & 0.937665069 & -2.122309152 & 0.242283015 \\ \text { H } & 4.607861332 & 0.102340007 & 0.413066029 \\ \text { H } & 3.326316237 & 0.726196051 & -1.605503114 \\ \text { C } & 3.325142242 & -2.002997145 & 1.693177122 \\ \text { H } & 2.907031212 & -3.005633216 & 1.703334123 \\ \text { H } & 4.405356316 & -2.094703150 & 1.762836126 \\ \text { H } & 2.977426216 & -1.489256108 & 2.584219188 \\ \text { C } & 3.451512251 & -2.038782148 & -0.788915059 \\ \text { H } & 4.531600328 & -2.137091152 & -0.730982052 \\ \text { H } & 3.031046218 & -3.040156221 & -0.806056057 \\ \text { H } & 3.222267232 & -1.573964113 & -1.740501125 \\ \text { H } & 1.168647086 & -0.621331043 & -1.680835121\end{array}$

\begin{tabular}{lrrr}
\multicolumn{4}{l}{1.7 angstrom } \\
C & -4.553722329 & -0.389337028 & -1.849330131 \\
C & -3.210917232 & -0.379378029 & -1.505330110 \\
C & -2.786011200 & 0.207487013 & -0.301964022 \\
C & -3.734818271 & 0.776391058 & 0.562410041 \\
C & -5.074605365 & 0.774968058 & 0.206531017 \\
C & -5.482224393 & 0.204934013 & -0.988734071 \\
H & -4.874693350 & -0.835147058 & -2.772806201 \\
H & -2.479674179 & -0.814213060 & -2.157891157 \\
H & -5.778751415 & 1.229639087 & 0.877577061 \\
H & -6.523192470 & 0.227764016 & -1.253010089 \\
C & -1.356808096 & 0.269608019 & 0.073443008 \\
O & -0.988096069 & 0.730786055 & 1.127304082 \\
O & -3.395351246 & 1.335789096 & 1.724748122 \\
H & -2.443944174 & 1.257753089 & 1.829317134 \\
O & -0.544528041 & -0.206121016 & -0.845483059 \\
C & 0.890397063 & -0.209957016 & -0.724502054 \\
C & 1.371880101 & -1.139996080 & 0.384613026 \\
& & & \\
& & & $S 130$
\end{tabular}




$\begin{array}{lrrr}\text { C } & 1.481550107 & 1.190738088 & -0.610632042 \\ \text { C } & 2.912873212 & -1.246923091 & 0.430240032 \\ \text { H } & 1.016888074 & -0.766139053 & 1.337263098 \\ \text { C } & 3.015790217 & 1.120276079 & -0.625633043 \\ \text { H } & 1.123180082 & 1.799557132 & -1.436325106 \\ \text { C } & 3.515101253 & 0.173931010 & 0.476284036 \\ \text { H } & 3.272961237 & 0.619762043 & 1.439950102 \\ \text { C } & 3.629549263 & 2.513508180 & -0.474384035 \\ \text { H } & 3.351679243 & 2.961991211 & 0.475432034 \\ \text { H } & 4.713749339 & 2.469476177 & -0.516079038 \\ \text { H } & 3.294475235 & 3.177982229 & -1.265285091 \\ \text { H } & 1.151686082 & 1.660453118 & 0.308026023 \\ \text { H } & 0.933858068 & -2.124117155 & 0.240509017 \\ \text { H } & 4.599807333 & 0.105772007 & 0.434577031 \\ \text { H } & 3.329391239 & 0.729069055 & -1.591423115 \\ \text { C } & 3.311834238 & -2.002005144 & 1.706238121 \\ \text { H } & 2.894929208 & -3.005153215 & 1.713433122 \\ \text { H } & 4.391680318 & -2.092331150 & 1.782772127 \\ \text { H } & 2.957764212 & -1.488971109 & 2.595184185 \\ \text { C } & 3.453837249 & -2.037355147 & -0.774940054 \\ \text { H } & 4.533616324 & -2.134586154 & -0.710321051 \\ \text { H } & 3.034471217 & -3.039140216 & -0.794432055 \\ \text { H } & 3.229975233 & -1.572946114 & -1.728025126 \\ \text { H } & 1.174554085 & -0.622188046 & -1.681787121\end{array}$

1.6 angstrom

$\begin{array}{lrrr}\text { C } & -4.579049329 & -0.381738027 & -1.843050131 \\ \text { C } & -3.231114234 & -0.381181025 & -1.519527110 \\ \text { C } & -2.789690199 & 0.198173017 & -0.319294021 \\ \text { C } & -3.720404266 & 0.769400055 & 0.562884041 \\ \text { C } & -5.066032365 & 0.777053054 & 0.227588017 \\ \text { C } & -5.491692395 & 0.213864016 & -0.965119071 \\ \text { H } & -4.916912355 & -0.821143060 & -2.763513198 \\ \text { H } & -2.510477180 & -0.816890057 & -2.183734158 \\ \text { H } & -5.758832416 & 1.232074091 & 0.910013066 \\ \text { H } & -6.536172469 & 0.242823019 & -1.214635087 \\ \text { C } & -1.362668099 & 0.26116021 & 0.053747004 \\ \text { O } & -1.005288074 & 0.727959052 & 1.111104082 \\ \text { O } & -3.339923242 & 1.316075095 & 1.715522123 \\ \text { H } & -2.377810173 & 1.217707088 & 1.771660130 \\ \text { O } & -0.543851038 & -0.212994017 & -0.856489062 \\ \text { C } & 0.891702062 & -0.212138015 & -0.727027055 \\ \text { C } & 1.368362099 & -1.140539080 & 0.385228030 \\ \text { C } & 1.477518104 & 1.190288086 & -0.609297045 \\ \text { C } & 2.909388209 & -1.244289090 & 0.438420033 \\ \text { H } & 1.007614073 & -0.766263055 & 1.335638095 \\ \text { C } & 3.012058216 & 1.123263083 & -0.617238042 \\ & & & \\ & & & S 131\end{array}$




$\begin{array}{lrrr}\text { H } & 1.121520079 & 1.798800128 & -1.436183101 \\ \mathrm{C} & 3.508337251 & 0.177893013 & 0.486921033 \\ \mathrm{H} & 3.260820234 & 0.623248046 & 1.449420105 \\ \mathrm{C} & 3.622136263 & 2.517786181 & -0.463213035 \\ \mathrm{H} & 3.339078241 & 2.965729214 & 0.485307037 \\ \mathrm{H} & 4.706578337 & 2.475906178 & -0.500027033 \\ \mathrm{H} & 3.289321239 & 3.181511228 & -1.255676090 \\ \mathrm{H} & 1.141939082 & 1.658141121 & 0.308252024 \\ \mathrm{H} & 0.932928065 & -2.125536154 & 0.239677018 \\ \mathrm{H} & 4.593339332 & 0.112091008 & 0.450183035 \\ \mathrm{H} & 3.330806238 & 0.732777053 & -1.581638115 \\ \mathrm{C} & 3.303741240 & -1.998191141 & 1.716505123 \\ \mathrm{H} & 2.888924205 & -3.002198215 & 1.721983123 \\ \mathrm{H} & 4.383385316 & -2.086220152 & 1.798106128 \\ \mathrm{H} & 2.944446213 & -1.485658104 & 2.603627187 \\ \mathrm{C} & 3.457578247 & -2.034152149 & -0.763899057 \\ \mathrm{H} & 4.537183328 & -2.129364153 & -0.693938051 \\ \mathrm{H} & 3.040181219 & -3.036713219 & -0.785006059 \\ \mathrm{H} & 3.237616235 & -1.570614114 & -1.718324122 \\ \mathrm{H} & 1.181798083 & -0.623485043 & -1.682799120\end{array}$

1.5 angstrom

$\begin{array}{lrrr}\text { C } & -4.599284331 & -0.376328025 & -1.838080135 \\ \text { C } & -3.247051232 & -0.384636029 & -1.531724111 \\ \text { C } & -2.794755204 & 0.190130015 & -0.334324026 \\ \text { C } & -3.709777265 & 0.764619055 & 0.561876043 \\ \text { C } & -5.059801362 & 0.780796057 & 0.243480019 \\ \text { C } & -5.498706396 & 0.221806014 & -0.947257068 \\ \text { H } & -4.951877355 & -0.810887059 & -2.755361196 \\ \text { H } & -2.535934183 & -0.822429061 & -2.205095159 \\ \text { H } & -5.743961413 & 1.237057089 & 0.933551065 \\ \text { H } & -6.545591469 & 0.255804018 & -1.186203086 \\ \text { C } & -1.371166098 & 0.254620021 & 0.039562000 \\ \text { O } & -1.026367072 & 0.726993050 & 1.101185078 \\ \text { O } & -3.292891238 & 1.299740092 & 1.703512123 \\ \text { H } & -2.318335164 & 1.180098085 & 1.713983122 \\ \text { O } & -0.545082042 & -0.217151017 & -0.861906065 \\ \text { C } & 0.890453066 & -0.213687017 & -0.726965051 \\ \text { C } & 1.363326099 & -1.141075083 & 0.387462026 \\ \text { C } & 1.472783105 & 1.189797084 & -0.606770043 \\ \text { C } & 2.904353209 & -1.242603089 & 0.446663032 \\ \text { H } & 0.998288070 & -0.767127054 & 1.336507094 \\ \text { C } & 3.007540217 & 1.125084082 & -0.608775043 \\ \text { H } & 1.119048080 & 1.797808127 & -1.434973105 \\ \text { C } & 3.501089253 & 0.180445013 & 0.497252033 \\ \text { H } & 3.249856232 & 0.625546046 & 1.458887107 \\ \text { C } & 3.614978262 & 2.520522181 & -0.452733034 \\ & & & \\ & & & \text { S132 }\end{array}$




$\begin{array}{lrrr}\mathrm{H} & 3.327819238 & 2.968201212 & 0.494661033 \\ \mathrm{H} & 4.699582338 & 2.480058177 & -0.485521036 \\ \mathrm{H} & 3.284223235 & 3.183616231 & -1.246565092 \\ \mathrm{H} & 1.132960081 & 1.657077121 & 0.309614020 \\ \mathrm{H} & 0.929845068 & -2.126675155 & 0.240345020 \\ \mathrm{H} & 4.586256332 & 0.116025006 & 0.464117034 \\ \mathrm{H} & 3.330202240 & 0.735010054 & -1.572012111 \\ \mathrm{C} & 3.294712237 & -1.996048143 & 1.726213123 \\ \mathrm{H} & 2.881262206 & -3.000612218 & 1.730030126 \\ \mathrm{H} & 4.374129313 & -2.082615148 & 1.811946129 \\ \mathrm{H} & 2.931341212 & -1.484016104 & 2.611953188 \\ \mathrm{C} & 3.458138248 & -2.031735147 & -0.753585053 \\ \mathrm{H} & 4.537565325 & -2.125431155 & -0.679375051 \\ \mathrm{H} & 3.042230220 & -3.034869216 & -0.776361054 \\ \mathrm{H} & 3.241359233 & -1.568419114 & -1.708852124 \\ \mathrm{H} & 1.184677087 & -0.624561046 & -1.681456121\end{array}$

1.4 angstrom

$\begin{array}{lrrr}\text { C } & -4.632426332 & -0.343472024 & -1.846167133 \\ \text { C } & -3.275335238 & -0.362586028 & -1.565504114 \\ \text { C } & -2.801413201 & 0.202751014 & -0.371641029 \\ \text { C } & -3.693180266 & 0.780290057 & 0.546446038 \\ \text { C } & -5.050998366 & 0.804903060 & 0.252845017 \\ \text { C } & -5.512145397 & 0.254393019 & -0.933270067 \\ \text { H } & -5.005765358 & -0.770392056 & -2.758785200 \\ \text { H } & -2.578181186 & -0.801910060 & -2.252860164 \\ \text { H } & -5.721091410 & 1.260163089 & 0.957253068 \\ \text { H } & -6.563188470 & 0.293601021 & -1.152806080 \\ \text { C } & -1.380064097 & 0.258611017 & -0.004228999 \\ \text { O } & -1.041461075 & 0.739760054 & 1.059183078 \\ \text { O } & -3.236066232 & 1.301396094 & 1.672961122 \\ \text { H } & -2.246175163 & 1.161463082 & 1.634365119 \\ \text { O } & -0.548549042 & -0.221411015 & -0.891648064 \\ \text { C } & 0.887257063 & -0.221581014 & -0.735222053 \\ \text { C } & 1.338473099 & -1.147315083 & 0.389131030 \\ \text { C } & 1.471607104 & 1.180183087 & -0.607832044 \\ \text { C } & 2.878005210 & -1.254815092 & 0.472195035 \\ \text { H } & 0.961362071 & -0.767325056 & 1.330891095 \\ \text { C } & 3.006083218 & 1.110264082 & -0.586968040 \\ \text { H } & 1.131591084 & 1.789820128 & -1.440497102 \\ \text { C } & 3.479679250 & 0.166027012 & 0.528213040 \\ \text { H } & 3.216387231 & 0.614390042 & 1.485085106 \\ \text { C } & 3.614182258 & 2.504541181 & -0.422694028 \\ \text { H } & 3.313089240 & 2.953622215 & 0.519676036 \\ \text { H } & 4.699044340 & 2.461641179 & -0.438311033 \\ \text { H } & 3.297467235 & 3.167713227 & -1.222141090 \\ \text { H } & 1.121203081 & 1.648215116 & 0.303851020 \\ & & & \\ & & & 5133\end{array}$




$\begin{array}{lrrr}\mathrm{H} & 0.902811064 & -2.131541152 & 0.239874015 \\ \mathrm{H} & 4.564913327 & 0.097197007 & 0.511002037 \\ \mathrm{H} & 3.342751239 & 0.718041054 & -1.544491110 \\ \mathrm{C} & 3.244565232 & -2.006597143 & 1.759779127 \\ \mathrm{H} & 2.826977203 & -3.009437217 & 1.759341127 \\ \mathrm{H} & 4.322074311 & -2.097328149 & 1.863131132 \\ \mathrm{H} & 2.869090208 & -1.490890106 & 2.638286191 \\ \mathrm{C} & 3.447165250 & -2.049003148 & -0.717285050 \\ \mathrm{H} & 4.524837327 & -2.147111155 & -0.625789044 \\ \mathrm{H} & 3.027421216 & -3.050407220 & -0.744606053 \\ \mathrm{H} & 3.247538234 & -1.586714115 & -1.676804118 \\ \mathrm{H} & 1.192341087 & -0.635794045 & -1.684686123\end{array}$

1.3 angstrom

$\begin{array}{lrrr}\text { C } & -4.651433336 & -0.336272022 & -1.845008134 \\ \text { C } & -3.291938237 & -0.363667026 & -1.577841115 \\ \text { C } & -2.807867200 & 0.197981015 & -0.385392027 \\ \text { C } & -3.684032263 & 0.779498054 & 0.547127042 \\ \text { C } & -5.047785366 & 0.811116061 & 0.263503017 \\ \text { C } & -5.519529399 & 0.265027021 & -0.920687068 \\ \text { H } & -5.037423361 & -0.758415055 & -2.754541199 \\ \text { H } & -2.603085188 & -0.805059056 & -2.272476161 \\ \text { H } & -5.709917409 & 1.267976090 & 0.974122069 \\ \text { H } & -6.572224473 & 0.309718024 & -1.131750081 \\ \text { C } & -1.392053100 & 0.253792017 & -0.020115001 \\ \text { O } & -1.062745076 & 0.739412052 & 1.048834075 \\ \text { O } & -3.200677229 & 1.288097092 & 1.659757118 \\ \text { H } & -2.184472159 & 1.126245081 & 1.579959115 \\ \text { O } & -0.551714040 & -0.221769014 & -0.894783064 \\ \text { C } & 0.885073064 & -0.222302018 & -0.733505052 \\ \mathrm{C} & 1.330755096 & -1.148959084 & 0.391940030 \\ \text { C } & 1.468335106 & 1.179491087 & -0.604410045 \\ \text { C } & 2.870053205 & -1.256705091 & 0.480401035 \\ \text { H } & 0.949812068 & -0.769775057 & 1.332757096 \\ \mathrm{C} & 3.002725217 & 1.108708079 & -0.577716043 \\ \mathrm{H} & 1.131917084 & 1.788378130 & -1.439012104 \\ \mathrm{C} & 3.471949251 & 0.163932010 & 0.538861039 \\ \mathrm{H} & 3.205957232 & 0.612153046 & 1.495031109 \\ \mathrm{C} & 3.611270259 & 2.502517179 & -0.411741027 \\ \mathrm{H} & 3.307364236 & 2.952309215 & 0.529359037 \\ \mathrm{H} & 4.696092340 & 2.458436174 & -0.423640033 \\ \mathrm{H} & 3.297994237 & 3.165525228 & -1.212646089 \\ \mathrm{H} & 1.114041079 & 1.648116118 & 0.305706020 \\ \mathrm{H} & 0.895509063 & -2.132874154 & 0.239869019 \\ \mathrm{H} & 4.557131326 & 0.094525006 & 0.524921039 \\ \mathrm{H} & 3.342035241 & 0.716365054 & -1.534228109 \\ \mathrm{C} & 3.231918234 & -2.009041143 & 1.768915129\end{array}$

S134 


$\begin{array}{llll}\mathrm{H} & 2.814197202 & -3.011794215 & 1.766619129 \\ \mathrm{H} & 4.309038310 & -2.099941152 & 1.875651137 \\ \mathrm{H} & 2.853706205 & -1.493648109 & 2.646422190 \\ \mathrm{C} & 3.442750250 & -2.050953145 & -0.707478051 \\ \mathrm{H} & 4.519987327 & -2.149730154 & -0.612098043 \\ \mathrm{H} & 3.022466217 & -3.052054221 & -0.736529052 \\ \mathrm{H} & 3.247109235 & -1.588378113 & -1.667709122 \\ \mathrm{H} & 1.191906088 & -0.636346046 & -1.682271122\end{array}$

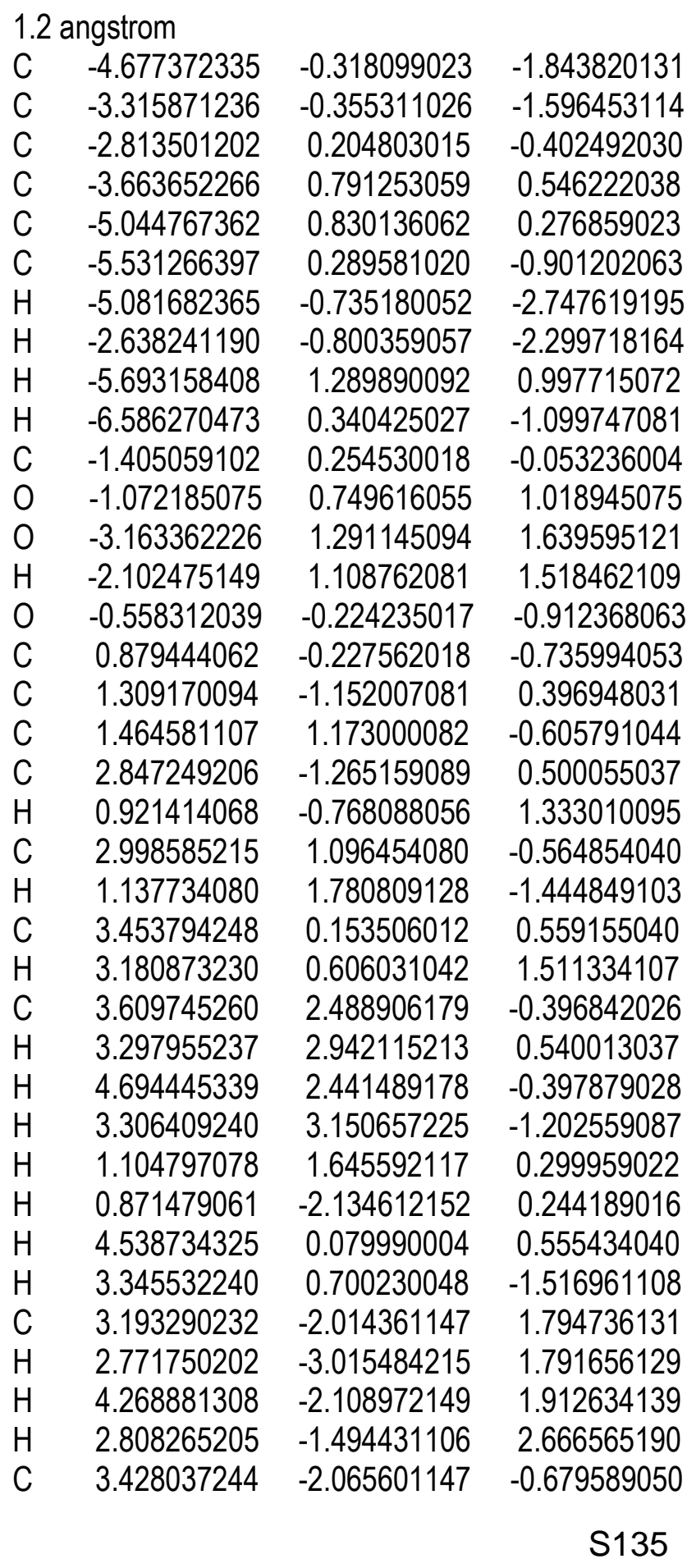




$\begin{array}{llll}H & 4.503778326 & -2.168673154 & -0.573119043 \\ H & 3.003793214 & -3.064955218 & -0.709892052 \\ H & 3.244179234 & -1.605054118 & -1.643136121 \\ H & 1.192798085 & -0.645740048 & -1.680339123\end{array}$

\section{1 angstrom}

$\begin{array}{lrrr}\text { C } & -4.666390336 & -0.319842021 & -1.854589132 \\ \text { C } & -3.323855240 & -0.359041027 & -1.606729118 \\ \text { C } & -2.800088199 & 0.210557013 & -0.390025027 \\ \text { C } & -3.669290263 & 0.809255056 & 0.579279042 \\ \text { C } & -5.079112368 & 0.836202058 & 0.266409021 \\ \text { C } & -5.541046397 & 0.300365023 & -0.903586065 \\ \text { H } & -5.073699366 & -0.737697052 & -2.756663200 \\ \text { H } & -2.643259192 & -0.808337060 & -2.304435165 \\ \text { H } & -5.734149414 & 1.298180093 & 0.979999069 \\ \text { H } & -6.593053472 & 0.348872024 & -1.119434078 \\ \mathrm{C} & -1.428836104 & 0.250667019 & -0.072378007 \\ \text { O } & -1.046377074 & 0.755386055 & 1.017705076 \\ \text { O } & -3.226611232 & 1.300918093 & 1.646921119 \\ \text { H } & -1.966150142 & 1.095691080 & 1.515918111 \\ \text { O } & -0.568957043 & -0.225381014 & -0.912925064 \\ \mathrm{C} & 0.870115064 & -0.230645016 & -0.729974054 \\ \mathrm{C} & 1.293863092 & -1.154325084 & 0.405503028 \\ \mathrm{C} & 1.459752105 & 1.168201085 & -0.604613043 \\ \mathrm{C} & 2.831322204 & -1.271830093 & 0.512211038 \\ \mathrm{H} & 0.903335066 & -0.767075057 & 1.338460097 \\ \mathrm{C} & 2.993295215 & 1.085945080 & -0.559420041 \\ \mathrm{H} & 1.137704081 & 1.772676129 & -1.448020103 \\ \mathrm{C} & 3.442365247 & 0.145037012 & 0.568776043 \\ \mathrm{H} & 3.168378228 & 0.601273045 & 1.518831110 \\ \mathrm{C} & 3.608719259 & 2.476869180 & -0.394068026 \\ \mathrm{H} & 3.295502236 & 2.934168209 & 0.540308037 \\ \mathrm{H} & 4.693293336 & 2.425858176 & -0.391696026 \\ \mathrm{H} & 3.310001237 & 3.136978226 & -1.202883087 \\ \mathrm{H} & 1.097424078 & 1.645069120 & 0.297121024 \\ \mathrm{H} & 0.853995061 & -2.135999152 & 0.252516017 \\ \mathrm{H} & 4.527107328 & 0.067851006 & 0.567933042 \\ \mathrm{H} & 3.341952240 & 0.685560048 & -1.509213107 \\ \mathrm{C} & 3.171973226 & -2.018569143 & 1.809740132 \\ \mathrm{H} & 2.747593197 & -3.018519216 & 1.808268130 \\ \mathrm{H} & 4.247032304 & -2.115930153 & 1.930603141 \\ \mathrm{H} & 2.786100203 & -1.495210108 & 2.679119195 \\ \mathrm{C} & 3.412514248 & -2.077244147 & -0.663878048 \\ \mathrm{H} & 4.487670324 & -2.183766157 & -0.554499042 \\ \mathrm{H} & 2.984885213 & -3.075224220 & -0.692797050 \\ \mathrm{H} & 3.232595232 & -1.618489119 & -1.629053116 \\ \mathrm{H} & 1.181687082 & -0.653112048 & -1.673043118 \\ & & & \\ & & 5136\end{array}$




\begin{tabular}{lrrr}
\multicolumn{5}{l}{1.0} & angstrom & & \\
$\mathrm{C}$ & -4.610451331 & -0.333190025 & -1.884683135 \\
$\mathrm{C}$ & -3.280776238 & -0.355678026 & -1.590423113 \\
$\mathrm{C}$ & -2.789202203 & 0.234047017 & -0.351665027 \\
$\mathrm{C}$ & -3.720886267 & 0.834376057 & 0.595443041 \\
$\mathrm{C}$ & -5.125902368 & 0.836931063 & 0.208878013 \\
$\mathrm{C}$ & -5.534945396 & 0.289287020 & -0.970099068 \\
$\mathrm{H}$ & -4.980404359 & -0.765237055 & -2.796171203 \\
$\mathrm{H}$ & -2.574416185 & -0.806235057 & -2.260086164 \\
$\mathrm{H}$ & -5.813629418 & 1.298289093 & 0.891996062 \\
$\mathrm{H}$ & -6.577430471 & 0.324215026 & -1.229951090 \\
$\mathrm{C}$ & -1.447438103 & 0.268564021 & -0.052413006 \\
$\mathrm{O}$ & -0.993270073 & 0.777511057 & 1.046318076 \\
$\mathrm{O}$ & -3.365788240 & 1.334659096 & 1.666753121 \\
$\mathrm{H}$ & -1.776922125 & 1.111518079 & 1.570087112 \\
$\mathrm{O}$ & -0.576353041 & -0.213518014 & -0.885288063 \\
$\mathrm{C}$ & 0.860655063 & -0.226751017 & -0.710777053 \\
$\mathrm{C}$ & 1.291836094 & -1.150975085 & 0.422043029 \\
$\mathrm{C}$ & 1.467019106 & 1.166913083 & -0.602174044 \\
$\mathrm{C}$ & 2.829509205 & -1.281756093 & 0.509114039 \\
$\mathrm{H}$ & 0.917852065 & -0.760659053 & 1.360488100 \\
$\mathrm{C}$ & 3.000086216 & 1.069431077 & -0.575595042 \\
$\mathrm{H}$ & 1.140483082 & 1.767978125 & -1.446241104 \\
$\mathrm{C}$ & 3.454033251 & 0.129677007 & 0.551657037 \\
$\mathrm{H}$ & 3.195963228 & 0.592897043 & 1.502902107 \\
$\mathrm{C}$ & 3.631423260 & 2.454912177 & -0.424913032 \\
$\mathrm{H}$ & 3.334400240 & 2.919993212 & 0.510983034 \\
$\mathrm{H}$ & 4.715395340 & 2.393240172 & -0.435562031 \\
$\mathrm{H}$ & 3.329231242 & 3.114011225 & -1.233259088 \\
$\mathrm{H}$ & 1.123057080 & 1.656530121 & 0.299870020 \\
$\mathrm{H}$ & 0.841555061 & -2.128960153 & 0.276238022 \\
$\mathrm{H}$ & 4.537939327 & 0.042509004 & 0.538177041 \\
$\mathrm{H}$ & 3.332908241 & 0.660970049 & -1.527558110 \\
$\mathrm{C}$ & 3.180707228 & -2.026401147 & 1.805073127 \\
$\mathrm{H}$ & 2.747429199 & -3.022499217 & 1.813048130 \\
$\mathrm{H}$ & 4.256326308 & -2.132953155 & 1.912409139 \\
$\mathrm{H}$ & 2.810920203 & -1.496359106 & 2.677462193 \\
$\mathrm{C}$ & 3.388476244 & -2.097080150 & -0.670941051 \\
$\mathrm{H}$ & 4.463913319 & -2.213063161 & -0.574563041 \\
$\mathrm{H}$ & 2.951360210 & -3.091143221 & -0.690542051 \\
$\mathrm{H}$ & 3.200686230 & -1.640492118 & -1.635583116 \\
$\mathrm{H}$ & 1.162566083 & -0.656163046 & -1.653938121 \\
& & &
\end{tabular}

$\mathrm{S}_{1}$ state minimum-energy proton transfer path 1.7 angstrom C $\quad-4.646267333 \quad-0.327544022 \quad-1.870410134$ 


\begin{tabular}{|c|c|c|c|}
\hline C & 3.234457232 & 5025 & -1. \\
\hline C & -2.772213200 & 0.231847015 & \\
\hline C & -3.784914275 & 0.802716060 & .55205504 \\
\hline C & -5.137127371 & 0.827955058 & 0.218980017 \\
\hline C & -5.598293405 & 0.278861022 & -1.013820073 \\
\hline $\mathrm{H}$ & -4.956669357 & -0.757702052 & $-\angle$ \\
\hline$H_{2}$ & -2.537658184 & -0.823961061 & -2 \\
\hline$H_{\text {- }}$ & -5.810501420 & 1.277510091 & 0.9210610 \\
\hline$H$ & -6.633632477 & 0.331427024 & -1.2798480 \\
\hline C & -1.393372100 & 0.293265020 & \\
\hline 0 & -1.000808070 & 0.772013053 & $1.12 \angle 6880$ \\
\hline 0 & -3.442130250 & 1.322206095 & 1.694645 \\
\hline $\mathrm{H}_{\text {- }}$ & -2.480553180 & 1.259147090 & 1.803184129 \\
\hline U & -0.567989040 & -0.220547017 & -0.852260063 \\
\hline C & 0.852945062 & -0.255518018 & -0.69509 \\
\hline C & 1.288044095 & -1.181206087 & 0.438144 \\
\hline C & 1.481959108 & 1.129998081 & -0.58465604 \\
\hline c & 2.823296204 & -1.326572093 & 0.526721 \\
\hline $\mathrm{H}$ & 0.915429068 & -0.785 & 1.37 \\
\hline C & 3.013296216 & 1.021042073 & -0.559942 \\
\hline $\mathrm{H}_{\mathrm{H}}$ & 1.160571085 & 1.736086126 & -1.4278271 \\
\hline C & 3.461031250 & 0.078 & 0.56 \\
\hline 11 & 3.2 & 0.5 & \\
\hline C & 3.659432263 & 2.400 & -0.4 \\
\hline $\mathrm{H}_{2}$ & 3.367582241 & 2.869568209 & 0.52319903 \\
\hline $\mathrm{H}_{\mathrm{H}}$ & 4.743067341 & 2.328640166 & -0.42357202 \\
\hline $\mathrm{H}$ & 3.36 & 3.06 & -1. \\
\hline H & 1.138760 & 1.620 & 0.31 \\
\hline $\mathrm{H}$ & 0.829643059 & -2.156597155 & 0.2955550 \\
\hline H & 4.544582327 & -0.017430003 & 0.554471 \\
\hline $\mathrm{H}$ & 3.342 & 0.607 & -1.5 \\
\hline C & 3.170 & -2.07 & 1.823 \\
\hline $\mathrm{H}$ & 2.727825199 & -3.064087220 & 1.834327130 \\
\hline $\mathrm{H}$ & 4.244997306 & -2.187926157 & 1.93118014 \\
\hline $\mathrm{H}$ & 2.805 & -1.536 & \\
\hline$C$ & 3.376 & -2.14 & -0.6 \\
\hline $\mathrm{H}$ & 4.451089322 & -2.271988164 & -0.55720004 \\
\hline $\mathrm{H}$ & 2.931966213 & -3.139847224 & -0.66752004 \\
\hline $\mathrm{H}$ & 3.188200229 & -1.693129120 & -1.61648 \\
\hline $\mathrm{H}$ & 1.157941084 & -0.688189048 & -1.638120118 \\
\hline \multicolumn{4}{|c|}{1.6 angstrom } \\
\hline C & -4.664275336 & -0.321311023 & -1.8580071 \\
\hline C & -3.248114233 & -0.361094029 & -1.534621113 \\
\hline C & -2.767257201 & 0.222738017 & -0.3408580 \\
\hline C & -3.76556 & 0.796186055 & 0.55608904 \\
\hline C & -5.121156368 & 0.827948062 & 0.2471 \\
\hline
\end{tabular}




$\begin{array}{lrrr}\mathrm{C} & -5.599636404 & 0.283880019 & -0.981206070 \\ \mathrm{H} & -4.993675362 & -0.747576055 & -2.786494202 \\ \mathrm{H} & -2.562870183 & -0.820953058 & -2.215880157 \\ \mathrm{H} & -5.783011419 & 1.277044092 & 0.960929072 \\ \mathrm{H} & -6.639082479 & 0.337597024 & -1.231482090 \\ \mathrm{C} & -1.390614097 & 0.284731021 & 0.036177002 \\ \mathrm{O} & -1.006874071 & 0.765619057 & 1.104216078 \\ \mathrm{O} & -3.378377245 & 1.301691094 & 1.685224122 \\ \mathrm{H} & -2.401867174 & 1.218258088 & 1.743837128 \\ \mathrm{O} & -0.558541041 & -0.222123018 & -0.873775061 \\ \mathrm{C} & 0.862923064 & -0.245070017 & -0.713694053 \\ \mathrm{C} & 1.303440094 & -1.168823085 & 0.418850032 \\ \mathrm{C} & 1.479815109 & 1.145431080 & -0.598868041 \\ \mathrm{C} & 2.839726205 & -1.302602094 & 0.508307038 \\ \mathrm{H} & 0.926891068 & -0.776200057 & 1.355170096 \\ \mathrm{C} & 3.012027217 & 1.048782073 & -0.573415044 \\ \mathrm{H} & 1.153793081 & 1.751412125 & -1.440330104 \\ \mathrm{C} & 3.466603248 & 0.107626006 & 0.552087041 \\ \mathrm{H} & 3.207336231 & 0.569783041 & 1.503543110 \\ \mathrm{C} & 3.647368265 & 2.432416175 & -0.422225029 \\ \mathrm{H} & 3.351613244 & 2.897582207 & 0.514148038 \\ \mathrm{H} & 4.731615342 & 2.369443172 & -0.433658030 \\ \mathrm{H} & 3.345487239 & 3.092996220 & -1.229778086 \\ \mathrm{H} & 1.131611082 & 1.629520115 & 0.304896024 \\ \mathrm{H} & 0.852250062 & -2.147354153 & 0.274967018 \\ \mathrm{H} & 4.550968327 & 0.019975002 & 0.539675041 \\ \mathrm{H} & 3.344481241 & 0.640218046 & -1.525903108 \\ \mathrm{C} & 3.191390231 & -2.047598146 & 1.804054128 \\ \mathrm{H} & 2.756447200 & -3.043190222 & 1.812642129 \\ \mathrm{H} & 4.267155307 & -2.155901154 & 1.911857138 \\ \mathrm{H} & 2.821934202 & -1.516828110 & 2.676193194 \\ \mathrm{C} & 3.399534244 & -2.118282150 & -0.671147049 \\ \mathrm{H} & 4.475551324 & -2.233539162 & -0.576423040 \\ \mathrm{H} & 2.962939211 & -3.112858222 & -0.689122051 \\ \mathrm{H} & 3.208705229 & -1.662619120 & -1.635495119 \\ \mathrm{H} & 1.172982085 & -0.673281046 & -1.657154121 \\ & & & \\ 1.5 & a n g s t r o m & & \\ \mathrm{C} & -4.686882338 & -0.300897021 & -1.858273131 \\ \mathrm{C} & -3.268711235 & -0.350581023 & -1.560313113 \\ \mathrm{C} & -2.767966198 & 0.228833017 & -0.376689025 \\ \mathrm{C} & -3.750636268 & 0.817055059 & 0.539228039 \\ \mathrm{C} & -5.108926367 & 0.856328060 & 0.249922017 \\ \mathrm{C} & -5.603623406 & 0.311428024 & -0.969023071 \\ \mathrm{H} & -5.035231360 & -0.727207055 & -2.779932197 \\ \mathrm{H} & -2.597254189 & -0.815652057 & -2.252257161 \\ \mathrm{H} & -5.759674416 & 1.312204095 & 0.969775068 \\ & & & \\ & & & \\ & & & \end{array}$




$\begin{array}{lrrr}\text { H } & -6.646316480 & 0.369685025 & -1.205376088 \\ \mathrm{C} & -1.393798099 & 0.285922019 & -0.001989003 \\ \mathrm{O} & -1.017274071 & 0.778323058 & 1.069599075 \\ \mathrm{O} & -3.320574241 & 1.316301096 & 1.648954118 \\ \mathrm{H} & -2.326065168 & 1.208936086 & 1.662595122 \\ \mathrm{O} & -0.555656040 & -0.229775016 & -0.898943064 \\ \mathrm{C} & 0.864258062 & -0.256646018 & -0.721150049 \\ \mathrm{C} & 1.286127092 & -1.172652085 & 0.424627033 \\ \mathrm{C} & 1.484517108 & 1.132487082 & -0.608732042 \\ \mathrm{C} & 2.820573201 & -1.311724094 & 0.535466039 \\ \mathrm{H} & 0.899196063 & -0.770162055 & 1.352404097 \\ \mathrm{C} & 3.015978218 & 1.030482076 & -0.563121038 \\ \mathrm{H} & 1.171293083 & 1.733728122 & -1.458414107 \\ \mathrm{C} & 3.452797246 & 0.096280006 & 0.575283039 \\ \mathrm{H} & 3.184310231 & 0.567321038 & 1.519778109 \\ \mathrm{C} & 3.653958263 & 2.413133173 & -0.413576029 \\ \mathrm{H} & 3.347692241 & 2.885682206 & 0.515690040 \\ \mathrm{H} & 4.738044343 & 2.346654169 & -0.410606032 \\ \mathrm{H} & 3.364650245 & 3.069092220 & -1.229442091 \\ \mathrm{H} & 1.126946083 & 1.623942116 & 0.287230018 \\ \mathrm{H} & 0.832853059 & -2.150604153 & 0.283417021 \\ \mathrm{H} & 4.536845327 & 0.003922002 & 0.576512043 \\ \mathrm{H} & 3.359394239 & 0.613806042 & -1.508203108 \\ \mathrm{C} & 3.151386225 & -2.046861150 & 1.842324131 \\ \mathrm{H} & 2.712264196 & -3.040583219 & 1.853441134 \\ \mathrm{H} & 4.225093304 & -2.158526154 & 1.966006142 \\ \mathrm{H} & 2.772026199 & -1.507037109 & 2.704586194 \\ \mathrm{C} & 3.392844242 & -2.139732154 & -0.629246043 \\ \mathrm{H} & 4.466985320 & -2.258519164 & -0.518957037 \\ \mathrm{H} & 2.952438213 & -3.132656224 & -0.644969047 \\ \mathrm{H} & 3.217094233 & -1.691316122 & -1.599870113 \\ \mathrm{H} & 1.184356083 & -0.693381050 & -1.657327120\end{array}$

1.4 angstrom

$\begin{array}{lrrr}\text { C } & -4.703912336 & -0.295835022 & -1.850978132 \\ \text { C } & -3.285191238 & -0.352465026 & -1.569301115 \\ \text { C } & -2.772899199 & 0.220330017 & -0.393268026 \\ \text { C } & -3.745691271 & 0.814820058 & 0.541584037 \\ \text { C } & -5.104737368 & 0.858787062 & 0.262223017 \\ \text { C } & -5.607094401 & 0.316380022 & -0.953429069 \\ \text { H } & -5.064258362 & -0.718434052 & -2.769861198 \\ \text { H } & -2.622322188 & -0.818450061 & -2.269589166 \\ \text { H } & -5.749686413 & 1.316093092 & 0.986268073 \\ \text { H } & -6.651646480 & 0.376874025 & -1.181848085 \\ \text { C } & -1.401237103 & 0.277419019 & -0.019692003 \\ \text { O } & -1.033773072 & 0.775619057 & 1.061218077 \\ \text { O } & -3.283327238 & 1.300487094 & 1.635533119 \\ & & & \\ & & & S 140\end{array}$




$\begin{array}{lrrr}\text { H } & -2.259733160 & 1.172746082 & 1.608313114 \\ \text { O } & -0.554524040 & -0.234731015 & -0.908249064 \\ \text { C } & 0.864916062 & -0.259934018 & -0.723368053 \\ \text { C } & 1.281286094 & -1.175216086 & 0.424870031 \\ \text { C } & 1.482373104 & 1.130122083 & -0.608183042 \\ \text { C } & 2.815280200 & -1.312430096 & 0.543485038 \\ \text { H } & 0.888472064 & -0.772928053 & 1.350398098 \\ \text { C } & 3.013672217 & 1.029610077 & -0.554440039 \\ \text { H } & 1.173196084 & 1.730395126 & -1.460006105 \\ \text { C } & 3.445667250 & 0.096278006 & 0.586428041 \\ \text { H } & 3.171791226 & 0.567089042 & 1.529473107 \\ \text { C } & 3.649752261 & 2.412856175 & -0.402662029 \\ \text { H } & 3.338520240 & 2.885992209 & 0.524631040 \\ \text { H } & 4.733842342 & 2.347038167 & -0.394268029 \\ \text { H } & 3.364086242 & 3.067850220 & -1.220583087 \\ \text { H } & 1.118675079 & 1.621279116 & 0.285609021 \\ \text { H } & 0.830006059 & -2.153697157 & 0.281090022 \\ \text { H } & 4.529772328 & 0.005092003 & 0.593152043 \\ \text { H } & 3.362096240 & 0.612791043 & -1.497612107 \\ \text { C } & 3.140529228 & -2.047172147 & 1.851918136 \\ \text { H } & 2.702640193 & -3.041454219 & 1.860866132 \\ \text { H } & 4.213774303 & -2.157458158 & 1.980651143 \\ \text { H } & 2.756400198 & -1.507897106 & 2.712390194 \\ \text { C } & 3.394096244 & -2.139952154 & -0.618422045 \\ \text { H } & 4.467755321 & -2.257857163 & -0.502789037 \\ \text { H } & 2.954579211 & -3.133233223 & -0.636174047 \\ \text { H } & 3.222834229 & -1.691819121 & -1.589979112 \\ \text { H } & 1.189237087 & -0.696666050 & -1.657923122\end{array}$

1.3 angstrom

$\begin{array}{lrrr}\text { C } & -4.716962339 & -0.290083019 & -1.844304132 \\ \text { C } & -3.298511238 & -0.348835024 & -1.570742112 \\ \text { C } & -2.776635201 & 0.215335018 & -0.404724031 \\ \text { C } & -3.743740269 & 0.816538059 & 0.549351042 \\ \text { C } & -5.102981368 & 0.859971064 & 0.269075021 \\ \text { C } & -5.607824401 & 0.318152025 & -0.945110069 \\ \text { H } & -5.081312364 & -0.710979051 & -2.762504200 \\ \text { H } & -2.640687190 & -0.814364061 & -2.277033166 \\ \text { H } & -5.746849414 & 1.317913097 & 0.993824074 \\ \text { H } & -6.654050479 & 0.379409028 & -1.167184086 \\ \text { C } & -1.404939100 & 0.268841019 & -0.037022002 \\ \text { O } & -1.038756075 & 0.773374055 & 1.057691074 \\ \text { O } & -3.259265236 & 1.288634090 & 1.626896115 \\ \text { H } & -2.177472155 & 1.140599083 & 1.566093115 \\ \text { O } & -0.551130040 & -0.240725017 & -0.918805065 \\ \text { C } & 0.867760061 & -0.262229018 & -0.726848050 \\ \text { C } & 1.280460090 & -1.176294084 & 0.423575029 \\ & & & \\ & & & S 141\end{array}$




$\begin{array}{lrrr}\text { C } & 1.481078107 & 1.129239082 & -0.607931042 \\ \text { C } & 2.814304202 & -1.312103096 & 0.546010039 \\ \text { H } & 0.883826063 & -0.772918057 & 1.347019095 \\ \text { C } & 3.012433218 & 1.030813076 & -0.550832039 \\ \text { H } & 1.172979084 & 1.729962127 & -1.459829106 \\ \text { C } & 3.443014246 & 0.097367005 & 0.590537044 \\ \text { H } & 3.166346230 & 0.567505039 & 1.533063109 \\ \text { C } & 3.646964264 & 2.414548171 & -0.397352031 \\ \text { H } & 3.333738239 & 2.887103206 & 0.529543037 \\ \text { H } & 4.731104342 & 2.349601168 & -0.387174027 \\ \text { H } & 3.362163244 & 3.069557219 & -1.215575085 \\ \text { H } & 1.113115083 & 1.617492117 & 0.285668019 \\ \text { H } & 0.830389061 & -2.155214155 & 0.279025019 \\ \text { H } & 4.527220328 & 0.007487001 & 0.599695044 \\ \text { H } & 3.363303240 & 0.614754042 & -1.493475105 \\ \text { C } & 3.137605227 & -2.046586147 & 1.855050135 \\ \text { H } & 2.700685195 & -3.041309219 & 1.863061132 \\ \text { H } & 4.210726305 & -2.155850157 & 1.985807145 \\ \text { H } & 2.751264200 & -1.507717107 & 2.714768194 \\ \text { C } & 3.396483246 & -2.139220154 & -0.614597046 \\ \text { H } & 4.469940320 & -2.256461162 & -0.496447038 \\ \text { H } & 2.957640215 & -3.132795223 & -0.633342044 \\ \text { H } & 3.227330230 & -1.691367124 & -1.586653112 \\ \text { H } & 1.197174084 & -0.697918052 & -1.660051118\end{array}$

\begin{tabular}{lrrr}
\multicolumn{4}{l}{1.2 angstrom } \\
C & -4.732225341 & -0.285872022 & -1.842336133 \\
C & -3.306350237 & -0.335713026 & -1.552787112 \\
C & -2.793098201 & 0.222353014 & -0.398083026 \\
C & -3.750557267 & 0.826931057 & 0.563170040 \\
C & -5.111088369 & 0.861140059 & 0.264382018 \\
C & -5.611108406 & 0.312487024 & -0.956056069 \\
H & -5.083489367 & -0.709139049 & -2.764442200 \\
H & -2.643284191 & -0.798492056 & -2.257142160 \\
H & -5.763115412 & 1.318699095 & 0.982134072 \\
H & -6.658733481 & 0.372172026 & -1.174918085 \\
C & -1.407082099 & 0.262053019 & -0.046550003 \\
O & -1.020463073 & 0.769185056 & 1.066746074 \\
O & -3.265743233 & 1.291284093 & 1.629050116 \\
H & -2.060949150 & 1.118892079 & 1.551612111 \\
O & -0.554326038 & -0.247238019 & -0.927576067 \\
C & 0.864470061 & -0.271196022 & -0.733031052 \\
C & 1.271826093 & -1.184954085 & 0.419740028 \\
C & 1.477672105 & 1.120209079 & -0.612177044 \\
C & 2.805050204 & -1.320084094 & 0.550482041 \\
H & 0.870675061 & -0.780842058 & 1.340653097 \\
C & 3.008702216 & 1.022134072 & -0.546726042 \\
& & & \\
& & & S142
\end{tabular}




$\begin{array}{llll}\mathrm{H} & 1.173676085 & 1.720830125 & -1.465604107 \\ \mathrm{C} & 3.433260245 & 0.089558005 & 0.597642043 \\ \mathrm{H} & 3.151653227 & 0.560300042 & 1.538373112 \\ \mathrm{C} & 3.640787263 & 2.406661171 & -0.389788030 \\ \mathrm{H} & 3.321291239 & 2.878939208 & 0.535086037 \\ \mathrm{H} & 4.724903343 & 2.343040169 & -0.372981027 \\ \mathrm{H} & 3.360155244 & 3.061194218 & -1.209817087 \\ \mathrm{H} & 1.105421078 & 1.608676114 & 0.279303022 \\ \mathrm{H} & 0.822589058 & -2.164068153 & 0.273940017 \\ \mathrm{H} & 4.517392327 & -0.000225001 & 0.612510045 \\ \mathrm{H} & 3.365430241 & 0.606017045 & -1.487127106 \\ \mathrm{C} & 3.120321224 & -2.053639148 & 1.862049134 \\ \mathrm{H} & 2.683672194 & -3.048491218 & 1.867888134 \\ \mathrm{H} & 4.192600302 & -2.162458158 & 1.999767142 \\ \mathrm{H} & 2.728239195 & -1.514351111 & 2.718868196 \\ \mathrm{C} & 3.394325245 & -2.147429153 & -0.606225044 \\ \mathrm{H} & 4.467062321 & -2.264331165 & -0.481521033 \\ \mathrm{H} & 2.955940212 & -3.141149228 & -0.627506044 \\ \mathrm{H} & 3.231004234 & -1.699644123 & -1.579354112 \\ \mathrm{H} & 1.195028085 & -0.707772052 & -1.665325121\end{array}$

\begin{tabular}{lrrr}
\multicolumn{4}{l}{1.1 angstrom } \\
C & -4.701210336 & -0.299107020 & -1.865494136 \\
C & -3.281960235 & -0.334336022 & -1.526443107 \\
C & -2.802274204 & 0.237864015 & -0.364449028 \\
C & -3.775527274 & 0.834107061 & 0.558943041 \\
C & -5.127073369 & 0.855493061 & 0.233193015 \\
C & -5.596652400 & 0.290022021 & -1.019264074 \\
H & -5.012463361 & -0.730688051 & -2.798634200 \\
H & -2.599428187 & -0.805042059 & -2.207288160 \\
H & -5.809988420 & 1.306132093 & 0.926181066 \\
H & -6.638665476 & 0.344482022 & -1.267476091 \\
C & -1.427031102 & 0.268930021 & -0.029887000 \\
O & -1.004575071 & 0.771398054 & 1.074546078 \\
O & -3.291478239 & 1.308917094 & 1.648110117 \\
H & -1.929579141 & 1.113830081 & 1.561485114 \\
O & -0.575269042 & -0.229875015 & -0.889429065 \\
C & 0.855028062 & -0.263583018 & -0.708515054 \\
C & 1.265533090 & -1.183412084 & 0.436596033 \\
C & 1.475832108 & 1.124241082 & -0.600146041 \\
C & 2.800019200 & -1.327128094 & 0.548146041 \\
H & 0.879610065 & -0.782281055 & 1.365364098 \\
C & 3.007077219 & 1.013946076 & -0.551225038 \\
H & 1.166776085 & 1.723105122 & -1.452562105 \\
C & 3.436795249 & 0.078831006 & 0.589130041 \\
H & 3.168966229 & 0.551424040 & 1.532966111 \\
C & 3.649414265 & 2.394545171 & -0.402272030 \\
& & & \\
& & & S143
\end{tabular}




$\begin{array}{rrrr}\mathrm{H} & 3.343404239 & 2.869619206 & 0.525652040 \\ \mathrm{H} & 4.732915340 & 2.323032168 & -0.397145028 \\ \mathrm{H} & 3.364650245 & 3.050135218 & -1.219835089 \\ \mathrm{H} & 1.120444082 & 1.621109118 & 0.293662019 \\ \mathrm{H} & 0.809217061 & -2.158999154 & 0.291565022 \\ \mathrm{H} & 4.520087326 & -0.018059999 & 0.592031044 \\ \mathrm{H} & 3.349660241 & 0.595007042 & -1.495316108 \\ \mathrm{C} & 3.125101224 & -2.064140147 & 1.855296134 \\ \mathrm{H} & 2.682551196 & -3.056212221 & 1.864972134 \\ \mathrm{H} & 4.198018304 & -2.179569157 & 1.980116140 \\ \mathrm{H} & 2.746689198 & -1.523444112 & 2.717336196 \\ \mathrm{C} & 3.369495243 & -2.156701154 & -0.616773044 \\ \mathrm{H} & 4.442635319 & -2.280282166 & -0.504650037 \\ \mathrm{H} & 2.924866210 & -3.147555225 & -0.634136048 \\ \mathrm{H} & 3.198278230 & -1.706668125 & -1.587593116 \\ \mathrm{H} & 1.162861083 & -0.702849052 & -1.645919117\end{array}$

\section{0 angstrom}

$\begin{array}{lrrr}\text { C } & -4.665797334 & -0.401169029 & -1.919069136 \\ \text { C } & -3.270367237 & -0.408156031 & -1.475118105 \\ \text { C } & -2.855772203 & 0.224393014 & -0.363814026 \\ \text { C } & -3.840367278 & 0.874848063 & 0.494636035 \\ \text { C } & -5.173953372 & 0.868090062 & 0.085028005 \\ \text { C } & -5.579674399 & 0.237254016 & -1.150877081 \\ \text { H } & -4.922806352 & -0.883605062 & -2.842512207 \\ \text { H } & -2.549390181 & -0.934061065 & -2.073535149 \\ \text { H } & -5.893054426 & 1.379718100 & 0.695037052 \\ \text { H } & -6.611341478 & 0.280022021 & -1.444561104 \\ \text { C } & -1.449951102 & 0.255790021 & -0.000237003 \\ \text { O } & -1.004671075 & 0.684609048 & 1.161058081 \\ \text { O } & -3.427850249 & 1.407880103 & 1.573532114 \\ \text { H } & -1.792749129 & 1.078538075 & 1.634086115 \\ \text { O } & -0.576500041 & -0.140104011 & -0.912731068 \\ \text { C } & 0.842130060 & -0.214739016 & -0.711266050 \\ \text { C } & 1.228903086 & -1.197651085 & 0.391573029 \\ \text { C } & 1.484497105 & 1.155617081 & -0.525164038 \\ \text { C } & 2.759879200 & -1.365596100 & 0.519530036 \\ \text { H } & 0.835302059 & -0.842182062 & 1.335086095 \\ \text { C } & 3.012683217 & 1.021604075 & -0.465797034 \\ \text { H } & 1.192235088 & 1.800454130 & -1.349452098 \\ \text { C } & 3.415012245 & 0.028079002 & 0.633844048 \\ \text { H } & 3.140426224 & 0.459895035 & 1.595245114 \\ \text { C } & 3.675449262 & 2.383284173 & -0.247887018 \\ \text { H } & 3.367900240 & 2.821529202 & 0.697580050 \\ \text { H } & 4.757669342 & 2.294069165 & -0.235665018 \\ \text { H } & 3.409295246 & 3.079593224 & -1.037745076 \\ \text { H } & 1.124345081 & 1.612470114 & 0.388593026\end{array}$




$\begin{array}{llrr}\mathrm{H} & 0.764159057 & -2.159766154 & 0.191062012 \\ \mathrm{H} & 4.496869325 & -0.084489006 & 0.647022048 \\ \mathrm{H} & 3.358524240 & 0.640698047 & -1.424898105 \\ \mathrm{C} & 3.056880222 & -2.161908157 & 1.798549129 \\ \mathrm{H} & 2.601082188 & -3.147383227 & 1.760949127 \\ \mathrm{H} & 4.126460295 & -2.297399166 & 1.932351141 \\ \mathrm{H} & 2.673888194 & -1.652463118 & 2.677515190 \\ \mathrm{C} & 3.336728239 & -2.151197155 & -0.671878049 \\ \mathrm{H} & 4.407365317 & -2.290130165 & -0.552948039 \\ \mathrm{H} & 2.882769207 & -3.136054225 & -0.735988052 \\ \mathrm{H} & 3.180871230 & -1.659036119 & -1.624693119 \\ \mathrm{H} & 1.162351083 & -0.610802046 & -1.664639122\end{array}$

$T_{1}$ state minimum-energy proton transfer path 1.9 angstrom

$\begin{array}{lrrr}\text { C } & -4.476113321 & -0.481194033 & -1.949121138 \\ \text { C } & -3.209544228 & -0.603623042 & -1.484494105 \\ \text { C } & -2.786358198 & 0.138406008 & -0.260524019 \\ \text { C } & -3.866226281 & 0.785224058 & 0.562952040 \\ \text { C } & -5.094509367 & 0.878948064 & 0.076434007 \\ \text { C } & -5.451021393 & 0.316153021 & -1.236240087 \\ \text { H } & -4.771547343 & -0.991176071 & -2.848319207 \\ \text { H } & -2.479074177 & -1.206181089 & -1.984044143 \\ \text { H } & -5.852999423 & 1.349294096 & 0.674056047 \\ \text { H } & -6.414597462 & 0.515493038 & -1.658327117 \\ \text { C } & -1.381386098 & 0.319620021 & 0.057922006 \\ \text { O } & -1.003388074 & 0.863891061 & 1.070240078 \\ \text { O } & -3.574797257 & 1.239668092 & 1.790889129 \\ \text { H } & -2.632705191 & 1.276946094 & 1.916856138 \\ \text { O } & -0.568331042 & -0.183185013 & -0.861294061 \\ \mathrm{C} & 0.862295063 & -0.197715014 & -0.729067054 \\ \mathrm{C} & 1.320058096 & -1.103579081 & 0.410293029 \\ \mathrm{C} & 1.476384104 & 1.195708083 & -0.647131048 \\ \mathrm{C} & 2.858045205 & -1.234964087 & 0.475077035 \\ \mathrm{H} & 0.962351072 & -0.697319049 & 1.349033095 \\ \mathrm{C} & 3.009424215 & 1.100439081 & -0.644105049 \\ \mathrm{H} & 1.136261084 & 1.787896129 & -1.492464107 \\ \mathrm{C} & 3.483203253 & 0.176587014 & 0.488094034 \\ \mathrm{H} & 3.239897232 & 0.652523047 & 1.436977105 \\ \mathrm{C} & 3.643276264 & 2.487666179 & -0.522701040 \\ \mathrm{H} & 3.361662240 & 2.965313211 & 0.411696031 \\ \mathrm{H} & 4.727130343 & 2.426479176 & -0.550645039 \\ \mathrm{H} & 3.327173240 & 3.136000227 & -1.334546096 \\ \mathrm{H} & 1.145795080 & 1.695632123 & 0.254701016 \\ \mathrm{H} & 0.867174061 & -2.084333149 & 0.290127020 \\ \mathrm{H} & 4.567027327 & 0.089726008 & 0.458472035 \\ \mathrm{H} & 3.327511241 & 0.678922049 & -1.595609114 \\ & & & \\ & & & \\ & & & \end{array}$




$\begin{array}{llll}\mathrm{C} & 3.231870232 & -1.962628139 & 1.774339127 \\ \mathrm{H} & 2.798272200 & -2.958309212 & 1.803291128 \\ \mathrm{H} & 4.309255310 & -2.068659151 & 1.864546136 \\ \mathrm{H} & 2.877638209 & -1.420743100 & 2.645981190 \\ \mathrm{C} & 3.397049244 & -2.066275150 & -0.703109053 \\ \mathrm{H} & 4.474243319 & -2.180604159 & -0.624505043 \\ \mathrm{H} & 2.960265215 & -3.060807220 & -0.701092051 \\ \mathrm{H} & 3.191162230 & -1.622997116 & -1.670141120 \\ \mathrm{H} & 1.151761082 & -0.640378048 & -1.671388121\end{array}$

1.8 angstrom

$\begin{array}{lrrr}\text { C } & -4.494690321 & -0.477123036 & -1.950358138 \\ \text { C } & -3.223647231 & -0.603430046 & -1.501248111 \\ \text { C } & -2.790611201 & 0.134247008 & -0.277523018 \\ \text { C } & -3.856575278 & 0.782824054 & 0.558435041 \\ \text { C } & -5.091113367 & 0.878059063 & 0.088668008 \\ \text { C } & -5.459508390 & 0.320268025 & -1.225599089 \\ \text { H } & -4.801734344 & -0.983500069 & -2.847778203 \\ \text { H } & -2.499025181 & -1.207569089 & -2.007507143 \\ \text { H } & -5.843713420 & 1.346661096 & 0.694810051 \\ \text { H } & -6.427557463 & 0.520467039 & -1.636554119 \\ \text { C } & -1.387809098 & 0.314288020 & 0.044954004 \\ \text { O } & -1.020883076 & 0.858577063 & 1.062115075 \\ \text { O } & -3.531696257 & 1.235038087 & 1.776733130 \\ \text { H } & -2.581775188 & 1.249386088 & 1.868891136 \\ \text { O } & -0.569030043 & -0.186857011 & -0.867695063 \\ \text { C } & 0.861563062 & -0.198876013 & -0.729707052 \\ \text { C } & 1.315394097 & -1.104173077 & 0.411452028 \\ \text { C } & 1.472832107 & 1.195499085 & -0.645297047 \\ \text { C } & 2.853247208 & -1.233708090 & 0.482635036 \\ \text { H } & 0.953102069 & -0.698304049 & 1.348651098 \\ \text { C } & 3.006032215 & 1.101751081 & -0.636280047 \\ \text { H } & 1.135452083 & 1.786971127 & -1.492232105 \\ \text { C } & 3.476461249 & 0.178653012 & 0.497936037 \\ \text { H } & 3.228786230 & 0.654406045 & 1.445760103 \\ \text { C } & 3.638463260 & 2.489437177 & -0.513244040 \\ \text { H } & 3.353049240 & 2.967445214 & 0.419790030 \\ \text { H } & 4.722430339 & 2.428769175 & -0.537072039 \\ \text { H } & 3.325093240 & 3.137054227 & -1.326713098 \\ \text { H } & 1.137637082 & 1.695161124 & 0.255095021 \\ \text { H } & 0.864183061 & -2.085400150 & 0.289096019 \\ \text { H } & 4.560479330 & 0.093185007 & 0.472573032 \\ \text { H } & 3.327736237 & 0.680031051 & -1.586472112 \\ \text { C } & 3.222389234 & -1.960645142 & 1.783623129 \\ \text { H } & 2.789755198 & -2.956781213 & 1.811054132 \\ \text { H } & 4.299486311 & -2.065460148 & 1.878365134 \\ \text { H } & 2.863912204 & -1.418927102 & 2.653625192 \\ & & & \\ & & 5146\end{array}$




$\begin{array}{llll}\mathrm{C} & 3.397966244 & -2.064891150 & -0.693045049 \\ \mathrm{H} & 4.474920322 & -2.178098155 & -0.609894047 \\ \mathrm{H} & 2.962208211 & -3.059879218 & -0.692189052 \\ \mathrm{H} & 3.195559227 & -1.622405115 & -1.661178117 \\ \mathrm{H} & 1.154949084 & -0.641048045 & -1.670962118\end{array}$

$\begin{array}{lrrr}\text { 1.7 } & \text { angstrom } & & \\ \text { C } & -4.518820327 & -0.440584029 & -1.968407141 \\ \text { C } & -3.245373232 & -0.585287040 & -1.533975109 \\ \text { C } & -2.795241200 & 0.139606013 & -0.308848020 \\ \text { C } & -3.841325279 & 0.801842060 & 0.538635038 \\ \text { C } & -5.080031363 & 0.913876065 & 0.083315005 \\ \text { C } & -5.466391393 & 0.365262024 & -1.230966089 \\ \text { H } & -4.841098347 & -0.937258068 & -2.866005205 \\ \text { H } & -2.532634180 & -1.196796088 & -2.049002148 \\ \text { H } & -5.819868418 & 1.394751099 & 0.695461050 \\ \text { H } & -6.435010464 & 0.580661041 & -1.633160119 \\ \text { C } & -1.394935100 & 0.303011020 & 0.022753002 \\ \text { O } & -1.040872075 & 0.844739063 & 1.048440076 \\ \text { O } & -3.475770249 & 1.250781089 & 1.744299126 \\ \text { H } & -2.517342180 & 1.229839090 & 1.797933129 \\ \text { O } & -0.568330042 & -0.201263015 & -0.877804063 \\ \text { C } & 0.862221063 & -0.211891016 & -0.727252050 \\ \text { C } & 1.306598091 & -1.116427081 & 0.417971031 \\ \text { C } & 1.470186105 & 1.183398087 & -0.636941047 \\ \text { C } & 2.844050202 & -1.244382087 & 0.501533035 \\ \text { H } & 0.936014068 & -0.710057050 & 1.351761099 \\ \text { C } & 3.003352218 & 1.091307080 & -0.616453045 \\ \text { H } & 1.138419085 & 1.775272130 & -1.485771105 \\ \text { C } & 3.465818251 & 0.168617012 & 0.521295037 \\ \text { H } & 3.210281229 & 0.644217044 & 1.467096106 \\ \text { C } & 3.633649260 & 2.479536181 & -0.488883037 \\ \text { H } & 3.341068239 & 2.957499211 & 0.441937030 \\ \text { H } & 4.717786337 & 2.419596173 & -0.504795037 \\ \text { H } & 3.325792241 & 3.126737223 & -1.304778092 \\ \text { H } & 1.127300081 & 1.681307120 & 0.261568017 \\ \text { H } & 0.857223063 & -2.098032151 & 0.292378018 \\ \text { H } & 4.550059327 & 0.084152005 & 0.504457035 \\ \text { H } & 3.332386239 & 0.669814046 & -1.564241111 \\ \text { C } & 3.203492229 & -1.970289144 & 1.805793131 \\ \text { H } & 2.771747201 & -2.966872215 & 1.830315131 \\ \text { H } & 4.279922306 & -2.073867150 & 1.909040137 \\ \text { H } & 2.837604204 & -1.428487101 & 2.672631191 \\ \text { C } & 3.398862247 & -2.075608148 & -0.669413046 \\ \text { H } & 4.475211322 & -2.187721159 & -0.577552040 \\ \text { H } & 2.964088214 & -3.071011223 & -0.671559050 \\ \text { H } & 3.203889229 & -1.633847118 & -1.639425117 \\ & & & \\ & & & \\ & & & \\ & & \end{array}$


$\begin{array}{llll}H & 1.163401082 & -0.653477048 & -1.666233120\end{array}$

1.6 angstrom

\begin{tabular}{lrrr}
$\mathrm{C}$ & -4.560684328 & -0.412077028 & -1.971507140 \\
$\mathrm{C}$ & -3.274929237 & -0.545899038 & -1.573506116 \\
$\mathrm{C}$ & -2.811733205 & 0.149855012 & -0.336380022 \\
$\mathrm{C}$ & -3.840070277 & 0.801576059 & 0.538671038 \\
$\mathrm{C}$ & -5.091093364 & 0.904781064 & 0.116267010 \\
$\mathrm{C}$ & -5.502481398 & 0.362436027 & -1.196049085 \\
$\mathrm{H}$ & -4.895990353 & -0.891384063 & -2.873775205 \\
$\mathrm{H}$ & -2.564809184 & -1.131756082 & -2.121054155 \\
$\mathrm{H}$ & -5.819009421 & 1.382155099 & 0.744770056 \\
$\mathrm{H}$ & -6.485927465 & 0.563422039 & -1.567884115 \\
$\mathrm{C}$ & -1.413920101 & 0.285816019 & 0.008234998 \\
$\mathrm{O}$ & -1.068252076 & 0.811005061 & 1.047733075 \\
$\mathrm{O}$ & -3.426723244 & 1.257759090 & 1.723793126 \\
$\mathrm{H}$ & -2.459479177 & 1.197728085 & 1.736903122 \\
$\mathrm{O}$ & -0.582795044 & -0.216032013 & -0.887473062 \\
$\mathrm{C}$ & 0.846822063 & -0.225085014 & -0.725889054 \\
$\mathrm{C}$ & 1.284274093 & -1.131685082 & 0.420165032 \\
$\mathrm{C}$ & 1.448822103 & 1.171946083 & -0.626059046 \\
$\mathrm{C}$ & 2.821542204 & -1.254161092 & 0.516611036 \\
$\mathrm{H}$ & 0.904543066 & -0.730100051 & 1.352065096 \\
$\mathrm{C}$ & 2.982074213 & 1.084920080 & -0.594210043 \\
$\mathrm{H}$ & 1.121421081 & 1.765922128 & -1.475115105 \\
$\mathrm{C}$ & 3.438557245 & 0.160862010 & 0.544851040 \\
$\mathrm{H}$ & 3.174681227 & 0.633452044 & 1.489843108 \\
$\mathrm{C}$ & 3.606316260 & 2.475120181 & -0.458449032 \\
$\mathrm{H}$ & 3.304513240 & 2.949882212 & 0.471042032 \\
$\mathrm{H}$ & 4.690741337 & 2.419196173 & -0.465726034 \\
$\mathrm{H}$ & 3.302708238 & 3.123050223 & -1.275359092 \\
$\mathrm{H}$ & 1.098072082 & 1.665682120 & 0.271681018 \\
$\mathrm{H}$ & 0.839804061 & -2.114586154 & 0.287596018 \\
$\mathrm{H}$ & 4.523129328 & 0.079746006 & 0.535864039 \\
$\mathrm{H}$ & 3.319715238 & 0.666873048 & -1.540468109 \\
$\mathrm{C}$ & 3.171293228 & -1.981707143 & 1.822608130 \\
$\mathrm{H}$ & 2.742699196 & -2.979769212 & 1.840881134 \\
$\mathrm{H}$ & 4.247018308 & -2.081867151 & 1.935927141 \\
$\mathrm{H}$ & 2.795310199 & -1.442996104 & 2.687020193 \\
$\mathrm{C}$ & 3.389710243 & -2.080443151 & -0.651303045 \\
$\mathrm{H}$ & 4.465587324 & -2.188806158 & -0.550040041 \\
$\mathrm{H}$ & 2.958696215 & -3.077429223 & -0.660028045 \\
$\mathrm{H}$ & 3.201866232 & -1.636713115 & -1.621843118 \\
$\mathrm{H}$ & 1.156705085 & -0.662824048 & -1.663664118 \\
& & & \\
\hline
\end{tabular}

1.5 angstrom

$\begin{array}{rrrr}\text { C } & -4.585839329 & -0.395504028 & -1.970481140 \\ & & S 148\end{array}$




$\begin{array}{lrrr}\text { C } & -3.291508239 & -0.527117040 & -1.602056117 \\ \text { C } & -2.812047203 & 0.152486012 & -0.361957027 \\ \text { C } & -3.820660278 & 0.802348060 & 0.535073040 \\ \text { C } & -5.081392365 & 0.903178064 & 0.140239009 \\ \text { C } & -5.516595397 & 0.361584025 & -1.166783086 \\ \text { H } & -4.936397354 & -0.864817063 & -2.872221207 \\ \text { H } & -2.588305188 & -1.101285082 & -2.170945155 \\ \text { H } & -5.797988415 & 1.380387101 & 0.781449056 \\ \text { H } & -6.509559468 & 0.556788040 & -1.515819107 \\ \text { C } & -1.415775100 & 0.276634022 & -0.013698002 \\ \text { O } & -1.080734076 & 0.796824058 & 1.034975077 \\ \text { O } & -3.363309241 & 1.260526089 & 1.699193121 \\ \text { H } & -2.387089169 & 1.170382084 & 1.670515122 \\ \text { O } & -0.578083043 & -0.220678015 & -0.902776064 \\ \text { C } & 0.851736061 & -0.226884015 & -0.730408053 \\ \text { C } & 1.282647090 & -1.135669083 & 0.416168029 \\ \text { C } & 1.448399105 & 1.171311086 & -0.620113047 \\ \text { C } & 2.819541204 & -1.254471090 & 0.523709038 \\ \text { H } & 0.895595065 & -0.735967055 & 1.345800098 \\ \text { C } & 2.981648215 & 1.088622077 & -0.578554043 \\ \text { H } & 1.124256079 & 1.769351128 & -1.467491105 \\ \text { C } & 3.432926249 & 0.161999010 & 0.560444041 \\ \text { H } & 3.161529225 & 0.630946046 & 1.505106109 \\ \text { C } & 3.600236257 & 2.480429178 & -0.433511030 \\ \text { H } & 3.291080235 & 2.950481215 & 0.495920036 \\ \text { H } & 4.684848340 & 2.428142174 & -0.434400032 \\ \text { H } & 3.299507240 & 3.130520227 & -1.249770091 \\ \text { H } & 1.090598077 & 1.658384120 & 0.278456021 \\ \text { H } & 0.841151061 & -2.119212153 & 0.278963021 \\ \text { H } & 4.517713325 & 0.083593008 & 0.558439042 \\ \text { H } & 3.327034241 & 0.674954050 & -1.523938109 \\ \text { C } & 3.161024226 & -1.984366142 & 1.830639130 \\ \text { H } & 2.734738196 & -2.983471214 & 1.843538133 \\ \text { H } & 4.236136307 & -2.082172148 & 1.951549141 \\ \text { H } & 2.777456198 & -1.448482106 & 2.693447193 \\ \text { C } & 3.398412245 & -2.076127149 & -0.642089047 \\ \text { H } & 4.473776321 & -2.182148155 & -0.533379038 \\ \text { H } & 2.969939215 & -3.074139222 & -0.656634045 \\ \text { H } & 3.216371229 & -1.630077116 & -1.612720118 \\ \text { H } & 1.167157082 & -0.660840046 & -1.668068122 \\ & & & \\ & & \end{array}$

1.4 angstrom

$\begin{array}{lrrr}\text { C } & -4.604420330 & -0.380420026 & -1.974044143 \\ \text { C } & -3.306705235 & -0.517042035 & -1.620919117 \\ \text { C } & -2.818994205 & 0.153734013 & -0.379921028 \\ \text { C } & -3.811243272 & 0.807222057 & 0.531167040 \\ \text { C } & -5.077759367 & 0.909733067 & 0.149276012\end{array}$




$\begin{array}{lrrr}\mathrm{C} & -5.525277396 & 0.371180025 & -1.154965081 \\ \mathrm{H} & -4.966042356 & -0.840803058 & -2.876233207 \\ \mathrm{H} & -2.609408189 & -1.087617079 & -2.200783159 \\ \mathrm{H} & -5.788011419 & 1.386228102 & 0.797992058 \\ \mathrm{H} & -6.521473470 & 0.567787042 & -1.494181107 \\ \mathrm{C} & -1.426320105 & 0.270492020 & -0.026673999 \\ \mathrm{O} & -1.104839079 & 0.789192057 & 1.031242075 \\ \mathrm{O} & -3.321041240 & 1.256275092 & 1.679968122 \\ \mathrm{H} & -2.328329169 & 1.144674081 & 1.611505117 \\ \mathrm{O} & -0.580213040 & -0.221338015 & -0.904933065 \\ \mathrm{C} & 0.850034063 & -0.228409014 & -0.726958050 \\ \mathrm{C} & 1.275343089 & -1.141201080 & 0.418268032 \\ \mathrm{C} & 1.445215103 & 1.169900083 & -0.610934044 \\ \mathrm{C} & 2.811874204 & -1.260048089 & 0.531046038 \\ \mathrm{H} & 0.883752063 & -0.745459055 & 1.347972095 \\ \mathrm{C} & 2.978284214 & 1.086749080 & -0.563181041 \\ \mathrm{H} & 1.124974082 & 1.768934126 & -1.459064106 \\ \mathrm{C} & 3.424909246 & 0.156362013 & 0.574600041 \\ \mathrm{H} & 3.150066224 & 0.622239043 & 1.519776109 \\ \mathrm{C} & 3.597265260 & 2.477656178 & -0.412056031 \\ \mathrm{H} & 3.284831239 & 2.945689213 & 0.517305036 \\ \mathrm{H} & 4.681777339 & 2.424208175 & -0.408678028 \\ \mathrm{H} & 3.300453239 & 3.129977227 & -1.227918087 \\ \mathrm{H} & 1.082714077 & 1.655313119 & 0.287035022 \\ \mathrm{H} & 0.834799061 & -2.124238151 & 0.274731021 \\ \mathrm{H} & 4.509628323 & 0.077805005 & 0.576372044 \\ \mathrm{H} & 3.326632241 & 0.675768047 & -1.508629107 \\ \mathrm{C} & 3.148912226 & -1.994376144 & 1.836563132 \\ \mathrm{H} & 2.722751198 & -2.993590216 & 1.844244134 \\ \mathrm{H} & 4.223576306 & -2.092469148 & 1.960769139 \\ \mathrm{H} & 2.762244199 & -1.461767106 & 2.700006192 \\ \mathrm{C} & 3.394753244 & -2.078229147 & -0.635390045 \\ \mathrm{H} & 4.469643319 & -2.184711157 & -0.522897037 \\ \mathrm{H} & 2.966186211 & -3.076098220 & -0.654523046 \\ \mathrm{H} & 3.216745230 & -1.629404119 & -1.605499114 \\ \mathrm{H} & 1.168659083 & -0.659144049 & -1.664809120 \\ & & & \\ 1.3 & a n g 5 t r o m & & \\ \mathrm{C} & -4.640906334 & -0.347340024 & -1.981822144 \\ \mathrm{C} & -3.332331242 & -0.470586035 & -1.665926118 \\ \mathrm{C} & -2.828732204 & 0.176197011 & -0.417712031 \\ \mathrm{C} & -3.800171275 & 0.822150057 & 0.519165036 \\ \mathrm{C} & -5.079796366 & 0.909872066 & 0.167099014 \\ \mathrm{C} & -5.553997401 & 0.369254026 & -1.124194080 \\ \mathrm{H} & -5.016950361 & -0.791606058 & -2.886235207 \\ \mathrm{H} & -2.639854191 & -1.012619074 & -2.278103162 \\ \mathrm{H} & -5.777557416 & 1.380660099 & 0.833192060 \\ & & & \\ & & & \\ & & & \end{array}$




$\begin{array}{lrrr}\text { H } & -6.563677472 & 0.546280041 & -1.433484101 \\ \mathrm{C} & -1.438746102 & 0.263386022 & -0.058036007 \\ \mathrm{O} & -1.123409083 & 0.773367054 & 1.011065071 \\ \mathrm{O} & -3.272998238 & 1.273744090 & 1.643090119 \\ \mathrm{H} & -2.258442163 & 1.123744079 & 1.539218113 \\ \mathrm{O} & -0.585952041 & -0.228663014 & -0.924621067 \\ \mathrm{C} & 0.844559063 & -0.238548018 & -0.727548051 \\ \mathrm{C} & 1.252366091 & -1.156297084 & 0.419799032 \\ \mathrm{C} & 1.437117104 & 1.159202082 & -0.596276040 \\ \mathrm{C} & 2.787315199 & -1.276208093 & 0.553588042 \\ \mathrm{H} & 0.848845060 & -0.762940055 & 1.345228099 \\ \mathrm{C} & 2.969455214 & 1.075805076 & -0.527526036 \\ \mathrm{H} & 1.128293082 & 1.763363128 & -1.444922103 \\ \mathrm{C} & 3.399881247 & 0.139892012 & 0.611956044 \\ \mathrm{H} & 3.111946222 & 0.601524045 & 1.555273112 \\ \mathrm{C} & 3.584847259 & 2.466433175 & -0.360000023 \\ \mathrm{H} & 3.258655232 & 2.928872209 & 0.567384042 \\ \mathrm{H} & 4.669205336 & 2.414058174 & -0.341457023 \\ \mathrm{H} & 3.298977237 & 3.123045222 & -1.176310086 \\ \mathrm{H} & 1.062976078 & 1.639260120 & 0.299619021 \\ \mathrm{H} & 0.812956058 & -2.138343154 & 0.267098020 \\ \mathrm{H} & 4.484422324 & 0.061249007 & 0.628488044 \\ \mathrm{H} & 3.331448241 & 0.669971047 & -1.470016106 \\ \mathrm{C} & 3.105094223 & -2.015705146 & 1.861092133 \\ \mathrm{H} & 2.678812193 & -3.014851219 & 1.858853136 \\ \mathrm{H} & 4.177835299 & -2.114351152 & 2.000337146 \\ \mathrm{H} & 2.706116194 & -1.486236109 & 2.720805197 \\ \mathrm{C} & 3.386513245 & -2.089500152 & -0.607808046 \\ \mathrm{H} & 4.459616321 & -2.196774159 & -0.480496032 \\ \mathrm{H} & 2.958044211 & -3.087111224 & -0.637539044 \\ \mathrm{H} & 3.222542229 & -1.636227119 & -1.578367111 \\ \mathrm{H} & 1.173297083 & -0.665286045 & -1.663427121 \\ & & & \\ 1.2 & a n g 5 t r o m & & \\ \mathrm{C} & -4.804223345 & -0.262004017 & -1.943630141 \\ \mathrm{C} & -3.372640244 & -0.372117029 & -1.671497121 \\ \mathrm{C} & -2.835695203 & 0.228459016 & -0.443750033 \\ \mathrm{C} & -3.698780269 & 0.815734058 & 0.478030035 \\ \mathrm{C} & -5.127262370 & 0.875357062 & 0.196768014 \\ \mathrm{C} & -5.645194406 & 0.340686022 & -1.063656076 \\ \mathrm{H} & -5.181595374 & -0.660884048 & -2.867799206 \\ \mathrm{H} & -2.716414195 & -0.848332060 & -2.369279169 \\ \mathrm{H} & -5.765256416 & 1.325274095 & 0.929652066 \\ \mathrm{H} & -6.692980483 & 0.438539029 & -1.271061093 \\ \mathrm{C} & -1.467787106 & 0.268862022 & -0.085178005 \\ \mathrm{O} & -1.110575080 & 0.779729055 & 1.001030071 \\ \mathrm{O} & -3.270011233 & 1.326392093 & 1.570514110 \\ & & & \\ & & & 5151\end{array}$




$\begin{array}{lrrr}\text { H } & -2.141901156 & 1.147135084 & 1.492337110 \\ \text { O } & -0.607294045 & -0.232096014 & -0.926698067 \\ \text { C } & 0.822227058 & -0.255164020 & -0.720903051 \\ \text { C } & 1.214515085 & -1.172834085 & 0.431787029 \\ \text { C } & 1.429736103 & 1.137131083 & -0.597395044 \\ \text { C } & 2.747100199 & -1.304324095 & 0.578066041 \\ \text { H } & 0.803443060 & -0.774777057 & 1.351169098 \\ \text { C } & 2.960305216 & 1.039302074 & -0.513544035 \\ \text { H } & 1.135862084 & 1.734912125 & -1.456021104 \\ \text { C } & 3.371438245 & 0.106449010 & 0.635236043 \\ \text { H } & 3.078044222 & 0.575575039 & 1.573181111 \\ \text { C } & 3.587131257 & 2.425142173 & -0.347404023 \\ \text { H } & 3.253550233 & 2.896669207 & 0.572839039 \\ \text { H } & 4.670773336 & 2.362481168 & -0.314650025 \\ \text { H } & 3.317897239 & 3.079160220 & -1.171503082 \\ \text { H } & 1.049318077 & 1.628750120 & 0.288785021 \\ \text { H } & 0.769931053 & -2.152139154 & 0.275760022 \\ \text { H } & 4.455192319 & 0.018119003 & 0.662913045 \\ \text { H } & 3.328778240 & 0.625003047 & -1.449939106 \\ \text { C } & 3.048507220 & -2.040608148 & 1.891240138 \\ \text { H } & 2.615820188 & -3.037089221 & 1.889045138 \\ \text { H } & 4.119382295 & -2.145611154 & 2.041025145 \\ \text { H } & 2.644608192 & -1.505226111 & 2.744994196 \\ \text { C } & 3.349655240 & -2.127406155 & -0.574800043 \\ \text { H } & 4.420889320 & -2.243144162 & -0.438019033 \\ \text { H } & 2.913019212 & -3.121549222 & -0.604174043 \\ \text { H } & 3.197329230 & -1.676723122 & -1.548473111 \\ \text { H } & 1.150077082 & -0.691137047 & -1.653168120\end{array}$

1.1 angstrom

$\begin{array}{lrrr}\text { C } & -4.700381338 & -0.249488017 & -1.964349140 \\ \text { C } & -3.301950239 & -0.323427023 & -1.613271114 \\ \text { C } & -2.816256204 & 0.255025016 & -0.387558029 \\ \text { C } & -3.806363274 & 0.858291064 & 0.516670039 \\ \text { C } & -5.186918373 & 0.892801062 & 0.121281007 \\ \text { C } & -5.620853406 & 0.347062026 & -1.148617082 \\ \text { H } & -5.010201361 & -0.668802047 & -2.904844207 \\ \text { H } & -2.612299190 & -0.794685060 & -2.282264162 \\ \text { H } & -5.876397423 & 1.348911099 & 0.804276059 \\ \text { H } & -6.654056480 & 0.420257029 & -1.425293104 \\ \text { C } & -1.492770109 & 0.280597019 & -0.048715004 \\ \text { O } & -1.029097076 & 0.784743057 & 1.068975075 \\ \text { O } & -3.486569249 & 1.351410096 & 1.613714114 \\ \text { H } & -1.906883136 & 1.152156081 & 1.620789119 \\ \text { O } & -0.610261042 & -0.224498019 & -0.883267061 \\ \text { C } & 0.812660057 & -0.254274018 & -0.686914049 \\ \text { C } & 1.220481088 & -1.175563083 & 0.458690035 \\ & & & \\ & & & S 152\end{array}$




$\begin{array}{lrrr}\text { C } & 1.439686106 & 1.131553084 & -0.578563044 \\ \text { C } & 2.754022198 & -1.322406098 & 0.575219041 \\ \text { H } & 0.832070062 & -0.776437054 & 1.387357101 \\ \text { C } & 2.970222214 & 1.017893071 & -0.523621036 \\ \text { H } & 1.135358080 & 1.729028124 & -1.433722104 \\ \text { C } & 3.393336245 & 0.082226006 & 0.618612046 \\ \text { H } & 3.121277225 & 0.555425039 & 1.561122114 \\ \text { C } & 3.616086259 & 2.396661171 & -0.372320026 \\ \text { H } & 3.307494239 & 2.872870206 & 0.554323037 \\ \text { H } & 4.699500337 & 2.322655166 & -0.362531025 \\ \text { H } & 3.336324239 & 3.052982218 & -1.191099087 \\ \text { H } & 1.083266078 & 1.632958116 & 0.312169020 \\ \text { H } & 0.762668053 & -2.149961156 & 0.310339025 \\ \text { H } & 4.476625321 & -0.016219002 & 0.627349043 \\ \text { H } & 3.316047241 & 0.597815041 & -1.466045107 \\ \text { C } & 3.075317223 & -2.060307147 & 1.882837137 \\ \text { H } & 2.631361187 & -3.051807222 & 1.891246134 \\ \text { H } & 4.147872299 & -2.177254155 & 2.010418147 \\ \text { H } & 2.695556192 & -1.519404108 & 2.744288199 \\ \text { C } & 3.325934240 & -2.152760154 & -0.588067041 \\ \text { H } & 4.398540318 & -2.279046166 & -0.472445035 \\ \text { H } & 2.878749206 & -3.142451226 & -0.607265046 \\ \text { H } & 3.158503225 & -1.702048122 & -1.559087110 \\ \text { H } & 1.131517084 & -0.694236052 & -1.620795114\end{array}$

\begin{tabular}{lrrr}
\multicolumn{4}{l}{1.0 angstrom } \\
C & -4.687064338 & -0.251310017 & -1.964054139 \\
C & -3.293419235 & -0.318215024 & -1.596345115 \\
C & -2.821159201 & 0.263729018 & -0.371918026 \\
C & -3.827388274 & 0.868739061 & 0.525781037 \\
C & -5.199425377 & 0.896024064 & 0.109630011 \\
C & -5.617529406 & 0.344734027 & -1.160986081 \\
H & -4.983321358 & -0.675442047 & -2.906764210 \\
H & -2.595614189 & -0.788678057 & -2.257554162 \\
H & -5.899434425 & 1.352732097 & 0.781661055 \\
H & -6.647800478 & 0.413427028 & -1.450084106 \\
C & -1.499222108 & 0.284891023 & -0.038458004 \\
O & -1.013291071 & 0.789411057 & 1.079687078 \\
O & -3.520760253 & 1.363069099 & 1.624766119 \\
H & -1.775990128 & 1.139231081 & 1.623669118 \\
O & -0.616051045 & -0.223953014 & -0.870952064 \\
C & 0.807704058 & -0.258446020 & -0.679177049 \\
C & 1.217219086 & -1.177740087 & 0.467502032 \\
C & 1.441807101 & 1.124861082 & -0.579270041 \\
C & 2.750668198 & -1.331086097 & 0.577182039 \\
H & 0.836458058 & -0.773825057 & 1.397144102 \\
C & 2.972109213 & 1.004100071 & -0.531365037 \\
& & & \\
& & & S153
\end{tabular}




$\begin{array}{lrrr}\mathrm{H} & 1.135823084 & 1.720862125 & -1.434785105 \\ \mathrm{C} & 3.396473245 & 0.070849007 & 0.612457042 \\ \mathrm{H} & 3.131198224 & 0.548827040 & 1.554533111 \\ \mathrm{C} & 3.624682262 & 2.380632170 & -0.388369030 \\ \mathrm{H} & 3.323047240 & 2.861472204 & 0.538186037 \\ \mathrm{H} & 4.707785337 & 2.302003167 & -0.384000027 \\ \mathrm{H} & 3.343454241 & 3.035238217 & -1.207996087 \\ \mathrm{H} & 1.093476077 & 1.631854120 & 0.311467024 \\ \mathrm{H} & 0.753978052 & -2.150448157 & 0.325383026 \\ \mathrm{H} & 4.479313324 & -0.032419002 & 0.616336044 \\ \mathrm{H} & 3.311546239 & 0.578845042 & -1.473752108 \\ \mathrm{C} & 3.074147223 & -2.065746147 & 1.886130138 \\ \mathrm{H} & 2.625142190 & -3.054894220 & 1.900217138 \\ \mathrm{H} & 4.146625298 & -2.187746157 & 2.009356146 \\ \mathrm{H} & 2.701073193 & -1.519741109 & 2.747305197 \\ \mathrm{C} & 3.313496236 & -2.168178156 & -0.585601043 \\ \mathrm{H} & 4.386103313 & -2.298452165 & -0.474670034 \\ \mathrm{H} & 2.862173205 & -3.156071227 & -0.598903046 \\ \mathrm{H} & 3.143209225 & -1.720432126 & -1.557505113 \\ \mathrm{H} & 1.120062079 & -0.703261048 & -1.612933118\end{array}$

LIIC path connecting FC point and S1-KETO-MIN Coord_1

$\begin{array}{lrrr}\text { C } & -4.515201326 & -0.400805029 & -1.855736134 \\ \text { C } & -3.181451230 & -0.375125026 & -1.481482107 \\ \text { C } & -2.778806198 & 0.220923013 & -0.272872021 \\ \text { C } & -3.754562271 & 0.783013055 & 0.566889039 \\ \text { C } & -5.085772365 & 0.765497056 & 0.179575014 \\ \text { C } & -5.467403392 & 0.188203016 & -1.019133071 \\ \text { H } & -4.809460348 & -0.854842062 & -2.784067199 \\ \text { H } & -2.434378173 & -0.806214059 & -2.117452151 \\ \text { H } & -5.806210417 & 1.216708086 & 0.835650062 \\ \text { H } & -6.503132467 & 0.201302015 & -1.303526096 \\ \text { C } & -1.344494099 & 0.280596019 & 0.100976010 \\ \text { O } & -0.954382070 & 0.733908052 & 1.147589081 \\ \text { O } & -3.481933248 & 1.359663097 & 1.742872125 \\ \text { H } & -2.548349183 & 1.317046093 & 1.923385137 \\ \text { O } & -0.544260039 & -0.199703016 & -0.830909057 \\ \text { C } & 0.890368064 & -0.207467015 & -0.722219051 \\ \text { C } & 1.380843099 & -1.138294082 & 0.382675025 \\ \text { C } & 1.486719105 & 1.192066085 & -0.615617046 \\ \text { C } & 2.921902209 & -1.247819088 & 0.414983032 \\ \text { H } & 1.035212074 & -0.764273053 & 1.338446095 \\ \text { C } & 3.020571217 & 1.118485078 & -0.643317046 \\ \text { H } & 1.122695080 & 1.800113128 & -1.439462105 \\ \text { C } & 3.527056256 & 0.172058014 & 0.455255034 \\ \text { H } & 3.293798237 & 0.619141043 & 1.420531101\end{array}$




$\begin{array}{lrrr}\mathrm{C} & 3.638651260 & 2.510523181 & -0.498054038 \\ \mathrm{H} & 3.369749243 & 2.960209212 & 0.453778032 \\ \mathrm{H} & 4.722405340 & 2.464154178 & -0.548779038 \\ \mathrm{H} & 3.298450240 & 3.175256231 & -1.286571093 \\ \mathrm{H} & 1.166035083 & 1.664744121 & 0.304595022 \\ \mathrm{H} & 0.940045065 & -2.121757151 & 0.242279020 \\ \mathrm{H} & 4.611296332 & 0.101698009 & 0.404701028 \\ \mathrm{H} & 3.325843238 & 0.726020052 & -1.611264114 \\ \mathrm{C} & 3.330888239 & -2.002916144 & 1.687826124 \\ \mathrm{H} & 2.912588208 & -3.005464218 & 1.699186120 \\ \mathrm{H} & 4.411244318 & -2.094817150 & 1.755189128 \\ \mathrm{H} & 2.985199216 & -1.488884106 & 2.579495186 \\ \mathrm{C} & 3.451961247 & -2.038805146 & -0.794613060 \\ \mathrm{H} & 4.532188329 & -2.137092152 & -0.738958054 \\ \mathrm{H} & 3.031487218 & -3.040188220 & -0.811097058 \\ \mathrm{H} & 3.220773232 & -1.573731111 & -1.745601124 \\ \mathrm{H} & 1.166358082 & -0.621012045 & -1.681525120\end{array}$

Coord_2

$\begin{array}{lrrr}\text { C } & -4.524623327 & -0.398375026 & -1.856388133 \\ \text { C } & -3.181997229 & -0.376448027 & -1.485355108 \\ \text { C } & -2.772994202 & 0.219610014 & -0.276608018 \\ \text { C } & -3.753759271 & 0.779318054 & 0.566783040 \\ \text { C } & -5.087366369 & 0.763812055 & 0.185492014 \\ \text { C } & -5.477394396 & 0.189793013 & -1.016749075 \\ \text { H } & -4.820105346 & -0.850280062 & -2.785241200 \\ \text { H } & -2.439781173 & -0.809322060 & -2.125395155 \\ \text { H } & -5.803257417 & 1.213076088 & 0.847588063 \\ \text { H } & -6.513396468 & 0.205129015 & -1.298429092 \\ \text { C } & -1.343858097 & 0.282200019 & 0.096747005 \\ \text { O } & -0.952648067 & 0.737726050 & 1.144380081 \\ \text { O } & -3.475197251 & 1.349847097 & 1.740329127 \\ \text { H } & -2.538890182 & 1.307740093 & 1.914156138 \\ \text { O } & -0.541852039 & -0.199254015 & -0.835506063 \\ \text { C } & 0.891723065 & -0.206751017 & -0.724653055 \\ \text { C } & 1.380806099 & -1.137125082 & 0.381388029 \\ \text { C } & 1.488581105 & 1.192614085 & -0.617584045 \\ \text { C } & 2.921738211 & -1.247012088 & 0.416262032 \\ \text { H } & 1.033437076 & -0.762594053 & 1.336314098 \\ \text { C } & 3.022426217 & 1.118752080 & -0.643003048 \\ \text { H } & 1.125972079 & 1.800471132 & -1.442234105 \\ \text { C } & 3.527202256 & 0.172709013 & 0.456686030 \\ \text { H } & 3.292552236 & 0.620271043 & 1.421407101 \\ \text { C } & 3.640656260 & 2.510696180 & -0.497391037 \\ \text { H } & 3.370187243 & 2.960910213 & 0.453757034 \\ \text { H } & 4.724512339 & 2.464141176 & -0.546236040 \\ \text { H } & 3.301903238 & 3.175138230 & -1.286798094 \\ & & & \\ & & & \text { S155 }\end{array}$




$\begin{array}{lrrr}\text { H } & 1.166307085 & 1.665542120 & 0.301899023 \\ \text { H } & 0.940077070 & -2.120609154 & 0.240706019 \\ \text { H } & 4.611535330 & 0.102148005 & 0.407784030 \\ \text { H } & 3.329115241 & 0.725800052 & -1.610336117 \\ \text { C } & 3.328601241 & -2.001513142 & 1.690150122 \\ \text { H } & 2.910062212 & -3.003974219 & 1.701376120 \\ \text { H } & 4.408849315 & -2.093612150 & 1.759346127 \\ \text { H } & 2.981539215 & -1.486937105 & 2.580975183 \\ \text { C } & 3.453567247 & -2.038880146 & -0.791994056 \\ \text { H } & 4.533703326 & -2.137437155 & -0.734633052 \\ \text { H } & 3.032829217 & -3.040165217 & -0.808468058 \\ \text { H } & 3.223885234 & -1.574355112 & -1.743609126 \\ \text { H } & 1.169888086 & -0.620683045 & -1.683297123\end{array}$

Coord_3

$\begin{array}{lrrr}\text { C } & -4.534031326 & -0.395944028 & -1.856955136 \\ \text { C } & -3.182542229 & -0.377764027 & -1.489219107 \\ \text { C } & -2.767172199 & 0.218330013 & -0.280377019 \\ \text { C } & -3.752940269 & 0.775610056 & 0.566653042 \\ \text { C } & -5.088920366 & 0.762081052 & 0.191424012 \\ \text { C } & -5.487377394 & 0.191372015 & -1.014290073 \\ \text { H } & -4.830723346 & -0.845738059 & -2.786316202 \\ \text { H } & -2.445200176 & -0.812399056 & -2.133294152 \\ \text { H } & -5.800226417 & 1.209356089 & 0.859517062 \\ \text { H } & -6.523643467 & 0.208913013 & -1.293249093 \\ \text { C } & -1.343216099 & 0.283827022 & 0.092471004 \\ \text { O } & -0.950909069 & 0.741577053 & 1.141118084 \\ \text { O } & -3.468470249 & 1.340021096 & 1.737710123 \\ \text { H } & -2.529486184 & 1.298472093 & 1.904817138 \\ \text { O } & -0.539431037 & -0.198784015 & -0.840140062 \\ \text { C } & 0.893087067 & -0.206023017 & -0.727110051 \\ \text { C } & 1.380757097 & -1.135948081 & 0.380080030 \\ \text { C } & 1.490455107 & 1.193170086 & -0.619560046 \\ \text { C } & 2.921557212 & -1.246204092 & 0.417537032 \\ \text { H } & 1.031643075 & -0.760908057 & 1.334153097 \\ \text { C } & 3.024289217 & 1.119020082 & -0.642681049 \\ \text { H } & 1.129277082 & 1.800841128 & -1.445016106 \\ \text { C } & 3.527336254 & 0.173357012 & 0.458126033 \\ \text { H } & 3.291286238 & 0.621396042 & 1.422287102 \\ \text { C } & 3.642674262 & 2.510866183 & -0.496709034 \\ \text { H } & 3.370632244 & 2.961607214 & 0.453751033 \\ \text { H } & 4.726630339 & 2.464121178 & -0.543660037 \\ \text { H } & 3.305384240 & 3.175020228 & -1.287003092 \\ \text { H } & 1.166584083 & 1.666348121 & 0.299187022 \\ \text { H } & 0.940095067 & -2.119451150 & 0.239103020 \\ \text { H } & 4.611761331 & 0.102589006 & 0.410889031 \\ \text { H } & 3.332405242 & 0.725583052 & -1.609394118 \\ & & & \\ & & & 5156\end{array}$




$\begin{array}{llll}\mathrm{C} & 3.326276237 & -2.000115145 & 1.692468119 \\ \mathrm{H} & 2.907492210 & -3.002487215 & 1.703551123 \\ \mathrm{H} & 4.406411316 & -2.092415151 & 1.763509128 \\ \mathrm{H} & 2.977833212 & -1.484995109 & 2.582443185 \\ \mathrm{C} & 3.455163251 & -2.038952146 & -0.789371056 \\ \mathrm{H} & 4.535204327 & -2.137785152 & -0.730292052 \\ \mathrm{H} & 3.034155219 & -3.040137218 & -0.805844058 \\ \mathrm{H} & 3.227001230 & -1.574973111 & -1.741612128 \\ \mathrm{H} & 1.173434087 & -0.620339042 & -1.685085123\end{array}$

Coord_4

$\begin{array}{lrrr}\text { C } & -4.543425328 & -0.393514030 & -1.857436132 \\ \text { C } & -3.183085228 & -0.379070026 & -1.493072105 \\ \text { C } & -2.761340200 & 0.217083018 & -0.284180020 \\ \text { C } & -3.752107270 & 0.771891057 & 0.566500041 \\ \text { C } & -5.090434369 & 0.760306054 & 0.197370017 \\ \text { C } & -5.497353396 & 0.192938014 & -1.011753075 \\ \text { H } & -4.841316347 & -0.841217060 & -2.787294201 \\ \text { H } & -2.450634176 & -0.815444059 & -2.141152154 \\ \text { H } & -5.797120417 & 1.205549087 & 0.871437060 \\ \text { H } & -6.533874469 & 0.212651015 & -1.287985091 \\ \text { C } & -1.342568095 & 0.285478018 & 0.088148007 \\ \text { O } & -0.949166070 & 0.745462056 & 1.137802080 \\ \text { O } & -3.461755249 & 1.330188094 & 1.735016124 \\ \text { H } & -2.520139184 & 1.289242094 & 1.895368139 \\ \text { O } & -0.536996039 & -0.198295013 & -0.844811062 \\ \text { C } & 0.894458064 & -0.205281016 & -0.729590051 \\ \text { C } & 1.380696099 & -1.134764084 & 0.378748027 \\ \text { C } & 1.492343105 & 1.193736089 & -0.621547043 \\ \text { C } & 2.921359209 & -1.245396091 & 0.418808031 \\ \text { H } & 1.029829077 & -0.759217055 & 1.331962097 \\ \text { C } & 3.026162219 & 1.119291079 & -0.642351048 \\ \text { H } & 1.132610084 & 1.801224130 & -1.447807104 \\ \text { C } & 3.527461256 & 0.174002015 & 0.459575031 \\ \text { H } & 3.290003237 & 0.622516046 & 1.423171103 \\ \text { C } & 3.644707260 & 2.511035181 & -0.496005038 \\ \text { H } & 3.371084241 & 2.962301214 & 0.453761035 \\ \text { H } & 4.728760341 & 2.464094180 & -0.541050039 \\ \text { H } & 3.308894241 & 3.174905228 & -1.287188092 \\ \text { H } & 1.166868082 & 1.667162122 & 0.296460023 \\ \text { H } & 0.940097067 & -2.118282150 & 0.237470016 \\ \text { H } & 4.611975330 & 0.103023005 & 0.414015029 \\ \text { H } & 3.335714240 & 0.725369053 & -1.608439117 \\ \text { C } & 3.323912238 & -1.998722144 & 1.694781121 \\ \text { H } & 2.904879212 & -3.001002216 & 1.705712124 \\ \text { H } & 4.403930316 & -2.091230149 & 1.767679129 \\ \text { H } & 2.974083213 & -1.483060109 & 2.583901184 \\ & & & \\ & & 5157\end{array}$




$\begin{array}{llll}\mathrm{C} & 3.456750248 & -2.039023146 & -0.786745057 \\ \mathrm{H} & 4.536692326 & -2.138137156 & -0.725935050 \\ \mathrm{H} & 3.035467218 & -3.040105219 & -0.803226060 \\ \mathrm{H} & 3.230123233 & -1.575585116 & -1.739611123 \\ \mathrm{H} & 1.176996085 & -0.619981043 & -1.686888120\end{array}$

Coord_5

$\begin{array}{lrrr}\text { C } & -4.552805328 & -0.391085027 & -1.857832136 \\ \text { C } & -3.183628228 & -0.380368029 & -1.496916107 \\ \text { C } & -2.755499200 & 0.215871016 & -0.288018021 \\ \text { C } & -3.751260269 & 0.768162056 & 0.566323042 \\ \text { C } & -5.091909366 & 0.758485055 & 0.203332013 \\ \text { C } & -5.507321396 & 0.194490012 & -1.009139072 \\ \text { H } & -4.851883350 & -0.836718058 & -2.788173202 \\ \text { H } & -2.456083177 & -0.818459057 & -2.148966154 \\ \text { H } & -5.793938416 & 1.201655088 & 0.883346062 \\ \text { H } & -6.544088474 & 0.216342016 & -1.282637094 \\ \text { C } & -1.341915095 & 0.287153023 & 0.083778009 \\ \text { O } & -0.947419071 & 0.749381052 & 1.134432083 \\ \text { O } & -3.455051251 & 1.320348095 & 1.732246124 \\ \text { H } & -2.510850181 & 1.280054090 & 1.885810134 \\ \text { O } & -0.534549038 & -0.197785013 & -0.849519062 \\ \text { C } & 0.895838063 & -0.204527017 & -0.732094054 \\ \text { C } & 1.380624099 & -1.133572080 & 0.377395027 \\ \text { C } & 1.494245106 & 1.194311087 & -0.623544047 \\ \text { C } & 2.921143210 & -1.244587091 & 0.420074029 \\ \text { H } & 1.027995075 & -0.757520052 & 1.329743098 \\ \text { C } & 3.028045216 & 1.119563081 & -0.642012047 \\ \text { H } & 1.135971084 & 1.801620129 & -1.450609102 \\ \text { C } & 3.527575252 & 0.174643013 & 0.461031031 \\ \text { H } & 3.288701239 & 0.623630043 & 1.424061100 \\ \text { C } & 3.646756261 & 2.511202179 & -0.495281033 \\ \text { H } & 3.371543244 & 2.962991213 & 0.453786033 \\ \text { H } & 4.730902339 & 2.464061175 & -0.538406037 \\ \text { H } & 3.312435241 & 3.174791227 & -1.287351095 \\ \text { H } & 1.167158082 & 1.667983120 & 0.293717022 \\ \text { H } & 0.940085066 & -2.117103154 & 0.235805018 \\ \text { H } & 4.612176333 & 0.103447008 & 0.417164031 \\ \text { H } & 3.339040241 & 0.725159054 & -1.607471114 \\ \mathrm{C} & 3.321509239 & -1.997334144 & 1.697088123 \\ \text { H } & 2.902221207 & -2.999520218 & 1.707859123 \\ \text { H } & 4.401406315 & -2.090055153 & 1.771857127 \\ \text { H } & 2.970287213 & -1.481132104 & 2.585348188 \\ \mathrm{C} & 3.458327249 & -2.039092145 & -0.784116056 \\ \text { H } & 4.538165328 & -2.138493154 & -0.721562051 \\ \text { H } & 3.036763221 & -3.040069219 & -0.800614057 \\ \text { H } & 3.233250231 & -1.576193114 & -1.737605124 \\ & & & \\ & & & \\ & & 5158\end{array}$


$\begin{array}{llll}H & 1.180575085 & -0.619608042 & -1.688708119\end{array}$

Coord_6

\begin{tabular}{lrrr} 
C & -4.571520327 & -0.386234028 & -1.858367134 \\
C & -3.184708231 & -0.382937026 & -1.504575106 \\
C & -2.743788195 & 0.213553014 & -0.295795022 \\
C & -3.749521271 & 0.760670054 & 0.565898038 \\
C & -5.094737368 & 0.754704057 & 0.215297018 \\
C & -5.527234400 & 0.197547016 & -1.003679074 \\
H & -4.872938350 & -0.827791060 & -2.789637202 \\
H & -2.467022176 & -0.824397060 & -2.164467158 \\
H & -5.787344417 & 1.193601085 & 0.907131066 \\
H & -6.564465470 & 0.223581018 & -1.271687094 \\
$\mathrm{C}$ & -1.340592094 & 0.290577022 & 0.074897007 \\
O & -0.943909070 & 0.757325056 & 1.127530083 \\
O & -3.441676248 & 1.300652092 & 1.726477124 \\
H & -2.492447179 & 1.261809090 & 1.866364135 \\
O & -0.529616037 & -0.196702015 & -0.859049063 \\
$\mathrm{C}$ & 0.898621065 & -0.202980015 & -0.737172055 \\
$\mathrm{C}$ & 1.380443099 & -1.131166081 & 0.374620027 \\
$\mathrm{C}$ & 1.498090108 & 1.195489083 & -0.627568043 \\
$\mathrm{C}$ & 2.920658208 & -1.242971089 & 0.422595030 \\
$\mathrm{H}$ & 1.024269075 & -0.754105055 & 1.325217098 \\
$\mathrm{C}$ & 3.031842217 & 1.120113081 & -0.641311046 \\
$\mathrm{H}$ & 1.142778082 & 1.802453128 & -1.456244105 \\
$\mathrm{C}$ & 3.527773254 & 0.175914012 & 0.463971034 \\
$\mathrm{H}$ & 3.286041234 & 0.625842046 & 1.425854101 \\
$\mathrm{C}$ & 3.650898263 & 2.511529179 & -0.493771036 \\
$\mathrm{H}$ & 3.372484243 & 2.964362211 & 0.453885032 \\
$\mathrm{H}$ & 4.735222341 & 2.463973178 & -0.533015038 \\
$\mathrm{H}$ & 3.319606238 & 3.174568226 & -1.287612090 \\
$\mathrm{H}$ & 1.167758084 & 1.669651118 & 0.288184019 \\
$\mathrm{H}$ & 0.940015066 & -2.114715151 & 0.232383019 \\
$\mathrm{H}$ & 4.612538332 & 0.104269006 & 0.423525032 \\
$\mathrm{H}$ & 3.345749240 & 0.724749053 & -1.605496113 \\
$\mathrm{C}$ & 3.316587240 & -1.994575141 & 1.701686122 \\
$\mathrm{H}$ & 2.896771211 & -2.996566213 & 1.712109126 \\
$\mathrm{H}$ & 4.396226315 & -2.087738150 & 1.780234129 \\
$\mathrm{H}$ & 2.962561215 & -1.477294109 & 2.588209185 \\
$\mathrm{C}$ & 3.461450247 & -2.039228149 & -0.778849055 \\
$\mathrm{H}$ & 4.541069325 & -2.139218154 & -0.712768051 \\
$\mathrm{H}$ & 3.039306219 & -3.039986217 & -0.795408059 \\
$\mathrm{H}$ & 3.239519236 & -1.577393113 & -1.733579127 \\
$\mathrm{H}$ & 1.187783088 & -0.618819044 & -1.692396120 \\
& & & \\
\hline
\end{tabular}

Coord_7

C $\quad-4.571520327 \quad-0.386234028 \quad-1.858367134$ 


$\begin{array}{lrrr}\text { C } & -3.184708231 & -0.382937026 & -1.504575106 \\ \text { C } & -2.743788195 & 0.213553014 & -0.295795022 \\ \text { C } & -3.749521271 & 0.760670054 & 0.565898038 \\ \text { C } & -5.094737368 & 0.754704057 & 0.215297018 \\ \text { C } & -5.527234400 & 0.197547016 & -1.003679074 \\ \text { H } & -4.872938350 & -0.827791060 & -2.789637202 \\ \text { H } & -2.467022176 & -0.824397060 & -2.164467158 \\ \text { H } & -5.787344417 & 1.193601085 & 0.907131066 \\ \text { H } & -6.564465470 & 0.223581018 & -1.271687094 \\ \text { C } & -1.340592094 & 0.290577022 & 0.074897007 \\ \text { O } & -0.943909070 & 0.757325056 & 1.127530083 \\ \text { O } & -3.441676248 & 1.300652092 & 1.726477124 \\ \text { H } & -2.492447179 & 1.261809090 & 1.866364135 \\ \text { O } & -0.529616037 & -0.196702015 & -0.859049063 \\ \text { C } & 0.898621065 & -0.202980015 & -0.737172055 \\ \text { C } & 1.380443099 & -1.131166081 & 0.374620027 \\ \text { C } & 1.498090108 & 1.195489083 & -0.627568043 \\ \text { C } & 2.920658208 & -1.242971089 & 0.422595030 \\ \text { H } & 1.024269075 & -0.754105055 & 1.325217098 \\ \text { C } & 3.031842217 & 1.120113081 & -0.641311046 \\ \text { H } & 1.142778082 & 1.802453128 & -1.456244105 \\ \text { C } & 3.527773254 & 0.175914012 & 0.463971034 \\ \text { H } & 3.286041234 & 0.625842046 & 1.425854101 \\ \text { C } & 3.650898263 & 2.511529179 & -0.493771036 \\ \text { H } & 3.372484243 & 2.964362211 & 0.453885032 \\ \text { H } & 4.735222341 & 2.463973178 & -0.533015038 \\ \text { H } & 3.319606238 & 3.174568226 & -1.287612090 \\ \text { H } & 1.167758084 & 1.669651118 & 0.288184019 \\ \text { H } & 0.940015066 & -2.114715151 & 0.232383019 \\ \text { H } & 4.612538332 & 0.104269006 & 0.423525032 \\ \text { H } & 3.345749240 & 0.724749053 & -1.605496113 \\ \text { C } & 3.316587240 & -1.994575141 & 1.701686122 \\ \text { H } & 2.896771211 & -2.996566213 & 1.712109126 \\ \text { H } & 4.396226315 & -2.087738150 & 1.780234129 \\ \text { H } & 2.962561215 & -1.477294109 & 2.588209185 \\ \text { C } & 3.461450247 & -2.039228149 & -0.778849055 \\ \text { H } & 4.541069325 & -2.139218154 & -0.712768051 \\ \text { H } & 3.039306219 & -3.039986217 & -0.795408059 \\ \text { H } & 3.239519236 & -1.577393113 & -1.733579127 \\ \text { H } & 1.187783088 & -0.618819044 & -1.692396120 \\ & & & \\ \text { Coord_8 } 8 & & \\ \text { C } & -4.580853331 & -0.383815026 & -1.858506133 \\ \text { C } & -3.185245229 & -0.384206030 & -1.508390109 \\ \text { C } & -2.737919196 & 0.212447017 & -0.299734021 \\ \text { C } & -3.748629268 & 0.756908054 & 0.565650039 \\ \text { C } & -5.096090369 & 0.752744053 & 0.221300015 \\ & & & \\ & & & \\ & & & \end{array}$




\begin{tabular}{|c|c|c|c|}
\hline ¿ & 5.537176397 & 0017 & 31074 \\
\hline$H$ & -4.883425351 & -0.823365059 & 21202 \\
\hline $\mathrm{H}$ & -2.472512178 & -0.827319061 & -2.1721531 \\
\hline$\theta$ & -5.783931414 & 189442085 & 0.919005068 \\
\hline & -6.574626472 & 0.227125018 & -1.26608409 \\
\hline C & -1.339921097 & 0.292328022 & 0.07 \\
\hline 0 & -0.942147068 & 0.761351052 & 1.123997( \\
\hline 0 & -3.435006249 & 1.290800092 & 1.7234781 \\
\hline $\mathrm{H}$ & -2.483334181 & 1.252756090 & 1.8564771 \\
\hline 0 & -0.527130037 & -0.196128017 & -0.8638700 \\
\hline C & 0.900026062 & -0.202185016 & -0.739747052 \\
\hline C & 1.380334099 & -1.129951079 & 0.3731980 \\
\hline C & 1.500033109 & 1.196092086 & -0.629595046 \\
\hline 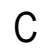 & 2.920388211 & -1.242163088 & 0.423848031 \\
\hline $\mathrm{H}$ & 1.022375075 & -0.7523 & 1.322909 \\
\hline C & 3.033755219 & 1.120391079 & -0.6409470 \\
\hline $\mathrm{H}$ & 1.146224084 & 1.802890128 & -1.459076102 \\
\hline C & 3.527856256 & 0.176544013 & 0.465454032 \\
\hline $\mathrm{H}$ & 3.284683238 & 0.6269 & 1.426757105 \\
\hline C & 3.652993260 & 2.511690181 & -0.492983033 \\
\hline $\mathrm{H}$ & 3.372967244 & 2.965042215 & 0.45395803 \\
\hline $\mathrm{H}$ & 4.737400340 & 2.46 & -0.5302680 \\
\hline$\Pi$ & 37241 & 3.17 & 709094 \\
\hline $\mathrm{H}$ & 1.168068081 & 1.67 & 94022 \\
\hline $\mathrm{H}$ & 0.939956068 & -2.113504150 & 0.2306250 \\
\hline $\mathrm{H}$ & 4.612 & 0.10 & 0.42673903 \\
\hline H & 3.3 & 0 & 115 \\
\hline C & 66239 & -1.993 & 1.703976121 \\
\hline $\mathrm{H}$ & 2.893977208 & -2.995094217 & 1.71421112 \\
\hline $\mathrm{H}$ & 4.393569316 & -2.086597149 & 1.784433130 \\
\hline $\mathrm{H}$ & 2.95 & & 2.5 \\
\hline C & 3.462995249 & -2.03 & -0.77 \\
\hline $\mathrm{H}$ & 4.542498326 & -2.139587154 & -0.7083470 \\
\hline $\mathrm{H}$ & 3.040553220 & -3.039938221 & -0.792814058 \\
\hline $\mathrm{H}$ & $3.24 t$ & -1.5 & -1.731559125 \\
\hline 列 & 1.1914 & & -1.6942 \\
\hline \multicolumn{4}{|c|}{ Coord_9 } \\
\hline C & -4.590 & -0.3 & -1.8585581 \\
\hline C & & -0.385466028 & -1.512194110 \\
\hline C & -2.732039196 & 0.211379016 & -0.3037080 \\
\hline C & -3.747721269 & 0.753138052 & 0.565377042 \\
\hline C & -5.097402369 & 0.750737053 & 0.227318015 \\
\hline C & -5.547109398 & 0.200535015 & -0.99790407 \\
\hline $\mathrm{H}$ & -4.893884355 & -0.818966061 & -2.7907061 \\
\hline $\mathrm{H}$ & -2.478013177 & -0.830210062 & -2.1797941 \\
\hline $\mathrm{H}$ & -5.780440416 & 1.185194083 & 0.9308 \\
\hline
\end{tabular}




$\begin{array}{lrrr}\text { H } & -6.584770472 & 0.230617017 & -1.260394091 \\ \mathrm{C} & -1.339244094 & 0.294104020 & 0.065825004 \\ \mathrm{O} & -0.940380066 & 0.765412054 & 1.120409082 \\ \mathrm{O} & -3.428347247 & 1.280945092 & 1.720401121 \\ \mathrm{H} & -2.474280180 & 1.243752091 & 1.846480130 \\ \mathrm{O} & -0.524631040 & -0.195533015 & -0.868729064 \\ \mathrm{C} & 0.901438066 & -0.201377016 & -0.742345053 \\ \mathrm{C} & 1.380212097 & -1.128729082 & 0.371753029 \\ \mathrm{C} & 1.501990107 & 1.196705085 & -0.631633045 \\ \mathrm{C} & 2.920100212 & -1.241355088 & 0.425097033 \\ \mathrm{H} & 1.020461073 & -0.750664053 & 1.320571096 \\ \mathrm{C} & 3.035677217 & 1.120671083 & -0.640575045 \\ \mathrm{H} & 1.149700085 & 1.803341130 & -1.461917107 \\ \mathrm{C} & 3.527928255 & 0.177170014 & 0.466945036 \\ \mathrm{H} & 3.283305234 & 0.628032047 & 1.427665104 \\ \mathrm{C} & 3.655103265 & 2.511849183 & -0.492174038 \\ \mathrm{H} & 3.373457241 & 2.965718212 & 0.454048034 \\ \mathrm{H} & 4.739590340 & 2.463855177 & -0.527485035 \\ \mathrm{H} & 3.326899237 & 3.174352227 & -1.287784094 \\ \mathrm{H} & 1.168385085 & 1.671354122 & 0.282589023 \\ \mathrm{H} & 0.939881068 & -2.112283153 & 0.228835018 \\ \mathrm{H} & 4.612848330 & 0.105054008 & 0.429975031 \\ \mathrm{H} & 3.352531239 & 0.724351053 & -1.603465115 \\ \mathrm{C} & 3.311504238 & -1.991839141 & 1.706259124 \\ \mathrm{H} & 2.891137209 & -2.993625216 & 1.716298124 \\ \mathrm{H} & 4.390867316 & -2.085466149 & 1.788638131 \\ \mathrm{H} & 2.954649210 & -1.473483106 & 2.591025185 \\ \mathrm{C} & 3.464529249 & -2.039359147 & -0.773570057 \\ \mathrm{H} & 4.543912325 & -2.139962156 & -0.703910053 \\ \mathrm{H} & 3.041783219 & -3.039887219 & -0.790227059 \\ \mathrm{H} & 3.245806232 & -1.578573115 & -1.729534123 \\ \mathrm{H} & 1.195056084 & -0.617969043 & -1.696148124 \\ & & & \\ \mathrm{Coord}-10 & & \\ \mathrm{C} & -4.633063335 & -0.331801025 & -1.868795133 \\ \mathrm{C} & -3.223365232 & -0.360891026 & -1.513388109 \\ \mathrm{C} & -2.763893200 & 0.237763016 & -0.305642021 \\ \mathrm{C} & -3.782943270 & 0.800950058 & 0.553650040 \\ \mathrm{C} & -5.131606370 & 0.820686057 & 0.209667016 \\ \mathrm{C} & -5.588237403 & 0.272216021 & -1.018426074 \\ \mathrm{H} & -4.936955356 & -0.769492058 & -2.800696203 \\ \mathrm{H} & -2.522336179 & -0.824436061 & -2.174906158 \\ \mathrm{H} & -5.808636419 & 1.269934093 & 0.909475067 \\ \mathrm{H} & -6.623034475 & 0.319895024 & -1.287803091 \\ \mathrm{C} & -1.378402099 & 0.302581022 & 0.075209004 \\ \mathrm{O} & -0.979869072 & 0.777618056 & 1.130651080 \\ \mathrm{O} & -3.459293246 & 1.326144095 & 1.704652124 \\ & & & \\ & & & \\ & & & \end{array}$




$\begin{array}{lrrr}\text { H } & -2.504746179 & 1.274509090 & 1.832740133 \\ \text { O } & -0.562139038 & -0.209438015 & -0.848354063 \\ \text { C } & 0.861324060 & -0.238336019 & -0.706716052 \\ \text { C } & 1.312616096 & -1.164443085 & 0.419964029 \\ \text { C } & 1.485229106 & 1.149940083 & -0.600791044 \\ \text { C } & 2.849608204 & -1.303258094 & 0.490878035 \\ \text { H } & 0.949123069 & -0.772306053 & 1.361601097 \\ \text { C } & 3.017343215 & 1.047402074 & -0.592781042 \\ \text { H } & 1.152350083 & 1.755727127 & -1.439699103 \\ \text { C } & 3.481574249 & 0.104802009 & 0.527530037 \\ \text { H } & 3.234666230 & 0.567817041 & 1.481867105 \\ \text { C } & 3.659307266 & 2.428828173 & -0.449076033 \\ \text { H } & 3.375396242 & 2.895420211 & 0.490266036 \\ \text { H } & 4.743142341 & 2.361879170 & -0.472172032 \\ \text { H } & 3.351188240 & 3.090232222 & -1.253602091 \\ \text { H } & 1.149985084 & 1.637665116 & 0.305871022 \\ \text { H } & 0.856870059 & -2.141453155 & 0.280313021 \\ \text { H } & 4.565442330 & 0.013695001 & 0.502737035 \\ \text { H } & 3.337449238 & 0.637660046 & -1.548986111 \\ \text { C } & 3.214340231 & -2.049568146 & 1.782318130 \\ \text { H } & 2.776546199 & -3.043829219 & 1.796079130 \\ \text { H } & 4.290995308 & -2.161139157 & 1.877156133 \\ \text { H } & 2.857092204 & -1.517691108 & 2.658865190 \\ \text { C } & 3.392817244 & -2.120745153 & -0.695160049 \\ \text { H } & 4.469507321 & -2.239466164 & -0.613111042 \\ \text { H } & 2.952877213 & -3.113903225 & -0.707982050 \\ \text { H } & 3.192196232 & -1.664410120 & -1.657204118 \\ \text { H } & 1.158526084 & -0.667788048 & -1.653815119\end{array}$

LIIC path connecting S1-KETO-MIN and T1-KETO-MIN Coord_1
C $\quad-4.633063335$
$-0.331801025$
$-1.868795133$
C -3.223365232
$-0.360891026$
$-1.513388109$
C -2.763893200
0.237763016
$-0.305642021$
C -3.782943270
0.800950058
0.553650040
C -5.131606370
0.820686057
0.209667016
C -5.588237403
0.272216021
$-1.018426074$
$\mathrm{H} \quad-4.936955356$
$-0.769492058$
$-2.800696203$
H $\quad-2.522336179$
$-0.824436061$
$-2.174906158$
H $\quad-5.808636419$
1.269934093
0.909475067
H $\quad-6.623034475$
0.319895024
$-1.287803091$
C -1.378402099
0.302581022
0.075209004
O $\quad-0.979869072$
0.777618056
1.130651080
O $\quad-3.459293246$
1.326144095
1.704652124
H $\quad-2.504746179$
1.274509090
1.832740133
O -0.562139038
$-0.209438015$
$-0.848354063$ 


$\begin{array}{lrrr}\text { C } & 0.861324060 & -0.238336019 & -0.706716052 \\ \text { C } & 1.312616096 & -1.164443085 & 0.419964029 \\ \text { C } & 1.485229106 & 1.149940083 & -0.600791044 \\ \text { C } & 2.849608204 & -1.303258094 & 0.490878035 \\ \text { H } & 0.949123069 & -0.772306053 & 1.361601097 \\ \text { C } & 3.017343215 & 1.047402074 & -0.592781042 \\ \text { H } & 1.152350083 & 1.755727127 & -1.439699103 \\ \text { C } & 3.481574249 & 0.104802009 & 0.527530037 \\ \text { H } & 3.234666230 & 0.567817041 & 1.481867105 \\ \text { C } & 3.659307266 & 2.428828173 & -0.449076033 \\ \text { H } & 3.375396242 & 2.895420211 & 0.490266036 \\ \text { H } & 4.743142341 & 2.361879170 & -0.472172032 \\ \text { H } & 3.351188240 & 3.090232222 & -1.253602091 \\ \text { H } & 1.149985084 & 1.637665116 & 0.305871022 \\ \text { H } & 0.856870059 & -2.141453155 & 0.280313021 \\ \text { H } & 4.565442330 & 0.013695001 & 0.502737035 \\ \text { H } & 3.337449238 & 0.637660046 & -1.548986111 \\ \text { C } & 3.214340231 & -2.049568146 & 1.782318130 \\ \text { H } & 2.776546199 & -3.043829219 & 1.796079130 \\ \text { H } & 4.290995308 & -2.161139157 & 1.877156133 \\ \text { H } & 2.857092204 & -1.517691108 & 2.658865190 \\ \text { C } & 3.392817244 & -2.120745153 & -0.695160049 \\ \text { H } & 4.469507321 & -2.239466164 & -0.613111042 \\ \text { H } & 2.952877213 & -3.113903225 & -0.707982050 \\ \text { H } & 3.192196232 & -1.664410120 & -1.657204118 \\ \text { H } & 1.158526084 & -0.667788048 & -1.653815119 \\ & & & \\ \text { Coord_2 } & & & \\ \text { C } & -4.619991334 & -0.340849025 & -1.870588134 \\ \text { C } & -3.224782231 & -0.382621028 & -1.504711110 \\ \text { C } & -2.767387199 & 0.228867017 & -0.293575023 \\ \text { C } & -3.791610272 & 0.800621058 & 0.564666040 \\ \text { C } & -5.128529368 & 0.831074060 & 0.206147014 \\ \text { C } & -5.577567401 & 0.283936022 & -1.031949072 \\ \text { H } & -4.923823357 & -0.785523054 & -2.799411201 \\ \text { H } & -2.519845183 & -0.861684064 & -2.151039153 \\ \text { H } & -5.813657417 & 1.281758093 & 0.897396067 \\ \text { H } & -6.607388476 & 0.350251023 & -1.316192096 \\ \text { C } & -1.378800099 & 0.304043022 & 0.079739005 \\ \text { O } & -0.980812071 & 0.783986059 & 1.131333084 \\ \text { O } & -3.468128247 & 1.315861096 & 1.724660125 \\ \text { H } & -2.514249181 & 1.272415093 & 1.849624131 \\ \text { O } & -0.564284042 & -0.205744014 & -0.845238061 \\ \text { C } & 0.860061062 & -0.235215017 & -0.705847053 \\ \text { C } & 1.311794093 & -1.162415082 & 0.419636029 \\ \text { C } & 1.484753105 & 1.152597082 & -0.599703045 \\ \text { C } & 2.848778205 & -1.302546091 & 0.488292036 \\ & & & \\ & & & \\ & & & \end{array}$




$\begin{array}{lrrr}\text { H } & 0.950215069 & -0.770541057 & 1.362217097 \\ \mathrm{C} & 3.016847217 & 1.048882077 & -0.593730042 \\ \mathrm{H} & 1.151169081 & 1.759355129 & -1.437589104 \\ \mathrm{C} & 3.481834250 & 0.105021009 & 0.525243038 \\ \mathrm{H} & 3.236698234 & 0.567463043 & 1.480315108 \\ \mathrm{C} & 3.659809264 & 2.429797176 & -0.449648031 \\ \mathrm{H} & 3.377516242 & 2.895691208 & 0.490520036 \\ \mathrm{H} & 4.743544341 & 2.362157168 & -0.474318036 \\ \mathrm{H} & 3.351002240 & 3.092116225 & -1.253136092 \\ \mathrm{H} & 1.151391082 & 1.639896117 & 0.307896020 \\ \mathrm{H} & 0.854991062 & -2.138934155 & 0.280133021 \\ \mathrm{H} & 4.565561326 & 0.013058999 & 0.498817038 \\ \mathrm{H} & 3.335424240 & 0.639751047 & -1.550680113 \\ \mathrm{C} & 3.214607232 & -2.050412146 & 1.778500127 \\ \mathrm{H} & 2.776008200 & -3.044314221 & 1.791878130 \\ \mathrm{H} & 4.291278306 & -2.162978154 & 1.871684134 \\ \mathrm{H} & 2.859090208 & -1.519102112 & 2.656096191 \\ \mathrm{C} & 3.389504245 & -2.119375155 & -0.699318048 \\ \mathrm{H} & 4.466164323 & -2.239188160 & -0.618811043 \\ \mathrm{H} & 2.948624210 & -3.112100223 & -0.712558052 \\ \mathrm{H} & 3.188105231 & -1.661880119 & -1.660650120 \\ \mathrm{H} & 1.155080082 & -0.664205047 & -1.653718120 \\ & & & \\ \mathrm{C} \text { oord_3 } & & & \\ \mathrm{C} & -4.606636334 & -0.349812028 & -1.872279135 \\ \mathrm{C} & -3.226173232 & -0.404369027 & -1.495890106 \\ \mathrm{C} & -2.770840197 & 0.219872015 & -0.281443020 \\ \mathrm{C} & -3.800230273 & 0.800219058 & 0.575657041 \\ \mathrm{C} & -5.125098368 & 0.841420063 & 0.202629012 \\ \mathrm{C} & -5.566178400 & 0.295785020 & -1.045571073 \\ \mathrm{H} & -4.910574351 & -0.801113055 & -2.798081199 \\ \mathrm{H} & -2.517776180 & -0.898856067 & -2.126832151 \\ \mathrm{H} & -5.818286416 & 1.293622094 & 0.885098062 \\ \mathrm{H} & -6.590376475 & 0.380848030 & -1.344885097 \\ \mathrm{C} & -1.379269098 & 0.305429022 & 0.084287008 \\ \mathrm{O} & -0.981879072 & 0.790258058 & 1.132026084 \\ \mathrm{O} & -3.477302249 & 1.305211091 & 1.744733125 \\ \mathrm{H} & -2.524228184 & 1.270031092 & 1.867060135 \\ \mathrm{O} & -0.566505042 & -0.202133014 & -0.842095060 \\ \mathrm{C} & 0.858721063 & -0.232146016 & -0.704974048 \\ \mathrm{C} & 1.310928095 & -1.160400081 & 0.419327032 \\ \mathrm{C} & 1.484175106 & 1.155215080 & -0.598657042 \\ \mathrm{C} & 2.847902205 & -1.301815091 & 0.485704036 \\ \mathrm{H} & 0.951270068 & -0.768775055 & 1.362844098 \\ \mathrm{C} & 3.016246215 & 1.050353073 & -0.594744041 \\ \mathrm{H} & 1.149863082 & 1.762916127 & -1.435531102 \\ \mathrm{C} & 3.482022251 & 0.105271008 & 0.522906038 \\ & & & \\ & & & \\ & & & \end{array}$




$\begin{array}{lrrr}\text { H } & 3.238665233 & 0.567156041 & 1.478700106 \\ \mathrm{C} & 3.660183266 & 2.430772174 & -0.450333030 \\ \mathrm{H} & 3.379515241 & 2.895986208 & 0.490651034 \\ \mathrm{H} & 4.743815344 & 2.362461170 & -0.476591032 \\ \mathrm{H} & 3.350665244 & 3.093979225 & -1.252793090 \\ \mathrm{H} & 1.152702081 & 1.642103119 & 0.309866025 \\ \mathrm{H} & 0.853085061 & -2.136439153 & 0.280007019 \\ \mathrm{H} & 4.565607327 & 0.012474999 & 0.494832037 \\ \mathrm{H} & 3.333287237 & 0.641817045 & -1.552431113 \\ \mathrm{C} & 3.214859232 & -2.051197149 & 1.774690130 \\ \mathrm{H} & 2.775474202 & -3.044746220 & 1.787718130 \\ \mathrm{H} & 4.291546308 & -2.164732154 & 1.866205133 \\ \mathrm{H} & 2.861078205 & -1.520440110 & 2.653324191 \\ \mathrm{C} & 3.386140244 & -2.118003152 & -0.703461051 \\ \mathrm{H} & 4.462771323 & -2.238885159 & -0.624510044 \\ \mathrm{H} & 2.944338213 & -3.110302221 & -0.717086053 \\ \mathrm{H} & 3.183942231 & -1.659377121 & -1.664088121 \\ \mathrm{H} & 1.151549083 & -0.660690046 & -1.653606120\end{array}$

Coord_4

$\begin{array}{lrrr}\text { C } & -4.592984328 & -0.358699025 & -1.873865133 \\ \text { C } & -3.227539234 & -0.426135030 & -1.486916107 \\ \text { C } & -2.774255200 & 0.210782014 & -0.269230017 \\ \text { C } & -3.808813274 & 0.799745058 & 0.586625043 \\ \text { C } & -5.121314370 & 0.851729060 & 0.199111015 \\ \text { C } & -5.554049398 & 0.307758021 & -1.059292078 \\ \text { H } & -4.897181351 & -0.816272057 & -2.796714202 \\ \text { H } & -2.516131179 & -0.935955065 & -2.102276152 \\ \text { H } & -5.822522417 & 1.305543092 & 0.872562065 \\ \text { H } & -6.571950471 & 0.411675028 & -1.373878100 \\ \text { C } & -1.379810097 & 0.306742022 & 0.088860005 \\ \text { O } & -0.983068070 & 0.796441055 & 1.132737081 \\ \text { O } & -3.486848252 & 1.294198093 & 1.764872124 \\ \text { H } & -2.534720181 & 1.267354090 & 1.885069138 \\ \text { O } & -0.568805042 & -0.198602015 & -0.838918060 \\ \text { C } & 0.857300063 & -0.229127018 & -0.704096053 \\ \text { C } & 1.310015095 & -1.158398082 & 0.419038032 \\ \text { C } & 1.483494108 & 1.157797084 & -0.597653044 \\ \text { C } & 2.846979204 & -1.301066094 & 0.483110036 \\ \text { H } & 0.952291068 & -0.767007058 & 1.363484101 \\ \text { C } & 3.015540218 & 1.051817074 & -0.595826044 \\ \text { H } & 1.148430081 & 1.766413126 & -1.433521101 \\ \text { C } & 3.482139252 & 0.105550006 & 0.520515035 \\ \text { H } & 3.240571235 & 0.566897040 & 1.477022106 \\ \text { C } & 3.660430264 & 2.431753174 & -0.451133035 \\ \text { H } & 3.381397244 & 2.896304207 & 0.490657035 \\ \text { H } & 4.743958343 & 2.362793171 & -0.478998032 \\ & & & \\ & & & \text { S166 }\end{array}$




$\begin{array}{llrr}\mathrm{H} & 3.350176242 & 3.095823223 & -1.252575090 \\ \mathrm{H} & 1.153917083 & 1.644287118 & 0.311785023 \\ \mathrm{H} & 0.851149061 & -2.133968154 & 0.279935019 \\ \mathrm{H} & 4.565579329 & 0.011941001 & 0.490775036 \\ \mathrm{H} & 3.331034239 & 0.643858045 & -1.554242111 \\ \mathrm{C} & 3.215097229 & -2.051924148 & 1.770884128 \\ \mathrm{H} & 2.774944199 & -3.045127217 & 1.783597130 \\ \mathrm{H} & 4.291801308 & -2.166407154 & 1.860713136 \\ \mathrm{H} & 2.863061207 & -1.521707109 & 2.650545190 \\ \mathrm{C} & 3.382721246 & -2.116629154 & -0.707595052 \\ \mathrm{H} & 4.459322320 & -2.238557159 & -0.630217046 \\ \mathrm{H} & 2.940014210 & -3.108509226 & -0.721568052 \\ \mathrm{H} & 3.179700230 & -1.656901122 & -1.667521122 \\ \mathrm{H} & 1.147927082 & -0.657241048 & -1.653479118\end{array}$

\section{Coord_5}

$\begin{array}{lrrr}\text { C } & -4.579018330 & -0.367520029 & -1.875342135 \\ \text { C } & -3.228878233 & -0.447919034 & -1.477775104 \\ \text { C } & -2.777632197 & 0.201598016 & -0.256922021 \\ \text { C } & -3.817369277 & 0.799202058 & 0.597576044 \\ \text { C } & -5.117174367 & 0.862004063 & 0.195586012 \\ \text { C } & -5.541153396 & 0.319850023 & -1.073109076 \\ \text { H } & -4.883614352 & -0.831007061 & -2.795318201 \\ \text { H } & -2.514914183 & -0.972979067 & -2.077365149 \\ \text { H } & -5.826365418 & 1.317530094 & 0.859769062 \\ \text { H } & -6.552062471 & 0.442719032 & -1.403159101 \\ \text { C } & -1.380424102 & 0.307988022 & 0.093468006 \\ \text { O } & -0.984375069 & 0.802540056 & 1.133472082 \\ \text { O } & -3.496800250 & 1.282826095 & 1.785077128 \\ \text { H } & -2.545759184 & 1.264381093 & 1.903669136 \\ \text { O } & -0.571190044 & -0.195148012 & -0.835703059 \\ \text { C } & 0.855794062 & -0.226156016 & -0.703209051 \\ \text { C } & 1.309055093 & -1.156407084 & 0.418770031 \\ \text { C } & 1.482706105 & 1.160344083 & -0.596689042 \\ \text { C } & 2.846008206 & -1.300299094 & 0.480508034 \\ \text { H } & 0.953280069 & -0.765236055 & 1.364139100 \\ \text { C } & 3.014727217 & 1.053274074 & -0.596977042 \\ \text { H } & 1.146866082 & 1.769849126 & -1.431557102 \\ \text { C } & 3.482185253 & 0.105858009 & 0.518065035 \\ \text { H } & 3.242418233 & 0.566684041 & 1.475276107 \\ \text { C } & 3.660549266 & 2.432741174 & -0.452051030 \\ \text { H } & 3.383162246 & 2.896645208 & 0.490536033 \\ \text { H } & 4.743971340 & 2.363150169 & -0.481544036 \\ \text { H } & 3.349535239 & 3.097647223 & -1.252483092 \\ \text { H } & 1.155039081 & 1.646452120 & 0.313657024 \\ \text { H } & 0.849180061 & -2.131519154 & 0.279919022 \\ \text { H } & 4.565478330 & 0.011453999 & 0.486640034 \\ & & & \\ & & & 5167\end{array}$




$\begin{array}{lrrr}\mathrm{H} & 3.328661239 & 0.645875046 & -1.556117113 \\ \mathrm{C} & 3.215324230 & -2.052595145 & 1.767077127 \\ \mathrm{H} & 2.774419197 & -3.045458217 & 1.779509130 \\ \mathrm{H} & 4.292045307 & -2.168004158 & 1.855199135 \\ \mathrm{H} & 2.865041208 & -1.522906108 & 2.647756188 \\ \mathrm{C} & 3.379241244 & -2.115254150 & -0.711724053 \\ \mathrm{H} & 4.455814320 & -2.238208161 & -0.635940045 \\ \mathrm{H} & 2.935646212 & -3.106722226 & -0.726011050 \\ \mathrm{H} & 3.175372227 & -1.654451121 & -1.670956122 \\ \mathrm{H} & 1.144207082 & -0.653855050 & -1.653334118\end{array}$

Coord_6

$\begin{array}{lrrr}\text { C } & -4.564720331 & -0.376284030 & -1.876703137 \\ \text { C } & -3.230188232 & -0.469718036 & -1.468459108 \\ \text { C } & -2.780973200 & 0.192322015 & -0.244502019 \\ \text { C } & -3.825906276 & 0.798592060 & 0.608514042 \\ \text { C } & -5.112675366 & 0.872247061 & 0.192052013 \\ \text { C } & -5.527461396 & 0.332055025 & -1.087019077 \\ \text { H } & -4.869842350 & -0.845326063 & -2.793900201 \\ \text { H } & -2.514128180 & -1.009928075 & -2.052090146 \\ \text { H } & -5.829809420 & 1.329599098 & 0.846698061 \\ \text { H } & -6.530657468 & 0.473965033 & -1.432719101 \\ \text { C } & -1.381111101 & 0.309169024 & 0.098119008 \\ \text { O } & -0.985796068 & 0.808560061 & 1.134238082 \\ \text { O } & -3.507193255 & 1.271099093 & 1.805346130 \\ \text { H } & -2.557381186 & 1.261110089 & 1.922877138 \\ \text { O } & -0.573662042 & -0.191766014 & -0.832442057 \\ \text { C } & 0.854200064 & -0.223229014 & -0.702313049 \\ \text { C } & 1.308045095 & -1.154427083 & 0.418522032 \\ \text { C } & 1.481811107 & 1.162859083 & -0.595763045 \\ \text { C } & 2.844988206 & -1.299515091 & 0.477893036 \\ \text { H } & 0.954236070 & -0.763461057 & 1.364811097 \\ \text { C } & 3.013804215 & 1.054724077 & -0.598200044 \\ \text { H } & 1.145166084 & 1.773226129 & -1.429637104 \\ \text { C } & 3.482159249 & 0.106193010 & 0.515554036 \\ \text { H } & 3.244209233 & 0.566515043 & 1.473459108 \\ \text { C } & 3.660540264 & 2.433736175 & -0.453091033 \\ \text { H } & 3.384815242 & 2.897009208 & 0.490287034 \\ \text { H } & 4.743853344 & 2.363531172 & -0.484235035 \\ \text { H } & 3.348738239 & 3.099454225 & -1.252519092 \\ \text { H } & 1.156067082 & 1.648598119 & 0.315482024 \\ \text { H } & 0.847175061 & -2.129091151 & 0.279959022 \\ \text { H } & 4.565303331 & 0.011011999 & 0.482420036 \\ \text { H } & 3.326161241 & 0.647870044 & -1.558058114 \\ \text { C } & 3.215540230 & -2.053213150 & 1.763264129 \\ \text { H } & 2.773899201 & -3.045741221 & 1.775452129 \\ \text { H } & 4.292277308 & -2.169526156 & 1.849657131 \\ & & & \\ & & & 5168\end{array}$




$\begin{array}{llll}\mathrm{H} & 2.867024205 & -1.524037108 & 2.644955189 \\ \mathrm{C} & 3.375694243 & -2.113879152 & -0.715854054 \\ \mathrm{H} & 4.452239320 & -2.237838160 & -0.641686047 \\ \mathrm{H} & 2.931226211 & -3.104940222 & -0.730418054 \\ \mathrm{H} & 3.170950226 & -1.652027119 & -1.674395118 \\ \mathrm{H} & 1.140384084 & -0.650529049 & -1.653172121\end{array}$

Coord_7

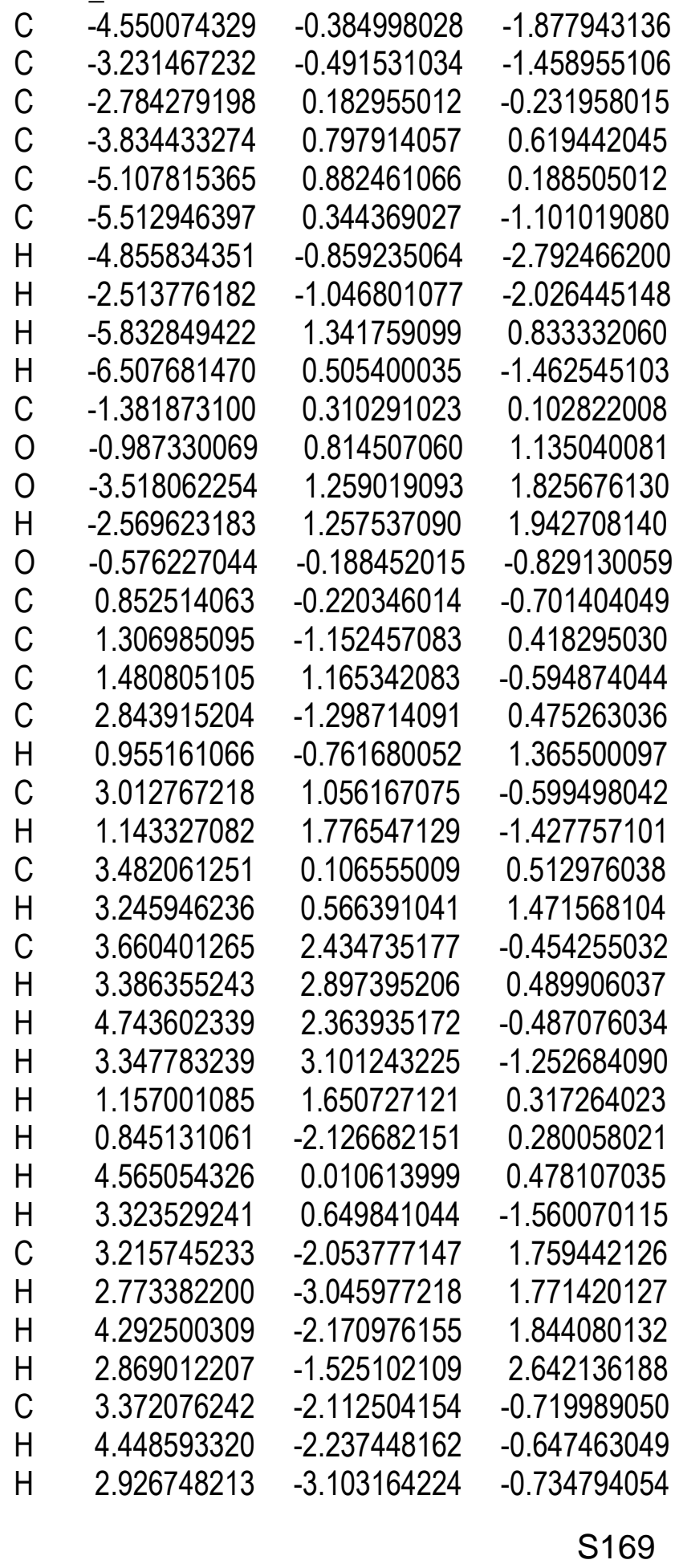




$\begin{array}{llrr}\text { H } & 3.166426226 & -1.649627120 & -1.677843121 \\ \text { H } & 1.136449079 & -0.647262046 & -1.652990121 \\ & & & \\ \text { Coord_8 } & & \\ \text { C } & -4.535060327 & -0.393670026 & -1.879054133 \\ \text { C } & -3.232714233 & -0.513357035 & -1.449254102 \\ \text { C } & -2.787552202 & 0.173501011 & -0.219273018 \\ \text { C } & -3.842958277 & 0.797170055 & 0.630364046 \\ \text { C } & -5.102587369 & 0.892647066 & 0.184943014 \\ \text { C } & -5.497576396 & 0.356784028 & -1.115103080 \\ \text { H } & -4.841556350 & -0.872740064 & -2.791023202 \\ \text { H } & -2.513863178 & -1.083593078 & -2.000424142 \\ \text { H } & -5.835477422 & 1.354021099 & 0.819651060 \\ \text { H } & -6.483075469 & 0.537007040 & -1.492622109 \\ \text { C } & -1.382711100 & 0.311356024 & 0.107583010 \\ \text { O } & -0.988974069 & 0.820385061 & 1.135886082 \\ \text { O } & -3.529441252 & 1.246592090 & 1.846065134 \\ \text { H } & -2.582523186 & 1.253658088 & 1.963174139 \\ \text { O } & -0.578888043 & -0.185204015 & -0.825762057 \\ \text { C } & 0.850732064 & -0.217503015 & -0.700481053 \\ \text { C } & 1.305870092 & -1.150494084 & 0.418091033 \\ \text { C } & 1.479685106 & 1.167795084 & -0.594020041 \\ \text { C } & 2.842788204 & -1.297896094 & 0.472615033 \\ \text { H } & 0.956054069 & -0.759894053 & 1.366209099 \\ \text { C } & 3.011615215 & 1.057604077 & -0.600872045 \\ \text { H } & 1.141345080 & 1.779815127 & -1.425915104 \\ \text { C } & 3.481889253 & 0.106943007 & 0.510329035 \\ \text { H } & 3.247630232 & 0.566308040 & 1.469600104 \\ \text { C } & 3.660130263 & 2.435741175 & -0.455546034 \\ \text { H } & 3.387785244 & 2.897802207 & 0.489392037 \\ \text { H } & 4.743217342 & 2.364361170 & -0.490074035 \\ \text { H } & 3.346666241 & 3.103015223 & -1.252982091 \\ \text { H } & 1.157842086 & 1.652841120 & 0.319005022 \\ \text { H } & 0.843045060 & -2.124291153 & 0.280215022 \\ \text { H } & 4.564727327 & 0.010259001 & 0.473697036 \\ \text { H } & 3.320760241 & 0.651790047 & -1.562155110 \\ \text { C } & 3.215938230 & -2.054290148 & 1.755604125 \\ \text { H } & 2.772870200 & -3.046166219 & 1.767410127 \\ \text { H } & 4.292713308 & -2.172356159 & 1.838459132 \\ \text { H } & 2.871007206 & -1.526102111 & 2.639297189 \\ \text { C } & 3.368379240 & -2.111128150 & -0.724134053 \\ \text { H } & 4.444869320 & -2.237040161 & -0.653278045 \\ \text { H } & 2.922205210 & -3.101394221 & -0.739144055 \\ \text { H } & 3.161793226 & -1.647253120 & -1.681304120 \\ \text { H } & 1.132397079 & -0.644051046 & -1.652787118\end{array}$

Coord_9 


$\begin{array}{lrrr}\text { C } & -4.519660327 & -0.402309030 & -1.880028137 \\ \text { C } & -3.233925234 & -0.535192036 & -1.439346104 \\ \text { C } & -2.790791200 & 0.163959014 & -0.206434013 \\ \text { C } & -3.851491277 & 0.796359059 & 0.641283047 \\ \text { C } & -5.096987367 & 0.902806063 & 0.181363014 \\ \text { C } & -5.481318394 & 0.369294027 & -1.129263080 \\ \text { H } & -4.826976347 & -0.885846065 & -2.789576199 \\ \text { H } & -2.514393181 & -1.120301082 & -1.974022140 \\ \text { H } & -5.837683419 & 1.366397100 & 0.805639060 \\ \text { H } & -6.456781467 & 0.568769042 & -1.522930111 \\ \text { C } & -1.383626100 & 0.312370023 & 0.112412006 \\ \text { O } & -0.990725069 & 0.826200057 & 1.136780080 \\ \text { O } & -3.541364256 & 1.233820090 & 1.866508135 \\ \text { H } & -2.596119189 & 1.249468089 & 1.984286141 \\ \text { O } & -0.581649042 & -0.182017013 & -0.822333057 \\ \text { C } & 0.848848060 & -0.214699016 & -0.699542049 \\ \text { C } & 1.304700091 & -1.148539081 & 0.417909027 \\ \text { C } & 1.478449107 & 1.170221087 & -0.593201044 \\ \text { C } & 2.841604202 & -1.297062095 & 0.469944032 \\ \text { H } & 0.956917067 & -0.758101052 & 1.366938099 \\ \text { C } & 3.010344216 & 1.059035078 & -0.602325044 \\ \text { H } & 1.139215084 & 1.783032128 & -1.424109102 \\ \text { C } & 3.481642249 & 0.107356009 & 0.507607037 \\ \text { H } & 3.249263236 & 0.566268039 & 1.467552104 \\ \text { C } & 3.659727263 & 2.436752174 & -0.456967034 \\ \text { H } & 3.389104245 & 2.898230211 & 0.488743038 \\ \text { H } & 4.742697340 & 2.364806171 & -0.493233038 \\ \text { H } & 3.345385240 & 3.104770224 & -1.253414090 \\ \text { H } & 1.158589083 & 1.654940118 & 0.320707025 \\ \text { H } & 0.840913058 & -2.121915153 & 0.280432022 \\ \text { H } & 4.564323326 & 0.009943002 & 0.469181032 \\ \text { H } & 3.317847237 & 0.653717045 & -1.564316111 \\ \text { C } & 3.216121230 & -2.054754146 & 1.751747126 \\ \text { H } & 2.772359200 & -3.046311219 & 1.763417125 \\ \text { H } & 4.292915311 & -2.173668159 & 1.832789130 \\ \text { H } & 2.873012205 & -1.527040109 & 2.636434192 \\ \text { C } & 3.364597242 & -2.109753152 & -0.728294053 \\ \text { H } & 4.441061318 & -2.236615163 & -0.659138048 \\ \text { H } & 2.917590208 & -3.099629224 & -0.743472053 \\ \text { H } & 3.157042225 & -1.644902117 & -1.684782121 \\ \text { H } & 1.128221082 & -0.640893049 & -1.652562117 \\ & & & \\ \text { Coord } 10 & & \\ \text { C } & -4.476113321 & -0.481194033 & -1.949121138 \\ \text { C } & -3.209544228 & -0.603623042 & -1.484494105 \\ \text { C } & -2.786358198 & 0.138406008 & -0.260524019 \\ \text { C } & -3.866226281 & 0.785224058 & 0.562952040 \\ & & & \\ & & & \text { S171 }\end{array}$




$\begin{array}{lrrr}\text { C } & -5.094509367 & 0.878948064 & 0.076434007 \\ \text { C } & -5.451021393 & 0.316153021 & -1.236240087 \\ \text { H } & -4.771547343 & -0.991176071 & -2.848319207 \\ \text { H } & -2.479074177 & -1.206181089 & -1.984044143 \\ \text { H } & -5.852999423 & 1.349294096 & 0.674056047 \\ \text { H } & -6.414597462 & 0.515493038 & -1.658327117 \\ \text { C } & -1.381386098 & 0.319620021 & 0.057922006 \\ \text { O } & -1.003388074 & 0.863891061 & 1.070240078 \\ \text { O } & -3.574797257 & 1.239668092 & 1.790889129 \\ \text { H } & -2.632705191 & 1.276946094 & 1.916856138 \\ \text { O } & -0.568331042 & -0.183185013 & -0.861294061 \\ \text { C } & 0.862295063 & -0.197715014 & -0.729067054 \\ \text { C } & 1.320058096 & -1.103579081 & 0.410293029 \\ \text { C } & 1.476384104 & 1.195708083 & -0.647131048 \\ \text { C } & 2.858045205 & -1.234964087 & 0.475077035 \\ \text { H } & 0.962351072 & -0.697319049 & 1.349033095 \\ \text { C } & 3.009424215 & 1.100439081 & -0.644105049 \\ \text { H } & 1.136261084 & 1.787896129 & -1.492464107 \\ \text { C } & 3.483203253 & 0.176587014 & 0.488094034 \\ \text { H } & 3.239897232 & 0.652523047 & 1.436977105 \\ \text { C } & 3.643276264 & 2.487666179 & -0.522701040 \\ \text { H } & 3.361662240 & 2.965313211 & 0.411696031 \\ \text { H } & 4.727130343 & 2.426479176 & -0.550645039 \\ \text { H } & 3.327173240 & 3.136000227 & -1.334546096 \\ \text { H } & 1.145795080 & 1.695632123 & 0.254701016 \\ \text { H } & 0.867174061 & -2.084333149 & 0.290127020 \\ \text { H } & 4.567027327 & 0.089726008 & 0.458472035 \\ \text { H } & 3.327511241 & 0.678922049 & -1.595609114 \\ \text { C } & 3.231870232 & -1.962628139 & 1.774339127 \\ \text { H } & 2.798272200 & -2.958309212 & 1.803291128 \\ \text { H } & 4.309255310 & -2.068659151 & 1.864546136 \\ \text { H } & 2.877638209 & -1.420743100 & 2.645981190 \\ \text { C } & 3.397049244 & -2.066275150 & -0.703109053 \\ \text { H } & 4.474243319 & -2.180604159 & -0.624505043 \\ \text { H } & 2.960265215 & -3.060807220 & -0.701092051 \\ \text { H } & 3.191162230 & -1.622997116 & -1.670141120 \\ \text { H } & 1.151761082 & -0.640378048 & -1.671388121\end{array}$

LIIC path connecting T1-KETO-MIN and $\mathrm{T}_{1} / \mathrm{S}_{0}-\mathrm{KETO}$ Coord_1
C -4.476113321
$-0.481194033$
$-1.949121138$
C -3.209544228
$-0.603623042-1.484494105$
C $\quad-2.786358198$
0.138406008
$-0.260524019$
C -3.866226281
0.785224058
0.562952040
C -5.094509367
0.878948064
0.076434007
C $\quad-5.451021393$
0.316153021
$-1.236240087$
H $\quad-4.771547343$
$-0.991176071$
$-2.848319207$ 


$\begin{array}{lrrr}\text { H } & -2.479074177 & -1.206181089 & -1.984044143 \\ \text { H } & -5.852999423 & 1.349294096 & 0.674056047 \\ \text { H } & -6.414597462 & 0.515493038 & -1.658327117 \\ \text { C } & -1.381386098 & 0.319620021 & 0.057922006 \\ \text { O } & -1.003388074 & 0.863891061 & 1.070240078 \\ \text { O } & -3.574797257 & 1.239668092 & 1.790889129 \\ \text { H } & -2.632705191 & 1.276946094 & 1.916856138 \\ \text { O } & -0.568331042 & -0.183185013 & -0.861294061 \\ \text { C } & 0.862295063 & -0.197715014 & -0.729067054 \\ \text { C } & 1.320058096 & -1.103579081 & 0.410293029 \\ \text { C } & 1.476384104 & 1.195708083 & -0.647131048 \\ \text { C } & 2.858045205 & -1.234964087 & 0.475077035 \\ \text { H } & 0.962351072 & -0.697319049 & 1.349033095 \\ \text { C } & 3.009424215 & 1.100439081 & -0.644105049 \\ \text { H } & 1.136261084 & 1.787896129 & -1.492464107 \\ \mathrm{C} & 3.483203253 & 0.176587014 & 0.488094034 \\ \text { H } & 3.239897232 & 0.652523047 & 1.436977105 \\ \text { C } & 3.643276264 & 2.487666179 & -0.522701040 \\ \text { H } & 3.361662240 & 2.965313211 & 0.411696031 \\ \text { H } & 4.727130343 & 2.426479176 & -0.550645039 \\ \text { H } & 3.327173240 & 3.136000227 & -1.334546096 \\ \text { H } & 1.145795080 & 1.695632123 & 0.254701016 \\ \text { H } & 0.867174061 & -2.084333149 & 0.290127020 \\ \text { H } & 4.567027327 & 0.089726008 & 0.458472035 \\ \text { H } & 3.327511241 & 0.678922049 & -1.595609114 \\ \text { C } & 3.231870232 & -1.962628139 & 1.774339127 \\ \text { H } & 2.798272200 & -2.958309212 & 1.803291128 \\ \text { H } & 4.309255310 & -2.068659151 & 1.864546136 \\ \text { H } & 2.877638209 & -1.420743100 & 2.645981190 \\ \text { C } & 3.397049244 & -2.066275150 & -0.703109053 \\ \text { H } & 4.474243319 & -2.180604159 & -0.624505043 \\ \text { H } & 2.960265215 & -3.060807220 & -0.701092051 \\ \text { H } & 3.191162230 & -1.622997116 & -1.670141120 \\ \text { H } & 1.151761082 & -0.640378048 & -1.671388121\end{array}$

Coord_2

$\begin{array}{lrrr}\text { C } & -4.495952324 & -0.518793040 & -1.927922139 \\ \text { C } & -3.206016230 & -0.578689041 & -1.494150108 \\ \text { C } & -2.785365202 & 0.162566013 & -0.273929021 \\ \text { C } & -3.851619279 & 0.799695056 & 0.546875037 \\ \text { C } & -5.099254367 & 0.897643066 & 0.056648006 \\ \text { C } & -5.482674394 & 0.244824018 & -1.209264086 \\ \text { H } & -4.793449344 & -1.065991076 & -2.804402200 \\ \text { H } & -2.466019179 & -1.156723082 & -2.008508145 \\ \text { H } & -5.798378419 & 1.523366111 & 0.582177044 \\ \text { H } & -6.477214466 & 0.365314027 & -1.587616114 \\ \text { C } & -1.377397097 & 0.324446022 & 0.056028007 \\ & & & S 173\end{array}$




$\begin{array}{lrrr}\text { O } & -1.000749072 & 0.851831059 & 1.076609076 \\ \text { O } & -3.579531255 & 1.268303090 & 1.772683130 \\ \text { H } & -2.638944191 & 1.294104095 & 1.915082140 \\ \text { O } & -0.565158042 & -0.172125013 & -0.864819064 \\ \text { C } & 0.865833063 & -0.191299015 & -0.730649051 \\ \text { C } & 1.320442093 & -1.105337082 & 0.403369031 \\ \text { C } & 1.481260107 & 1.200766087 & -0.638687046 \\ \text { C } & 2.858231205 & -1.238773089 & 0.469676035 \\ \text { H } & 0.961468070 & -0.704784052 & 1.343927096 \\ \text { C } & 3.014091215 & 1.103607080 & -0.634395043 \\ \text { H } & 1.142944085 & 1.798835128 & -1.480608108 \\ \text { C } & 3.485144248 & 0.171896011 & 0.492402034 \\ \text { H } & 3.241225234 & 0.642111045 & 1.443975104 \\ \text { C } & 3.649510263 & 2.489216177 & -0.503403036 \\ \text { H } & 3.367133240 & 2.961305212 & 0.433584030 \\ \text { H } & 4.733319341 & 2.426818172 & -0.530234037 \\ \text { H } & 3.335313240 & 3.142998226 & -1.311618094 \\ \text { H } & 1.149547084 & 1.694770120 & 0.266027018 \\ \text { H } & 0.866770061 & -2.084801148 & 0.275806018 \\ \text { H } & 4.568896328 & 0.083759006 & 0.463614035 \\ \text { H } & 3.332708238 & 0.687727050 & -1.588225112 \\ \text { C } & 3.229131232 & -1.974498140 & 1.765251126 \\ \text { H } & 2.794502199 & -2.969903216 & 1.787596128 \\ \text { H } & 4.306269311 & -2.082098147 & 1.856635132 \\ \text { H } & 2.873885205 & -1.437366103 & 2.639404189 \\ \text { C } & 3.398283243 & -2.063507151 & -0.712630052 \\ \text { H } & 4.475245322 & -2.179387157 & -0.633026046 \\ \text { H } & 2.960486215 & -3.057589219 & -0.717279049 \\ \text { H } & 3.194327229 & -1.614140118 & -1.677288120 \\ \text { H } & 1.155350084 & -0.628234044 & -1.675609120\end{array}$

Coord_3

$\begin{array}{lrrr}\text { C } & -4.516273323 & -0.555033040 & -1.902798137 \\ \text { C } & -3.205747228 & -0.553202039 & -1.503179110 \\ \text { C } & -2.784967203 & 0.186967011 & -0.287419019 \\ \text { C } & -3.836228275 & 0.815654057 & 0.531732037 \\ \text { C } & -5.102284367 & 0.918357064 & 0.036136001 \\ \text { C } & -5.509944396 & 0.176567011 & -1.175338083 \\ \text { H } & -4.814755348 & -1.138788080 & -2.755161199 \\ \text { H } & -2.459194178 & -1.107650079 & -2.033843146 \\ \text { H } & -5.732976413 & 1.680230123 & 0.461657031 \\ \text { H } & -6.528537468 & 0.220198014 & -1.503584111 \\ \text { C } & -1.374288101 & 0.329454023 & 0.053780003 \\ \text { O } & -0.999227074 & 0.840161060 & 1.082263075 \\ \text { O } & -3.580943259 & 1.295916093 & 1.755379124 \\ \text { H } & -2.642106189 & 1.308655093 & 1.913238137 \\ \text { O } & -0.562770040 & -0.161055011 & -0.868478065 \\ & & & \\ & & & S 174\end{array}$




$\begin{array}{lrrr}\text { C } & 0.868563061 & -0.184851011 & -0.732313054 \\ \text { C } & 1.319950096 & -1.106784080 & 0.396524028 \\ \text { C } & 1.485297105 & 1.205812088 & -0.630587047 \\ \text { C } & 2.857532204 & -1.242259092 & 0.464466031 \\ \text { H } & 0.959640070 & -0.711750052 & 1.338755097 \\ \text { C } & 3.017915219 & 1.106767078 & -0.624926046 \\ \text { H } & 1.148838082 & 1.809559132 & -1.469204105 \\ \text { C } & 3.486175250 & 0.167474010 & 0.496639034 \\ \text { H } & 3.241572232 & 0.632150046 & 1.450752102 \\ \text { C } & 3.654868262 & 2.490728179 & -0.484608036 \\ \text { H } & 3.371658245 & 2.957441213 & 0.454818035 \\ \text { H } & 4.738631343 & 2.427112173 & -0.510253034 \\ \text { H } & 3.342625242 & 3.149763228 & -1.289312093 \\ \text { H } & 1.152389083 & 1.694068124 & 0.276841020 \\ \text { H } & 0.865516064 & -2.084938152 & 0.261748017 \\ \text { H } & 4.569855329 & 0.078057005 & 0.468762035 \\ \text { H } & 3.337134240 & 0.696339050 & -1.580940114 \\ \text { C } & 3.225426235 & -1.985747141 & 1.756479125 \\ \text { H } & 2.789780202 & -2.980834213 & 1.772400126 \\ \text { H } & 4.302309308 & -2.094900152 & 1.849122133 \\ \text { H } & 2.869102205 & -1.453199102 & 2.632979189 \\ \text { C } & 3.398722243 & -2.060641148 & -0.721712052 \\ \text { H } & 4.475445324 & -2.178056155 & -0.641027047 \\ \text { H } & 2.959929214 & -3.054235220 & -0.732845052 \\ \text { H } & 3.196758232 & -1.605403116 & -1.684065123 \\ \text { H } & 1.158202085 & -0.616236045 & -1.679767119 \\ & & & \\ \text { Coord_4 } & & & \\ \text { C } & -4.536825329 & -0.589477044 & -1.873982134 \\ \text { C } & -3.208496230 & -0.526733037 & -1.511804107 \\ \text { C } & -2.785112202 & 0.211929016 & -0.301076020 \\ \text { C } & -3.820181277 & 0.833237062 & 0.517304036 \\ \text { C } & -5.103644369 & 0.940986069 & 0.014422001 \\ \text { C } & -5.532751401 & 0.111953009 & -1.134778081 \\ \text { H } & -4.835143346 & -1.208988088 & -2.700924193 \\ \text { H } & -2.458246179 & -1.058502075 & -2.060240148 \\ \text { H } & -5.658303407 & 1.815032132 & 0.315276021 \\ \text { H } & -6.568447471 & 0.081390006 & -1.406777102 \\ \text { C } & -1.372002097 & 0.334881022 & 0.051127005 \\ \text { O } & -0.998742072 & 0.829093059 & 1.087171078 \\ \text { O } & -3.579316256 & 1.322785094 & 1.738911122 \\ \text { H } & -2.642499188 & 1.320906097 & 1.911310138 \\ \text { O } & -0.561128040 & -0.149780011 & -0.872319061 \\ \text { C } & 0.870520065 & -0.178266014 & -0.734087052 \\ \text { C } & 1.318547094 & -1.107858077 & 0.389730027 \\ \text { C } & 1.488615105 & 1.210920088 & -0.622836045 \\ \text { C } & 2.855905207 & -1.245456090 & 0.459438032 \\ & & & \\ & & & \\ & & & \end{array}$




$\begin{array}{rrrr}\text { H } & 0.956845067 & -0.718142052 & 1.333492096 \\ \mathrm{C} & 3.021010218 & 1.109897082 & -0.615683045 \\ \mathrm{H} & 1.154112085 & 1.820174131 & -1.458256105 \\ \mathrm{C} & 3.486339252 & 0.163255013 & 0.500823038 \\ \mathrm{H} & 3.240995233 & 0.622581045 & 1.457332104 \\ \mathrm{C} & 3.659548264 & 2.492147178 & -0.466272034 \\ \mathrm{H} & 3.375450244 & 2.953672212 & 0.475444036 \\ \mathrm{H} & 4.743260343 & 2.427241176 & -0.490646033 \\ \mathrm{H} & 3.349357239 & 3.156268229 & -1.267587092 \\ \mathrm{H} & 1.154459082 & 1.693611121 & 0.287144022 \\ \mathrm{H} & 0.863321063 & -2.084656148 & 0.247908016 \\ \mathrm{H} & 4.569940331 & 0.072488007 & 0.473948036 \\ \mathrm{H} & 3.340889239 & 0.704728049 & -1.573742113 \\ \mathrm{C} & 3.220650230 & -1.996448143 & 1.748018125 \\ \mathrm{H} & 2.783939202 & -2.991151217 & 1.757679124 \\ \mathrm{H} & 4.297260311 & -2.107204152 & 1.842013134 \\ \mathrm{H} & 2.863203207 & -1.468299106 & 2.626704189 \\ \mathrm{C} & 3.398290244 & -2.057731149 & -0.730370053 \\ \mathrm{H} & 4.474757320 & -2.176734159 & -0.648508047 \\ \mathrm{H} & 2.958455212 & -3.050778221 & -0.747821054 \\ \mathrm{H} & 3.198419229 & -1.596820115 & -1.690487123 \\ \mathrm{H} & 1.160341083 & -0.604287043 & -1.683895120\end{array}$

Coord_5

$\begin{array}{lrrr}\text { C } & -4.557206326 & -0.621768043 & -1.841726134 \\ \text { C } & -3.213964229 & -0.498798035 & -1.520164107 \\ \text { C } & -2.785750200 & 0.237847017 & -0.314903025 \\ \text { C } & -3.803675275 & 0.852626063 & 0.503388034 \\ \text { C } & -5.103417368 & 0.965355068 & -0.009063002 \\ \text { C } & -5.550882400 & 0.051417005 & -1.088015079 \\ \text { H } & -4.854024348 & -1.276143094 & -2.642066189 \\ \text { H } & -2.462713175 & -1.008705072 & -2.087731149 \\ \text { H } & -5.575993400 & 1.923230136 & 0.146204011 \\ \text { H } & -6.596630477 & -0.050123002 & -1.297982091 \\ \text { C } & -1.370482099 & 0.341014022 & 0.048077002 \\ \text { O } & -0.999200070 & 0.818870058 & 1.091363077 \\ \text { O } & -3.575085256 & 1.349196098 & 1.723215122 \\ \text { H } & -2.640610189 & 1.331217095 & 1.909322135 \\ \text { O } & -0.560213040 & -0.138057010 & -0.876352064 \\ \text { C } & 0.871719064 & -0.171409015 & -0.735984052 \\ \text { C } & 1.316181095 & -1.108484078 & 0.382958029 \\ \text { C } & 1.491330107 & 1.216181089 & -0.615415043 \\ \text { C } & 2.853286208 & -1.248403088 & 0.454558034 \\ \text { H } & 0.953059068 & -0.723876053 & 1.328121095 \\ \text { C } & 3.023484217 & 1.112976078 & -0.606653042 \\ \text { H } & 1.158925084 & 1.830816129 & -1.447736104 \\ \text { C } & 3.485676251 & 0.159156011 & 0.504949038 \\ & & & \\ & & & \text { S176 }\end{array}$




$\begin{array}{llll}\text { H } & 3.239569232 & 0.613320046 & 1.463722104 \\ \mathrm{C} & 3.663758265 & 2.493402180 & -0.448355034 \\ \mathrm{H} & 3.378753242 & 2.949932214 & 0.495518036 \\ \mathrm{H} & 4.747408341 & 2.427054175 & -0.471375033 \\ \mathrm{H} & 3.355766242 & 3.162487226 & -1.246390087 \\ \mathrm{H} & 1.155910081 & 1.693499121 & 0.296969023 \\ \mathrm{H} & 0.860060062 & -2.083847152 & 0.234237018 \\ \mathrm{H} & 4.569185327 & 0.066887004 & 0.479161035 \\ \mathrm{H} & 3.344051242 & 0.712865049 & -1.566631114 \\ \mathrm{C} & 3.214685233 & -2.006696147 & 1.739818127 \\ \mathrm{H} & 2.776790202 & -3.000922215 & 1.743365128 \\ \mathrm{H} & 4.290996308 & -2.119190155 & 1.835256133 \\ \mathrm{H} & 2.856112205 & -1.482753107 & 2.620545190 \\ \mathrm{C} & 3.396859242 & -2.054838148 & -0.738661054 \\ \mathrm{H} & 4.473045320 & -2.175560158 & -0.655530049 \\ \mathrm{H} & 2.955865212 & -3.047246217 & -0.762285055 \\ \mathrm{H} & 3.199216228 & -1.588410112 & -1.696604121 \\ \mathrm{H} & 1.161747086 & -0.592256045 & -1.688018120\end{array}$

Coord_6

$\begin{array}{lrrr}\text { C } & -4.576792330 & -0.651649047 & -1.806312132 \\ \text { C } & -3.221749231 & -0.468842036 & -1.528269108 \\ \text { C } & -2.786838199 & 0.265232019 & -0.328763024 \\ \text { C } & -3.787036270 & 0.874074062 & 0.489816034 \\ \text { C } & -5.101753370 & 0.991173070 & -0.035021003 \\ \text { C } & -5.563959401 & -0.004781000 & -1.035621075 \\ \text { H } & -4.870413349 & -1.339959097 & -2.579025186 \\ \text { H } & -2.471941180 & -0.957530071 & -2.116119154 \\ \text { H } & -5.487832396 & 2.000493142 & -0.042058002 \\ \text { H } & -6.612537477 & -0.173672015 & -1.178277085 \\ \text { C } & -1.369673098 & 0.348218024 & 0.044735004 \\ \text { O } & -1.000471074 & 0.809796060 & 1.094958080 \\ \text { O } & -3.568956256 & 1.375526100 & 1.708249122 \\ \text { H } & -2.637210188 & 1.340128095 & 1.907377139 \\ \text { O } & -0.560045042 & -0.125566009 & -0.880516064 \\ \text { C } & 0.872141062 & -0.164104014 & -0.737983051 \\ \text { C } & 1.312773092 & -1.108567079 & 0.376185026 \\ \text { C } & 1.493558107 & 1.221710086 & -0.608276045 \\ \text { C } & 2.849582205 & -1.251157091 & 0.449750030 \\ \text { H } & 0.948266067 & -0.728854050 & 1.322645095 \\ \text { C } & 3.025440220 & 1.115970079 & -0.597836044 \\ \text { H } & 1.163431081 & 1.841660130 & -1.437566106 \\ \text { C } & 3.484227253 & 0.155061010 & 0.508970039 \\ \text { H } & 3.237409231 & 0.604244042 & 1.469902106 \\ \text { C } & 3.667740266 & 2.494399182 & -0.430831033 \\ \text { H } & 3.381878245 & 2.946123212 & 0.515093038 \\ \text { H } & 4.751309340 & 2.426351174 & -0.452449035 \\ & & & \\ & & & \text { S177 }\end{array}$




$\begin{array}{lrrr}\mathrm{H} & 3.362135240 & 3.168381229 & -1.225663088 \\ \mathrm{H} & 1.156930086 & 1.693849124 & 0.306390023 \\ \mathrm{H} & 0.855556059 & -2.082370150 & 0.220693017 \\ \mathrm{H} & 4.567621328 & 0.061036002 & 0.484320037 \\ \mathrm{H} & 3.346659240 & 0.720718050 & -1.559634110 \\ \mathrm{C} & 3.207403230 & -2.016628148 & 1.731773125 \\ \mathrm{H} & 2.768107197 & -3.010241217 & 1.729328125 \\ \mathrm{H} & 4.283379309 & -2.131097151 & 1.828707130 \\ \mathrm{H} & 2.847774202 & -1.496689106 & 2.614426187 \\ \mathrm{C} & 3.394227242 & -2.052030148 & -0.746706056 \\ \mathrm{H} & 4.470095321 & -2.174713157 & -0.662251050 \\ \mathrm{H} & 2.951861214 & -3.043668217 & -0.776378056 \\ \mathrm{H} & 3.198964229 & -1.580194112 & -1.702516121 \\ \mathrm{H} & 1.162334086 & -0.579964041 & -1.692141120\end{array}$

\section{Coord_7}

$\begin{array}{lrrr}\text { C } & -4.594641330 & -0.679006050 & -1.768054125 \\ \text { C } & -3.231293233 & -0.436205029 & -1.535926112 \\ \text { C } & -2.788344200 & 0.294784023 & -0.342294022 \\ \text { C } & -3.770791270 & 0.897957064 & 0.476484033 \\ \text { C } & -5.098920366 & 1.017979073 & -0.064335005 \\ \text { C } & -5.571412402 & -0.056613007 & -0.978367071 \\ \text { H } & -4.882739353 & -1.400334099 & -2.512333179 \\ \text { H } & -2.484958178 & -0.904031066 & -2.144859157 \\ \text { H } & -5.395737388 & 2.042545149 & -0.245721016 \\ \text { H } & -6.615343476 & -0.288984018 & -1.049124075 \\ \text { C } & -1.369517097 & 0.356992026 & 0.041367003 \\ \text { O } & -1.002365074 & 0.802269059 & 1.098227078 \\ \text { O } & -3.562067258 & 1.402342099 & 1.694008120 \\ \text { H } & -2.633527189 & 1.348515094 & 1.905724138 \\ \text { O } & -0.560694041 & -0.111876008 & -0.884632063 \\ \text { C } & 0.871715063 & -0.156113009 & -0.740015054 \\ \text { C } & 1.308204097 & -1.107982079 & 0.369405027 \\ \text { C } & 1.495420107 & 1.227658086 & -0.601327043 \\ \text { C } & 2.844661206 & -1.253799093 & 0.444890034 \\ \text { H } & 0.942467067 & -0.732946051 & 1.317107097 \\ \text { C } & 3.026986216 & 1.118828080 & -0.589259044 \\ \text { H } & 1.167773086 & 1.852943131 & -1.427600105 \\ \text { C } & 3.482051250 & 0.150803012 & 0.512783036 \\ \text { H } & 3.234696235 & 0.595175045 & 1.475812105 \\ \text { C } & 3.671792266 & 2.494993178 & -0.413702031 \\ \text { H } & 3.385252242 & 2.942093210 & 0.534210037 \\ \text { H } & 4.755250340 & 2.424849173 & -0.433955031 \\ \text { H } & 3.368796243 & 3.173884228 & -1.205356085 \\ \text { H } & 1.157765085 & 1.694815121 & 0.315554024 \\ \text { H } & 0.849554062 & -2.080032150 & 0.207254016 \\ \text { H } & 4.565288329 & 0.054627004 & 0.489238035 \\ & & & \\ & & & 5178\end{array}$




$\begin{array}{lrrr}\mathrm{H} & 3.348697239 & 0.728240051 & -1.552821111 \\ \mathrm{C} & 3.198657232 & -2.026431146 & 1.723693123 \\ \mathrm{H} & 2.757615200 & -3.019242215 & 1.715365121 \\ \mathrm{H} & 4.274255309 & -2.143263153 & 1.822092129 \\ \mathrm{H} & 2.838176202 & -1.510291110 & 2.608210190 \\ \mathrm{C} & 3.390069242 & -2.049404148 & -0.754713053 \\ \mathrm{H} & 4.465573322 & -2.174433158 & -0.668964050 \\ \mathrm{H} & 2.945985214 & -3.040082221 & -0.790324057 \\ \mathrm{H} & 3.197334231 & -1.572196111 & -1.708397122 \\ \mathrm{H} & 1.161909083 & -0.567165042 & -1.696238121\end{array}$

Coord_8

$\begin{array}{lrrr}\text { C } & -4.609341329 & -0.703916048 & -1.727319124 \\ \text { C } & -3.241793232 & -0.400088031 & -1.542642112 \\ \text { C } & -2.790249201 & 0.327486024 & -0.354796025 \\ \text { C } & -3.755777268 & 0.924864065 & 0.463374032 \\ \text { C } & -5.095347367 & 1.045068074 & -0.098171005 \\ \text { C } & -5.572421401 & -0.104357008 & -0.917304064 \\ \text { H } & -4.888585354 & -1.457411104 & -2.442658178 \\ \text { H } & -2.500293179 & -0.846970063 & -2.172905154 \\ \text { H } & -5.301655383 & 2.044910147 & -0.460703031 \\ \text { H } & -6.603905474 & -0.396276029 & -0.912489065 \\ \text { C } & -1.369952097 & 0.368029029 & 0.038477002 \\ \text { O } & -1.004599075 & 0.796840060 & 1.101659078 \\ \text { O } & -3.556217256 & 1.430537102 & 1.680548121 \\ \text { H } & -2.631470192 & 1.357802097 & 1.904856139 \\ \text { O } & -0.562311042 & -0.096378005 & -0.888345062 \\ \text { C } & 0.870295064 & -0.147107010 & -0.741926051 \\ \text { C } & 1.302299093 & -1.106551078 & 0.362646026 \\ \text { C } & 1.497048110 & 1.234229086 & -0.594407045 \\ \text { C } & 2.838325203 & -1.256441090 & 0.439778034 \\ \text { H } & 0.935682067 & -0.735971055 & 1.311612093 \\ \text { C } & 3.028230217 & 1.121467082 & -0.580986041 \\ \text { H } & 1.172092083 & 1.864982136 & -1.417592104 \\ \text { C } & 3.479220252 & 0.146145008 & 0.516197039 \\ \text { H } & 3.231718232 & 0.585853043 & 1.481324106 \\ \text { C } & 3.676304264 & 2.494969180 & -0.397031027 \\ \text { H } & 3.389473246 & 2.937617212 & 0.552880040 \\ \text { H } & 4.759601341 & 2.422130175 & -0.416110031 \\ \text { H } & 3.376162242 & 3.178891229 & -1.185441087 \\ \text { H } & 1.158759081 & 1.696601121 & 0.324699022 \\ \text { H } & 0.841673062 & -2.076560149 & 0.193945012 \\ \text { H } & 4.562238331 & 0.047220005 & 0.493563038 \\ \text { H } & 3.350073243 & 0.735352055 & -1.546329112 \\ \text { C } & 3.188278230 & -2.036370147 & 1.715279125 \\ \text { H } & 2.744958196 & -3.028102219 & 1.701170122 \\ \text { H } & 4.263441308 & -2.156157154 & 1.814954131 \\ & & & \\ & & & 5179\end{array}$




$\begin{array}{llll}\mathrm{H} & 2.827365201 & -1.523815107 & 2.601688186 \\ \mathrm{C} & 3.383878244 & -2.047095147 & -0.763014055 \\ \mathrm{H} & 4.458954320 & -2.175061154 & -0.676161051 \\ \mathrm{H} & 2.937541212 & -3.036536220 & -0.804461059 \\ \mathrm{H} & 3.193780229 & -1.564450115 & -1.714520126 \\ \mathrm{H} & 1.160122083 & -0.553519037 & -1.700228123\end{array}$

Coord_9

$\begin{array}{lrrr}\text { C } & -4.618838330 & -0.726719053 & -1.684555120 \\ \text { C } & -3.252096234 & -0.359544026 & -1.547521110 \\ \text { C } & -2.792557202 & 0.364704028 & -0.365117024 \\ \text { C } & -3.743264268 & 0.955688068 & 0.450561031 \\ \text { C } & -5.091641364 & 1.071402077 & -0.138113008 \\ \text { C } & -5.565868403 & -0.148652012 & -0.853876059 \\ \text { H } & -4.884382353 & -1.511629108 & -2.370844168 \\ \text { H } & -2.515750182 & -0.784765055 & -2.198532160 \\ \text { H } & -5.207280373 & 2.002512143 & -0.682470050 \\ \text { H } & -6.576748473 & -0.496294037 & -0.770998054 \\ \text { C } & -1.370911098 & 0.382290027 & 0.036885004 \\ \text { O } & -1.006772073 & 0.794272058 & 1.106038078 \\ \text { O } & -3.554124254 & 1.461479107 & 1.667989121 \\ \text { H } & -2.633892189 & 1.370178098 & 1.905590139 \\ \text { O } & -0.565158042 & -0.078234004 & -0.891062064 \\ \text { C } & 0.867631064 & -0.136630010 & -0.743459051 \\ \text { C } & 1.294792094 & -1.104025077 & 0.355983028 \\ \text { C } & 1.498582110 & 1.241696089 & -0.587278043 \\ \text { C } & 2.830281206 & -1.259238093 & 0.434125029 \\ \text { H } & 0.927953069 & -0.737668053 & 1.306355093 \\ \text { C } & 3.029280216 & 1.123765082 & -0.573137040 \\ \text { H } & 1.176514084 & 1.878205137 & -1.407171101 \\ \text { C } & 3.475824252 & 0.140753009 & 0.518898039 \\ \text { H } & 3.228893235 & 0.575921042 & 1.486225107 \\ \text { C } & 3.681781265 & 2.494013179 & -0.380962025 \\ \text { H } & 3.395376244 & 2.932367213 & 0.571064041 \\ \text { H } & 4.764841344 & 2.417602175 & -0.399318031 \\ \text { H } & 3.384745243 & 3.183229228 & -1.165937085 \\ \text { H } & 1.160374082 & 1.699486121 & 0.334184027 \\ \text { H } & 0.831357059 & -2.071565149 & 0.180862015 \\ \text { H } & 4.558523327 & 0.038206005 & 0.496725036 \\ \text { H } & 3.350580242 & 0.741938053 & -1.540390113 \\ \mathrm{C} & 3.176047229 & -2.046797146 & 1.706092121 \\ \text { H } & 2.729653199 & -3.037056216 & 1.686316122 \\ \text { H } & 4.250707308 & -2.170418158 & 1.806594131 \\ \text { H } & 2.815452204 & -1.537600111 & 2.594548188 \\ \text { C } & 3.374885242 & -2.045286149 & -0.772096055 \\ \text { H } & 4.449455318 & -2.177066159 & -0.684588050 \\ \text { H } & 2.925492211 & -3.033090218 & -0.819264057 \\ & & & \\ & & & \\ & & & \\ & & \end{array}$




\begin{tabular}{|c|c|c|c|}
\hline & 3.187454227 & -1.557011110 & -1.721286123 \\
\hline & 1.156402083 & -0.538556037 & -1.703954123 \\
\hline \multicolumn{4}{|c|}{ Coord_10 } \\
\hline 0 & -4.603230332 & -0.719979054 & -1.903864138 \\
\hline C & -3.255434236 & -0.281120021 & -1.712338122 \\
\hline C & -2.862792205 & 0.371422029 & -0.469667034 \\
\hline$\tau$ & -3.851508279 & 0.897261063 & 0.320994024 \\
\hline C & -5.170152371 & 1.028167074 & -0.369388025 \\
\hline$C$ & -5.584156404 & -0.222391015 & -1.073803076 \\
\hline H & -4.802985345 & -1.496096110 & -2.621813189 \\
\hline $\mathrm{H}$ & -2.483353178 & -0.635941045 & -2.364181171 \\
\hline $\mathrm{H}$ & -5.165166373 & 1.883688133 & -1.038534074 \\
\hline $\mathrm{H}$ & -6.568807475 & -0.638906046 & -0.990238072 \\
\hline C & -1.464137106 & 0.350570027 & 0.015913000 \\
\hline 0 & -1.163424085 & 57050 & 751084 \\
\hline 0 & -3.748081268 & 1.329093093 & 277113 \\
\hline $\mathrm{H}$ & -2.847158203 & 1.212758090 & 698134 \\
\hline 0 & -0.607126042 & -0.0 & 1062 \\
\hline C & 0.816382057 & -0.10 & -0.6 \\
\hline C & 1.189260085 & -1.145757085 & 0.381987026 \\
\hline C & 1.427956104 & 1.258412090 & -0.390813030 \\
\hline C & 2.719290194 & -1.300439093 & 0.534087040 \\
\hline $\mathrm{H}$ & 0.768153054 & -0.84 & 57097 \\
\hline C & 2.956322215 & 1.144983084 & -0.300784020 \\
\hline $\mathrm{H}$ & 1.146534081 & 1.947008139 & -1.183119084 \\
\hline C & 3.349503239 & 0.092994006 & 0.746490051 \\
\hline $\mathrm{H}$ & 3.047278222 & 0.46 & 3122 \\
\hline C & 3.587823256 & 2.50 & 0.017052001 \\
\hline $\mathrm{H}$ & 3.246828232 & 2.874787209 & 0.978810072 \\
\hline $\mathrm{H}$ & 4.670772336 & 2.430146173 & 0.053136005 \\
\hline $\mathrm{H}$ & 3.32 & 3.2 & -0.7 \\
\hline $\mathrm{H}$ & 1.03 & 1.65 & 0.5 \\
\hline $\mathrm{H}$ & 0.743118054 & -2.101065151 & 0.117533008 \\
\hline $\mathrm{H}$ & 4.432491319 & -0.004335999 & 0.776898058 \\
\hline $\mathrm{H}$ & 3.332279239 & 0.829349059 & -1.272218091 \\
\hline C & 3.0004 & -2.1 & 1.76 \\
\hline $\mathrm{H}$ & 2.562910183 & -3.157407225 & 1.659296120 \\
\hline $\mathrm{H}$ & 4.068828294 & -2.295159164 & 1.920013140 \\
\hline $\mathrm{H}$ & 2.587933187 & -1.720811123 & 2.667357194 \\
\hline C & 3.334740242 & -2.003061144 & -0.689407050 \\
\hline $\mathrm{H}$ & 4.403838319 & -2.136511153 & -0.551713040 \\
\hline $\mathrm{H}$ & 2.895692208 & -2.987173217 & -0.826166058 \\
\hline $\mathrm{H}$ & 3.195992232 & -1.453697106 & -1.613264118 \\
\hline 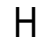 & 1.159202082 & -0.444621032 & -1.640508121 \\
\hline
\end{tabular}




\begin{tabular}{lrrr}
\multicolumn{4}{l}{ Coord_1 } \\
C & -4.603230332 & -0.719979054 & -1.903864138 \\
C & -3.255434236 & -0.281120021 & -1.712338122 \\
C & -2.862792205 & 0.371422029 & -0.469667034 \\
C & -3.851508279 & 0.897261063 & 0.320994024 \\
C & -5.170152371 & 1.028167074 & -0.369388025 \\
C & -5.584156404 & -0.222391015 & -1.073803076 \\
H & -4.802985345 & -1.496096110 & -2.621813189 \\
H & -2.483353178 & -0.635941045 & -2.364181171 \\
H & -5.165166373 & 1.883688133 & -1.038534074 \\
H & -6.568807475 & -0.638906046 & -0.990238072 \\
C & -1.464137106 & 0.350570027 & 0.015913000 \\
O & -1.163424085 & 0.681957050 & 1.130751084 \\
O & -3.748081268 & 1.329093093 & 1.575277113 \\
H & -2.847158203 & 1.212758090 & 1.870698134 \\
O & -0.607126042 & -0.043193003 & -0.894221062 \\
C & 0.816382057 & -0.108512007 & -0.672541050 \\
C & 1.189260085 & -1.145757085 & 0.381987026 \\
C & 1.427956104 & 1.258412090 & -0.390813030 \\
C & 2.719290194 & -1.300439093 & 0.534087040 \\
H & 0.768153054 & -0.844745063 & 1.332657097 \\
C & 2.956322215 & 1.144983084 & -0.300784020 \\
H & 1.146534081 & 1.947008139 & -1.183119084 \\
C & 3.349503239 & 0.092994006 & 0.746490051 \\
H & 3.047278222 & 0.462098032 & 1.725513122 \\
C & 3.587823256 & 2.501586182 & 0.017052001 \\
H & 3.246828232 & 2.874787209 & 0.978810072 \\
H & 4.670772336 & 2.430146173 & 0.053136005 \\
H & 3.329210239 & 3.240366231 & -0.735788055 \\
H & 1.036320076 & 1.652217120 & 0.539055041 \\
H & 0.743118054 & -2.101065151 & 0.117533008 \\
H & 4.432491319 & -0.004335999 & 0.776898058 \\
H & 3.332279239 & 0.829349059 & -1.272218091 \\
C & 3.000423217 & -2.169030158 & 1.768698129 \\
H & 2.562910183 & -3.157407225 & 1.659296120 \\
H & 4.068828294 & -2.295159164 & 1.920013140 \\
H & 2.587933187 & -1.720811123 & 2.667357194 \\
C & 3.334740242 & -2.003061144 & -0.689407050 \\
H & 4.403838319 & -2.136511153 & -0.551713040 \\
H & 2.895692208 & -2.987173217 & -0.826166058 \\
H & 3.195992232 & -1.453697106 & -1.613264118 \\
H & 1.159202082 & -0.444621032 & -1.640508121 \\
& & & \\
& & &
\end{tabular}

Coord_2

$\begin{array}{cccc}C & -4.591334332 & -0.691860052 & -1.940754142 \\ C & -3.241718232 & -0.297551022 & -1.707527123 \\ C & -2.861985205 & 0.345770024 & -0.462844034 \\ & & & S 182\end{array}$




$\begin{array}{lrrr}\text { C } & -3.856571278 & 0.872026061 & 0.325376025 \\ \text { C } & -5.183955372 & 0.991396071 & -0.328276022 \\ \text { C } & -5.586435401 & -0.192034016 & -1.124795083 \\ \text { H } & -4.800534345 & -1.433959101 & -2.690480192 \\ \text { H } & -2.463282179 & -0.657386048 & -2.349107170 \\ \text { H } & -5.301602381 & 1.927642141 & -0.858104064 \\ \text { H } & -6.588064473 & -0.575195042 & -1.106014080 \\ \text { C } & -1.462184103 & 0.334103025 & 0.020664001 \\ \text { O } & -1.159200082 & 0.676038049 & 1.132194082 \\ \text { O } & -3.758631269 & 1.320676095 & 1.575047112 \\ \text { H } & -2.857862205 & 1.218002088 & 1.874748134 \\ \text { O } & -0.604775045 & -0.065463004 & -0.887879063 \\ \text { C } & 0.819053058 & -0.123222008 & -0.668609046 \\ \text { C } & 1.198456085 & -1.151988084 & 0.391926028 \\ \text { C } & 1.426514101 & 1.247860089 & -0.397637030 \\ \text { C } & 2.729404195 & -1.300110093 & 0.541248041 \\ \text { H } & 0.778809054 & -0.846398060 & 1.341848096 \\ \text { C } & 2.955540213 & 1.140570084 & -0.310088020 \\ \text { H } & 1.140890083 & 1.929986137 & -1.194015087 \\ \text { C } & 3.354824243 & 0.097008006 & 0.743348056 \\ \text { H } & 3.053181220 & 0.471383034 & 1.720546121 \\ \text { C } & 3.582859256 & 2.501522178 & -0.002607002 \\ \text { H } & 3.242886232 & 2.879814207 & 0.957521070 \\ \text { H } & 4.666145337 & 2.434200174 & 0.031339004 \\ \text { H } & 3.319850236 & 3.234459232 & -0.759619055 \\ \text { H } & 1.035994076 & 1.647079121 & 0.530387038 \\ \text { H } & 0.755064056 & -2.110603153 & 0.134998011 \\ \text { H } & 4.438224320 & 0.003981000 & 0.772205054 \\ \text { H } & 3.330666238 & 0.819887057 & -1.280160094 \\ \text { C } & 3.016852218 & -2.160030155 & 1.780459130 \\ \text { H } & 2.582622184 & -3.150625226 & 1.678306119 \\ \text { H } & 4.086081293 & -2.281419167 & 1.929771137 \\ \text { H } & 2.605126187 & -1.707797125 & 2.677466194 \\ \text { C } & 3.344494243 & -2.008043146 & -0.679379046 \\ \text { H } & 4.414415318 & -2.136619152 & -0.543484037 \\ \text { H } & 2.908846210 & -2.994630213 & -0.808952059 \\ \text { H } & 3.201472233 & -1.465035104 & -1.606310115 \\ \text { H } & 1.161437083 & -0.464525034 & -1.634912119 \\ & & & \end{array}$

Coord_3

$\begin{array}{crrr}\text { C } & -4.579048329 & -0.661064047 & -1.976429140 \\ \text { C } & -3.229529232 & -0.312052024 & -1.704335120 \\ \text { C } & -2.861491207 & 0.321562022 & -0.457852035 \\ \text { C } & -3.862216276 & 0.847985062 & 0.327995023 \\ \text { C } & -5.194939372 & 0.954533070 & -0.292893019 \\ \text { C } & -5.585291404 & -0.157205014 & -1.172497083 \\ \text { H } & -4.795869345 & -1.367606100 & -2.757125198 \\ & & & S 183\end{array}$




$\begin{array}{lrrr}\text { H } & -2.445938177 & -0.677185051 & -2.336708167 \\ \text { H } & -5.430362392 & 1.937799137 & -0.669152050 \\ \text { H } & -6.600103473 & -0.502441034 & -1.215107086 \\ \mathrm{C} & -1.460767104 & 0.318721023 & 0.024043999 \\ \mathrm{O} & -1.155914081 & 0.670951047 & 1.132341082 \\ \mathrm{O} & -3.767776272 & 1.311937093 & 1.573201114 \\ \mathrm{H} & -2.866956206 & 1.221946088 & 1.875611137 \\ \mathrm{O} & -0.602661046 & -0.086650007 & -0.882586063 \\ \mathrm{C} & 0.821359059 & -0.137182010 & -0.665253046 \\ \mathrm{C} & 1.206663084 & -1.157652085 & 0.401198028 \\ \mathrm{C} & 1.424841102 & 1.237808087 & -0.404609030 \\ \mathrm{C} & 2.738413199 & -1.299561092 & 0.548252041 \\ \mathrm{H} & 0.788163056 & -0.847548062 & 1.350229100 \\ \mathrm{C} & 2.954448213 & 1.136324082 & -0.319020024 \\ \mathrm{H} & 1.135468080 & 1.913606138 & -1.205008088 \\ \mathrm{C} & 3.359212239 & 0.101003008 & 0.740493055 \\ \mathrm{H} & 3.057834218 & 0.480542034 & 1.715779123 \\ \mathrm{C} & 3.577706255 & 2.501371182 & -0.021462000 \\ \mathrm{H} & 3.238448234 & 2.884661206 & 0.936934069 \\ \mathrm{H} & 4.661283336 & 2.437931175 & 0.010717998 \\ \mathrm{H} & 3.310745239 & 3.228597234 & -0.782582056 \\ \mathrm{H} & 1.035142074 & 1.642362120 & 0.521443038 \\ \mathrm{H} & 0.765953053 & -2.119385151 & 0.151493011 \\ \mathrm{H} & 4.442976321 & 0.012052001 & 0.768165056 \\ \mathrm{H} & 3.329065239 & 0.810681056 & -1.287606095 \\ \mathrm{C} & 3.031517217 & -2.151015155 & 1.791968127 \\ \mathrm{H} & 2.600431189 & -3.143668228 & 1.696750121 \\ \mathrm{H} & 4.101468296 & -2.267913161 & 1.939630138 \\ \mathrm{H} & 2.620293191 & -1.694832123 & 2.687214194 \\ \mathrm{C} & 3.353483240 & -2.012718147 & -0.669356048 \\ \mathrm{H} & 4.424128318 & -2.136665154 & -0.534912038 \\ \mathrm{H} & 2.921108210 & -3.001615215 & -0.792055059 \\ \mathrm{H} & 3.206662229 & -1.475922106 & -1.599280117 \\ \mathrm{H} & 1.163623083 & -0.483581035 & -1.629783116\end{array}$

Coord_4

$\begin{array}{lrrr}\text { C } & -4.566556327 & -0.627608043 & -2.010666146 \\ \text { C } & -3.218925234 & -0.324591022 & -1.702424124 \\ \text { C } & -2.861417207 & 0.298868023 & -0.454338034 \\ \text { C } & -3.868359278 & 0.825335059 & 0.329362026 \\ \text { C } & -5.203420374 & 0.917995064 & -0.262450018 \\ \text { C } & -5.581133399 & -0.118222008 & -1.216607087 \\ \text { H } & -4.789198347 & -1.297159093 & -2.821355203 \\ \text { H } & -2.431260176 & -0.695273049 & -2.326583170 \\ \text { H } & -5.549570400 & 1.913973138 & -0.477164036 \\ \text { H } & -6.605507473 & -0.421278029 & -1.317011093 \\ \text { C } & -1.459953103 & 0.304480022 & 0.026317001\end{array}$




$\begin{array}{lrrr}\text { O } & -1.153547082 & 0.666752046 & 1.131443084 \\ \text { O } & -3.774893272 & 1.303181093 & 1.570244114 \\ \text { H } & -2.873695209 & 1.224702086 & 1.873538133 \\ \text { O } & -0.600924042 & -0.106720007 & -0.878132063 \\ \text { C } & 0.823180059 & -0.150376013 & -0.662353049 \\ \text { C } & 1.213816090 & -1.162736086 & 0.409915027 \\ \text { C } & 1.422870102 & 1.228273090 & -0.411661032 \\ \text { C } & 2.746268195 & -1.298798093 & 0.555115041 \\ \text { H } & 0.796222055 & -0.848171063 & 1.357943096 \\ \text { C } & 2.952987213 & 1.132245084 & -0.327612026 \\ \text { H } & 1.130155082 & 1.897888139 & -1.216020086 \\ \text { C } & 3.362662242 & 0.104974007 & 0.737888053 \\ \text { H } & 3.061310219 & 0.489580037 & 1.711194125 \\ \text { C } & 3.572357258 & 2.501131179 & -0.039594005 \\ \text { H } & 3.233582232 & 2.889341208 & 0.916989066 \\ \text { H } & 4.656186338 & 2.441324175 & -0.008879002 \\ \text { H } & 3.301849235 & 3.222780232 & -0.804753059 \\ \text { H } & 1.033769076 & 1.638097120 & 0.512319038 \\ \text { H } & 0.775679056 & -2.127400154 & 0.167166013 \\ \text { H } & 4.446746322 & 0.019855000 & 0.764669057 \\ \text { H } & 3.327344238 & 0.801716058 & -1.294613095 \\ \text { C } & 3.044439218 & -2.141993154 & 1.803237131 \\ \text { H } & 2.616330187 & -3.136545227 & 1.714679123 \\ \text { H } & 4.115023298 & -2.254664161 & 1.949535140 \\ \text { H } & 2.633524188 & -1.681914124 & 2.696640196 \\ \text { C } & 3.361560241 & -2.017112144 & -0.659361049 \\ \text { H } & 4.432843317 & -2.136690152 & -0.526085039 \\ \text { H } & 2.932301208 & -3.008155216 & -0.775462056 \\ \text { H } & 3.211359232 & -1.486387110 & -1.592199117 \\ \text { H } & 1.165570084 & -0.501784034 & -1.625025119\end{array}$

Coord_5

$\begin{array}{lrrc}\text { C } & -4.554000327 & -0.591577042 & -2.043218146 \\ \text { C } & -3.209952229 & -0.335197025 & -1.701470123 \\ \text { C } & -2.861881205 & 0.277701018 & -0.451970034 \\ \text { C } & -3.874941279 & 0.804210060 & 0.329971024 \\ \text { C } & -5.209647377 & 0.882063061 & -0.236214019 \\ \text { C } & -5.574270399 & -0.075446007 & -1.256795088 \\ \text { H } & -4.780716344 & -1.222805086 & -2.882768207 \\ \text { H } & -2.419204174 & -0.711652053 & -2.318365169 \\ \text { H } & -5.657554405 & 1.856861133 & -0.287388020 \\ \text { H } & -6.604726476 & -0.332384025 & -1.411165103 \\ \text { C } & -1.459825105 & 0.291389023 & 0.027731999 \\ \text { O } & -1.152101084 & 0.663463046 & 1.129732079 \\ \text { O } & -3.779284274 & 1.294653095 & 1.566631114 \\ \text { H } & -2.877234209 & 1.226319088 & 1.868658134 \\ \text { O } & -0.599706046 & -0.125675009 & -0.874320065 \\ & & & \\ & & & S 185\end{array}$




\begin{tabular}{|c|c|c|c|}
\hline & 060 & 8011 & 48 \\
\hline & 1.219853087 & -1.167244084 & \\
\hline $0+2+$ & 1.420507103 & 1.219258090 & -0.4187320 \\
\hline$C$ & 2.752920197 & -1.297829095 & 0.56185403 \\
\hline $\mathrm{H}$ & 0.802985057 & -0.848258059 & 36512210 \\
\hline C & 2.951075211 & 1.128328082 & 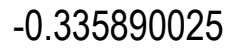 \\
\hline $\mathrm{H}$ & 1.124813080 & 2833136 & 1 \\
\hline C & 3.365153244 & 0.108918008 & 0.7354970 \\
\hline H & 3.063653220 & 0.498508035 & 1.706772124 \\
\hline C & 3.566765256 & 2.500806180 & \\
\hline $\mathrm{H}$ & 3.228302234 & 2.893867208 & $0.0910<0$ \\
\hline $\mathrm{H}$ & 4.650812336 & 2.444379173 & -0.027 \\
\hline $\mathrm{H}$ & 3.293071238 & 3.217012232 & -0.82620905 \\
\hline $\mathrm{H}$ & 1.031845073 & 1.634297120 & 0.5030990 \\
\hline $\mathrm{H}$ & 0.784155057 & -2.1346 & $0.1 \varepsilon$ \\
\hline $\mathrm{H}$ & 4.449520322 & 0.027385002 & 0.7616190 \\
\hline $\mathrm{H}$ & 3.325356241 & 0.792978056 & -1.301229096 \\
\hline C & 3.055644218 & -2.132966152 & 1.814283129 \\
\hline $\mathrm{H}$ & 2.630 & -3.12 & 1.7 \\
\hline $\mathrm{H}$ & 4.126786294 & -2.2416 & 1.95944014 \\
\hline $\mathrm{H}$ & 2.644906193 & -1.669033119 & 2.705783193 \\
\hline C & 3.368595240 & -2.0212 & \\
\hline $\mathrm{H}$ & 4.44 & & \\
\hline $\mathrm{H}$ & 2.94 & -3.0 & -0 . \\
\hline $\mathrm{H}$ & 3.215 & -1.49 & -1.58508 \\
\hline $\mathrm{H}$ & 1.16 & & \\
\hline \multicolumn{4}{|c|}{ Coord_6 } \\
\hline C & -4.541 & -0.55 & -2.07383 \\
\hline C & -3.202636232 & -0.343952025 & -1.701183124 \\
\hline C & -2.8 & 0.258 & -0.45 \\
\hline C & -3.881913280 & 0.784695056 & 0.330295024 \\
\hline C & -5.213815373 & 0.846919061 & -0.21349701 \\
\hline C & -5.564932399 & -0.029288003 & -1.292713094 \\
\hline $\mathrm{H}$ & -4.770 & 88082 & -2.94096 \\
\hline $\mathrm{H}$ & -2.4097 & -0.7 & -2 \\
\hline $\mathrm{H}$ & -5.752821412 & 1.767915126 & -0.104711006 \\
\hline $\mathrm{H}$ & -6.598107475 & -0.236491017 & -1.496968109 \\
\hline C & -1.460472104 & 0.279422018 & 0.028512001 \\
\hline 0 & 080 & 48 & 1.127415082 \\
\hline 0 & -3.780153273 & 1.286544094 & 1.562755 \\
\hline $\mathrm{H}$ & -2.876623205 & 1.226780091 & 1.86095613 \\
\hline 0 & -0.599152045 & -0.143549013 & -0.870971061 \\
\hline C & 0.824868060 & -0.174503014 & -0.657478046 \\
\hline C & 1.224713087 & -1.171188084 & 0.42610903 \\
\hline C & 1.417641100 & 1.210750090 & -0.42576602 \\
\hline C & 2.758322197 & -1.296658095 & $0.5684 c$ \\
\hline
\end{tabular}




$\begin{array}{lrrr}\text { H } & 0.808446060 & -0.847815059 & 1.371887097 \\ \mathrm{C} & 2.948608213 & 1.124572082 & -0.343880025 \\ \mathrm{H} & 1.119279082 & 1.868421137 & -1.237847087 \\ \mathrm{C} & 3.366646243 & 0.112843011 & 0.733288053 \\ \mathrm{H} & 3.064880218 & 0.507344036 & 1.702496123 \\ \mathrm{C} & 3.560841255 & 2.500407180 & -0.074011005 \\ \mathrm{H} & 3.222578235 & 2.898261210 & 0.878781061 \\ \mathrm{H} & 4.645078336 & 2.447112178 & -0.045568003 \\ \mathrm{H} & 3.284274237 & 3.211295234 & -0.847026061 \\ \mathrm{H} & 1.029311076 & 1.630960117 & 0.493855038 \\ \mathrm{H} & 0.791304056 & -2.141174157 & 0.196616013 \\ \mathrm{H} & 4.451262321 & 0.034656003 & 0.758928056 \\ \mathrm{H} & 3.322940240 & 0.784454058 & -1.307501095 \\ \mathrm{C} & 3.065160222 & -2.123926153 & 1.825126130 \\ \mathrm{H} & 2.642446191 & -3.121817224 & 1.749205128 \\ \mathrm{H} & 4.136794296 & -2.228934161 & 1.969310139 \\ \mathrm{H} & 2.654518190 & -1.656172122 & 2.714682198 \\ \mathrm{C} & 3.374470245 & -2.025142144 & -0.639508044 \\ \mathrm{H} & 4.446815322 & -2.136761152 & -0.507960035 \\ \mathrm{H} & 2.950908213 & -3.020005215 & -0.743088055 \\ \mathrm{H} & 3.218540231 & -1.506155108 & -1.577941113 \\ \mathrm{H} & 1.167991086 & -0.535715038 & -1.616254118\end{array}$

Coord_7

$\begin{array}{lrrc}\text { C } & -4.529102326 & -0.512402039 & -2.102264150 \\ \text { C } & -3.196980232 & -0.350988024 & -1.701315122 \\ \text { C } & -2.864922207 & 0.239731015 & -0.449536031 \\ \text { C } & -3.889230282 & 0.766832053 & 0.330787026 \\ \text { C } & -5.216078373 & 0.812681060 & -0.193645016 \\ \text { C } & -5.553276397 & 0.019796002 & -1.324002096 \\ \text { H } & -4.759070344 & -1.063390075 & -2.995543213 \\ \text { H } & -2.402813174 & -0.739563052 & -2.306465168 \\ \text { H } & -5.834012421 & 1.649217119 & 0.066413004 \\ \text { H } & -6.585922475 & -0.134394008 & -1.573786114 \\ \text { C } & -1.462001103 & 0.268516019 & 0.028847002 \\ \text { O } & -1.152098084 & 0.659528047 & 1.124667080 \\ \text { O } & -3.776583274 & 1.278998090 & 1.558921112 \\ \text { H } & -2.870787206 & 1.225988087 & 1.850238136 \\ \text { O } & -0.599408045 & -0.160408013 & -0.867929065 \\ \text { C } & 0.824477061 & -0.185502016 & -0.655308049 \\ \text { C } & 1.228331088 & -1.174595086 & 0.433779032 \\ \text { C } & 1.414138101 & 1.202725085 & -0.432722032 \\ \text { C } & 2.762420198 & -1.295286092 & 0.575053043 \\ \text { H } & 0.812581056 & -0.846859063 & 1.378350097 \\ \text { C } & 2.945462212 & 1.120976079 & -0.351608024 \\ \text { H } & 1.113372078 & 1.854614135 & -1.248558090 \\ \text { C } & 3.367084243 & 0.116767008 & 0.731241052 \\ & & & \\ & & & \text { S187 }\end{array}$




$\begin{array}{llll}\text { H } & 3.064976221 & 0.516117038 & 1.698353121 \\ \mathrm{C} & 3.554461256 & 2.499951177 & -0.090446007 \\ \mathrm{H} & 3.216327233 & 2.902549207 & 0.860396063 \\ \mathrm{H} & 4.638864334 & 2.449559178 & -0.062915005 \\ \mathrm{H} & 3.275289236 & 3.205635233 & -0.867277060 \\ \mathrm{H} & 1.026075072 & 1.628069116 & 0.484646036 \\ \mathrm{H} & 0.797061055 & -2.146996154 & 0.210658017 \\ \mathrm{H} & 4.451919321 & 0.041701003 & 0.756524056 \\ \mathrm{H} & 3.319931238 & 0.776134058 & -1.313465092 \\ \mathrm{C} & 3.073006222 & -2.114855151 & 1.835797132 \\ \mathrm{H} & 2.652714193 & -3.114206226 & 1.765927129 \\ \mathrm{H} & 4.145081296 & -2.216401158 & 1.979131140 \\ \mathrm{H} & 2.662415192 & -1.643304119 & 2.723381194 \\ \mathrm{C} & 3.379084242 & -2.028806146 & -0.629647048 \\ \mathrm{H} & 4.451876320 & -2.136811154 & -0.498761035 \\ \mathrm{H} & 2.958103214 & -3.025363219 & -0.727232053 \\ \mathrm{H} & 3.220697232 & -1.515509109 & -1.570768116 \\ \mathrm{H} & 1.168110082 & -0.551530040 & -1.612072114\end{array}$

\section{Coord_8}

$\begin{array}{lrrr}\text { C } & -4.516893323 & -0.469665034 & -2.128262152 \\ \text { C } & -3.192964232 & -0.356485027 & -1.701670120 \\ \text { C } & -2.867771207 & 0.222695016 & -0.449026031 \\ \text { C } & -3.896834279 & 0.750627053 & 0.331872025 \\ \text { C } & -5.216562374 & 0.779442056 & -0.176002014 \\ \text { C } & -5.539405397 & 0.071306005 & -1.350288098 \\ \text { H } & -4.746327343 & -0.978913071 & -3.046128219 \\ \text { H } & -2.398424172 & -0.751366054 & -2.302372167 \\ \text { H } & -5.899826423 & 1.503403106 & 0.222004017 \\ \text { H } & -6.568390473 & -0.026945002 & -1.640946121 \\ \text { C } & -1.464530105 & 0.258568016 & 0.028883002 \\ \text { O } & -1.153677085 & 0.658767047 & 1.121619083 \\ \text { O } & -3.767494273 & 1.272090094 & 1.555317114 \\ \text { H } & -2.858509203 & 1.223730088 & 1.836083131 \\ \text { O } & -0.600617045 & -0.176349011 & -0.865072063 \\ \text { C } & 0.823087061 & -0.195864015 & -0.653205045 \\ \text { C } & 1.230638089 & -1.177498084 & 0.441283030 \\ \text { C } & 1.409846103 & 1.195149087 & -0.439565030 \\ \text { C } & 2.765158198 & -1.293706095 & 0.581578042 \\ \text { H } & 0.815351061 & -0.845417060 & 1.384605099 \\ \text { C } & 2.941492213 & 1.117542079 & -0.359088023 \\ \text { H } & 1.106894080 & 1.841358134 & -1.259092093 \\ \text { C } & 3.366384242 & 0.120716009 & 0.729344052 \\ \text { H } & 3.063887222 & 0.524864036 & 1.694339121 \\ \text { C } & 3.547462257 & 2.499466181 & -0.106451010 \\ \text { H } & 3.209410230 & 2.906768210 & 0.842413059 \\ \text { H } & 4.632010335 & 2.451771176 & -0.079734004 \\ & & & \\ & & & \text { S188 }\end{array}$




$\begin{array}{lrrr}\mathrm{H} & 3.265906236 & 3.200041232 & -0.887031062 \\ \mathrm{H} & 1.022009075 & 1.625596117 & 0.475511035 \\ \mathrm{H} & 0.801369055 & -2.152173153 & 0.224405016 \\ \mathrm{H} & 4.451415322 & 0.048574005 & 0.754366056 \\ \mathrm{H} & 3.316154240 & 0.768012055 & -1.319146096 \\ \mathrm{C} & 3.079191224 & -2.105720154 & 1.846343132 \\ \mathrm{H} & 2.661156189 & -3.106409223 & 1.782387129 \\ \mathrm{H} & 4.151663298 & -2.204018157 & 1.988915146 \\ \mathrm{H} & 2.668629193 & -1.630392120 & 2.731932196 \\ \mathrm{C} & 3.382351245 & -2.032242147 & -0.619798043 \\ \mathrm{H} & 4.455546323 & -2.136843153 & -0.489487034 \\ \mathrm{H} & 2.963792213 & -3.030369220 & -0.711522050 \\ \mathrm{H} & 3.221714231 & -1.524538112 & -1.563541110 \\ \mathrm{H} & 1.167283084 & -0.566662043 & -1.607928117\end{array}$

\section{Coord_9}

$\begin{array}{lrrr}\text { C } & -4.504914322 & -0.425152030 & -2.151579157 \\ \text { C } & -3.190557227 & -0.360674026 & -1.702122122 \\ \text { C } & -2.871701206 & 0.206709016 & -0.448813032 \\ \text { C } & -3.904630282 & 0.736048051 & 0.333955025 \\ \text { C } & -5.215360375 & 0.747293051 & -0.159870014 \\ \text { C } & -5.523375396 & 0.124710006 & -1.371165098 \\ \text { H } & -4.732682338 & -0.891654065 & -3.092330224 \\ \text { H } & -2.396580175 & -0.762008052 & -2.299426164 \\ \text { H } & -5.948927431 & 1.333651098 & 0.358592026 \\ \text { H } & -6.545699469 & 0.084959005 & -1.697722124 \\ \text { C } & -1.468199108 & 0.249438021 & 0.028708003 \\ \text { O } & -1.156469082 & 0.658677045 & 1.118349080 \\ \text { O } & -3.751545268 & 1.265793091 & 1.551971110 \\ \text { H } & -2.838348206 & 1.219594086 & 1.817746129 \\ \text { O } & -0.602921041 & -0.191503013 & -0.862318061 \\ \text { C } & 0.820564060 & -0.205665013 & -0.651114049 \\ \text { C } & 1.231555090 & -1.179938083 & 0.448694030 \\ \text { C } & 1.404588102 & 1.187973084 & -0.446277034 \\ \text { C } & 2.766464197 & -1.291902093 & 0.588120043 \\ \text { H } & 0.816680058 & -0.843524061 & 1.390729098 \\ \text { C } & 2.936527213 & 1.114280082 & -0.366324025 \\ \text { H } & 1.099630080 & 1.828580133 & -1.269440091 \\ \text { C } & 3.364432240 & 0.124737010 & 0.727613050 \\ \text { H } & 3.061502220 & 0.533642039 & 1.690465120 \\ \text { C } & 3.539631254 & 2.498991181 & -0.122069010 \\ \text { H } & 3.201621228 & 2.910966211 & 0.824788059 \\ \text { H } & 4.624309335 & 2.453831179 & -0.096084004 \\ \text { H } & 3.255874236 & 3.194529231 & -0.906341068 \\ \text { H } & 1.016940076 & 1.623502115 & 0.466469036 \\ \text { H } & 0.804175060 & -2.156767153 & 0.237961018 \\ \text { H } & 4.449638318 & 0.055360005 & 0.752450053 \\ & & & \\ & & & 5189\end{array}$




$\begin{array}{llll}\mathrm{H} & 3.311434239 & 0.760091055 & -1.324548096 \\ \mathrm{C} & 3.083696221 & -2.096471152 & 1.856830134 \\ \mathrm{H} & 2.667787193 & -3.098395225 & 1.798683127 \\ \mathrm{H} & 4.156529300 & -2.191691158 & 1.998715143 \\ \mathrm{H} & 2.673141191 & -1.617378116 & 2.740403197 \\ \mathrm{C} & 3.384203244 & -2.035438145 & -0.609904043 \\ \mathrm{H} & 4.457765322 & -2.136803153 & -0.480096033 \\ \mathrm{H} & 2.967937211 & -3.035029218 & -0.695872051 \\ \mathrm{H} & 3.221488230 & -1.533254111 & -1.556215111 \\ \mathrm{H} & 1.165370082 & -0.581198040 & -1.603764116\end{array}$

$\begin{array}{lrrr}\text { Coord_10 } & & \\ \text { C } & -4.515201326 & -0.400805029 & -1.855736134 \\ \text { C } & -3.181451230 & -0.375125026 & -1.481482107 \\ \text { C } & -2.778806198 & 0.220923013 & -0.272872021 \\ \text { C } & -3.754562271 & 0.783013055 & 0.566889039 \\ \text { C } & -5.085772365 & 0.765497056 & 0.179575014 \\ \text { C } & -5.467403392 & 0.188203016 & -1.019133071 \\ \text { H } & -4.809460348 & -0.854842062 & -2.784067199 \\ \text { H } & -2.434378173 & -0.806214059 & -2.117452151 \\ \text { H } & -5.806210417 & 1.216708086 & 0.835650062 \\ \text { H } & -6.503132467 & 0.201302015 & -1.303526096 \\ \text { C } & -1.344494099 & 0.280596019 & 0.100976010 \\ \text { O } & -0.954382070 & 0.733908052 & 1.147589081 \\ \text { O } & -3.481933248 & 1.359663097 & 1.742872125 \\ \text { H } & -2.548349183 & 1.317046093 & 1.923385137 \\ \text { O } & -0.544260039 & -0.199703016 & -0.830909057 \\ \text { C } & 0.890368064 & -0.207467015 & -0.722219051 \\ \text { C } & 1.380843099 & -1.138294082 & 0.382675025 \\ \text { C } & 1.486719105 & 1.192066085 & -0.615617046 \\ \text { C } & 2.921902209 & -1.247819088 & 0.414983032 \\ \text { H } & 1.035212074 & -0.764273053 & 1.338446095 \\ \text { C } & 3.020571217 & 1.118485078 & -0.643317046 \\ \text { H } & 1.122695080 & 1.800113128 & -1.439462105 \\ \text { C } & 3.527056256 & 0.172058014 & 0.455255034 \\ \text { H } & 3.293798237 & 0.619141043 & 1.420531101 \\ \text { C } & 3.638651260 & 2.510523181 & -0.498054038 \\ \text { H } & 3.369749243 & 2.960209212 & 0.453778032 \\ \text { H } & 4.722405340 & 2.464154178 & -0.548779038 \\ \text { H } & 3.298450240 & 3.175256231 & -1.286571093 \\ \text { H } & 1.166035083 & 1.664744121 & 0.304595022 \\ \text { H } & 0.940045065 & -2.121757151 & 0.242279020 \\ \text { H } & 4.611296332 & 0.101698009 & 0.404701028 \\ \text { H } & 3.325843238 & 0.726020052 & -1.611264114 \\ \text { C } & 3.330888239 & -2.002916144 & 1.687826124 \\ \text { H } & 2.912588208 & -3.005464218 & 1.699186120 \\ \text { H } & 4.411244318 & -2.094817150 & 1.755189128 \\ & & & \\ & & & 5 \\ & & & \\ & & & \\ & & \end{array}$




$\begin{array}{llll}\text { H } & 2.985199216 & -1.4888884106 & 2.579495186 \\ \mathrm{C} & 3.451961247 & -2.038805146 & -0.794613060 \\ \mathrm{H} & 4.532188329 & -2.137092152 & -0.738958054 \\ \mathrm{H} & 3.031487218 & -3.040188220 & -0.811097058 \\ \mathrm{H} & 3.220773232 & -1.573731111 & -1.745601124 \\ \mathrm{H} & 1.166358082 & -0.621012045 & -1.681525120\end{array}$

LIIC path connecting T1-ENOL-MIN and $\mathrm{T}_{1} / \mathrm{S}_{0}-\mathrm{ENOL}$ Coord_1

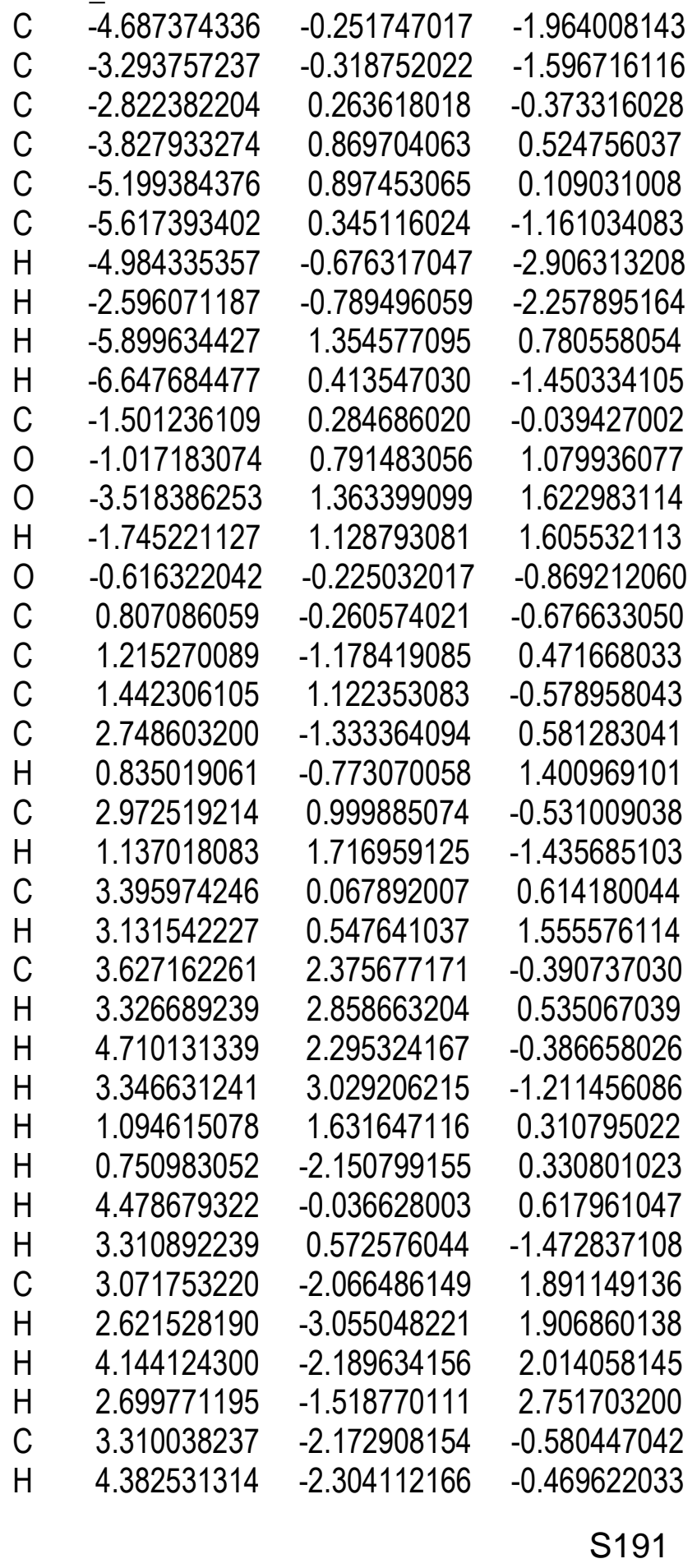




$\begin{array}{llll}\mathrm{H} & 2.857691206 & -3.160348228 & -0.592034044 \\ \mathrm{H} & 3.140012227 & -1.726577123 & -1.553032110 \\ \mathrm{H} & 1.120173079 & -0.707047052 & -1.609240116\end{array}$

Coord_2

\begin{tabular}{|c|c|c|c|}
\hline & -4.689484340 & -0.262902020 & -1.954305139 \\
\hline & -3.298205236 & -0.335481024 & -1.578214116 \\
\hline & -2.828109203 & 0.266810021 & -0.378718028 \\
\hline & -3.823011274 & 0.910009065 & 0.502220034 \\
\hline & -5.194055376 & 0.949293067 & 0.083734007 \\
\hline & -5.615281403 & 0.368941029 & -1.167655085 \\
\hline & -4.988078360 & -0.704629051 & -2.888015206 \\
\hline & -2.603254186 & -0.829500060 & -2.225779158 \\
\hline & -5.889051422 & 1.433867104 & 0.741492051 \\
\hline & -6.643617480 & 0.442246032 & -1.463000105 \\
\hline & -1.492151109 & 0.284574019 & -0.031908002 \\
\hline & -1.048520078 & 0.728018050 & 1.135745083 \\
\hline & -3.504315250 & 1.418895102 & 1.590627116 \\
\hline & -1.785046129 & 1.105049077 & 1.619270116 \\
\hline & -0.617289045 & -0.251790018 & -0.860243062 \\
\hline & 0.806372056 & -0.276803020 & -0.675326046 \\
\hline & 1.225858089 & -1.195098085 & 0.468964033 \\
\hline & 1.430189105 & 1.110850082 & -0.571574041 \\
\hline & 2.760480197 & -1.337057095 & 0.576584043 \\
\hline & 0.842704058 & -0.796262056 & 1.399813103 \\
\hline & 2.961439211 & 1.001 & -0.52800 \\
\hline & 1083 & 1.70 & -1.4 \\
\hline & 3.395960244 & 0.069564006 & 0.612739046 \\
\hline & 3.129672225 & 0.543838041 & 1.556412114 \\
\hline & 3.604909257 & 2.382543171 & -0.385142028 \\
\hline & 6240 & 2.86 & 0.5 \\
\hline & 4.688538335 & 2.311251169 & -0.384202029 \\
\hline & 3.316682237 & 3.036251221 & -1.203069087 \\
\hline & 1.080104080 & 1.611738114 & 0.322515023 \\
\hline & 0.769747058 & -2.171024156 & 0.325827021 \\
\hline & 4.479576320 & -0.025525002 & 0.613557043 \\
\hline & 3.300632239 & 0.580429039 & -1.472269105 \\
\hline & 3.091644220 & -2.070558152 & 1.884299137 \\
\hline & 2.649947188 & -3.063002221 & 1.898192136 \\
\hline & 4.165219300 & -2.184711157 & 2.005735145 \\
\hline & 2.715820194 & -1.528121113 & 2.746534197 \\
\hline & 3.327971239 & -2.168595159 & -0.587933043 \\
\hline & 4.401839314 & -2.290264163 & -0.479201035 \\
\hline & 2.884602208 & -3.160105225 & -0.601108043 \\
\hline & 3.152020227 & -1.721443124 & -1.559125110 \\
\hline & & & -1.6106 \\
\hline
\end{tabular}




$\begin{array}{lrrr}\text { Coord } 3 & & & \\ \text { C } & -4.691928340 & -0.279760020 & -1.941021139 \\ \text { C } & -3.302941240 & -0.355440024 & -1.556053114 \\ \text { C } & -2.833760202 & 0.271622020 & -0.383831029 \\ \text { C } & -3.817601273 & 0.954123069 & 0.476632033 \\ \text { C } & -5.188138375 & 1.002535072 & 0.055067005 \\ \text { C } & -5.613046401 & 0.389579026 & -1.174293085 \\ \text { H } & -4.992427361 & -0.742610055 & -2.863665205 \\ \text { H } & -2.611019190 & -0.875011065 & -2.187297156 \\ \text { H } & -5.877572424 & 1.516427110 & 0.696383051 \\ \text { H } & -6.639450479 & 0.465868033 & -1.475873104 \\ \text { C } & -1.483072105 & 0.287248021 & -0.024279001 \\ \text { O } & -1.079480079 & 0.663261048 & 1.186249088 \\ \text { O } & -3.489663253 & 1.482364108 & 1.552779111 \\ \text { H } & -1.814229132 & 1.083605079 & 1.633712119 \\ \text { O } & -0.618600044 & -0.275606021 & -0.850684062 \\ \text { C } & 0.805185058 & -0.290973021 & -0.673648046 \\ \text { C } & 1.235664088 & -1.210919088 & 0.465653034 \\ \text { C } & 1.418364099 & 1.100834080 & -0.562574038 \\ \text { C } & 2.771406199 & -1.340820095 & 0.570933043 \\ \text { H } & 0.850036063 & -0.819315059 & 1.398470099 \\ \text { C } & 2.950474214 & 1.004329073 & -0.523696036 \\ \text { H } & 1.098267078 & 1.702172124 & -1.409121103 \\ \text { C } & 3.395736244 & 0.070675003 & 0.611622043 \\ \text { H } & 3.128064225 & 0.538701037 & 1.558046112 \\ \text { C } & 3.583514256 & 2.389465170 & -0.376999028 \\ \text { H } & 3.280445238 & 2.860930205 & 0.553937040 \\ \text { H } & 4.667684338 & 2.326662165 & -0.379414028 \\ \text { H } & 3.287815238 & 3.044203220 & -1.191449085 \\ \text { H } & 1.066417074 & 1.592613113 & 0.336338027 \\ \text { H } & 0.787198059 & -2.189928156 & 0.319359025 \\ \text { H } & 4.480127321 & -0.015555001 & 0.609241042 \\ \text { H } & 3.290042238 & 0.589639041 & -1.470822107 \\ \mathrm{C} & 3.110392224 & -2.076060150 & 1.875727133 \\ \text { H } & 2.676708195 & -3.072083220 & 1.886897138 \\ \text { H } & 4.185053303 & -2.181910158 & 1.995436144 \\ \text { H } & 2.731207197 & -1.539562110 & 2.740210195 \\ \text { C } & 3.344239243 & -2.163483158 & -0.597240043 \\ \text { H } & 4.419346318 & -2.276288163 & -0.490860038 \\ \text { H } & 2.909336207 & -3.158724226 & -0.612911045 \\ \text { H } & 3.162446226 & -1.714542124 & -1.566569115 \\ \text { H } & 1.122214079 & -0.723196050 & -1.612130117 \\ & & & \end{array}$

Coord_4

$\begin{array}{crrr}C & -4.694900337 & -0.301448021 & -1.924069136 \\ C & -3.308119239 & -0.377927030 & -1.530273112 \\ C & -2.839358205 & 0.277913022 & -0.388823027 \\ & & & S 193\end{array}$




$\begin{array}{lrrr}\text { C } & -3.811654273 & 1.001468071 & 0.447716031 \\ \text { C } & -5.181632374 & 1.056825075 & 0.022796003 \\ \text { C } & -5.610801404 & 0.407442028 & -1.180996083 \\ \text { H } & -4.997688362 & -0.788878059 & -2.833044204 \\ \text { H } & -2.619599191 & -0.924950067 & -2.142411156 \\ \text { H } & -5.865137420 & 1.601536116 & 0.644902048 \\ \text { H } & -6.635328479 & 0.484958034 & -1.488910105 \\ \text { C } & -1.474010103 & 0.292467021 & -0.016701003 \\ \text { O } & -1.109682082 & 0.596770043 & 1.231159091 \\ \text { O } & -3.474258252 & 1.552689114 & 1.509086109 \\ \text { H } & -1.832425130 & 1.062855076 & 1.650115117 \\ \text { O } & -0.620253046 & -0.296747022 & -0.840666059 \\ \text { C } & 0.803535057 & -0.303249023 & -0.671663049 \\ \text { C } & 1.244701091 & -1.225892089 & 0.461782034 \\ \text { C } & 1.406787104 & 1.092180079 & -0.552162042 \\ \text { C } & 2.781419201 & -1.344578095 & 0.564467043 \\ \text { H } & 0.856978059 & -0.842143062 & 1.396916101 \\ \text { C } & 2.939602214 & 1.007466073 & -0.518195038 \\ \text { H } & 1.079518080 & 1.698077123 & -1.392686102 \\ \text { C } & 3.395287242 & 0.071335003 & 0.610830045 \\ \text { H } & 3.126647226 & 0.532440039 & 1.560401110 \\ \text { C } & 3.562899257 & 2.396450172 & -0.366563028 \\ \text { H } & 3.259176234 & 2.861156206 & 0.567581039 \\ \text { H } & 4.647506333 & 2.341619170 & -0.372488024 \\ \text { H } & 3.259948234 & 3.052962220 & -1.176931086 \\ \text { H } & 1.053455073 & 1.574233115 & 0.351968023 \\ \text { H } & 0.803388057 & -2.207565158 & 0.311545025 \\ \text { H } & 4.480332324 & -0.006553003 & 0.605075041 \\ \text { H } & 3.279153236 & 0.600067045 & -1.468536104 \\ \text { C } & 3.128028225 & -2.082752152 & 1.865661134 \\ \text { H } & 2.701880194 & -3.082071224 & 1.873299135 \\ \text { H } & 4.203670304 & -2.180926158 & 1.983452142 \\ \text { H } & 2.745909196 & -1.552764114 & 2.732871195 \\ \text { C } & 3.358955239 & -2.157615154 & -0.608096045 \\ \text { H } & 4.435182318 & -2.262163163 & -0.504262039 \\ \text { H } & 2.932042213 & -3.156276225 & -0.627072045 \\ \text { H } & 3.171414229 & -1.706040124 & -1.575143115 \\ \text { H } & 1.121886079 & -0.727301052 & -1.613673114 \\ & & & \end{array}$

Coord_5

$\begin{array}{lrrr}\text { C } & -4.698538340 & -0.326784023 & -1.903514135 \\ \text { C } & -3.313844239 & -0.402021031 & -1.501070106 \\ \text { C } & -2.844919207 & 0.285525020 & -0.393867029 \\ \text { C } & -3.805130275 & 1.051288077 & 0.415339030 \\ \text { C } & -5.174530371 & 1.111725082 & -0.013166999 \\ \text { C } & -5.608616405 & 0.423091033 & -1.187815087 \\ \text { H } & -5.004074362 & -0.841546060 & -2.796191200 \\ & & & S 194\end{array}$




$\begin{array}{lrrr}\text { H } & -2.629161191 & -0.977880069 & -2.091352150 \\ \text { H } & -5.851688423 & 1.688261123 & 0.586985043 \\ \text { H } & -6.631341478 & 0.500275038 & -1.502061107 \\ \text { C } & -1.464981107 & 0.299938019 & -0.009308000 \\ \text { O } & -1.138794081 & 0.527878039 & 1.270153093 \\ \text { O } & -3.457968250 & 1.628363116 & 1.459450104 \\ \text { H } & -1.839481132 & 1.040638072 & 1.669625120 \\ \text { O } & -0.622254045 & -0.315570021 & -0.830261059 \\ \text { C } & 0.801424058 & -0.313858022 & -0.669401049 \\ \text { C } & 1.253003088 & -1.240028091 & 0.457478034 \\ \text { C } & 1.395388102 & 1.084711076 & -0.540583040 \\ \text { C } & 2.790571200 & -1.348232096 & 0.557378041 \\ \text { H } & 0.863518060 & -0.864625062 & 1.395185099 \\ \text { C } & 2.928769210 & 1.011124074 & -0.511685036 \\ \text { H } & 1.061089074 & 1.695755120 & -1.374580101 \\ \mathrm{C} & 3.394592242 & 0.071689007 & 0.610337042 \\ \text { H } & 3.125353224 & 0.525340037 & 1.563350114 \\ \mathrm{C} & 3.542937257 & 2.403491171 & -0.354228028 \\ \mathrm{H} & 3.238924234 & 2.860937206 & 0.583424040 \\ \mathrm{H} & 4.627891331 & 2.356185170 & -0.363778026 \\ \mathrm{H} & 3.232931233 & 3.062365219 & -1.160020084 \\ \mathrm{H} & 1.041092075 & 1.556544112 & 0.369050029 \\ \mathrm{H} & 0.818406060 & -2.223996159 & 0.302658022 \\ \mathrm{H} & 4.480187324 & 0.001693002 & 0.601082044 \\ \mathrm{H} & 3.267958237 & 0.611506042 & -1.465503103 \\ \mathrm{C} & 3.144624224 & -2.090296151 & 1.854413132 \\ \mathrm{H} & 2.725586197 & -3.092656224 & 1.857865136 \\ \mathrm{H} & 4.221158305 & -2.181335159 & 1.970146144 \\ \mathrm{H} & 2.759948200 & -1.567266110 & 2.724718198 \\ \mathrm{C} & 3.372250241 & -2.151053155 & -0.620141045 \\ \mathrm{H} & 4.449492318 & -2.247869160 & -0.518995037 \\ \mathrm{H} & 2.952903211 & -3.152857227 & -0.643081048 \\ \mathrm{H} & 3.179045230 & -1.696172122 & -1.584559115 \\ \mathrm{H} & 1.120732081 & -0.729209054 & -1.615208114 \\ & & & \end{array}$

Coord_6

$\begin{array}{lrrr}\text { C } & -4.702910339 & -0.354365026 & -1.879598133 \\ \text { C } & -3.320170241 & -0.426647030 & -1.468807106 \\ \text { C } & -2.850452204 & 0.294319019 & -0.399124029 \\ \text { C } & -3.798007274 & 1.102737081 & 0.379543026 \\ \text { C } & -5.166821369 & 1.166756082 & -0.052734005 \\ \text { C } & -5.606520402 & 0.437196030 & -1.194809085 \\ \text { H } & -5.011701362 & -0.898377066 & -2.753441198 \\ \text { H } & -2.639794192 & -1.032118075 & -2.034635145 \\ \text { H } & -5.837186421 & 1.775546127 & 0.522869038 \\ \text { H } & -6.627529476 & 0.512742036 & -1.515287109 \\ \text { C } & -1.456006107 & 0.309351024 & -0.002194001 \\ & & & \\ & & & S 195\end{array}$




$\begin{array}{rrrr}\text { O } & -1.166512083 & 0.455667031 & 1.302833096 \\ \text { O } & -3.440718247 & 1.707646124 & 1.404069101 \\ \text { H } & -1.835452130 & 1.014168071 & 1.693225123 \\ \text { O } & -0.624617043 & -0.332478023 & -0.819475061 \\ \text { C } & 0.798844059 & -0.323065023 & -0.666851050 \\ \text { C } & 1.260615091 & -1.253328088 & 0.452936032 \\ \text { C } & 1.384076102 & 1.078221077 & -0.528113037 \\ \text { C } & 2.798927200 & -1.351659095 & 0.549904037 \\ \text { H } & 0.869668063 & -0.886589066 & 1.393381102 \\ \text { C } & 2.917905211 & 1.015213074 & -0.504408039 \\ \text { H } & 1.042861077 & 1.694802124 & -1.355199095 \\ \text { C } & 3.393633246 & 0.071912008 & 0.610091043 \\ \text { H } & 3.124126226 & 0.517754037 & 1.566735113 \\ \text { C } & 3.523473255 & 2.410569171 & -0.340523025 \\ \text { H } & 3.219495232 & 2.860429206 & 0.600831045 \\ \text { H } & 4.608694331 & 2.370414170 & -0.353787027 \\ \text { H } & 3.206572231 & 3.072204222 & -1.141376085 \\ \text { H } & 1.029198075 & 1.539505112 & 0.387190029 \\ \text { H } & 0.832355061 & -2.239283164 & 0.293099022 \\ \text { H } & 4.479686320 & 0.009432002 & 0.597240043 \\ \text { H } & 3.256421236 & 0.623691047 & -1.461866105 \\ \text { C } & 3.160288225 & -2.098275149 & 1.842369132 \\ \text { H } & 2.747991196 & -3.103447222 & 1.841184130 \\ \text { H } & 4.237638308 & -2.182625156 & 1.955934142 \\ \text { H } & 2.773389201 & -1.582493112 & 2.716016195 \\ \text { C } & 3.384249245 & -2.143881152 & -0.632950045 \\ \text { H } & 4.462419320 & -2.233396162 & -0.534600040 \\ \text { H } & 2.972105212 & -3.148584226 & -0.660308049 \\ \text { H } & 3.185443232 & -1.685234123 & -1.594481114 \\ \text { H } & 1.118778078 & -0.729360054 & -1.616623118\end{array}$

\section{Coord_7}

$\begin{array}{lrrr}\text { C } & -4.708021339 & -0.382664029 & -1.852742134 \\ \text { C } & -3.327093239 & -0.450642032 & -1.434004102 \\ \text { C } & -2.855958204 & 0.304203024 & -0.404726032 \\ \text { C } & -3.790279275 & 1.154959086 & 0.340577022 \\ \text { C } & -5.158505370 & 1.221451085 & -0.095618006 \\ \text { C } & -5.604506401 & 0.450502033 & -1.202041086 \\ \text { H } & -5.020584364 & -0.956941070 & -2.705447192 \\ \text { H } & -2.651507192 & -1.085852077 & -1.973068145 \\ \text { H } & -5.821623419 & 1.862309134 & 0.453128033 \\ \text { H } & -6.623891477 & 0.523383039 & -1.528563108 \\ \text { C } & -1.447104102 & 0.320415025 & 0.004602001 \\ \text { O } & -1.192531088 & 0.378964027 & 1.328634096 \\ \text { O } & -3.422488244 & 1.788708130 & 1.343492096 \\ \text { H } & -1.820594129 & 0.980007070 & 1.721619123 \\ \text { O } & -0.627369046 & -0.347888024 & -0.808229060 \\ & & & \\ & & & S 196\end{array}$




\begin{tabular}{|c|c|c|c|}
\hline & 5775059 & -0.331150026 & -0.663951048 \\
\hline C & 1.267589092 & -1.265770093 & 130034 \\
\hline C & 1.372750100 & 1.072502078 & -0.515040035 \\
\hline $\mathrm{C}$ & 2.806557200 & -1.354714099 & 0.542327039 \\
\hline H & 0.875464062 & -0.907809064 & 1.391685099 \\
\hline C & 2.906921211 & 1.019630074 & -0.496650036 \\
\hline $\mathrm{H}$ & 1.024687072 & 1.694782122 & -1.334965098 \\
\hline C & 3.392396246 & 0.072203008 & 0.610020044 \\
\hline $\mathrm{H}$ & 3.122934227 & 0.510095038 & 1.570370111 \\
\hline C & 3.504338253 & 2.417664173 & -0.326079021 \\
\hline $\mathrm{H}$ & 3.200702232 & 2.859817208 & 0.619056047 \\
\hline$H$ & 4.589756331 & 2.384355170 & -0.343149025 \\
\hline I & 3.180651230 & 3.082245222 & -1.121783080 \\
\hline $\mathrm{H}$ & 1.017651073 & 01110 & 0.405981028 \\
\hline - & 0.845342060 & -2.253472162 & 0.283385022 \\
\hline 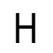 & 4.478825322 & 0.016931000 & 0.593480042 \\
\hline H & 3.244468234 & 0.636312046 & -1.457812104 \\
\hline C & 3.175152226 & -2.106205150 & 1.829980130 \\
\hline$\theta$ & 2.769290200 & -3.11 & $1.823 \varsigma$ \\
\hline H & 4.253259308 & -2.184216159 & 1.941276139 \\
\hline H & 2.786 & -1.597 & 2.707088197 \\
\hline U & 3.395062246 & -2.136206156 & -0.646047045 \\
\hline$H$ & 4.474 & -2.2 & -0.550591041 \\
\hline$H$ & 2.98981 & -3.143598228 & -0.678031047 \\
\hline H & 3.190673228 & 77121 & -1.604536117 \\
\hline & 1.116022081 & -0.72 & -1.617750117 \\
\hline \multicolumn{4}{|c|}{ Coord_8 } \\
\hline C & -4.713 & -0.41 & -1.82 \\
\hline C & -3.3 & -0.47 & -1.397324102 \\
\hline C & 9203 & 1024 & 760028 \\
\hline C & -3.781968271 & 1.207149086 & 0.298920020 \\
\hline C & -5.149591369 & 1.275403093 & -0.141320012 \\
\hline C & -5.602542402 & 0.463799035 & -1.209563087 \\
\hline $\mathrm{H}$ & 62 & -1.01 & -2.653182192 \\
\hline $\mathrm{H}$ & -2.664227190 & -1.137260081 & -1.907730137 \\
\hline $\mathrm{H}$ & -5.805029420 & 1.947527140 & 0.378694030 \\
\hline $\mathrm{H}$ & -6.620390478 & 0.533285040 & -1.541873112 \\
\hline C & -1.438300101 & 0.332882023 & 0.011108002 \\
\hline 0 & -1.216487085 & 0.296370021 & 1.346697095 \\
\hline 0 & -3.403323243 & 1.869757135 & 1.278639091 \\
\hline $\mathrm{H}$ & -1.795340129 & 0.934081068 & 1.755045128 \\
\hline 0 & -0.630555047 & -0.362202025 & -0.796347057 \\
\hline C & 0.792179056 & -0.338391023 & -0.660581046 \\
\hline C & 1.273979092 & -1.277308094 & 0.444311030 \\
\hline C & 1.361304097 & 1.067354078 & -0.501644035 \\
\hline & 2.813531201 & -1.357234099 & 0.5349 \\
\hline
\end{tabular}




$\begin{array}{rrrr}\text { H } & 0.880970061 & -0.928006066 & 1.390362098 \\ \mathrm{C} & 2.895722207 & 1.024272075 & -0.488733036 \\ \mathrm{H} & 1.006400071 & 1.695256121 & -1.314311093 \\ \mathrm{C} & 3.390876243 & 0.072782007 & 0.610039046 \\ \mathrm{H} & 3.121776223 & 0.502827037 & 1.574058111 \\ \mathrm{C} & 3.485366249 & 2.424758175 & -0.311617025 \\ \mathrm{H} & 3.182384227 & 2.859323205 & 0.637266047 \\ \mathrm{H} & 4.570916330 & 2.398051171 & -0.332615021 \\ \mathrm{H} & 3.154935227 & 3.092240222 & -1.102127077 \\ \mathrm{H} & 1.006344074 & 1.507364108 & 0.425032028 \\ \mathrm{H} & 0.857470062 & -2.266589165 & 0.274148021 \\ \mathrm{H} & 4.477608321 & 0.024472003 & 0.589692044 \\ \mathrm{H} & 3.231996235 & 0.649026048 & -1.453569103 \\ \mathrm{C} & 3.189376230 & -2.113559153 & 1.817748129 \\ \mathrm{H} & 2.789695200 & -3.123787224 & 1.806965132 \\ \mathrm{H} & 4.268190308 & -2.185484157 & 1.926655140 \\ \mathrm{H} & 2.798965200 & -1.612368115 & 2.698311195 \\ \mathrm{C} & 3.404765246 & -2.128152152 & -0.658924049 \\ \mathrm{H} & 4.484578320 & -2.203981157 & -0.566476043 \\ \mathrm{H} & 3.006159218 & -3.138062225 & -0.695453049 \\ \mathrm{H} & 3.194752232 & -1.661597120 & -1.614329114 \\ \mathrm{H} & 1.112418082 & -0.726373050 & -1.618372118\end{array}$

Coord_9

$\begin{array}{lrrr}\text { C } & -4.720184341 & -0.435186030 & -1.792740128 \\ \text { C } & -3.342470241 & -0.492071033 & -1.359543095 \\ \text { C } & -2.866846206 & 0.327206025 & -0.417256028 \\ \text { C } & -3.773123269 & 1.258614092 & 0.255288017 \\ \text { C } & -5.140116371 & 1.328299095 & -0.189111015 \\ \text { C } & -5.600575403 & 0.477882034 & -1.217399085 \\ \text { H } & -5.041721365 & -1.069451075 & -2.597909189 \\ \text { H } & -2.677808190 & -1.184606088 & -1.839943130 \\ \text { H } & -5.787485416 & 2.030309147 & 0.300859021 \\ \text { H } & -6.616967479 & 0.543544041 & -1.555197112 \\ \text { C } & -1.429616101 & 0.346555026 & 0.017436999 \\ \text { O } & -1.237904090 & 0.206340016 & 1.355720097 \\ \text { O } & -3.383330244 & 1.949146142 & 1.210811088 \\ \text { H } & -1.760241125 & 0.871760065 & 1.793002129 \\ \text { O } & -0.634240047 & -0.375787026 & -0.783545058 \\ \text { C } & 0.787999059 & -0.345050025 & -0.656552049 \\ \text { C } & 1.279834090 & -1.287867090 & 0.441009033 \\ \text { C } & 1.349626097 & 1.062600076 & -0.488176035 \\ \text { C } & 2.819912205 & -1.359044097 & 0.528172040 \\ \text { H } & 0.886271063 & -0.946849067 & 1.389770102 \\ \text { C } & 2.884202209 & 1.029041074 & -0.480994036 \\ \text { H } & 0.987817070 & 1.695800121 & -1.293650093 \\ \text { C } & 3.389075246 & 0.073886003 & 0.610051043 \\ & & & \\ & & & \text { S198 }\end{array}$




$\begin{array}{lrrr}\text { H } & 3.120691225 & 0.496455034 & 1.577597111 \\ \mathrm{C} & 3.466398250 & 2.431842176 & -0.297915022 \\ \mathrm{H} & 3.164414225 & 2.859199208 & 0.654580049 \\ \mathrm{H} & 4.552018325 & 2.411538174 & -0.323024022 \\ \mathrm{H} & 3.129189224 & 3.101939226 & -1.083357081 \\ \mathrm{H} & 0.995196071 & 1.492371109 & 0.443987030 \\ \mathrm{H} & 0.868822062 & -2.278637165 & 0.266130017 \\ \mathrm{H} & 4.476042321 & 0.032335000 & 0.585725040 \\ \mathrm{H} & 3.218862230 & 0.661470048 & -1.449389105 \\ \mathrm{C} & 3.203133230 & -2.119775155 & 1.806224130 \\ \mathrm{H} & 2.809426204 & -3.132306226 & 1.791083131 \\ \mathrm{H} & 4.282622311 & -2.185787159 & 1.912574136 \\ \mathrm{H} & 2.811457202 & -1.625471115 & 2.690120192 \\ \mathrm{C} & 3.413391248 & -2.119868152 & -0.671042049 \\ \mathrm{H} & 4.493947324 & -2.189145159 & -0.581763042 \\ \mathrm{H} & 3.021219216 & -3.132160226 & -0.711721053 \\ \mathrm{H} & 3.197638232 & -1.649723118 & -1.623443117 \\ \mathrm{H} & 1.107873079 & -0.724279053 & -1.618210115 \\ & & & \\ \mathrm{C} 00 \mathrm{rd}-10 & & \\ \mathrm{C} & -4.712976338 & -0.504409034 & -2.014659143 \\ \mathrm{C} & -3.341945239 & -0.534929041 & -1.557043110 \\ \mathrm{C} & -2.934262212 & 0.185111012 & -0.523456039 \\ \mathrm{C} & -3.898448280 & 0.975761069 & 0.238681017 \\ \mathrm{C} & -5.260825379 & 1.028362076 & -0.224292015 \\ \mathrm{C} & -5.654672405 & 0.285693023 & -1.352816099 \\ \mathrm{H} & -4.983413361 & -1.050931078 & -2.898876209 \\ \mathrm{H} & -2.629687187 & -1.125752079 & -2.101667154 \\ \mathrm{H} & -5.954358430 & 1.629772120 & 0.332043023 \\ \mathrm{H} & -6.667247477 & 0.335061027 & -1.704847120 \\ \mathrm{C} & -1.493656105 & 0.228757017 & -0.044331004 \\ \mathrm{O} & -1.327785094 & -0.227073016 & 1.230523089 \\ \mathrm{O} & -3.559260258 & 1.556060110 & 1.282488093 \\ \mathrm{H} & -1.839361130 & 0.334256026 & 1.803562130 \\ \mathrm{O} & -0.651423046 & -0.328637021 & -0.930014066 \\ \mathrm{C} & 0.762687056 & -0.216606017 & -0.774557057 \\ \mathrm{C} & 1.315606096 & -1.292331092 & 0.160055009 \\ \mathrm{C} & 1.216092086 & 1.178796087 & -0.359225027 \\ \mathrm{C} & 2.855673205 & -1.265442093 & 0.268015021 \\ \mathrm{H} & 0.888165062 & -1.145306083 & 1.143347085 \\ \mathrm{C} & 2.748614197 & 1.256461092 & -0.326323024 \\ \mathrm{H} & 0.813765059 & 1.912480138 & -1.052381076 \\ \mathrm{C} & 3.315136241 & 0.170788015 & 0.599828042 \\ \mathrm{H} & 3.007915219 & 0.404095030 & 1.618641114 \\ \mathrm{C} & 3.222724235 & 2.647078190 & 0.099863007 \\ \mathrm{H} & 2.882093210 & 2.885592209 & 1.103957078 \\ \mathrm{H} & 4.307001311 & 2.709988195 & 0.093789005 \\ & & & \\ & & & \\ & & & \end{array}$




$\begin{array}{lrrr}\mathrm{H} & 2.842503205 & 3.413219244 & -0.569579043 \\ \mathrm{H} & 0.822994058 & 1.416177100 & 0.625302048 \\ \mathrm{H} & 0.981708068 & -2.266831163 & -0.186387012 \\ \mathrm{H} & 4.402349314 & 0.213080014 & 0.591328043 \\ \mathrm{H} & 3.117254224 & 1.081954078 & -1.335600095 \\ \mathrm{C} & 3.282876236 & -2.199538158 & 1.409996103 \\ \mathrm{H} & 2.966359215 & -3.221079234 & 1.218278086 \\ \mathrm{H} & 4.363248312 & -2.204087156 & 1.527585109 \\ \mathrm{H} & 2.846691204 & -1.889724135 & 2.354870170 \\ \mathrm{C} & 3.516085253 & -1.769546125 & -1.027692074 \\ \mathrm{H} & 4.597974332 & -1.773728128 & -0.927943067 \\ \mathrm{H} & 3.201857230 & -2.786681202 & -1.244335091 \\ \mathrm{H} & 3.273801238 & -1.163728082 & -1.893147134 \\ \mathrm{H} & 1.118461080 & -0.403437030 & -1.779500129\end{array}$

LIIC path connecting $\mathrm{T}_{1} / \mathrm{S}_{0}$-ENOL and SO-ENOL-MIN Coord_1

$\begin{array}{lrrr}\text { C } & -4.712976338 & -0.504409034 & -2.014659143 \\ \text { C } & -3.341945239 & -0.534929041 & -1.557043110 \\ \text { C } & -2.934262212 & 0.185111012 & -0.523456039 \\ \text { C } & -3.898448280 & 0.975761069 & 0.238681017 \\ \text { C } & -5.260825379 & 1.028362076 & -0.224292015 \\ \text { C } & -5.654672405 & 0.285693023 & -1.352816099 \\ \text { H } & -4.983413361 & -1.050931078 & -2.898876209 \\ \text { H } & -2.629687187 & -1.125752079 & -2.101667154 \\ \text { H } & -5.954358430 & 1.629772120 & 0.332043023 \\ \text { H } & -6.667247477 & 0.335061027 & -1.704847120 \\ \text { C } & -1.493656105 & 0.228757017 & -0.044331004 \\ \text { O } & -1.327785094 & -0.227073016 & 1.230523089 \\ \text { O } & -3.559260258 & 1.556060110 & 1.282488093 \\ \text { H } & -1.839361130 & 0.334256026 & 1.803562130 \\ \text { O } & -0.651423046 & -0.328637021 & -0.930014066 \\ \text { C } & 0.762687056 & -0.216606017 & -0.774557057 \\ \text { C } & 1.315606096 & -1.292331092 & 0.160055009 \\ \text { C } & 1.216092086 & 1.178796087 & -0.359225027 \\ \text { C } & 2.855673205 & -1.265442093 & 0.268015021 \\ \text { H } & 0.888165062 & -1.145306083 & 1.143347085 \\ \text { C } & 2.748614197 & 1.256461092 & -0.326323024 \\ \text { H } & 0.813765059 & 1.912480138 & -1.052381076 \\ \text { C } & 3.315136241 & 0.170788015 & 0.599828042 \\ \text { H } & 3.007915219 & 0.404095030 & 1.618641114 \\ \text { C } & 3.222724235 & 2.647078190 & 0.099863007 \\ \text { H } & 2.882093210 & 2.885592209 & 1.103957078 \\ \text { H } & 4.307001311 & 2.709988195 & 0.093789005 \\ \text { H } & 2.842503205 & 3.413219244 & -0.569579043 \\ \text { H } & 0.822994058 & 1.416177100 & 0.625302048 \\ \text { H } & 0.981708068 & -2.266831163 & -0.186387012\end{array}$




$\begin{array}{lrrr}\mathrm{H} & 4.402349314 & 0.213080014 & 0.591328043 \\ \mathrm{H} & 3.117254224 & 1.081954078 & -1.335600095 \\ \mathrm{C} & 3.282876236 & -2.199538158 & 1.409996103 \\ \mathrm{H} & 2.966359215 & -3.221079234 & 1.218278086 \\ \mathrm{H} & 4.363248312 & -2.204087156 & 1.527585109 \\ \mathrm{H} & 2.846691204 & -1.889724135 & 2.354870170 \\ \mathrm{C} & 3.516085253 & -1.769546125 & -1.027692074 \\ \mathrm{H} & 4.597974332 & -1.773728128 & -0.927943067 \\ \mathrm{H} & 3.201857230 & -2.786681202 & -1.244335091 \\ \mathrm{H} & 3.273801238 & -1.163728082 & -1.893147134 \\ \mathrm{H} & 1.118461080 & -0.403437030 & -1.779500129\end{array}$

\section{Coord_2}

$\begin{array}{lrrr}\text { C } & -4.737835339 & -0.474041033 & -2.036674149 \\ \text { C } & -3.364818243 & -0.519939037 & -1.617181114 \\ \text { C } & -2.932776209 & 0.159991010 & -0.550686039 \\ \text { C } & -3.880493280 & 0.915358068 & 0.265555019 \\ \text { C } & -5.254191380 & 0.978982070 & -0.167729010 \\ \text { C } & -5.667275406 & 0.286425023 & -1.314811097 \\ \text { H } & -5.032788361 & -0.985189070 & -2.934163209 \\ \text { H } & -2.661977192 & -1.084746076 & -2.200036156 \\ \text { H } & -5.937400426 & 1.554482114 & 0.427471033 \\ \text { H } & -6.687915479 & 0.346444026 & -1.641611117 \\ \text { C } & -1.500974108 & 0.202619016 & -0.088517008 \\ \text { O } & -1.304706092 & -0.162638013 & 1.202711088 \\ \text { O } & -3.522427251 & 1.460243108 & 1.321041095 \\ \text { H } & -1.873762133 & 0.378702026 & 1.744692125 \\ \text { O } & -0.637640048 & -0.322529025 & -0.965809070 \\ \text { C } & 0.776751057 & -0.213973016 & -0.790310055 \\ \text { C } & 1.313517095 & -1.285331093 & 0.157881011 \\ \text { C } & 1.229038090 & 1.183543087 & -0.381396030 \\ \text { C } & 2.852661207 & -1.262712089 & 0.281344022 \\ \text { H } & 0.876903064 & -1.129793083 & 1.135914081 \\ \text { C } & 2.761531196 & 1.255729092 & -0.330949023 \\ \text { H } & 0.837947062 & 1.912000137 & -1.086369078 \\ \text { C } & 3.313152239 & 0.174140014 & 0.609003044 \\ \text { H } & 2.995121216 & 0.415214028 & 1.622635117 \\ \text { C } & 3.234983234 & 2.647496193 & 0.092280008 \\ \text { H } & 2.883203207 & 2.893286208 & 1.090720079 \\ \text { H } & 4.319421313 & 2.706844194 & 0.098736008 \\ \text { H } & 2.865329208 & 3.410668244 & -0.586365043 \\ \text { H } & 0.825283062 & 1.431131104 & 0.595804041 \\ \text { H } & 0.980026068 & -2.260929164 & -0.185742013 \\ \text { H } & 4.400474318 & 0.212488013 & 0.612686044 \\ \text { H } & 3.141733228 & 1.073639079 & -1.334519097 \\ \text { C } & 3.264797234 & -2.191535156 & 1.433032104 \\ \text { H } & 2.947020210 & -3.213136231 & 1.243878088\end{array}$




$\begin{array}{llll}\mathrm{H} & 4.343835312 & -2.198834156 & 1.561649111 \\ \mathrm{H} & 2.820104201 & -1.874888133 & 2.371638172 \\ \mathrm{C} & 3.524422255 & -1.776866128 & -1.004570071 \\ \mathrm{H} & 4.605094333 & -1.784765130 & -0.893168063 \\ \mathrm{H} & 3.208503231 & -2.793941202 & -1.218864086 \\ \mathrm{H} & 3.294183235 & -1.175045082 & -1.876052137 \\ \mathrm{H} & 1.142268082 & -0.409848028 & -1.789670127\end{array}$

\section{Coord_3}

$\begin{array}{lrrr}\text { C } & -4.755773342 & -0.446031030 & -2.056392145 \\ \text { C } & -3.382728241 & -0.501839037 & -1.671370119 \\ \text { C } & -2.929790210 & 0.139196011 & -0.573623042 \\ \text { C } & -3.863969276 & 0.856502064 & 0.290731023 \\ \text { C } & -5.247325379 & 0.926182066 & -0.116659008 \\ \text { C } & -5.675243408 & 0.281373021 & -1.279891092 \\ \text { H } & -5.071861364 & -0.923720069 & -2.965100212 \\ \text { H } & -2.687218195 & -1.038716074 & -2.288180163 \\ \text { H } & -5.922463425 & 1.473813107 & 0.513081037 \\ \text { H } & -6.702660484 & 0.348197026 & -1.584012115 \\ \text { C } & -1.507767109 & 0.181661014 & -0.127260011 \\ \text { O } & -1.284066095 & -0.092507004 & 1.173830086 \\ \text { O } & -3.491209249 & 1.368352097 & 1.356588096 \\ \text { H } & -1.906180135 & 0.420750032 & 1.689301121 \\ \text { O } & -0.626325042 & -0.312275025 & -0.996928074 \\ \text { C } & 0.788462057 & -0.208800012 & -0.803473059 \\ \text { C } & 1.309165093 & -1.276801094 & 0.156639011 \\ \text { C } & 1.242020089 & 1.190014083 & -0.400941028 \\ \text { C } & 2.847450203 & -1.260253092 & 0.293239022 \\ \text { H } & 0.865168061 & -1.112790078 & 1.130049083 \\ \text { C } & 2.774436200 & 1.254993091 & -0.335257023 \\ \text { H } & 0.862043063 & 1.913953140 & -1.116540082 \\ \mathrm{C} & 3.311221239 & 0.176459012 & 0.616848044 \\ \text { H } & 2.984330217 & 0.425115030 & 1.625777117 \\ \text { C } & 3.249555236 & 2.647102188 & 0.085041006 \\ \text { H } & 2.888560206 & 2.900035208 & 1.078355080 \\ \text { H } & 4.334086312 & 2.701683197 & 0.102394009 \\ \text { H } & 2.890391206 & 3.408019247 & -0.601676046 \\ \text { H } & 0.829565058 & 1.447726102 & 0.569508043 \\ \text { H } & 0.974363072 & -2.252889163 & -0.184194011 \\ \text { H } & 4.398560315 & 0.209649014 & 0.631068048 \\ \text { H } & 3.164330229 & 1.065419078 & -1.333647098 \\ \text { C } & 3.245149232 & -2.184833158 & 1.453299106 \\ \text { H } & 2.924591212 & -3.205991232 & 1.266608093 \\ \text { H } & 4.322934309 & -2.196166156 & 1.591264114 \\ \text { H } & 2.793865202 & -1.861363135 & 2.386400170 \\ \text { C } & 3.527822256 & -1.784507130 & -0.984068072 \\ \text { H } & 4.607311331 & -1.797391130 & -0.862687061 \\ & & & \\ & & & \end{array}$




$\begin{array}{llll}H & 3.208664233 & -2.801006200 & -1.196127086 \\ H & 3.309020238 & -1.186001084 & -1.860734134 \\ H & 1.161833082 & -0.413569028 & -1.797781128\end{array}$

Coord_4

$\begin{array}{lrrr}\mathrm{C} & -4.766709345 & -0.420260030 & -2.074499152 \\ \mathrm{C} & -3.395443244 & -0.480925036 & -1.719425123 \\ \mathrm{C} & -2.925613212 & 0.122597007 & -0.591409044 \\ \mathrm{C} & -3.849795279 & 0.799474056 & 0.314872020 \\ \mathrm{C} & -5.241184378 & 0.870541063 & -0.071350006 \\ \mathrm{C} & -5.679106407 & 0.271064018 & -1.249011092 \\ \mathrm{H} & -5.100206368 & -0.866516061 & -2.992963215 \\ \mathrm{H} & -2.704777196 & -0.988240074 & -2.365762172 \\ \mathrm{H} & -5.910939426 & 1.388624100 & 0.588458045 \\ \mathrm{H} & -6.712076484 & 0.341081027 & -1.533695111 \\ \mathrm{C} & -1.514297108 & 0.165554013 & -0.159887011 \\ \mathrm{O} & -1.265590093 & -0.017266001 & 1.144644082 \\ \mathrm{O} & -3.466949251 & 1.280666094 & 1.390377100 \\ \mathrm{H} & -1.935418141 & 0.460966032 & 1.639066118 \\ \mathrm{O} & -0.617641043 & -0.298215023 & -1.022901072 \\ \mathrm{C} & 0.797748059 & -0.201339015 & -0.813831058 \\ \mathrm{C} & 1.302796096 & -1.266881090 & 0.156580013 \\ \mathrm{C} & 1.254900088 & 1.198013084 & -0.417784030 \\ \mathrm{C} & 2.840351206 & -1.258097092 & 0.303683023 \\ \mathrm{H} & 0.853362064 & -1.094396078 & 1.126119079 \\ \mathrm{C} & 2.787247200 & 1.254155091 & -0.339443022 \\ \mathrm{H} & 0.885667065 & 1.918066139 & -1.142839080 \\ \mathrm{C} & 3.309574239 & 0.177758015 & 0.623197044 \\ \mathrm{H} & 2.975930216 & 0.433866029 & 1.628001117 \\ \mathrm{C} & 3.266306236 & 2.645853193 & 0.077807005 \\ \mathrm{H} & 2.898179209 & 2.905849209 & 1.066629079 \\ \mathrm{H} & 4.350897315 & 2.694531192 & 0.104230005 \\ \mathrm{H} & 2.917330207 & 3.405151244 & -0.615876046 \\ \mathrm{H} & 0.835851060 & 1.465813106 & 0.546611041 \\ \mathrm{H} & 0.964996069 & -2.242890163 & -0.181392012 \\ \mathrm{H} & 4.396874314 & 0.204649014 & 0.646113049 \\ \mathrm{H} & 3.184807229 & 1.057148074 & -1.333298095 \\ \mathrm{C} & 3.224568232 & -2.179349157 & 1.470824107 \\ \mathrm{H} & 2.899768206 & -3.199589230 & 1.286604092 \\ \mathrm{H} & 4.301237311 & -2.195922158 & 1.616275115 \\ \mathrm{H} & 2.768739199 & -1.849034136 & 2.399290171 \\ \mathrm{H} & 3.526427255 & -1.792540130 & -0.966357070 \\ \mathrm{H} & 3.202539229 & -2.807971204 & -1.176184084 \\ \mathrm{H} & -176925085 & -0.414897030 & -1.803729128\end{array}$




\begin{tabular}{lrrr}
\multicolumn{5}{l}{ Coord 5} & & \\
C & -4.770351344 & -0.396594027 & -2.091642150 \\
C & -3.402570245 & -0.457476034 & -1.761070129 \\
C & -2.920541213 & 0.110035006 & -0.603059045 \\
C & -3.838962275 & 0.744475055 & 0.338677022 \\
C & -5.236729378 & 0.812557058 & -0.032132002 \\
C & -5.679270410 & 0.255995019 & -1.223171086 \\
H & -5.117096367 & -0.813509059 & -3.018994216 \\
H & -2.713803197 & -0.933848066 & -2.432281176 \\
H & -5.904284424 & 1.299656096 & 0.653054045 \\
H & -6.716595484 & 0.325829022 & -1.492407109 \\
C & -1.520832109 & 0.153989013 & -0.185591013 \\
O & -1.248943092 & 0.062530002 & 1.115957082 \\
O & -3.451181250 & 1.197340087 & 1.423668101 \\
H & -1.960289138 & 0.500081036 & 1.595676113 \\
O & -0.611812044 & -0.280647021 & -1.043154077 \\
C & 0.804485057 & -0.191811015 & -0.821118061 \\
C & 1.294623091 & -1.255691092 & 0.157984010 \\
C & 1.267505090 & 1.207360085 & -0.431809032 \\
C & 2.831638202 & -1.256270091 & 0.312633024 \\
H & 0.841896063 & -1.074716076 & 1.124534082 \\
C & 2.799844201 & 1.253125090 & -0.343724023 \\
H & 0.908386068 & 1.924094140 & -1.165161083 \\
C & 3.308430237 & 0.178042014 & 0.627837045 \\
H & 2.970332214 & 0.441501030 & 1.629202116 \\
C & 3.285093234 & 2.643694192 & 0.070201003 \\
H & 2.912098211 & 2.910704210 & 1.055284074 \\
H & 4.369743312 & 2.685394195 & 0.103632009 \\
H & 2.945765213 & 3.401943244 & -0.629347047 \\
H & 0.844148061 & 1.485239107 & 0.527357038 \\
H & 0.952152069 & -2.231082160 & -0.176943013 \\
H & 4.395663318 & 0.197551017 & 0.657375047 \\
H & 3.202852232 & 1.048701077 & -1.333815096 \\
C & 3.203679230 & -2.175019159 & 1.485590105 \\
H & 2.873227205 & -3.193882228 & 1.303969091 \\
H & 4.279425308 & -2.197967158 & 1.636442118 \\
H & 2.745518198 & -1.837819134 & 2.410408174 \\
C & 3.520294254 & -1.801010131 & -0.951650070 \\
H & 4.597733329 & -1.827366132 & -0.816107060 \\
H & 3.190227232 & -2.814899203 & -1.159126081 \\
H & 3.321593240 & -1.207403086 & -1.836339131 \\
H & 1.187226087 & -0.414079028 & -1.807364131 \\
& & & \\
& & &
\end{tabular}

Coord_6

$\begin{array}{lrrr}\text { C } & -4.766109343 & -0.374893029 & -2.108425150 \\ \text { C } & -3.403487246 & -0.431747034 & -1.795874127 \\ \text { C } & -2.914865210 & 0.101344005 & -0.607391043\end{array}$




$\begin{array}{lrrr}\text { C } & -3.832580276 & 0.691641052 & 0.362888024 \\ \text { C } & -5.234939377 & 0.752648055 & 0.000557001 \\ \text { C } & -5.675972408 & 0.236621020 & -1.203456085 \\ \text { H } & -5.121364366 & -0.764590056 & -3.044400217 \\ \text { H } & -2.713131195 & -0.876007061 & -2.486970179 \\ \text { H } & -5.904064424 & 1.207524088 & 0.706092051 \\ \text { H } & -6.716418484 & 0.303125021 & -1.462090105 \\ \text { C } & -1.527661109 & 0.146690013 & -0.203364013 \\ \text { O } & -1.233727087 & 0.146358013 & 1.088673079 \\ \text { O } & -3.445744250 & 1.118434081 & 1.457744105 \\ \text { H } & -1.979579141 & 0.538999038 & 1.560896113 \\ \text { O } & -0.609142043 & -0.259818018 & -1.056948077 \\ \text { C } & 0.808472059 & -0.180400011 & -0.824986061 \\ \text { C } & 1.284823093 & -1.243342090 & 0.161183014 \\ \text { C } & 1.279621090 & 1.217893088 & -0.442850030 \\ \text { C } & 2.821544204 & -1.254800090 & 0.320008024 \\ \text { H } & 0.831194062 & -1.053852078 & 1.125773082 \\ \text { C } & 2.812059204 & 1.251813091 & -0.348347027 \\ \text { H } & 0.929689066 & 1.931820138 & -1.183324087 \\ \text { C } & 3.307994237 & 0.177295011 & 0.630489043 \\ \text { H } & 2.967988213 & 0.448024034 & 1.629223119 \\ \text { C } & 3.305753240 & 2.640555192 & 0.061782005 \\ \text { H } & 2.930401213 & 2.914547211 & 1.044007074 \\ \text { H } & 4.390491315 & 2.674256194 & 0.099865007 \\ \text { H } & 2.975270216 & 3.398273246 & -0.642516046 \\ \text { H } & 0.854475061 & 1.505855107 & 0.512056037 \\ \text { H } & 0.935991070 & -2.217588161 & -0.170377013 \\ \text { H } & 4.395156314 & 0.188394012 & 0.664279048 \\ \text { H } & 3.218051229 & 1.039972076 & -1.335593094 \\ \text { C } & 3.183117228 & -2.171800158 & 1.497512109 \\ \text { H } & 2.845630204 & -3.188836232 & 1.318761093 \\ \text { H } & 4.258186308 & -2.202200158 & 1.651397117 \\ \text { H } & 2.725059194 & -1.827672134 & 2.419809172 \\ \text { C } & 3.509358251 & -1.809946130 & -0.940227065 \\ \text { H } & 4.586034331 & -1.844664132 & -0.801013056 \\ \text { H } & 3.171685226 & -2.821827203 & -1.145088083 \\ \text { H } & 3.318773239 & -1.218041088 & -1.827808132 \\ \text { H } & 1.192288085 & -0.411315029 & -1.808466132 \\ & & & \end{array}$

Coord_7

$\begin{array}{lrrr}\text { C } & -4.752938344 & -0.355023027 & -2.125391154 \\ \text { C } & -3.397235244 & -0.403958031 & -1.823149129 \\ \text { C } & -2.908889211 & 0.096380005 & -0.602908044 \\ \text { C } & -3.831967277 & 0.641066047 & 0.388304026 \\ \text { C } & -5.236834377 & 0.691148049 & 0.026076003 \\ \text { C } & -5.669194410 & 0.213331013 & -1.191132087 \\ \text { H } & -5.111166369 & -0.719642053 & -3.070342222 \\ & & & S 205\end{array}$




$\begin{array}{lrrr}\text { H } & -2.701129196 & -0.815108058 & -2.528613185 \\ \text { H } & -5.912029425 & 1.112716078 & 0.746395053 \\ \text { H } & -6.711393481 & 0.273556020 & -1.445042105 \\ \text { C } & -1.535114110 & 0.143441013 & -0.211884015 \\ \text { O } & -1.219451087 & 0.233723017 & 1.063901075 \\ \text { O } & -3.452967250 & 1.043959078 & 1.493918106 \\ \text { H } & -1.991982146 & 0.578810043 & 1.536671109 \\ \text { O } & -0.610065045 & -0.235909017 & -1.063291076 \\ \text { C } & 0.809394060 & -0.167249010 & -0.824958057 \\ \text { C } & 1.273515094 & -1.229919091 & 0.166590015 \\ \text { C } & 1.290957094 & 1.229474090 & -0.450664030 \\ \text { C } & 2.810242200 & -1.253722092 & 0.325676026 \\ \text { H } & 0.821718058 & -1.031897075 & 1.130422084 \\ \text { C } & 2.823646202 & 1.250133090 & -0.353620024 \\ \text { H } & 0.948944070 & 1.941073141 & -1.197032085 \\ \text { C } & 3.308452240 & 0.175484013 & 0.630770047 \\ \text { H } & 2.969427215 & 0.453403030 & 1.627819117 \\ \text { C } & 3.328101237 & 2.636352191 & 0.052000005 \\ \text { H } & 2.953231211 & 2.917296208 & 1.032404074 \\ \text { H } & 4.412973320 & 2.661065192 & 0.091990009 \\ \text { H } & 3.005349217 & 3.394021244 & -0.655884047 \\ \text { H } & 0.866865064 & 1.527523110 & 0.501125034 \\ \text { H } & 0.916584066 & -2.202498159 & -0.161107013 \\ \text { H } & 4.395558314 & 0.177181010 & 0.666055046 \\ \text { H } & 3.229819231 & 1.030874075 & -1.339106095 \\ \text { C } & 3.163538225 & -2.169681157 & 1.506412108 \\ \text { H } & 2.817634203 & -3.184437229 & 1.330976097 \\ \text { H } & 4.238230303 & -2.208571161 & 1.660581121 \\ \text { H } & 2.708327197 & -1.818591129 & 2.427478173 \\ \text { C } & 3.493373251 & -1.819367131 & -0.932465067 \\ \text { H } & 4.569526329 & -1.863470133 & -0.792328057 \\ \text { H } & 3.146656227 & -2.828766203 & -1.134266080 \\ \text { H } & 3.309158237 & -1.228714091 & -1.822192133 \\ \text { H } & 1.191454086 & -0.406743028 & -1.806709132\end{array}$

Coord_8

$\begin{array}{lrrr}\text { C } & -4.729040339 & -0.336885027 & -2.143003152 \\ \text { C } & -3.382305244 & -0.374268028 & -1.841746132 \\ \text { C } & -2.902953208 & 0.095083007 & -0.587559041 \\ \text { C } & -3.838802278 & 0.592842045 & 0.415827032 \\ \text { C } & -5.243517378 & 0.628286046 & 0.043434001 \\ \text { C } & -5.658491408 & 0.186403014 & -1.187793084 \\ \text { H } & -5.083520366 & -0.678581047 & -3.097925221 \\ \text { H } & -2.675391195 & -0.751431053 & -2.555207183 \\ \text { H } & -5.930241425 & 1.015547075 & 0.772097055 \\ \text { H } & -6.700769480 & 0.237526018 & -1.444218102 \\ \text { C } & -1.543600113 & 0.144136008 & -0.209299016 \\ & & & S 206\end{array}$




$\begin{array}{lrrr}\text { O } & -1.205465085 & 0.324169024 & 1.043130074 \\ \text { O } & -3.476021248 & 0.973932068 & 1.533568109 \\ \text { H } & -1.995958146 & 0.620846047 & 1.525325109 \\ \text { O } & -0.615230042 & -0.208997015 & -1.060765075 \\ \text { C } & 0.806738056 & -0.152438010 & -0.820345060 \\ \text { C } & 1.260731092 & -1.215481088 & 0.174757013 \\ \text { C } & 1.301106094 & 1.242005092 & -0.454878033 \\ \text { C } & 2.797810202 & -1.253079089 & 0.329413023 \\ \text { H } & 0.813992060 & -1.008923072 & 1.139252084 \\ \text { C } & 2.834227201 & 1.247994092 & -0.359944026 \\ \text { H } & 0.965314067 & 1.951743143 & -1.205806087 \\ \text { C } & 3.309968238 & 0.172538014 & 0.628120043 \\ \text { H } & 2.975310216 & 0.457557035 & 1.624599116 \\ \text { C } & 3.351901243 & 2.630973189 & 0.040121002 \\ \text { H } & 2.980843214 & 2.918830208 & 1.019929075 \\ \text { H } & 4.436967317 & 2.645716189 & 0.078725006 \\ \text { H } & 3.035382217 & 3.389059244 & -0.670085048 \\ \text { H } & 0.881380063 & 1.550134113 & 0.495147035 \\ \text { H } & 0.893844065 & -2.185850158 & -0.148342009 \\ \text { H } & 4.397034316 & 0.163849014 & 0.661594050 \\ \text { H } & 3.237286235 & 1.021322071 & -1.344969099 \\ \text { C } & 3.145637228 & -2.168694157 & 1.511954107 \\ \text { H } & 2.789892202 & -3.180700227 & 1.340515099 \\ \text { H } & 4.220306303 & -2.217103160 & 1.663121119 \\ \text { H } & 2.696480194 & -1.810624128 & 2.433288174 \\ \text { C } & 3.471787248 & -1.829291131 & -0.928898068 \\ \text { H } & 4.547709325 & -1.883798133 & -0.791127057 \\ \text { H } & 3.114530226 & -2.835719206 & -1.126954078 \\ \text { H } & 3.291730239 & -1.239451087 & -1.819986131 \\ \text { H } & 1.183720086 & -0.400431028 & -1.801589130\end{array}$

Coord_9

$\begin{array}{lrrr}\text { C } & -4.691224338 & -0.320462021 & -2.161578157 \\ \text { C } & -3.356172243 & -0.342724027 & -1.849625131 \\ \text { C } & -2.897486209 & 0.097597007 & -0.558246040 \\ \text { C } & -3.855457280 & 0.547126042 & 0.446546031 \\ \text { C } & -5.256243376 & 0.564128041 & 0.050964002 \\ \text { C } & -5.642627404 & 0.155890014 & -1.195695087 \\ \text { H } & -5.033321364 & -0.641465047 & -3.128168224 \\ \text { H } & -2.632094187 & -0.685051049 & -2.563242184 \\ \text { H } & -5.961356428 & 0.916061064 & 0.780017055 \\ \text { H } & -6.682678482 & 0.195066016 & -1.463847106 \\ \text { C } & -1.553680114 & 0.148879013 & -0.192778012 \\ \text { O } & -1.190802086 & 0.417325027 & 1.028595072 \\ \text { O } & -3.519663253 & 0.908494068 & 1.578219111 \\ \text { H } & -1.989425146 & 0.666794047 & 1.530014112 \\ \text { O } & -0.625683045 & -0.178967015 & -1.047172078\end{array}$




$\begin{array}{lrrr}\text { C } & 0.799623056 & -0.135937010 & -0.810070057 \\ \text { C } & 1.246334090 & -1.200022085 & 0.186486016 \\ \text { C } & 1.309438096 & 1.255456090 & -0.454891035 \\ \text { C } & 2.784140199 & -1.252943090 & 0.330828022 \\ \text { H } & 0.808655059 & -0.984945072 & 1.153366082 \\ \text { C } & 2.843181203 & 1.245291092 & -0.367909027 \\ \text { H } & 0.977580068 & 1.963824143 & -1.208833087 \\ \text { C } & 3.312658236 & 0.168327012 & 0.621649047 \\ \text { H } & 2.986540215 & 0.460333035 & 1.618894114 \\ \text { C } & 3.376839245 & 2.624247187 & 0.025056003 \\ \text { H } & 3.013698216 & 2.918962211 & 1.005751073 \\ \text { H } & 4.462143322 & 2.627988190 & 0.058140006 \\ \text { H } & 3.064528220 & 3.383235246 & -0.686006049 \\ \text { H } & 0.898140064 & 1.573645115 & 0.495017037 \\ \text { H } & 0.867394061 & -2.167592156 & -0.130922007 \\ \text { H } & 4.399674318 & 0.148215012 & 0.649165047 \\ \text { H } & 3.239050231 & 1.011224072 & -1.354055099 \\ \text { C } & 3.130184226 & -2.168941156 & 1.513510111 \\ \text { H } & 2.763040198 & -3.177684228 & 1.347097095 \\ \text { H } & 4.205238303 & -2.227950161 & 1.657556121 \\ \text { H } & 2.691032192 & -1.803893131 & 2.436909175 \\ \text { C } & 3.443471249 & -1.839763130 & -0.930364069 \\ \text { H } & 4.519503326 & -1.905740135 & -0.799078056 \\ \text { H } & 3.074026221 & -2.842681205 & -1.123631083 \\ \text { H } & 3.264696235 & -1.250274090 & -1.821913130 \\ \text { H } & 1.167426084 & -0.392330028 & -1.792277129 \\ & & & \\ \text { Coord_10 } & & & \\ \text { C } & -4.611562334 & -0.333859022 & -1.886868134 \\ \text { C } & -3.282183236 & -0.356643023 & -1.592134113 \\ \text { C } & -2.790555203 & 0.234685019 & -0.353389028 \\ \text { C } & -3.723649266 & 0.836482061 & 0.592918040 \\ \text { C } & -5.128749368 & 0.839068060 & 0.204941014 \\ \text { C } & -5.537092400 & 0.290274020 & -0.973681069 \\ \text { H } & -4.980988359 & -0.766878055 & -2.798165201 \\ \text { H } & -2.575890187 & -0.808617058 & -2.260952162 \\ \text { H } & -5.816977417 & 1.301218095 & 0.887116063 \\ \text { H } & -6.579261472 & 0.325136022 & -1.234926087 \\ \text { C } & -1.450121106 & 0.269661021 & -0.054218003 \\ \text { O } & -0.995568072 & 0.780885054 & 1.044869077 \\ \text { O } & -3.369633242 & 1.337184097 & 1.663357121 \\ \text { H } & -1.758676126 & 1.109712081 & 1.561620112 \\ \text { O } & -0.576987043 & -0.213360018 & -0.884903065 \\ \text { C } & 0.859982061 & -0.228992014 & -0.708958049 \\ \text { C } & 1.288269090 & -1.150774082 & 0.426993032 \\ \text { C } & 1.468879106 & 1.163779084 & -0.603727041 \\ \text { C } & 2.825561204 & -1.284398090 & 0.515862038 \\ & & & \\ & & & 5208\end{array}$




$\begin{array}{rrrr}\text { H } & 0.914149067 & -0.756897052 & 1.363881100 \\ \mathrm{C} & 3.001741218 & 1.063296077 & -0.575537039 \\ \mathrm{H} & 1.144252084 & 1.763118129 & -1.449730102 \\ \mathrm{C} & 3.452777249 & 0.125919007 & 0.554801038 \\ \mathrm{H} & 3.194472229 & 0.592350043 & 1.504409109 \\ \mathrm{C} & 3.636087264 & 2.447786175 & -0.428619030 \\ \mathrm{H} & 3.339625241 & 2.916377212 & 0.505695035 \\ \mathrm{H} & 4.719917339 & 2.383543174 & -0.438433029 \\ \mathrm{H} & 3.335932240 & 3.105132223 & -1.239145090 \\ \mathrm{H} & 1.124887080 & 1.656453120 & 0.296690019 \\ \mathrm{H} & 0.836079061 & -2.128236154 & 0.283650023 \\ \mathrm{H} & 4.536533329 & 0.036725002 & 0.542798039 \\ \mathrm{H} & 3.334353239 & 0.651227044 & -1.526024110 \\ \mathrm{C} & 3.174392227 & -2.025724144 & 1.814356129 \\ \mathrm{H} & 2.739113195 & -3.020915220 & 1.825318131 \\ \mathrm{H} & 4.249745306 & -2.134081155 & 1.922580137 \\ \mathrm{H} & 2.805284201 & -1.492141108 & 2.684871193 \\ \mathrm{C} & 3.383601241 & -2.104576152 & -0.661274046 \\ \mathrm{H} & 4.458723323 & -2.222561163 & -0.563815038 \\ \mathrm{H} & 2.944370212 & -3.097761223 & -0.677889047 \\ \mathrm{H} & 3.197335231 & -1.650727121 & -1.627500118 \\ \mathrm{H} & 1.161593085 & -0.661679046 & -1.650685120\end{array}$

LIIC path connecting S1-ENOL-MIN and $\mathrm{S}_{1} / \mathrm{S}_{0}-\mathrm{ENOL}$ Coord_1

$\begin{array}{lrrr}\text { C } & -4.659676336 & -0.343543024 & -1.903248138 \\ \text { C } & -3.258364232 & -0.339190022 & -1.473542104 \\ \text { C } & -2.845738204 & 0.255102016 & -0.339823024 \\ \text { C } & -3.827907275 & 0.869480062 & 0.543017039 \\ \text { C } & -5.162575373 & 0.868914065 & 0.138394011 \\ \text { C } & -5.572413399 & 0.263157020 & -1.110107080 \\ \text { H } & -4.917736352 & -0.807560059 & -2.835634204 \\ \text { H } & -2.534540181 & -0.823480060 & -2.101963149 \\ \text { H } & -5.885426425 & 1.339336097 & 0.776295055 \\ \text { H } & -6.607669475 & 0.301148020 & -1.392479098 \\ \text { C } & -1.436313104 & 0.283971022 & 0.014883999 \\ \text { O } & -0.966125068 & 0.778832058 & 1.139273081 \\ \text { O } & -3.412341244 & 1.365570096 & 1.637587120 \\ \text { H } & -1.726542123 & 1.126622083 & 1.634599116 \\ \text { O } & -0.579210042 & -0.209899013 & -0.862877063 \\ \text { C } & 0.844857058 & -0.257910016 & -0.689359049 \\ \text { C } & 1.259062089 & -1.187943085 & 0.447808034 \\ \text { C } & 1.482936107 & 1.122930082 & -0.580746043 \\ \text { C } & 2.793000203 & -1.345150099 & 0.547531037 \\ \text { H } & 0.883376066 & -0.791895057 & 1.382905101 \\ \text { C } & 3.013229217 & 0.998794069 & -0.542474039 \\ \text { H } & 1.173694082 & 1.726579123 & -1.429778103 \\ & & & \\ & & & \text { S209 }\end{array}$




$\begin{array}{lrrr}\mathrm{C} & 3.442014249 & 0.055032005 & 0.590935044 \\ \mathrm{H} & 3.183445228 & 0.525530038 & 1.538469110 \\ \mathrm{C} & 3.670088262 & 2.372461170 & -0.392494031 \\ \mathrm{H} & 3.374761245 & 2.846986205 & 0.539221038 \\ \mathrm{H} & 4.752958341 & 2.290744164 & -0.394739028 \\ \mathrm{H} & 3.386196242 & 3.034011219 & -1.205616086 \\ \mathrm{H} & 1.138782084 & 1.622673117 & 0.315945023 \\ \mathrm{H} & 0.793846060 & -2.158957153 & 0.299620021 \\ \mathrm{H} & 4.524569325 & -0.050857003 & 0.587389043 \\ \mathrm{H} & 3.345765242 & 0.580485042 & -1.490505109 \\ \mathrm{C} & 3.122973222 & -2.091413149 & 1.848263135 \\ \mathrm{H} & 2.672399195 & -3.079929220 & 1.856741131 \\ \mathrm{H} & 4.195968303 & -2.216249157 & 1.963699141 \\ \mathrm{H} & 2.756102198 & -1.552055112 & 2.716142198 \\ \mathrm{C} & 3.347050243 & -2.173445158 & -0.625777048 \\ \mathrm{H} & 4.420100316 & -2.306197166 & -0.522832038 \\ \mathrm{H} & 2.894325211 & -3.160668227 & -0.644434049 \\ \mathrm{H} & 3.171539226 & -1.717258126 & -1.592892117 \\ \mathrm{H} & 1.147894083 & -0.695794051 & -1.629625120\end{array}$

Coord_2

$\begin{array}{lrrr}\text { C } & -4.689960340 & -0.427682032 & -1.849334132 \\ \text { C } & -3.291957235 & -0.445906034 & -1.437984103 \\ \text { C } & -2.847869206 & 0.217672013 & -0.350236026 \\ \text { C } & -3.798966275 & 0.928696065 & 0.495886036 \\ \text { C } & -5.138619371 & 0.956156068 & 0.092491007 \\ \text { C } & -5.573775401 & 0.278717022 & -1.098351080 \\ \text { H } & -4.980552359 & -0.953333071 & -2.738913198 \\ \text { H } & -2.591519188 & -1.000727074 & -2.033273148 \\ \text { H } & -5.837534419 & 1.504828106 & 0.693085049 \\ \text { H } & -6.608210474 & 0.337072022 & -1.380611097 \\ \text { C } & -1.440906103 & 0.236397019 & 0.014499002 \\ \text { O } & -1.000496073 & 0.712866049 & 1.162125082 \\ \text { O } & -3.368257244 & 1.471303108 & 1.557573113 \\ \text { H } & -1.766808125 & 1.085098079 & 1.625929118 \\ \text { O } & -0.569127041 & -0.187534014 & -0.883424063 \\ \text { C } & 0.854117062 & -0.237787019 & -0.699675052 \\ \text { C } & 1.261543088 & -1.182455083 & 0.427428032 \\ \text { C } & 1.489364107 & 1.142034080 & -0.564223039 \\ \text { C } & 2.794515201 & -1.342697098 & 0.532913039 \\ \text { H } & 0.882699063 & -0.797777058 & 1.365810099 \\ \text { C } & 3.019928219 & 1.016033071 & -0.523896039 \\ \text { H } & 1.183111083 & 1.761115125 & -1.403057102 \\ \text { C } & 3.444844247 & 0.056048004 & 0.597286044 \\ \text { H } & 3.184509229 & 0.513961038 & 1.550496113 \\ \text { C } & 3.680945265 & 2.385490170 & -0.354991027 \\ \text { H } & 3.385354241 & 2.849589206 & 0.581863041\end{array}$




$\begin{array}{llrr}\mathrm{H} & 4.763560344 & 2.300087164 & -0.355783026 \\ \mathrm{H} & 3.401458243 & 3.058121222 & -1.160452083 \\ \mathrm{H} & 1.141691083 & 1.625804116 & 0.340463027 \\ \mathrm{H} & 0.796045056 & -2.150988156 & 0.264408022 \\ \mathrm{H} & 4.527277326 & -0.051053005 & 0.595053043 \\ \mathrm{H} & 3.352957243 & 0.609307046 & -1.476833105 \\ \mathrm{C} & 3.115700226 & -2.104789151 & 1.826641133 \\ \mathrm{H} & 2.662274192 & -3.092040225 & 1.821119131 \\ \mathrm{H} & 4.187662300 & -2.233802163 & 1.946458138 \\ \mathrm{H} & 2.745268198 & -1.574422111 & 2.698521193 \\ \mathrm{C} & 3.353672240 & -2.157545155 & -0.647203048 \\ \mathrm{H} & 4.426033319 & -2.292706163 & -0.540510040 \\ \mathrm{H} & 2.899814208 & -3.143873226 & -0.679644048 \\ \mathrm{H} & 3.183213231 & -1.689842120 & -1.609739115 \\ \mathrm{H} & 1.165360086 & -0.660468045 & -1.644140118\end{array}$

\section{Coord 3}

$\begin{array}{lrrr}\text { C } & -4.722319338 & -0.508331036 & -1.788967131 \\ \text { C } & -3.328755242 & -0.551026041 & -1.394140101 \\ \text { C } & -2.850108208 & 0.179355013 & -0.359399026 \\ \text { C } & -3.766371269 & 0.985073069 & 0.441748034 \\ \text { C } & -5.109655367 & 1.040442076 & 0.036985002 \\ \text { C } & -5.572829402 & 0.295212022 & -1.089696079 \\ \text { H } & -5.048059363 & -1.091812079 & -2.629442189 \\ \text { H } & -2.654603191 & -1.174632086 & -1.949911142 \\ \text { H } & -5.781352416 & 1.664514120 & 0.592952045 \\ \text { H } & -6.605603477 & 0.373671027 & -1.373512099 \\ \text { C } & -1.445415106 & 0.188086015 & 0.013391000 \\ \text { O } & -1.032538073 & 0.643321046 & 1.183042083 \\ \text { O } & -3.319196237 & 1.573752114 & 1.466864105 \\ \text { H } & -1.800498131 & 1.041739073 & 1.617612119 \\ \text { O } & -0.560420038 & -0.164758014 & -0.900628065 \\ \text { C } & 0.862020060 & -0.217424014 & -0.707867050 \\ \text { C } & 1.263661089 & -1.176488085 & 0.408690029 \\ \text { C } & 1.494414109 & 1.160941085 & -0.546317041 \\ \text { C } & 2.795733203 & -1.339832095 & 0.518559038 \\ \text { H } & 0.882455065 & -0.803126056 & 1.350498096 \\ \text { C } & 3.025282218 & 1.033112076 & -0.505293035 \\ \text { H } & 1.190376088 & 1.795155129 & -1.374475102 \\ \text { C } & 3.447351251 & 0.057215004 & 0.603167045 \\ \text { H } & 3.186052230 & 0.502560036 & 1.562061112 \\ \text { C } & 3.690521267 & 2.398147174 & -0.318250023 \\ \text { H } & 3.395462246 & 2.851842205 & 0.623838047 \\ \text { H } & 4.772868344 & 2.309077166 & -0.318613023 \\ \text { H } & 3.414710243 & 3.081656222 & -1.115719079 \\ \text { H } & 1.143959084 & 1.628684115 & 0.366310028 \\ \text { H } & 0.797794055 & -2.142362154 & 0.231375016\end{array}$




$\begin{array}{lrrr}\mathrm{H} & 4.529660327 & -0.051082004 & 0.601290043 \\ \mathrm{H} & 3.357997243 & 0.637943044 & -1.463240103 \\ \mathrm{C} & 3.109264224 & -2.117535153 & 1.804848132 \\ \mathrm{H} & 2.653031191 & -3.103343223 & 1.785824130 \\ \mathrm{H} & 4.180266302 & -2.250779164 & 1.928045141 \\ \mathrm{H} & 2.736019196 & -1.596104117 & 2.680890193 \\ \mathrm{C} & 3.358996240 & -2.141285152 & -0.668592049 \\ \mathrm{H} & 4.430742320 & -2.278894166 & -0.559110038 \\ \mathrm{H} & 2.904026210 & -3.126554223 & -0.714314053 \\ \mathrm{H} & 3.192741232 & -1.662219120 & -1.626292118 \\ \mathrm{H} & 1.180675084 & -0.624949044 & -1.656445119\end{array}$

$\begin{array}{lrrr}\text { Coord_4 } & & \\ \text { C } & -4.756494341 & -0.585157042 & -1.721799123 \\ \text { C } & -3.368605241 & -0.653779049 & -1.341350098 \\ \text { C } & -2.852423205 & 0.140422009 & -0.367140027 \\ \text { C } & -3.730215271 & 1.038128074 & 0.380259029 \\ \text { C } & -5.075800364 & 1.120634083 & -0.028542000 \\ \text { C } & -5.569469401 & 0.311904024 & -1.084322078 \\ \text { H } & -5.119778369 & -1.222117087 & -2.506724180 \\ \text { H } & -2.723548198 & -1.343751097 & -1.850787131 \\ \text { H } & -5.717178414 & 1.816435129 & 0.475191036 \\ \text { H } & -6.599770478 & 0.409795031 & -1.371420098 \\ \text { C } & -1.449825105 & 0.139235012 & 0.011926999 \\ \text { O } & -1.062312077 & 0.571106042 & 1.202201088 \\ \text { O } & -3.265255237 & 1.672479121 & 1.364675099 \\ \text { H } & -1.827184132 & 0.999396072 & 1.608985117 \\ \text { O } & -0.553241039 & -0.141476008 & -0.914261068 \\ \text { C } & 0.868451061 & -0.196756012 & -0.713817050 \\ \text { C } & 1.265354091 & -1.170180086 & 0.391524028 \\ \text { C } & 1.498044106 & 1.179697085 & -0.526696037 \\ \text { C } & 2.796612203 & -1.336705097 & 0.504269035 \\ \text { H } & 0.882650061 & -0.808257059 & 1.337003097 \\ \text { C } & 3.029252217 & 1.050078076 & -0.486455034 \\ \text { H } & 1.195414088 & 1.828896131 & -1.343571097 \\ \text { C } & 3.449547246 & 0.058377003 & 0.608590043 \\ \text { H } & 3.188159229 & 0.490992036 & 1.573280115 \\ \text { C } & 3.698832266 & 2.410453176 & -0.281865018 \\ \text { H } & 3.405175247 & 2.853589204 & 0.665657046 \\ \text { H } & 4.780900345 & 2.317747169 & -0.282909021 \\ \text { H } & 3.425930246 & 3.104787226 & -1.070876075 \\ \text { H } & 1.145624082 & 1.631193119 & 0.393941029 \\ \text { H } & 0.798987059 & -2.133190153 & 0.200305014 \\ \text { H } & 4.531731326 & -0.051091005 & 0.606019046 \\ \text { H } & 3.360771243 & 0.666620048 & -1.449611106 \\ \text { C } & 3.103695221 & -2.130036156 & 1.782539130 \\ \text { H } & 2.644669190 & -3.114223224 & 1.750368127 \\ & & & \\ & & & \\ & & 5212\end{array}$




$\begin{array}{llll}\mathrm{H} & 4.173827300 & -2.267582161 & 1.908021137 \\ \mathrm{H} & 2.728452194 & -1.617651119 & 2.663036192 \\ \mathrm{C} & 3.362888243 & -2.124587154 & -0.690345049 \\ \mathrm{H} & 4.434108321 & -2.264698160 & -0.579130041 \\ \mathrm{H} & 2.906796209 & -3.108634223 & -0.748994054 \\ \mathrm{H} & 3.199934232 & -1.634136117 & -1.642865118 \\ \mathrm{H} & 1.193650087 & -0.588996043 & -1.666540122\end{array}$

\section{Coord_5}

$\begin{array}{lrrr}\text { C } & -4.792318344 & -0.657501049 & -1.647578119 \\ \text { C } & -3.411412247 & -0.753182053 & -1.279116091 \\ \text { C } & -2.854788203 & 0.101036008 & -0.373393029 \\ \text { C } & -3.690556267 & 1.087134078 & 0.311079021 \\ \text { C } & -5.037194364 & 1.195480087 & -0.104394008 \\ \text { C } & -5.563672402 & 0.328215024 & -1.082358079 \\ \text { H } & -5.195352373 & -1.342825095 & -2.370445169 \\ \text { H } & -2.798191199 & -1.506293107 & -1.735081123 \\ \text { H } & -5.645313408 & 1.958406141 & 0.339332027 \\ \text { H } & -6.590744476 & 0.444553033 & -1.374446097 \\ \text { C } & -1.454138106 & 0.089873009 & 0.010368001 \\ \text { O } & -1.089957080 & 0.497031038 & 1.219705086 \\ \text { O } & -3.206411229 & 1.766547129 & 1.250223088 \\ \text { H } & -1.846060133 & 0.960614068 & 1.599326113 \\ \text { O } & -0.547716037 & -0.117729010 & -0.924141066 \\ \text { C } & 0.873318063 & -0.175798011 & -0.717417053 \\ \text { C } & 1.266592090 & -1.163706084 & 0.375864027 \\ \text { C } & 1.500181109 & 1.198274085 & -0.505048036 \\ \text { C } & 2.797143201 & -1.333439094 & 0.489878033 \\ \text { H } & 0.883293065 & -0.813524061 & 1.325335094 \\ \text { C } & 3.031772217 & 1.066970075 & -0.467162036 \\ \text { H } & 1.198116089 & 1.862426135 & -1.309913096 \\ \text { C } & 3.451419248 & 0.059411005 & 0.613587042 \\ \text { H } & 3.190862229 & 0.478962032 & 1.584266115 \\ \text { C } & 3.705808267 & 2.422436172 & -0.245432016 \\ \text { H } & 3.414463244 & 2.854684204 & 0.707801051 \\ \text { H } & 4.787589346 & 2.326172168 & -0.248333019 \\ \text { H } & 3.435005249 & 3.127655224 & -1.025398074 \\ \text { H } & 1.146657083 & 1.633132120 & 0.423753028 \\ \text { H } & 0.799588056 & -2.123630153 & 0.170995013 \\ \text { H } & 4.533482326 & -0.051151003 & 0.609209042 \\ \text { H } & 3.361172243 & 0.695553053 & -1.435798103 \\ \text { C } & 3.099054226 & -2.142613154 & 1.759415127 \\ \text { H } & 2.637256190 & -3.125013227 & 1.714318123 \\ \text { H } & 4.168429300 & -2.284503164 & 1.886016138 \\ \text { H } & 2.722659196 & -1.639552120 & 2.644765188 \\ \text { C } & 3.365298243 & -2.107350152 & -0.712787054 \\ \text { H } & 4.436100319 & -2.249985160 & -0.600966043\end{array}$




\begin{tabular}{|c|c|c|c|}
\hline $\mathrm{H}$ & 2.908077210 & -3.090013222 & -0.784148056 \\
\hline $\mathrm{H}$ & 3.204693229 & -1.605341117 & -1.659697120 \\
\hline & 1.204155086 & -0.552452041 & 1671 \\
\hline \multicolumn{4}{|c|}{ Coord_6 } \\
\hline C & -4.829691350 & -0.724429054 & -1.566170110 \\
\hline & -3.457129250 & -0.848069059 & -1.207115086 \\
\hline & -2.857192208 & 0.061265004 & -0.378180029 \\
\hline & -3.647416261 & 1.131144083 & 0.233907018 \\
\hline & -4.993980359 & 1.263629089 & -0.190734012 \\
\hline & -5.555472398 & 0.343689024 & -1.083873076 \\
\hline & -5.274506378 & -1.452067102 & -2.220510161 \\
\hline & -2.878423207 & -1.660169119 & -1.602275117 \\
\hline r & -5.566059399 & 2.088054149 & 0.185184012 \\
\hline ⺊ & -6.578635472 & 0.477263035 & -1.382567100 \\
\hline & -1.458375107 & 0.039886005 & 870000 \\
\hline C & -1.115676078 & 0.421828029 & 1.235580086 \\
\hline C & -3.142535228 & 1.854588131 & 1.122768080 \\
\hline ト & -1.855820135 & 0.927628069 & 1.587841115 \\
\hline & -0.543956037 & -0.093678005 & 122066 \\
\hline C & 0.876539064 & -0.154632011 & -0.718572051 \\
\hline C & 1.267367092 & -1.157278083 & 42029 \\
\hline $\mathrm{C}$ & 1.500711107 & 1.216583089 & -0.481079032 \\
\hline C & 2.797339203 & -1.330139097 & 0.475253034 \\
\hline $\mathrm{H}$ & 0.884384064 & -0.819317059 & 1.315480093 \\
\hline C & 3.032733219 & 1.083820079 & -0.447179033 \\
\hline $\mathrm{H}$ & 1.198328088 & 1.895741135 & 88090 \\
\hline C & 3.452929250 & 0.060233002 & 0.618219047 \\
\hline r & 3.194130232 & 0.466208035 & 34114 \\
\hline C & 3.711289268 & 2.434136175 & -0.208540017 \\
\hline 卜 & 3.423170247 & 2.854990206 & 0.750737053 \\
\hline $\mathrm{H}$ & 4.792777347 & 2.334487167 & -0.214515016 \\
\hline ⺊ & 3.441724250 & 3.150391229 & -0.978755069 \\
\hline $\mathrm{H}$ & 1.146960085 & 1.6342 & 0.456103030 \\
\hline $\mathrm{H}$ & 0.799623056 & -2.113888153 & 0.143244011 \\
\hline $\mathrm{H}$ & 4.534877327 & -0.051259003 & 0.610875046 \\
\hline $\mathrm{H}$ & 3.359079241 & 0.724955051 & -1.421614100 \\
\hline$C$ & 3.095429224 & -2.155533154 & 1.735210126 \\
\hline $\mathrm{H}$ & 2.630919191 & -3.135998227 & 1.677277123 \\
\hline ト & 4.164174302 & -2.301742166 & 1.861714133 \\
\hline $\mathrm{H}$ & 2.718724197 & -1.662247119 & 2.625894188 \\
\hline C & 3.366247243 & -2.089436148 & -0.736178054 \\
\hline $\mathrm{H}$ & 4.436753318 & -2.234549160 & -0.624929046 \\
\hline $\mathrm{H}$ & 2.907932210 & -3.070571223 & -0.820174057 \\
\hline$\cdots$ & 3.206988228 & -1.575568113 & -1.676963120 \\
\hline 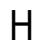 & 1.212098089 & -0.515223035 & -1.680032121 \\
\hline
\end{tabular}




$\begin{array}{lrrr}\text { Coord_7 } & & & \\ \text { C } & -4.868554349 & -0.784784058 & -1.477553104 \\ \text { C } & -3.505735255 & -0.937108068 & -1.125195083 \\ \text { C } & -2.859634208 & 0.021097000 & -0.381595027 \\ \text { C } & -3.600792258 & 1.169025083 & 0.148490010 \\ \text { C } & -4.946306357 & 1.323649098 & -0.287566019 \\ \text { C } & -5.544941401 & 0.357974026 & -1.088866080 \\ \text { H } & -5.357014388 & -1.547609112 & -2.057049145 \\ \text { H } & -2.964180211 & -1.803027132 & -1.452148103 \\ \text { H } & -5.479714394 & 2.202848156 & 0.012866003 \\ \text { H } & -6.563612473 & 0.507424037 & -1.395622099 \\ \text { C } & -1.462570106 & -0.010969003 & 0.007484000 \\ \text { O } & -1.139705081 & 0.346187026 & 1.249774091 \\ \text { O } & -3.073409222 & 1.934852138 & 0.981628073 \\ \text { H } & -1.854499134 & 0.902292063 & 1.573624112 \\ \text { O } & -0.542066038 & -0.069590004 & -0.932103067 \\ \text { C } & 0.878032064 & -0.133402007 & -0.717199053 \\ \text { C } & 1.267681089 & -1.151135082 & 0.348776026 \\ \text { C } & 1.499472107 & 1.234485086 & -0.454504031 \\ \text { C } & 2.797223202 & -1.326883096 & 0.460280033 \\ \text { H } & 0.885906063 & -0.826069059 & 1.307389095 \\ \text { C } & 3.031978220 & 1.100664082 & -0.426246030 \\ \text { H } & 1.195843087 & 1.928764139 & -1.232693091 \\ \text { C } & 3.453998246 & 0.060790004 & 0.622569043 \\ \text { H } & 3.197864228 & 0.452495031 & 1.606005113 \\ \text { C } & 3.715016268 & 2.445613178 & -0.170751015 \\ \text { H } & 3.431015247 & 2.854384203 & 0.794943055 \\ \text { H } & 4.796210347 & 2.342881167 & -0.181037014 \\ \text { H } & 3.445769249 & 3.173126229 & -0.930388067 \\ \text { H } & 1.146357082 & 1.634184119 & 0.491314035 \\ \text { H } & 0.799160058 & -2.104206152 & 0.116837008 \\ \text { H } & 4.535842324 & -0.051334003 & 0.611082044 \\ \text { H } & 3.354350244 & 0.755054055 & -1.406824104 \\ \text { C } & 3.092916225 & -2.169018156 & 1.709675122 \\ \text { H } & 2.625827189 & -3.147429228 & 1.638863120 \\ \text { H } & 4.161179302 & -2.319416167 & 1.834835130 \\ \text { H } & 2.716707195 & -1.686140123 & 2.606235190 \\ \text { C } & 3.365809243 & -2.070666151 & -0.760730052 \\ \text { H } & 4.436158322 & -2.218103162 & -0.651258049 \\ \text { H } & 2.906509210 & -3.050151220 & -0.857421060 \\ \text { H } & 3.206848229 & -1.544523110 & -1.694786122 \\ \text { H } & 1.217419088 & -0.477266035 & -1.683365122 \\ & & & \\ & & & \end{array}$

Coord_8

$\begin{array}{llll}\text { C } & -4.908865352 & -0.837215062 & -1.381797100 \\ \text { C } & -3.557222254 & -1.018809072 & -1.033365076 \\ \text { C } & -2.862131205 & -0.019536002 & -0.383798029\end{array}$




$\begin{array}{lrrr}\text { C } & -3.550662255 & 1.199478085 & 0.054628005 \\ \text { C } & -4.894341352 & 1.374041097 & -0.394725026 \\ \text { C } & -5.532189398 & 0.370794027 & -1.097245078 \\ \text { H } & -5.442653391 & -1.626911118 & -1.880385136 \\ \text { H } & -3.055402220 & -1.932268139 & -1.284764090 \\ \text { H } & -5.386609388 & 2.300126164 & -0.177195012 \\ \text { H } & -6.545890470 & 0.534674041 & -1.413275102 \\ \text { C } & -1.466773107 & -0.063039007 & 0.006141001 \\ \text { O } & -1.162289085 & 0.270805018 & 1.262134090 \\ \text { O } & -2.998750218 & 2.005261145 & 0.826166058 \\ \text { H } & -1.839281134 & 0.885872063 & 1.555609114 \\ \text { O } & -0.542153040 & -0.045810001 & -0.930038069 \\ \text { C } & 0.877705064 & -0.112290010 & -0.713237050 \\ \text { C } & 1.267533089 & -1.145536085 & 0.337158024 \\ \text { C } & 1.496250106 & 1.251800089 & -0.425048031 \\ \text { C } & 2.796811200 & -1.323724093 & 0.444854034 \\ \text { H } & 0.887814064 & -0.834246060 & 1.300969095 \\ \text { C } & 3.029293217 & 1.117552080 & -0.404074027 \\ \text { H } & 1.190393085 & 1.961359140 & -1.188325087 \\ \text { C } & 3.454501250 & 0.061068002 & 0.626747045 \\ \text { H } & 3.201892230 & 0.437615033 & 1.617006115 \\ \text { C } & 3.716629269 & 2.456950176 & -0.131580008 \\ \text { H } & 3.437588248 & 2.852754205 & 0.840923060 \\ \text { H } & 4.797532343 & 2.351603167 & -0.147408011 \\ \text { H } & 3.446717249 & 3.195999228 & -0.879693061 \\ \text { H } & 1.144594080 & 1.632585120 & 0.529678036 \\ \text { H } & 0.798298060 & -2.094852150 & 0.091523004 \\ \text { H } & 4.536257326 & -0.051228004 & 0.609947044 \\ \text { H } & 3.346817241 & 0.786098058 & -1.391148101 \\ \text { C } & 3.091607220 & -2.183246156 & 1.682564123 \\ \text { H } & 2.622174188 & -3.159518230 & 1.598683115 \\ \text { H } & 4.159553299 & -2.337564169 & 1.805128130 \\ \text { H } & 2.716637196 & -1.711610122 & 2.585586185 \\ \text { C } & 3.364096244 & -2.050809146 & -0.786615059 \\ \text { H } & 4.434442322 & -2.200275159 & -0.680126049 \\ \text { H } & 2.904022209 & -3.028551216 & -0.896216065 \\ \text { H } & 3.204340231 & -1.511872111 & -1.713241126 \\ \text { H } & 1.220070086 & -0.438561032 & -1.684375121\end{array}$

Coord_9

$\begin{array}{crrc}C & -4.950568356 & -0.880188064 & -1.279016093 \\ \text { C } & -3.611557258 & -1.091508077 & -0.931758065 \\ \text { C } & -2.864719204 & -0.060732006 & -0.385012025 \\ \text { C } & -3.497024251 & 1.221063087 & -0.047859002 \\ \text { C } & -4.838308350 & 1.413239102 & -0.511888039 \\ \text { C } & -5.517362397 & 0.381935029 & -1.108799081 \\ H & -5.531145401 & -1.687157122 & -1.690954122 \\ & & & S 216\end{array}$




$\begin{array}{lrrr}\text { H } & -3.151972226 & -2.045031149 & -1.100418078 \\ \text { H } & -5.287171378 & 2.377101171 & -0.384292027 \\ \text { H } & -6.525749471 & 0.558776038 & -1.434994103 \\ \text { C } & -1.471045107 & -0.116729008 & 0.004640001 \\ \text { O } & -1.183630084 & 0.196460012 & 1.272374093 \\ \text { O } & -2.918246208 & 2.063439146 & 0.655732047 \\ \text { H } & -1.806332130 & 0.878697065 & 1.532509108 \\ \text { O } & -0.544332039 & -0.022736000 & -0.923944064 \\ \text { C } & 0.875452066 & -0.091498006 & -0.706651053 \\ \text { C } & 1.266906094 & -1.140747083 & 0.326643023 \\ \text { C } & 1.490775106 & 1.268322092 & -0.392433027 \\ \text { C } & 2.796103203 & -1.320682096 & 0.428875030 \\ \text { H } & 0.890022061 & -0.844338063 & 1.296066094 \\ \text { C } & 3.024409218 & 1.134547084 & -0.380330029 \\ \text { H } & 1.181657083 & 1.993349143 & -1.139574083 \\ \mathrm{C} & 3.454259247 & 0.061082004 & 0.630893043 \\ \text { H } & 3.205958232 & 0.421376033 & 1.628278115 \\ \mathrm{C} & 3.715682270 & 2.468248179 & -0.090474006 \\ \mathrm{H} & 3.442359246 & 2.849992207 & 0.889232063 \\ \mathrm{H} & 4.796300344 & 2.360954168 & -0.113041008 \\ \mathrm{H} & 3.444057249 & 3.219157231 & -0.825984058 \\ \mathrm{H} & 1.141336084 & 1.629012115 & 0.571463041 \\ \mathrm{H} & 0.797146057 & -2.086101151 & 0.066995004 \\ \mathrm{H} & 4.535945328 & -0.050728005 & 0.607650044 \\ \mathrm{H} & 3.336281238 & 0.818368059 & -1.374243100 \\ \mathrm{C} & 3.091566225 & -2.198358156 & 1.653615121 \\ \mathrm{H} & 2.620150191 & -3.172445231 & 1.556310109 \\ \mathrm{H} & 4.159376300 & -2.356162172 & 1.772352129 \\ \mathrm{H} & 2.718485194 & -1.739018127 & 2.563713183 \\ \mathrm{C} & 3.361240242 & -2.029573146 & -0.813980058 \\ \mathrm{H} & 4.431751318 & -2.180604159 & -0.711656049 \\ \mathrm{H} & 2.900731208 & -3.005510214 & -0.936875066 \\ \mathrm{H} & 3.199560231 & -1.477219108 & -1.732365126 \\ \mathrm{H} & 1.220013089 & -0.399088029 & -1.683028121\end{array}$

$\begin{array}{llll}\text { Coord_10 } & & \\ \text { C } & -5.007863361 & -0.976640069 & -1.491434106 \\ \text { C } & -3.683587267 & -1.211023087 & -1.135607084 \\ \text { C } & -2.945969211 & -0.204399015 & -0.520909040 \\ \text { C } & -3.587354257 & 1.055661076 & -0.119057007 \\ \text { C } & -4.919941355 & 1.275558094 & -0.609594045 \\ \text { C } & -5.577305400 & 0.282089019 & -1.270055090 \\ \text { H } & -5.587662404 & -1.758647127 & -1.950868143 \\ \text { H } & -3.220272234 & -2.154237156 & -1.345352096 \\ \text { H } & -5.370686386 & 2.232788158 & -0.445775030 \\ \text { H } & -6.577884474 & 0.476027035 & -1.610566118 \\ \text { C } & -1.563985111 & -0.272487018 & -0.098237010\end{array}$




$\begin{array}{lrrr}\text { O } & -1.343608097 & -0.159823012 & 1.217913086 \\ \text { O } & -3.039536221 & 1.850917134 & 0.653971045 \\ \text { H } & -1.931882136 & 0.529708040 & 1.530914110 \\ \text { O } & -0.620439046 & 0.074183004 & -0.945511069 \\ \text { C } & 0.794360055 & 0.032514005 & -0.691584049 \\ \text { C } & 1.209674087 & -1.155794085 & 0.166465012 \\ \text { C } & 1.330965095 & 1.345758097 & -0.131252008 \\ \text { C } & 2.742454198 & -1.283510093 & 0.288034018 \\ \text { H } & 0.795525060 & -1.041441072 & 1.158807083 \\ \text { C } & 2.869091208 & 1.280303094 & -0.094622009 \\ \text { H } & 1.003885072 & 2.173626158 & -0.753278057 \\ \text { C } & 3.327654241 & 0.072723004 & 0.736801054 \\ \text { H } & 3.039552218 & 0.249968018 & 1.772106126 \\ \text { C } & 3.490866252 & 2.575244183 & 0.431801031 \\ \text { H } & 3.178542231 & 2.777470200 & 1.452481104 \\ \text { H } & 4.575561331 & 2.518759179 & 0.424016029 \\ \text { H } & 3.201450230 & 3.425733249 & -0.177646014 \\ \text { H } & 0.940857066 & 1.519840108 & 0.868725064 \\ \text { H } & 0.792944056 & -2.064261149 & -0.261175018 \\ \text { H } & 4.413747316 & 0.014271000 & 0.728444054 \\ \text { H } & 3.217142229 & 1.147781082 & -1.117603083 \\ \text { C } & 3.047399218 & -2.338342171 & 1.361316098 \\ \text { H } & 2.624496191 & -3.301728239 & 1.089978077 \\ \text { H } & 4.117965296 & -2.468684179 & 1.487773109 \\ \text { H } & 2.629765188 & -2.051677150 & 2.321437169 \\ \text { C } & 3.372679245 & -1.751473124 & -1.034855075 \\ \text { H } & 4.446290320 & -1.870559135 & -0.923492067 \\ \text { H } & 2.963033214 & -2.712677193 & -1.331272097 \\ \text { H } & 3.207501229 & -1.062376076 & -1.854967133 \\ \text { H } & 1.181788086 & -0.089342006 & -1.692367121\end{array}$

LIIC path connecting $\mathrm{S}_{1} / \mathrm{S}_{0}-\mathrm{ENOL}$ and S0-ENOL-MIN Coord_1

$\begin{array}{lrrr}\text { C } & -4.854739351 & -0.810520059 & -1.413566102 \\ \text { C } & -3.447410249 & -0.856604063 & -1.139168082 \\ \text { C } & -2.871991205 & 0.172662011 & -0.460299036 \\ \text { C } & -3.658184262 & 1.255013089 & -0.028111001 \\ \text { C } & -5.012425361 & 1.335946098 & -0.316447021 \\ \text { C } & -5.604595404 & 0.244707017 & -0.995236072 \\ \text { H } & -5.308365382 & -1.604915114 & -1.977851145 \\ \text { H } & -2.855641205 & -1.677337121 & -1.501133110 \\ \text { H } & -5.592391402 & 2.186500157 & -0.012157001 \\ \text { H } & -6.655520481 & 0.281089022 & -1.211321088 \\ \text { C } & -1.425882105 & 0.382213027 & -0.077965007 \\ \text { O } & -1.146903082 & 0.363529027 & 1.245618092 \\ \text { O } & -2.975686213 & 2.182043156 & 0.645294047 \\ \text { H } & -1.711336125 & 1.011798071 & 1.658396121\end{array}$




$\begin{array}{lrrr}\text { O } & -0.543849037 & -0.171280012 & -0.909802067 \\ \text { C } & 0.866638063 & -0.175305014 & -0.697884052 \\ \text { C } & 1.283068093 & -1.206431088 & 0.348879025 \\ \text { C } & 1.465544104 & 1.197037086 & -0.410640032 \\ \text { C } & 2.814995202 & -1.358801099 & 0.453853032 \\ \text { H } & 0.890851065 & -0.903863063 & 1.311220095 \\ \text { C } & 2.998481216 & 1.088845077 & -0.395679027 \\ \text { H } & 1.142098084 & 1.901607139 & -1.171803083 \\ \text { C } & 3.449870251 & 0.037984004 & 0.630348044 \\ \text { H } & 3.198064231 & 0.407900031 & 1.623528119 \\ \text { C } & 3.657057262 & 2.442120174 & -0.122374006 \\ \text { H } & 3.375307245 & 2.826961201 & 0.854072061 \\ \text { H } & 4.739793343 & 2.358653168 & -0.146129011 \\ \text { H } & 3.365394242 & 3.177682228 & -0.866305062 \\ \text { H } & 1.114346081 & 1.566526114 & 0.547567037 \\ \text { H } & 0.826721059 & -2.162686154 & 0.107047006 \\ \text { H } & 4.533650324 & -0.053767001 & 0.604584044 \\ \text { H } & 3.318729238 & 0.768631055 & -1.385473098 \\ \text { C } & 3.136076228 & -2.214639157 & 1.687850122 \\ \text { H } & 2.683692192 & -3.199169232 & 1.606689117 \\ \text { H } & 4.207866303 & -2.351115171 & 1.802628127 \\ \text { H } & 2.760275199 & -1.751875124 & 2.595418187 \\ \text { C } & 3.389529243 & -2.074517149 & -0.781424058 \\ \text { H } & 4.461835319 & -2.212822158 & -0.676913049 \\ \text { H } & 2.940155210 & -3.057058222 & -0.895126066 \\ \text { H } & 3.222109230 & -1.532085110 & -1.704514125 \\ \text { H } & 1.221133087 & -0.495696035 & -1.667901118\end{array}$

Coord_2

$\begin{array}{lrrr}\text { C } & -4.858919349 & -0.767519058 & -1.470011106 \\ \text { C } & -3.455897246 & -0.828023057 & -1.219378087 \\ \text { C } & -2.865082204 & 0.160523013 & -0.478583036 \\ \text { C } & -3.645718264 & 1.217148086 & 0.038273004 \\ \text { C } & -5.011478361 & 1.305052095 & -0.230763017 \\ \text { C } & -5.607544403 & 0.265698018 & -0.972449070 \\ \text { H } & -5.322427384 & -1.525340111 & -2.075021148 \\ \text { H } & -2.867316205 & -1.623962119 & -1.636963120 \\ \text { H } & -5.587458401 & 2.132538153 & 0.137929007 \\ \text { H } & -6.660985479 & 0.313499022 & -1.174399084 \\ \text { C } & -1.427624103 & 0.350344026 & -0.105126008 \\ \text { O } & -1.139924081 & 0.398453027 & 1.209482086 \\ \text { O } & -2.976723215 & 2.102927151 & 0.757289056 \\ \text { H } & -1.750742123 & 1.015437075 & 1.609509118 \\ \text { O } & -0.537466038 & -0.193363013 & -0.928562067 \\ \text { C } & 0.874271064 & -0.193599015 & -0.708869052 \\ \text { C } & 1.286153090 & -1.210078087 & 0.353447026 \\ \text { C } & 1.467900107 & 1.184395083 & -0.438784033\end{array}$




$\begin{array}{lrrr}\mathrm{C} & 2.818218203 & -1.355330098 & 0.470019032 \\ \mathrm{H} & 0.886866064 & -0.897007064 & 1.309581095 \\ \mathrm{C} & 3.000958215 & 1.081622078 & -0.409099031 \\ \mathrm{H} & 1.148810083 & 1.875665134 & -1.213846089 \\ \mathrm{C} & 3.446614250 & 0.045796005 & 0.634509044 \\ \mathrm{H} & 3.184553230 & 0.427410029 & 1.620549116 \\ \mathrm{C} & 3.650519261 & 2.441343178 & -0.146204011 \\ \mathrm{H} & 3.357687241 & 2.836350202 & 0.822865061 \\ \mathrm{H} & 4.733788340 & 2.363164171 & -0.158902011 \\ \mathrm{H} & 3.362109241 & 3.166484229 & -0.901518067 \\ \mathrm{H} & 1.108283081 & 1.568349114 & 0.510206038 \\ \mathrm{H} & 0.835159060 & -2.170924158 & 0.120081007 \\ \mathrm{H} & 4.530830328 & -0.042510004 & 0.619644042 \\ \mathrm{H} & 3.331944239 & 0.750450054 & -1.391676098 \\ \mathrm{C} & 3.134399228 & -2.195812157 & 1.715736122 \\ \mathrm{H} & 2.687530193 & -3.183444228 & 1.642468119 \\ \mathrm{H} & 4.206006304 & -2.325611166 & 1.839296131 \\ \mathrm{H} & 2.750238199 & -1.724811126 & 2.615514186 \\ \mathrm{C} & 3.403555245 & -2.082988149 & -0.753219053 \\ \mathrm{H} & 4.475683322 & -2.215494159 & -0.640035046 \\ \mathrm{H} & 2.959178211 & -3.068704222 & -0.858459062 \\ \mathrm{H} & 3.240310234 & -1.552149109 & -1.683761122 \\ \mathrm{H} & 1.232110091 & -0.527018036 & -1.673004123\end{array}$

Coord_3

$\begin{array}{lrrr}\text { C } & -4.858025352 & -0.721625049 & -1.526249111 \\ \text { C } & -3.460259249 & -0.792288056 & -1.295445094 \\ \text { C } & -2.857455204 & 0.152809011 & -0.493413037 \\ \text { C } & -3.635295261 & 1.176450085 & 0.103923009 \\ \text { C } & -5.011650359 & 1.267546091 & -0.150349009 \\ \text { C } & -5.608043401 & 0.282977021 & -0.952913068 \\ \text { H } & -5.328470382 & -1.441434106 & -2.170947156 \\ \text { H } & -2.872583207 & -1.559074113 & -1.765030126 \\ \text { H } & -5.585718402 & 2.066582147 & 0.279102020 \\ \text { H } & -6.663340477 & 0.339466025 & -1.143469081 \\ \text { C } & -1.429474102 & 0.322465021 & -0.127705007 \\ \text { O } & -1.132310082 & 0.435862032 & 1.174286083 \\ \text { O } & -2.983312212 & 2.019234145 & 0.868452061 \\ \text { H } & -1.784512131 & 1.018781073 & 1.566018115 \\ \text { O } & -0.532832038 & -0.212194018 & -0.943809066 \\ \text { C } & 0.880341065 & -0.210073017 & -0.717820053 \\ \text { C } & 1.287647095 & -1.212384088 & 0.359167024 \\ \text { C } & 1.470499108 & 1.172625086 & -0.465320033 \\ \text { C } & 2.819745202 & -1.351971098 & 0.485564037 \\ \text { H } & 0.882644066 & -0.888702066 & 1.309426094 \\ \text { C } & 3.003478216 & 1.073927078 & -0.422654033 \\ \text { H } & 1.155778083 & 1.850888135 & -1.253539092\end{array}$




$\begin{array}{llll}\mathrm{C} & 3.443315247 & 0.052667006 & 0.637583045 \\ \mathrm{H} & 3.172467229 & 0.445897032 & 1.616623118 \\ \mathrm{C} & 3.645713263 & 2.439237174 & -0.170735012 \\ \mathrm{H} & 3.343274241 & 2.844354204 & 0.791153056 \\ \mathrm{H} & 4.729310341 & 2.365414170 & -0.173642011 \\ \mathrm{H} & 3.360576241 & 3.154284227 & -0.936808067 \\ \mathrm{H} & 1.103901080 & 1.571028111 & 0.474635035 \\ \mathrm{H} & 0.840737059 & -2.177194157 & 0.134634011 \\ \mathrm{H} & 4.527768328 & -0.033132005 & 0.632391044 \\ \mathrm{H} & 3.343780240 & 0.731816051 & -1.398223100 \\ \mathrm{C} & 3.131413224 & -2.177570158 & 1.742279124 \\ \mathrm{H} & 2.688947192 & -3.167728229 & 1.677255120 \\ \mathrm{H} & 4.202764304 & -2.301707166 & 1.873342136 \\ \mathrm{H} & 2.740368197 & -1.698249122 & 2.634649187 \\ \mathrm{C} & 3.413787247 & -2.091735153 & -0.726229050 \\ \mathrm{H} & 4.485657323 & -2.219458157 & -0.605634043 \\ \mathrm{H} & 2.973256215 & -3.080039220 & -0.822669059 \\ \mathrm{H} & 3.254325234 & -1.572196111 & -1.663773118 \\ \mathrm{H} & 1.240152088 & -0.556600040 & -1.676336119\end{array}$

Coord_4

$\begin{array}{lrrr}\text { C } & -4.851568347 & -0.672907050 & -1.582272111 \\ \text { C } & -3.459897249 & -0.749599052 & -1.366483096 \\ \text { C } & -2.849254205 & 0.149593011 & -0.503702037 \\ \text { C } & -3.627945258 & 1.133228083 & 0.169024013 \\ \text { C } & -5.013979359 & 1.223818090 & -0.076032007 \\ \text { C } & -5.606426405 & 0.296623020 & -0.937483069 \\ \text { H } & -5.325505386 & -1.353484095 & -2.265786165 \\ \text { H } & -2.870283207 & -1.483091108 & -1.883873134 \\ \text { H } & -5.588851402 & 1.989249141 & 0.409871031 \\ \text { H } & -6.662959480 & 0.359101025 & -1.120061079 \\ \text { C } & -1.431550101 & 0.298700020 & -0.144938013 \\ \text { O } & -1.123599079 & 0.475785032 & 1.140782083 \\ \text { O } & -2.997076213 & 1.931322140 & 0.978899069 \\ \text { H } & -1.811881130 & 1.022677072 & 1.529198110 \\ \text { O } & -0.530198037 & -0.227724015 & -0.954971070 \\ \text { C } & 0.884671063 & -0.224742017 & -0.724462053 \\ \text { C } & 1.287649090 & -1.213363087 & 0.366268027 \\ \text { C } & 1.473232107 & 1.161711086 & -0.490099033 \\ \text { C } & 2.819748203 & -1.348809095 & 0.500401035 \\ \text { H } & 0.878511065 & -0.878907064 & 1.311090092 \\ \text { C } & 3.005995215 & 1.065671077 & -0.436515032 \\ \text { H } & 1.162671082 & 1.827261132 & -1.290701094 \\ \text { C } & 3.440209247 & 0.058506006 & 0.639350047 \\ \text { H } & 3.162277228 & 0.463330036 & 1.611620118 \\ \text { C } & 3.642686263 & 2.435714176 & -0.196264015 \\ \text { H } & 3.332341238 & 2.850945207 & 0.758737054\end{array}$




$\begin{array}{llll}\mathrm{H} & 4.726450339 & 2.365260168 & -0.190873011 \\ \mathrm{H} & 3.360643241 & 3.140995227 & -0.972441069 \\ \mathrm{H} & 1.101323077 & 1.574602116 & 0.441107031 \\ \mathrm{H} & 0.843485061 & -2.181512158 & 0.151023012 \\ \mathrm{H} & 4.524734327 & -0.025776002 & 0.642377047 \\ \mathrm{H} & 3.353962240 & 0.712577050 & -1.405391102 \\ \mathrm{C} & 3.127603227 & -2.159988154 & 1.767363126 \\ \mathrm{H} & 2.688391196 & -3.152095228 & 1.711025122 \\ \mathrm{H} & 4.198688301 & -2.279529162 & 1.904432136 \\ \mathrm{H} & 2.731358198 & -1.672209119 & 2.652809190 \\ \mathrm{C} & 3.420114244 & -2.100923152 & -0.700695052 \\ \mathrm{H} & 4.491707322 & -2.224926161 & -0.574171042 \\ \mathrm{H} & 2.982235215 & -3.091225223 & -0.787903055 \\ \mathrm{H} & 3.263809233 & -1.592404115 & -1.644785116 \\ \mathrm{H} & 1.244846091 & -0.584495041 & -1.677708122\end{array}$

Coord_5

$\begin{array}{lrrr}\text { C } & -4.838846349 & -0.621452045 & -1.638058120 \\ \text { C } & -3.454068251 & -0.700222052 & -1.431474101 \\ \text { C } & -2.840635204 & 0.150898009 & -0.508165039 \\ \text { C } & -3.624806258 & 1.087820077 & 0.233828017 \\ \text { C } & -5.019517362 & 1.174338086 & -0.008728001 \\ \text { C } & -5.602914403 & 0.306773021 & -0.927136067 \\ \text { H } & -5.312226381 & -1.261791093 & -2.359708168 \\ \text { H } & -2.859063204 & -1.396539099 & -1.991806142 \\ \text { H } & -5.598608404 & 1.901289135 & 0.528567039 \\ \text { H } & -6.660054477 & 0.372612026 & -1.105935079 \\ \text { C } & -1.433997101 & 0.279147021 & -0.155885013 \\ \text { O } & -1.113285081 & 0.518202039 & 1.109844079 \\ \text { O } & -3.019882218 & 1.839604134 & 1.088788080 \\ \text { H } & -1.832015134 & 1.027941073 & 1.500462108 \\ \text { O } & -0.529878038 & -0.239910015 & -0.961343068 \\ \text { C } & 0.887025066 & -0.237625017 & -0.728459051 \\ \text { C } & 1.286263090 & -1.213039088 & 0.375015026 \\ \text { C } & 1.475915105 & 1.151627084 & -0.512931036 \\ \text { C } & 2.818403204 & -1.345936096 & 0.514410039 \\ \text { H } & 0.874837061 & -0.867610061 & 1.314964093 \\ \text { C } & 3.008389219 & 1.056761077 & -0.450881030 \\ \text { H } & 1.169037084 & 1.804762130 & -1.325087095 \\ \text { C } & 3.437517248 & 0.063218007 & 0.639536048 \\ \text { H } & 3.154478229 & 0.479655032 & 1.605372117 \\ \text { C } & 3.641400262 & 2.430681177 & -0.223137017 \\ \text { H } & 3.325113238 & 2.856078205 & 0.725389050 \\ \text { H } & 4.725213340 & 2.362557168 & -0.211219014 \\ \text { H } & 3.362030241 & 3.126525224 & -1.008711073 \\ \text { H } & 1.100629077 & 1.579086115 & 0.409937030 \\ \text { H } & 0.843441060 & -2.183898155 & 0.169622010\end{array}$




$\begin{array}{lrrr}\mathrm{H} & 4.521988326 & -0.020589001 & 0.649053047 \\ \mathrm{H} & 3.362100240 & 0.692593052 & -1.413506104 \\ \mathrm{C} & 3.123523223 & -2.143154153 & 1.790817129 \\ \mathrm{H} & 2.686392192 & -3.136630224 & 1.743711125 \\ \mathrm{H} & 4.194404304 & -2.259209164 & 1.932127140 \\ \mathrm{H} & 2.724007195 & -1.646736119 & 2.669950194 \\ \mathrm{C} & 3.422381244 & -2.110697151 & -0.676919049 \\ \mathrm{H} & 4.493741321 & -2.232092158 & -0.546215042 \\ \mathrm{H} & 2.985931217 & -3.102406225 & -0.754359054 \\ \mathrm{H} & 3.268325237 & -1.612926116 & -1.627081116 \\ \mathrm{H} & 1.245678090 & -0.610745043 & -1.676881119\end{array}$

$\begin{array}{lrrr}\text { Coord_6 } & & \\ \text { C } & -4.818828346 & -0.567370040 & -1.693563119 \\ \text { C } & -3.441804245 & -0.644483045 & -1.489199105 \\ \text { C } & -2.831769205 & 0.156726013 & -0.505246039 \\ \text { C } & -3.627179258 & 1.040611074 & 0.298670021 \\ \text { C } & -5.029338363 & 1.119652078 & 0.050504005 \\ \text { C } & -5.597541402 & 0.313606022 & -0.923022068 \\ \text { H } & -5.286832382 & -1.166705085 & -2.452893175 \\ \text { H } & -2.837253206 & -1.300048094 & -2.086799152 \\ \text { H } & -5.616859404 & 1.803575132 & 0.633238045 \\ \text { H } & -6.654562479 & 0.380265025 & -1.103180081 \\ \text { C } & -1.436995102 & 0.263908018 & -0.159364009 \\ \text { O } & -1.100791079 & 0.563051038 & 1.082522076 \\ \text { O } & -3.053969218 & 1.744581124 & 1.198333088 \\ \text { H } & -1.843946133 & 1.035368075 & 1.481429105 \\ \text { O } & -0.532278037 & -0.248697019 & -0.962039069 \\ \text { C } & 0.887080064 & -0.248731019 & -0.729382052 \\ \text { C } & 1.283584093 & -1.211439089 & 0.385730029 \\ \text { C } & 1.478267107 & 1.142347083 & -0.533569039 \\ \text { C } & 2.815879203 & -1.343445095 & 0.527423037 \\ \text { H } & 0.872042063 & -0.854815063 & 1.321520096 \\ \text { C } & 3.010437219 & 1.047102073 & -0.465995036 \\ \text { H } & 1.174262085 & 1.783380130 & -1.356371096 \\ \mathrm{C} & 3.435441249 & 0.066693002 & 0.637791043 \\ \text { H } & 3.149602226 & 0.494786035 & 1.597653114 \\ \mathrm{C} & 3.641712260 & 2.424033176 & -0.251769020 \\ \text { H } & 3.321769240 & 2.859677203 & 0.690830050 \\ \text { H } & 4.725497339 & 2.357152168 & -0.235440018 \\ \text { H } & 3.364295241 & 3.110783222 & -1.045962077 \\ \text { H } & 1.101853080 & 1.584478114 & 0.381522027 \\ \text { H } & 0.840634060 & -2.184369155 & 0.190880012 \\ \text { H } & 4.519764326 & -0.017727999 & 0.651720047 \\ \text { H } & 3.367646240 & 0.671739050 & -1.422957104 \\ \mathrm{C} & 3.119807224 & -2.127180154 & 1.812393130 \\ \text { H } & 2.683569195 & -3.121437227 & 1.775188128\end{array}$




\begin{tabular}{|c|c|c|c|}
\hline $\mathrm{H}$ & 4.190618300 & -2.240907161 & 1.955839139 \\
\hline $\mathrm{H}$ & 2.719244198 & -1.621916118 & 2.685974195 \\
\hline $\mathrm{C}$ & 3.420352246 & -2.121191154 & -0.655288046 \\
\hline ト & 4.491589321 & -2.241134162 & -0.522497037 \\
\hline & 2.984087214 & -3.113704222 & -0.722297052 \\
\hline & 3.267290235 & -1.633885118 & -1.611014114 \\
\hline & 1.241988089 & -0.635366046 & -1.6735 \\
\hline \multicolumn{4}{|c|}{ Coord_7 } \\
\hline 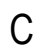 & -4.789937343 & -0.510829039 & -1.74 \\
\hline c & -3.421759244 & 55043 & \\
\hline C & -2.822862204 & 0.167094014 & 96303 \\
\hline C & -3.636637259 & 0.992063072 & 0.3 \\
\hline C & -5.044549362 & 1.060363075 & 0.1 \\
\hline C & -5.590028403 & 0.317301023 & -0.9 \\
\hline 11 & -5.246700379 & -1.068664077 & -2.5 \\
\hline $\mathrm{H}$ & -2.802661202 & -1.194331085 & -2.16 \\
\hline 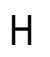 & -5.645671407 & 1121 & 0.7 \\
\hline $\mathrm{H}$ & -6.645977478 & 0.382320026 & -1.114392083 \\
\hline 0 & -1.440772105 & 122020 & -0.1 \\
\hline 0 & -1.085433080 & 0.610248045 & 1.06013207 \\
\hline 0 & -3.102177223 & & 1.30 \\
\hline$H$ & -1.846453132 & 1.045766075 & 043108 \\
\hline 0 & -0.537947039 & -0.253975017 & $-0 . \varsigma$ \\
\hline C & 0.884374063 & -0.258040019 & -0.7 \\
\hline C & 1.279674093 & -1.208584088 & 3814026 \\
\hline C & 1.479864106 & 1.133862080 & -0.5 \\
\hline C & 2.812307203 & -1.341446096 & 0.539199041 \\
\hline ⺊ & 0.870608062 & 39062 & 1.3 \\
\hline C & 3.011773217 & 1.036598074 & 160036 \\
\hline 卜 & 1.177511085 & 1.763136127 & -1.384101100 \\
\hline C & 3.434146246 & 0.068792005 & $\$ 45045$ \\
\hline $\mathrm{H}$ & 3.148239224 & 0.508594037 & 1.588142116 \\
\hline C & 3.643346264 & 2.415648172 & 38021 \\
\hline $\mathrm{H}$ & 3.322445237 & 2.861635207 & 0.65470304 \\
\hline $\mathrm{H}$ & 4.727059338 & 2.348861167 & 517022 \\
\hline $\mathrm{H}$ & 3.366785242 & 3.093679224 & -1.084607077 \\
\hline 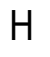 & 1.104980078 & 1.590774117 & 37902 \\
\hline $\mathrm{H}$ & 0.835052060 & -2.182927157 & 0.215361017 \\
\hline 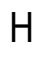 & 4.518252324 & -0.017393003 & 0.649453046 \\
\hline $\mathrm{H}$ & 3.369812242 & 0.649906049 & -1.434234104 \\
\hline 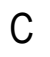 & 3.117175223 & -2.112218154 & 1.831724134 \\
\hline $\mathrm{H}$ & 2.680633193 & -3.106655222 & 1.805246131 \\
\hline $\mathrm{H}$ & 4.188122303 & -2.224838159 & 1.974754140 \\
\hline ト & 2.718177197 & -1.597892117 & 2.7007011 \\
\hline 0 & 3.413634246 & -2.132526152 & -0.63632004 \\
\hline & 4.484921323 & -2.252238163 & -0.50397803 \\
\hline
\end{tabular}




\begin{tabular}{|c|c|c|c|}
\hline & 216 & 226 & -0 \\
\hline $\mathrm{H}$ & 3.259870234 & -1.655378118 & \\
\hline & 81087 & -0.658325047 & -1.667272 \\
\hline \multicolumn{4}{|c|}{ Coord_8 } \\
\hline 0 & -4.749647343 & -0.452110033 & -1.803275131 \\
\hline$C$ & 5245 & 31039 & \\
\hline$C$ & -2.814165203 & 0.182130014 & -0.4686090 \\
\hline U & -3.655216265 & 0.942775069 & 0.430421032 \\
\hline C & & & \\
\hline C & -5.579550402 & 7948022 & -0.9395040 \\
\hline $\mathrm{H}$ & -5.187766374 & 300072 & -2.63767918 \\
\hline H & -2.752165198 & -1.080130078 & -2.226639161 \\
\hline H & -5.687475409 & 812116 & 0.790075058 \\
\hline $\mathrm{H}$ & -6.633014477 & 0. & -1.1 \\
\hline C & -1.445636106 & 0.247067015 & -0.137084012 \\
\hline 0 & -1.066316076 & 0.659727049 & 1.044451075 \\
\hline 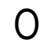 & -3.168395226 & 448110 & 1.4 \\
\hline $\mathrm{H}$ & -1.837 & 1.0 & 17106 \\
\hline 0 & -0.547680037 & -0.255506017 & 239069 \\
\hline C & 0.878203062 & -0.265453020 & -0.719519051 \\
\hline C & 1.274 & -1.2 & \\
\hline 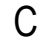 & & & 043 \\
\hline C & 2.807 & -1 . & 0.5 \\
\hline $\mathrm{H}$ & 0.871114061 & 784058 & 1.345218098 \\
\hline C & 3.01179 & 1.02 & -0.4 \\
\hline $\mathrm{H}$ & & & \\
\hline C & 3.433 & 0.06 & 0.62 \\
\hline $\mathrm{H}$ & 3.151083229 & 0.52087 & 1.57 \\
\hline C & 3.645836265 & 2.405366173 & -0.316620025 \\
\hline $\mathrm{H}$ & 3.327 & & \\
\hline $\mathrm{H}$ & $4.72 \varsigma$ & & -0.2 \\
\hline $\mathrm{H}$ & 3.368540243 & 3.07 & -1.125190082 \\
\hline $\mathrm{H}$ & 1.109941078 & 1.598001117 & 0.335227024 \\
\hline $\mathrm{H}$ & 0.826 & & 0.2 \\
\hline & 4.517 & & \\
\hline H & 3.367409243 & 0.626993044 & -1.447991104 \\
\hline C & 3.116453224 & -2.098495149 & 1.848237131 \\
\hline $\mathrm{H}$ & 2.678386195 & -3.092475224 & 1.833522130 \\
\hline 11 & 4.18 & & 145 \\
\hline $\mathrm{H}$ & 2.722179195 & 35111 & 2.71 \\
\hline & 3.401519246 & -2.144833153 & -0.620743043 \\
\hline $\mathrm{H}$ & 4.473095322 & -2.265632164 & -0.492019036 \\
\hline H & $2.9617 \mathrm{c}$ & -3.137 & \\
\hline $\mathrm{H}$ & 35 & -1.677469119 & -1.585776112 \\
\hline & & -0.6794 & -1.65 \\
\hline
\end{tabular}




$\begin{array}{lrrr}\text { Coord_9 } & & & \\ \text { C } & -4.693561338 & -0.391742027 & -1.856919136 \\ \text { C } & -3.348923240 & -0.442846034 & -1.599733114 \\ \text { C } & -2.806020202 & 0.202313014 & -0.428048032 \\ \text { C } & -3.685878266 & 0.893632066 & 0.498876036 \\ \text { C } & -5.096052369 & 0.930388067 & 0.164344012 \\ \text { C } & -5.564212401 & 0.315325023 & -0.964865071 \\ \text { H } & -5.103112366 & -0.866674063 & -2.729413196 \\ \text { H } & -2.680772192 & -0.958079071 & -2.262331161 \\ \text { H } & -5.745461414 & 1.461623107 & 0.834148061 \\ \text { H } & -6.612867477 & 0.369436026 & -1.194277088 \\ \text { C } & -1.452041104 & 0.246382017 & -0.105761005 \\ \text { O } & -1.042102073 & 0.711568052 & 1.038156072 \\ \text { O } & -3.258582232 & 1.447647107 & 1.528391109 \\ \text { H } & -1.815463131 & 1.079413080 & 1.505416106 \\ \text { O } & -0.562759038 & -0.252724021 & -0.915510064 \\ \text { C } & 0.867385060 & -0.270688022 & -0.706684053 \\ \text { C } & 1.267924093 & -1.198982087 & 0.434519033 \\ \text { C } & 1.477882104 & 1.119538083 & -0.578085043 \\ \text { C } & 2.801964201 & -1.339511096 & 0.557359038 \\ \text { H } & 0.874298062 & -0.807477057 & 1.364300097 \\ \text { C } & 3.009503216 & 1.012616073 & -0.519529035 \\ \text { H } & 1.172770086 & 1.726662124 & -1.425803104 \\ \text { C } & 3.434181246 & 0.067986005 & 0.615015043 \\ \text { H } & 3.159059227 & 0.531281041 & 1.561464111 \\ \mathrm{C} & 3.648433261 & 2.392933170 & -0.354684025 \\ \text { H } & 3.336387242 & 2.859841206 & 0.575420043 \\ \text { H } & 4.731905342 & 2.322392165 & -0.343433024 \\ \text { H } & 3.368105243 & 3.054961219 & -1.168522084 \\ \text { H } & 1.116626078 & 1.606287117 & 0.319169024 \\ \text { H } & 0.814778057 & -2.173932155 & 0.277469021 \\ \text { H } & 4.517640325 & -0.025666001 & 0.624089046 \\ \text { H } & 3.358488240 & 0.602900043 & -1.465206108 \\ \mathrm{C} & 3.118616226 & -2.086383150 & 1.860953134 \\ \text { H } & 2.677728195 & -3.079192219 & 1.859368131 \\ \text { H } & 4.190740302 & -2.200963158 & 1.992619143 \\ \text { H } & 2.733110198 & -1.553315114 & 2.724669196 \\ \mathrm{C} & 3.382630243 & -2.158279155 & -0.609701045 \\ \text { H } & 4.454783318 & -2.281690164 & -0.488748033 \\ \text { H } & 2.939131209 & -3.149294229 & -0.639484045 \\ \text { H } & 3.219741231 & -1.700202124 & -1.578159113 \\ \text { H } & 1.192362085 & -0.698526050 & -1.643082118 \\ & & & \end{array}$

Coord_10

$\begin{array}{llll}\text { C } & -4.611562334 & -0.333859022 & -1.886868134 \\ \text { C } & -3.282183236 & -0.356643023 & -1.592134113 \\ \text { C } & -2.790555203 & 0.234685019 & -0.353389028\end{array}$




$\begin{array}{lrrr}\text { C } & -3.723649266 & 0.836482061 & 0.592918040 \\ \text { C } & -5.128749368 & 0.839068060 & 0.204941014 \\ \text { C } & -5.537092400 & 0.290274020 & -0.973681069 \\ \text { H } & -4.980988359 & -0.766878055 & -2.798165201 \\ \text { H } & -2.575890187 & -0.808617058 & -2.260952162 \\ \text { H } & -5.816977417 & 1.301218095 & 0.887116063 \\ \text { H } & -6.579261472 & 0.325136022 & -1.234926087 \\ \text { C } & -1.450121106 & 0.269661021 & -0.054218003 \\ \text { O } & -0.995568072 & 0.780885054 & 1.044869077 \\ \text { O } & -3.369633242 & 1.337184097 & 1.663357121 \\ \text { H } & -1.758676126 & 1.109712081 & 1.561620112 \\ \text { O } & -0.576987043 & -0.213360018 & -0.884903065 \\ \text { C } & 0.859982061 & -0.228992014 & -0.708958049 \\ \text { C } & 1.288269090 & -1.150774082 & 0.426993032 \\ \text { C } & 1.468879106 & 1.163779084 & -0.603727041 \\ \text { C } & 2.825561204 & -1.284398090 & 0.515862038 \\ \text { H } & 0.914149067 & -0.756897052 & 1.363881100 \\ \text { C } & 3.001741218 & 1.063296077 & -0.575537039 \\ \text { H } & 1.144252084 & 1.763118129 & -1.449730102 \\ \text { C } & 3.452777249 & 0.125919007 & 0.554801038 \\ \text { H } & 3.194472229 & 0.592350043 & 1.504409109 \\ \text { C } & 3.636087264 & 2.447786175 & -0.428619030 \\ \text { H } & 3.339625241 & 2.916377212 & 0.505695035 \\ \text { H } & 4.719917339 & 2.383543174 & -0.438433029 \\ \text { H } & 3.335932240 & 3.105132223 & -1.239145090 \\ \text { H } & 1.124887080 & 1.656453120 & 0.296690019 \\ \text { H } & 0.836079061 & -2.128236154 & 0.283650023 \\ \text { H } & 4.536533329 & 0.036725002 & 0.542798039 \\ \text { H } & 3.334353239 & 0.651227044 & -1.526024110 \\ \text { C } & 3.174392227 & -2.025724144 & 1.814356129 \\ \text { H } & 2.739113195 & -3.020915220 & 1.825318131 \\ \text { H } & 4.249745306 & -2.134081155 & 1.922580137 \\ \text { H } & 2.805284201 & -1.492141108 & 2.684871193 \\ \text { C } & 3.383601241 & -2.104576152 & -0.661274046 \\ \text { H } & 4.458723323 & -2.222561163 & -0.563815038 \\ \text { H } & 2.944370212 & -3.097761223 & -0.677889047 \\ \text { H } & 3.197335231 & -1.650727121 & -1.627500118 \\ \text { H } & 1.161593085 & -0.661679046 & -1.650685120 \\ & & & \end{array}$

